\title{
Reservoir Characterization of Pennsylvanian Sandstone Reservoirs
}

\author{
ANNUAL REPORT \\ - Submitted by \\ Mohan Kelkar \\ Department of Petroleum Engineering \\ University of Tulsa \\ Tulsa, OK 74104
}

September 1992

Work Performed Under Contract No. DE-AC22-90BC14651

\author{
Prepared for \\ U.S. Department of Energy \\ Assistant Secretary for Fossil Energy \\ Contracting Officer's Representative
}

Ms. Rhonda L. Patterson

U.S. Department of Energy

Bartlesville Project Office

P.O. Box 1398

Bartlesville, OK 74005

\section{DISCLAIMER}

\begin{abstract}
This report was prepared as an account of work sponsored by an agency of the United States Government. Neither the United States Government nor any agency thereof, nor any of their employees, makes any warranty, express or implied, or assumes any legal liability or responsibility for the accuracy, completeness, or usefulness of any information, apparatus, product, or process disclosed, or represents that its use would not infringe privately owned rights. Reference herein to any specific commercial product, process, or service by trade name, trademark, manufacturer, or otherwise does not necessarily constitute or imply its endorsement, recommendation, or favoring by the United States Government or any agency thereof. The views and opinions of authors expressed herein do not necessarily state or reflect those of the United States Government or any agency thereof.
\end{abstract}

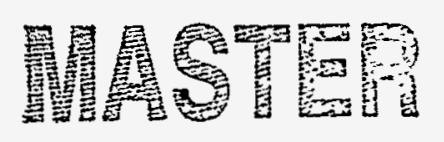




\section{DISCLAIMER}

Portions of this document may be illegible in electronic image products. Images are produced from the best available original document. 


\section{TABLE OF CONTENTS}

Page

TABLE OF CONTENTS $\ldots \ldots \ldots \ldots \ldots \ldots \ldots$ ii LIST OF FIGURES . . . . . . . . . . . . . . . . . iii LIST OF TABLES $\ldots \ldots \ldots \ldots \ldots \ldots \ldots \ldots \ldots \ldots$ xiii ACKNOWLEDGMENT ................... . xvi

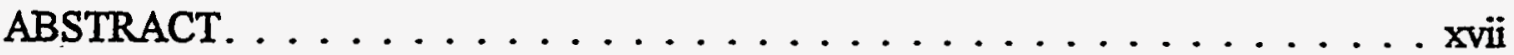
EXECUTIVE SUMMARY . . . . . . . . . . . . xviii

\section{SECTIONI}

Report I : An Approach to Generating Reservoir Property Description

Using a Stochastic Condition Simulation Method . . . . . . . . 1

Report II : Reservoir Characterization for Waterfloods Incorporating

Well Test Data in Stochastic Conditional Simulations . . . . . . . . 121

Report III : Effective Properties of Reservoir Simulator Grid Blocks

Part 1 : Analytical Upscaling of Small Scale Permeability

Using a Full Tensor . . . . . . . . . . . . . . . . . . . 145

Part 2 : Incorporating of Full Tensor Permeability in a Numerical

Simulator . . . . . . . . . . . . . . 177

Report IV : Result of Closed Chamber Tests

Part 1 : Oil Wells . . . . . . . . . . . . . . . . .225

Part 2: Gas Wells . . . . . . . . . . . . . . . . . . . .

\section{SECTION II}

Outcrop Studies Geologic and Engineering Report. . . . . . . . . . . . . . . 345

\section{SECTION III}

Application of Geostatistical Techniques in the Evaluation of

Infill Drilling Propect . . . . . . . . . . . . . . . . . .373 


\section{LIST OF FIGURES}

Figure $\quad$ Page

\section{SECTION I}

\section{Report 1}

1 Interpretation of standard integer dimension (after Peitgen) . . . . . . . . 61

2 Flowchart for the simulated annealing algorithm (after Perez) . . . . . . . . 62

3 Procedure for removing the local means . . . . . . . . . . . . . . 63

4 Example porosity trace before seasonal adjustments . . . . . . . . . . . 64

5 Example porosity trace after seasonal adjustments . . . . . . . . . . . . . 65

6 R/S analysis of porosity trace showing periodic trend $\ldots \ldots \ldots 6$

$7 \quad \mathrm{R} / \mathrm{S}$ analysis of porosity after seasonal adjustment . . . . . . . . . . 67

8 H exponent analysis using box-counting before seasonal adjustment . . . . . . 68

9 H exponent analysis using box-counting after seasonal adjustment . . . . . . . 69

10 Theoretical and analysis variogram matches for original porosity trace in Burbank Well 41 . . . . . . . . . . . . . . . . . . 70

11 Theoretical and analysis variogram matches for adjusted porosity trace

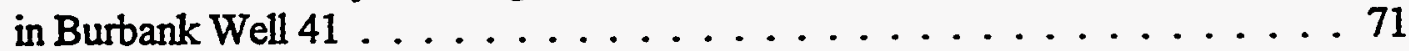

12 Theoretical and analysis variogram matches for original porosity trace in Hugoton Well R2 . . . . . . . . . . . . . . . . . 72

13 Theoretical and analysis variogram matches for adjusted porosity trace in Hugoton Well R2 . . . . . . . . . . . . . . . . . 73

14 Well location map of Hugoton Field . . . . . . . . . . . . . . 74

15 Example Hugoton porosity trace before seasonal adjustments . . . . . . . 75

16 Example Hugoton porosity trace after seasonal adjustments . . . . . . . . . 76

17 Distribution of $H$ values using box-counting for Hugoton Field before seasonal adjustment .................... 77

18 Distribution of $H$ values using box-counting for Hugoton Field after seasonal adjustment . . . . . . . . . . . . . . . . . 78

19 Well location map of Burbank Field. . . . . . . . . . . . . . . . . 79

20 Example Burbank porosity trace before seasonal adjustments . . . . . . . . . . . . . .

21 Example Burbank porosity trace after seasonal adjustments . . . . . . . . 81

22 Well location map of Sable Field ... . . . . . . . . . . . . . . . 82

23 Example Sable porosity trace before seasonal adjustments . . . . . . . . . 83

24 Example Sable porosity trace after seasonal adjustments . . . . . . . . . . . 84

25 Well location map of Loudon Field . . . . . . . . . . . . . . . . . . . . . . . .

26 Well location map for 2D Hugoton Field simulation . . . . . . . . . . 86

27 Gray scale plot of linearly interpolated values for Hugoton well pair A2 and E2 before adding Gaussian random element . . . . . . . . . . . . 87

28 Gray scale plot of linearly interpolated values for Hugoton well pair A2 and E2 after adding Gaussian random element . . . . . . . . . . . 88 


\section{LIST OF FIGURES (Continued)}

Figure

Page

29 Distribution of conditioning data for Hugoton well pair A2 and E2 . . . . . 89

30 Distribution of initial pattern for Hugoton well pair A2 and E2 . . . . . 90

31 Comparison plot for Hugoton well B2 using (i) $\mathrm{fBm}$ horizontal structure, and (ii) greedy algorithm . . . . . . . . . . . . . . . . . . 91

32 Comparison plot for Hugoton well B2 using (i) $\mathrm{fBm}$ horizontal structure, and (ii) conventional annealing . . . . . . . . . . . . . . 92

33 Comparison plot for Hugoton well B2 using (i) $\mathrm{EBm}$ horizontal structure, and (ii) conventional annealing with initial pattern . . . . . . . . . 93

34 Comparison plot for Hugoton well B2 using (i) fGn horizontal structure, and (ii) greedy algorithm . . . . . . . . . . . . . . . . 94

35 Comparison plot for Hugoton well B2 using (i) fGn horizontal structure, and (ii) conventional annealing . . . . . . . . . . . . . 95

36 Comparison plot for Hugoton well B2 using (i) fGn horizontal structure, and (ii) conventional annealing with initial pattern . . . . . . . 96

37 Field map of Burbank simulation region . . . . . . . . . . . . . . . 97

38 Comparison plot for Burbank well 35 using an fGn horizontal correlation structure . . . . . . . . . . . . . . . . . . . 98

39 Comparison plot for Burbank well 35 using an $\mathrm{fBm}$ horizontal correlation structure . . . . . . . . . . . . . . . . . . 99

40 Comparison plot for Burbank well 26 using an fGn horizontal correlation structure . . . . . . . . . . . . . . . . . . . 100

41 Comparison plot for Burbank well 26 using an $\mathrm{fBm}$ horizontal correlation structure . . . . . . . . . . . . . . . . . . 101

42 Well location map for $2 \mathrm{D}$ Loudon Field simulation . . . . . . . . . . . . . 102

43 Comparison plot for Loudon well 435 using an $\mathrm{fGn}$ horizontal correlation structure . . . . . . . . . . . . . . . . . . . . . .103

44 Comparison plot for Loudon well 435 using an $\mathrm{fBm}$ horizontal correlation structure. . . . . . . . . . . . . . . . . . . . 104

45 Well location map for 3D Burbank Field simulation . . . . . . . . 105

46 Fieldwide horizontal porosity variogram for Burbank Field . . . . . . . . . 106

47 Comparison plot for Burbank well 27 using an fGn horizontal correlation structure (4 conditioning wells) . . . . . . . . . . . . . 107

48 Comparison plot for Burbank well 27 using an $\mathrm{ABm}$ horizontal correlation structure (4 conditioning wells) . . . . . . . . . . . . 108

49 Comparison plot for Burbank well 27 using interpolated values (4 conditioning wells) . . . . . . . . . . . . . . . . . 109

50 Well location map for 3D Burbank Field simulation (8 conditioning wells) . . .110

51 Well location map for 3D Loudon Field simulation. . . . . . . . . . . . 111

52 Comparison plot for Loudon well 501 using an $\mathrm{fBm}$ horizontal correlation structure. . . . . . . . . . . . . . . . . . .112 


\section{LIST OF FIGURES (Continued)}

Figure

Page

53 Well location map for 3D Hugoton Field simulation (small scale) . . . . . . .113

54 Fieldwide horizontal porosity variogram for Hugoton Field . . . . . . . . . . 114

55 Comparison plot for Hugoton well $\mathrm{I} 2$ using (i) greedy algorithm, and (ii) $\mathrm{fGn}$ horizontal structure. . . . . . . . . . . . . . . . 115

56 Comparison plot for Hugoton well M2 using (i) greedy algorithm, and (ii) fGn horizontal structure. . . . . . . . . . . . . . . . . 116

57 Comparison plot for Hugoton well 12 using (i) linear interpolation, and (ii) $\mathrm{fGn}$ horizontal structure. . . . . . . . . . . . . . . . . . 117

58 Well location map for 3D Hugoton Field simulation (large scale) . . . . . . . 118

59 Comparison plot for Hugoton well C2 using (i) greedy algorithm, and (ii) fin horizontal structure. . . . . . . . . . . . . . . . . . . . 119

60 Comparison plot for Hugoton well K2 using (i) greedy algorithm, and (ii) $\mathrm{fG}$ horizontal structure. . . . . . . . . . . . . . . . . . 120

\section{Report $\mathbf{I}$}

1 Weighting function for the calculation of the pressure derivative in a reservoir

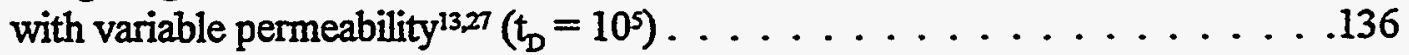

2 Permeability field generated by $\operatorname{TBM}^{23,24}$ (seed $=-6$, scale $=500 \mathrm{ft}$, mean $\left.=20, \sigma_{\text {lnk }}^{2}=0.5, \Delta X=\Delta Y=35 \mathrm{ft}, \mathrm{NX}=\mathrm{NY}=115\right) \ldots \ldots . . . . .137$

3 Comparison between analytical 7 and numerical ${ }^{25}$ drawdown solutions shows pressure differential and pressure derivative for a homogeneous reservoir . . . 138

4 Pressure differential and pressure derivative for a drawdown simulation of

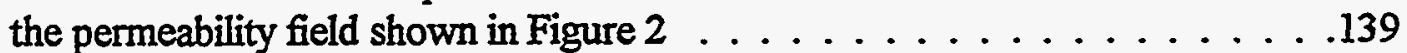

5 Permeability Distribution ( $\left.\mathrm{HI}=0.06, \mathrm{a}=1000 \mathrm{ft}, \sigma_{\mathrm{lnk}}^{2}=.5\right) \ldots \ldots . . . .140$

6 Permeability Distribution ( $\left.\mathrm{HI}=0.45, \mathrm{a}=695 \mathrm{ft}, \sigma_{\mathrm{lnk}}^{2}=1.6\right) \ldots \ldots . . . .140$

7 Pressure and pressure derivative response of the permeability field shown in Figure 5 . . . . . . . . . . . . . . . . . . . . 141

8 Pressure and pressure derivative response of the permeability field shown in Figure 6 . . . . . . . . . . . . . . . . . . . . . . 142

9 Comparison between the weighted harmonic average permeability and the instantaneous well test permeability. . . . . . . . . . . . . . . . . 143

10 Comparison between the weighted geometric average permeability and the instantaneous well test permeability . . . . . . . . . . . . . . . . . . 143

11 Comparison between the weighted arithmetic average permeability and the instantaneous well test permeability . . . . . . . . . . . . . . . . . 144 
LIST OF FIGURES (Continued)

Figure

Page

\section{Report III}

\section{Part 1}

1 Top : Cross-bedded permeability distribution $\left(-45^{\circ}\right.$ structure orientation, dark grid block : $20 \mathrm{md}$, light grid block $=1000 \mathrm{md}$ )

Bottom : Pressure map indicating non-zero transverse pressure gradient.

Boundary conditions : closed horizontal boundaries;

left face : injection; right face : production . . . . . . . . . 148

2 Top : Permeability field with low permeability section (dark grid block :

$1 \mathrm{md}$, light grid block $=1000 \mathrm{md}$ )

Bottom : Pressure map indicating non-zero transverse pressure gradient.

Boundary conditions : closed horizontal boundaries;

left face : injection; right face : production . . . . . . . . . . 149

3 Field of small scale heterogeneities divided into four quadrants . . . . . . . 150

4 Directions and data points to consider in calculating maximum and minimum permeabilities along $45^{\circ}$ and $-45^{\circ}$ from the horizontal axis. . . . . . 152

5 System containing four blocks and four permeability tensors . . . . . . . . 153

6 Schematic for no cross-flow case; $\hat{k}_{x a p p t b}$ (injection along $\mathrm{x}$-axis) $\ldots \ldots .156$

7 Schematic for cross-flow case; $\hat{k}_{x a p p f s}$ (injection along $x$-axis) $\ldots \ldots \ldots . .157$

8 Schematic for cross-flow case; $\hat{k}_{\text {yaprb }}$ (injection along $\mathrm{y}$-axis) $\ldots \ldots \ldots 158$

9 Schematic for cross-flow case; $\hat{k}_{\text {yappfs }}$ (injection along $\mathrm{x}$-axis) . . . . . . . . 159

10 Permeability contrast yielding a "Global" non-zero transverse pressure gradient . . . . . . . . . . . . . . . 163

11 Non-zero off-diagonal elements in permeability tensor of local grid block yielding "Local" transverse pressure gradient . . . . . . . . . . . . . 164

12 Effective permeability tensors calculated by analytical and numerical methods for anisotropic and full local permeability tensors (units : $m d$ ) . . . . . . 168

13 Effective permeability tensors calculated by analytical and numerical methods for cross-bedded permeability distribution (units : md) . . . . . . . . . . . 169

14 Effective permeability tensors calculated by analytical and numerical methods for cross-bedded stochastic permeability distribution (units $: \mathrm{md}$ ) $\ldots \ldots .171$

15 Effective permeability tensors calculated by analytical and numerical methods for minipermeameter measured permeability data from

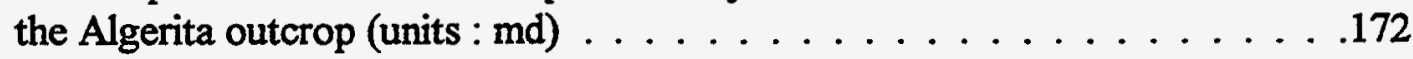


LIST OF FIGURES (Continued)

Figure

Page

16 Initial permeability distribution (dark : $20 \mathrm{md}$; light : $1000 \mathrm{md}$ ) and 0.5 concentration contours after 49.7 days of injection using the initial permeability distribution and the permeability distributions obtained using various upscaling methods . . . . . . . . . . . . . . 173

17 Initial permeability distribution $(V=0.7)$ and 0.5 concentration contours after 94.8 days of injection using the initial permeability distribution and the permeability distributions obtained using various upscaling methods . . . . . . .175

\section{Part 2}

18 Linear trial function in (a) $\mathrm{x}$-direction and (b) $\mathrm{y}$-direction . . . . . . . . 179

19 Quadrilateral element with labeled transmiscibilities . . . . . . . . . . . . . . . . . . . . . . . . . . . .

20 Element mesh superimposed on point-centered grid . . . . . . . . . . . . . . . . . . . . . . . . . . . .

21 Relative permeability curve ${ }^{3}$. . . . . . . . . . . . . . . . . . . . . . . . . . . . . . . . . . . . .

22 Cross-bedded permeability distribution . . . . . . . . . . . . . . . 191

23 Pressure maps after 0.5 days of water injection using (a) the initial cross-bedded permeability distribution (b) the effective permeability tensor and (c) the real-space renormalized permeability (units : psi) . . . . . . 192

24 Water saturation maps after 0.5 days of water injection using (a) the initial cross-bedded permeability distribution (b) the effective permeability tensor and (c) the real-space renormalized permeability . . . . . . . . . . . . 194

25 Water saturation maps after 1.0 days of water injection using (a) the initial cross-bedded permeability distribution (b) the effective permeability tensor and (c) the real-space renormalized permeability . . . . . . . . . . 195

26 Water saturation maps after 1.5 days of water injection using (a) the initial cross-bedded permeability distribution (b) the effective permeability tensor and (c) the real-space renormalized permeability . . . . . . . . . . 196

27 Cumulative oil recovery versus time for rectilinear waterflood. Cross-bedded initial permeability distribution. Effect of permeability upscaling . . . . . . . . 197

28 Isotropic stochastic permeability distribution $\left(\lambda_{D}=0.5,40 \times 40\right.$ values $) . \ldots .198$

29 Cumulative oil recovery versus time for waterflood in a 1/4 of a 5-spot. Isotropic initial permeability distribution. Effect of permeability upscaling . . . .200

30 Anisotropic (cross-bedded) stochastic permeability distribution. $\left(\lambda_{D}=1.0\right.$, $\lambda_{D}=0.025, \mathrm{~V}=0.7,45^{\circ}$ structure orientation $64 \times 64$ values) $\ldots . \ldots . . .201$

31 Water saturation maps after 20 days of water injection using (a) the initial cross-bedded permeability distribution (b) the effective permeability tensor and (c) the geometrically averaged permeability. . . . . . . . . . . . 202

32 Cumulative oil recovery versus pore volume injected for waterflood in a 1/4 of a 5-spot. Anisotropic (cross-bedded) initial permeability distribution. Effect of permeability upscaling. . . . . . . . . . . . . . . 203 


\section{LIST OF FIGURES (Continued)}

Figure

Page

33 Anisotropic (cross-bedded) stochastic permeability distribution $\left(\lambda_{D}=1.0\right.$, $\lambda_{D}=0.016, V=0.7,45^{\circ}$ structure orientation $63 \times 63$ values) $\ldots . . . .205$

34 Water saturation maps after 8 days of water injection using (a) the initial permeability distribution (b) the effective permeability tensor and

(c) the geometrically averaged permeability. . . . . . . . . . . . . . . . . 206

35 Cumulative oil recovery versus pore volume injected for producing well P-1 in a 5-spot waterflood. Anisotropic (cross-bedded) initial permeability distribution. Effect of permeability upscaling . . . . . . . . . . . . . . . . . . . 207

36 Fractional flow of water versus pore volume injected for producing well P-1 in a 5-spot waterflood. Anisotropic (cross-bedded) initial permeability distribution. Effect of permeability upscaling. . . . . . . . . . . . . . . . . . 208

37 Cumulative oil recovery versus pore volume injected for producing well $\mathrm{P}-2$ in a 5-spot waterflood. Anisotropic (cross-bedded) initial permeability distribution. Effect of permeability upscaling. . . . . . . . . . . . . . . . . . . . 209

38 Fractional flow of water versus pore volume injected for producing well P-2 in a 5-spot waterflood. Anisotropic (cross-bedded) initial permeability distribution. Effect of permeability upscaling. . . . . . . . . . . . . . . . 210

\section{Report IV}

\section{Part 1}

1 Schematic of closed chamber test . . . . . . . . . . . . . . . 270

2 Discrete representation of the pressure response of a closed chamber test . . . 271

3 BHP and chamber gas pressure for base case CCT . . . . . . . . . . . . . . . . . . . .

4 Liquid level as a function of time for base case CCT . . . . . . . . . . . . . . . . . . . . . . . . . . . .

5 Wellbore storage coefficient for base case CCT . . . . . . . . . . . . 274

6 Comparison of slug test and base case closed chamber test solution . . . . . . 275

7 Correlation of base case CCT solution with analogous slug test solution,

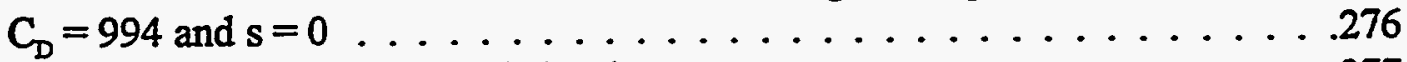

8 Effect of skin factor on closed chamber test . . . . . . . . . . . 277

9 Correlation of base case CCT solution with analogous slug test solution,

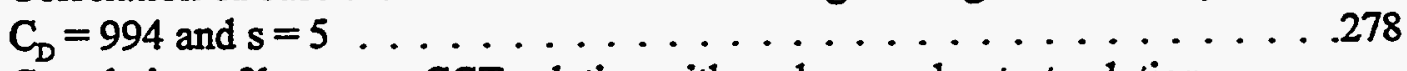

10 Correlation of base case CCT solution with analogous slug test solution,

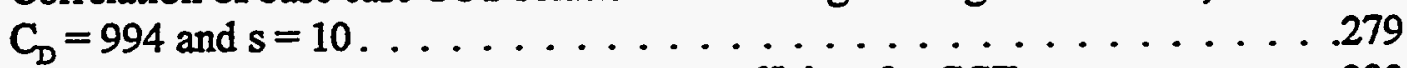

11 Effect of skin factor on wellbore storage coefficient for CCT . . . . . . . 280

12 Effect of initial chamber gas pressure on dimensionless wellbore storage coefficient for closed chamber tests . . . . . . . . . . . . . .281

13 Use of Pseudotime for closed chamber test, $\mathrm{p}_{\mathrm{chi}}=0.0 \mathrm{psig}$,

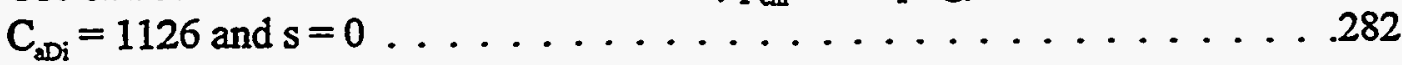




\section{LIST OF FIGURES (Continued)}

Figure

Page

14 Use of Pseudotime for closed chamber test, $\mathrm{p}_{\mathrm{cti}}=100.0 \mathrm{psig}$,

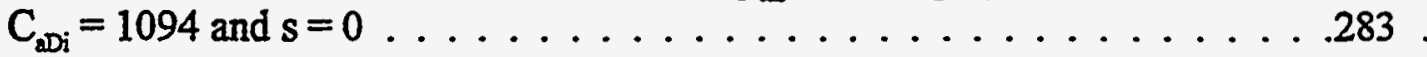

15 Use of Pseudotime for closed chamber test, $\mathrm{p}_{\mathrm{chi}}=1000.0 \mathrm{psig}$,

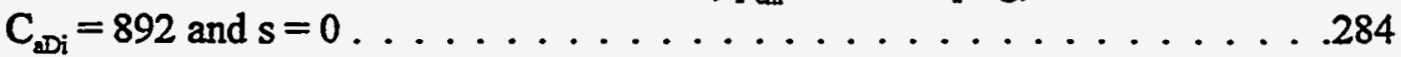

16 Use of Pseudotime for closed chamber test, $\mathrm{p}_{\mathrm{chi}}=2000.0 \mathrm{psig}$,

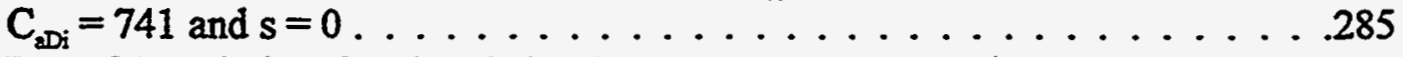

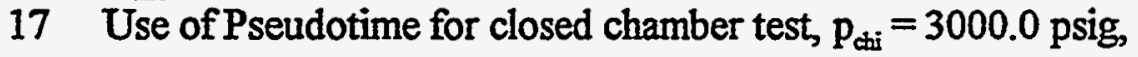
$\mathrm{C}_{\mathrm{aDi}}=663$ and $\mathrm{s}=0 \ldots \ldots \ldots \ldots \ldots \ldots \ldots \ldots \ldots \ldots \ldots \ldots \ldots \ldots . .286$

18 Use of Pseudotime for closed chamber test, $\mathrm{p}_{\mathrm{chi}}=4000.0 \mathrm{psig}$, $\mathrm{C}_{\mathrm{aDi}}=553$ and $\mathrm{s}=0$.

19 Effect of initial level on dimesionless wellbore storage coefficient for closed chamber tests. . . . . . . . . . . . . . . . .288

20 Use of Pseudotime for closed chamber test, $L_{\mathrm{i}}=2500.0 \mathrm{ft}$, $\mathrm{C}_{\mathrm{aDi}}=959$ and $\mathrm{s}=0$.

21 Use of Pseudotime for closed chamber test, $\mathrm{L}_{\mathrm{i}}=5000.0 \mathrm{ft}$, $\mathrm{C}_{\mathrm{aDi}}=892$ and $\mathrm{s}=0$.

22 Effect of total chamber length on dimesionless wellbore storage coefficient for closed chamber tests. . . . . . . . . . . . . . . . . . 291

23 Use of Pseudotime for closed chamber test, $L_{c}=2500.0 \mathrm{ft}$, $\mathrm{C}_{\mathrm{aDi}}=\mathbf{7 2 8}$ and $\mathrm{s}=0$.

24 Use of Pseudotime for closed chamber test, $L_{c}=5000.0 \mathrm{ft}$, $\mathrm{C}_{\mathrm{aDi}}=\mathbf{8 8 8}$ and $\mathrm{s}=0$.

25 Use of Pseudotime for closed chamber test, $p_{i}=4000.0 \mathrm{psig}$, $\mathrm{C}_{\mathrm{aDi}}=995$ and $\mathrm{s}=0$.

26 Effect of intial chamber gas pressure and initial reservoir pressure on dimesionless wellbore storage coefficient for closed chamber tests. . . . . . .295

27 Use of Pseudotime for closed chamber test, $p_{i}=6000.0 \mathrm{psig}$,

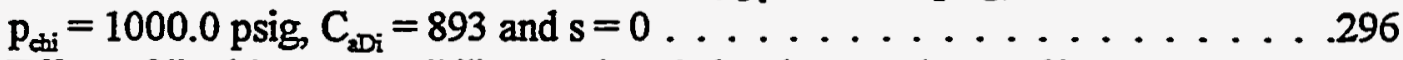

28 Effect of liquid compressibility on closed chamber test $\left(\mathrm{L}_{\mathrm{i}}=0 \mathrm{ft}\right) \ldots \ldots .297$

29 Effect of wellbore friction on closed chamber test $\left(L_{\mathrm{i}}=0 \mathrm{ft}\right)$, in $t_{D}$ and $p_{\text {wD }} \ldots .298$

30 Effect of wellbore friction on closed chamber test $\left(L_{i}=0 \mathrm{ft}\right) \ldots \ldots . \ldots 29$

31 Comparison of converted closed chamber test response with wellbore storage and skin constant surface rate solution,

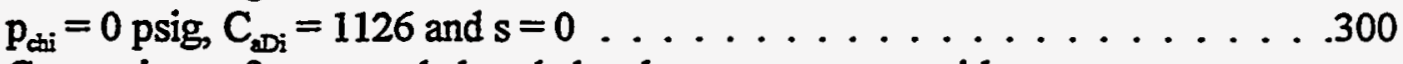

32 Comparison of converted closed chamber test response with wellbore storage and skin constant surface rate solution, $\mathrm{p}_{\mathrm{chi}}=100$ psig, $\mathrm{C}_{2 \mathrm{Di}}=1094$ and $\mathrm{s}=0$.

33 Comparison of converted closed chamber test response with wellbore storage and skin constant surface rate solution, $\mathrm{p}_{\mathrm{chi}}=500$ psig, $\mathrm{C}_{\mathrm{aDi}}=994$ and $\mathrm{s}=0$. 


\section{LIST OF FIGURES (Continued)}

Figure

Page

34 Comparison of converted closed chamber test response with wellbore storage and skin constant surface rate solution,

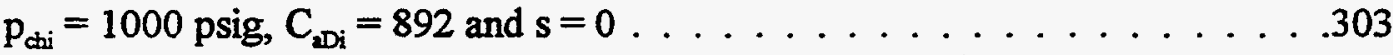

35 Comparison of converted closed chamber test response with wellbore storage and skin constant surface rate solution, $\mathrm{P}_{\mathrm{chi}}=2000 \mathrm{psig}, \mathrm{C}_{\mathrm{Di}}=741$ and $\mathrm{s}=0$.

36 Comparison of converted closed chamber test response with wellbore storage and skin constant surface rate solution, $\mathrm{P}_{\mathrm{cu}}=3000$ psig, $\mathrm{C}_{2 \mathrm{Di}}=663$ and $\mathrm{s}=0$.

37 Comparison of converted closed chamber test response with wellbore storage and skin constant surface rate solution,

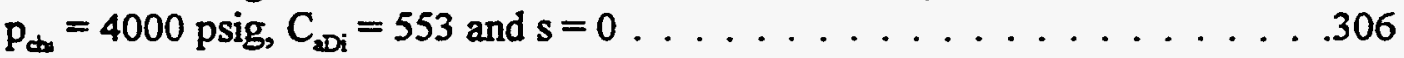

38 Use of pseudotime for slug test with step change in wellbore storage . . . . . 307

39 Buildup response of closed chamber DST . . . . . . . . . . . . . . . . . . . . . . .

40 Effect of skin factor on buildup response of closed chamber DST . . . . . . . . 309

41 Convolution method for buildup data for a closed chamber DST, $\mathrm{p}_{\text {chi }}=0 \mathrm{psig}$,

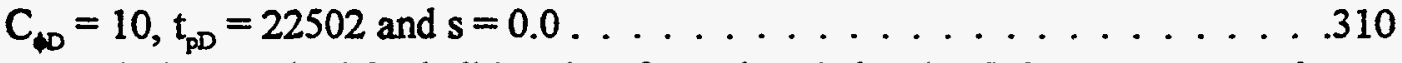

42 Convolution method for buildup data for a closed chamber DST, $\mathrm{p}_{\mathrm{cbi}}=500 \mathrm{psig}$,

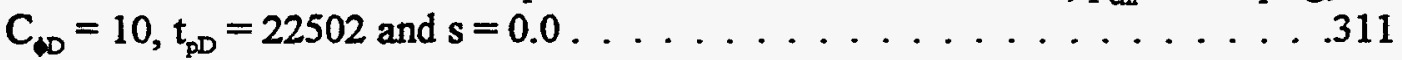

43 Convolution method for buildup data for a closed chamber DST, $\mathrm{p}_{\text {chi }}=3000 \mathrm{psig}$,

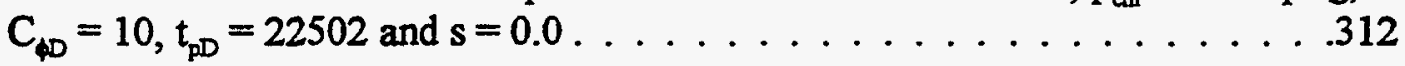

44 Convolution method for buildup data for a closed chamber DST, $\mathrm{p}_{\mathrm{chi}}=500 \mathrm{psig}$,

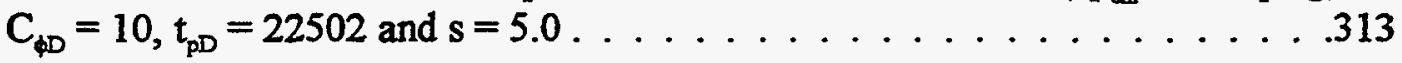

45 Convolution method for buildup data for a closed chamber DST, $\mathrm{p}_{c h}=500 \mathrm{psig}$, $C_{\phi D}=10, t_{p D}=22502$ and $s=10.0 \ldots \ldots . \ldots . \ldots . \ldots . \ldots . \ldots 14$

\section{Part 2}

1 Comparison of gas well CCT with Ramey et al. slug test solution . . . . . . . .327

$2 a_{D}$ as a function of time and position, base case . . . . . . . . . . 328

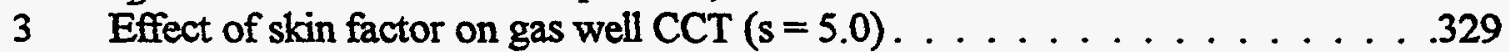

4 Effect of skin factor on gas well CCT $(s=-2.0) \ldots \ldots . \ldots . \ldots . \ldots$

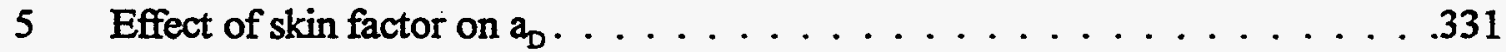

6 Effect of initial chamber pressure on gas well CCT $\left(p_{0}=2000 \mathrm{psig}\right) \ldots . . .332$

7 Effect of initial chamber pressure on gas well CCT $\left(p_{0}=4000 \mathrm{psig}\right) \ldots . . .333$

8 Effect of wellbore initial pressure . . . . . . . . . . . . . . . . 334

9 Effect of initial reservoir pressure on gas well CCT $\left(p_{i}=4000 \mathrm{psig}\right) \ldots . . .335$

10 Effect of initial reservoir pressure on gas well CCT $\left(p_{i}=2500 \mathrm{psig}\right) \ldots . . .336$

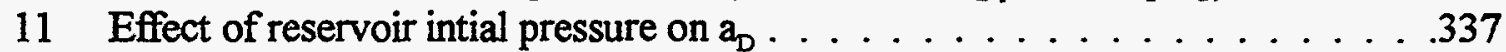

12 Effect of chamber volume on gas well CCT $\left(\mathrm{V}_{\mathrm{w}}=60 \mathrm{bbl}\right) \ldots . . \ldots 38$ 


\section{LIST OF FIGURES (Continued)}

Figure

Page

13 Effect of chamber volume on gas well CCT $\left(\mathrm{V}_{\mathrm{w}}=500 \mathrm{psig}\right) \ldots . \ldots . . . .339$

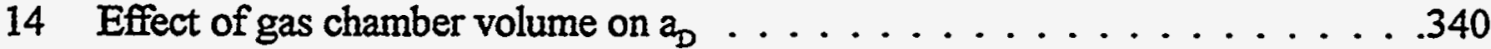

15 Comparison of converted gas well CCT response with wellbore storage

and skin constant surface rate solution, $C_{D}=9309, s=0.0$,

$\mathrm{p}_{\mathrm{i}}=5000 \mathrm{psig}, \mathrm{p}_{\mathrm{o}}=500 \mathrm{psig}, \mathrm{V}_{\mathrm{w}}=130 \mathrm{bbl}$.

16 Comparison of converted gas well CCT response with wellbore storage

and skin constant surface rate solution, $C_{D}=9309, s=5.0$,

$\mathrm{p}_{\mathrm{i}}=5000 \mathrm{psig}, \mathrm{p}_{\mathrm{o}}=500 \mathrm{psig}, \mathrm{v}_{\mathrm{w}}=130 \mathrm{bbl} \ldots \ldots \ldots \ldots . . \ldots . . \ldots . . \ldots 2$

17 Convolution analysis for gas well CCT, base case . . . . . . . . . . . . . . . . . . . . . . . .

18 Convolution analysis for gas well $\mathrm{CCT}_{2}(\mathrm{~s}=5.0) \ldots \ldots . \ldots . \ldots . \ldots . \ldots$

\section{SECTION II}

1 Base map of well location . . . . . . . . . . . . . . . . . . . . . . . . . . . . . .

2 Photograph of portions of Holeman \#5 on dry and wet. . . . . . . . . 349

3 Grid survey outcrop surface. . . . . . . . . . . . . . . . . . . . . . . . . . . . . . .

4 Sample photograph of part of the outcrop face, showing possible contacts

between discrete genetic unit . . . . . . . . . . . . . . . . . . . . . . . . . . . .

5 Permeability and Gamma ray profiles and geologic of Holeman \#3.

Discrete genetic unit are represented by number. Associated facies are

indentified by small letters. . . . . . . . . . . . . . . . . . . 353

6 Graph Ka minipermeameter vs. Ka laboratory . . . . . . . . . . . . . . . 370

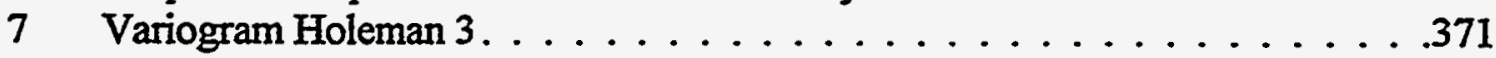

\section{SECTION III}

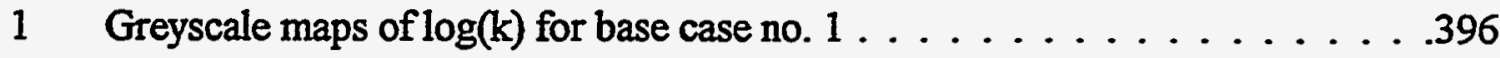

2 Waterflood performance plots for base case no. $1: \mu=2.0, \sigma_{\log (k)}=0.4$, conventional simulated annealing (truth case highlighted) . . . . . . . . . . . . . . . . . . .

3 Greyscale maps of $\log (k)$ for case study no. $1 \ldots \ldots$. . . . . . . . . . . . . . . . . . . . . .

4 Waterflood performance plots for case study no. $1: \mu=2.0, \sigma_{\log (k)}=0.4$, simulated annealing with connectivity, $\mathrm{p}_{\mathrm{uH}}=0.319$ (truth case highlighted) . . .399

5 Greyscale maps of $\log (k)$ for base case no. $2 \ldots . . . . . . . .400$

6 Waterflood performance plots for base case no. $2: \mu=2.5, \sigma_{\log (k)}=0.2$, conventional simulated annealing (truth case highlighted) .........401

7 Greyscale maps of $\log (\mathrm{k})$ for case study no. $2 \ldots . . . . . . . . . .402$

8 Waterflood performance plots for case study no. $2: \mu=2.5, \sigma_{\log (k)}=0.2$, simulated annealing with connectivity, $\mathrm{p}_{\mathrm{tH}}=0.298$ (truth case highlighted) . . .403

9 Greyscale maps of $\log (k)$ for case study no. $3 \ldots . . . . . . . . . .404$ 


\section{LIST OF FIGURES (Continued)}

Figure

Page

10 Waterflood performance plots for case study no. $3: \mu=2.0, \sigma_{\log (k)}=0.4$, simulated annealing with parameter constraints $\mathrm{p}_{\mathrm{EH}}=0.319, \log \left(\mathrm{k}_{\mathrm{N} w \mathrm{w}}\right)=2.57$,

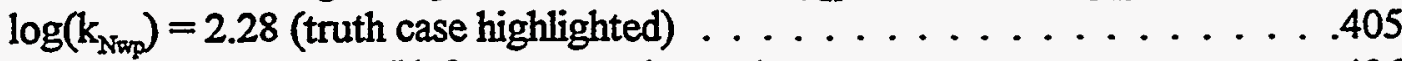

11 Greyscale maps of $\log (k)$ for case study no. $4 \ldots . . . . . . . .406$

12 Waterflood performance plots for case study no. $4: \mu=2.5, \sigma_{\log (k)}=0.2$, simulated annealing with parameter constraints $\mathrm{p}_{\mathrm{ut}}=0.298, \log \left(\mathrm{k}_{\mathrm{Nw}}\right)=2.76$, $\log \left(k_{\mathrm{Nwp}}\right)=2.53$ (truth case highlighted) . . . . . . . . . . . . 407

13 Waterflood performance plots for case study no. $5: \mu=2.0, \sigma_{\log (x)}=0.4$, $\mathrm{CV}_{\mathrm{k}^{*}}$ sensitivity $(0.05,0.10,0.186,0.30,0.40), \mathrm{p}_{\mathrm{uH}}=0.319, \log \left(\mathrm{k}_{\mathrm{N} * \mathrm{i}}\right)=2.57$, $\log \left(\mathrm{k}_{\mathrm{N} \text { ip }}\right)=2.28, \mathrm{k}_{\mathrm{mD}}=0.610$ (truth case highlighted) . . . . . . . 408

14 Waterflood performance plots for case study no. $5: \mu=2.0, \sigma_{\log (x)}=0.4$, $\mathrm{CV}_{\mathrm{k}^{*}}$ sensitivity $(0.05,0.10,0.186,0.30,0.40), \mathrm{p}_{\mathrm{tH}}=0.319, \log \left(\mathrm{k}_{\mathrm{Nkxi}}\right)=2.57$, $\log \left(\mathrm{k}_{\mathrm{Nwp}}\right)=2.28, \mathrm{k}_{\mathrm{mD}}=0.610$ (truth case highlighted) - one realization per sensitivity . . . . . . . . . . . . . . . . . . . . . . . . . . . . . . . . . . . .

15 Correlation between $\mathrm{CV}_{\mathrm{k}^{*}}$ and water breakthrough time ......... . . . . . . . . . .

16 Greyscale maps of $\log (k)$ for case study no. $5 \ldots \ldots . . . . . . . . . . .411$

17 Waterflood performance plots for case study no. $5: \mu=2.0, \sigma_{\log (x)}=0.4$, $\mathrm{k}_{\mathrm{mD}}$ sensitivity $(0.50,0.61,0.65,0.80,1.0), \mathrm{p}_{\mathrm{zH}}=0.319, \log \left(\mathrm{k}_{\mathrm{Nzi}}\right)=2.57$, $\log \left(\mathrm{k}_{\mathrm{Nwp}}\right)=2.28, \mathrm{CV}_{\mathrm{k}^{*}}=0.186$ (truth case highlighted). ..............

18 Waterflood performance plots for case study no. $5: \mu=2.0, \sigma_{\log (x)}=0.4$, $\mathrm{p}_{\mathrm{rH}}$ sensitivity $(0.10,0.20,0.319,0.40,0.50), \log \left(\mathrm{k}_{\mathrm{N}+\mathrm{i}}\right)=2.57$, $\log \left(\mathrm{k}_{\mathrm{Nwp}}\right)=2.28, \mathrm{k}_{\mathrm{mD}}=0.610, \mathrm{CV}_{\mathrm{k}^{*}}=0.186$ (truth case highlighted). . . . . 413

19 Greyscale maps of $\log (\mathrm{k})$ for case study no. $6 \ldots . . \ldots . . . . . . .414$

20 Waterflood performance plots for case study no. $6: \mu=2.0, \sigma_{\log (k)}=0.4$, simulated annealing with parameter constraints $\mathrm{p}_{\mathrm{tH}}=0.319, \log \left(\mathrm{k}_{\mathrm{N}_{\mathrm{H} i}}\right)=2.57$, $\log \left(\mathrm{k}_{\mathrm{Nwp}}\right)=2.28, \mathrm{k}_{\mathrm{mD}}=0.610, \mathrm{CV}_{\mathrm{k}^{*}}=0.186$ (truth case highlighted) . . . . . 415

21 Greyscale maps of $\log (\mathrm{k})$ for case study no. $7 \ldots . . \ldots . . . . . .416$

22 Waterflood performance plots for case study no. $7: \mu=2.5, \sigma_{\log (x)}=0.2$, simulated annealing with parameter constraints $\mathrm{p}_{\mathrm{LH}}=0.298, \log \left(\mathrm{k}_{\mathrm{N} \text { riv }}\right)=2.76$, $\log \left(\mathrm{k}_{\mathrm{Nap}}\right)=2.53, \mathrm{k}_{\mathrm{mD}}=1.02, \mathrm{CV}_{\mathrm{k}^{*}}=0.300$ (truth case highlighted) $\ldots . . .417$

23 Waterflood performance plots for case study no. $8: \mu=2.0, \sigma_{\log (k)}=0.4$, $\mathrm{CV}_{\mathrm{k}}$ sensitivity $(0.05,0.10,0.186,0.30,0.40), \mathrm{p}_{\mathrm{HH}}$ varies, $\log \left(\mathrm{k}_{\mathrm{N} \text { ti }}\right)=2.57$, $\log \left(\mathrm{k}_{\mathrm{N} \text { wop }}\right)=2.28, \mathrm{k}_{\mathrm{mD}}=0.610$ (truth case highlighted $) \ldots \ldots . . . . .418$ 


\section{LIST OF TABLES}

Table

Page

\section{SECTION I}

\section{Report I}

1 Summary of available data for Hugoton field . . . . . . . . . . . . . . . 14

2 Summary of statistics of intermittency exponent values for Hugoton field with box-counting method. . . . . . . . . . . . . 16

3 Summary of statistics of intermittency exponent values for Hugoton field with $\mathrm{R} / \mathrm{S}$ analysis method. . . . . . . . . . . . . . 17

4 Summary of available data for Burbank field . . . . . . . . . . . . . 19

5 Summary of statistics of intermittency exponent values for Burbank field with box-counting method . . . . . . . . . . . 20

6 Summary of statistics of intermittency exponent values for Burbank field

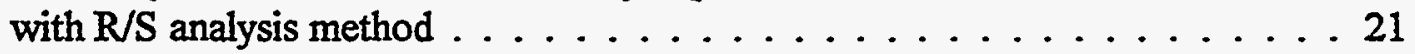

7 Summary of available data for Sable field . . . . . . . . . . . . . 23

8 Summary of statistics of intermittency exponent values for Sable field with box-counting method . . . . . . . . . . . 25

9 Summary of statistics of intermittency exponent values for Sable field with $\mathrm{R} / \mathrm{S}$ analysis method . . . . . . . . . . . 26

10 Summary of available data for Loudon field . . . . . . . . . . . 27

11 Summary of simulation parameters for 2-D stochastic conditional simulation in Hugoton field . . . . . . . . . . . . . . 34

12 Correlation coefficients and CPU usage for two-dimensional stochastic conditional simulations for Hugoton field . . . . . . . . . . . . 36

13 Correlation coefficients for two-dimensional stochastic conditional simulations runs for Hugoton field $\ldots \ldots \ldots \ldots \ldots \ldots \ldots$

14 Summary of simulation parameters for 2-D stochastic conditional simulation in the Burbank field . . . . . . . . . . . . . . . . 39

15 Correlation coefficients for two-dimensional stochastic conditional simulations runs for Burbank field . . . . . . . . . . . . . 40

16 Summary of simulation parameters for 2-D stochastic conditional simulation in the Loudon field . . . . . . . . . . . . . . 42

17 Correlation coefficients for two-dimensional stochastic conditional simulations runs for Loudon field . . . . . . . . . . . . . . . 43

18 Summary of simulation parameters for 3-D stochastic conditional simulation in the Burbank field using 4 conditioning wells. . . . . . . 45

19 Correlation coefficients for three-dimensional stochastic conditional simulations for Burbank field using 4 conditioning wells . . . . . . . . 46

20 Correlation coefficients for three-dimensional stochastic conditional simulations for Burbank field using 8 conditioning wells . . . . . . . 47 


\section{LIST OF TABLES (Continued)}

Table

Page

21 Summary of simulation parameters for 3-D stochastic conditional simulation in the Loudon field using 4 conditioning wells . . . . . . . . . 49

22 Correlation coefficients for three-dimensional stochastic conditional simulations for Loudon field ... . . . . . . . . . . . . . . . 50

23 Correlation coefficients for three-dimensional stochastic conditional simulations for Hugoton field with small-scale simulation . . . . . . . . . 52

24 Correlation coefficients for three-dimensional stochastic conditional simulations for Hugoton field with large-scale simulation . . . . . . . . . 53

\section{Report II}

1 Variability of the permeability fields and the differences in the averaging methods and the well test permeability . . . . . . . . 135

\section{Report III}

\section{Part 1}

1 Flow rates of case 1 and case $2 \ldots \ldots \ldots \ldots$

\section{Part 2}

2 Simulation input parameters. . . . . . . . . . . . . . . . . . . . . . . .

\section{Report IV}

\section{Part 1}

1 Parameters used for slug test comparison. . . . . . . . . . . . . 261

2 Comparison with analytical slug test solutions . . . . . . . . . . . . 262

3 Parameters used for comparison with Simmons and Grader solutions . . . . . .263

4 Comparison with Simmons and Grader solutions . . . . . . . . . . . . . . . . . . . . . . . . . . . . . .

5 Comparison with Salas and Sageev solutions . . . . . . . . . . . . . 266

6 Comparison with analytical DST buildup solutions . . . . . . . . . . . 268

7 Parameters for base case closed chamber test. . . . . . . . . . . . . 269

\section{Part 2}

1 Gas physical properties as functions of pressure . . . . . . . . . . . . . . . . . . . . .

2 Parameters for base case gas well closed chamber test . . . . . . . . . . 326 


\section{LIST OF TABLES (Continued)}

Table

Page

\section{SECTION II}

1 Summary of coring and logging activity $\ldots \ldots \ldots \ldots \ldots . \ldots . \ldots . \ldots$

2 Core description, Holeman \#3 well . . . . . . . . . . . . . . . .355

3 Summary of permeability values for all wells . . . . . . . . . . . 356

4 Measured core data . . . . . . . . . . . . . . . . . . . . 357

5 Porosity and permeability data, Holeman $3 \ldots \ldots . \ldots . . . . . . .369$

\section{SECTION III}

1 Fluid properties used in flow simulation runs . . . . . . . . . . . . 390

2 Pertinent data used in flow simulation runs . . . . . . . . . . . . 391

3 Values of parameters used in sensitivity study . . . . . . . . . . 392 


\section{Acknowledgment}

The research effort described in this report was supported by the U.S. Department of Energy under contract DE-AC22-90BC14651 and by Tulsa University Petroleum Reservoir Exploitation Projects (TUPREP) member companies: Amoco, Arco, Aramco, Conoco, Intevep, Marathon, Mobil, Pertamina, Petrobras, Phillips Petroleum and Unocal. Additionally, the computer facilities and the lab space was provided by the University of Tulsa. We gratefully acknowledge all this support.

Our special thanks go to Rhonda Patterson, representative of the contracting officer for this project, for her enthusiasm, willingness to interact, and valuable suggestions. We also acknowledge the comments and suggestions provided by the representatives of the member companies during our bi-annual meetings.

Mohan Kelkar

September 1992 


\begin{abstract}
This annual report describes the progress during the second year of a project on Reservoir Characterization of Pennsylvanian Sandstone Reservoirs. The report is divided into three sections:

(i) reservoir description and scale-up procedures

(ii) outcrop investigation

(iii) in-fill drilling potential

The first section describes the methods by which a reservoir can be characterized, can be described in three dimensions, and can be scaled up with respect to its properties, appropriate for simulation purposes. In the first report it is shown that the fractal model adequately describes the reservoir properties for both the sandstone and the carbonate reservoirs. The results are validated by comparing the simulated well data with the actual well bore data. In the second report, the method of simulated annealing is extended to incorporate the well test data in reservoir description process. Oliver's weighting functions are used to incorporate the well test data. The third report in this section addresses the issue of scale-up of reservoir properties once the spatial descriptions of properties are created. A new analytical method is proposed to estimate a permeability tensor based on small scale permeability variations. The method has been validated by comparing the results with the numerical results. Further, a technique is proposed to incorporate full permeability tensors in finite difference and finite element models. The last report in the first section deals with the analysis of DST test data. DST tests are the only tests which may be available for determining the large scale permeability values. The report proposes new versions of pseudo time for analyzing closed chamber DST tests. It shows that the flow period data can be analyzed using the procedures for the slug tests provided the appropriate pseudo time is incorporated in the analysis.

The second section describes the progress on investigation of an outcrop. The outcrop is an analog of Bartlesville sandstone. We have drilled ten wells behind the outcrop and collected extensive log and core data. The cores have been slabbed, photographed and the several plugs have been taken. In addition, minipermeameter is used to measure permeabilities on the core surface at six inch intervals. The plugs have been analyzed for the permeability and porosity values. The variations in property values will be tied to the geological descriptions as well as the subsurface data collected from the Glen Pool field.

The third section discusses the application of geostatistical techniques to infer infill well locations. The geostatistical technique used is the simulated annealing technique because of its flexibility. One of the important reservoir data is the production data. Use of production data will allow us to define the reservoir continuities, which may in turn, determine the in-fill well locations. The proposed technique allows us to incorporate some of the production data as constraints in the reservoir descriptions. The technique has been validated by comparing the results with numerical simulations.
\end{abstract}




\section{Executive Summary}

This annual report contains several progress reports related to Reservoir Characterization of Pennsylvanian sandstone reservoirs. The report is divided into three sections. The first section discusses the characterization, description and the scale-up processes. The second section describes the investigation of an analogous outcrop, and the third section describes the application of geostatistical techniques for inferring in-fill well locations in existing reservoirs.

The first section is divided into four reports. The first report describes the characterization of reservoir using fractals. The second report discusses the incorporation of well test data in a conditional simulation process. The third report explains a new analytical technique for estimating up scaled permeability values to be used as input in a reservoir simulator. The final repoit establishes a procedure to analyze the drill stem tests (DST) for oil and gas wells.

The first report, titled "An Approach to - Generating Reservoir Property Descriptions using Stochastic Conditional Simulation Method," develops a methodology to generate three dimensional description of reservoir properties using fractal distributions. The first step in the method is to remove the seasonal effects found in the well bore data. These seasonal effects may be due to differences in depositional environment. After removal of these effects, the well bore data are analyzed using the box counting method to estimate the intermittency exponent, $\mathrm{H}$. We observed that even for different types of depositional environments, the average values of $\mathrm{H}$ are always between 0.75 and 0.8 . Therefore, in the absence of actual data, this value may be used to describe the reservoir properties. The next step in the method is to generate several two dimensional cross sections between the two wells and compare the simulated results at another well located between these two wells with the actual data. After conducting a study of several cross sections, we may have a better idea about the horizontal correlation structures. Based on the four field studies, we observed that fractional Brownian motion ( $\mathrm{EBm}$ ) describes the sandstone reservoirs the best; whereas, the fractional Gaussian noise (fGn) describes the carbonate reservoirs. Once the horizontal structure is known, three dimensional descriptions can be generated using the available well bore data. We observe that the most efficient way to generate this description is to generate preliminary description using linear interpolation layer by layer, add a random component based on the variance between the wells, and use simulated annealing as a post processor to obtain the final results.

The second report, titled "Reservoir Characterization for Water floods Incorporating Well Test Data in Stochastic Conditional Simulations," describes a method to incorporate the well test data in the simulated annealing process to generate the reservoir descriptions. One of the advantages of using the simulated annealing techniques is its flexibility in using varying constraints in generating the reservoir descriptions. To define the proper constraint for incorporating the well test data, we first need to identify a proper averaging technique which represents the well test data. We have observed that 
Oliver's approach (SPEFE, Spet. 1990, pp 319-24) is adequate to describe the proper averaging functions to describe the well test data. If the reservoir is divided into several concentric rings, then after calculating the geometric averages of permeability values within each ring, we can apply Oliver's weighting functions to estimate instantaneous permeability value represented by the pressure - time data. This method can be applied to many different times so that the pressure - time characteristics can be reproduced when the generated description is used to simulate the well test data. At present, we are in the process of incorporating the weighting functions in the existing simulated annealing program (Perez, 1991).

The third report, titled "Effective Properties of Reservoir Simulator Grid Blocks" proposes a new analytical method to estimate an up-scaled permeability tensor based on small scale heterogeneties. The method involves dividing the small scale permeability field into four smaller blocks, estimating the effective permeability tensors for individual blocks, and depending upon the local and global transverse pressure gradients as well as the permeability values of individual blocks, determine the effective permeability tensor of the entire field. The method has been validated by comparing the results with the numerical results. Further, unit mobility ratio displacements were conducted in up scaled permeability media, and it is shown that the proposed method outperforms geometric averaging as well as renormalization methods in terms of determining the flood front locations at constant pressure injection.

In the second part of the third report, a method is developed to incorporate the full tensor permeability into finite element and finite difference methods. Based on earlier work, the derivations of transmissibility equations are derived starting from the continuity equations without boundary conditions. It is further shown that the finite element and finite difference methods can be reconciled if appropriate linear approximations are used to describe pressure and mobility trial functions. The method is validated by comparing numerically simulated water flooding results in the presence of small scale heterogeneities as well as upscaled permeability values. The comparison shows that the proposed method of upscaling the permeability is superior to other up scaling methods.

The fourth report, titled "Results for Closed Chamber Tests," explains a new method to analyze drill stem tests. A proper analysis of a DST is important to determine reservoir properties on a large scale. For many wells, a DST may be the only well test run on the well. This report develops a new method to analyze closed chamber DST's. Closed chamber tests are the DST's where the well is shut-in during the entire test and nitrogen cushion is used. This type of testing is supposed to provide more reliable results. The report derives an analytical expression for the effective well bore storage coefficient for a closed chamber test and investigates its effects on the pressure response under various test conditions. New versions of pseudo time for analyzing such tests are presented. In particular, it is shown that, in most cases, flow period data can be analyzed using procedures for slug tests provided the appropriate pseudo time is incorporated in the analysis. In the second part of the report, the analysis has been extended for gas wells. For gas wells, it is shown that the real gas pseudo pressure function should also be used. 
In addition, a rate-convolution method is derived for the analysis of pressure data obtained using the shut-in period of a DST.

In the second section, we discuss the investigation of the outcrop. The outcrop selected is a Bluejacket sandstone. This is an outcrop of Bartlesville sand, one of the most prolific sands in Oklahoma. To study the outcrop carefully, we have drilled twelve wells behind the outcrop. These wells are approximately seventy feet in depth. All the wells have been logged and cored. In one well, FMS (formation micro scanner survey) log has been run. The cores have been slabbed and photographed. Additionally, with the help of minipermeameter, several permeability measurements were made. To validate the minipermeameter data, approximately two hundred core plugs are taken. These plugs will be analyzed for permeability and porosity data, and the measurements will be compared with the minipermeameter and the log data. We are also in the process of conducting the geological description of the outcrop. Cores from two wells have been geologically described. In addition, several vertical transsects on the outcrop surface will also be investigated for geological description. We hope to compare the results of the outcrop study with the subsurface information in the next year.

In the last section, we discuss the application of conditional simulation method to investigate potential in fill well locations. We have chosen the method of simulated annealing to incorporate the production constraints in reservoir description process. Traditionally most of the conditional simulation methods incorporate static information such as core and log data as well as geological description. However, no methods currently exist which will allow incorporation of production data in reservoir description. To decide an in fill well location, it is very important to understand the reservoir continuities. More continuous the reservoir, less is the need to drill additional well. One of the ways by which the continuity can be determined is by incorporating production information in the reservoir description. For example, early water breakthrough may indicate better communication between two wells. In this report, we present a method to incorporate the production information in the reservoir description. It is observed that a simple stream tube model which incorporates variance in the permeability values is adequate to capture many of the production parameters. The incorporation of this additional constraint does not increase the computational time of the conditional simulation method significantly. The results are validated by conducting numerical simulations under various conditions.

Looking ahead, we expect to apply various techniques developed in the first section to optimize a water flooding performance in a sandstone reservoir. We have selected Glen Pool reservoir for such validation. Currently, we are in the process of constructing the reservoir description of the field. We will also incorporate the information gathered from the outcrop in the describing small scale permeability values.

The outcrop work has lagged behind the projected targets. However, over the last six months, we have collected a lot of data on the outcrop. We intend to finish the collection of data before December 1992 and concentrate on the analysis and evaluation of 
the data over the last eight months. In the mean time, we have collected sub surface data from the Glen Pool field. We intend to compare the geological as well as engineering information from the subsurface data with the outcrop information.

The in fill potential work has shown a lot of promise in terms of incorporating production information in describing the reservoirs. We intend to extend this work for primary production over the next year. Specifically, we are going to look at the key parameters observed during the primary production which may be important in describing the reservoir continuity. Currently, such effort is underway. 


\title{
Section I
}

Report I

\section{An Approach to Generating Reservoir Property Descriptions Using a Stochastic Conditional Simulation Method}

\author{
S. Shibli and M. Kelkar.
}

\section{Summary}

This work investigates the utility of applying fractal geometry for describing the spatial correlation structure of rock properties. It also provides a proper framework within which to apply a stochastic conditional simulation technique to generate equiprobable distributions of a rock property.

A normalization technique is developed to remove the seasonal effects found in the wellbore data. It is found that where the seasonal effects are unusually strong, as in the case of one carbonate reservoir, removal of the local means will result in a narrower distribution of the Hurst $(H)$ exponent values. Theoretical and analytical variogram plots of the results of these estimations indicate that the box-counting method gives slightly improved estimates over $R / S$ analysis.

Conditional simulation runs using simulated annealing are performed for three fields. The results indicate that a combination of the greedy algorithm and a bilinear interpolation technique to generate the initial pattern decrease CPU usage by as much as 85 percent. More importantly, the simulations also indicate that an $\mathrm{fGn}$ horizontal correlation structure gives better predictions of interwell distributions in a carbonate environment. In contrast, in a sandstone field, the use of a $\mathrm{fBm}$ horizontal correlation structure gives more reliable results.

\subsection{Introduction}

Reservoir description encompasses a diverse number of disciplines. Geophysicists, geologists, and petroleum and mining engineers all have as their primary goal a better understanding of a reservoir so as to be able to describe it as accurately as possible on all scales. Although a reservoir is essentially deterministic, due to the lack of complete knowledge, a host of statistical and mathematical tools have been developed within the last two decades which add a random element to the description of a geologic formation. ${ }^{1-5}$

One of the commonly used techniques is a method of geostatistics. Geostatistics attempts to capture the spatial variability of a variable. Conventional statistics, although useful for describing the summary statistics of a sampled distribution, fails to account for the spatial relationship of most natural phenomena. It is a 
well known fact that measurements of reservoir properties at close distances tend to be similar to each other. As the distance between two points increases, the similarity between any two measurements decreases. Geostatistics is primarily a tool for capturing and quantifying such a spatial relationship.

This study is an attempt to determine whether a representative spatial correlation structure can be obtained by analyzing wellbore data with the help of fractal models. The motivation for using fractal statistics is that several studies have been conducted in the past that show it to be a promising and simple tool for determining the spatial correlation structure of geological data. ${ }^{3,6-9}$ Although different geostatistical techniques could have been used, only fractals offer the convenience of describing the spatial correlation structure in terms of only one major variable: the $H$ or intermittency exponent of the data.

Several major drawbacks are associated with the determination of the $H$ exponent. Most obvious is the limited data typically available at wellbore locations. An ideal situation would be to have a very large sample size in order to obtain the most reliable value for the $H$ exponent. One study has indicated that the quality of the estimates tend to deteriorate as the sample size decreases. ${ }^{11}$

Numerous techniques exist for estimating the $H$ exponent of a time series, which, in the case of hydrocarbon wells, is the vertical or horizontal well data. They can roughly be divided into two groups: graded, and non-graded methods. ${ }^{12}$ Graded methods include box-counting and semi-variogram analysis of a graded sequence. Non-graded methods include the rescaled-range analysis and semi-variogram analysis of a raw sequence. Graded sequences have the advantage of being more regular than the original data and are often easier to characterize. ${ }^{3}$ The reliability of these different techniques have not been examined in the existing literature. Wallis and Matalas $^{11}$ have shown that different parent distributions can also affect the estimates.

It is fairly common practice today to analyze wellbore data without taking into account alternating sand and shale sequences that might be present in the zone of interest. Mixing zones together is tantamount to mixing two different populations, and would lead to biased estimates of the intermittency exponent. Such a problem, however, is not particular only to geologic description. Feder ${ }^{13}$ presents a case study whereby wave height data had to be seasonally adjusted before an $H$ exponent analysis was done. In this study, a normalization technique is devised to remove any local trends in the sample data and improve the $H$ exponent estimations.

The primary source of information used in this study is the vertical well data that is obtained for most reservoirs. Two drastically different sedimentary depositional environments are also considered: that of a carbonate field, and a sandstone. This study involves a total of more than 90 well log and core porosity data from three different fields. All the data are analyzed after being adjusted for seasonal variations. The effects of the adjustments on the values of the intermittency exponents are also investigated. The analyses are carried out using only the methods 
that are found to be more reliable than other estimation algorithms.

The last part of this study dealt with the conditional simulation of reservoir properties based on the simulated annealing technique. $4,5,12$ The characteristic spatial correlation obtained from fractal models is used as input into the conditional simulator. The method of simulated annealing is used for conditional simulation purposes. ${ }^{12}$

Stochastic conditional simulation using simulated annealing is an immensely flexible technique for the generation of random rock properties given a specified number of constraints. Usually, these constraints will include the cumulative distribution function of the conditioning data, the conditioning data itself, and the spatial correlation structure of the conditioning data. Given different seed values for the random number generator, one can create different realizations of the reservoir for the same set of constraints. This, in effect, facilitates the calculation of the range of uncertainties in future performance curves.

Once the alternate descriptions of reservoir properties are created, validation of the reliability of the predictions involves comparing the actual well log data with the simulated data. A close agreement between actual and simulated logs will indicate a reliable prediction for a particular set of constraints. In this study, several comparisons are done in order to determine to what extent fractal correlation structures can be usefully utilized in predicting rock properties at unsampled locations.

This study is also an attempt to provide a few necessary guidelines for the effective characterization of a reservoir. A basic framework for attacking the different problems of stochastic conditional simulation is presented. Reservoir description via conditional simulation is a relatively new approach; while a lot of studies extol the virtues of its application, few actually present an effective framework by which to apply the technology. A lot of problems and kinks in the analysis procedure still need to be studied, modified, and improved. It is hoped that this report will encourage further work in the area of stochastic conditional simulation and its application to reservoir property description.

\subsection{Literature Review}

This section presents a brief literature review of the application of fractals for modeling the spatial correlation structure of rock properties and the use of different techniques for generating equiprobable descriptions of rock properties.

\subsection{Fractals}

Hewett laid the groundwork for the application of fractal geometry to reservoir description. ${ }^{3}$ He also presents a case study where a fractal generation algorithm is used as a conditional simulation technique for generating equiprobable distributions of reservoir properties. His work is motivated by the fact that the structure of spatial correlations in permeability distributions largely affect fluid flow in heterogeneous porous media. 
Hewett presents examples where property distributions in sedimentary environments show a fractal character with long-range correlation. ${ }^{3}$ Based on the assumption that a graded trace of vertical wellbore data (porosity) can approximate the correlation structure in the horizontal direction, Hewett outlines a procedure to investigate the effects of fractal property distributions on reservoir performance.

Hewett and Behrens present two uses of conditional simulation. ${ }^{6}$ One is for scaling flow processes and properties. They review different scales within the reservoir and indicate that fractal distributions have scaling properties. Different numbers of layers are used in the simulation to observe the effect of scaling in assigning reservoir and rock properties. The second use of conditional simulation is to estimate uncertainties of outcomes as a result of sampling of data. Flow simulations in various possible reservoir descriptions (realizations of property distributions) generate a probability distribution of reservoir performance which may be used to evaluate the risk associated with a project.

\subsubsection{Theory}

Fractals are distinguished by the fact that they exhibit variations at all scales of observation. Thus, on a closer examination, a fractal will appear to have more resolution or detail. Fractals can be characterized by their fractal dimension, which need not be an integer. For example, in a $d$-dimensional Euclidean space, the number of objects of scale $r L$ to fill a space of scale $L$ is

$$
N=r^{-d}
$$

Therefore, if $r=1 / 3$, it will take 3 line segments $(d=1)$ of length $1 / 3 L$ to fill a line of length $L$. Similarly, it will take 9 squares with sides $1 / 3 L$ to fill a square $(d=2)$ of side $L$, and 27 cubes with sides $1 / 3 L$ to fill a cube $(d=3)$ of side $L$ (see Figure 1).

A similar equation can be written for the fractal dimension. Fractal dimensions do not fill space and can be written in the following form:

$$
N=r^{-D}
$$

where $D$ is the fractal dimension and is less than $d$.

The application of fractals can be extended to random natural sequences. For example, it can be shown that the probability of variations in property $Z(x)$ in a distribution with fractal geometry is of the form ${ }^{3}$

$$
P\left(\frac{Z(x+h)-Z(x)}{h^{H}} \leq y\right)=F(y)
$$

where $F(y)$ is a normalized cumulative distribution function, $h$ is the lag distance, and $H$ is the intermittency exponent. 
$H$ is a dimension of self-similarity and is related to the fractal dimension, $D$, by

$$
H=d-D
$$

The typical value of $H$ varies between 0 and 1 , where $H=1 / 2$ denotes the wellknown process of Brownian motion, which in itself is the sum of a sequence of uncorrelated Gaussian random variables. If $H<1 / 2$, the correlation is negative, or high values will be more likely be followed by low values. If $H>1 / 2$, the correlation is positive, or high values; will be more likely be followed by high values and low values will more likely be followed by more low values.

The intermittency exponent is useful in that it can indicate the degree of variability of a record of a property in time. Various studies of geological records have shown that the value of $H$ will generally fall in the range from 0.60 to $0.95 .^{3,21,22,23}$ The intermittency exponent for $\mathrm{fBm}$ can also be related to the variance of increments. The semivariogram equation can be written $a^{3}$

$$
2 \gamma(h)=E\left\{[Z(x+h)-Z(x)]^{2}\right\}=V_{H} h^{2 H}
$$

where $V_{H}$ is a constant, and $E\{X\}$ denotes the mathematical expectation of a random variable, $X$.

Distributions having the form of (5) are said to be statistically self-affine, since the variations over any scale $r h$ are related to the variations over a scale $h$ by

$$
\gamma(r h)=r^{2 H} \gamma(h)
$$

This implies that the variance at any scale can be determined from the variance measured at any other scale.

It is interesting to see that fractional Brownian motion does not have a derivative. One can, of course, smooth out the trace over an arbitrarily small interval $\delta$, which is also called the regularizing interval. If $\delta$ is small compared to the scale of observation, both the smoothed and original functions are indistinguishable for all practical purposes. ${ }^{3}$ Smoothing removes the highest frequency variations in the original function and renders it differentiable.

After smoothing, the derivative of Brownian motion is just the sequence of uncorrelated Gaussian random variables called white noise. Extending this reasoning further, the derivative of fractional Brownian motion is called fractional Gaussian noise. Unlike $\mathrm{fBm}, \mathrm{fGn}$ is a stationary process, and has an autocovariance given by

$$
\operatorname{COV}(h, \delta)=\frac{1}{2} V_{H} \delta^{2 H-2}\left(\left|\frac{h}{\delta}+1\right|^{2 H}-2\left|\frac{h}{\delta}\right|^{2 H}+\left|\frac{h}{\delta}-1\right|^{2 H}\right)
$$

An interesting point to note in the above relation is that $\delta$ provides an insight on the effects of sample size on the determination of spatial or temporal structure. It 
can easily be adjusted to account for the influence of sample size on the statistical measurements.

Persistency reflects the non-random spatial correlation structure of natural phenomena. Values tend to be clustered together, creating intermittent regions of potentially long-range persistence. The value of $H$ is an indicator of such a correlation and determines the variability of the values.

\subsubsection{Determination of Intermittency Exponent}

Numerous $H$ estimation methods exist in the literature for analyzing the spatial or temporal correlation of a data set. ${ }^{3,13,24}$ These methods can roughly be divided into graded and non-graded methods. ${ }^{12}$ Graded sequences have the advantage of being smoother to analyze. Graded methods include the box-counting method and the semivariogram analysis of a graded sequence. Non-graded methods include Hurst's rescaled-range analysis ${ }^{20}$ and the semivariogram analysis of raw data.

\subsection{Conditional Simulation Techniques}

This section will present a brief overview of some conditional simulation techniques that have been extensively used for generating reservoir property descriptions.

\subsubsection{Introduction}

Stochastic conditional simulation techniques offer several significant advantages over conventional reservoir description. Chief among these is that most available information concerning the reservoir is honored in the simulation model. Properties such as conditioning data, the original distribution function, and the spatial correlation of the rock properties are honored. Stochastic conditional simulation also makes use of random or stochastic variables; therefore, several equiprobable descriptions of the actual rock property may be generated. This, in effect, permits a reservoir simulation engineer to quantify uncertainties with respect to future performance curves.

\subsubsection{Different Techniques}

Several conditional simulation algorithms have been discussed in the literature. Some of the popular ones include kriging, ${ }^{1,2,31,32,38}$ fractal interpolation, ${ }^{3}$ sequential indicator simulation, ${ }^{30}$ and simulated annealing. ${ }^{12,27,28,41}$ In addition, some unconditional simulation techniques have also been developed. An example is the Turning Bands method, ${ }^{33}$ which only accounts for a prescribed spatial correlation model. Haldorsen and Damsleth ${ }^{36}$ give a general overview of these various stochastic simulation techniques. Haldorsen et $\mathrm{al} .{ }^{37}$ present a review of the stochastic nature of petroleum reservoirs.

Hewett ${ }^{3}$ gives a detailed description of using a fractal interpolation technique called successive random additions for generating distributions of porosity in an interwell region. He assumed that the vertical and horizontal correlation structures of the porosity can be based on two statistical fractal models, namely fractional Gaussian noise and fractional Brownian motion. Hewett also presented a hybrid 
approach to extend the use of the conditional simulation technique for predicting the performance of large fields. Aasum et $\mathrm{al}^{35}$ and Crane and Tubman ${ }^{22}$ present case studies of the application of fractals for spatial modeling. Williams and Dawe $\mathrm{e}^{39}$ and Sahimi and Yortsos ${ }^{40}$ present brief overviews of the application of fractals in porous media.

Journel and Alabert ${ }^{30}$ investigate the use of sequential indicator simulation as a method for stochastic conditional simulation. Indicator kriging has the advantage of accounting for the spatial connectivity of extreme values of a property, such as permeability. Journel and Gomez-Hernandez ${ }^{34}$ present a study using the sequential indicator simulation method to generate sand-shale sequences in a reservoir. In indicator kriging, the spatial correlation of rock properties is represented by the variogram.

\subsubsection{Simulated Annealing}

The stochastic conditional simulation method used in this study is based on the technique of simulated annealing. Simulated annealing is a simple and flexible optimization technique which has been applied to a diverse number of disciplines. Aarts and Korst ${ }^{26}$ cite Kirkpatrick et al. ${ }^{27}$ and Cerny ${ }^{28}$ as independently introducing the simulated annealing optimization method.

Applying the technique of simulated annealing requires that the system be divided into three components: the objective function, which represents the energy of the system and is also the function to be minimized; the control parameter, which defines the rate at which the system will reach equilibrium; and the interchange mechanism, which is a finite set of perturbations to the variables of the system which tend to result in a change in the objective function. ${ }^{12}$

The goal of simulated annealing is to find a configuration of variables that will yield a global minimum of the objective function. Changes are applied to the system through the interchange mechanism, and the objective function is then evaluated: an increase in the magnitude of the objective function may or may not be accepted according to the probability function proposed by Metropolis et al. ${ }^{29}$ Perez ${ }^{12}$ provides an extensive discussion on the use and derivation of the Metropolis condition. Figure 2 shows a schematic and the steps involved in simulated annealing.

The specified constraints in the simulated annealing method are the conditioning data, the distribution function, and the semi-variogram models for several directions. In the first set of constraints, the conditioning data values are assigned at specified locations. The conditioning data remain fixed at the specified locations throughout the conditional simulation. Mathematically, the constraints corresponding to a number $N_{c}$ of conditioning data can be expressed as ${ }^{12}$

$$
V\left(\vec{\omega}_{c, l}\right)=V_{c}\left(\vec{\omega}_{c, l}\right)
$$

where $V_{c}$ are the values of the conditioning data and $\vec{\omega}_{c, l}$ for $l=1, \ldots, N_{c}$ are the set of locations corresponding to the conditioning data. The conditioning data can be measurements usually taken in a well location such as log or core porosities. 
The specified discrete cumulative distribution function, $F_{0}$, for the simulated variable should obey the following constraint ${ }^{12}$

$$
F\left(V_{f, k}\right)=F_{o}\left(V_{f, k}\right)
$$

for $k=1, \ldots, N_{f}$. The cumulative distribution function represents the probability that the variable is smaller than a given value, and must be a strictly increasing function. In practice, the discrete cumulative distribution function is given by ${ }^{12}$

$$
F\left(V_{f, k}\right)=\frac{1}{N_{f}} \sum_{l=1}^{N_{f}} i\left(\vec{\omega}_{l} ; V_{f, k}\right)
$$

where $N_{f}$ is the number of classes used to discretize the cumulative distribution function and $i$ is an indicator variable defined as

$$
i\left(\vec{\omega}_{l} ; V_{f, k}\right)= \begin{cases}1, & V\left(\vec{\omega}_{l}\right) \leq V_{f, k} \\ 0, & V\left(\vec{\omega}_{l}\right)>V_{f, k}\end{cases}
$$

In the above equation, $V_{f, k}$ is an upper class limit.

The third set of constraints is honoring the spatial correlation structure of the conditioning data. We can specify the semi-variogram of the simulated variable, $V$, as

$$
\gamma_{s}\left(\vec{h}_{l, i}\right)=\frac{1}{2 N_{p}\left(\vec{h}_{l, i}\right)} \sum_{j=1}^{N_{p}\left(\vec{h}_{l, i}\right)}\left[V\left(\vec{\omega}_{j}+\vec{h}_{l, i}\right)-V\left(\vec{\omega}_{j}\right)\right]^{2}
$$

where $\vec{h}_{l, i}=\left(h_{x, l, i}, h_{y, l, i}, h_{z, l, i}\right)$ is the lag distance vector and $N_{p}\left(\vec{h}_{l, i}\right)$ is the number of pairs within the simulation grid corresponding to the lag $\vec{h}_{l, i}$. Indices $i$ and $l$ refer to the direction and the lag distances for that particular direction. The semivariograms models specified in the conditional simulations are denoted by $\gamma_{0}$ and require that

$$
\gamma_{s}\left(\vec{h}_{l, i}\right)=\gamma_{0}\left(\vec{h}_{l, i}\right)
$$

where, as noted previously, $i$ is the direction of the variogram and $l$ is the lag distance for that particular direction.

The objective function is the function to be minimized with the simulated annealing method and it is analogous to the energy of the system. The objective function is defined as a function of the difference between the sample semi-variograms of the simulation variable and the required semi-variogram models. Therefore, by minimizing this objective function, the requirements in Equation 13 can be satisfied.

The objective function, $E$, is defined as ${ }^{12}$

$$
E^{k}=\frac{1}{E_{0}} \sqrt{\sum_{i=1}^{N_{d}} \sum_{l=1}^{N_{h, i}}\left[\frac{\gamma_{s}^{k}\left(\vec{h}_{l, i}\right)-\gamma_{o}\left(\vec{h}_{l, i}\right)}{\gamma_{o}\left(\vec{h}_{l, i}\right)}\right]^{2}},
$$


where, the index $k$ refers to the iteration number within a step and $E_{0}$ is a normalizing constant equal to ${ }^{12}$

$$
E_{0}=\sqrt{\sum_{i=1}^{N_{d}} \sum_{l=1}^{N_{h, i}}\left[\frac{\gamma_{s}^{0}\left(\vec{h}_{l, i}\right)-\gamma_{0}\left(\vec{h}_{l, i}\right)}{\gamma_{0}\left(\vec{h}_{l, i}\right)}\right]^{2}}
$$

In Equation 15, $\gamma_{s}^{0}\left(\vec{h}_{l, i}\right)$ are the semi-variograms of the simulation variable at initial conditions calculated from the initial distribution of the variable. The purpose of the normalizing constant used in Equation 14 is to assign a value of one to the objective function at the initial conditions for any distribution or specifications.

In simulated annealing, the Metropolis condition is used to calculate the probability of the transition between two states of the objective function at a given value of a control parameter analogous to the temperature of the system. The Metropolis condition is given by ${ }^{12}$

$$
P\left(\Delta E^{k}, T^{r}\right)= \begin{cases}1, & \Delta E^{k} \leq 0 \\ \exp \left(\frac{-\Delta E^{k}}{T^{r}}\right), & \Delta E^{k}>0\end{cases}
$$

where, the index $k$ refers to an iteration within a step represented by the index $r$. In Equation (16), the change of the objective function, $\Delta E^{k}$, due to one interchange is ${ }^{12}$

$$
\Delta E^{k}=E^{\prime}-E^{k}
$$

where $E^{\prime}$ is the new objective function and $E^{k}$ is the objective function for iteration $k$ within a step. The control parameter, $T^{r}$, in Equation (16) is calculated according to the following equation ${ }^{12}$

$$
T^{r}=\alpha T^{r-1}
$$

where the index $r$ is the step number, and $\alpha$ is the convergence rate factor. The convergence rate factor is a constant value in the range $0<\alpha<1$ throughout the simulation.

An approach proposed by Perez ${ }^{12}$ to measure the quality of the stochastic conditional simulation was adopted in this study. This approach requires one to visually compare the simulated distribution to the actual distribution as well as using a coefficient to measure the certainty of the simulated distribution.

The certainty coefficient, $C$, between a simulated variable, $V_{s}$, and the true value, $V_{t}$, is defined using the linear correlation coefficient and it is defined as ${ }^{12}$

$$
C=\frac{\sum_{i=1}^{N_{s}}\left[V_{s}\left(\vec{\omega}_{i}\right)-\bar{V}_{s}\right]\left[V_{t}\left(\vec{\omega}_{i}\right)-\bar{V}_{t}\right]}{\sqrt{\sum_{i=1}^{N_{s}}\left[V_{s}\left(\vec{\omega}_{i}\right)-\bar{V}_{s}\right]^{2} \sum_{i=1}^{N_{s}}\left[V_{t}\left(\vec{\omega}_{i}\right)-\bar{V}_{t}\right]^{2}}}
$$


where,

$$
\bar{V}_{s}=\frac{I}{N_{s}} \sum_{i=1}^{N_{s}} V_{s}\left(\vec{\omega}_{i}\right)
$$

and

$$
\bar{V}_{t}=\frac{1}{N_{s}} \sum_{i=1}^{N_{s}} V_{t}\left(\vec{\omega}_{i}\right) .
$$

The certainty coefficient varies between 1 and -1 . The extreme values of the certainty coefficient indicate the following relations between the true and the simulated distributions: (1) for $C=1$ there is a perfect positive linear relation, (2) for $C=0$ there is no correlation and (3) $C=-1$ there is a perfect negative correlation.

\subsubsection{The Greedy Algorithm}

The Metropolis condition effectively enables one to perform exchanges of two values of the simulated variable, even if the change results in an increase of the objective function. The probability of approving an exchange which results in an increase of the magnitude of the objective function decreases as the magnitude of the control parameter is decreased from step to step according to Equation (9). Therefore, for a fixed value of the change of objective function, the probability of accepting an exchange decreases as the control parameter becomes smaller.

The greedy algorithm is a limiting case of simulated annealing because the Metropolis condition is not used. Instead, only the iterations which reduce the magnitude of the objective function are accepted. The greedy algorithm corresponds to the limit when the control parameter approaches zero and the exponential probability function of the Metropolis condition in Equation (16) becomes equal to zero, or

$$
P\left(\Delta E^{k}, T^{r}\right)= \begin{cases}1, & \Delta E^{k} \leq 0 \\ 0, & \Delta E^{k}>0,\end{cases}
$$

Therefore, the convergence rate factor and the maximum number of accepted iterations per step are meaningless in the greedy algorithm.

The advantage of using the greedy algorithm is that computational costs are reduced significantly. This is because all interchanges which result in an increase of the objective function are ignored. This, in effect, reduces the number of variogram calculations that have to be performed for a particular simulation run.

\subsection{Determination of Intermittency Exponent}

This section presents the results of the analysis of actual wellbore data collected from three fields, using the $R / S$ analysis and box-counting techniques.

\subsection{Application to Actual Data}

\subsubsection{Removal of Trends}

Little has been done in the literature that shows the effects of seasonal variations of rock properties on the value of the intermittency exponent. Alternating sand 
and shale sequences create a non-stationarity in the resulting sequence of values, thus making an analysis based on fractional Gaussian noise invalid. One solution is to remove the local means from the trace to be studied in order to eliminate most of the non-stationarity.

To achieve this, zones of different properties based on geology can be visually identified. These different zones will have a local mean associated with each one, which can easily be calculated. For each point in a zone, the local mean is subtracted from the value. The result will become the value for that particular point. Figure 3 illustrates this procedure graphically.

Mathematically, for each value $x_{i N}$ in a particular zone $N$, its new value is given by

$$
x_{i N}=x_{i N}-\overline{x_{N}}
$$

where $\overline{x_{N}}$ is the mean value for zone $N$. The total number of zones is arbitrary and dependent on the judgment of the person doing the adjusting. However, if seasonal or "hole effects" are to be removed from a trace, it is important that the width of a chosen zone does not exceed the actual width of the anomaly on the well log. Doing so will defeat the whole purpose of making the seasonal adjustments since more than one zone of differing means will be accounted for in that particular chosen zone.

An example of a porosity log where each zone is determined and adjusted for seasonal effects is given in Figure 4. In this well log, which is taken from a carbonate field, one can discern three zones of changing local means: a zone of moderate porosity at the bottom, a zone of high porosity in the middle, and a zone of low porosity at the top (regions $\mathrm{A}, \mathrm{B}$, and $\mathrm{C}$ on the graph). A preliminary study of the geology of the region also indicated that three different flow units are present in the reservoir. ${ }^{44}$ Figure 5 shows the $\log$ after seasonal adjustments were done based on the three flow units. There is not much discernable change in the shape of the log from the original, but an $\mathrm{R} / \mathrm{S}$ analysis of the $\log$ before and after seasonal adjustments reveals several interesting observations.

Figures 6 and 7 show the R/S analysis of the log before and after adjustments. A slight anomaly in the $R / S$ analysis plot at a lag distance of around 120 feet reflects the seasonal nature of the data. The measured value of the slope is 1.0742 . This means the data can be described using an $\mathrm{fBm}$ model with an intermittency exponent of 0.0742 . Figure 7 shows the $R / S$ analysis plot after seasonal adjustments have been made. One can observe that the anomaly has been eliminated. The plot, however, also shows that the long-term persistence starts to deviate to a slope of 0.5 (corresponding to white noise) at a lag distance of about 150 feet. This behavior is consistent with the behavior observed by Feder. ${ }^{13}$ The measured $H$ exponent value is 0.0359 .

Figures 8 and 9 show the same log analyzed using the box-counting method. Figure 8 shows the plot of number of boxes versus the box scaling factor on a loglog scale for the original data trace. The calculated $H$ exponent value is 0.8796 . 
In Figure 9, after seasonal adjustments, the measured $H$ exponent value is 0.7832 . There seems to be a discrepancy between the $H$ values measured using box-counting and $\mathrm{R} / \mathrm{S}$ analysis for this particular porosity trace. The reasons for this are not clear, except that the box-counting method, after grading the sequence, would result in values of the slope less than one. Perhaps the analysis of raw data using boxcounting method may result in a value of $H$ closer to the one predicted by R/S analysis. Considering that the number of sample points in the trace is limited, it is difficult to say which method is better based on the determination of $H$ only. As explained later, the approximation of $H$ is based on the comparison between the experimental and model variograms using the $H$ values determined from both methods.

One point that should be noted is that no attempt has been made to analyze the data on the basis of different porosity zones. (This entails analyzing the data for different porosity zones and then finding the average $H$ exponent for each of the different zones.) One limiting factor with this approach is that each well $\log$ can be divided into different regions that might not necessarily correspond with other wells in the region. Therefore, the approach is to treat each well log separately.

Perhaps a better indicator of the ability of the different methods to reliably estimate the $H$ exponent would be to compare the $\mathrm{fGn}$ (or $\mathrm{fBm}$ ) variogram fit to the actual data being studied. This also serves another purpose: to observe the effects of removing the seasonal trends on the shape of the variogram itself. A zone with severe "hole effects" indicates the presence of a series of different porosity or permeability zones in the reservoir. If a zone has been seasonally adjusted properly, most, if not all, of the hole effects would be eliminated.

Calculation of the $\mathrm{fBm}$ or $\mathrm{fGn}$ models involves one or two parameters: the smoothing factor, $\delta$, applicable only to the $f \mathrm{Gn}$ model, and the variance of an infinite sample, $V_{H}$, applicable to both models. For simplicity, the resolution of the porosity log is assumed to be 3 feet; therefore $\delta$ can be set equal to this value. Determination of $V_{H}$ involves back calculating it from Equations (5) and (7). The lag distance for which this calculation is made is assumed to be the thickness of the zone itself. This, in effect, requires that $\gamma(h)$ should then be set equal to the variance or "sill" calculated from the well log. $H$ is of course determined from R/S analysis or box-counting.

Figures 10 and 11 show the calculated variograms for a porosity log taken from a sandstone environment. Only the raw porosity values are used ( $\mathrm{fGn}$ model). From Figure 10, a hole effect can be observed between lag distances 20 and 65 feet. The estimated $H$ values calculated from R/S analysis and box-counting were $0.1490(\mathrm{fBm})$ and $0.9929(\mathrm{fGn})$, respectively. Even for this non-adjusted data, boxcounting seems to better fit the variogram plot. Figure 11 show the variogram fit after seasonal adjustments are done. The variogram now appears more regular, and the $H$ exponents are recalculated to be 0.6808 for box-counting, and 0.9183 for $\mathrm{R} / \mathrm{S}$ analysis. The box-counting method still gives the better variogram fit. 
Figures 12 and 13 show the variogram fits for two well logs taken from a carbonate environment. In all of the cases, it can be observed that the box-counting method provides the most reliable fit through the variogram plots. In cases where the seasonal trends are not too discernable (Figure 12), the effect of seasonal adjustments would not have much of an effect on the shape of the variogram plots. However, the $H$ exponent values are affected significantly. For box-counting, the mean intermittency exponent values shift from 0.8833 without seasonal adjustments, to 0.7597 after seasonal adjustments. Because of the reliability of box-counting to estimate the best values of $H$, it is subsequently chosen for further use in the stochastic conditional simulations in the next main section. The next three sections present the $H$ exponent analyses of three different fields.

\subsection{Analysis of Hugoton Field}

\subsubsection{Geology}

The Hugoton Field is composed of repeated sequences of interlayered carbonates, siliciclastics, and evaporites which were deposited on a gently dipping, lowrelief, shallow marine carbonate ramp. The producing formation consists of three reservoir flow units or layers which are mainly composed of dolostone, each capped with siltstones, mudstones, and shale intervals that act as barriers to vertical flow, thus no crossflow occurs between the separate flow units. ${ }^{44}$

Good drainage occurs within each individual flow unit because of the areal continuity of each unit. A laterally continuous intercrystalline pore system within each flow unit, which resulted from widespread dolomitization that was not constrained by depositional facies boundaries, promotes good areal continuity of reservoir porosity and permeability within each of the dolomitized carbonate intervals. ${ }^{44}$

The producing formation is characterized by interlayered carbonates, layers of contrasting permeability, and no crossflow between the flow units. Wells generally produce from depths between 2700 and 3000 feet and are located within a 640-acre spacing pattern. In the study area, layer 1 ranges from 60 to 65 feet thick, layer 2 ranges from 50 to 60 feet thick, and layer 3 ranges from 40 to 50 feet thick. ${ }^{44}$ Figure 14 shows a field map of the zone to be studied. Table 1 summarizes the available data and the geology of the field, as well as pertinent parameters related to the field.

\subsubsection{Analysis}

The data studied are obtained from the 31 porosity logs provided. $H$ exponent estimations are done for all the logs before and after adjustments for seasonal effects were made. For this field, the zones of varying local means chosen for adjustments are largely affected by the presence of three flow units in the reservoir. Figures 15 and 16 show one of the porosity logs before and after seasonal adjustments. The three zones roughly correspond to the three layers $\mathrm{A}, \mathrm{B}$, and $\mathrm{C}$. The $H$ exponents for this porosity log before adjustment are 0.9060 for box-counting and 1.0807 for $\mathrm{R} / \mathrm{S}$ analysis. After adjustments, the values are 0.7675 for box-counting and 0.9863 
Table 1

Summary of available data for Hugoton Field

Number of wells

Average depth

Average thickness

of producing zone

Formation type

Available data
31

2785 feet

150 feet

interlayered carbonates

3 flow units

no cross-flow

gamma ray

$\log$ porosity

core porosity

core permeability 
for $R / S$ analysis.

Table 2 presents the results of the field-wide analyses of Hugoton Field using box-counting. A total of 31 porosity logs are studied and their $H$ exponents estimated. The porosity logs are then adjusted for seasonal effects using the procedure delineated in the previous section and analyzed again. It can be seen the mean value of the $H$ exponent is significantly affected by the normalization procedure. In particular, a decrease in the mean value is observed. Because of the strong seasonal trends observed in Hugoton Field, removal of the means result in a narrower range of $H$ values. The variance without adjustment is found to be 0.001374 . After applying seasonal adjustments, the variance decreased significantly to 0.000388 . A similar behavior can be observed in Table 3, which presents the results for $\mathrm{R} / \mathrm{S}$ analysis. However, the decrease in variance is not as apparent as for box-counting.

Much of the effects of seasonal adjustments can be better appreciated by studying a histogram of the $H$ exponent values before and after normalization. Figures 17 and 18 present histogram plots of the distribution of $H$ exponent values before and after seasonal adjustments for box-counting. Figure 17 is a distribution of the $H$ values before adjustment for box-counting; Figure 18 presents the results after the logs have been adjusted. A narrower range of the $H$ exponent values is observed. A shift to a lower mean $H$ value is also discernable from the plots.

\subsection{Analysis of Burbank Field}

\subsubsection{Geology}

The North Burbank Field was discovered near Burbank, Oklahoma, in May, 1920, by the Marland Oil Company. The field was developed rapidly on a 10 acre spacing. Wells were drilled with cable tools and were produced at maximum capacity by open flow. Some wells were stimulated with nitroglycerin during completion or when they stopped flowing. By July 1923, the field production reached its peak of 122,000 barrels per day with the reservoir energy being supplied by solution gas drive. Production declined rapidly as a result of the flush production. Gas injection was started in some sections in 1926, but proved to be rather unsuccessful. By November 1, 1949, the cumulative production from the North Burbank Field and the Stanley Stringer amounted to $221,104,298$ barrels. Only 17,000,000 barrels were produced as a result of the gas injection.

The reservoir produces from the Burbank Sandstone member of the Cherokee Shale at a depth of about 3000 feet. The field lies in a north-northwest trend and is 12 miles long. 4 to 5 miles wide, and covers an area of 23,000 productive acres. ${ }^{46}$ In 1937, a study concluded that the Burbank sand was deposited as a series of overlapping beach deposits during the Teeter-Quincy and Sallyards-Lamont stages of the Cherokee Sea. ${ }^{47}$ However, recent geologic study has shown the reservoir to be of fluvial origin with some contribution from marine incursions having occurred during deposition. The massive sand body was created by the superimposed effect of a series of channels cut laterally into each other. The resulting structure is an undulating monocline dipping at a rate of $35 \mathrm{ft} /$ mile in a westerly direction. ${ }^{48}$ The 


\section{Table 2}

Summary statistics of intermittency exponent values

for Hugoton field

Box-counting method

\begin{tabular}{llll} 
Parameter & Without normalization & With normalization \\
\cline { 2 - 3 } & 31 & 31 \\
Mean & 0.885157 & 0.771484 \\
Variance & 0.001374 & 0.000388 \\
Standard Deviation & 0.037074 & 0.019708 \\
Average Deviation & 0.026698 & 0.014858 \\
Skewness & -0.260090 & -0.800875 \\
Kurtosis & 1.1179027 & 0.549853 \\
& $\cdot$ & \\
\hline
\end{tabular}


Table 3

Summary statistics of intermittency exponent values for Hugoton field

$R / S$ analysis method

Parameter

Number

Mean

Variance

Standard Deviation

Average Deviation

Skewness

Kurtosis

\section{Without normalization}

31

1.046313

0.001832

0.042807

0.034381

0.052231

$-0.585267$
With normalization

31

0.971304

0.001175

0.034278

0.027428

0.094578

$-0.927165$ 
limits of the field are determined by an abrupt sand to shale lithology change on the east and a tilted oil-water contact in combination with a permeability barrier on the west. ${ }^{46}$

The producing zone consists of fine-grained quartz sand, loosely cemented by magnesium, iron, and calcium carbonate, and locally by silica, dolomite, or calcite. In addition to quartz the sand contains one percent mica; traces of feldspar, zircon, chlorite, glauconite, hornblende, rutile, magnetite, pyrite, and epidote; $10-20$ percent detrital rock fragments (chert, shale, and schist); and a trace to 10 percent of carboneceous material. ${ }^{47}$.

The sand averages 42 feet in thickness, with average porosity of 16.8 percent and permeability of $50 \mathrm{md}$. The formation is vertically and areally heterogeneous. Also, east-west tending natural fractures are thought to exist throughout the field. These are indicated by an aerial study of the joints which occur on the surface above the North Burbank Field and by the rapid channeling of water which was detected during waterflooding. ${ }^{49}$ Figure 19 shows a field map of the zone to be simulated. Table 4 summarizes the available data and the geology of the field, as well as pertinent parameters related to the field.

\subsubsection{Analysis}

Core data for 45 wells are available for analysis. Data for each well log is not abundant, however, due to the thinness of the producing zone. Figure 20 shows an unadjusted well porosity trace taken from the field. The zones $\mathrm{A}, \mathrm{B}$, and $\mathrm{C}$ refer to the three discernable layers present from the log. A high porosity section exists somewhere around the middle of the zone, resulting in region of varying mean. After applying the seasonal adjustments discussed previously, the porosity trace is plotted again in Figure 21. It can be observed that the technique does an outstanding job of removing a lot of the seasonal effects.

The measured $H$ exponent value of the trace in Figure 20 is 0.9265 . This means the trace can be described using an $\mathrm{fGn}$ model. After adjustments, the measured $H$ exponent value decreases to 0.8311 . The remaining 44 wells are adjusted similarly and the results are as tabulated in Tables 5 and 6 . The mean value of the $H$ exponents before seasonal adjustments are performed is found to be 0.9312 using box-counting (Table 5). For R/S analysis, the mean value is 1.1846 (Table 6) After seasonal adjustments are made, the mean value of the $H$ exponent decreases to 0.9748 for $R / S$ analysis and 0.7731 for box-counting. Both values are remarkably similar to the mean values of the $H$ exponent after seasonal adjustments for Hugoton Field, which is a carbonate environment.

Since the seasonal effects in Burbank are not particularly strong, the calculated variances of the $H$ exponent before and after seasonal adjustments do not differ very much. For box-counting, variance before and after adjustments is calculated to be 0.007247 and 0.006684 respectively. For $R / S$ analysis, the variance decreases from 0.031311 to 0.030812 . The significant difference in the values of the variances 
Table 4

Summary of available data for Burbank Field

Number of wells

Average depth

Average thickness

of producing zone

Formation type

Available data
45

3000 feet

42 feet

Burbank sandstone

of fluvial origin

fine-grained quartz sand

single flow unit

core porosity

core permeability

$\log$ porosity 


\section{Table 5}

Summary statistics of intermittency exponent values for Burbank field

Box-counting method

\begin{tabular}{llll} 
Parameter & \multicolumn{1}{l}{ Without normalization } & With normalization \\
\cline { 2 - 3 } & 45 & 45 \\
Mean & 0.931220 & 0.773067 \\
Variance & 0.007247 & 0.006684 \\
Standard Deviation & 0.085128 & 0.081758 \\
Average Deviation & 0.068381 & 0.064981 \\
Skewness & -1.142375 & 0.334954 \\
Kurtosis & 0.0519910 & -0.068210
\end{tabular}


Table 6

Summary statistics of intermittency exponent values for Burbank field

$R / S$ analysis method

Parameter

Number

Mean

Variance

Standard Deviation

Average Deviation

Skewness

Kurtosis
Without normalization With normalization

45

45

1.184589

0.974761

0.031311

0.030812

0.176948

0.175533

0.144614

0.131661

$-0.384180$

0.394912

$-0.580046$

0.516459 
for both methods indicate the consistency of box-counting in estimating the $H$ exponent. A larger variance in $H$ exponent values for $\mathrm{R} / \mathrm{S}$ analysis reflects the larger spread in measured estimates of the $H$ exponent compared to box-counting.

\subsection{Analysis of Sable Field}

\subsubsection{Geology}

The Sable (San Andreas) Unit is operated by the Atlantic Richfield Company, and is located approximately 6 miles northwest of Plains, in Yoakum County, Texas. The unit comprises about 1920 surface acres. The producing reservoir is the San Andreas dolomite (STOOIP 22.7 MMbbl) which has an average depth of approximately 5200 feet.

Production by solution gas drive first began in December of 1957 upon completion of the Mattie B. Lewis No. 1, the now Sable No. 1. In February of 1967, the field was unitized and secondary operations began on a peripheral waterflood pattern. Cumulative oil production by January 1, 1969, was 1.755 MMbbl. Continued development and interior injection began in mid-1971 and resulted in a peak production rate of 1600 BOPD achieved during April of 1977. The next year a CO2 flood economic feasibility study was performed, and in $1984 \mathrm{CO} 2$ injection began with infill drilling covering about 20 acres. ${ }^{45}$

There are currently 65 wells in the Sable Field, and 32 are active producers. In addition, 17 wells have been cored. Current oil production rate is 660 BOPD.

The Sable Unit (San Andreas) reservoir lies in a structural anticline trap with the oil column reaching a maximum thickness of 108 feet in Sable No. 12. Production data, supported by resistivity logs, indicate that the water-oil-contact dips slightly from west to east across the reservoir. The reservoir itself is capped by a dense dolomite. A study done by ARCO concluded that:45

(1) The reservoir is composed of average quality San Andreas carbonate with a highly random distribution of permeability and porosity, with no evidence of significant permeability channels.

(2) There are zones of random, but higher than average permeability; and there are zones of random, but lower than average permeability.

(3) There is a low porosity region at the top of each section, and there is a high porosity region somewhere in the middle of each section.

The study also found that the carbonate is heterogeneous at every scale, micro to macro, and that randomness of the unit also occurs areally, aside from vertically. A reservoir description with constant permeability and porosity layers is obviously inappropriate for a reservoir with this degree of heterogeneity. Figure 22 presents a field location of the the wells chosen for the study. Table 7 summarizes the data and pertinent information with regard to the field.

\subsubsection{Analysis}

All 17 core porosities are analyzed and adjusted for seasonal effects. Figures 23 
Table 7

Summary of available data for Sable Field

Number of wells

Average depth

Average thickness

of producing zone

Formation type

Available data
16

5200 feet

40 feet

San Andreas carbonate

dolomitic cap rock

single flow unit

anticline structure

gamma ray

neutron $\log$

core porosity

core permeability 
and 24 provide an example of a porosity trace for one of the wells in the field before and after seasonal adjustments, respectively. The producing zone is also divided into three discernable sections $A, B$, and $C$. The shape of the trace after adjustments (Figure 24) shows that most of the seasonal effects have been eliminated, with an equal amount of excursions around the mean value of zero for the porosity peaks. This attests to the effectiveness of the normalization technique.

Tables 8 and 9 show the summary statistics of the $H$ exponent analysis using the box-counting method and R/S analysis. In Table 8 , as in the previous two fields, it can be observed that the value of the mean decreases after seasonal adjustments are made. The variance displays an increase from 0.003973 to 0.005922 , however. Most interesting is the fact that the value of the mean after seasonal adjustments calculated using box-counting is very similar to the values of the mean for the previous two fields. The mean intermittency values all fall in the range between 0.75 to 0.80 after seasonal adjustments. These values indicate that such a range can be used for generating stochastic conditional distributions of property values regardless the type of the sedimentary environment.

\subsection{Loudon Field}

Although no $H$ exponent analyses are done for the Loudon Field, a brief discussion of its geology will be presented here for the benefit of the section in Section 4 that deals with the stochastic conditional simulation of the field.

The Loudon Field is located in Fayatte County, Illinois. The field produces from the Weiler sand which is of marine origin, and several shale stringers impede vertical communication between 4 small, though discernable, layers. The average thichness of the producing zone is approximately 60 feet. Barlow limestone marks the top of the producing zone, at around a depth of 1530 feet. The north-east of the Weiler base consists of a sand-filled erosional channel cutting into the underlying Point Creek shale, while the south-east portion represents a steepened depositional slope. The top of the Weiler sand is also marked by sand-filled erosional channels, created during sea-level lowering.

Table 10 summarizes the geology of the field and the available data. Figure 25 presents the part of the field considered in the three-dimensional stochastic conditional simulations.

\section{$\underline{3.6 \text { Summary }}$}

Several observations can be noted from the results in this section. We can state that::

(1) Based on the match between the experimental and theoretical variograms of the field data, the box-counting method more accurately predicts the trend. Therefore it should be used to analyze the wellbore data. The $R / S$ analysis can still be used for detecting periodic elements in a data set. 
Table 8

Summary statistics of intermittency exponent values

for Sable field

Box-counting method

Parameter

Number

Mean

Variance

Standard Deviation

Average Deviation

Skewness

Iurtosis
Without normalization With normalization

16

0.835006

0.003973

0.063029

0.049983

0.295944

$-1.076510$
16

0.793954

0.005922

0.076951

0.064584

$-0.394313$

$-1.238816$ 


\section{Table 9}

Summary statistics of intermittency exponent values for Sable field

$R / S$ analysis method

\begin{tabular}{lll} 
Parameter & Without normalization & With normalization \\
\cline { 2 - 3 } Number & 16 & 16 \\
Mean & 0.921266 & 0.884810 \\
Variance & 0.013791 & 0.008082 \\
Standard Deviation & 0.117435 & 0.089899 \\
Average Deviation & 0.094818 & 0.075791 \\
Skewness & 0.099087 & -0.013495 \\
Kurtosis & -1.140491 & -1.574277 \\
\multicolumn{1}{l}{} & & \\
\hline
\end{tabular}


Table 10

Summary of available data for Loudon Field

Number of wells

Average depth

Average thickness

of producing zone

Formation type

Available data
45

1530 feet

60 feet

sandstone

marine origin

4 small layers

core porosity

$\log$ porosity 
Therefore it should be used to analyze the wellbore data. The $R / S$ analysis can be still be used for detecting periodic elements in a data set.

(2) The seasonal effects have a significant impact on the value of the intermittency exponents. For reservoirs with significant seasonal trends, the intermittency exponents show a narrower range after removal of seasonal trends. Also, the value of $H$ is much more consistent.

(3) If seasonal trends are not significant, the variation of $H$ is not affected. The average value of $H$, however, may still be affected. The results seem to indicate a decrease in the means and variances of the $H$ exponent values once seasonal adjustments have been made.

(4) Regardless the geological makeup of the field, the average value of $H$ calculated using box-counting is surprisingly very close to 0.75 to 0.80 for all the fields once the seasonal trends are removed. One may therefore use this value for generating stochastic conditional distributions of rock properties.

\subsection{Stochastic Conditional Simulation}

This study provides a set of procedures to be followed in the stochastic conditional simulation of reservoir properties. Factors to be taken into account are the removal of the non-stationarity in the vertical well data and the selection of a correct horizontal spatial correlation structure based on the structure determined from vertical well data.

\subsection{Framework for Stochastic Conditional Simulation}

Frequently, the determination of a reliable horizontal correlation model can be a trial-and-error procedure. This is primarily due to the fact that not much is known about the distribution of properties in the horizontal direction. Therefore, different models have been assumed by numerous researchers. However, in real life, taking into account the relative uncertainty in the distribution of horizontal properties, it would make more sense to experiment with some likely correlation structure than to adopt one model and use that throughout the entire study. This procedure will help to pinpoint the most reliable structure to be used in the final three dimensional simulation of the field.

The framework for applying stochastic conditional simulation consists of the following steps:

(1) Removal of trends observed in vertical well logs that might result in biased estimates of the intermittency exponent.

(2) The use of reliable intermittency exponent estimation techniques that are both robust and fairly insensitive to small sample distributions.

(3) The use of a combination of the greedy algorithm and a bilinear interpolation technique to improve CPU usage in simulated annealing runs.

(4) Determining the most reliable horizontal correlation model based on different, small, two-dimensional simulations of the field.

(5) Using the same correlation model determined from Step (3) to perform a regular three-dimensional stochastic conditional simulation of the entire field. 
The importance of Step (1) has been discussed in the previous chapter. Removing the local means in different zones of varying property distributions will enable one to remove the bias in the intermittency exponent calculations. It has already been shown that seasonal adjustments can significantly affect the value of the estimated $H$ exponent, as well as provide more consistent results for a particular region of interest. Also, no technique can be robust enough to take into account the periodic effects in a data trace.

Step (2) derives from the conclusions reached in the previous chapter. Although many techniques exist for the estimation of the $H$ exponent, some have been found to be more reliable than the others. Preliminary results from Chapter 3 have indicated that the $R / S$ analysis and box-counting method can reliably predict the value of the intermittency exponent. However, based on the match between analytical and experimental variograms for a real dataset, the box-counting method appears to predict the $H$ exponent value more accurately. It is recommended that the box-counting be used for all subsequent analysis of property traces.

Step (3) will be discussed in the next section. Suffice it to say that significant savings in CPU usage can be achieved through the use of a combination of the greedy algorithm and a linear or bilinear interpolation technique to generate an initial pattern for a stochastic conditional simulation run. As discussed in Chapter 2, the greedy algorithm is a limiting case of the simulated annealing algorithm, in which all swaps resulting in an increase in the energy function are rejected by the Metropolis condition (refer to Equation 2.39). The linear or bilinear interpolation technique enables one to generate an initial pattern of values that honors the original conditioning data and its distribution function.

As noted at the beginning of the chapter, the determination of a horizontal spatial correlation structure is frequently a trial-and-error procedure. Step (4) achnowledges this fact by recommending that small, two-dimensional stochastic conditional simulations should be done beforehand in order to have a feel for the best correlation model to use. The advantage to doing this is obviously to save on CPU usage before extensive three-dimensional stochastic conditional runs are performed. These two-dimensional runs should preferably be field-wide in the sense that different spatial directions should be considered.

Step (4) involves a pitfall that most people tend to encounter. If an fGn model is to be used in both the vertical and horizontal directions, it is imperative that similar values of the smoothing factor, $\delta$, be used. Doing otherwise would result in the support volume being unequal for well log comparison purposes. Step (5) follows from Step (4). Once the best horizontal correlation model has been determined, the same horizontal model should therefore be used for the field-wide three-dimensional stochastic conditional simulations.

In this study, all simulated annealing runs were performed by using SAN3D, a FORTRAN program developed by Perez ${ }^{12}$. The original program was modified to use the greedy algorithm and to read in an initial pattern prepared by bilinear or 
linear interpolation.

4.2 Bilinear Interpolation

Optimized simulated annealing is somewhat of a misnomer. Annealing requires extensive CPU usage and the time taken to perform simulation runs tend to increase dramatically as the number of grid blocks is increased. This makes the algorithm almost useless unless the area to be simulated is small, or powerful, vectorized computers are utilized. This study attempts to retain the flexibility and reliability of the simulated annealing algorithm while decreasing CPU usage significantly.

The problem was attacked in two different ways: the use of the greedy algorithm, and using a bilinear interpolation technique with an added Gaussian random element to generate an initial pattern for the distribution. Use of the added random element will hopefully retain the original distribution of the conditioning data. For two dimensional simulations, simple linear interpolation can be used.

The interpolation technique simply involves linearly or bilinearly interpolating the unknown values in the interwell distribution and then adding a random Gaussian element multiplied by the standard deviation of the the difference between two farthest locations in the simulation region. This also allows one to back calculate the variance of an infinite sample associated with the $\mathrm{fGn}$ or $\mathrm{fBm}$ spatial correlation structure. The program interpolates layer by layer, starting from the top of the zone of interest.

The problem of two-dimensional interpolation can be defined in the following manner: $:^{50}$

$$
y a[j][k]=f(x 1 a[j], x 2 a[k])
$$

where $f$, the function at some untabulated point $(x 1, x 2)$, is to be estimated. $y a$ is a matrix of functional values with $j$ varying from 1 to $m$ and $k$ varying from 1 to $n$. The arrays $x 1 a$ and $x 2 a$ are location arrays of lengths $m$ and $n$ respectively.

The desired point to be estimated is surrounded by a grid square of four tabulated points, which for convenience will be numbered 1 to 4 . More precisely, if

$$
\begin{aligned}
& x 1 a[j] \leq x_{1} \leq x 1 a[j+1] \\
& x 2 a[k] \leq x_{2} \leq x 2 a[k+1]
\end{aligned}
$$

defines $j$ and $k$, then

$$
\begin{aligned}
& f_{1} \equiv y a[j][k] \\
& f_{2} \equiv y a[j+1][k] \\
& f_{3} \equiv y a[j+1][k+1] \\
& f_{4} \equiv y a[j][k+1]
\end{aligned}
$$

The formulas for bilinear interpolation are as follows: $:^{50}$

$$
\begin{aligned}
t & \equiv\left(x_{1}-x 1 a[j]\right) /(x 1 a[j+1]-x 1 a[j]) \\
u & \equiv\left(x_{2}-x 2 a[k]\right) /(x 2 a[k+1]-x 2 a[k])
\end{aligned}
$$


such that $t$ and $u$ each lie between 0 and 1 . The estimated value within the interior grid is then

$$
f\left(x_{1}, x_{2}\right)=(1-t)(1-u) f_{1}+t(1-u) f_{2}+t u f_{3}+(1-t) u f_{4}
$$

For the one-dimensional case (linear interpolation), Equation (28) decomposes into

$$
f\left(x_{1}, x_{2}\right)=(1-t) f_{1}+t f_{2}
$$

since $u=0$. As the interpolating point wanders from grid square to grid square, the interpolated function value changes continuously. However, the gradient of the interpolated function changes discontinuously at the boundaries of each grid square.

The initial pattern is an array of values calculated from bilinear (or linear, for the one-dimensional case) interpolation with an added random Gaussian element. For each level, the random component is added to each of the interpolated values according to the following formula

$$
f_{r}\left(x_{1}, x_{2}\right)=f\left(x_{1}, x_{2}\right)+z \sigma
$$

where $f_{r}$ is the interpolated value with the random component, $f$ is as defined by Equation (28), $\sigma$ is the standard deviation of the difference of values between the longest well pair in the region to be simulated, and $z$ is a random number drawn from a normal distribution having a mean of zero and unit variance.

As discussed in Section 2, the approach used by Perez ${ }^{12}$ will be used to measure the quality of the stochastic conditional simulations. Several wells will be chosen as "test" cases, which means that they will be visually compared to synthetic wells obtained using the conditional simulation technique. Also, a statistical measure of the accuracy of the predictions will be used (refer to Equation 19).

\subsection{Determination of Simulation Parameters}

\subsubsection{Parameters in 2-D Simulations}

The variance of an infinite sample associated with the $\mathrm{fBm}$ or $\mathrm{fGn}$ horizontal correlation structure can be calculated from Equations (5) and (7). For the twodimensional stochastic conditional simulations, one can use $\bar{H}$ as simply the average intermittency exponent for the pair of wells, or

$$
\bar{H}=\frac{1}{2}\left(H_{1}+H_{2}\right)
$$

where $H_{1}$ and $H_{2}$ are the estimated intermittency exponent values for the pair of wells in the two-dimensional region.

In the vertical direction, the lag distance $h$ is the length of the zone of interest, and $V_{H}$ is calculated from Equation (7) assuming a smoothing interval of 3 feet and an fGn correlation model. The average variance, $\overline{\sigma^{2}}$, between the two wells is 
calculated and set equal to the variogram value at which the interval distance is reached, or

$$
\overline{\sigma^{2}}=\frac{1}{2}\left(\sigma_{1}^{2}+\sigma_{2}^{2}\right)
$$

and

$$
\begin{aligned}
\gamma(h) & =\frac{1}{2} V_{H} \delta^{2 \bar{H}-2}\left[2-\left(\frac{\left|h_{\max }\right|}{\delta}+1\right)^{2 \bar{H}}+2\left|\frac{h_{\max }}{\delta}\right|^{2 \bar{H}}-\left(\frac{\left|h_{\max }\right|}{\delta}-1\right)^{2 \bar{H}}\right] \\
& =\overline{\sigma^{2}}
\end{aligned}
$$

where $h_{\max }$ is the distance between two wells. Equation (33) permits one to back calculate the value of $V_{H}$, which is needed in as input into the simulated annealing program. A reasonable value of $\delta$ can be assumed, based on the an estimate of the resolution of the property trace. For porosity logs, $\delta$ can be assumed to be 3 feet.

For the horizontal case, the variance of the difference between the two wells is first calculated and set equal to $\gamma(h)$ in either of Equations (5) or (7). The lag distance $h$ is the horizontal distance between the two wells. If an $\mathrm{fGn}$ model is used in the horizontal direction, the same smoothing factor as that used in the vertical direction would have to be incorporated in order to be consistent in the comparisons.

For each level, $i$, in the two-dimensional cross-section, the variance of the difference can be calculated using the following formula

$$
\sigma^{2}=\frac{1}{N} \sum_{i=1}^{N}\left(\delta_{i}-\bar{\delta}\right)^{2}
$$

where $N$ is the total number of layers (or the depth index). The mean difference between the two wells, $\bar{\delta}$, is defined as

$$
\bar{\delta}=\frac{1}{N} \sum_{i=1}^{N}\left|x_{i 1}-x_{i 2}\right|
$$

where $x_{i 1}$ and $x_{i 2}$ are the values of the property at each level $i$ between the well pair. The value of $\delta_{i}$ is defined as

$$
\delta_{i}=\left|x_{i 1}-x_{i 2}\right|
$$

which is the difference of the property at each level $i$.

\subsubsection{Parameters in 3-D Simulations}

For three-dimensional stochastic simulations, field-wide horizontal porosity variograms should first be generated. This will serve to identify the lag distance at 
which the variance of an infinite sample can be calculated. For example, a pure nugget will indicate that the minimum interwell distance can be used to calculate $V_{H}$, since the sill would have been reached at that lag distance. Calculation of $V_{H}$. for the vertical vertical direction for three-dimensional simulations is similar to that for the two-dimensional simulation.

For the horizontal direction, the variance of the difference between the two longest well pairs in the region to be simulated is calculated and set equal to $\gamma(h)$ in either of Equations (5) and (7). Equation (34) can be used to calculate the variance of the difference. If an $\mathrm{fGn}$ model is used in the horizontal direction, the same smoothing factor as that used in the vertical direction would have to be incorporated in order to be consistent in the comparisons. The scaling factor, $V_{H}$ can be back calculated from Equations (5) or (7).

\subsection{2-D Simulations}

\subsubsection{Simulation of Hugoton Field}

As discussed in Section 2, one of the advantages of the greedy algorithm is that computational costs are reduced significantly. Since all interchanges of the properties which result in an increase of the objective function are ignored, the effective number of variogram calculations that have to be performed are significantly reduced. To illustrate this, each of the simulation runs for the Hugoton Field is timed using a program profiler. A profiler measures the total execution times of each simulation run in units of CPU seconds.

Figure 26 shows a field map of the region under study. A well pair is taken from the northern section of the field (Wells A2 and E2). The spacing between the well pair is 21120 feet. Well B2 is chosen as the test well for the simulation. The logs are seasonally adjusted to remove the local trends, and a $H$ exponent analysis is performed. Table 11 summarizes the simulation parameters used in the simulations.

Since an $\mathrm{fGn}$ model is assumed for the vertical direction with a smoothing factor of 3 feet, $V_{H}$ can be calculated from Equation (7) by setting it equal to the average variance calculated from the two wells. This variance is found to be 24.59. The variance of the difference between the well pair is calculated using Equation (34) and is found to be 34.2956. Two horizontal structures are assumed in the simulations: $\mathrm{fBm}$ and $\mathrm{fGn}$. This is done in order to determine the best model to use in the horizontal direction. $V_{H}$ is again calculated using Equations (5) and (7). For an $\mathrm{fBm}$ model and a lag distance of 21120 feet, it is found to be $5.3053 \mathrm{E}-06$. For an fGn model, using $\delta=3$ feet, it is calculated to be 56.2045 (refer to Table 11). In both of the above cases, the variogram value is set equal to the variance of the difference between the two wells.

Figures 27 and 28 illustrate gray scale plots of the linearly interpolated values before and after adding the Gaussian element. The added Gaussian element results in a more heterogeneous distribution of values, as to be expected. Figures 29 and 30 illustrate the distribution of the conditioning data, and the generated initial pattern, respectively. From the figures, the linear interpolation technique seems to 
Table 11

Summary of simulation parameters for 2-D stochastic conditional simulation in Hugoton Field

Well pair - A2 and E2

Distance between well pair

Variance of difference

Average $H$ value

Blocks in $x$ direction

Blocks in $z$ direction

Blocks in $y$ direction

Average vertical variance

Smoothing factor

Scaling factor $(\mathrm{fGn})$

Scaling factor ( $\mathrm{fBm}$ )
21120 feet

34.2956

0.7874

51

399

1

24.5958

3 feet

$56.204 \mathrm{E}+00$

5.3053E-06 
retain the original distribution of the conditioning data fairly accurately. In this case, the standard deviation of the difference between the two wells was multiplied by the random Gaussian element and added back to the interpolated values.

In addition to simulations performed using the greedy algorithm, two other algorithms are also studied: conventional annealing algorithm, and interpolation with the conventional annealing algorithm. For the first case, the program is instructed to generate its own initial pattern based on the distribution of the conditioning data, and the conventional algorithm is used. In the second case, an initial pattern is generated using linear interpolation with added random element; however, the same conventional algorithm is also used. The two horizontal correlation structures studied are $\mathrm{fGn}$ and $\mathrm{fBm}$.

In Figures 31 to 36, comparisons are made between the greedy algorithm, no interpolation, and simulation with interpolation. Certainty coefficients are also calculated for two horizontal correlation structures, $\mathrm{fBm}$ and $\mathrm{fGn}$. From the figures, predictions obtained using a $\mathrm{fGn}$ horizontal structure seem to be more reliable than those obtained using an $\mathrm{fBm}$ structure. The plots for the $\mathrm{fBm}$ horizontal structure display erratic behavior and do not capture the trends. The certainty coefficients for $\mathrm{fGn}$ are also markedly higher as the figures indicate. The calculated CPU time for the greedy algorithm is 611.12 seconds, compared to 4248.50 seconds for the simulation with interpolation and 5573.60 seconds for normal simulation without any interpolation (see Table 12).

Simulations in other principal directions should preferably be done in order to determine the best horizontal correlation structure for the entire region. The previous example only represents the direction along the east-west plane of the field. Two other directions are also simulated in two dimensions: north-south, and diagonally. Referring to Figure 26 again, two well pairs are taken from each of these directions. In the north-south direction, the region between well pair A2 and U2 is simulated. In the diagonal direction, the region between well pair J2 and V2 is simulated similarly. Wells F2 and N2 represent the test wells for these 2D simulations. Table 13 presents the results for the simulations. Interesting enough, the correlation coefficients for both directions show that $\mathrm{fGn}$ is the best horizontal correlation structure to use for this field, where the coefficients are larger than that for $\mathrm{fBm}$.

Several observations that can be noted from these simulation runs are that the best model to use for a carbonate environment seems to be the fGn structure. The greedy algorithm apparently seems to save CPU usage significantly. The savings estimated from the previous runs amount to as high as 85 percent. The conventional annealing algorithm takes the longest CPU times. It can also be noted that the greedy algorithm with interpolation retains the best overall certainty coefficients for the $\mathrm{fGn}$ model, resulting in the best predictions.

\subsubsection{Simulation of Burbank Field}

Preliminary two-dimensional simulations of the Burbank are performed in order 
Table 12

Correlation coefficients and CPU usage for

two-dimensional stochastic conditional simulations

for Hugoton Field

Well B2

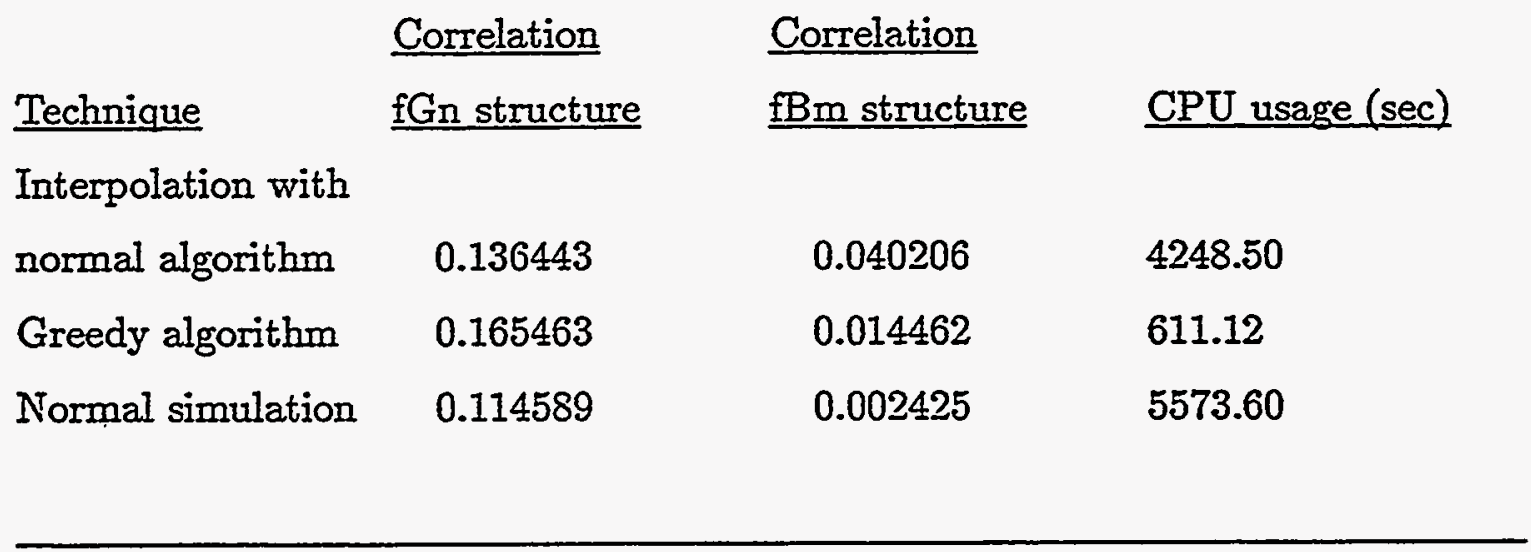




\section{Table 13}

Correlation coefficients for two-dimensional stochastic conditional simulation runs for Hugoton Field

Test well

F2

N2
fBm structure

0.102891

0.091213
$\underline{\mathrm{fGn} \text { structure }}$

0.281018

0.132636 
to have a better feel for the horizontal spatial correlation structure. The horizontal correlation structure in the vertical direction is assumed to be $\mathrm{fGn}$, with a smoothing interval of 3 feet. Figure 37 provides a field map of the conditioning wells and test wells used in the simulations, with bold circles marking the conditioning wells and the striped circles marking the test wells. Two well pairs are chosen for the simulations: wells 25 and 28 , and wells 50 and 28 .

The estimated $H$ values for the two conditioning traces using box-counting are 0.7841 and 0.8260 , for an average $H$ value of 0.8051 . This value is used for both the vertical and horizontal correlation structure of the two-dimensional slice. The number of blocks consists of 24 in the $z$ direction and 40 in the $x$ direction. The horizontal distance between the two wells is 933 feet. Table 14 summarizes the pertinent simulation parameters for the well pair.

Since an $\mathrm{fGn}$ model is assumed for the vertical direction with a smoothing factor of 3 feet, $V_{H}$ is calculated from Equation (7) by setting it equal to the average variance calculated from the two wells. The value is found to be 0.0017 , giving a variance of an infinite sample of 0.003338 . The variance of the difference between the well pair is calculated using Equation (34) and is found to be 0.0015 . Two horizontal structures are assumed in the simulations: $\mathrm{fBm}$ and $\mathrm{fGn}$. This is done in order to determine the best model to use in the horizontal direction. $V_{H}$ is then calculated using Equations (5) and (7). For an $\mathrm{fBm}$ model and a lag distance of 933 feet, it is found to be 1.8614E-08. For an fGn model, using $\delta=3$ feet, it is calculated to be $2.3739 \mathrm{E}-03$. In both of the above cases, the variogram value is set equal to the variance of the difference between the two wells.

Two-dimensional stochastic conditional simulation runs using the greedy algorithm are performed on the two well pairs. Two models are assumed in the runs: $\mathrm{fGn}$ and $\mathrm{fBm}$. In both simulations, the vertical structure used is an $\mathrm{fGn}$ model. Figures 38 and 39 display the comparison plots between the test well 35 and the synthetic data generated from the simulation for well pair 50 and 28. Table 15 summarizes the certainty coefficients for the comparisons wells for both simulation runs. For the test cases, the subtracted means are first linearly interpolated and added back to the synthetic well logs.

The comparison plots in Figures 38 and 39 offer several interesting insights. If an $\mathrm{fBm}$ model is used for the horizontal direction, the the major trends in the porosity trace will be captured fairly accurately. The fGn model seems to give a poor performance in capturing the trends present in the region. The calculated correlation coefficients are also slightly higher when an $\mathrm{fBm}$ model is used (refer to Table 15). Figures 40 and 41 show the comparison plots for another well pair in the region. Again, the $\mathrm{fBm}$ model seems to predict the values in the interwell region fairly accurately, with a higher correlation coefficient. An interesting point to note is that fGn models tend to generate smoother distributions of the porosity in the interwell region. 
Table 14

Summary of simulation parameters for 2-D stochastic conditional simulation in the Burbank Field

Well pair -28 and 50

Distance between well pair

933 feet

Variance of difference

0.0015

Average $H$ value

0.8051

Blocks in $x$ direction

40

Blocks in $z$ direction

24

Blocks in $y$ direction

Average vertical variance

1

Smoothing factor

0.0017

Scaling factor $(\mathrm{fGn})$

3 feet

Scaling factor (fBm)

$2.3739 \mathrm{E}-03$

$1.8614 \mathrm{E}-08$ 
Table 15

Correlation coefficients for two-dimensional stochastic conditional simulation runs for Burbank Field

Test well

35

26
$\mathrm{BBm}$ structure

0.529878

0.386122
fGn structure

0.353443

0.291828 


\subsubsection{Simulation of Loudon Field}

A pair of wells is taken from the Loudon Field and simulated using the greedy algorithm. Figure 42 shows a map of the simulation region, with the bold squares denoting the conditioning well and striped square denoting the test well. It can be noted that some of the wells are comprised of core data, while more others are comprised of $\log$ data. Although log and core measurements are measured over different support volumes, both can be reconciled by matching their respective resolutions. For example, a series of core porosity measurements can be averaged over one-foot spacing to match the resolution of a log porosity trace.

Table 16 summarizes the pertinent simulation parameters calculated for the two-dimensional stochastic conditional simulation. The distance between the well pair is estimated at 652 feet, with a variance of the difference of approximately 2.5549. Measured $H$ exponent values average out at 0.7986 using box-counting. This indicates that the vertical section can be described using an $\mathrm{fGn}$ model. The same $H$ value is used in the horizontal direction. The number of grid nodes chosen for the $x$ direction is 40 , and in the $z$ direction, the number of grid nodes totaled 62. Using a smoothing factor of 3 feet, the variance of an infinite sample calculated for both $\mathrm{fGn}$ and $\mathrm{fBm}$ models were $4.2082 \mathrm{E}-00$ and $8.1744 \mathrm{E}-05$, respectively.

Two-dimensional distributions of properties in the interwell region are generated using the greedy algorithm. Both the $\mathrm{fGn}$ and $\mathrm{fBm}$ horizontal correlation structures are used in the simulations to determine the best model that can predict values in the interwell region. Figures 43 and 44 show the comparison plots for the test well 435 and the generated synthetic well at the same position. Both models seem to capture the major trends in the region fairly accurately. However, a better

estimate of the performance of both models can be quantitatively obtained using the correlation coefficient value. Table 17 presents correlation coefficient values for realization of the simulation. It can be noted that the fBm model resulted in sightly better predictions of the interwell distribution of properties.

\subsection{3-D Simulations}

\subsubsection{Simulation of Burbank Field}

Fieldwide three-dimensional stochastic conditional simulations in the Burbank Field are done in two stages. Simulations are started with 4 conditioning wells, and on the next stage, 8 conditioning wells are used. The purpose of this procedure is to observe the effects of sample data size on the certainty coefficients of the test well comparisons.

Three dimensional stochastic conditional simulations should progress naturally from the small two-dimensional simulations. The purpose of the later is to determine the best horizontal correlation structure to use in the three-dimensional runs. Fieldwide horizontal variograms should preferably be generated beforehand in order to study the extent of the horizontal correlation of rock properties.

Figure 45 illustrates a field map of the region under investigation. Four con- 
Table 16

Summary of simulation parameters for 2-D stochastic conditional simulation in the Loudon Field

Well pair -433 and 437

Distance between well pair

652 feet

Variance of difference

2.5549

Average $H$ value

0.7986

Blocks in $x$ direction

40

Blocks in $z$ direction

62

Blocks in $y$ direction

1

Average vertical variance

7.2159

Smoothing factor

3 feet

Scaling factor ( $\mathrm{fGn}$ )

$4.2082 \mathrm{E}-00$

Scaling factor (fBm)

8.1744E-05 


\section{Table 17}

Correlation coefficients for two-dimensional stochastic conditional simulation runs for Loudon Field

Test well 435
$\underline{\mathrm{fBm} \text { structure }}$

0.433074
$\underline{\mathrm{fGn} \text { structure }}$

0.359939 
ditioning wells (dark circles) are chosen from the four corners of the rectangular region. Three test wells are picked from the interior region (wells 27, 49, and 43). The simulation region covers an area of approximately 8 million square feet. Figure 46 shows a field-wide horizontal variogram of the field. A nugget can be observed in the variogram model plot, signifying a lack of information at the small lag distances.

Table 18 summarizes the pertinent simulation parameters used in the simulation run. Calculation of $V_{H}$ in three-dimensional cases involves finding the largest distance between any two wells in the region. If two pairs are found to be the same distance apart, then $V_{H}$ for both pairs is calculated. In the simulation, however, the average variance of an infinite sample can be used. This is the case for Burbank Field since the simulation region is rectangular in shape. After calculating $V_{H}$ for both well pairs at the four corners of the region, the average value of $V_{H}$ is calculated to be 0.0042 . For this case, the average $H$ value is 0.7946 , which is just the mean of the four $H$ values estimated from the four conditioning wells. Forty blocks in the $x$ and $y$ direction are used. In the $z$ or vertical direction, 36 blocks are used.

Although three test wells are chosen for comparison, for reasons of brevity, only two test cases will be shown. Simulations are done for both $\mathrm{fGn}$ and $\mathrm{fBm}$ models. Aside from these, synthetic logs are also obtained from the interpolated values themselves (without added random element). This procedure corresponds to linear interpolation of values without doing any simulation. Figures 47 to 49 show cases for test well 27 using $f \mathrm{Gn}, \mathrm{fBm}$, and interpolation, respectively. The quality of the predictions is poor, to say the least, although the $\mathrm{fBm}$ model captures part of the trends in the lower and upper sections of the region. Similar results are observed for well 40 .

Table 19 summarizes the certainty coefficients for all three test cases. The coefficients are generally low, with $\mathrm{fBm}$ performing the best among the three techniques. Linear interpolation performs almost as well as $\mathrm{fBm}$, however, thus suggesting that doing a stochastic conditional simulation of the entire field in the absence of good data is unnecessary.

The number of conditioning wells is increased to 8 (see Figure 50), and Table 20 summarizes the certainty coefficients for 5 test wells. A general increase in certainty coefficients for all methods is noted. This is especially so for the fBm horizontal correlation structure. This means that as the number of data points is increased, the certainty coefficient will most likely increase because of the availability of more data to describe the reservoir.

\subsubsection{Simulation of Loudon Field}

Figure 51 presents a field map of the region to be simulated. Four conditioning wells are chosen in the region (bold squares). The test wells consist of two cored wells and one logged well. Table 21 summarizes the simulation parameters in the simulation runs. Although the $\mathrm{fBm}$ model is found to be the best for the field based on the two dimensional simulations, $\mathrm{fGn}$ model will also be tested for the sake of 
Table 18

Summary of simulation parameters for 3-D stochastic conditional simulation in the Burbank Field using 4 conditioning wells

Largest between well pair

4226 feet

Variance of difference

- 0.0042

Average $H$ value

0.7946

Blocks in $x$ direction

40

Blocks in $z$ direction

36

Blocks in $y$ direction

40

Average vertical variance

0.0036

Smoothing factor

3 feet

Scaling factor $(\mathrm{fGn})$

$6.7646 \mathrm{E}-03$

Scaling factor ( $\mathrm{fBm}$ )

7.2598E-09 
Table 19

Correlation coefficients for

three-dimensional stochastic conditional simulations

for Burbank Field

4 conditioning wells

$\begin{array}{lcccc}\text { Well } & \frac{\text { Correlation }}{\text { fGn structure }} & & \begin{array}{l}\text { Correlation } \\ \text { fBm structure }\end{array} & \frac{\text { Correlation }}{\text { Interpolation }} \\ 40 & -0.45 & & -0.04 & -0.32 \\ 27 & -0.22 & & 0.09 & 0.08 \\ 43 & -0.01 & . & 0.02 & 0.02\end{array}$


Table 20

Correlation coefficients for

three-dimensional stochastic conditional simulations

for Burbank Field

8 conditioning wells

\begin{tabular}{|c|c|c|c|}
\hline & Correlation & Correlation & Correlation \\
\hline Well & fBm structure & fGn structure & Interpolation \\
\hline 25 & 0.23 & -0.08 & -0.02 \\
\hline 26 & 0.05 & -0.06 & 0.07 \\
\hline 27 & 0.13 & -0.21 & 0.06 \\
\hline 34 & 0.49 & 0.21 & 0.43 \\
\hline 42 & 0.12 & -0.05 & 0.09 \\
\hline
\end{tabular}


comparisons. All porosity traces are seasonally adjusted to remove the local trends in the zone of interest.

The maximum lag distance between any two wells in the region was found to be 728 feet (wells 453 and 437). This value of $h$ is therefore used to calculate the value of $V_{H}$. The average $H$ value calculated from all four conditioning wells is 0.8009 , and this is used as input into the simulator. The variance of the difference between wells 453 and 437 is found to be 4.8038 . Forty blocks are used in the $x$ direction and $y$ directions, and 62 blocks are used in the $z$ direction. Using the previous value of $h, H$, and assuming a smoothing factor of 3 feet, $V_{H}$ is calculated to be 7.8664 using an $\mathrm{fGn}$ model, and 1.2503E-04 using an $\mathrm{fBm}$ model (refer to Table 21).

Table 22 presents the correlation coefficients for both $\mathrm{fGn}$ and $\mathrm{fBm}$ models for all cases. It can be noted that the best model to use for this field is still $\mathrm{fBm}$, as the correlation coefficients are generally higher than those calculated for $\mathrm{fGn}$ model. In all the simulations, the greedy algorithm with bilinear interpolation and added random element is used.

Figure 52 shows an unusually close agreement between the synthetic log and the test well. All the major and minor trends are captured in the predictions. No such agreement is observed for the fGn models. The three-dimensional simulation results in this section still support the fact that the best model to use in a sandstone environment is the $\mathrm{fBm}$ model. Previous two-dimensional simulation runs for this field also indicate that $\mathrm{fBm}$ is the best model to use. However, for a carbonate environment, the previous runs performed on Hugoton indicate that $\mathrm{fGn}$ is the best structure to use in the simulations.

One notable thing to mention at this point is that the quality of the predictions has increased whenever the amount of data in a particular zone is abundant, or when the zone of interest is relatively thick (as in the case for Hugoton and Loudon). For Burbank, since the relative thickness of the producing zone is approximately 40 feet, that in itself left very little data to work with. Correlation coefficients also tend to generally increase as the size of sample data increases. This reflects the importance of getting as much data as possible for generating better and more reliable distributions of reservoir properties.

\subsubsection{Simulation of Hugoton Field}

The three dimensional simulations in the Hugoton Field are done in two parts: the first part presents the simulations for a small sub-section of the field, while the second part is a field-wide simulation of the field.

The previous, small 2-dimensional runs give a feel for the most reliable correlation structure to use in the simulation run. The next step is to perform a simulation for a 3-dimensional case. A 3 by 3 square region within the Hugoton Field is chosen for a tentative 3-dimensional run. The four wells in the corners are taken as the conditioning wells, leaving five wells as test cases.

Figure 53 shows a field map of the simulation region. A square, north-east corner of the field is chosen for the simulation. Figure 54 displays a variogram 
Table 21

Summary of simulation parameters for 3-D stochastic conditional simulation in Loudon Field using 4 conditioning wells

Largest between well pair

Variance of difference

Average $H$ value

Blocks in $x$ direction

Blocks in $z$ direction

Blocks in $y$ direction

Average vertical variance

Smoothing factor

Scaling factor ( $f G n$ )

Scaling factor ( $\mathrm{fBm}$ )
728 feet

4.8038

0.8009

40

62

40

4.4951

3 feet

$7.8664 \mathrm{E}+00$

1.2503E-04 


$$
\begin{gathered}
\text { Table } 22 \\
\text { Correlation coefficients for } \\
\text { three-dimensional stochastic conditional simulations } \\
\text { for Loudon Field }
\end{gathered}
$$

$\begin{array}{lcc}\text { Well } & \frac{\text { Correlation }}{\text { fBm structure }} & \frac{\text { Correlation }}{\text { fGn structure }} \\ 426 \mathrm{r} & 0.083683 & 0.060136 \\ 446 & -0.105691 & -0.116193 \\ 501 & 0.225909 & -0.144635\end{array}$


plot for the entire field. A pure nugget effect can be observed from the plot. This fact has one consequence. Since the value of the property has reached its sill value at the minimum interwell separation distance, one does need to use the maximum distance between any two wells to calculate the value of $V_{H}$. Therefore, in doing a three-dimensional simulation of the field, the value of $h$ in Equations (5) or (7) to be used is simply the minimum distance between two wells in the region under study.

Using the data at the four conditioning wells, an initial pattern is generated using bilinear interpolation with an added Gaussian element. It is observed that the bilinear interpolation technique retains much of the original distribution of the data. The shape of both plots appear to be bell-shaped, denoting a normal distribution. Twenty blocks were used in the $x$ and $y$ directions. In the $z$ direction, 389 blocks were used.

The fGn model is used in the horizontal direction since the previous twodimensional simulations have indicated that this is the best model to use. Figures 55 to 57 show the comparison plots for some of the test cases, first using the greedy algorithm with interpolation and added random element, and then with plain interpolation without any simulation. It can be seen that the $\mathrm{fGn}$ spatial correlation structure captures the major trends in the interwell region remarkably. Table 23 summarizes the certainty coefficients obtained in the simulations.

In the second part of the simulation, a large scale section of the field is chosen as the simulation region. An $\mathrm{fGn}$ model is used is the horizontal direction. Figure 58 shows a field map of the fieldwide region to be simulated in the Hugoton Field. Five conditioning wells are chosen: four in the corners of the field and one in the middle of the field. Figures 59 and 60 present comparison plots for the fieldwide simulation in two of the test wells. In Figure 60, a close agreement between simulated values and actual values is observed. The correlation for this test case is calculated to be 0.2332. However, a poor match is observed for test well K2. Table 24 summarizes the certainty coefficients obtained in the simulations.

\subsection{Summary}

Several significant observations can be made from the results in this section. They can be summarized as follows:

(1) The best method for generating a description of rock properties is by using a combination of interpolation and the greedy algorithm. The interpolation has been found to honor the original distribution of the conditioning data if an added random Gaussian element is used.

(2) The CPU times reduction compared to simulated annealing is close to 85 percent. Since the greedy algorithm ignores all interchanges that result in an increase of the objective function, a lot of variogram calculations are made unnecessary. This results in considerable savings of CPU usage.

(3) The type of structure required to describe the horizontal correlation are dif- 


$$
\begin{gathered}
\text { Table } 23 \\
\text { Correlation coefficients for } \\
\text { three-dimensional stochastic conditional simulations } \\
\text { for Hugoton Field }
\end{gathered}
$$

$\underline{\text { Small-scale simulation }}$

Correlation Correlation

Well $\underline{\mathrm{fG} n \text { structure }}$ Interpolation

I2

0.267669

0.217725

M2

0.035683

0.078009

N2

0.162906

0.285647 
Table 24

Correlation coefficients for

three-dimensional stochastic conditional simulations

for Hugoton Field

Large-scale simulation

Correlation

$\underline{\text { Well }}$

fGn structure

C2

0.219091

K2

0.045612 
ferent for both sandstone and carbonate environments. The extensive twodimensional and three-dimensional simulations in this section have indicated that the $\mathrm{fBm}$ model is best used for sandstone environments and that the fGn model should be used for carbonate environments. Determining the best type of structure requires one to perform small, two-dimensional simulations of the region.

(4) The number of wells as conditioning data has significant influence on the results of the stochastic conditional simulations. This is because the more data that is available, the less the uncertainty in the predictions. In the absence of data, however, one can still generate probable reservoir descriptions with some measure of confidence by using the range of $H$ exponent values in Section 3 regardless of the field. One needs to know only the sedimentary environment of the field.

\subsection{Conclusions}

This section will summarize the major findings and observations of this work. Several conclusions are drawn from these findings.

Section 3 of this report presents results related to $H$ exponent estimation of actual wellbore data. Where seasonal effects are observed in the data trace, the seasonal bias is removed using a normalizing technique which involves eliminating the local means. The distribution of $H$ exponent values after and before removal of sample means is compared. The results in Section 3 indicate that if the seasonal effects are unusually strong, removal of the local means will result in a narrower distribution of $H$ exponent values. Actual plots of variogram values favor the boxcounting method to give the most reliable fit. The results indicate that after seasonal adjustments, the range of $H$ exponent values calculated using box-counting narrow down to between 0.75 and 0.80 regardless the type of the sedimentary environment. Therefore, in the absence of data, this value can be used for the stochastic conditional simulation of a reservoir. If the seasonal effects are not strong, the variation of the value of $H$ exponent is not significantly affected, although the mean value might be affected.

Section 4 of this report presents results for the stochastic conditional simulation of three fields, a carbonate reservoir and two sandstone reservoirs. This part also delineates a set of procedures to be followed in the stochastic conditional simulation of the rock properties. Factors that should be taken into account include the removal of the non-stationarity in the vertical well data and the selection of a suitable horizontal correlation model based on the correlation structure determined from vertical well data. The results seem to indicate that using a fGn model in the horizontal direction for a carbonate field results in better predictions than using an $\mathrm{fBm}$ model. However, in a sandstone environment, the reverse is apparent. Techniques for decreasing the extensive CPU usage associated with most simulated annealing algorithms are also presented These techniques include generating an initial pattern using bilinear interpolation and adding a random Gaussian element, 
and using only the greedy version of the algorithm. The results obtained using these enhancements seem to provide accurate and reliable predictors of the actual rock properties, as well as saving CPU usage by as much as 85 percent. In general, the results of the simulations indicate that the greedy algorithm coupled with the linear or bilinear interpolation technique is the best method for generating reservoir descriptions.

The framework for applying stochastic conditional simulation to generate descriptions of a property will be summarized as follows:

(1) Removal of trends observed in vertical well logs that might result in biased estimates of the intermittency exponent - Removing the local means in different zones of varying property distributions will enable one to remove the bias in the intermittency exponent calculations. It has been shown that seasonal adjustments can significantly affect the value of the estimated $H$ exponent, as well as provide more consistent results for a particular region of interest. Also, no technique can be robust enough to take into account the periodic effects in a data trace.

(2) The use of reliable intermittency exponent estimation techniques that are both robust and fairly insensitive to small sample distributions - Although many techniques exist for the estimation of the $H$ exponent, some have been found to be more reliable than the others. The results have indicated that the R/S analysis and box-counting method can reliably predict the value of the intermittency exponent. However, based on the match between analytical and experimental variograms for a real dataset, the box-counting method appears to predict the $H$ exponent value more accurately. It is recommended that the box-counting be used for all subsequent analysis of property traces.

(3) The use of a combination of the greedy algorithm and a bilinear interpolation technique to improve CPU usage in annealing runs - that significant savings in CPU. usage can be achieved through the use of a combination of the greedy algorithm and a linear or bilinear interpolation technique to generate an initial pattern for a stochastic conditional simulation run. As discussed previously, the greedy algorithm is a limiting case of the simulated annealing algorithm, in which all swaps resulting in an increase in the energy function are rejected by the Metropolis' condition. The linear or bilinear interpolation technique enables one to generate an initial pattern of values that honors the original conditioning data and its distribution function.

(4) Determining the most reliable horizontal correlation model based on different, small, two-dimensional simulations of the field - The determination of a horizontal spatial correlation structure is frequently a trial-and-error procedure. This step acknowledges this fact by recommending that small, two-dimensional stochastic conditional simulations should be done beforehand in order to have a feel for the best correlation model to use. The advantage to doing this is obviously to save on CPU usage before extensive three-dimensional stochastic 
conditional runs are performed. These two-dimensional runs should preferably be field-wide in the sense that different spatial directions should be considered.

(5) Using the same correlation model determined from Step (3) to perform a regular three-dimensional stochastic conditional simulation of the entire field - Once the best horizontal correlation model has been determined, the same horizontal model should therefore be used for the field-wide three-dimensional stochastic conditional simulations.

An important consequence of this work is that in the absence of real data, an $H$ value in the range of 0.75 to $0.80 \mathrm{can}$ be used regardless the type of sedimentary environment considered.

\section{Nomenclature}

Symbol

$$
\begin{array}{ll}
\hline a & =\text { coefficient in discrete Fourier transform } \\
A & =\text { real random variable } \\
b & =\text { box scaling factor } \\
B & =\text { real random variable } \\
C & =\text { certainty coefficient } \\
C_{o} & =\text { variance of a population } \\
C O V & =\text { covariance function } \\
d & =\text { topological dimension } \\
D & =\text { fractal dimension } \\
E & =\text { mathematical expectation of a random variable } \\
f & =\text { frequency } \\
F & =\text { cumulative distribution function } \\
F & =\text { specified cumulative distribution function in simulation } \\
\vec{h} & =\text { lag distance vector for semi-variograms } \\
h & =\text { lag distance } \\
h_{c} & =\text { lag at which } C_{o} \text { is reached } \\
H & =\text { intermittency exponent } \\
I & =\text { integral function } \\
L & =\text { spectral density resolution } \\
N & =\text { number of boxes to cover a record } \\
N_{c} & =\text { number of conditioning data } \\
N_{f} & =\text { number of classes for distribution function } \\
N_{s} & =\text { total number of simulation grid points } \\
r & =\text { scaling factor } \\
R & =\text { range function } \\
S & =\text { sample variance } \\
S_{z} & =\text { spectral density function } \\
V & =\text { simulation variable } \\
V_{c} & =\text { conditioning data value } \\
V_{f} & =\text { upper class limit of distribution function } \\
&
\end{array}
$$


$V_{H} \quad=$ scaling factor for $\mathrm{fGn}$ and $\mathrm{fBm}$ semi-variogram models

$x=\mathrm{x}$ coordinate of a point

$\bar{X}=$ discrete Fourier transform

$y=y$ coordinate of a point

$Z \quad=$ realization of random variable

$Z(x)=$ realization of random variable at point $x$

$Z_{g} \quad=$ graded sequence

$\beta \quad=$ spectral density exponent

$\delta \quad=$ smoothing parameter for fGn semi-variogram model

$\gamma=$ semi-variogram

$\gamma_{0}=$ specified semi-variogram model for a conditional simulation

$\gamma_{s}=$ sample semi-variogram of simulation variable

$\sigma \quad=$ standard deviation

$\vec{\omega} \quad=$ coordinates of grid points in the simulation region

$\vec{\omega}_{\mathrm{c}} \quad=$ coordinates of the conditioning data

\section{References}

1. Matheron, G.: The Theory of Regionalized Variables and Its Applications, Les Cahiers du Cent. de Morph. Math., Fasc. 5, CG Fontainebleau (1971).

2. Journel, A.G. and Huijbregts, Ch.J.: Mining Geostatistics, Academic Press, New York (1978).

3. Hewett, T.A.: "Fractal Distributions of Reservoir Heterogeneity and Their Influence on Fluid Transport," paper SPE 15386 presented at the 1986 SPE Annual Technical Conference and Exhibition, New Orleans, Oct. 5-8.

4. Farmer, C.L.: "Numerical Rocks, The Mathematical Generation of Reservoir Geology," paper presented at the 1989 Joint IMA/SPE European Conference, Cambridge University, July 25-27.

5. Farmer, C.L.: "The Generation of Stochastic Fields of Reservoir Parameters With Specified Geostatistical Distributions," paper presented at the 1987 IMA on The Mathematics of Oil Production conference, Robinson College, Cambridge, July 6-7.

6. Hewett, T.A. and Behrens, R.A.: "Conditional Simulation of Reservoir Heterogeneity with Fractals," paper SPE 18326 presented at the 1988 SPE Annual Technical Conference and Exhibition, Houston, Oct. 2-5.

7. Hewett, T.A. and Behrens, R.A.: "Conditional Simulation of Reservoir Heterogeneity With Fractals," SPE Formation Evaluation Journal (Sept. 1990) 217-225.

8. Emanuel, A.S., Alameda, G.K., Behrens, R.A. and Hewett, T.A.: "Reservoir Performance Prediction Methods Based on Fractal Geostatistics," SPE Reservoir Engineering Journal (Aug. 1989) 311-318.

9. Mattews, J.L., Emanuel, A.S. and Edwards, K.A.: "A Modeling Study of the Mitsue Stage I Miscible Flood Using Fractal Geometries," paper SPE 18327 
presented at the 1988 Annual Technical Conference and Exhibition, Houston, Oct. 2-5.

10. Mandelbrot, B.B.: The Fractal Geometry of Nature, W.H. Freeman and Co., New York (1982).

11. Wallis, J.R. and Matalas, N.C.: "Small Sample Properties of $H$ and K Estimators of the Hurst Coefficient H," Water Resour. Res. (1970) 6, 1583-1594.

12. Perez, G.: "Stochastic Conditional Simulation for Description of Reservoir Properties," Ph.D. Dissertation, University of Tulsa, Oklahoma (1991).

13. Feder, J.: Fractals, Plenum Press, New York (1988).

14. Mandelbrot, B.B.: Fractals: Form, Chance, and Dimension, W.H. Freeman and Co., San Francisco (1977).

15. Mandelbrot, B.B.: "How long is the coast of Britain? Statistical self-similarity and fractal dimension," Science (1967) 155, 636-638.

16. Mandelbrot, B.B.: Les Objets Fractals: Forme, Hasard et Dimension, Flammarion, Paris (1975).

17. Mandelbrot, B.B.: Fractals and Multifractals: Noise, Turbulence and Galazies, Springer, New York (1988).

18. Mandelbrot, B.B: "Stochastic models of the Earth's relief, the shape and the fractal dimension of the coastlines, and the number-area rule for islands," Proc. Natl. Acad. Sci. USA (1975) 72, 3825-3828.

19. Mandelbrot, B.B. and Wallis, J.R.: "Noah, Joseph, and operational research," Water Resour. Res. (1968) 4, 909-918.

20. Hurst, H.E.: "Long-Term Storage Capacity of Reservoirs," Trans. Amer. Soc. Civil Eng., vol. 116 (1951) 770.

21. Mandelbrot, B.B. and Wallis, J.R.: "Some long-run properties of geophysical records," Water Resour. Res. (1969) 5, 321-340.

22. Crane, S.D. and Tubman, K.M.: "Reservoir Variability and Modeling With Fractals," paper SPE 20606 presented at the 1990 Annual Technical Conference and Exhibition, New Orleans, Sept. 23-26.

23. Goggin, D.J., Chandler, M.A., Kocurek, G. and Lake, L.W.: "Patterns of Permeability in Eolian Deposits: Page Sandstone (Jurassic), Northeastern Arizona," SPE Formation Evaluation Journal (June 1988) 297-306.

24. Mandelbrot, B. and Wallis, J.R.: "Robustness of the Rescaled Range R/S in the Measurement of Noncyclic Long Run Statistical Dependence," Water Resour. Res. (1969) 5, 967-988.

25. Perez, G: Personal Communication, Tulsa University, 1990.

26. Aarts, E. and Korst, J.: Simulated Annealing and Boltzmann Machines: A Stochastic Approach to Combinatorial Optimization and Neural Computing, John Wiley \& Sons Ltd, Chichester (1989).

27. Kirkpatrick, S., Gelatt, C.D. Jr. and Vecchi, M.P.: "Optimization by Simulated Annealing," Science (May 13, 1983) 671-80.

28. Cerny, V.: "Thermodynamical Approach to the Traveling Salesman Problem: 
An Efficient Simulation Algorithm," Journal of Optimization Theory and Applications (1985) vol. 45, no. 1, 41-51.

29. Metropolis, N., Rosenbluth, A.W., Rosenbluth, M.N., Teller, A.G. and Teller, E.: "Equation of State Calculations by Fast Computing Machines," The Journal of Chemical Physics (June 1953) 1087-92.

30. Journel, A.G. and Alabert, F.G.: "New Method for Reservoir Mapping," Journal of Petroleum Technology (Feb. 1990) 212-18.

31. Journel, A.G.: Fundamentals of Geostatistics in Five Lessons, American Geophysical Union, Washington, D.C. (1989).

32. Isaaks, E.H. and Srivastava, R.M.: Applied Geostatistics, Oxford University Press, New York (1989).

33. Mantoglou, A. and Wilson, J.L.: "The Turning Bands Method for Simulation of Random Fields Using Line Generation by a Spectral Method," Water Resources Research (Oct. 1982) 1379-94.

34. Journel, A.G. and Gómez-Hernández, J.J.: "Stochastic Imaging of the Wilmington Clastic Sequence," paper SPE 19857 presented at the 1989 Annual Technical Conference and Exhibition, San Antonio, Oct. 8-11.

35. Aasum, Y., Kelkar, M.G. and Gupta, S.P.: "An Application of Geostatistics and Fractal Geometry for Reservoir Characterization," SPE Formation Evaluation Journal (March 1991) 11-19.

36. Haldorsen, H.H. and Damsleth, E.: "Stochastic Modeling," Journal of Petroleum Technology (April 1990) 404-12.

37. Haldorsen, H.H., Brand, P.J. and Macdonald, C.J.: "Review of the Stochastic Nature of Reservoirs," Mathematics in Oil Production, S. Edwards and P. King (editors), Oxford Science Publications, Clarendon Press, Oxford (1988) 109209.

38. $\mathrm{Da}$ Costa e Silva, A.J.: "A New Approach to the Characterization of Reservoir Heterogeneity Based on the Geomathematical Model and Kriging Technique," paper SPE 14275 presented at the 1985 Annual Technical Conference and Exhibition, Las Vegas, Sept. 22-25.

39. Williams, J.I. and Dawe, R.A.: "Fractals - An Overview of Potential Applications to Transport in Porous Media," Transport in Porous Media, Reidel Publishing Company, vol. 1 (1986) 201-9.

40. Sahimi, M. and Yortsos, Y.C.: "Applications of Fractal Geometry to Porous Media: A Review," paper SPE 20476 presented at the 1990 Annual Technical Conference and Exhibition, New Orleans, Sept. 23-26.

41. Gómez-Hernández, J. J. and Srivastava, M.R.: "isim3d: A 3-dimensional Multiple Indicator Simulation Program," Computer and Geosciences (1990), vol. 16 , no. $4,396-440$.

42. Peitgen, Heinz-Otto, and Saupe, Dietmar (Editors): The Science of Fractal Images, Springer-Verlag, New York (1988). 
43. Mandelbrot, B.B.: "A Fast Fractional Gaussian Noise Generator," Water Resources Research (June 1971) 543-53.

44. Ben-Rached, L.: "An Application of Geostatistical Techniques for Reservoir Performance Prediction," M.S. Thesis, University of Tulsa, Oklahoma (1991).

45. MacAllister, D.J.: "Reservoir and Fluid Properties For The Sable Unit," Internal Report, ARCO, Midland (1987).

46. Hagen, K.B.: "Mapping of Surface Joints on Air Photos Can Help Understand Waterflood Performance Problems at North Burbank Unit, Osage and Kay Counties, Oklahoma," M.S. Thesis, University of Tulsa, Oklahoma (1972).

47. Bass, N.W., Leatherock, C., Dillard, W.R. and Kennedy, L.E.: "Origin and Distribution of Bartlesville and Burbank Shoestring Oil Sands in Parts of Oklahoma and Kansas," $A A P G$, Vol. 21 (January, 1937) 30-66.

48. Szpakiewicz, M.J., McGee, K. and Sharma, B.: "Geological Problems Related to Characterization of Clastic Reservoirs for Enhanced Oil Recovery," paper SPE/DOE 14888 presented at the 1986 SPE/DOE Fifth Symposium on Enhanced Oil Recovery, Tulsa, OK, April 20-23.

49. Trantham, J.C., Threlkeld, C.B. and Patterson, Jr., H.L.: "Reservoir Description for a Surfactant/Polymer Pilot in a Fractured, Oil-Wet Reservoir-North Burbank Unit Tract 97," JPT, (Sept. 1980), 1647-1656.

50. Press, W. H., Flannery, B. P., Teukolsky, S. A. and Vetterling, W. T.: Numerical Recipes: The Art of Scientific Computing, Cambridge University Press, Cambridge (1986). 


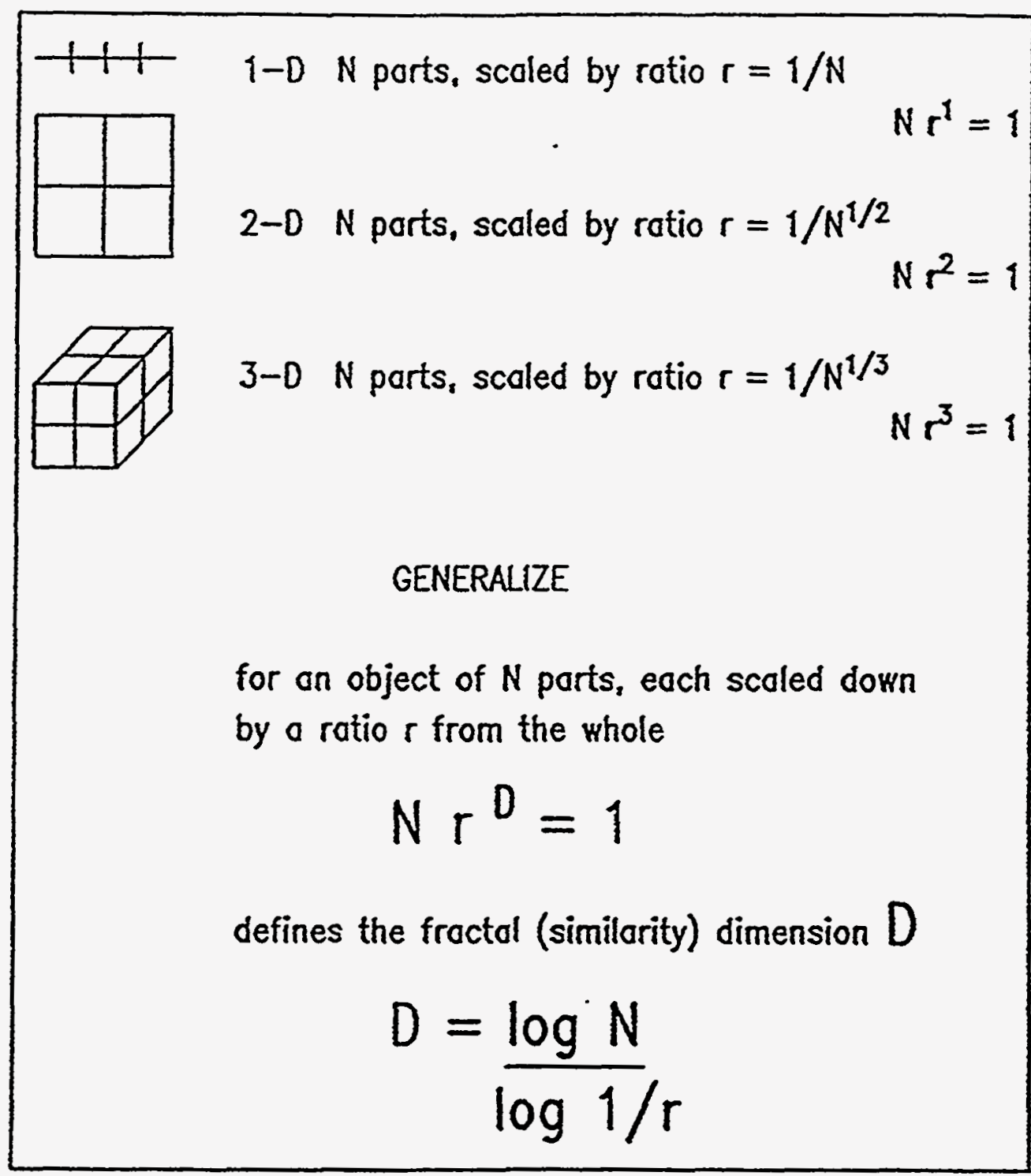

Figure 1: Interpretation of standard integer dimensions (after Peitgen) 
Simulation Process

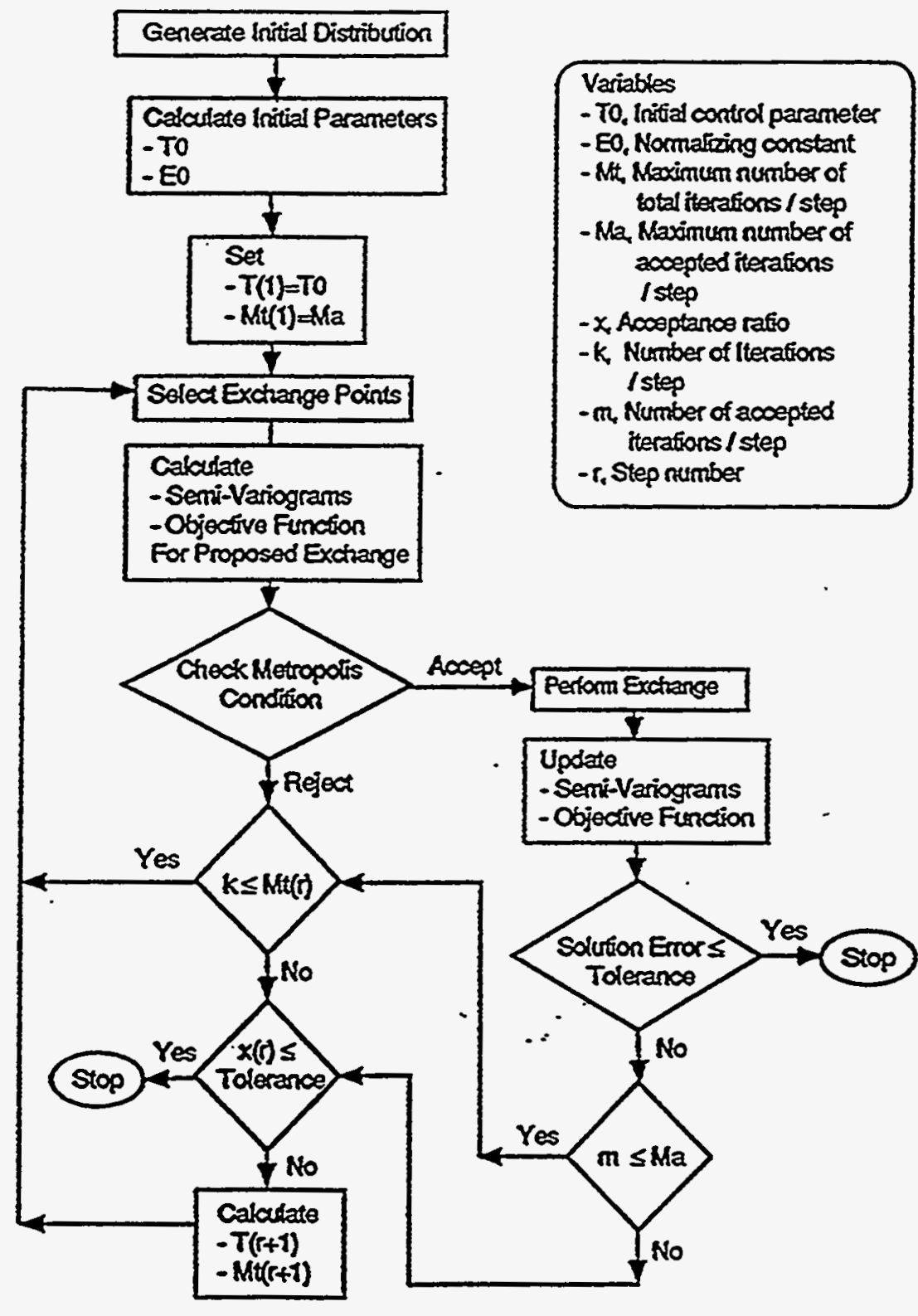

Figure. 2. Flowchart for the simulated annealing algorithm (after Perez) 


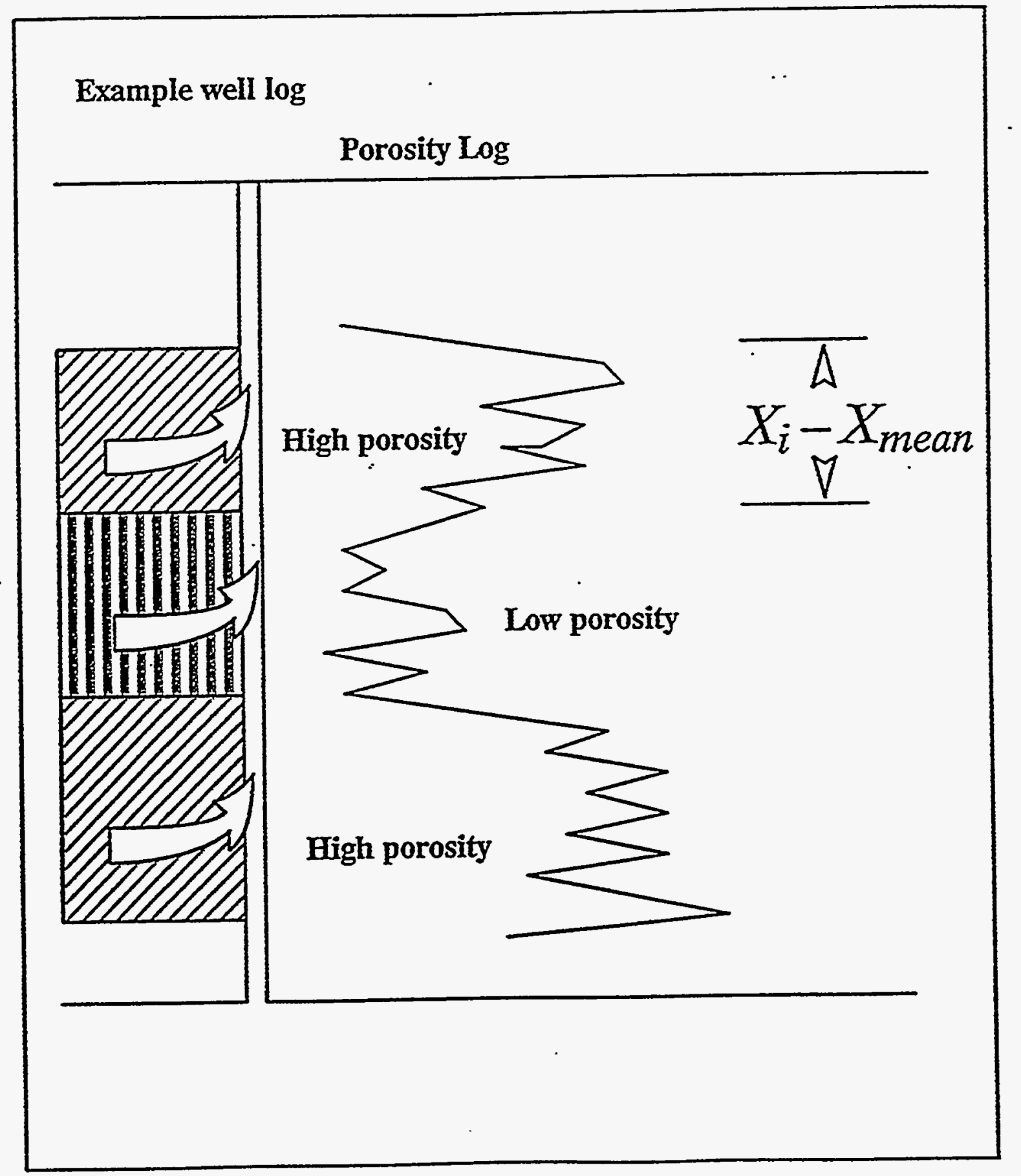

Figure 3. Procedure for removing the local means 


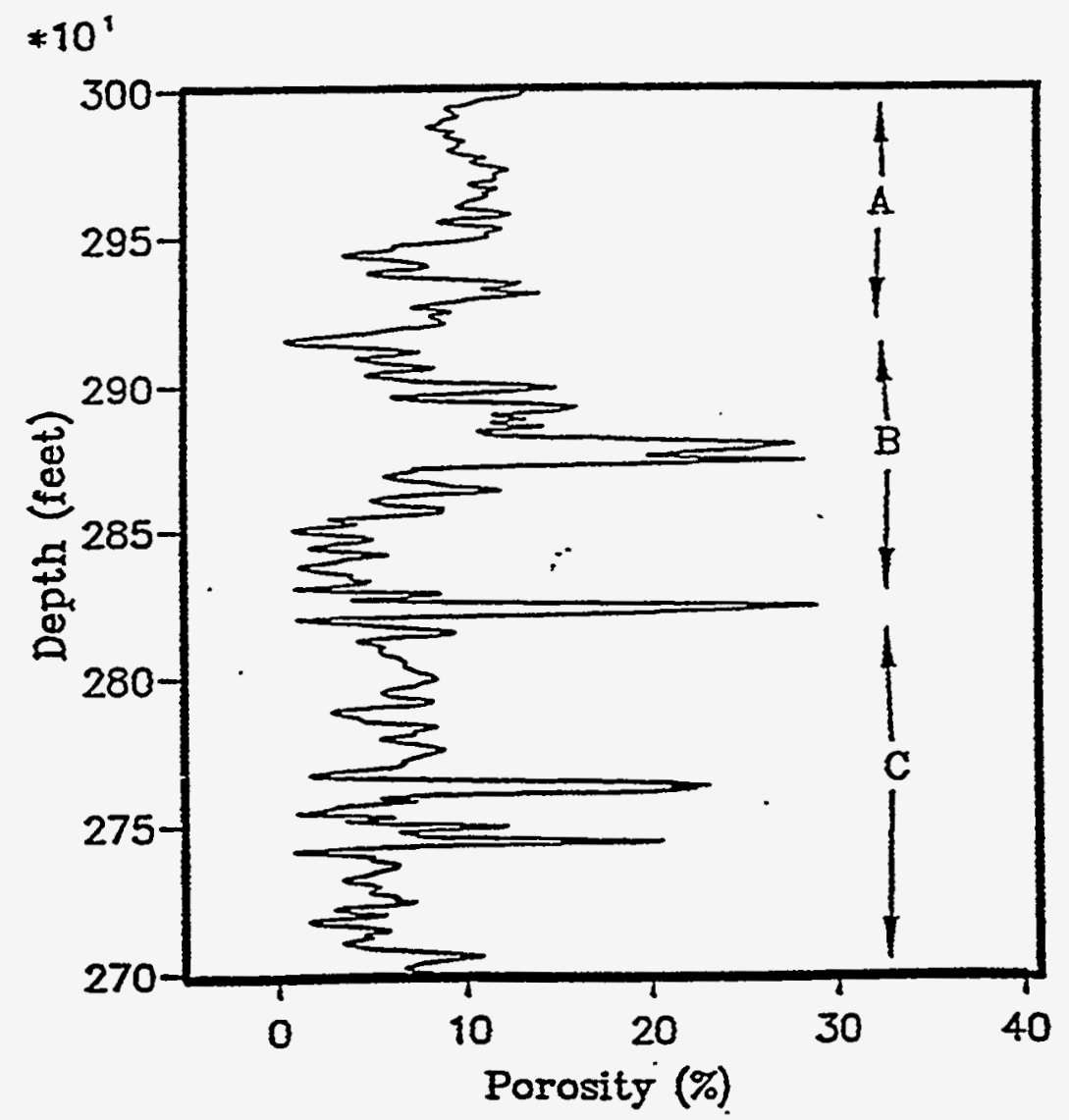

Figure 4.Example porosity trace before seasonal adjustments 


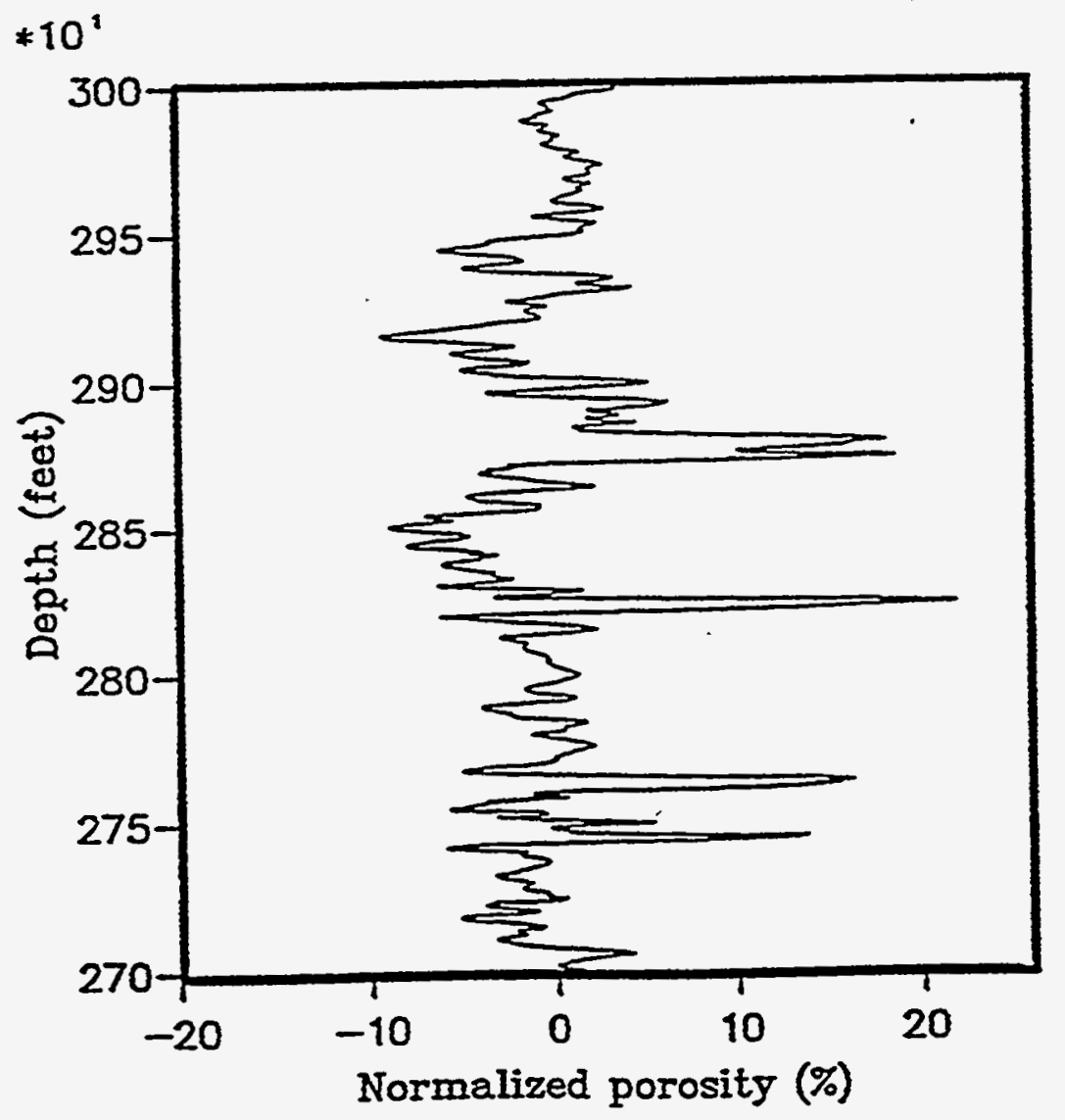

Figure $\dddot{5}$ Example porosity trace after seasonal adjustments 


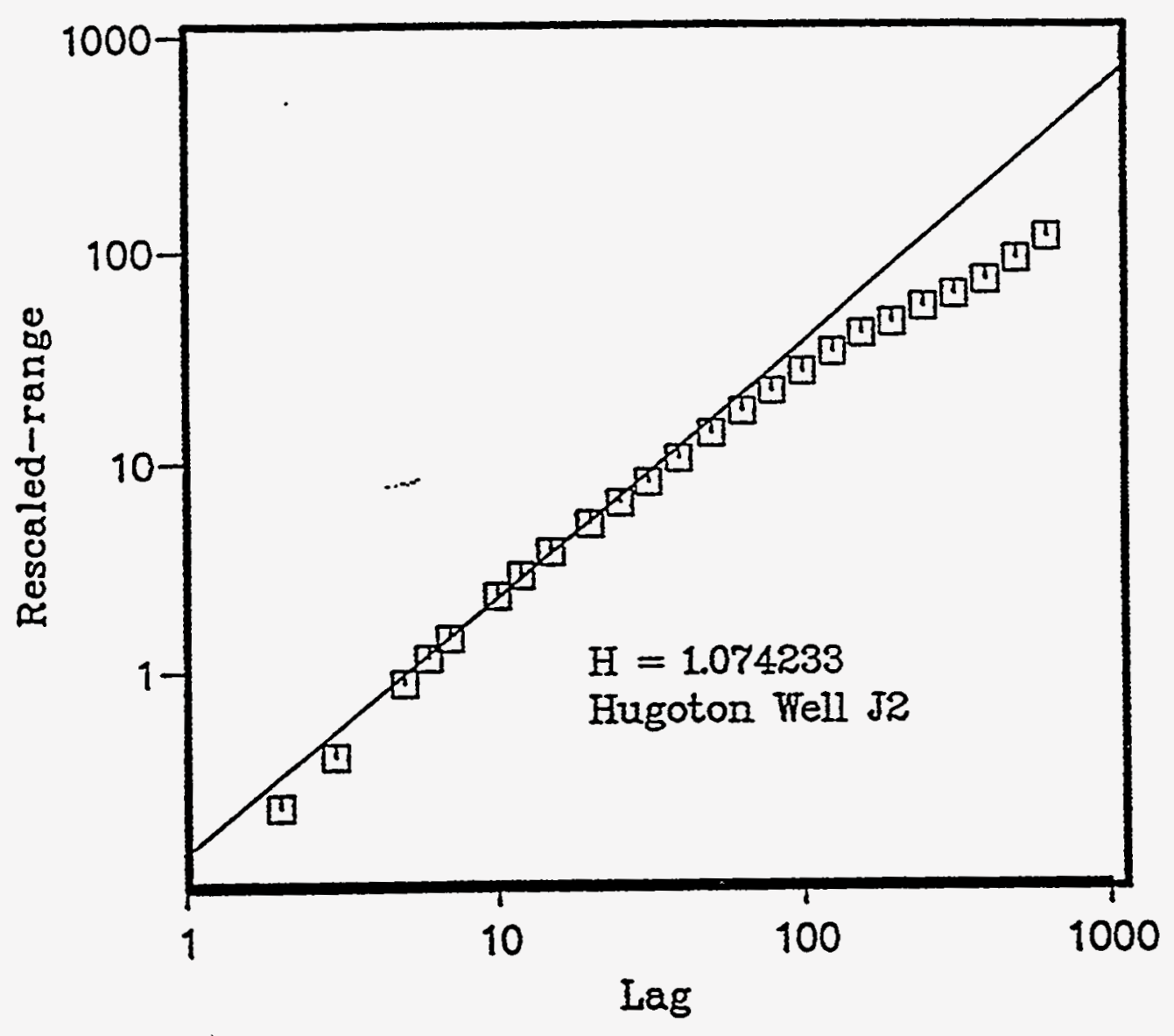

Figure. $6 . \mathrm{R} / \mathrm{S}$ analysis of porosity trace showing periodic trend 


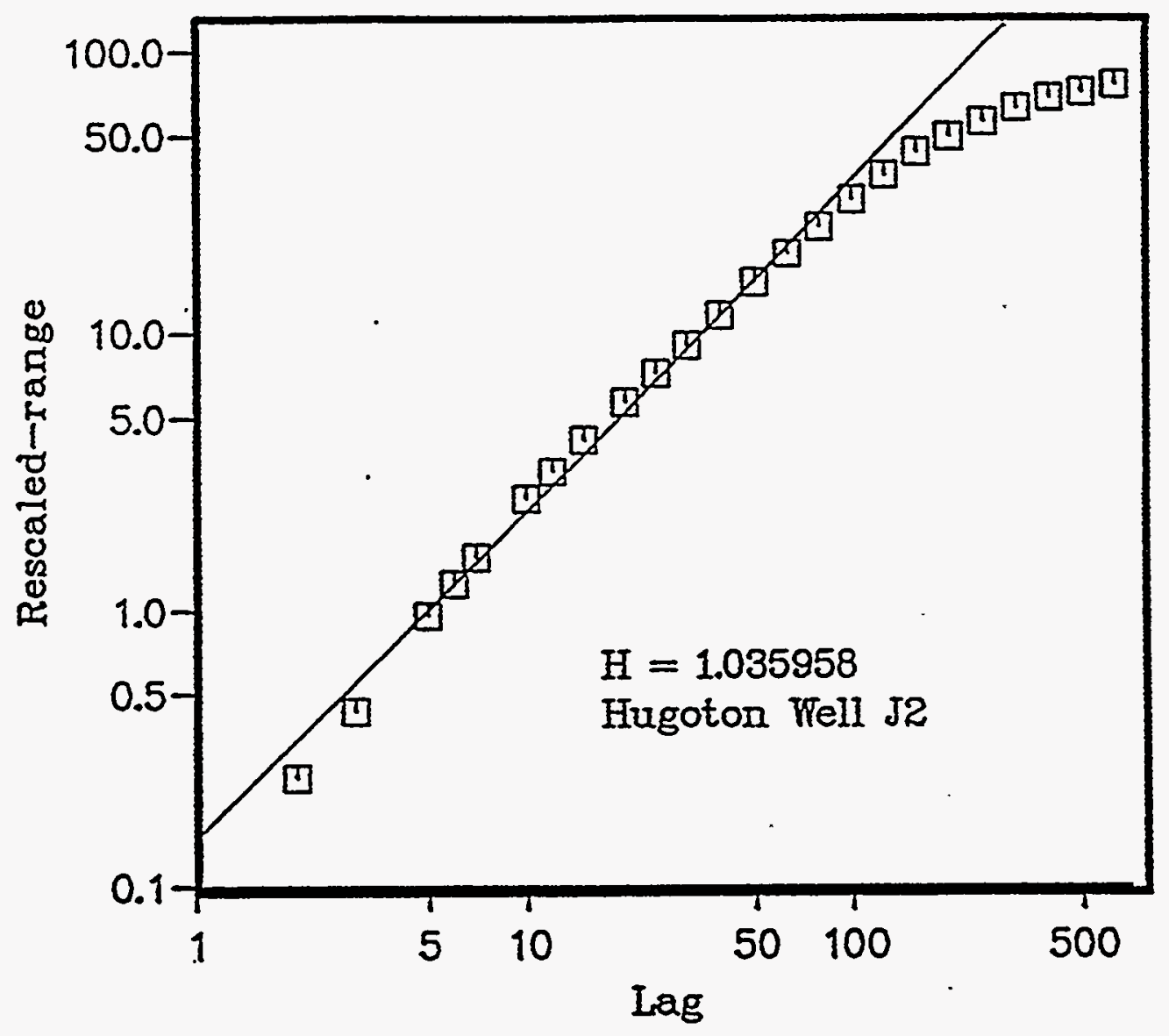

Figure $:-7 . ? / \mathrm{S}$ analysis of porosity trace after seasonal adjustments 


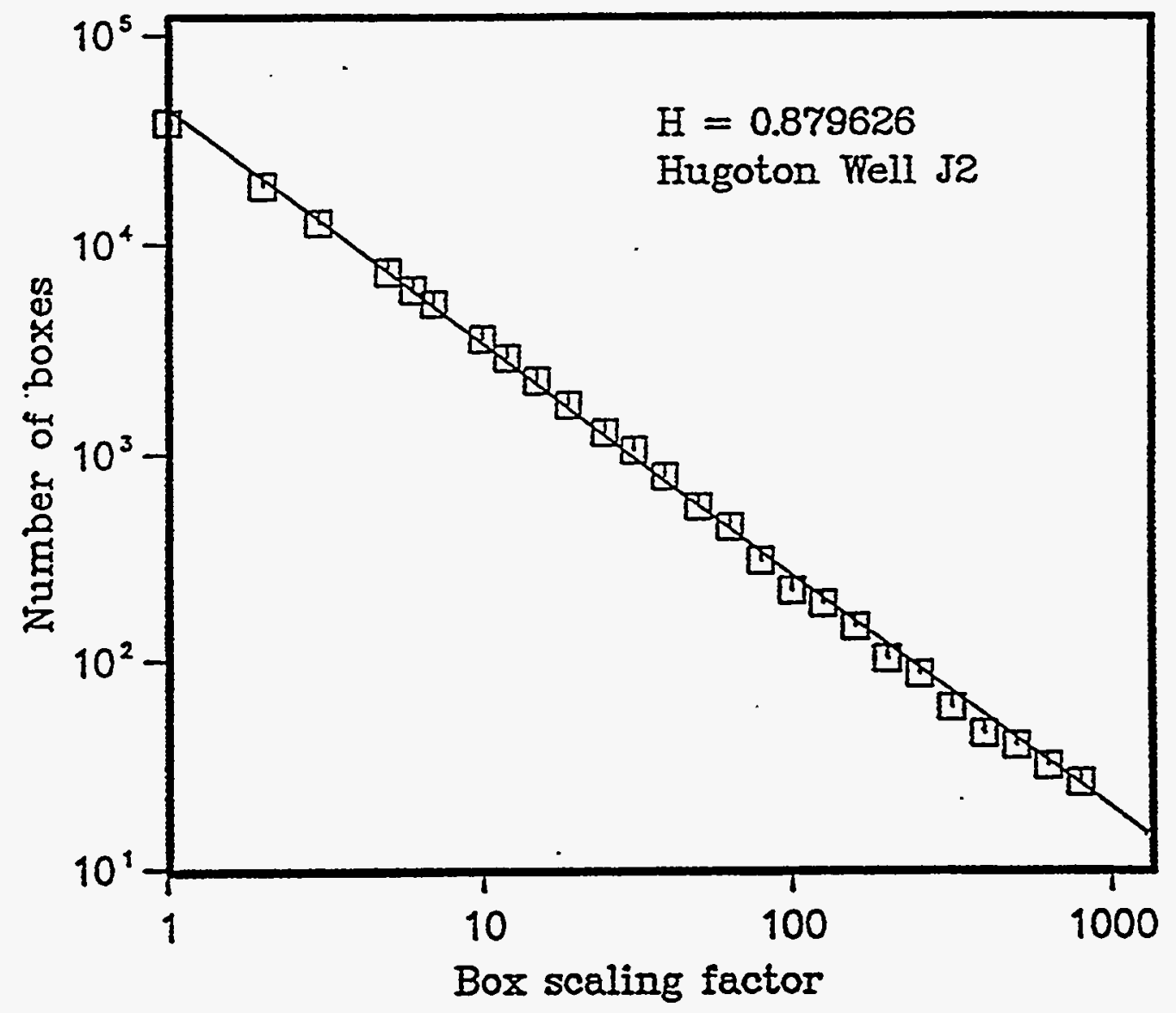

Figure 8. I exponent analysis using box-counting before seasonal adjustments 


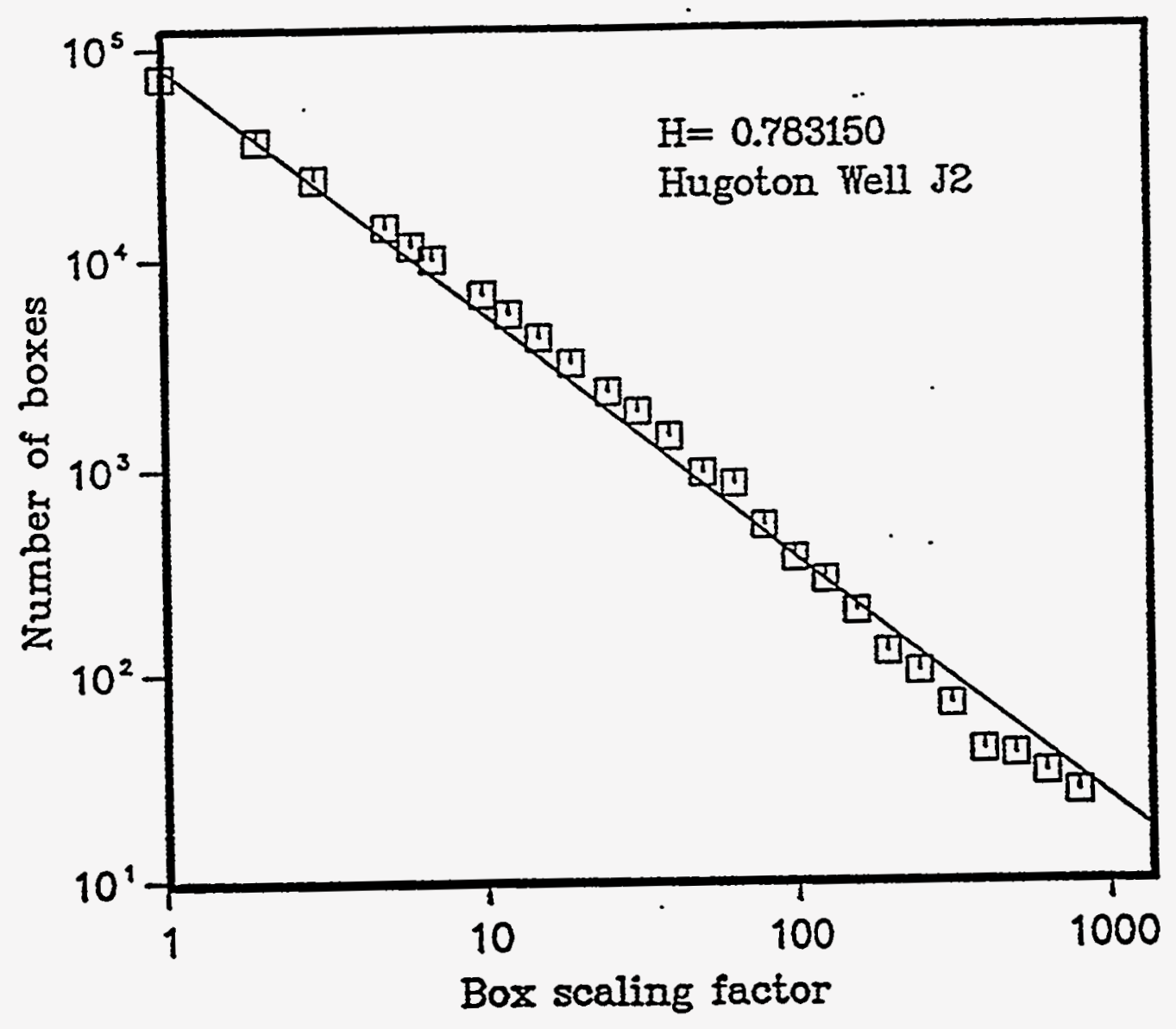

Figure 9. $H$ exponent analysis using box-counting after seasonal adjustments 


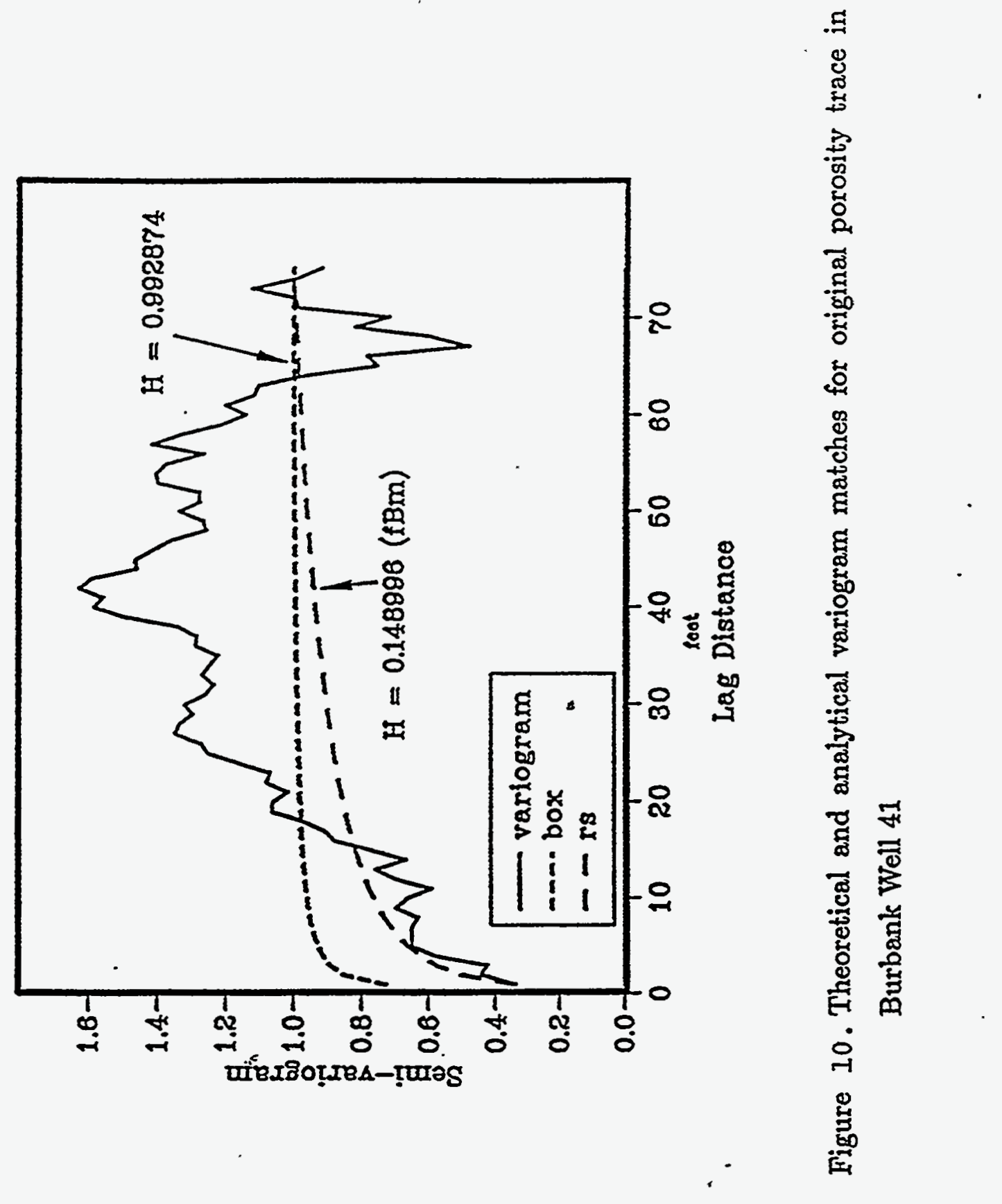




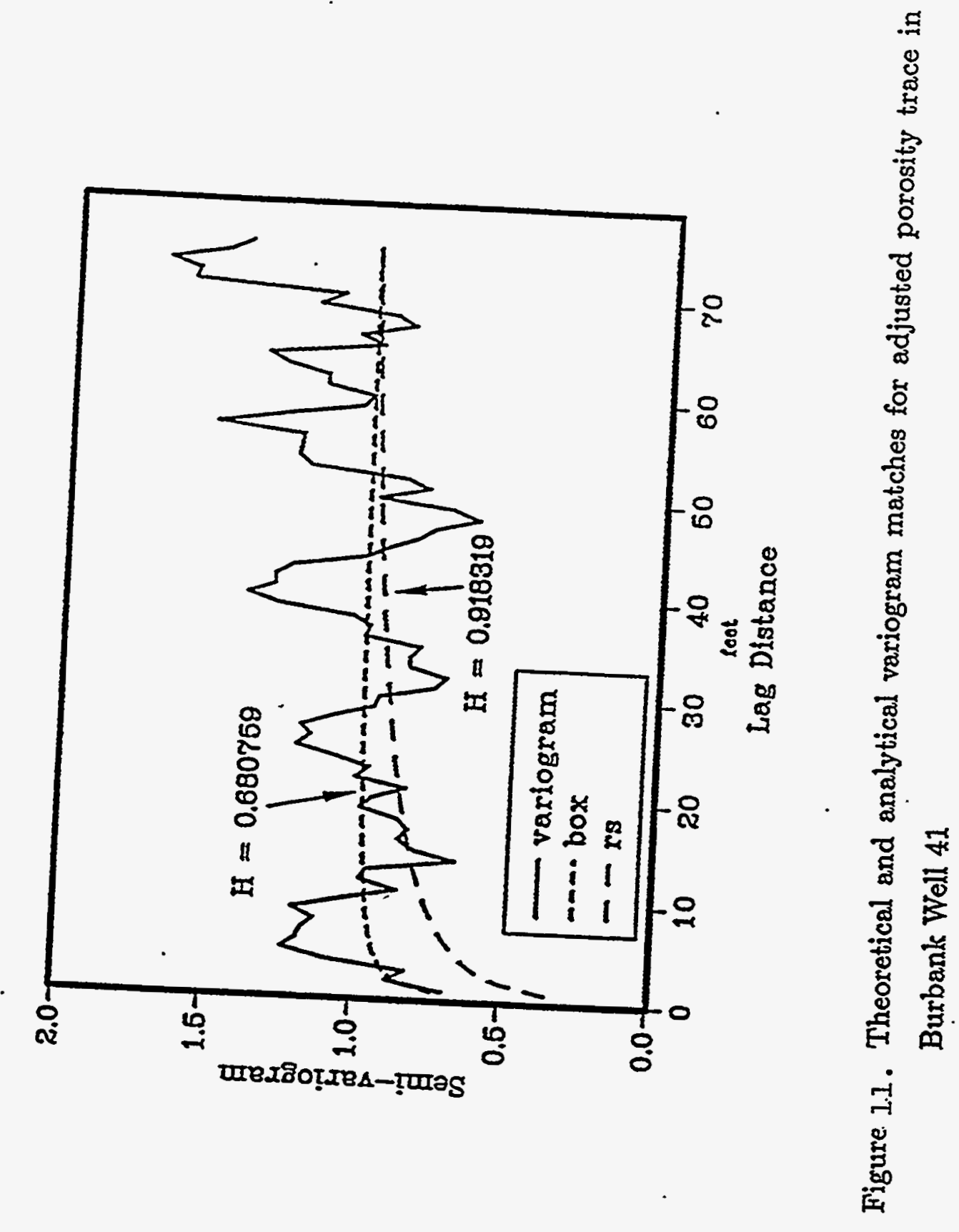




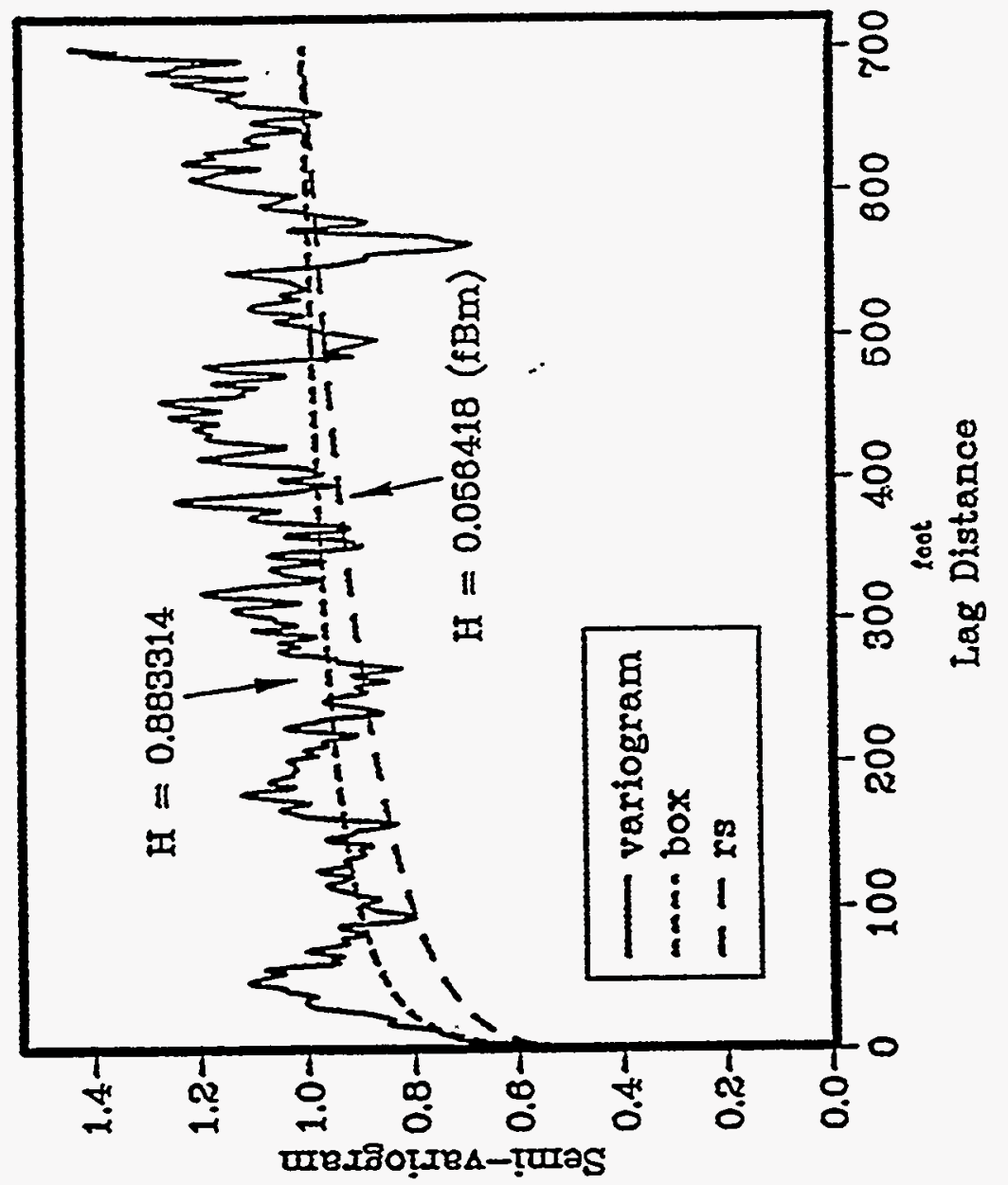

.

点

.

क्ष

总

要

范

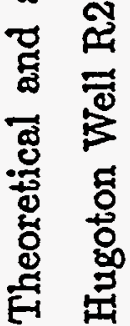

茫 


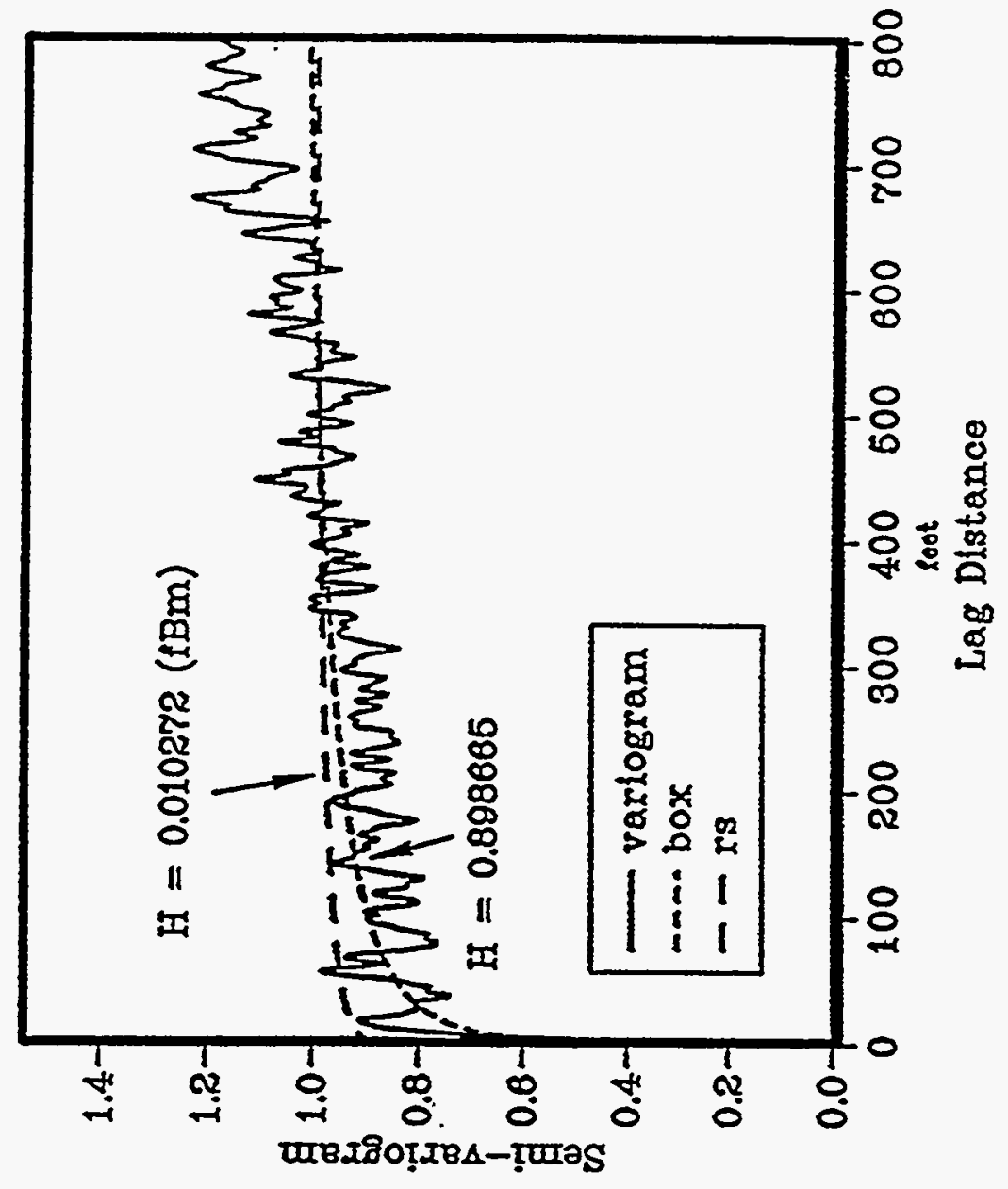


Northing (feet)

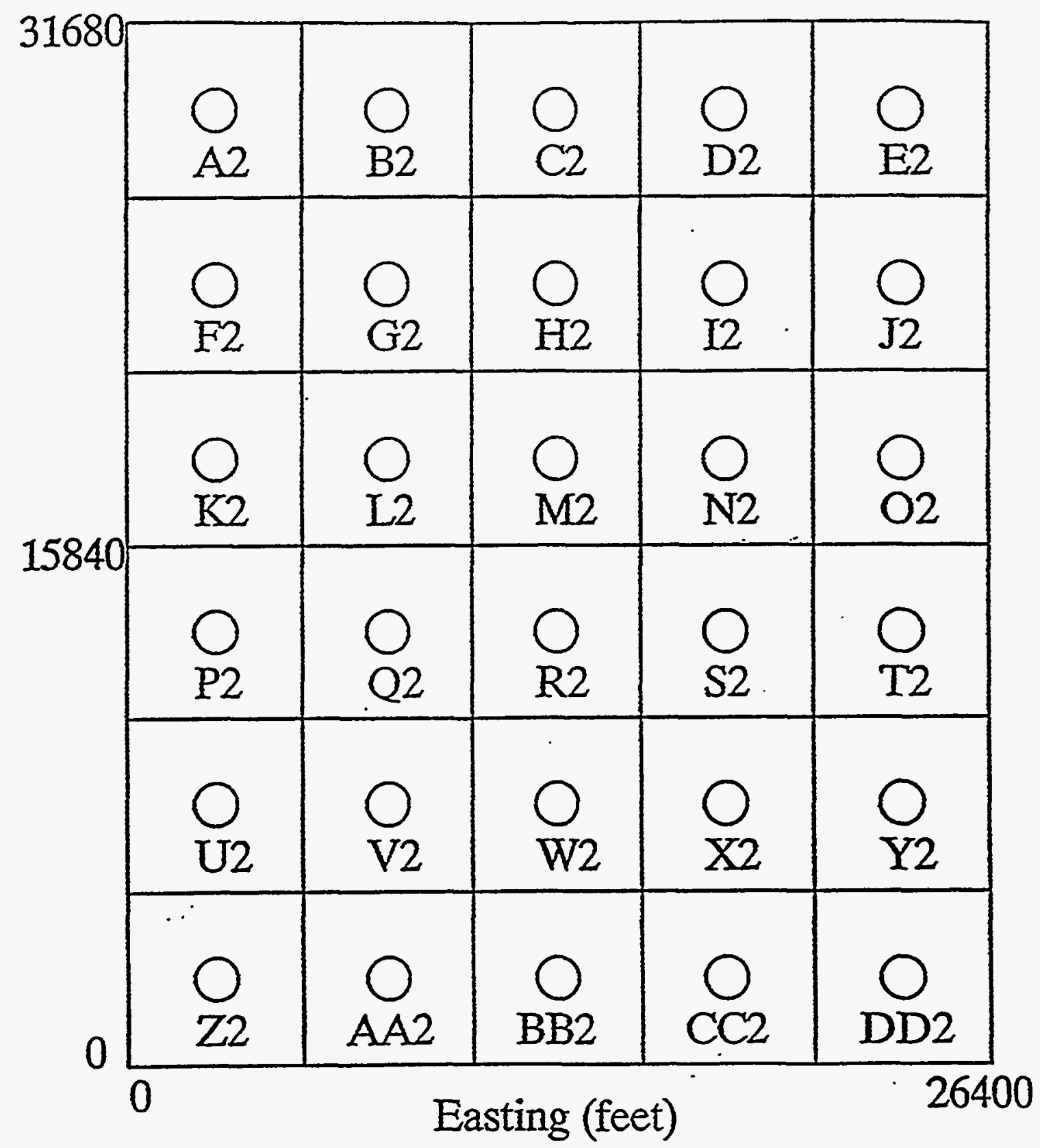

. Figure '14. Well location map of Hugoton Field 


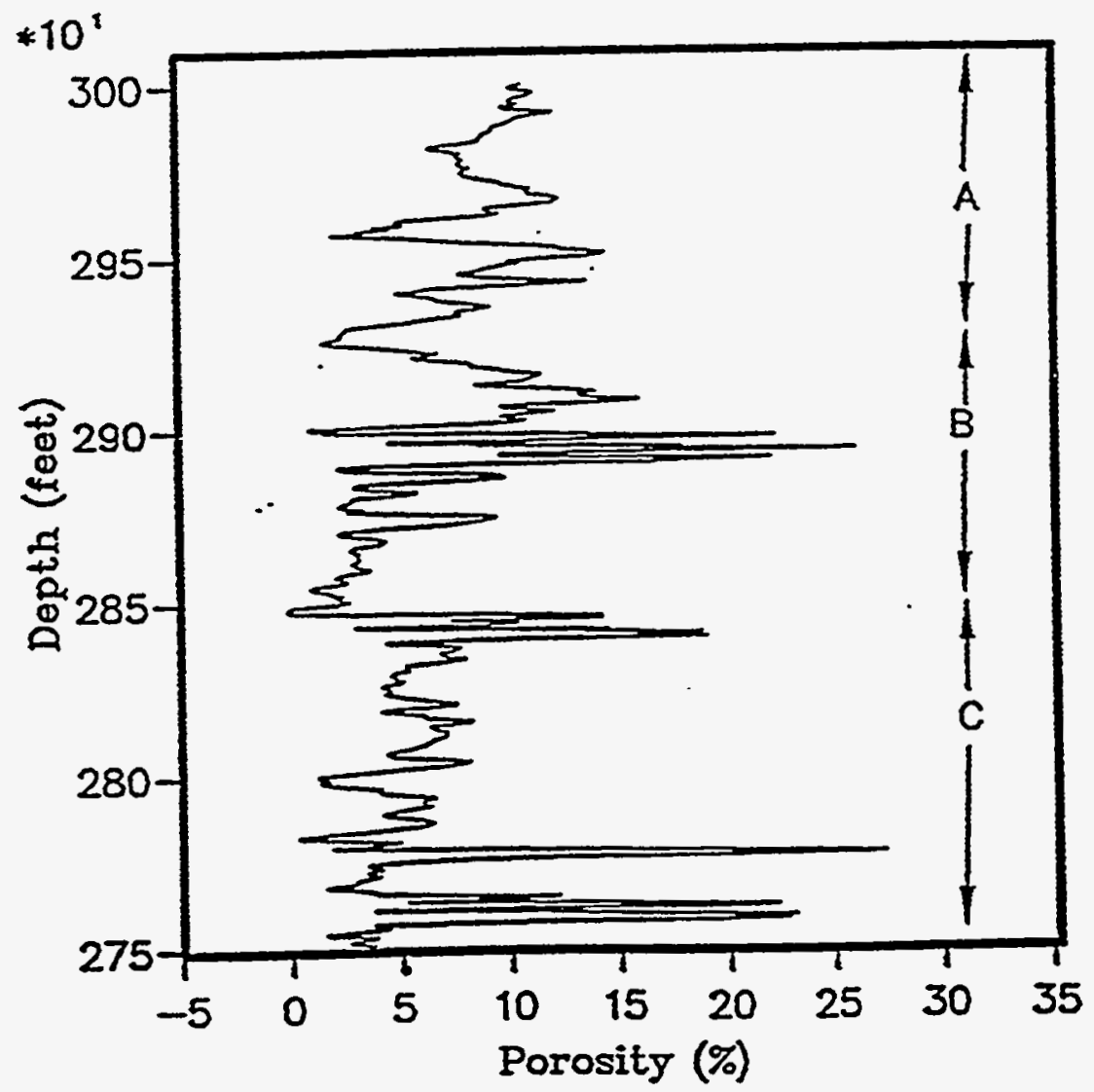

Figure 15. Example Hugoton porosity trace before seasonal adjustments 


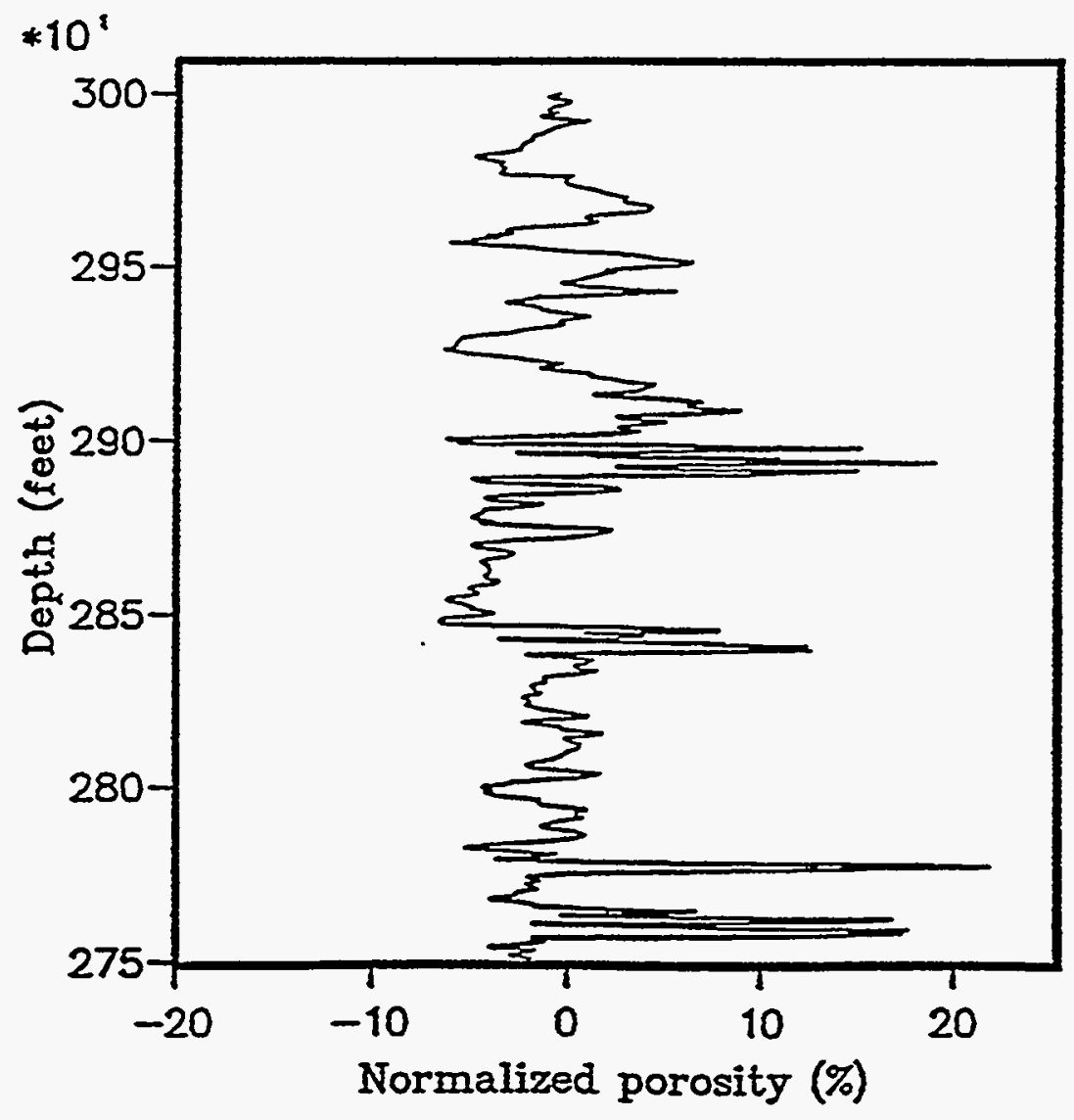

Figure .16. Example Hugoton porosity trace after seasonal adjustments 


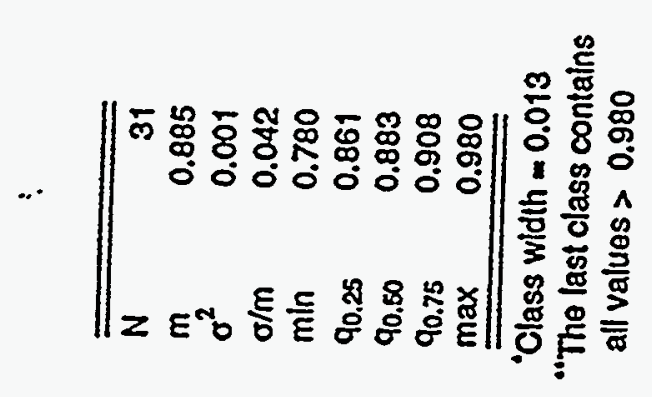

ב్ర

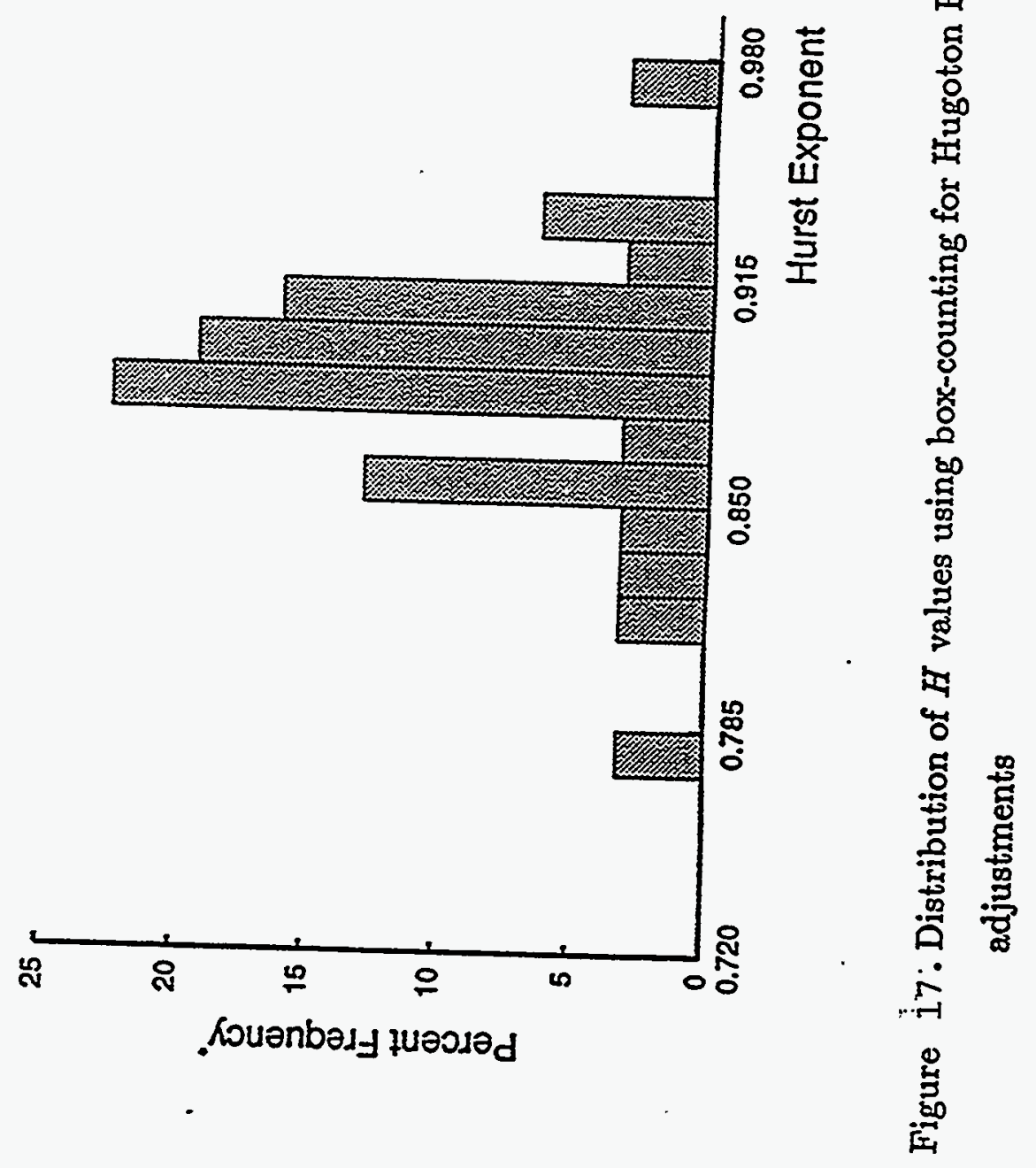




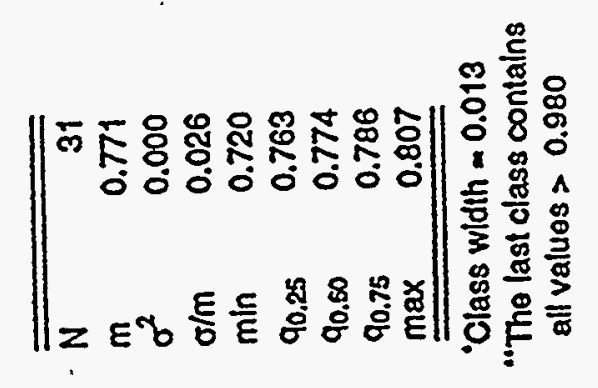

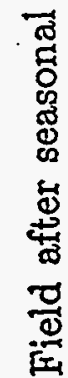

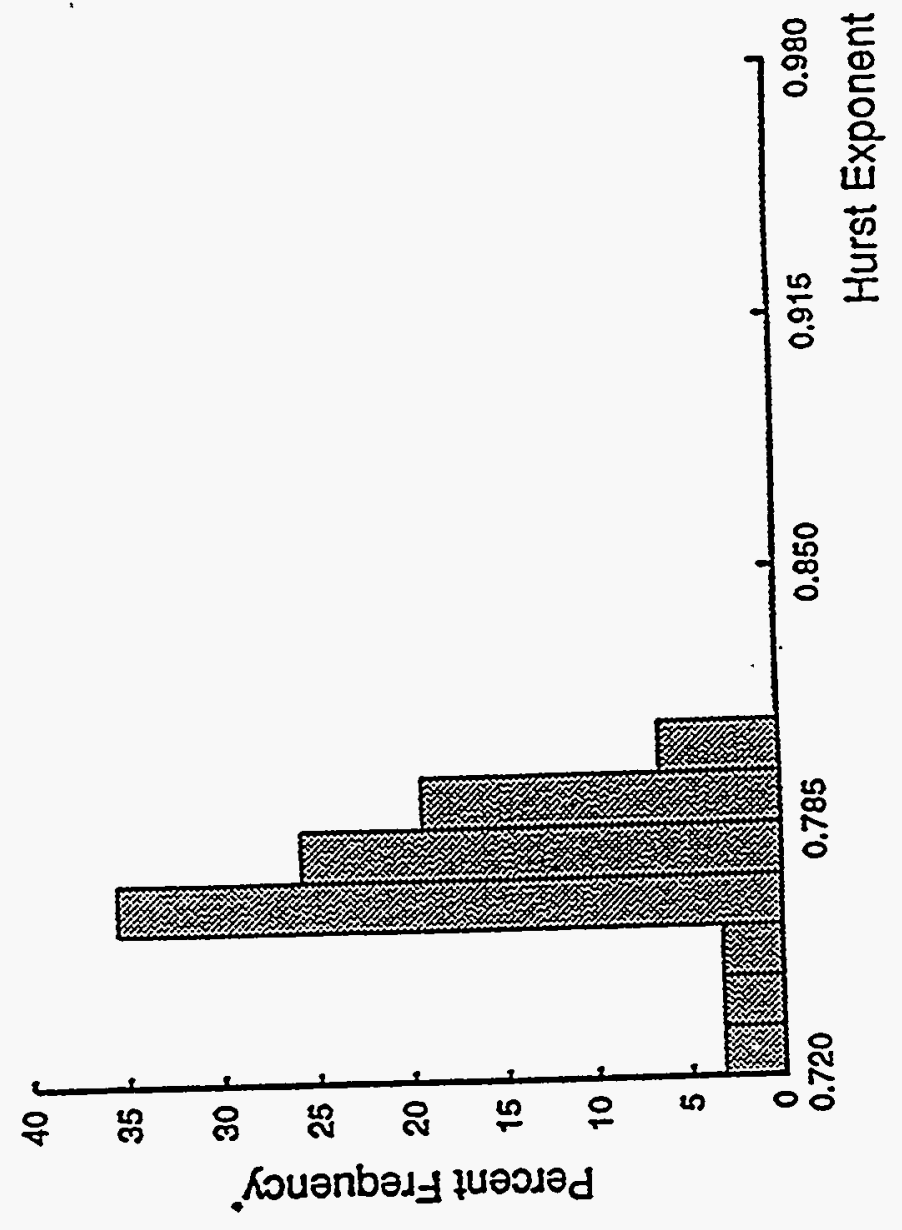

总

웅

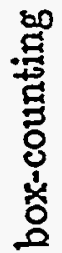

㿣

ङ

4

4

总

$\dot{\infty}$.

$\pi$

䓌 


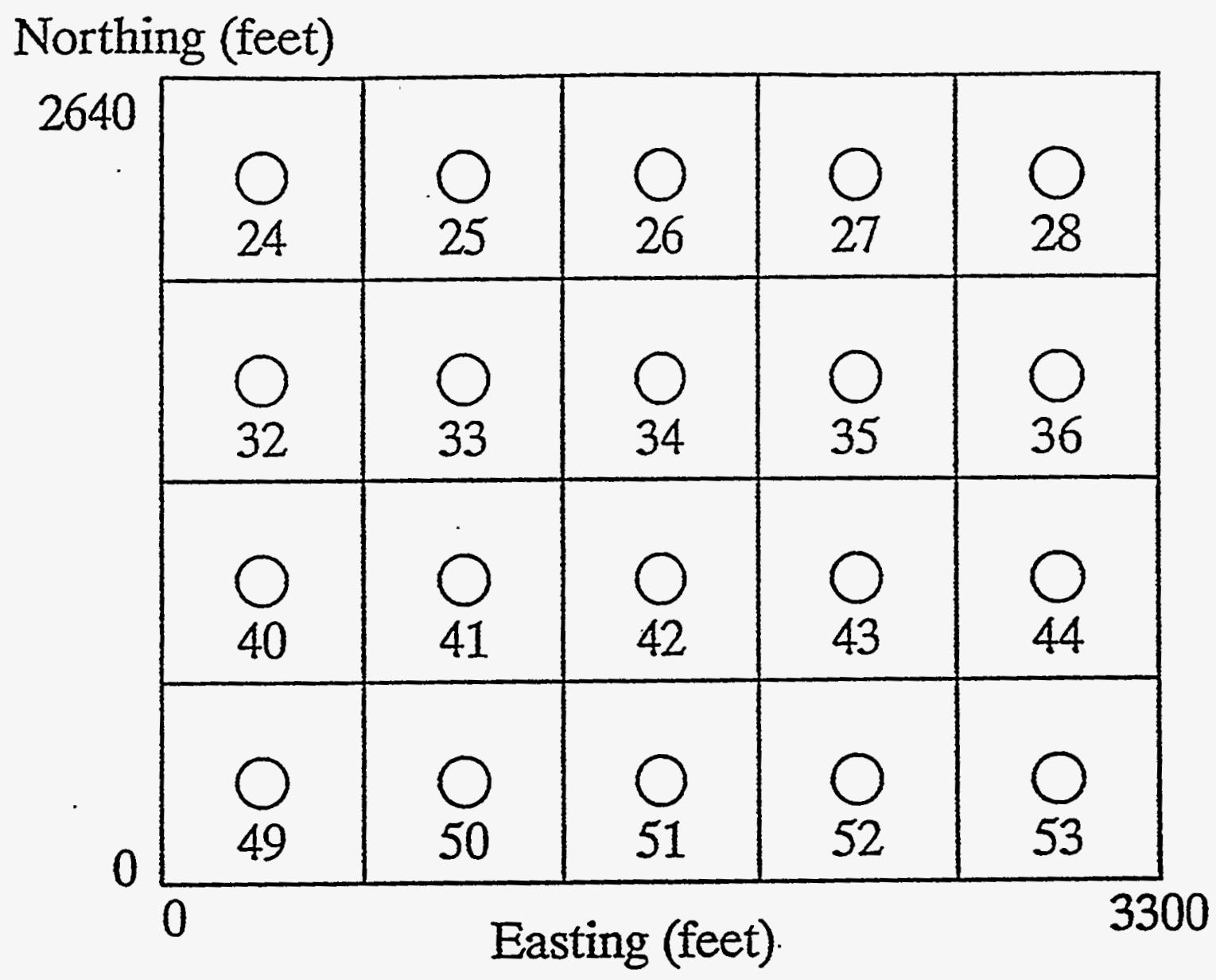

Figure 19. Well location map of Burbank Field 


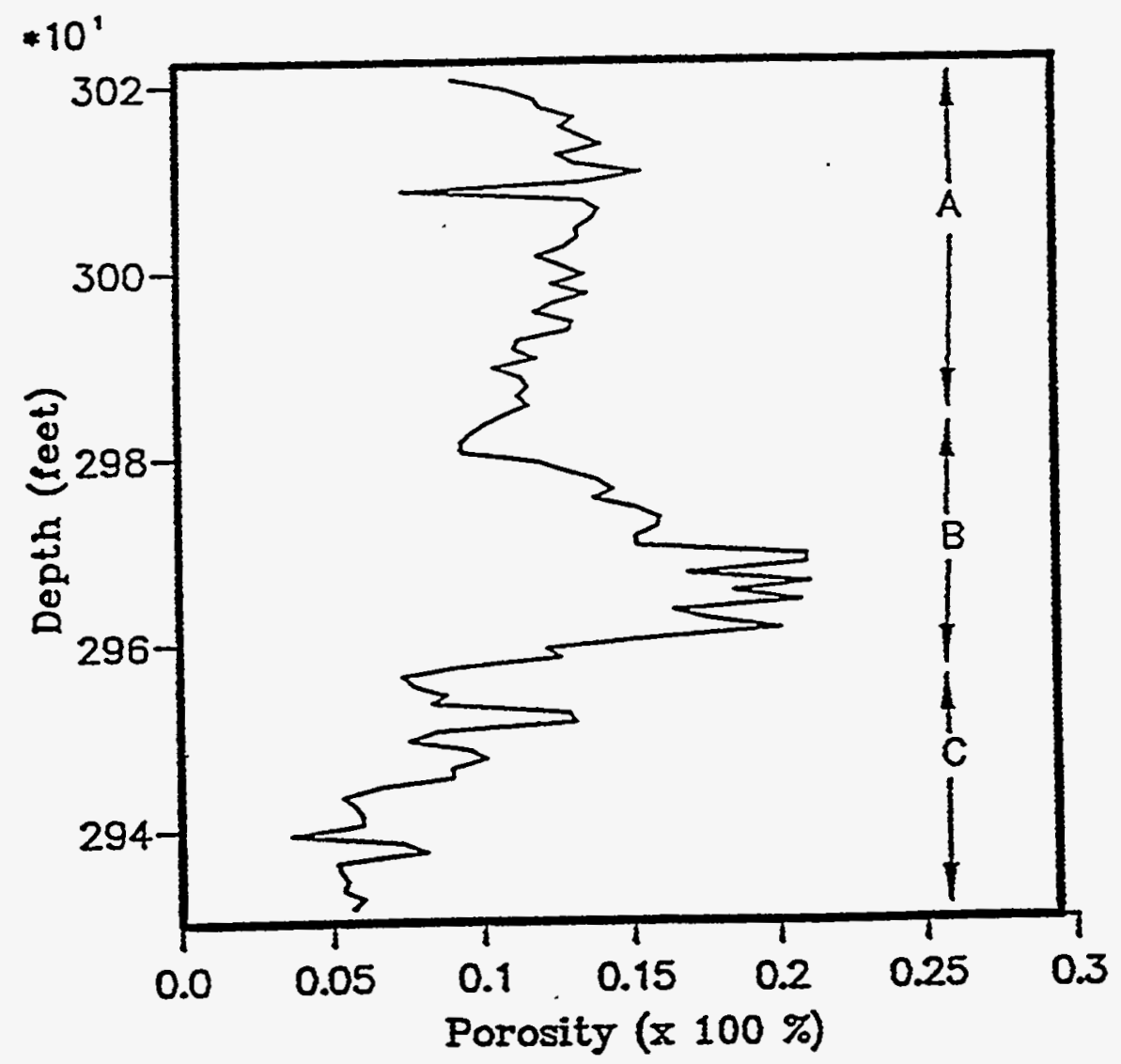

Figure 20. Example Burbank porosity trace before seasonal adjustments 


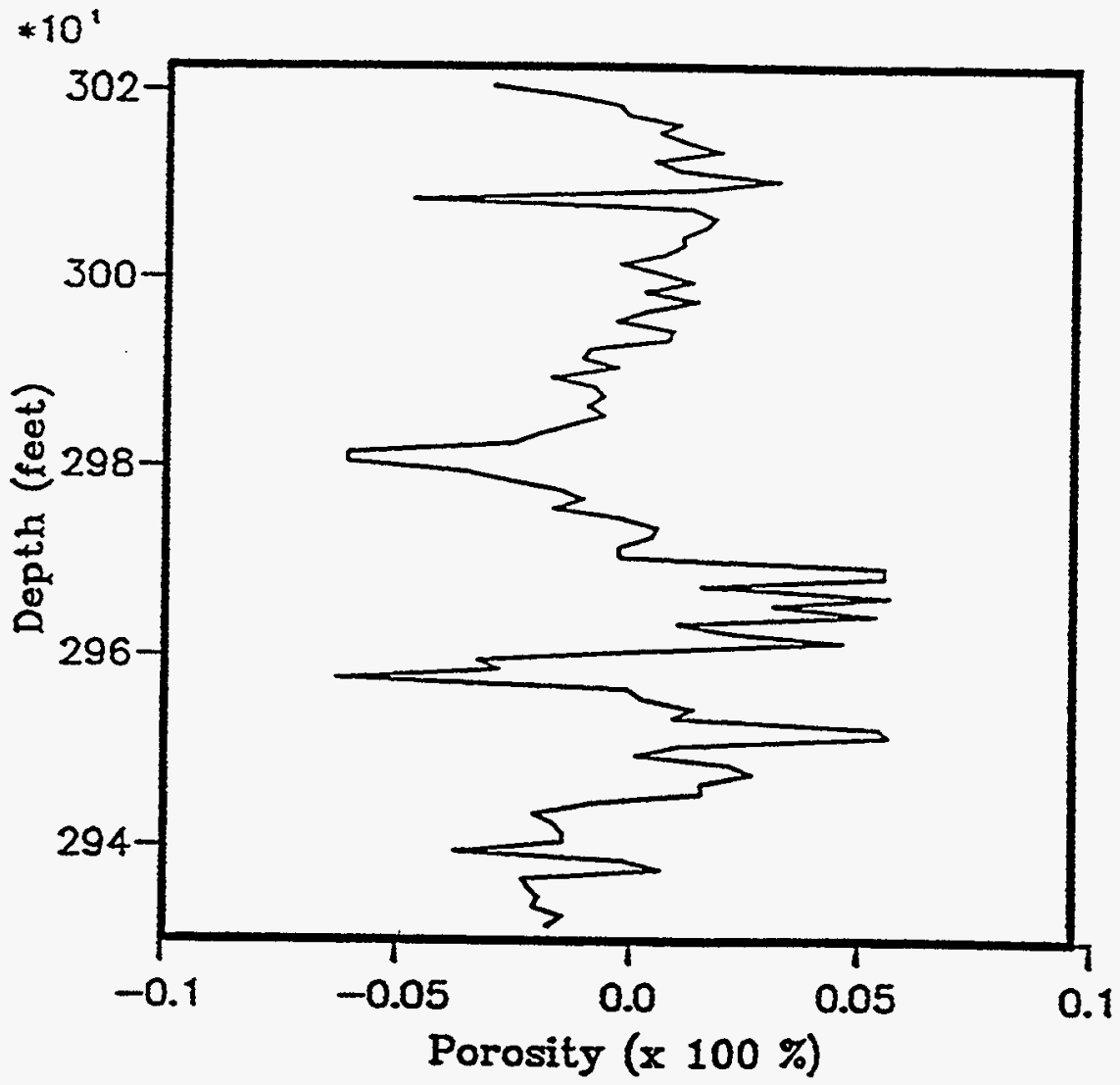

Figure 21: Example Burbank porosity trace after seasonal adjustments 
Northing (feet)

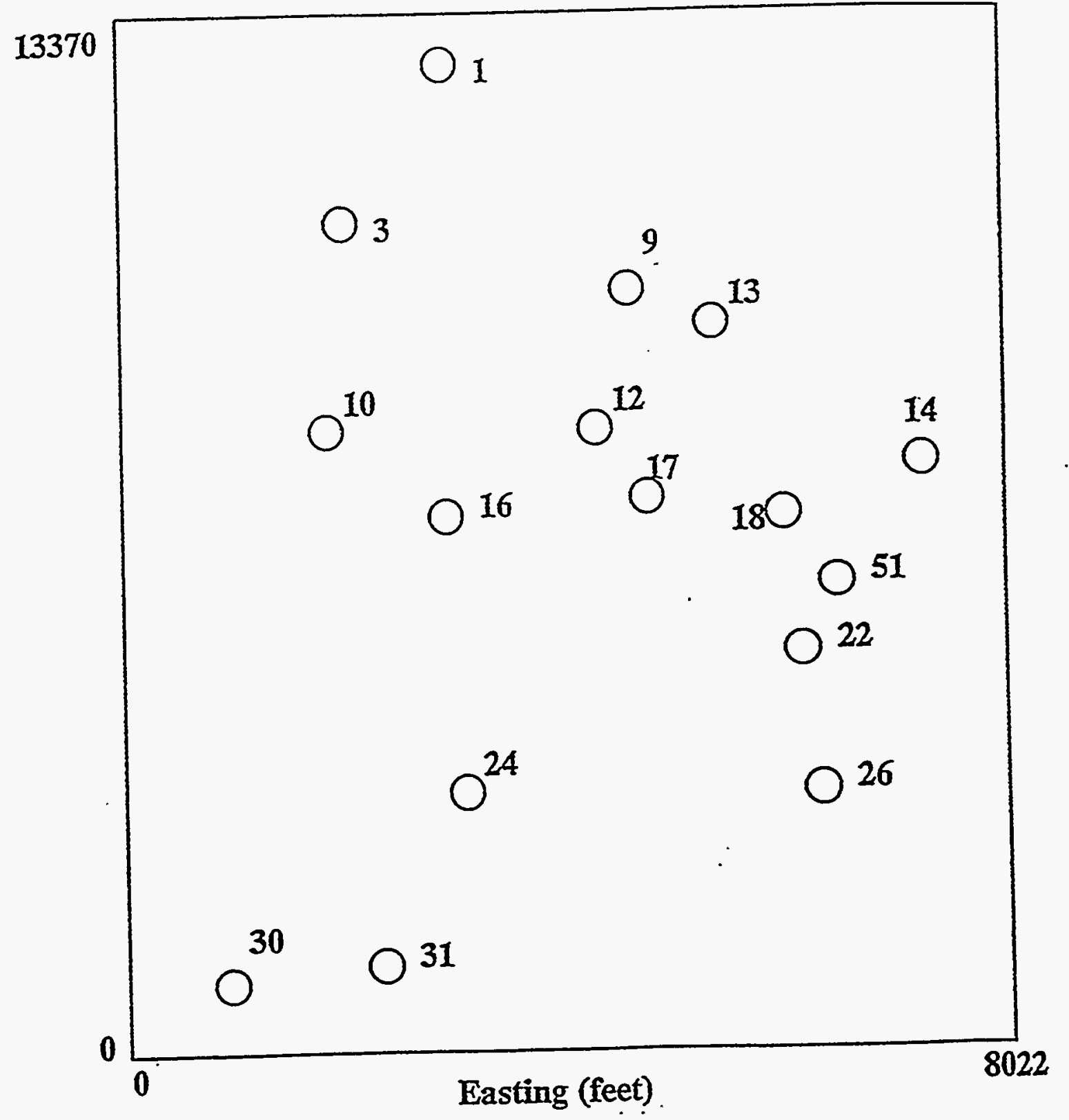

Figure 22. Well location map of Sable Field 


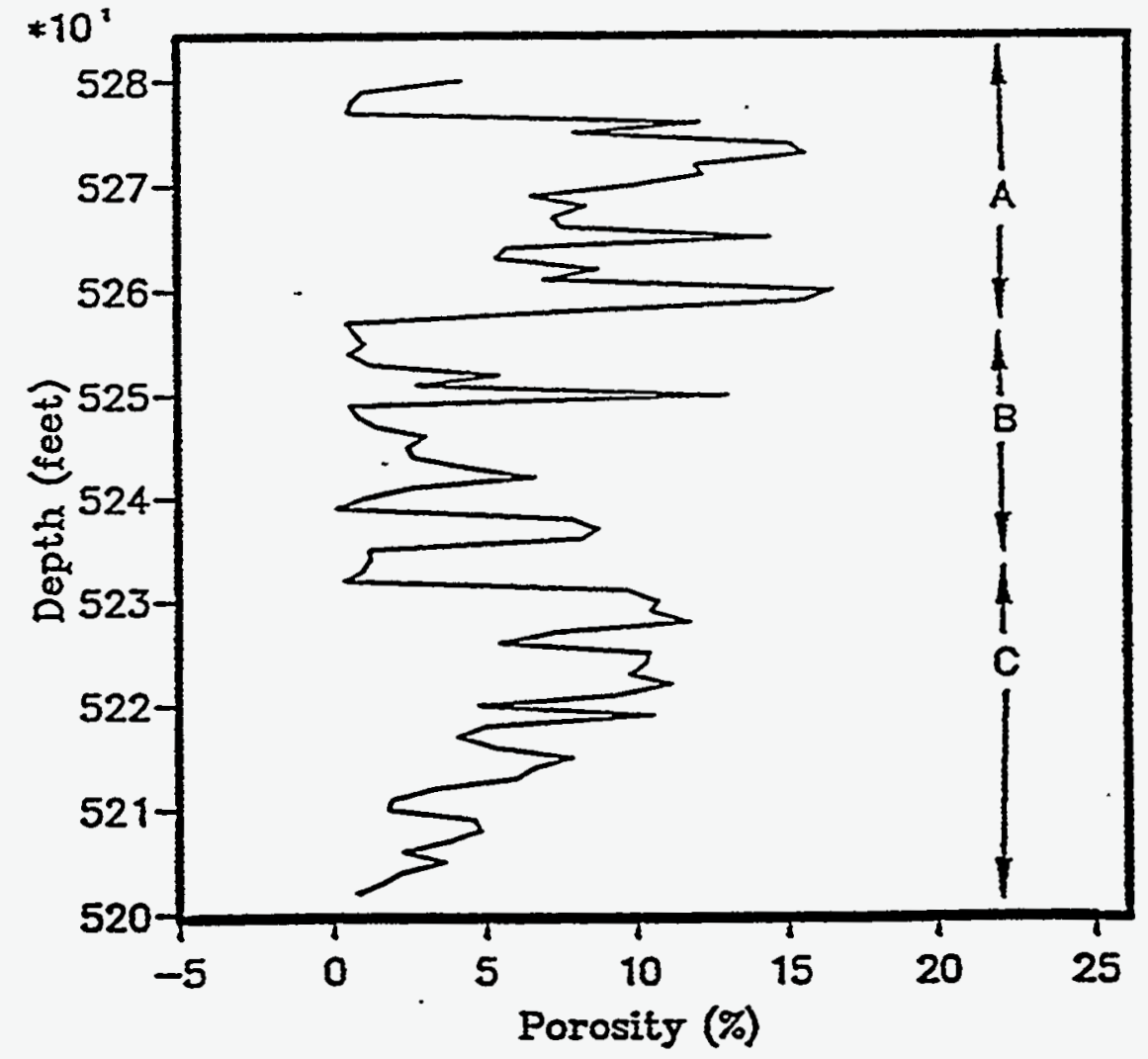

Figure $23:$ Sable porosity log before seasonal adjustments 


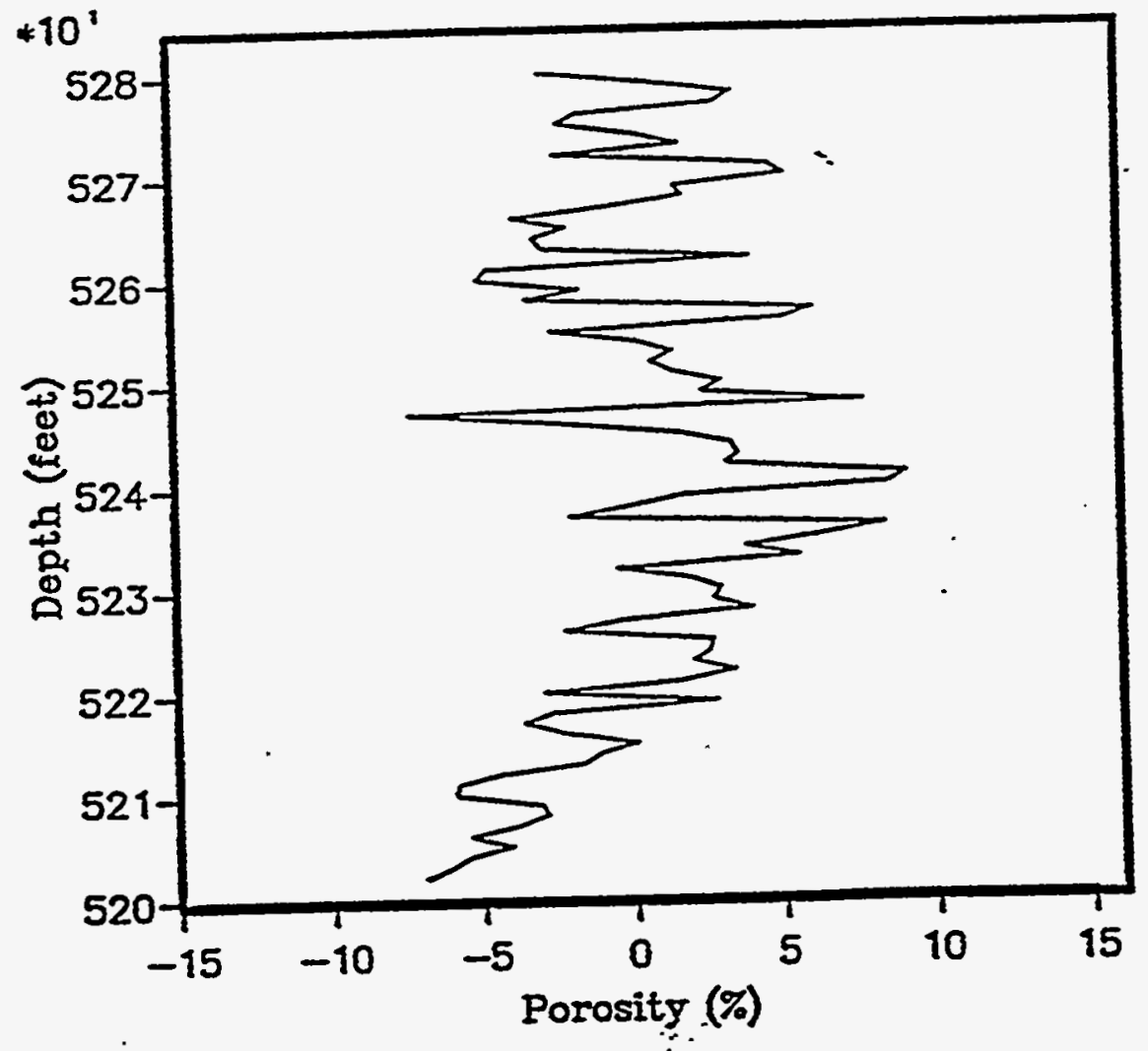

Figure; 24 . Example Sable porosity trace after seasonal adjustments 


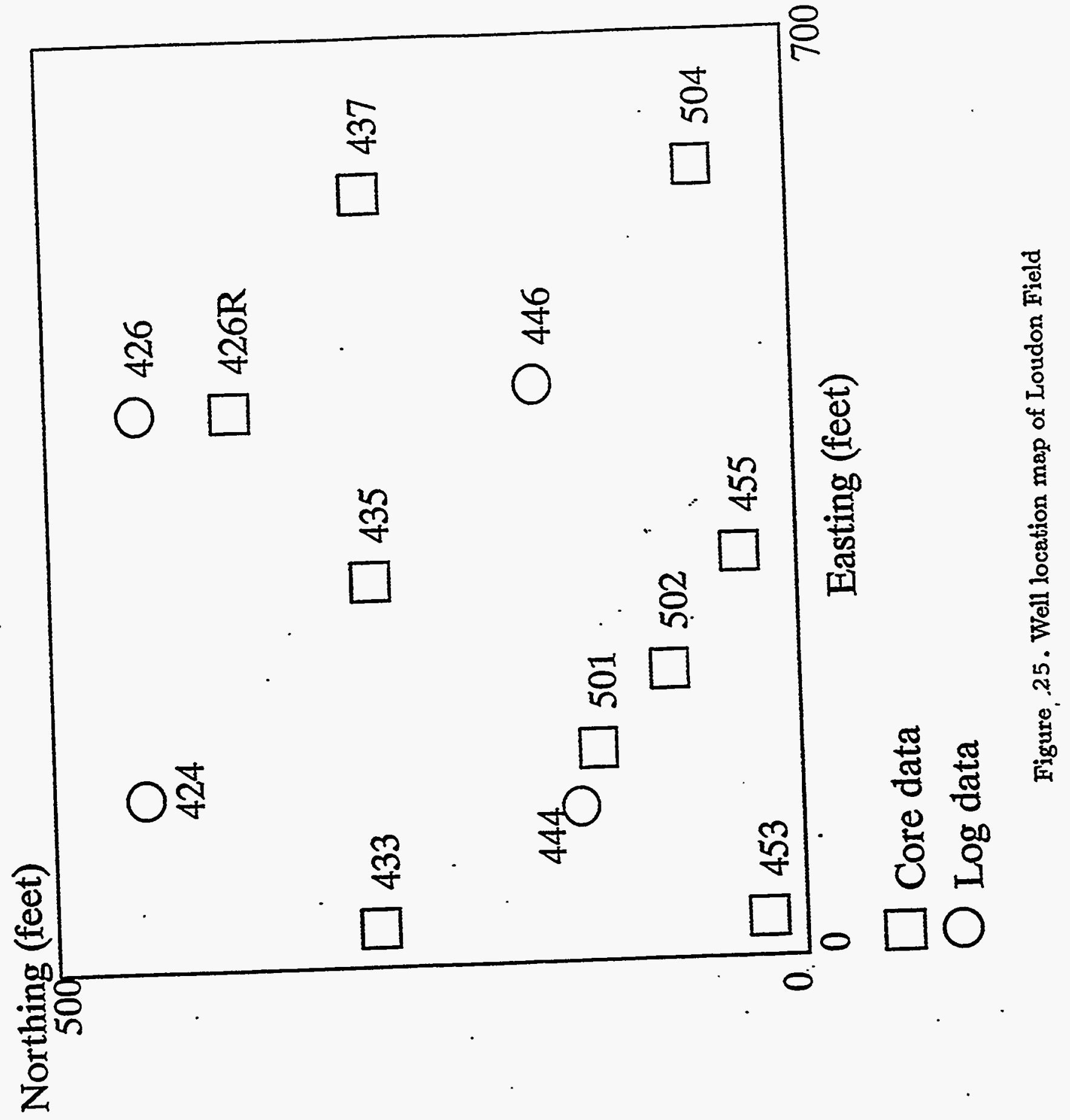


Northing (feet)

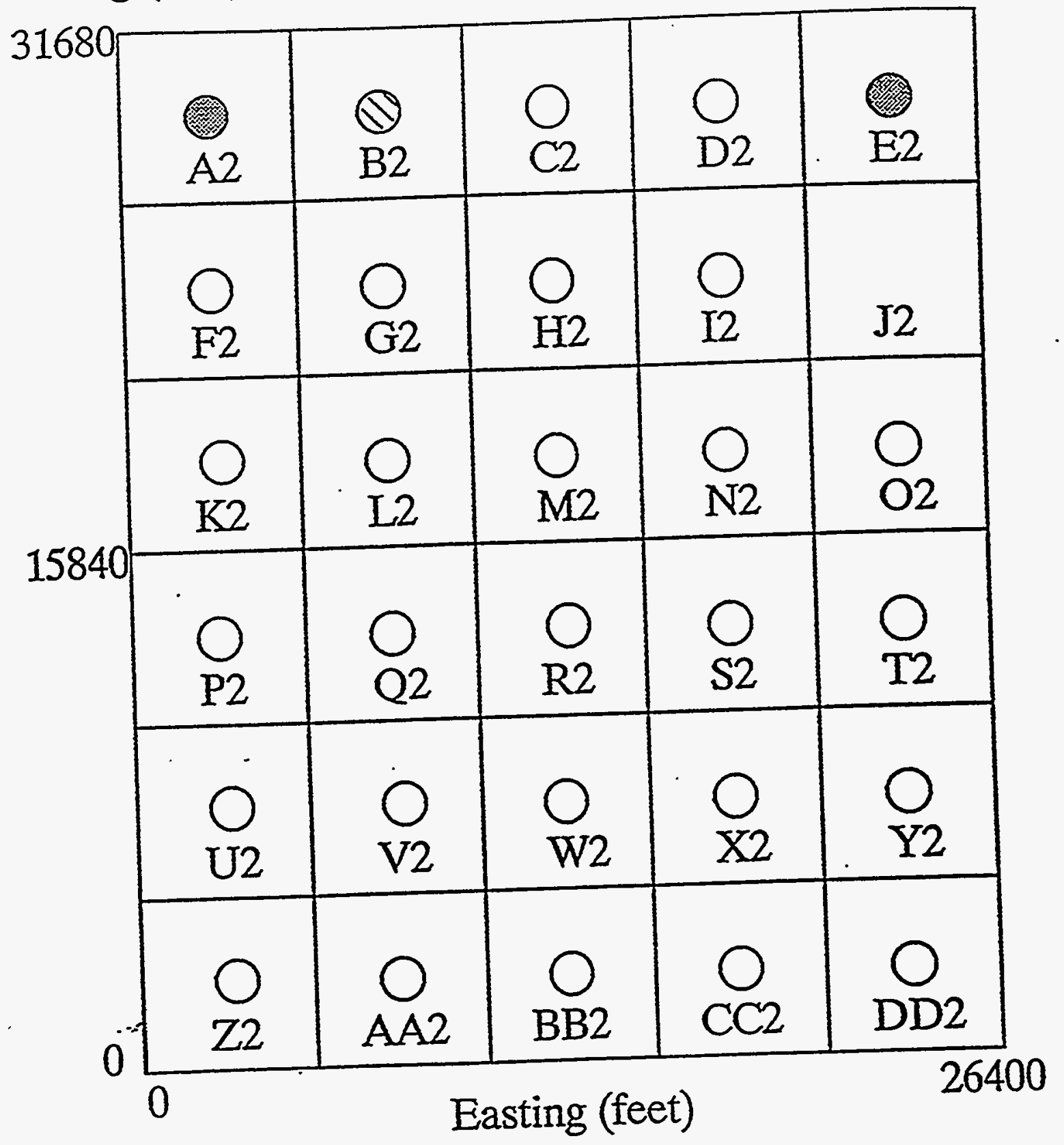

Figure. 26. Well location map for 2D Hugoton Field simulation 


\section{Before adding random component}

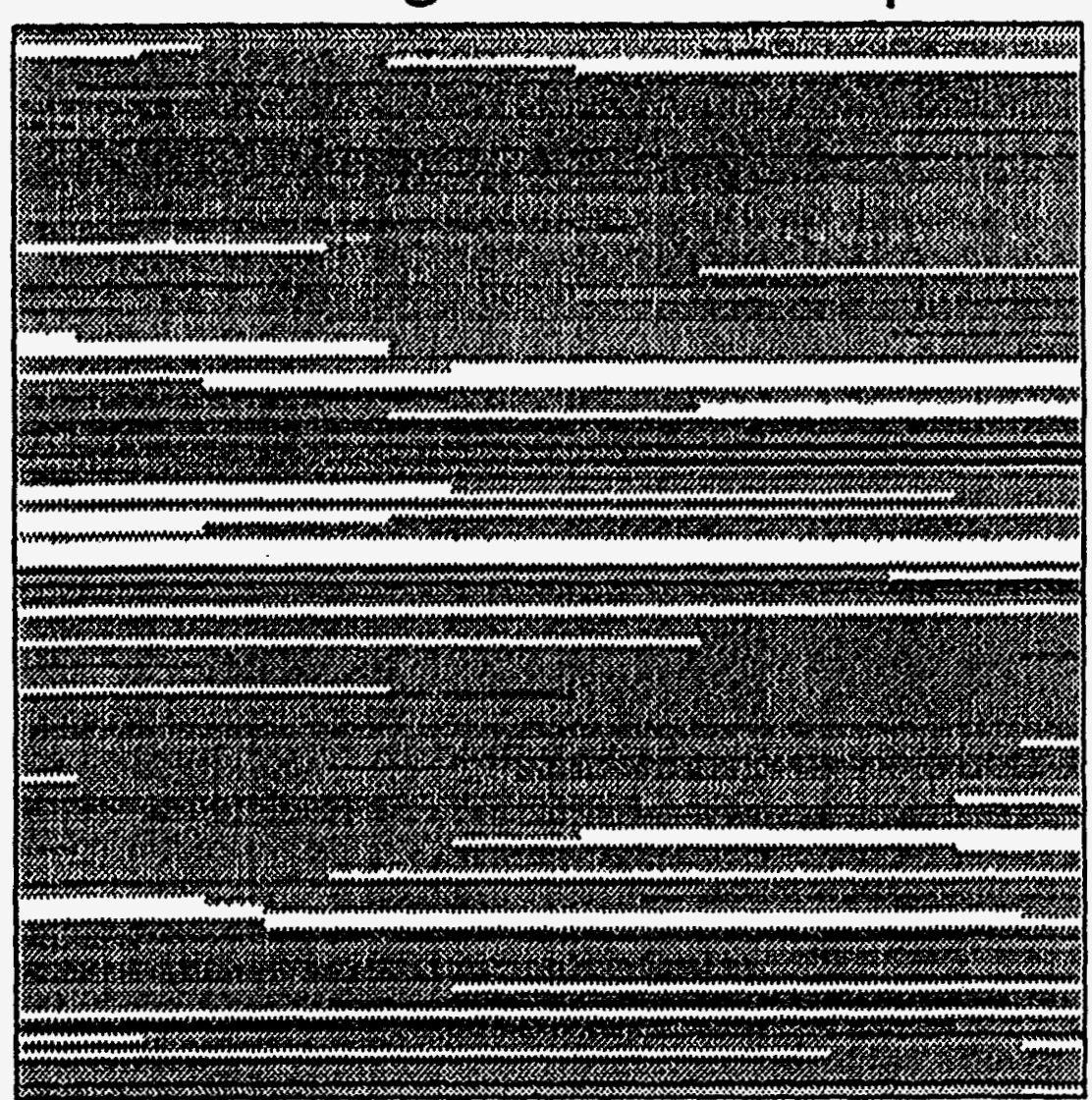

Figure 27. Gray scale plot of linearly interpolated values for Hugoton well pair A2 and E2 before adding Gaussian random element 


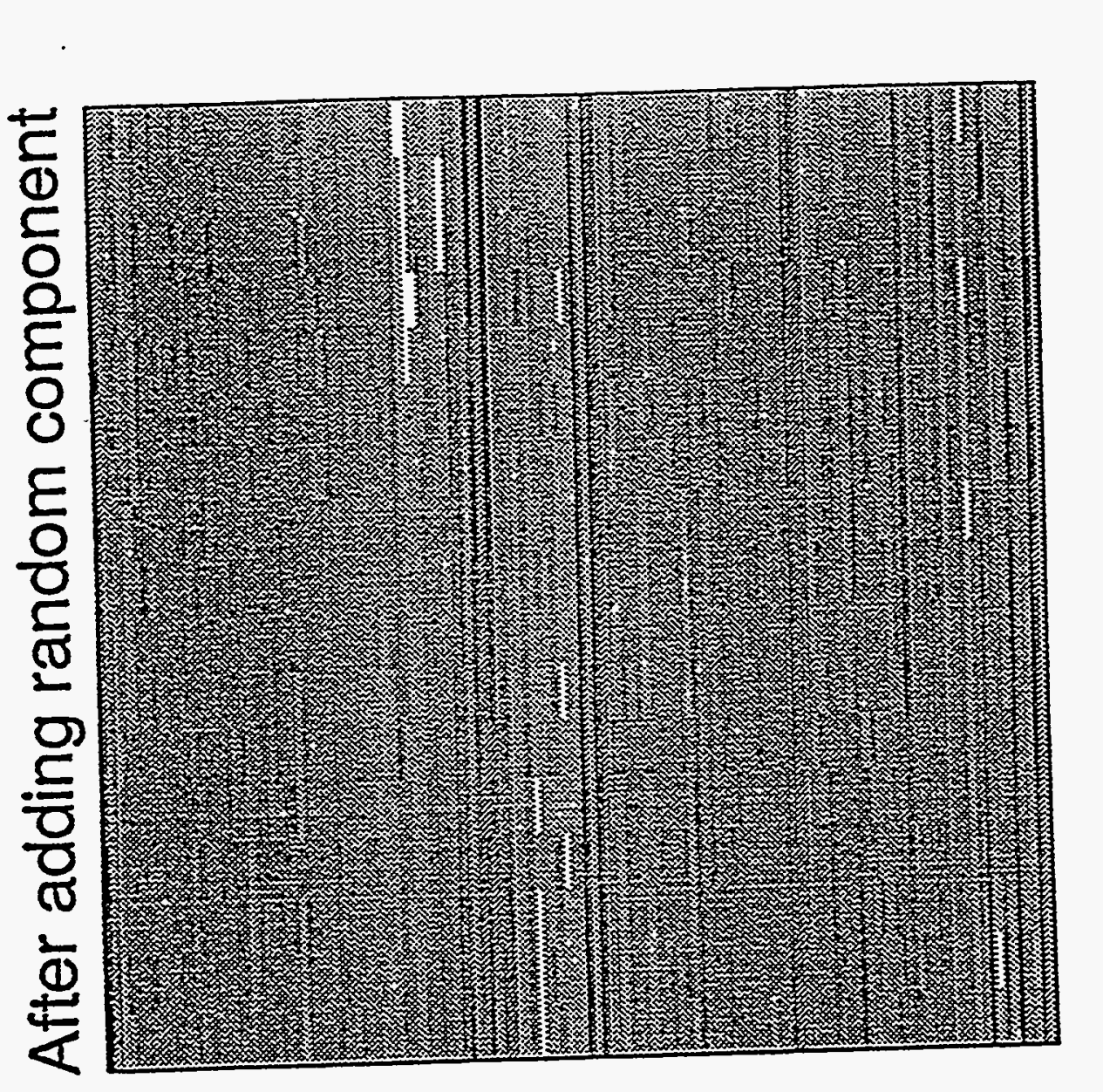

甶

器

व

娄

है

영

莒

$\frac{8}{5}$

总 范

过

.ृ.్ำ

站

.

을 임

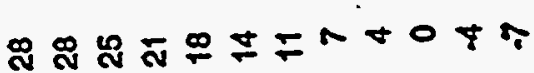

总

岩

密热

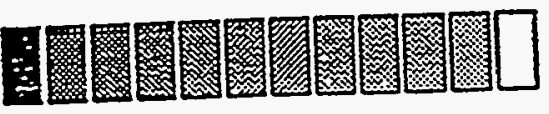

文 


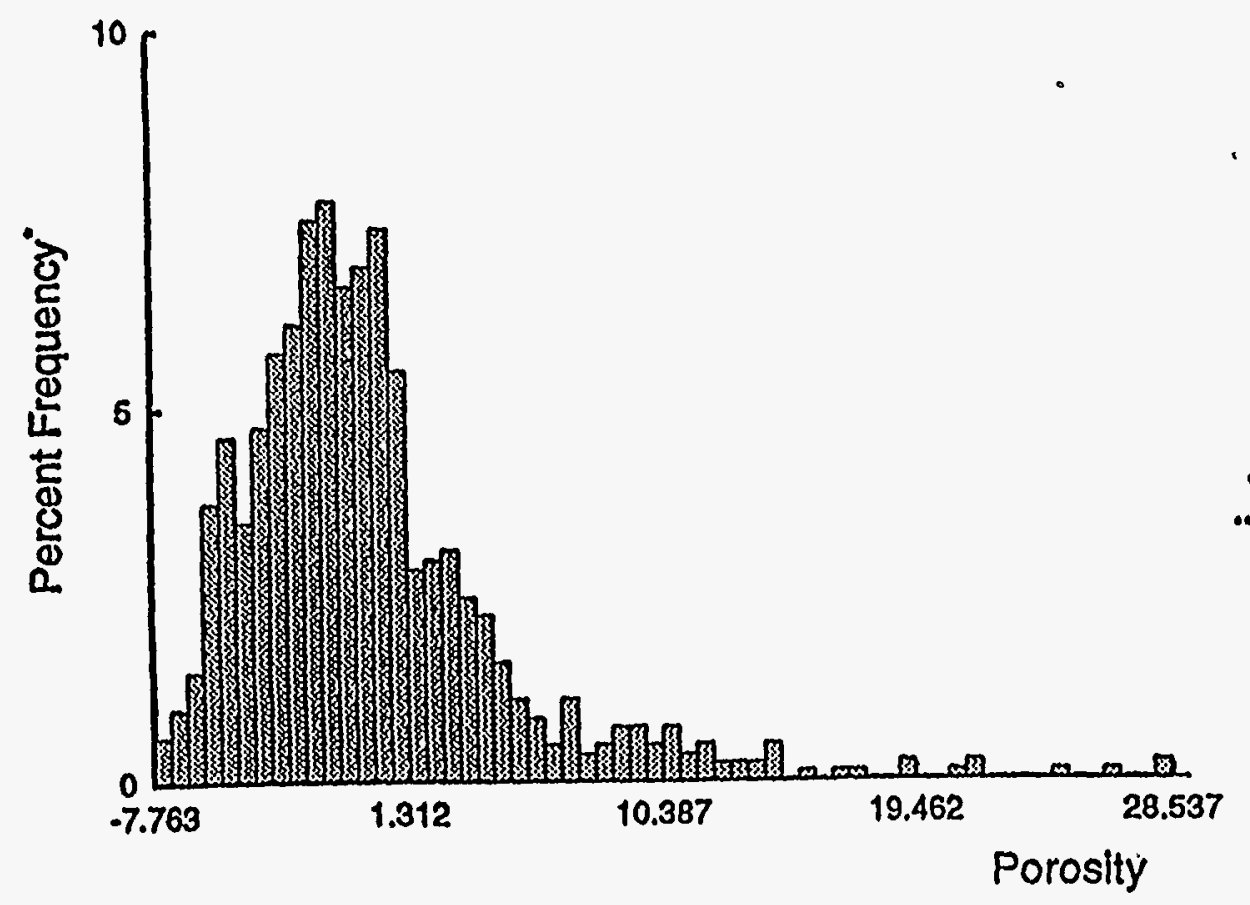




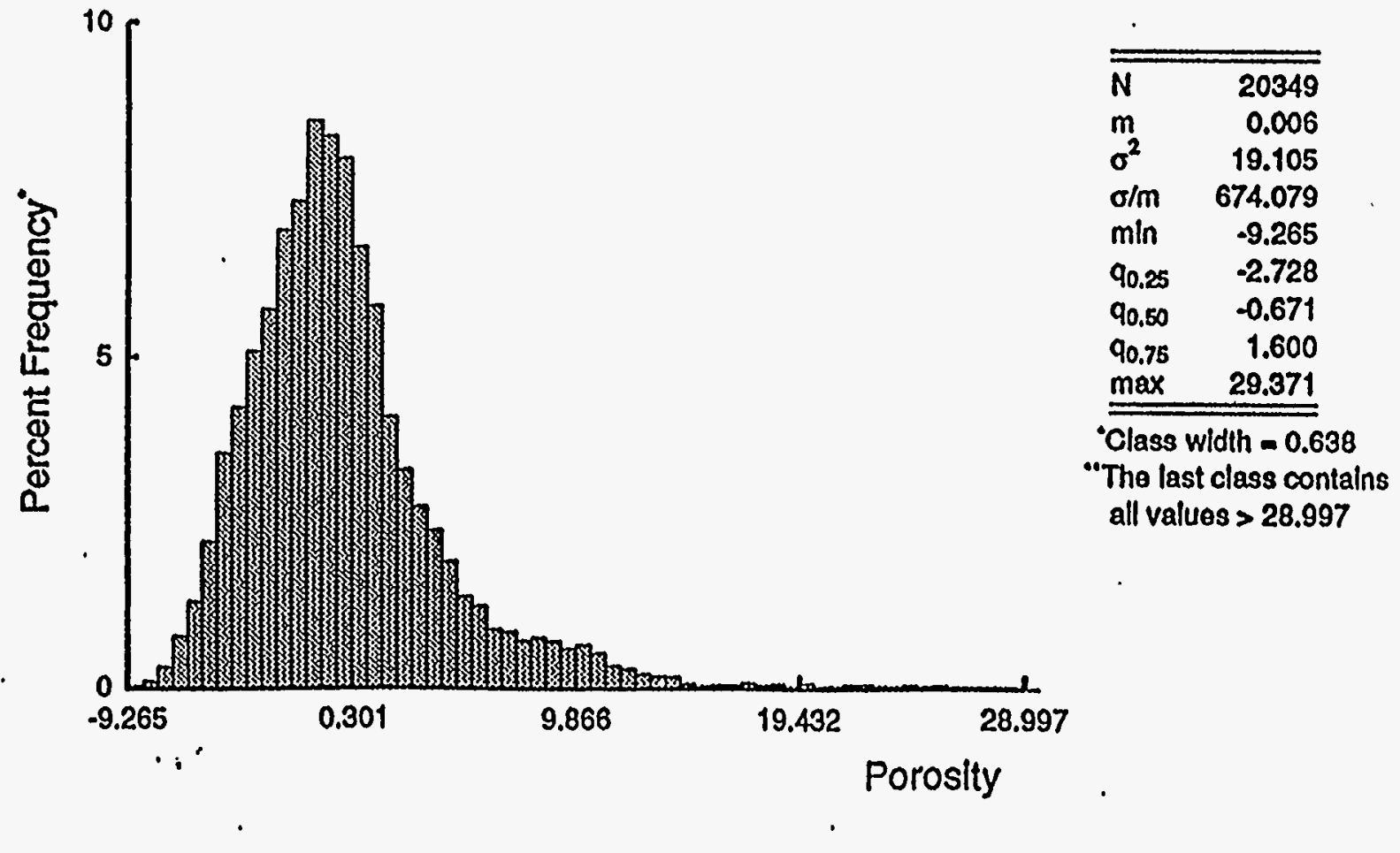

Figure 30.Distribution of initial pattern for Hugoton well pair A2 and $\mathrm{E} 2$ 


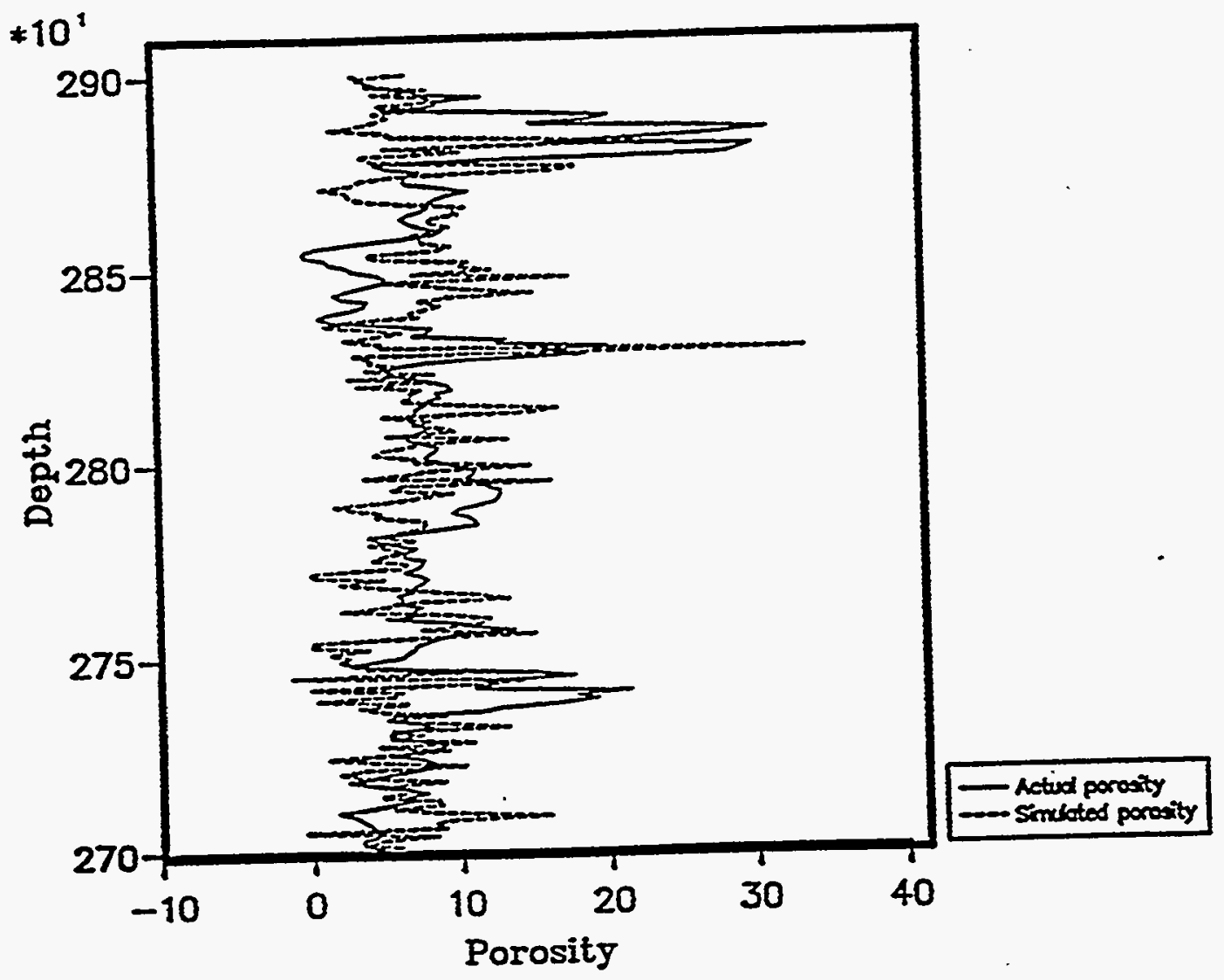

Figure $\cdot 3:$ Somparison plot for Hugoton well B2 using (i) fBm horizontal structure, and (ii) greedy algorithm 


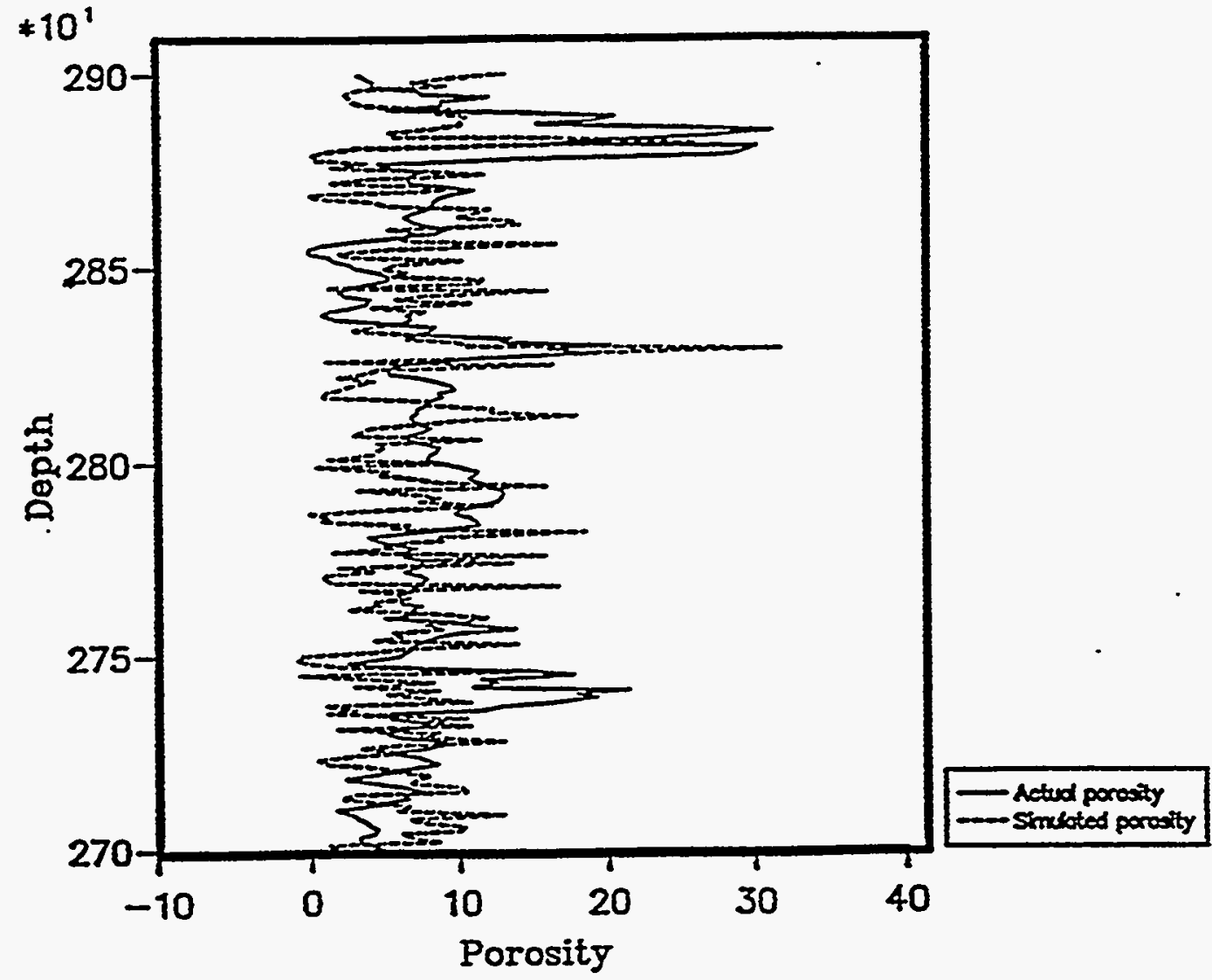

Figure 32 .Comparison plot for Hugoton well B2 using (i) $\mathrm{fBm}$ horizontal structure, and (ii) conventional annealing 


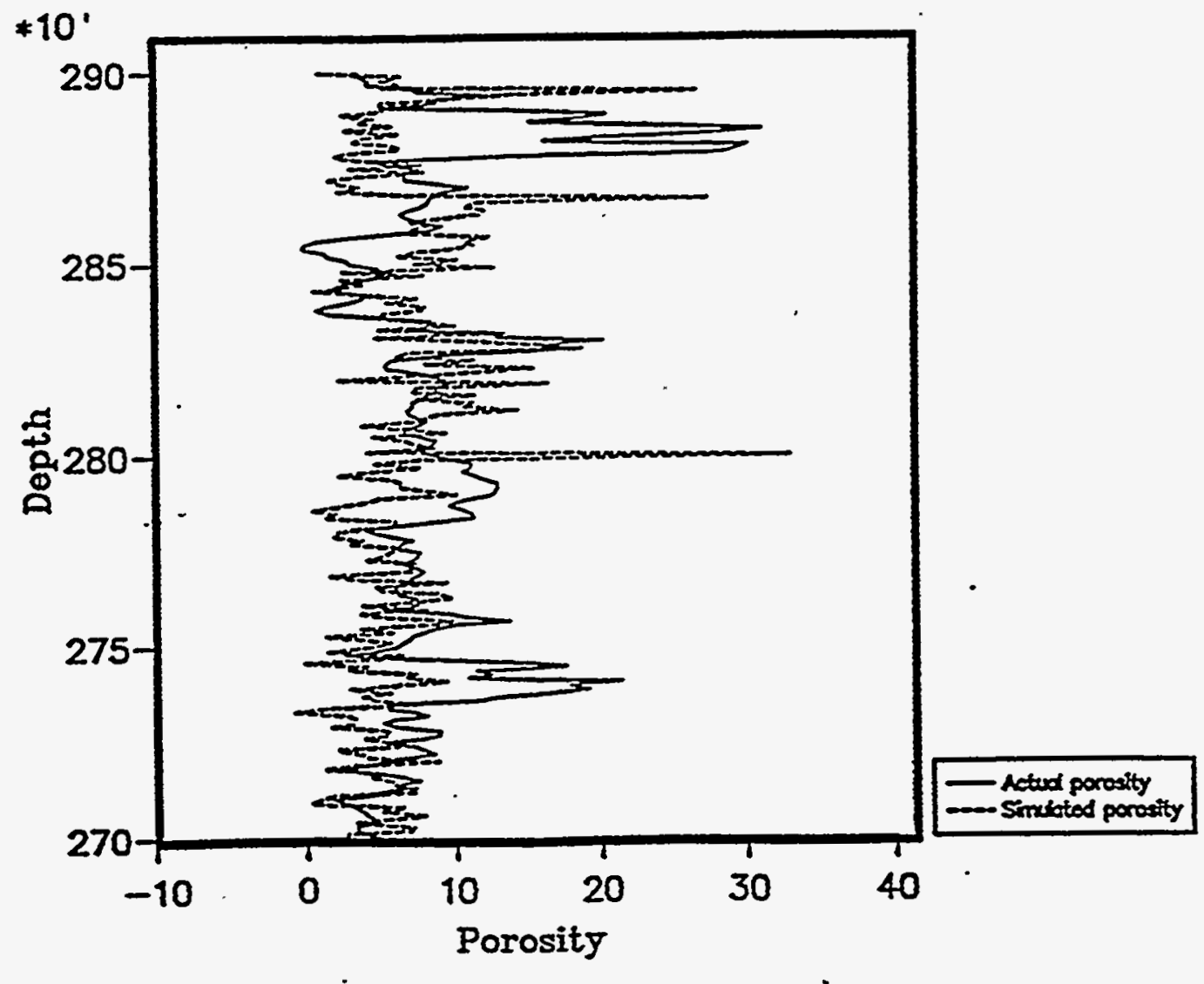

Figure 33. Comparison plot for Hugoton well B2 using (i) fBm horizontal structure, and (ii) conventional annealing with initial pattern 


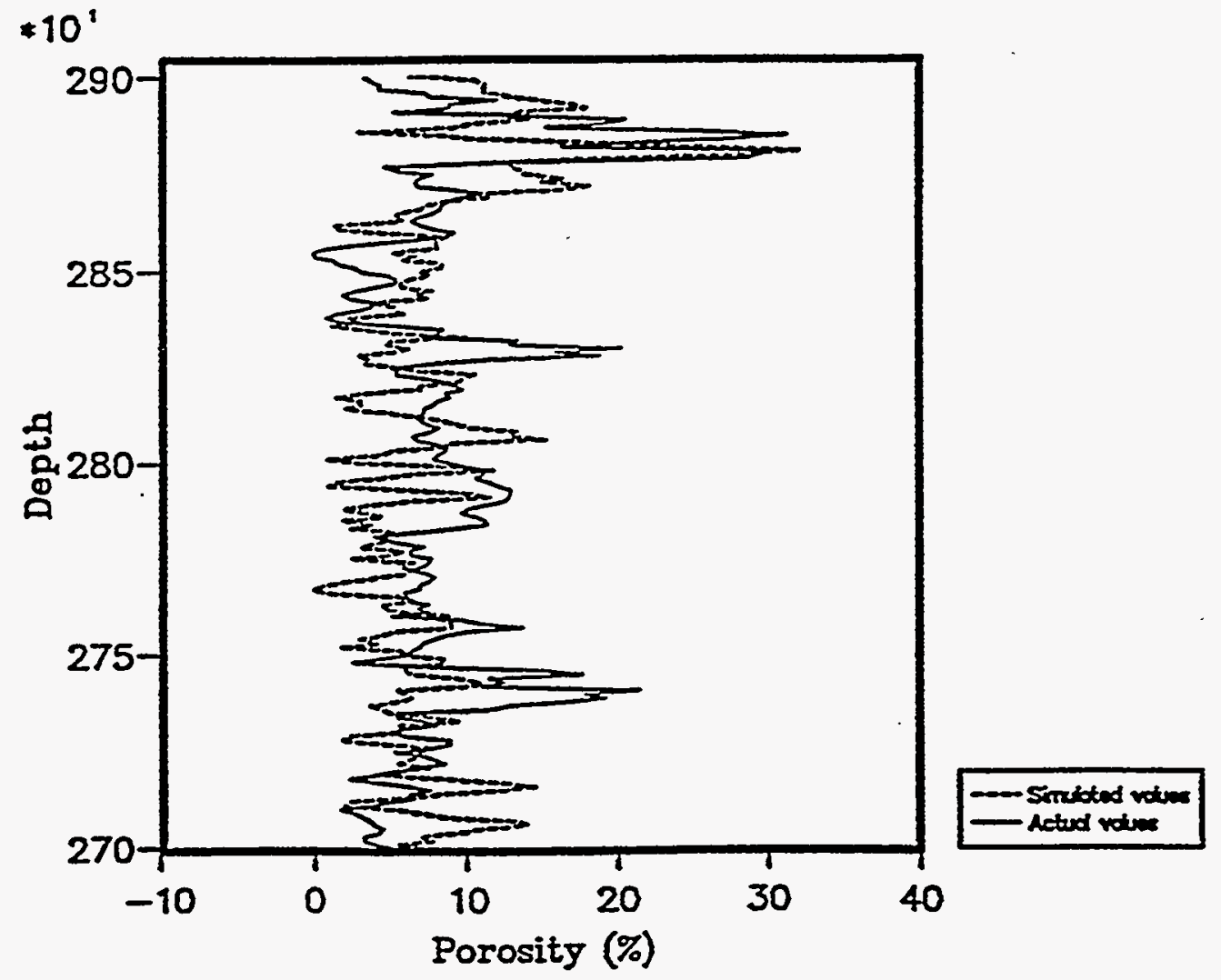

Figure 34. Comparison plot for Hugoton well $B 2$ using (i) $f G n$ horizontal structure, and (ii) greedy algorithm 


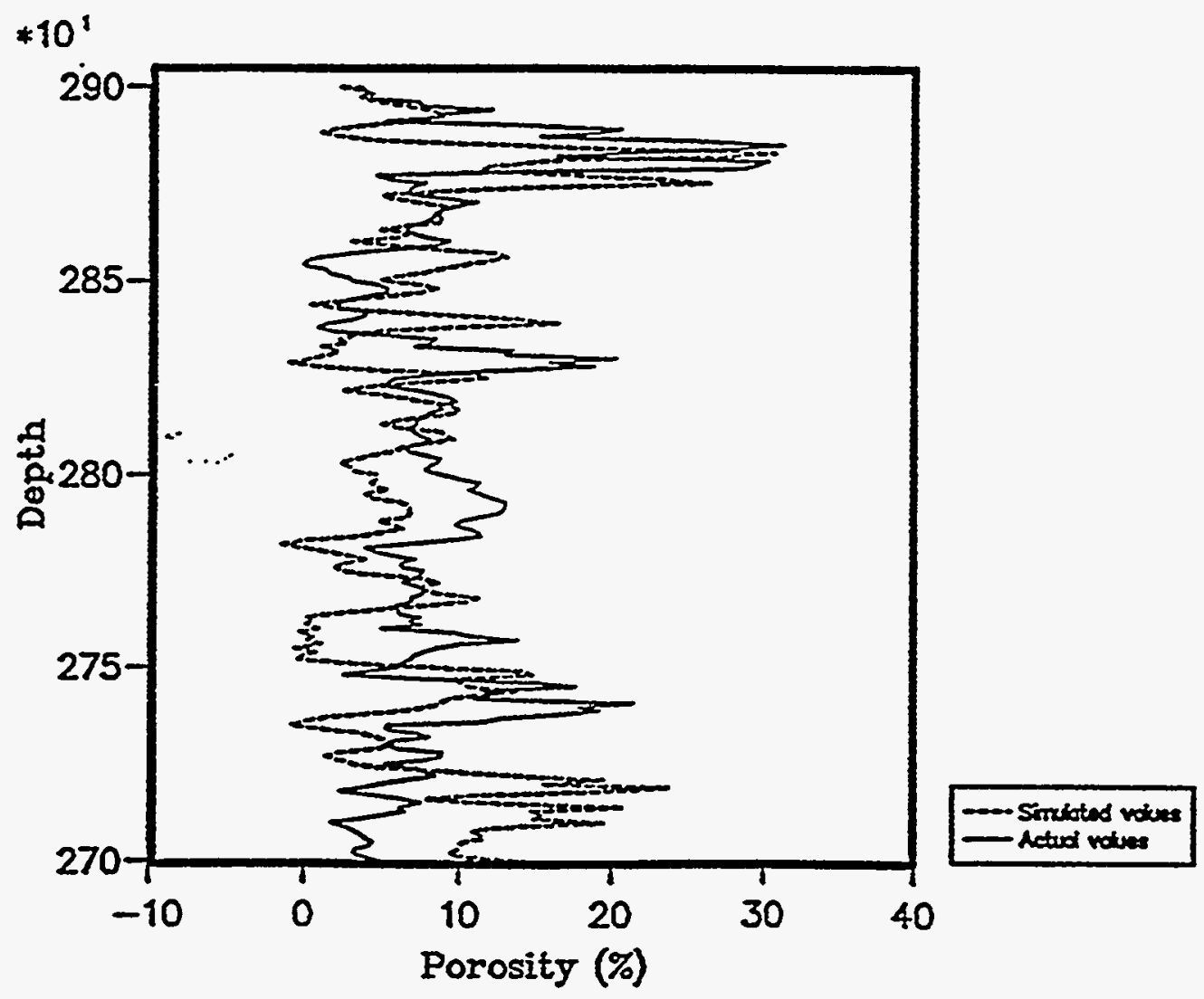

Figure 35: Jomparison plot for Hugoton well B2 using (i) fGn horizontal structure, and (ii) conventional annealing 


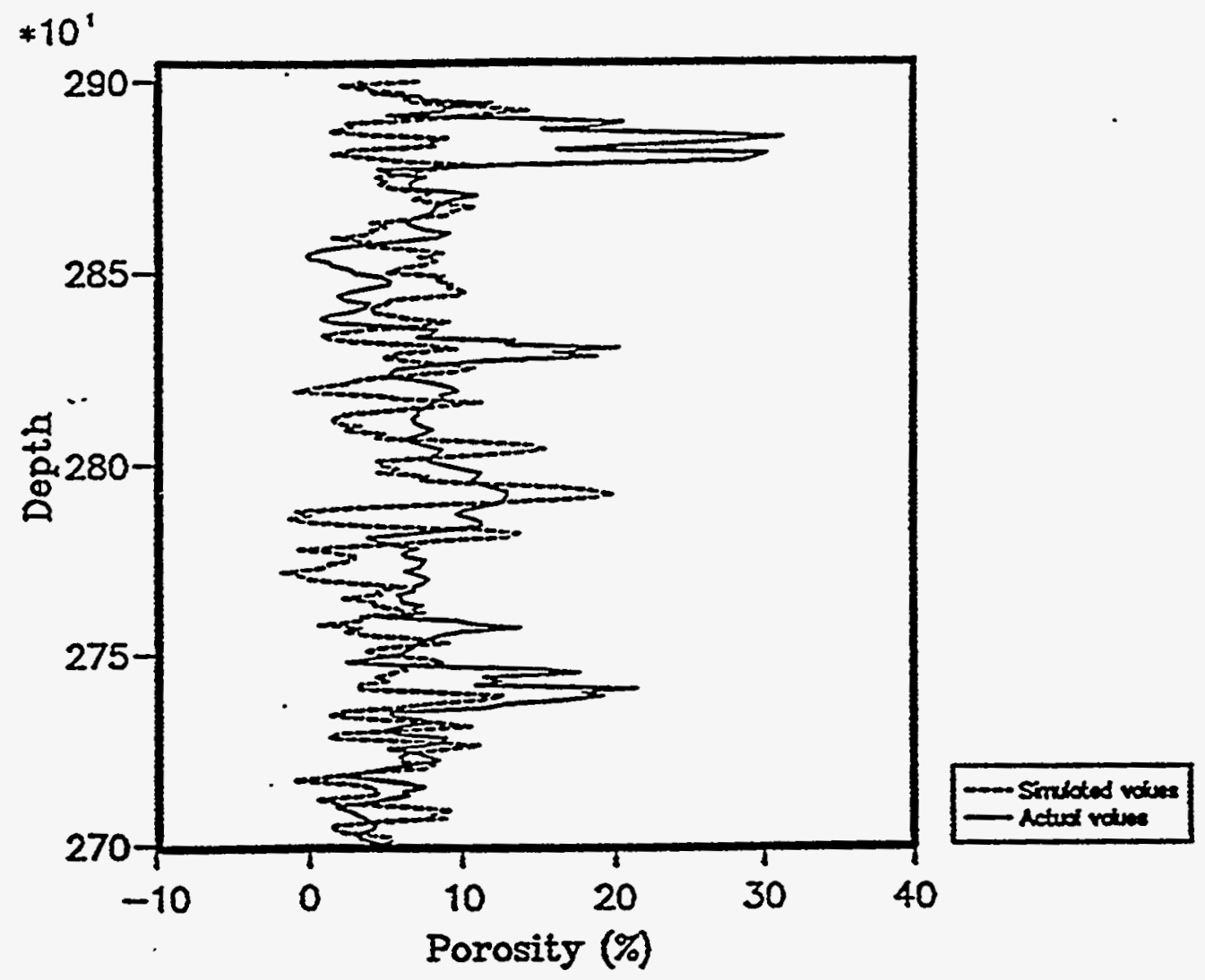

Figure 36: Comparison plot for Hugoton well B2 using (i) fGn horizontal structure, and (ii) conventional annealing with initial pattern 
Northing (feet)

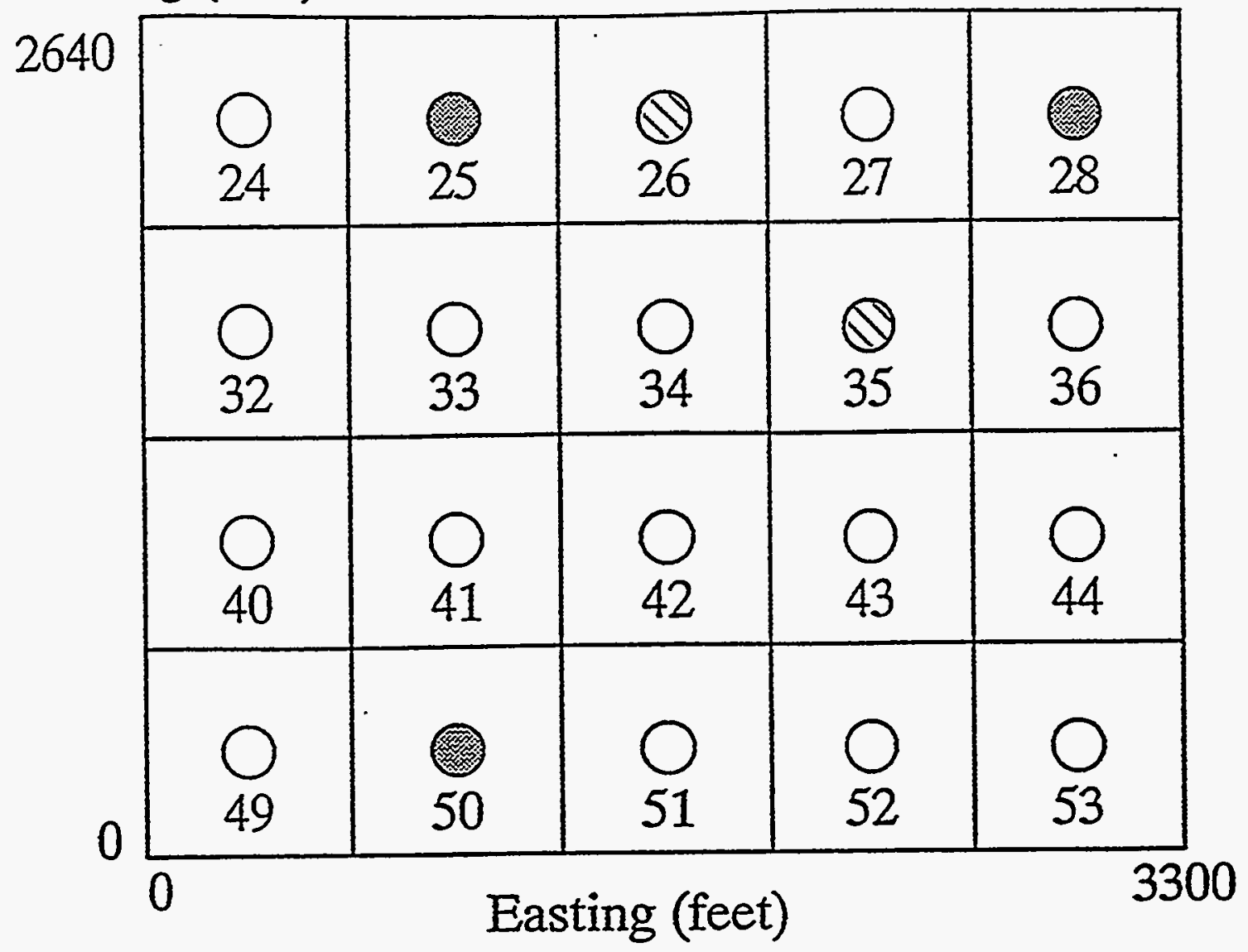

Figure37. Field map of Burbank simulation region 


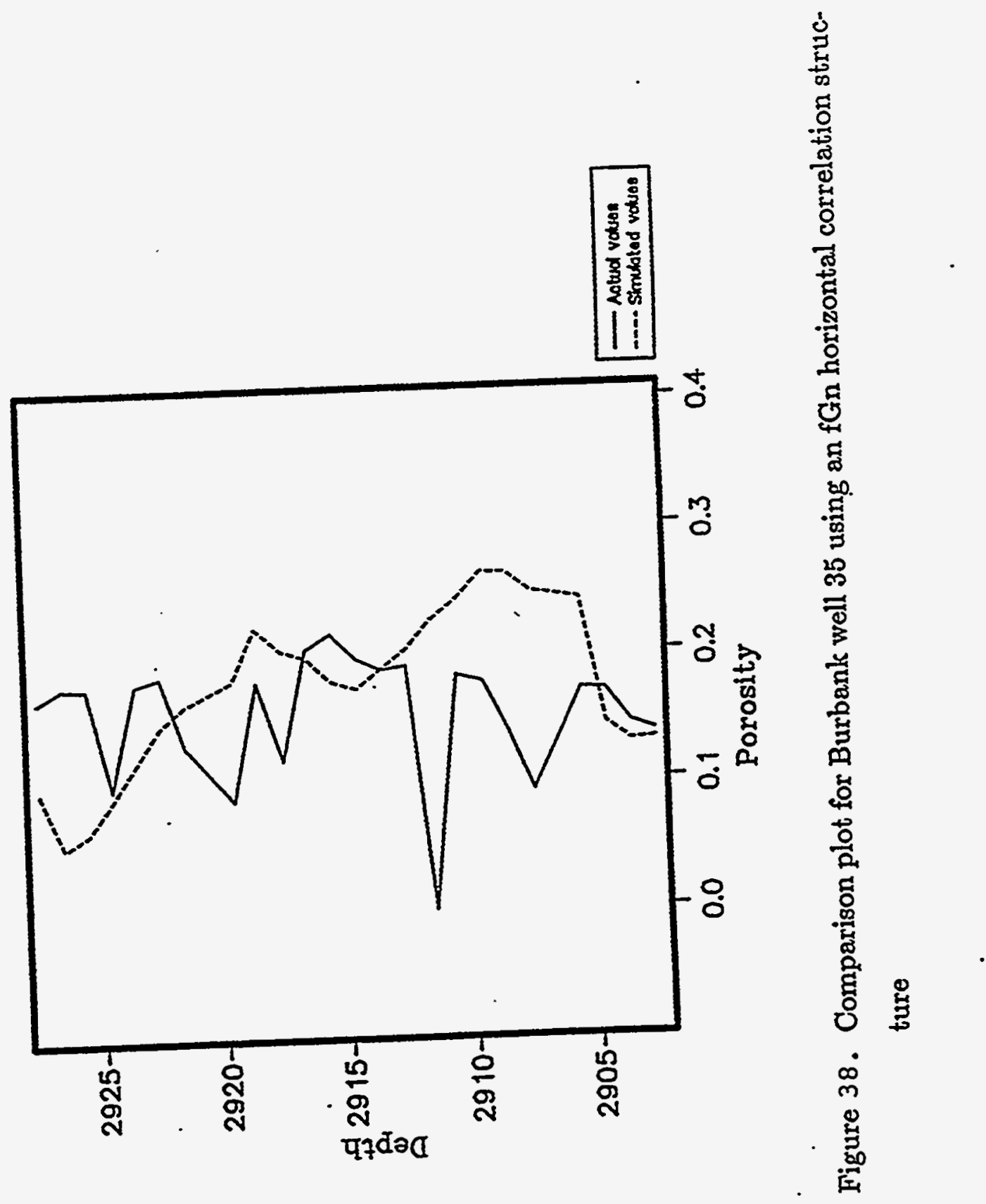




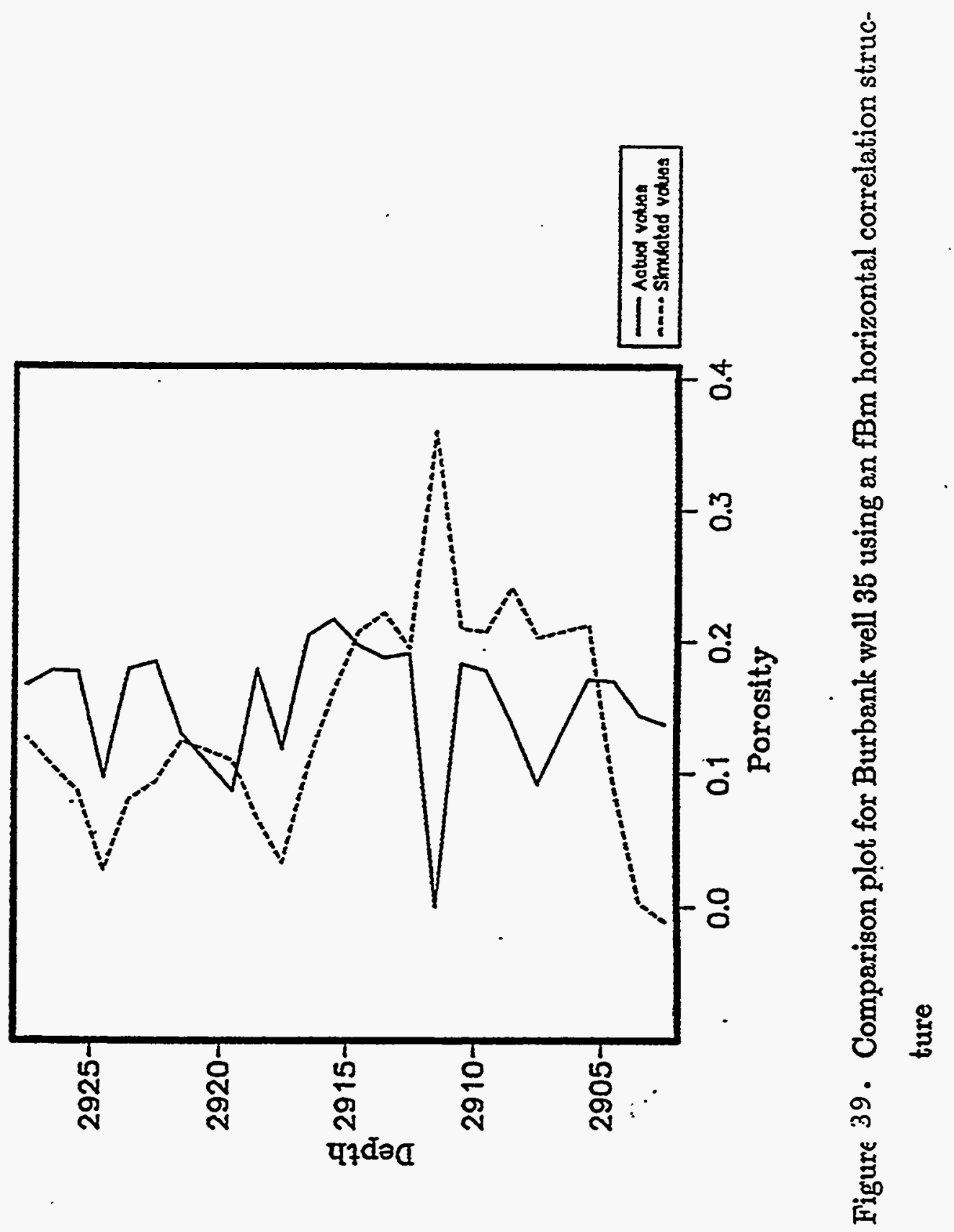




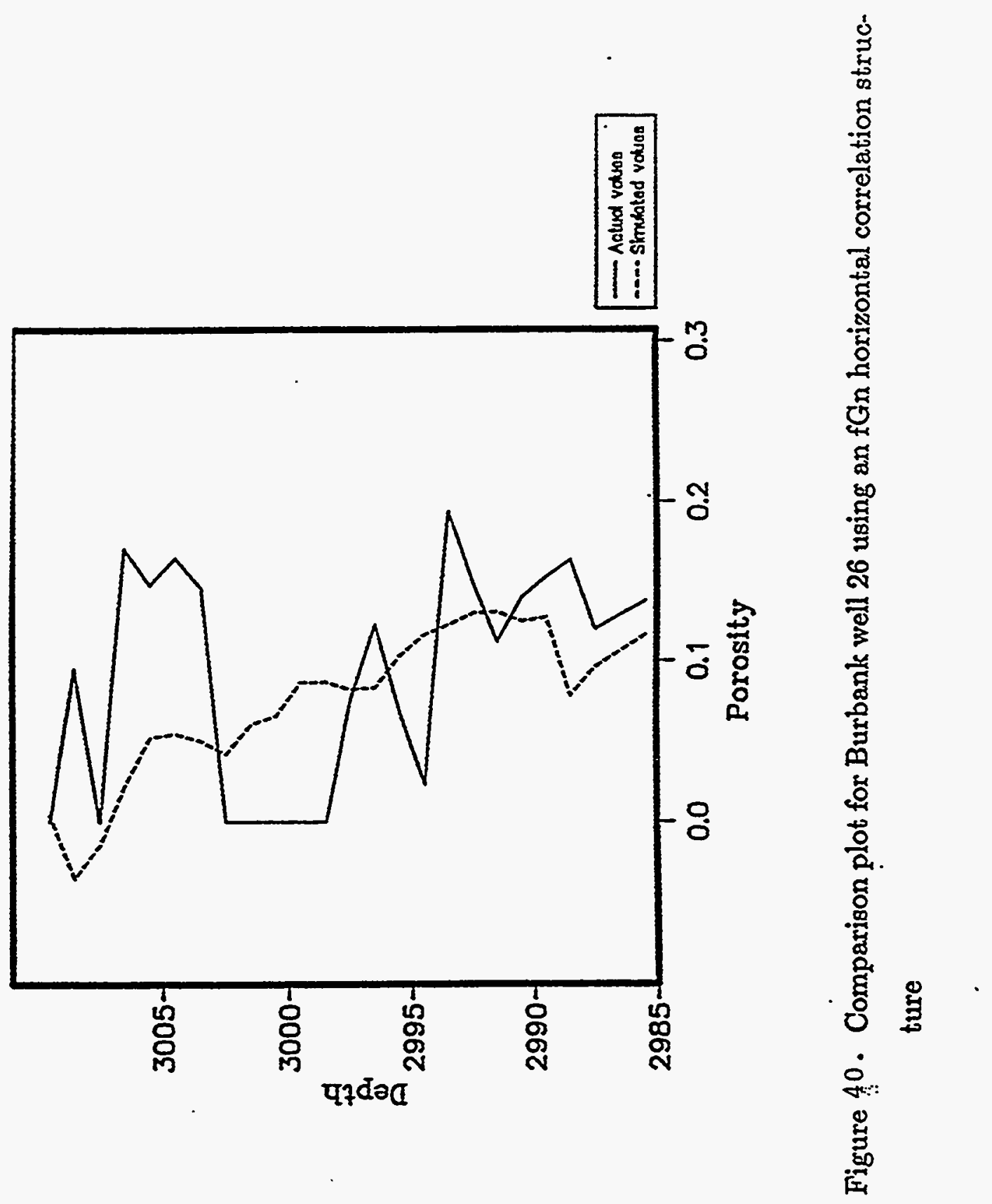




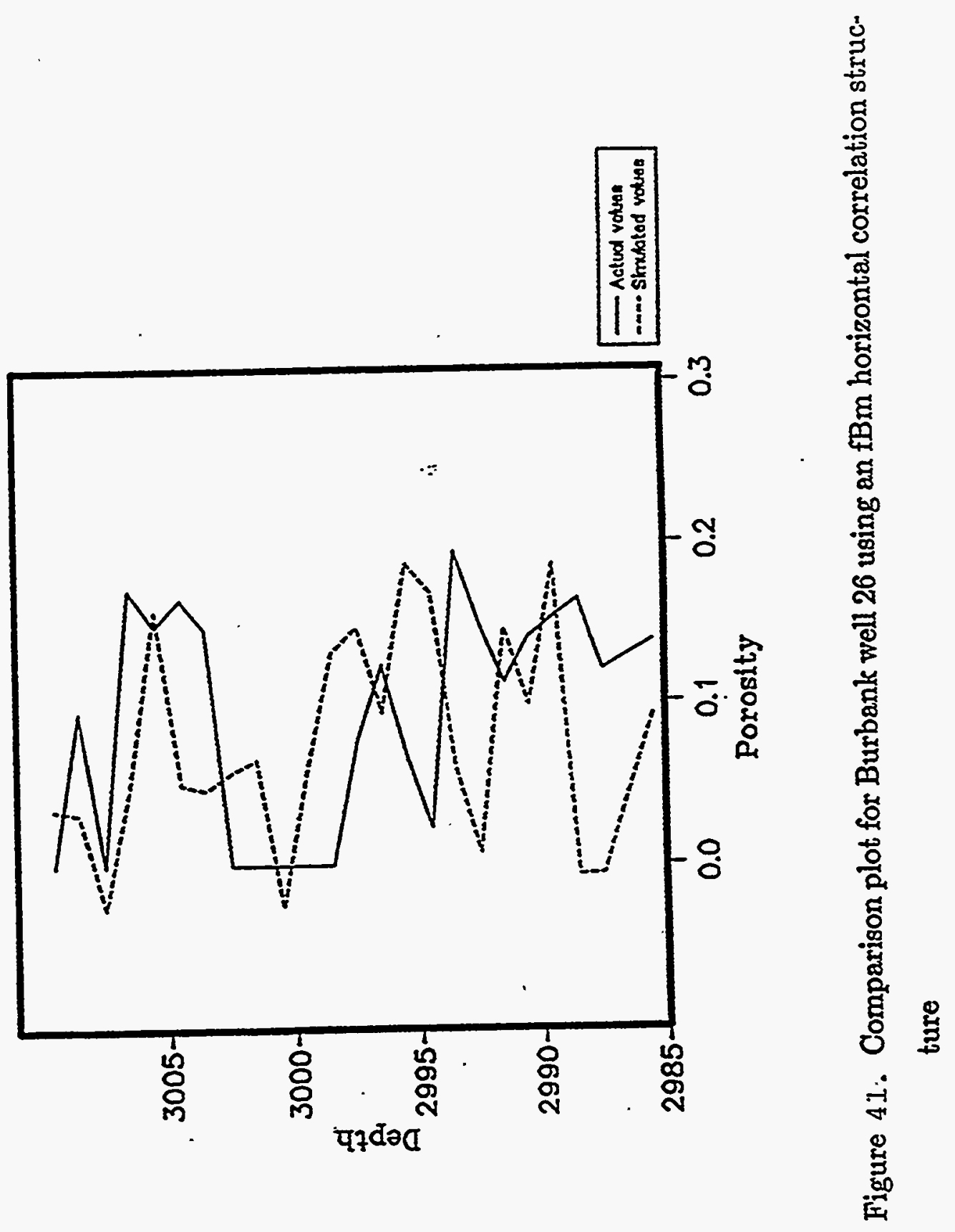




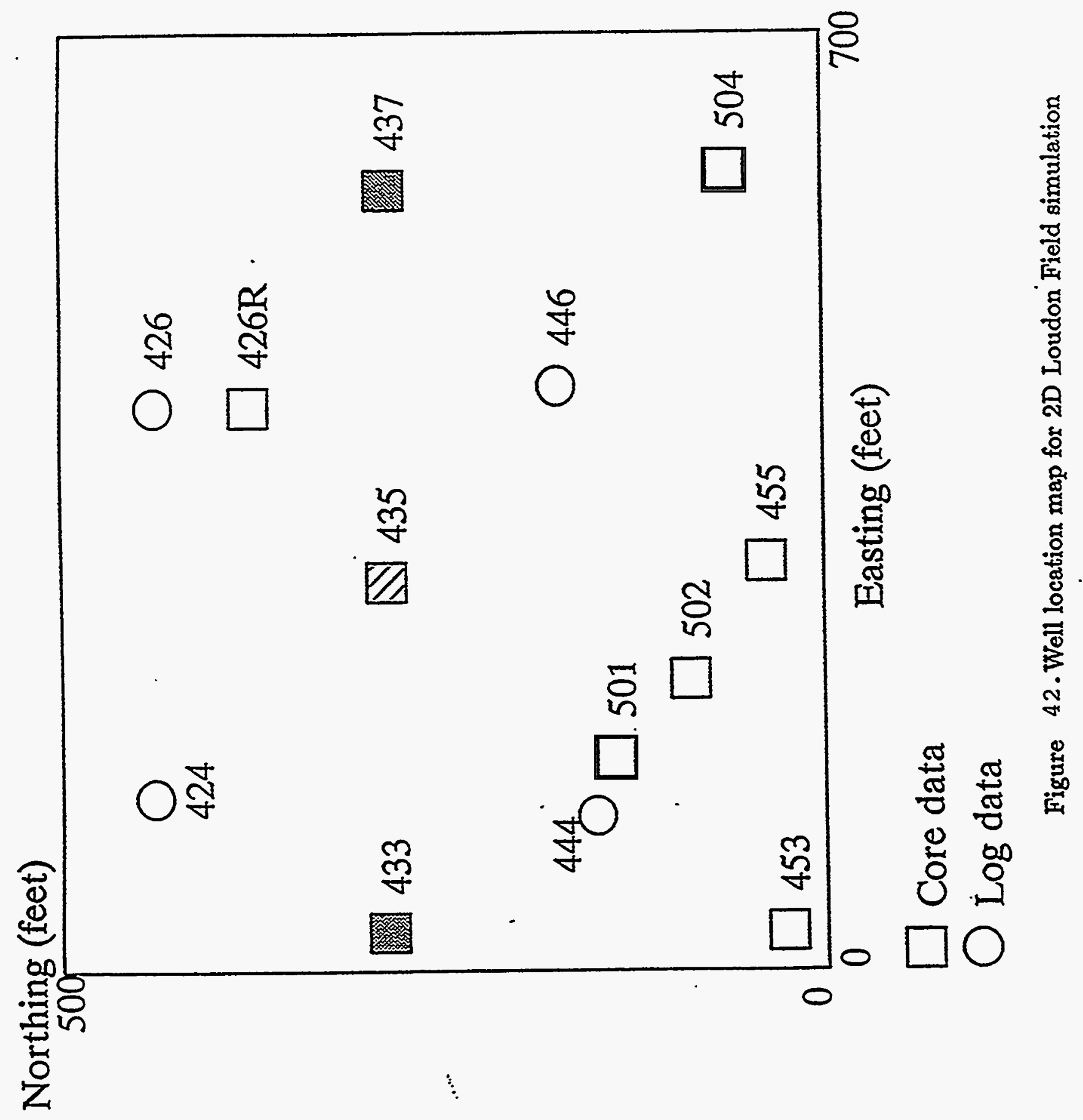




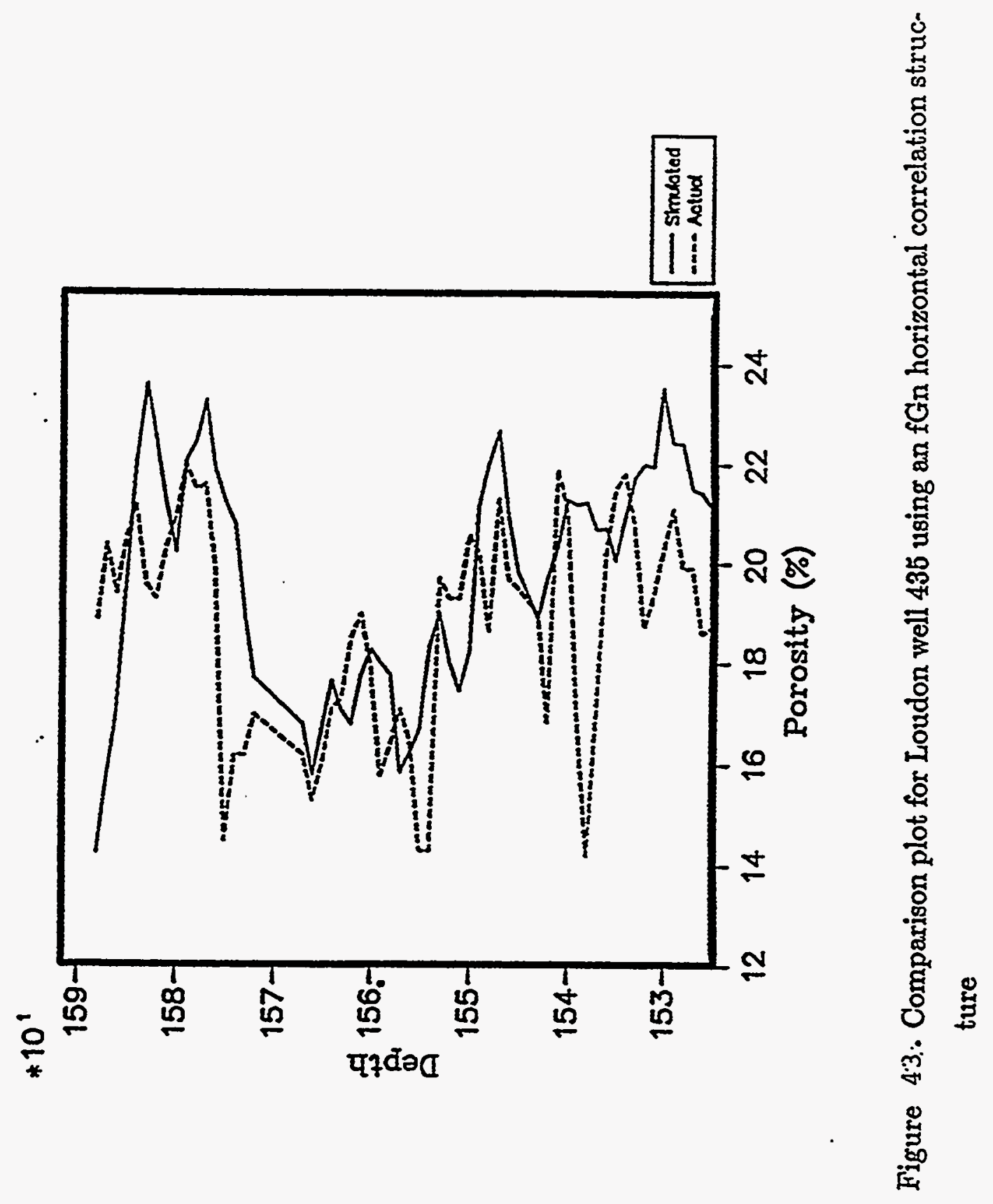




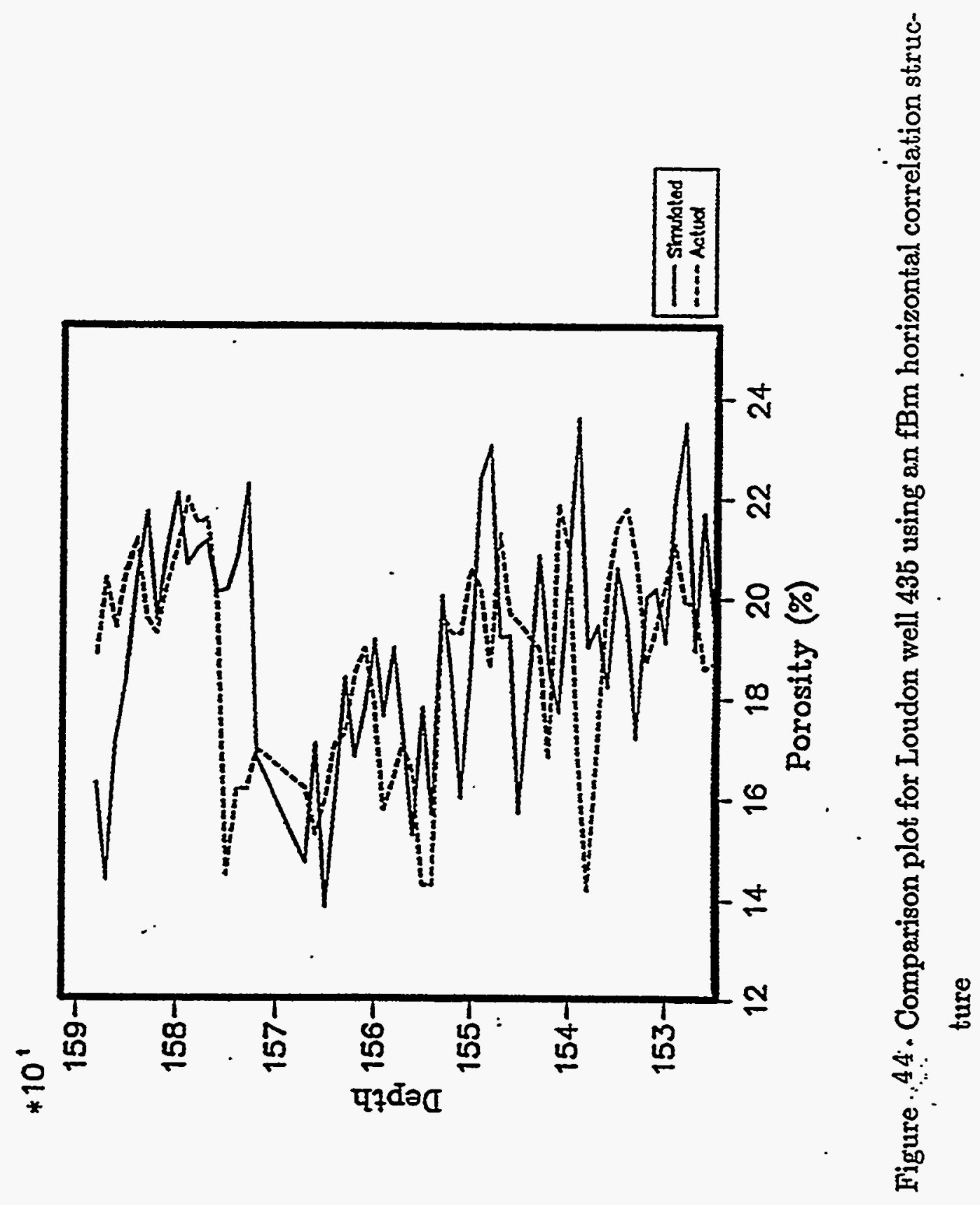




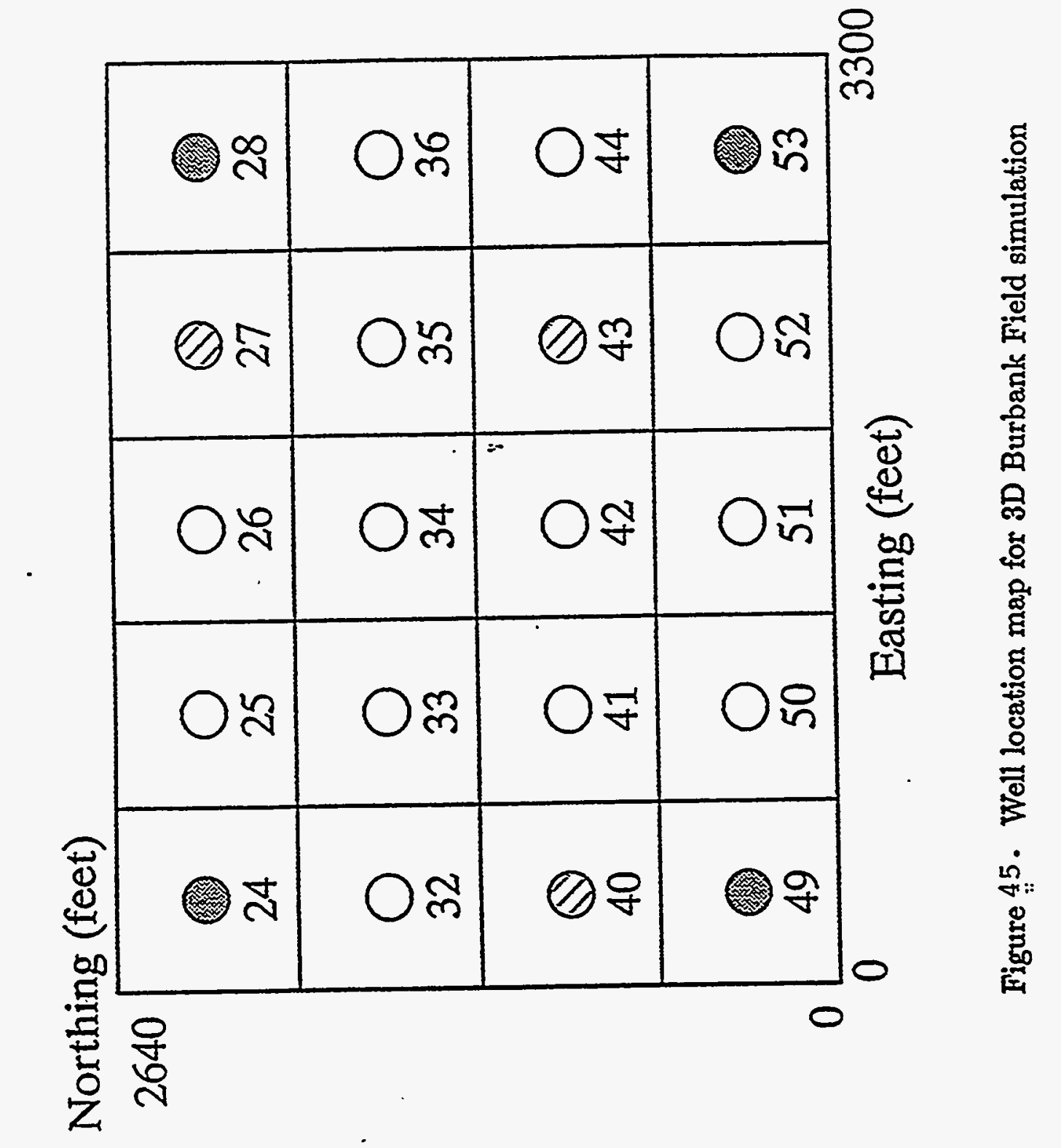




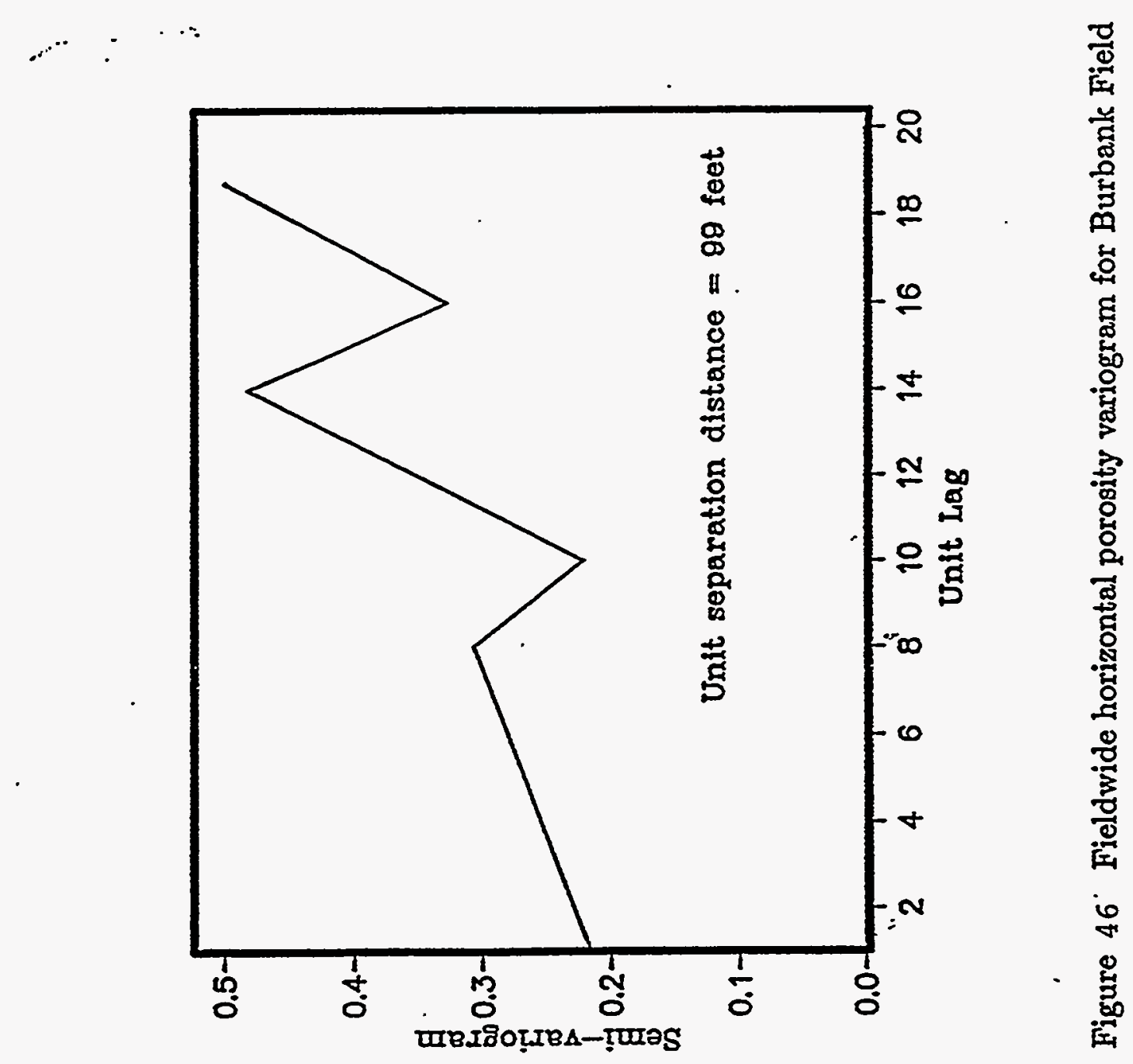




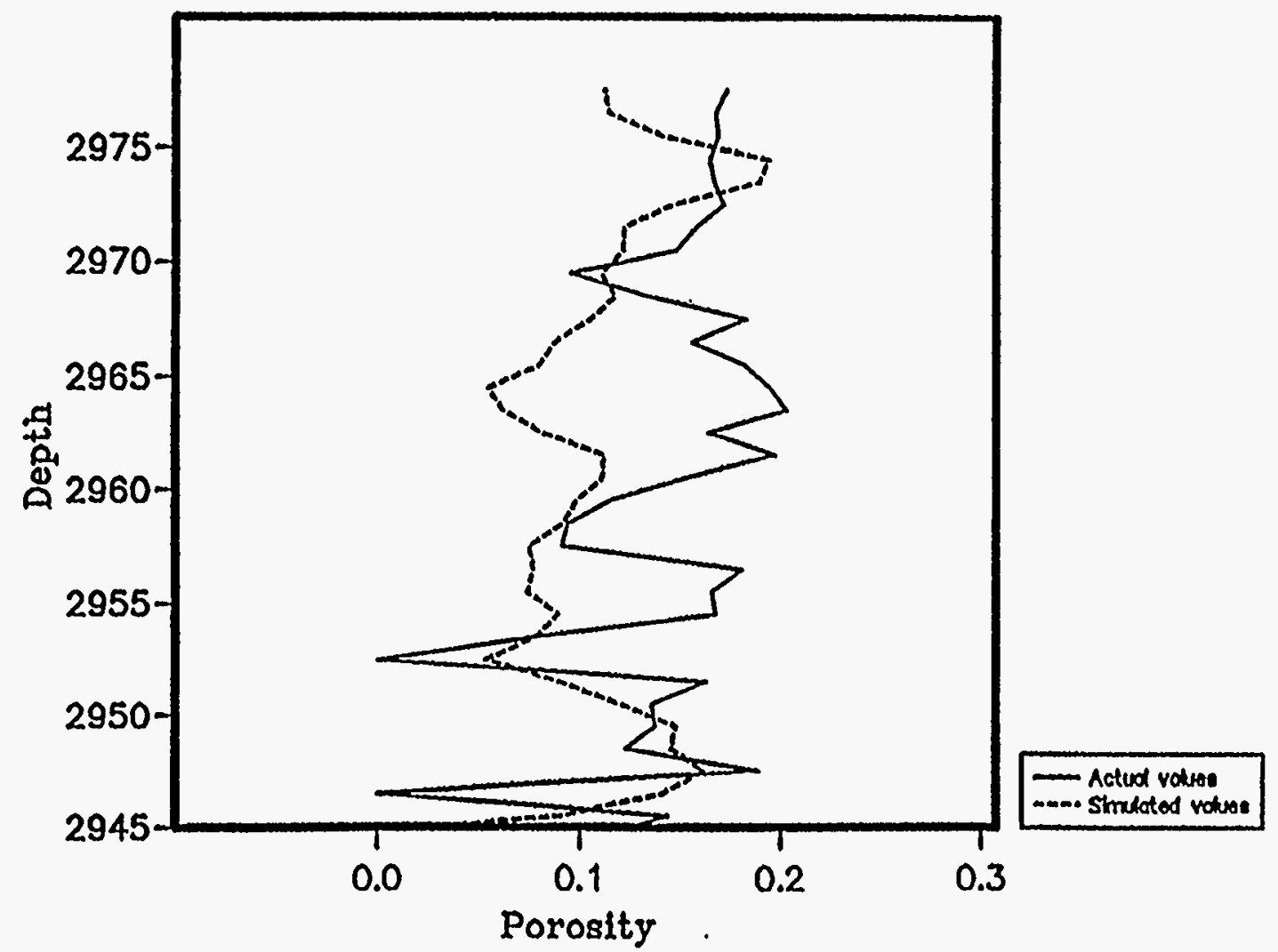

Figure 47 . Comparison plot for Burbank well 27 using an $f G n$ horizontal correlation structure ( 4 conditioning wells) 


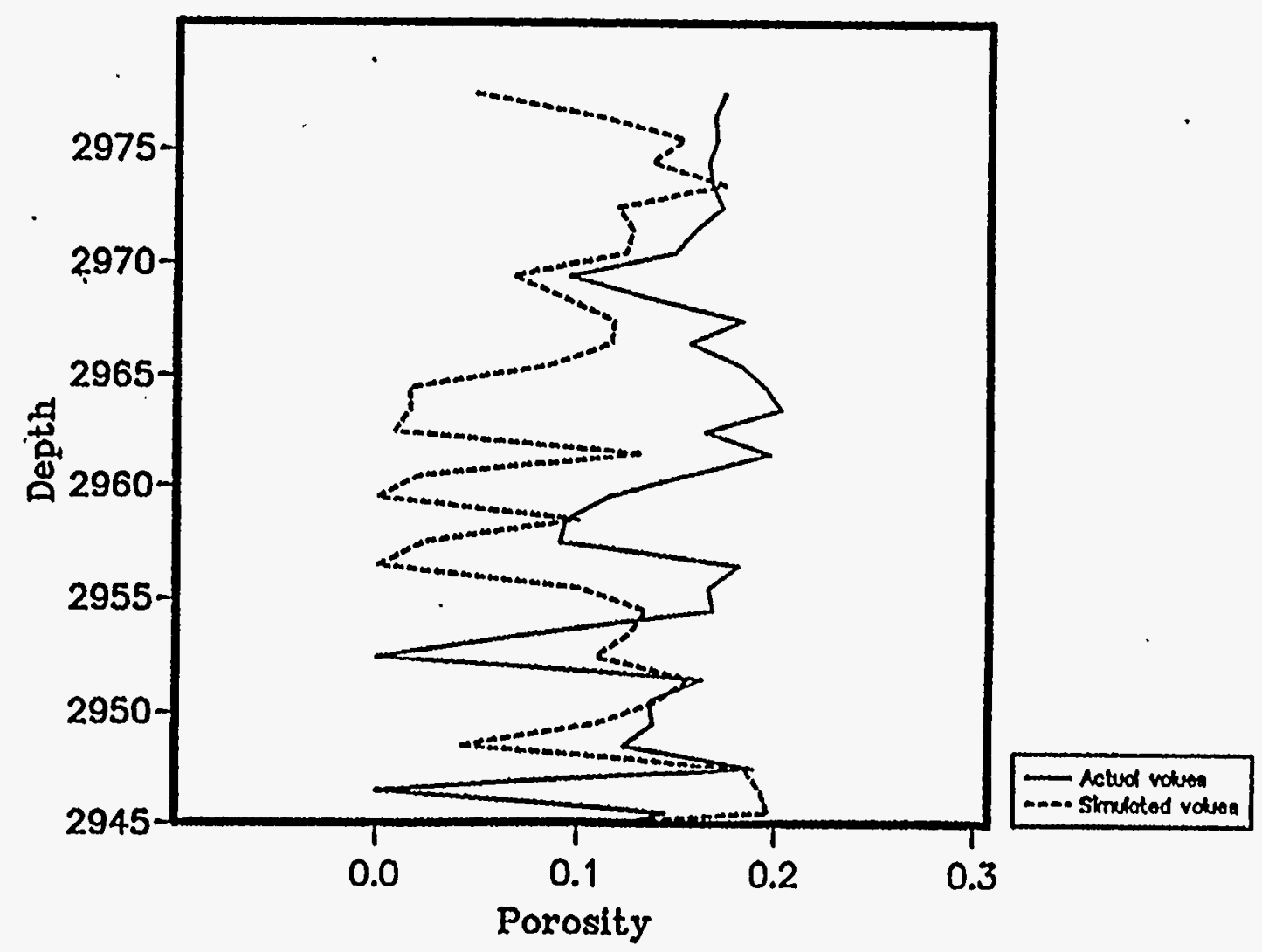

Figure 48. Comparison plot for Burbank well 27 using an $\mathrm{fBm}$ horizontal correlation structure (4 conditioning wells) 


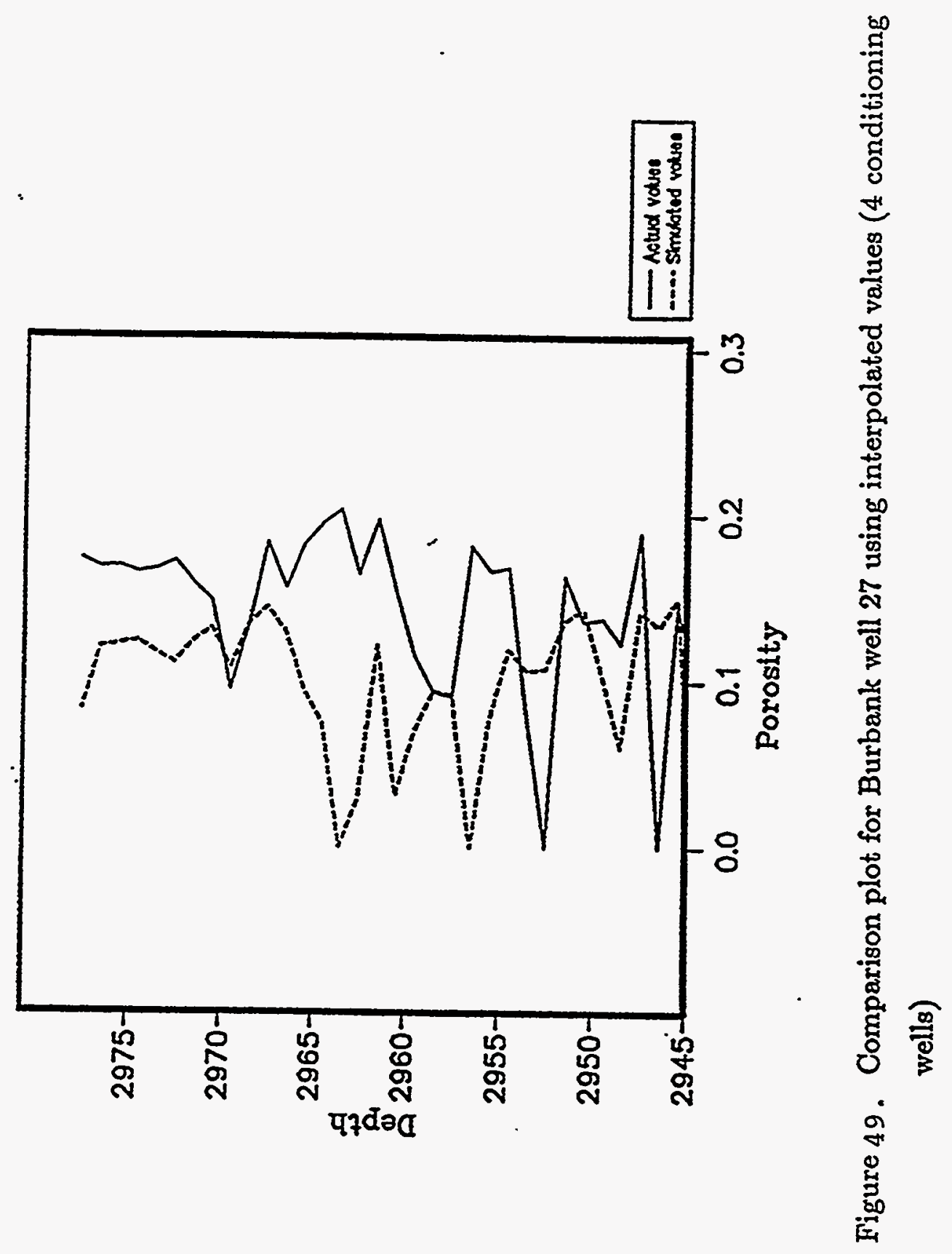




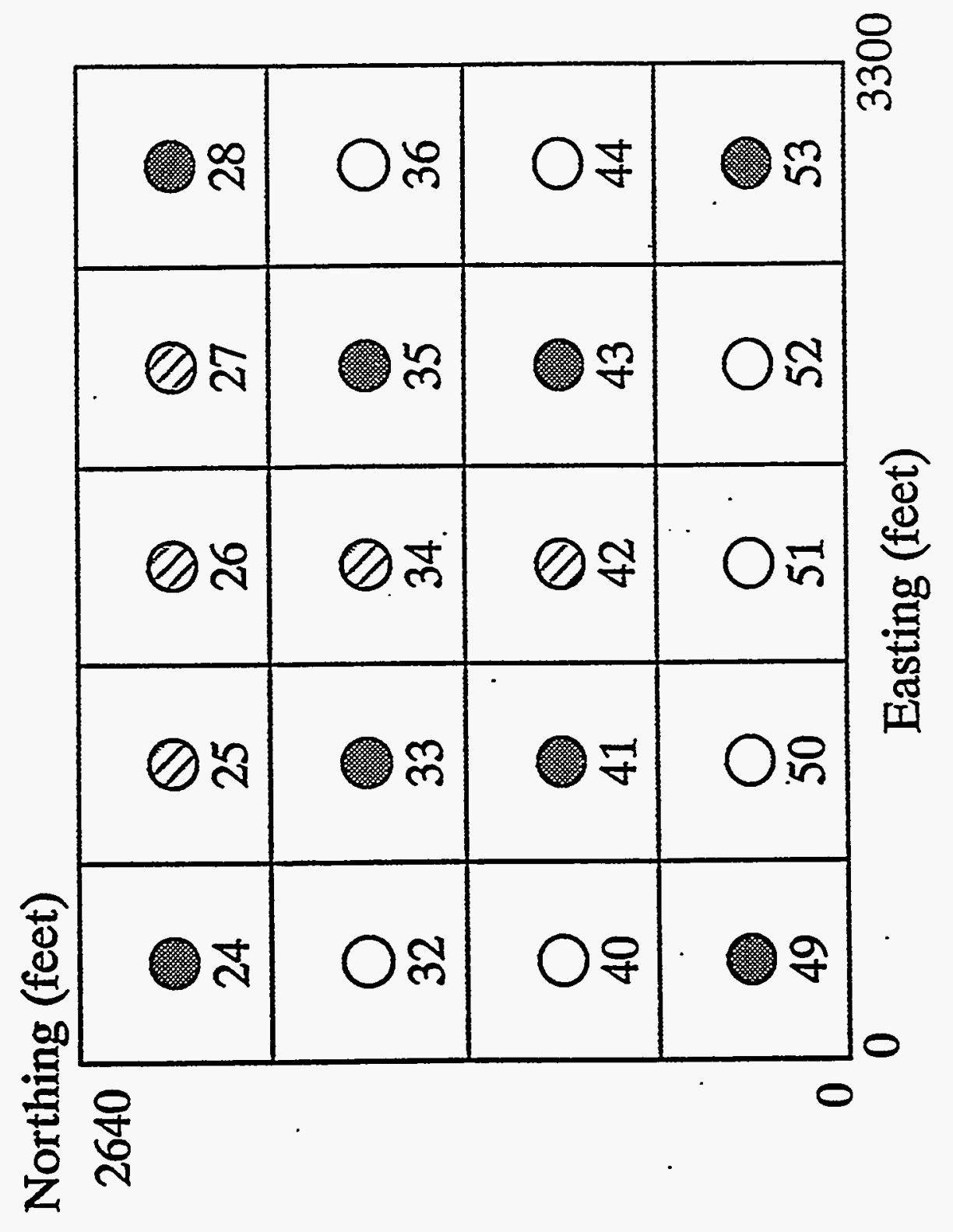

Oొ 


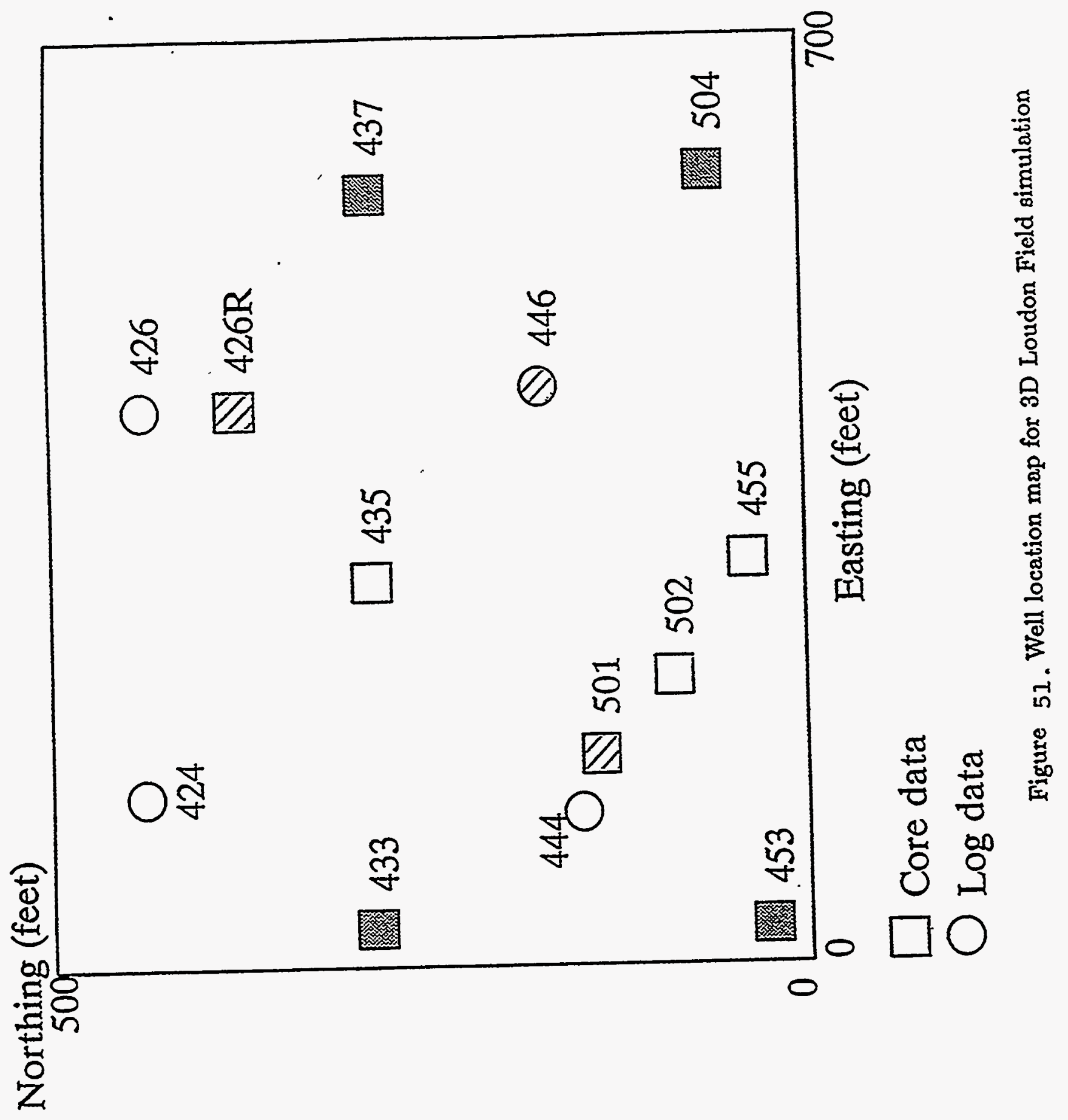




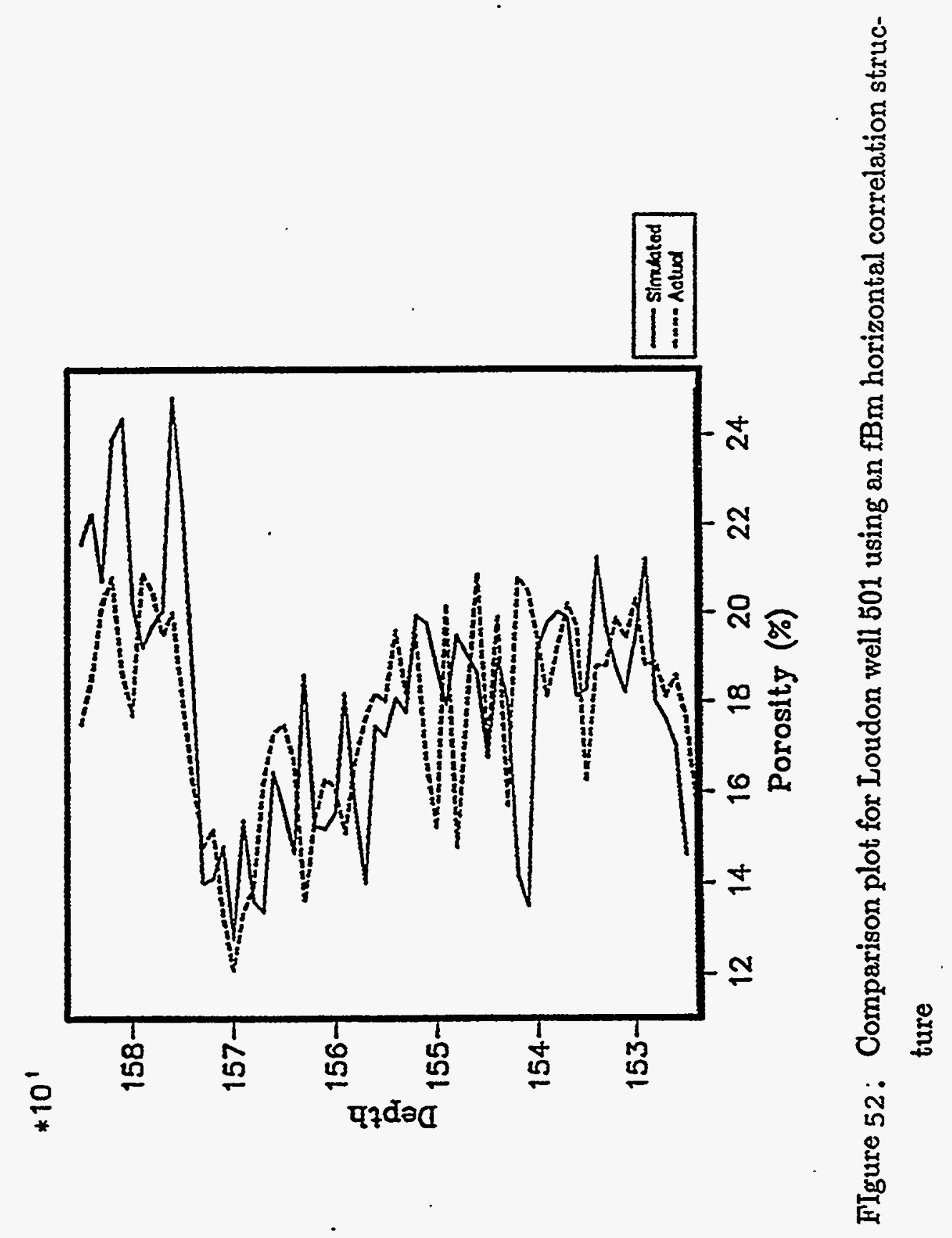


Northing (feet)

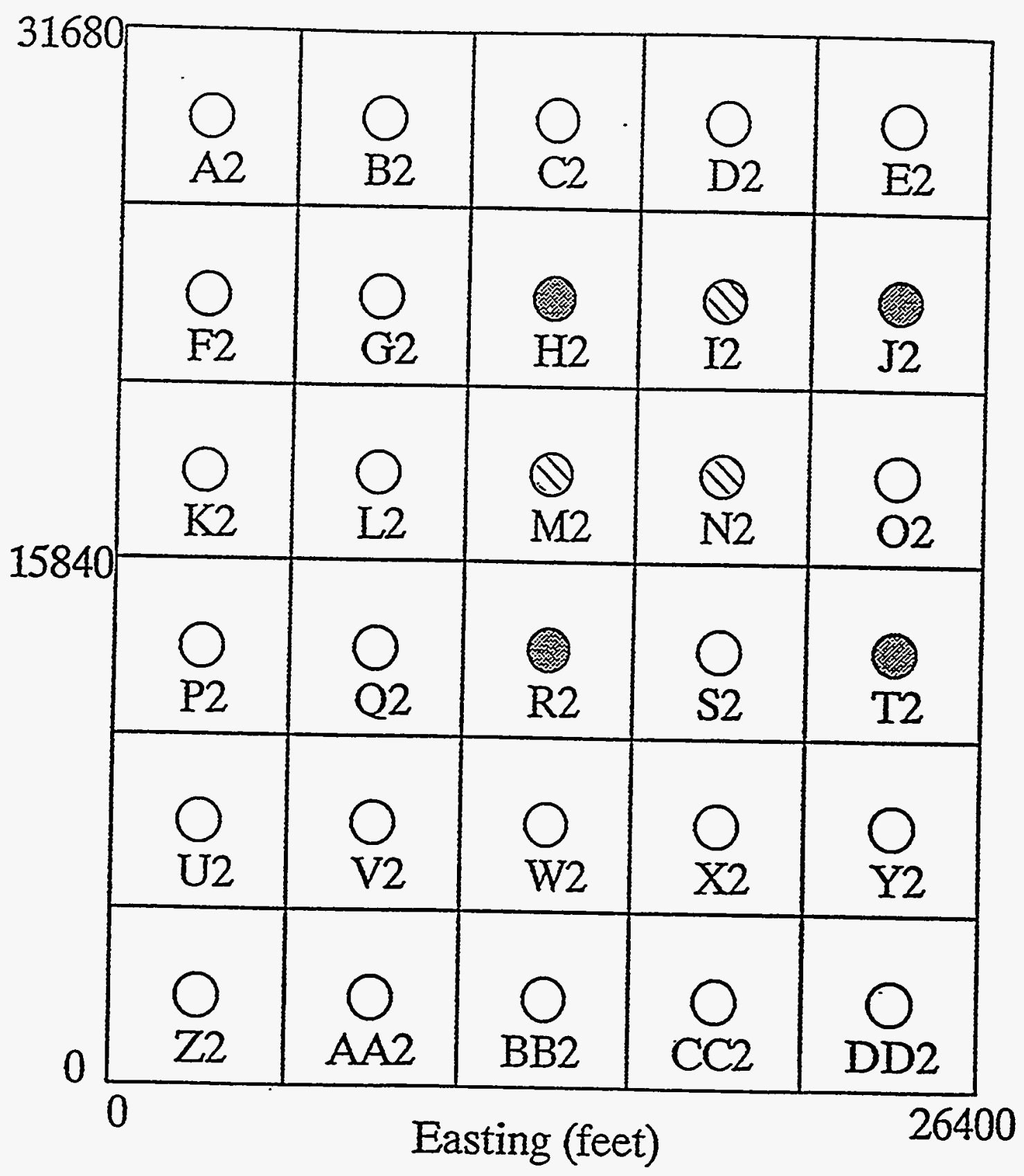

Figure 53. Well location map for 3D Hugoton Field simulation (small scale) 


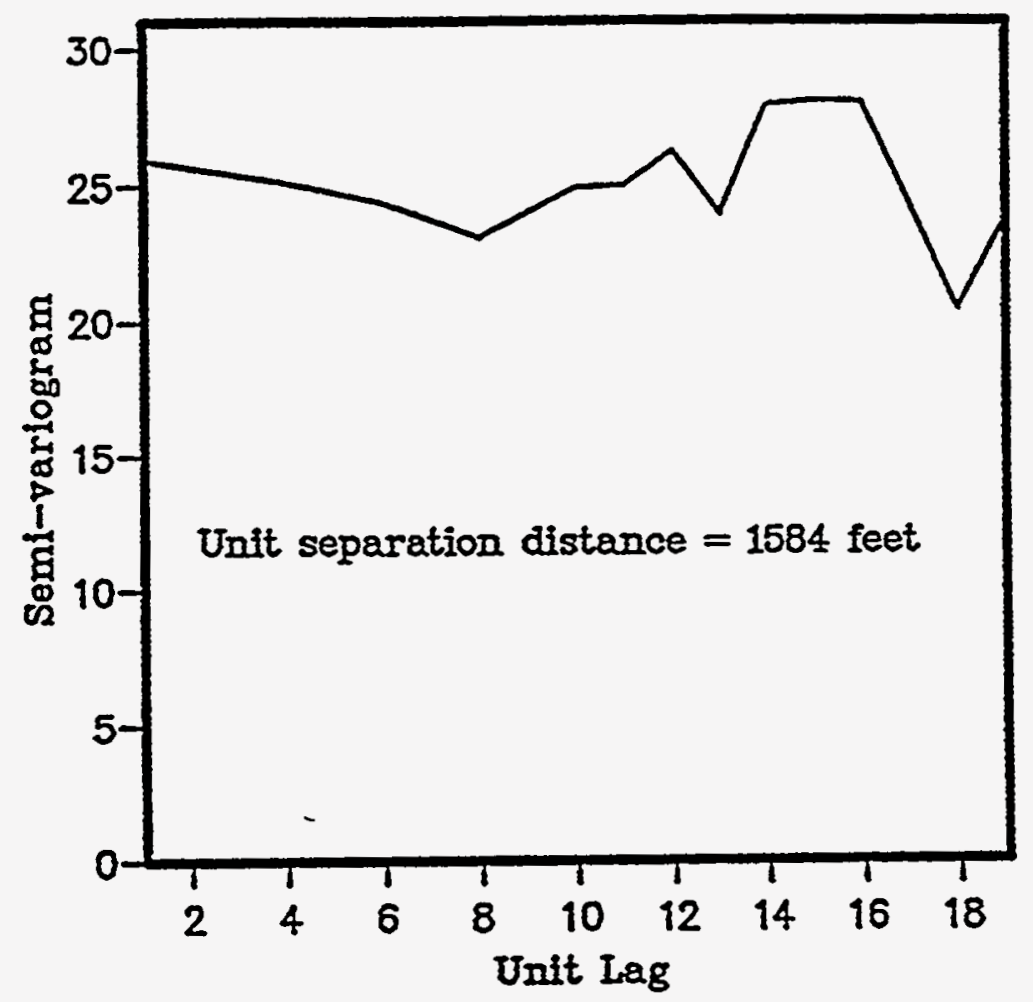

Figure 54: Fieldwide horizontal porosity variogram for Hugoton Field 


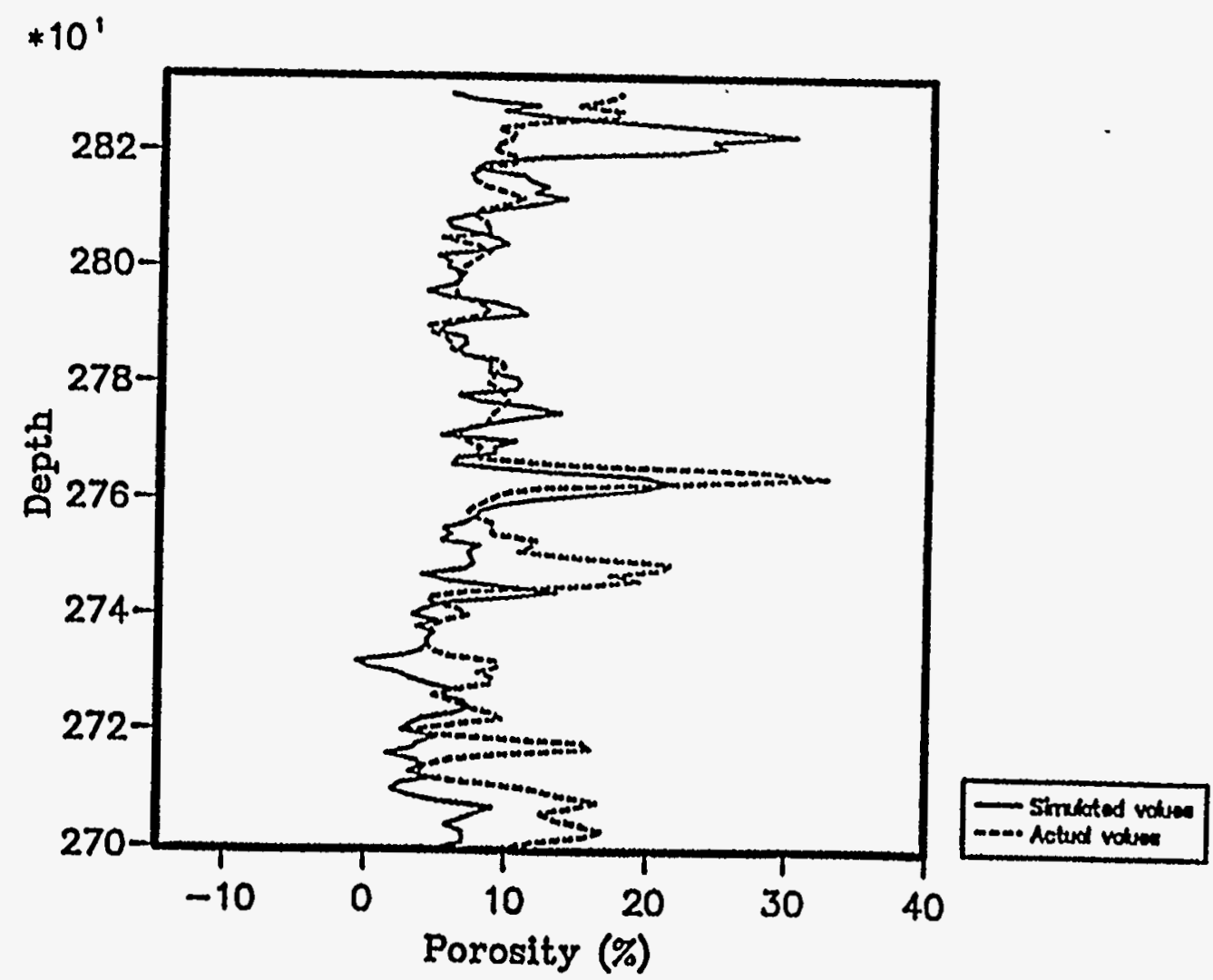

Figure 55. Comparison plot for Hugoton well I2 using (i) greedy algorithm, and (ii) fGn horizontal structure 


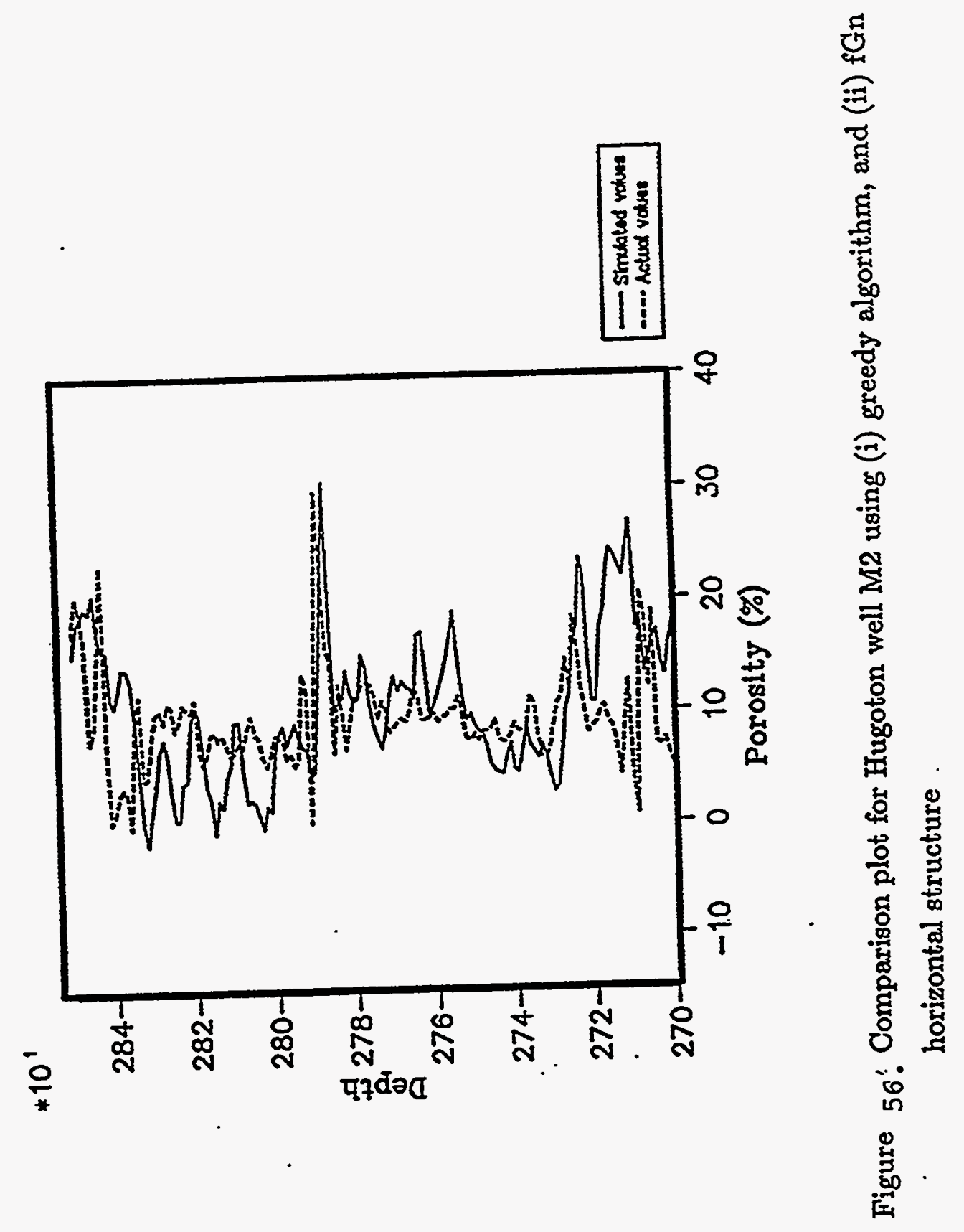




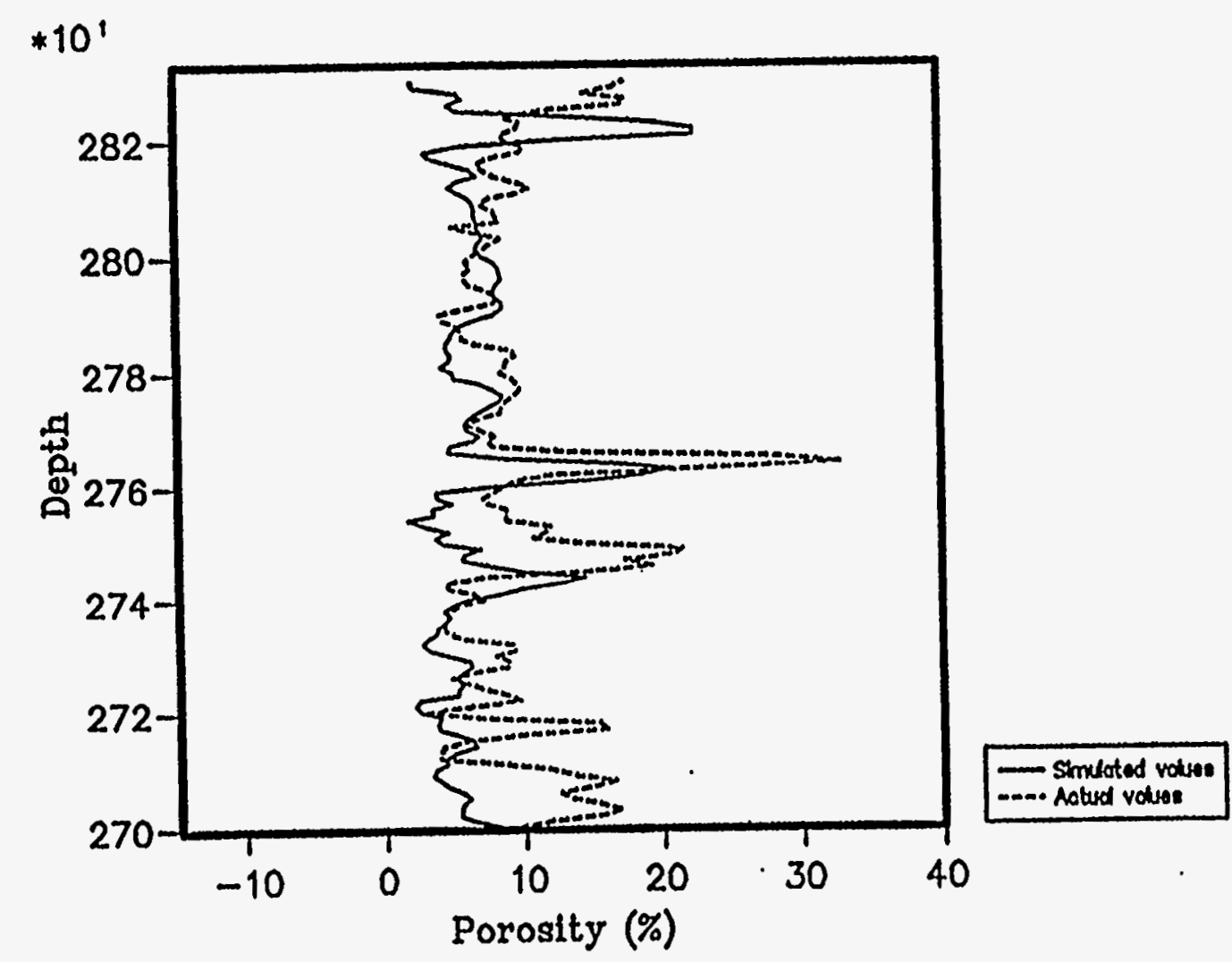

Figure 57. Comparison plot for Hugoton well I2 using (i) linear interpolation, and (ii) $\mathrm{fGn}$ horizontal structure 
Northing (feet)

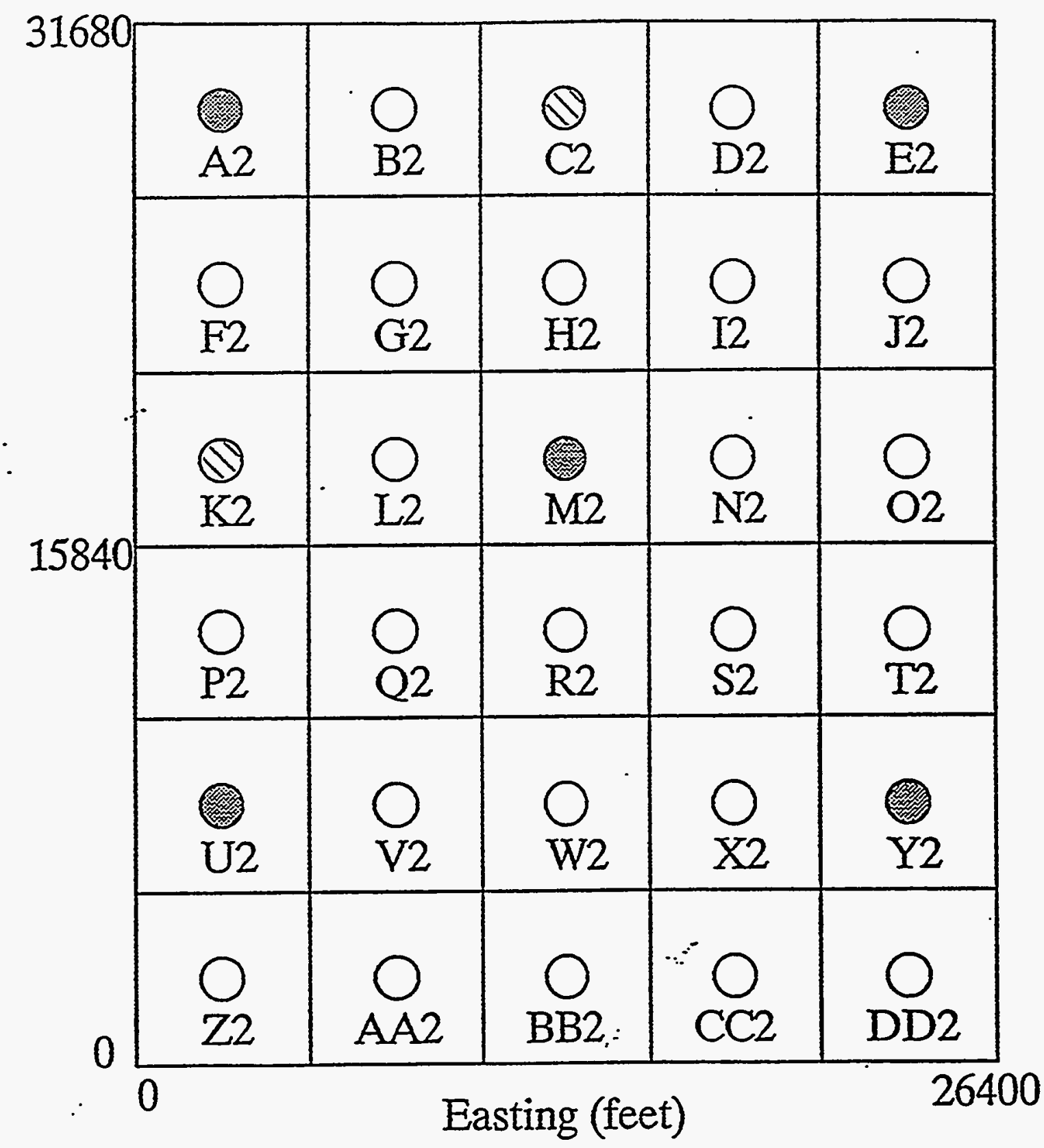

Figure 58. Well location map for 3D Hugoton Field simulation (large scale) 


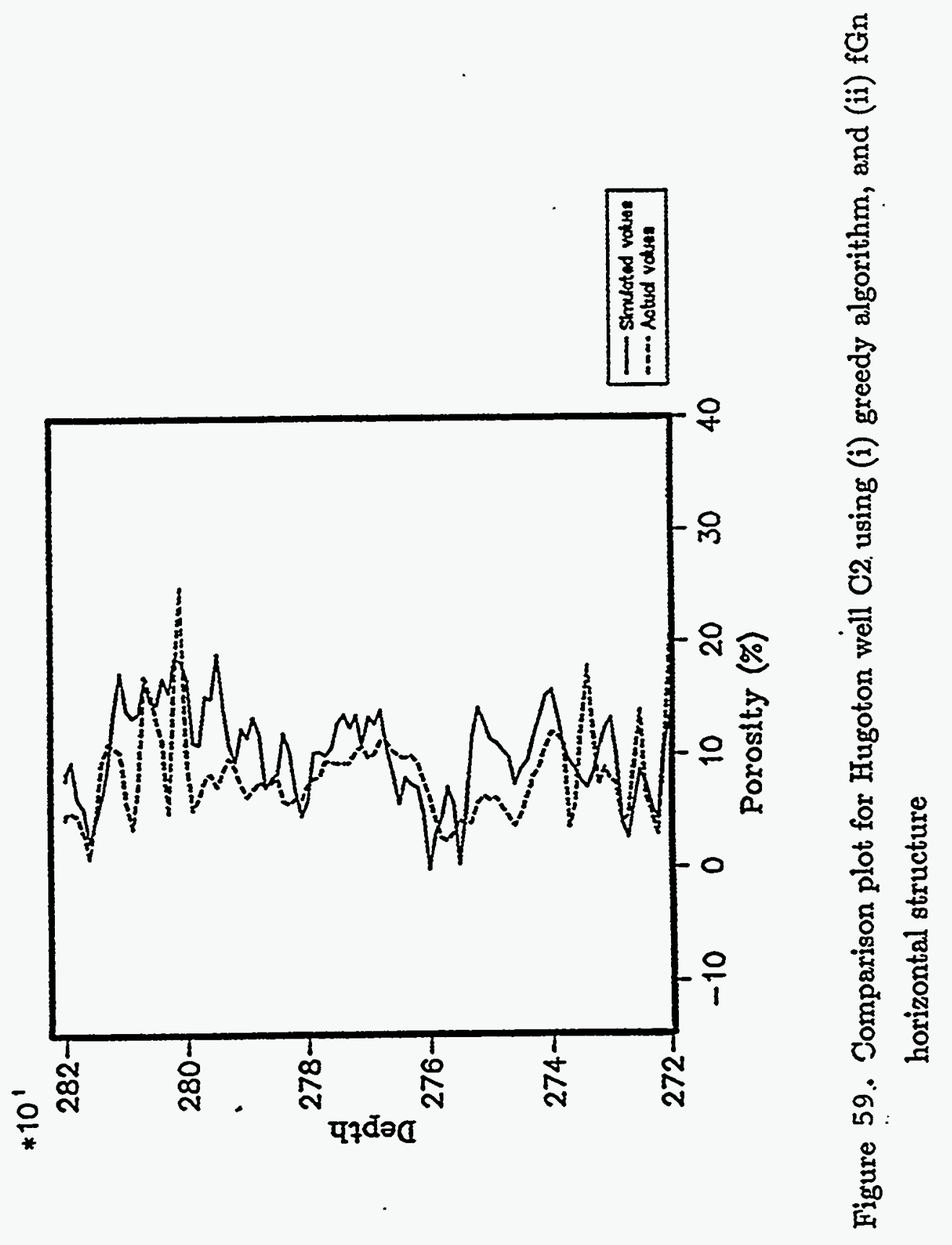




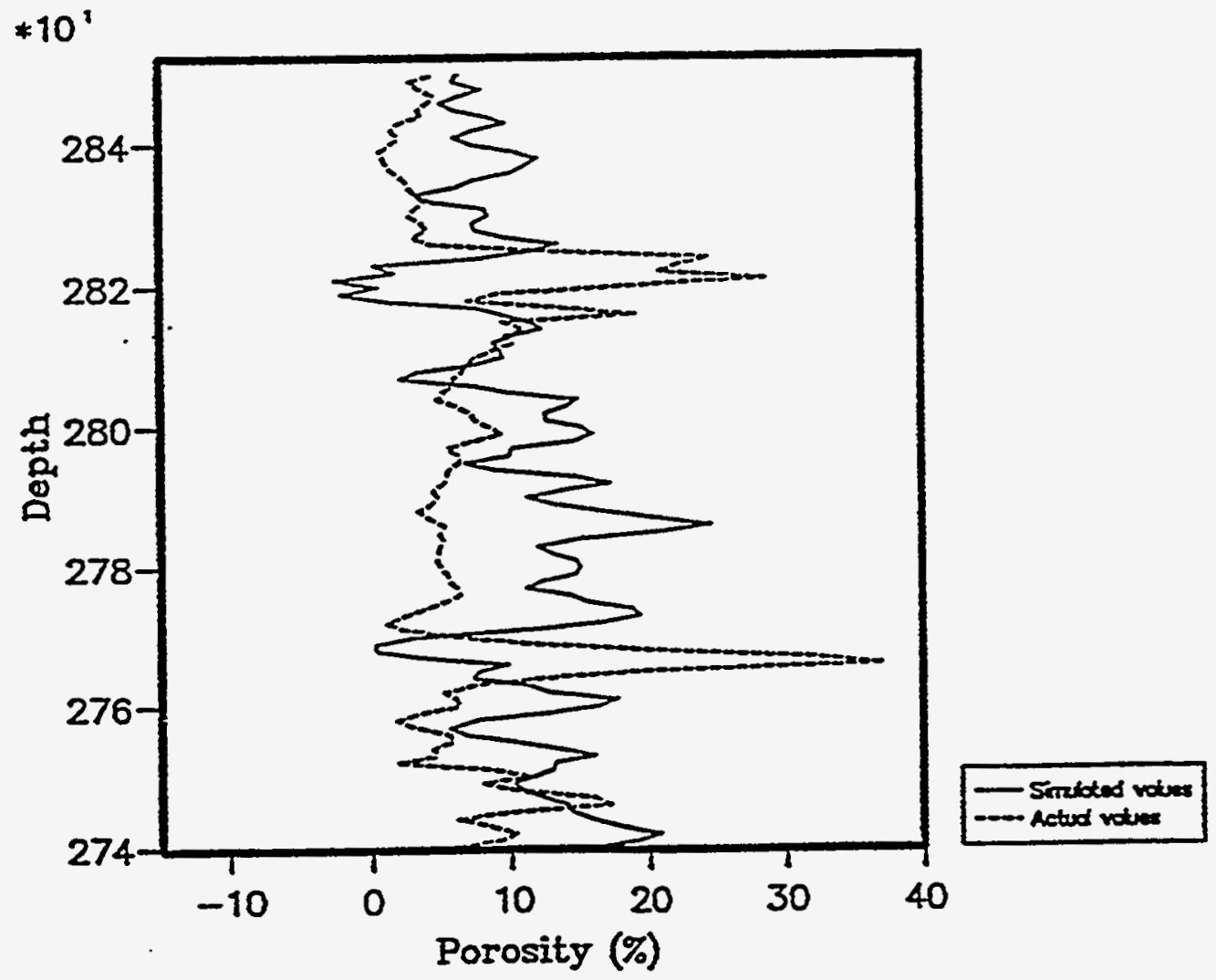

Figure 60. Comparison plot for Hugoton well K2 using (i) greedy algorithm, and (ii) fGn horizontal structure 


\title{
Section I
}

\author{
Report II \\ Reservoir Characterization for Waterfloods \\ Incorporating Well Test Data in \\ Stochastic Conditional Simulations
}

R. Sagar, M. Kelkar and L. Thompson

\begin{abstract}
Summary
A comprehensive reservoir description procedure must honor as much of the available data as possible. Geostatistical techniques provide a promising way to create an alternate reservoir descriptions. Unfortunately, conventional geostatistical techniques are unable to adequately incorporate information obtained from. well test analysis. This report presents an approach to incorporate well testing data in the method of simulated annealing, a geostatistical method reported earlier. ${ }^{1}$ Investigating the problem of incorporating well test data in a conditional simulation method is broken down into two major sections:

- The permeability derived from well test analysis represents, within some radius of investigation or support volume, some ill-defined average of a reservoir permeability distribution at a smaller scale. Based on available literature, a new method which describes the type of average and support volume represented by the well test permeability is determined.

- In geostatistics, descriptions of reservoir properties are determined as a function of univariate and the spatial statistics of the sample data. Incorporating well test data adds another constraint that the reservoir description must satisfy. Additional constraints may be considered if permeability porosity correlations have to satisfied. A stochastic conditional simulation procedure will be used in this study. Simulated annealing is a stochastic simulation method that is flexible and robust enough to include additional constraints. The second part of this study will focus on extending the simulated annealing method to incorporate the additional constraints.
\end{abstract}

\section{Introduction}

In recent years, developments in reservoir characterization have helped better describe reservoir properties. The methods have become increasingly important to the petroleum reservoir engineer. A better description of the reservoir properties, such as permeability and porosity, will improve estimates of hydrocarbon reserves, 
help select locations for infill well drilling, and yield better predictions of primary and secondary recovery processes.

Geostatistical ${ }^{1,2,3,18}$ methods are used to characterize reservoirs by describing the spatial distribution of properties which incorporate heterogeneity and variability inherent in the geologic complexities of any reservoir. The heterogeneity is a function of the lithology, and depositional and post depositional environment of the reservoir. Geostatistical methods allow to describe and model the spatial variability .and correlation of reservoir properties at the interwell or unsampled regions. The geology of a reservoir determines the spatial continuity or correlation of reservoir properties. In geostatistics, the spatial correlation and variability of a reservoir property is represented by the variogram. With the help of the variogram, kriging and conditional simulation methods are used to estimate the value of a property at unsampled locations. Kriging methods provide a smooth image of the reservoir properties, whereas, conditional simulation generates alternate equiprobable images which exhibit the variabilities representative of sampled data. We will use the method of simulated annealing, a conditional simulation method, in this study.

To develop a reservoir description using geostatistical techniques, sample data must be available. Sample data are obtained from cores, logs and well test analysis. In using sampled information the volume over which a sample is measured has to be accounted for. For example, a permeability obtained from a core sample typically represents a volume in the order of $10^{-3} \mathrm{ft}^{3}$, whereas, an effective well test permeability may represent a volume in the order of $10^{9} \mathrm{ft}^{3}$. This is known as scale or support effect.

A rigorous geostatistical procedure for the purpose of describing reservoir properties must be able to incorporate all the available information and account for the various volumes of investigation.

The conventional approach to reservoir description using geostatistics can readily accommodate the use of core and log data; specifically, permeability and porosity data. The statistics of the sample data can be well defined and the support volume is a function of the size of the retrieved core or the depth of investigation of the logging instrument. The methods, however, do not account for scaling these values to values representative of a grid block size for the purpose of reservoir simulation studies. Conventional geostatistical methods are also unable to incorporate well test data. The support volume represented by a well test permeability needs to be determined as does a procedure that relates the well test derived permeability to the distribution of small scale permeabilities. Ultimately, we need a geostatistical method that will incorporate the well test permeability and at the same time honor geological, core, and log data.

\section{Literature Review}

The permeability calculated by well test analysis is based on an analytical solution to the diffusivity equation. The solution for the infinite-acting or transient flow period can be written as: ${ }^{7}$ 


$$
p_{w f}=p_{i}-\frac{162.6 q B \mu}{k h}\left[\log t+\log \left(\frac{\bar{k}_{w t}}{\phi \mu c_{t} r_{w}^{2}}\right)-3.2275+0.86859 s\right] .
$$

The slope $(m)$ of the semilog straight line plot of pressure vs time determines the well test permeability,

$$
\bar{k}_{w t}=-162.6 \frac{q B \mu}{m h} .
$$

This solution is based on the assumption that the reservoir is homogeneous; however, no reservoir is homogeneous and the degree of heterogeneity is a function of the lithology, and depositional and post depositional environment of the reservoir. For practical purposes, it is assumed that the permeability determined by well test analysis is an effective permeability representing some radius of investigation or radius of drainage, which is influenced by the producing well.

The classical definition of radius of drainage is given by Van Poolen. ${ }^{8}$ In physical terms it defines the radius over which all the reservoir properties have influenced the well flowing pressure. ${ }^{9}$ It is given by the following equation:

$$
r=A \sqrt{\frac{\bar{k}_{w t} t}{\phi \mu c_{t}}}
$$

where $A$ is given as 0.03248 by Lee. ${ }^{9}$ As the time of the test increases more of the reservoir is influenced by the well and the radius of drainage increases. However, this definition of radius of drainage is questionable in the presence of heterogeneities. ${ }^{10}$

Well test derived permeabilities have been used by some authors for describing reservoirs using geostatistical techniques. De Costa e Silva ${ }^{5}$ assumed that the well test permeability represents the permeability at the center of a grid block. Ben Rached ${ }^{4}$ determined averages, between two arbitrary radii, of the distribution of permeability and thickness $\left(\bar{k}_{w t} h\right)$. He honored well test permeability by normalizing the log-derived average permeability to exactly match the permeability of the well test at the well. He did this to account for the support volume of a well test permeability.

Fasanino et al. ${ }^{11}$ described a procedure for determining the permeability distribution by an inverse modeling technique. The initial reservoir description is developed using kriging., ${ }^{2,3,6}$ Kriging generates one smoothed realization of a variable. An objective function based on a measured pressure distribution is defined (this objective function is not the same as in annealing). The transmissibility, $\bar{k}_{w t} h$, values are iteratively modified until the method of steepest gradient descends to the smallest value of the objective function. An optimum value of $\bar{k}_{w t} h$ is specified at a few grid points until a good match between calculated and observed pressures is obtained. In this method the univariate and spatial statistics are not necessarily honored once a match has been obtained and the number of flow simulations required to obtain a pressure match may not be cost effective in terms of CPU time. 


\section{The Radius of Investigation and the Averaging Process of a Well}

\section{Test Permeability}

Recently, some authors ${ }^{12,13}$ have investigated the concept of radius of investigation and the averaging process of small scale permeabilities within this radius as related to the well test permeability. The first is a stochastic approach to the problem, whereas the second is an analytical approach.

The stochastic approach is proposed by Alabert. ${ }^{12}$ For stochastic conditioning, a permeability model must explicitly relate the test permeability to the distribution of smaller scale permeabilities. Alabert proposed guidelines to determine an optimum averaging of the distribution. A permeability field honoring some spatial and univariate statistics was developed and a drawdown test was numerically simulated in the permeability field. A single well test permeability was matched to a power average of permeabilities as circular volumes around the well were increased. The power average of the permeability is

$$
\bar{k}(\omega, A)^{\omega}=\frac{1}{N(A)} \sum_{\vec{h} \in V(A)} k(\vec{h})^{\omega},
$$

where, $A$ is similar to $A$ defined in Equation 3. Alabert optimizes $A$ to be a variable between 0.005 and 0.035 . To eliminate boundary and wellbore effects he defines a minimum radius $\left(r_{m i n}\right)$ at $t_{D} / r_{D}^{2}=120$ and a maximum radius $\left(\dot{r}_{m a x}\right)$ at $t_{D} / r_{D}^{2}=$ 2500. Therefore, as $A$ varies $r_{\text {min }}$ and $r_{m a x}$ also vary. The averaging volume $(V(A))$ is defined by $r_{\min }$ and $r_{\max }$ and $\omega \in[-1,1] . N(A)$ is the number of grid blocks in $V(A)$ and $k(\vec{h})$ is the block permeability at location vector, $\vec{h}$. An optimum $A$ and $\omega$ were determined by minimizing the mean normalized absolute difference and yielding an unbiased fit between the well test permeability and the power averaged permeability. The well test permeability was determined from the semilog straight line. For each permeability distribution an optimum $A$ and $\omega$ were determined. However, there is no uniqueness to these optimum coefficients. Alabert also notes that as the heterogeneity of the reservoir increases $\omega$ also increases.

The reservoir permeability may be statistically distributed in a variety of ways ${ }^{14}$ and the distribution may not necessarily be log normally distributed. An understanding for the averaging process of layered permeabilities can be obtained from an analysis of flow through layered permeability fields. ${ }^{14}$ For the case when bed layers are parallel to the direction of flow an arithmetic average of the permeabilities in the bed is a more appropriate average. Layering in series with the beds perpendicular to the flow direction, a harmonic average is more representative. These conclusions are based on the assumption that the flow is steady state. In the numerical simulation of a transient well test, the flow is not steady state and the arrangement of grid blocks does not necessarily produce flows in series or parallel relative to the arrangement of beds. It has been shown that the most probable behavior for an uncorrelated (i.e., spatial continuity between variables is not present) heterogeneous system approaches that of a homogeneous system with a permeability equal to the geometric mean of the individual permeabilities. ${ }^{15}$ 
Gómez-Hernández and Gorelick ${ }^{16}$ observed that for small correlation lengths the effective permeability falls below the geometric average, but as the correlation length is increased the effective permeability approaches the harmonic average of the permeability distribution.

Recently, Oliver ${ }^{13}$ investigated the averaging process of permeability variations in $r$ and $\theta$ coordinates. He solved the diffusion equation for the pressure response of a well situated in an infinite reservoir where permeability is an arbitrary function of position and is solved for the case of small permeability variations about the mean. Some type of averaging is inherently present when an effective permeability is determined from the slope of the semilog plot, therefore, the averaged permeability of the small scale permeabilities within the radius of investigation are determined from the slope of the semilog plot at time, $t_{D}$. Neglecting wellbore storage and skin, the slope is written as:

$$
t_{D} \frac{\partial p_{D}}{\partial t_{D}}=+\frac{1}{2}-\frac{\epsilon}{2 \pi} \int_{1}^{\infty} K\left(\xi, t_{D}\right) \int_{-\pi}^{+\pi} f(\xi, \theta) d \theta d \xi,
$$

where, $\epsilon f\left(r_{D}, \theta\right)$ is the permeability distribution function for small variations in permeability and can be written as $\left(1-\bar{k}_{w t} / k(r, \theta)\right)$. Thus it can be shown that the integral over $\theta$ is a normalized form of the harmonic average of permeabilities over $\theta . K\left(r_{D}, t_{D}\right)$ is the weighting or kernel function and is given by:

$$
\sqrt{t_{D}} K\left(r_{D}, t_{D}\right)=0.5 \sqrt{\frac{\pi r_{D}^{2}}{t_{D}} \exp \left(-\frac{r_{D}^{2}}{2 t_{D}}\right) W_{\frac{1}{2}, \frac{1}{2}}\left(\frac{r_{D}^{2}}{t_{D}}\right)}
$$

and is valid for dimensionless times greater then 100.

For further developments of the complex mathematics of this equation, the reader is referred to Oliver's paper. ${ }^{13}$ Of more importance, is the physical significance of the weighting function. The shape of this function (Figure 1) is used to determine the radius of investigation. Oliver defines the radius of investigation as the radius at which the weighting function becomes so small that the permeabilities at greater distances do not contribute to the slope of the semilog plot. A normalized plot of the weighting function (Figure 1) shows that $98 \%$ of the contribution of the weighting function is from $r_{D}=0.12 \sqrt{t_{D}}$ and $r_{D}=2.34 \sqrt{t_{D}}$. So, the kernel function gives the weighted average of the well test permeability within these radii. Oliver claims that the weighting function represents the average permeability estimate; therefore, the integral of the area under the curve is a measure of the cumulative contribution to the permeability estimate. Thus the kernel function defines the radius of investigation or support volume for small variations in permeability in a radially heterogeneous system.

The inverse problem of analytically determining the permeability distribution from the pressure data was investigated in a second paper by Oliver. ${ }^{17} \mathrm{He}$ concludes that an infinite number of permeability fields can actually reproduce the pressure data and one should abandon hope of determining the true permeability from a finite set of data. He also investigates the effect of porosity variations. Small porosity 
variations result in greater permeability variations, so in most cases variation in porosity would have a small effect on the interpretation of well test data. For the purpose of most reservoir studies porosity variations are important and it may be important to consider some lind of porosity-permeability relation.

\section{Stochastic Conditional Simulation}

Stochastic conditional simulation methods are used to generate possible descriptions of reservoir properties. The methods are stochastic since reservoir properties are represented by random variables; it is conditional since available data are honored at sampled locations; and the method simulates several equiprobable distributions of a property in the reservoir. There are several conditional simulation techniques available in the literature. ${ }^{1,18,19}$ This study will focus on the method of simulated annealing. Simulated annealing is an algorithm approach to solving optimization problems. ${ }^{20}$ The method for geostatistics was proposed by Farmer. ${ }^{21}$ The method was extended by Pérez ${ }^{1}$ to develop reservoir descriptions; it comprises three steps.

1) An initial, uncorrelated distribution of the reservoir property is developed. The distribution must satisfy an input histogram or cumulative distribution function ( $c d f$ ). The $c d f$ is sampled with a random number and an uncorrelated distribution is developed. Since the method is conditional, the initial or conditioning data are honored.

2) In geostatistics the spatial relationship between data points is defined by the semi-variogram. The semi-variogram is defined by:

$$
\gamma\left(\vec{h}_{l, i}\right)=\frac{1}{2 N_{p}\left(\vec{h}_{l, i}\right)} \sum_{j=1}^{N\left(\vec{h}_{l, i}\right)}\left[V\left(\vec{w}_{j}+\vec{h}_{l, i}\right)-V\left(\vec{w}_{j}\right)\right]^{2},
$$

where, $\vec{h}_{l, i}=\left(h_{x, l, i}, h_{y, l, i}, h_{z, l, i}\right)$ is the lag distance vector (separation distance between points), $N_{p}\left(\vec{h}_{l, i}\right)$ is the number of pairs within the simulation grid corresponding to the lag $\vec{h}_{l, i}, N_{h, i}$ is the number of semi-variogram lags, $\vec{w}_{j}$ is the location vector, and $V$ is the variable being modeled. The index $i$ refers to the direction and the index $l$ refers to the lag distances considered for a given direction. Therefore, spatial relations can be defined in the three principle directions, so that anisotropy may be considered and three dimensional realizations can be generated. The efficiency and accuracy of a simulated annealing procedure depends on the number of constraints that must be satisfied. The total number of constraints, $N_{0}$ is:

$$
N_{o}=N_{c}+N_{f}+\sum_{i=1}^{N_{d}} N_{h, i}
$$

where, $N_{c}$ is the number of conditioning data, $N_{f}$ is the number of class intervals of the cumulative distribution function, and $N_{d}$ is the number of semivariogram directions. An objective or energy function provides a measure of 
the difference between the sample variogram of the distribution of properties and a specified model variogram. The objective function is defined by Pérez as:

$$
E^{k}=\frac{1}{E_{o}} \sqrt{\sum_{i=1}^{N_{d}} \sum_{l=1}^{N_{h, i}}\left[\frac{\gamma_{s}^{k}\left(\vec{h}_{l, i}\right)-\gamma_{o}\left(\vec{h}_{l, i}\right)}{\gamma_{s}^{\circ}\left(\vec{h}_{l, i}\right)}\right]^{2}},
$$

where, the index $k$ is an iteration number, $\gamma_{s}^{k}$ is the semi-variogram of the simulated variable, $\gamma_{0}$ is the variogram of the sample data, and $E_{0}$ is the normalizing constant equal to:

$$
E_{o}=\sqrt{\sum_{i=1}^{N_{d}} \sum_{l=1}^{N_{h, i}}\left[\frac{\gamma_{s}^{o}\left(\vec{h}_{l, i}\right)-\gamma_{o}\left(\vec{h}_{l, i}\right)}{\gamma_{s}^{o}\left(\vec{h}_{l, i}\right)}\right]^{2}},
$$

where, $\gamma_{s}^{\circ}\left(\vec{h}_{l, i}\right)$ are the semi-variograms of the simulated variable at initial conditions calculated from the initial distribution of the variable. By minimizing the objective function the difference between semi-variograms of the simulation variables $\left(\gamma_{s}\right)$ and the required semi-variogram models $\left(\gamma_{0}\right)$ is minimized.

3) Variables in the system are interchanged until the objective function is minimized. The interchange of variables can be done in several ways ${ }^{1}$. The Metropolis condition ${ }^{22}$ is used most often. Pérez uses the metropolis condition to minimize the objective function. The Metropolis condition calculates the probability of the transition between the two states of the objective function.

\section{Approach}

The following section proposes the approach that will be used to solve the inverse problem of determining the permeability distribution from limited available information such as univariate and spatial statistics and pressure transient data. The procedure that is being used can be broken down into three major sections:

1) The Base Case The first step will be to generate the base case permeability distribution and numerically simulate a pressure drawdown test in this reservoir. A two dimensional permeability field is generated using the Turning Bands Method (TBM). ${ }^{23,24}$ TBM generates an unconditional correlated distribution. The distribution is unconditional since there is no available data at gridblocks that must be honored. The specified univariate and spatial statistics of the distribution are honored. For simplicity a square reservoir with square grid blocks is considered. An example of a permeability field generated using this method is shown as a greyscale map in Figure 2. Using this permeability field, a numerical simulation of a drawdown is performed on the reservoir. It is assumed that the reservoir has uniform porosity, thickness and compressibility. A well producing at a constant rate is placed at the center of the grid block at the center of 
the reservoir. The outer boundaries are no flow boundaries. A finite difference numerical simulation of the reservoir is performed using ECL 100, a commercial reservoir simulation package. ${ }^{25}$ Grid block pressures are corrected to well flowing pressures using Peaceman's approximation. ${ }^{26} \mathrm{~A}$ Comparison between the analytical ${ }^{7}$ and numerical solution for a homogeneous square reservoir show excellent agreement (Figure 3). Figure 4 shows the transient pressure response for the permeability field shown in Figure 2. The pressure derivatives are also plotted. To draw general conclusions numerous base cases are investigated.

\section{2) The Radius of Investigation and Averaging Process of}

Permeabilities To effectively use a well test derived permeability in a stochastic conditional simulation method, the well test permeability must impose a deterministic constraint on the distribution of surrounding small scale permeabilities within the radius of investigation. Therefore, an averaging model that defines the permeability distribution within the radius of investigation has to be developed. The radius of investigation will be the annular region defined by the kernel function. For a dimensionless time, $t_{D}$, the kernel function is calculated betwreen $r_{D}=0.12 \sqrt{t_{D}}$ and $r_{D}=2.34 \sqrt{t_{D}}$. where,

$$
t_{D}=2.637 * 10^{-4} \frac{\bar{k}_{w t} t}{\phi \mu c_{t} r_{w}^{2}}
$$

and $\bar{k}_{w t}$ is the permeability calculated from the best fit semilog straight line slope, $m$, in Equation (2). Since the weighting or kernel function represents the weighted average of the well test permeability, the integral of the area under the curve is a measure of the cumulative contribution to the permeability estimate. Based on this discussion, the annular region in the radius of investigation is divided into $n$ more regions at a dimensionless time, $t_{D}$. A numerical integration using Simpson's rule of the kernel function gives the area under the curve between $r_{D}=0.12 \sqrt{t_{D}}$ and $r_{D}=2.34 \sqrt{t_{D}}$. The weight of each annular region is, therefore given by:

$$
w_{i}=\frac{\int_{r_{i-1}}^{r_{i}} K\left(r_{D}\right)}{\int_{0.12 \sqrt{t_{D}}}^{2.34 \sqrt{t_{D}}} K\left(r_{D}, t_{D}\right)},
$$

where, $i=1,2, \ldots \ldots \ldots . . ., n$.

If we are trying to represent an areal permeability distribution in a radial form, then, we need to first evaluate the correct type of averaging of permeability values within the annular rings. An area based power averaging scheme is considered. It accounts for segments of grid blocks that may partly fall into one of the specified radii. The equation to calculate this average, $\bar{k}$, is: 


$$
\left(\bar{k} A_{r}\right)^{\omega}=\sum_{\vec{h} \in A_{r}}\left(k(\vec{h}) A_{b}(\vec{h})\right)^{\omega},
$$

where, $\vec{h}$ is the location vector of the grid block, $A_{\tau}$ is the radial area between the $(i-1)$ and $i$ interval and $A_{b}$ is the area of the grid block that falls in between these radii. For $\omega=1$ the arithmetic average is calculated and when $\omega=-1$ the harmonic average is calculated. However, when $\omega=0$ the geometric average cannot be calculated by Equation 14; instead, the following area based geometric averaging equation is used:

$$
A_{r} \log \bar{k}=\sum_{\vec{h} \in A_{r}} A_{b}(\vec{h}) \log k(\vec{h})
$$

The equivalent permeability in the radius of investigation, $\bar{k}_{e}$, is then calculated as function of the average permeability within an annular region and the weight of the region:

$$
\bar{k}_{e}=\sum_{i=1}^{n} w_{i} k_{i}
$$

3) Simulated Annealing The next step will be to solve the inverse problem: assuming the permeability field is unknown, can we determine the truth case permeability field. The data available to do this will be the univariate and spatial statistics of the distribution and the transient pressure response of the permeability field. A stochastic conditional simulation method must be used. Simulated annealing is a general and flexible simulation procedure that will enable us to incorporate additional constraints. Equation 8 can be expanded to incorporate the constraint imposed by well test data:

$$
N_{o}=N_{c}+N_{f}+\sum_{i=1}^{N_{d}} N_{h, i}+N_{w},
$$

where, $N_{w}$ is the number of constraints that will be specified from results in section 2 . Since the well test permeability represents an effective permeability within a radius of investigation in the reservoir, we can specify another subregion. In this region a distribution function defined by our investigation of the averaging process can be used to constrain our permeability distribution in the radius of investigation. The objective function will be based on the variogram and an additional function describing the distribution of properties in the sub-region. Therefore, the reservoir description will honor more available information resulting in the best possible reservoir description. The developed reservoir description can be compared to the base case by checking the distribution of properties and the simulated pressure response. A drawback of simulated annealing is that it is computationally expensive and further work on making the algorithm more efficient needs to be done. 


\section{Results and Discussion}

Thirty-two permeability fields using the Turning Bands Method were generated. The heterogeneity of a reservoir can be described by the correlation range and the variance of the distribution. The heterogeneity index ${ }^{28}$ describes the overall variability and is given by the following equation:

$$
H I=\sigma_{l n k}^{2} \lambda_{D}
$$

where $\sigma_{l n k}^{2}$ is the variance of the logarithm of the permeability distribution and $\lambda_{D}$ is the dimensionless correlation length. Table I gives a summary of range of variability of the permeability fields. Figures 5 and 6 are grey scale maps of the permeability fields showing the lowest and highest variabilities, respectively.

A drawdown simulation of the 32 permeability fields was then performed. An example of the pressure response and derivatives of the permeability fields in Figures 5 and 6 are shown in Figures 7 and 8, respectively. Notice the more heterogeneous distribution shows more variability in its pressure derivative. In general similar trends in the pressure derivative data were observed for the 32 permeability fields.

The next step was to determine the kind of averaging that occurs in the radii of investigation defined by Oliver. ${ }^{13}$ The instantaneous derivative is used to calculate the instantaneous permeability $\left(k_{w t i}\right)$ at time, $t$, using Equation (2).

Using the procedure outlined in section 2 of the Approach, equivalent permeabilities, $\bar{k}_{e}$, using the harmonic, geometric, and arithmetic averages are calculated. Figures 9, 10, and 11 show the comparisons between harmonic, geometric, and arithmetic averaging methods to the instantaneous well test permeability, respectively. Note that the harmonic average under predicts the instantaneous well test permeability and the arithmetic average over predicts the permeability. The overall average difference of the results are summarized in Table I. The geometric averaging method yields the best overall results. Note that

Phase 3 of the approach is currently being implemented. The objective function is modified so that at time, $t$, we calculate Oliver's radius of investigation. The radius of investigation is defined between $r_{D}=0.12 \sqrt{t_{D}}$ and $r_{D}=2.34 \sqrt{t_{D}}$, where $t_{D}$ is defined by Equation (12). The radius of investigation is divided into 10 annular rings of equal area. Each region is assigned a weight according to the area under the curve of the weighting function, this is given by Equation (13). Within each of these ten annular rings an area based geometric average of the grid blocks is calculated (Equation 15). This averaging technique accounts for the part of a grid block that may fall on the perimeter of a ring. Therefore, we are able to overlay a radial coordinate system onto an areal coordinate system. Finally, at time, $t$, the equivalent permeability is calculated by the following equation:

$$
\bar{k}_{e}=\sum_{i=1}^{n} w_{i} k_{i}
$$

It was found that this equivalent permeability is approximately equal to the instantaneous well test permeability at time, $t$; therefore, inorder to honor well test 
information, we can simulate the annealing process until the equivalent permeability approaches the instantaneous well test permeability at time $t$. Oliver's solution is valid for a total of $N_{t}$ time steps; therefore, we can define $N_{t}$ different radii of investigation. It should be noted that there will be overlap of the different radii of investigation. The energy function incorporating well test information can be written as:

$$
E_{2}^{k}=\sqrt{\sum_{i=1}^{N t}\left[\frac{\bar{k}_{e}^{k}-k_{w t i}}{k_{w t i}}\right]^{2}}
$$

This is added to Equation 10 to yield the overall energy function:

$$
E^{k}=\frac{1}{2 E_{o 1}} \sqrt{\sum_{i=1}^{N_{d}} \sum_{l=1}^{N_{h, i}}\left[\frac{\gamma_{s}^{k}\left(\vec{h}_{l, i}\right)-\gamma_{o}\left(\vec{h}_{l, i}\right)}{\gamma_{s}^{o}\left(\vec{h}_{l, i}\right)}\right]^{2}}+\frac{1}{2 E_{o 2}} \sqrt{\sum_{i=1}^{N t}\left[\frac{\vec{k}_{e}^{k}-k_{w t i}}{k_{w t i}}\right]^{2}}
$$

where $\bar{k}_{e}^{k}$ is the equivalent permeability or the weighted area based geometric average at the iteration level $k$ and $E_{o 1}$ and $E_{o 2}$ are initial energy functions defined as:

$$
E_{o 1}=\sqrt{\sum_{i=1}^{N_{d}} \sum_{l=1}^{N_{h, i}}\left[\frac{\gamma_{s}^{o}\left(\vec{h}_{l, i}\right)-\gamma_{0}\left(\vec{h}_{l, i}\right)}{\gamma_{s}^{o}\left(\vec{h}_{l, i}\right)}\right]^{2}}
$$

and

$$
E_{o 2}=\sqrt{\sum_{i=1}^{N t}\left[\frac{\bar{k}_{\mathrm{e}}^{\circ}-k_{w t i}}{k_{w t i}}\right]^{2}}
$$

where $\bar{k}_{e}^{\circ}$ is the equivalent permeability or the weighted area based geometric average for the initial distribution. Thus the overall energy function converges to a value of 0 within some tolerance.

\section{Conclusions}

It was found that Oliver's solution can be used to define the radius of investigation using drawdown pressure transient data. The solution is not restricted to small variations in permeability and can be used to define the radius of investigation for a distribution with a high heterogeneity index. The weighted area based geometric average of permeabilities within the radius of investigation defines the equivalent permeability that is approximately equal to the instantaneous well-test permeability determined from the pressure derivative at time, $t$. Thus the instantaneous well test permeability can be explicitly related to the distribution of small scale permeabilities within annular regions of the reservoir. This observation can be incorporated 
into simulated annealing so that the reservoir description honors the spatial and univariate statistics as well as the pressure transient data. The annealing objective function is modified to include the variogram function and equivalent permeability within annular regions of the reservoir. The additional constraints imposed on the annealing schedule will require that the annealing algorithm be optimized so that it's computational cost is reduced.

\section{Nomenclature}

Symbol

$A=$ radius of investigation coefficient

$A_{b}=$ area of grid block in the annular ring

$A_{r}=$ area of annular region

$B=$ formation volume factor, $b b l / S T B$

$c_{i}=$ total compressibility $p s i^{-1}$

$E=$ objective function

$E_{0}=$ normalizing constant for objective function

$h=$ thickness, $f t$

$\vec{h}=$ lag distance vector for semi-variograms

$H I=$ Heterogeneity index

$\bar{k}=$ average permeability, md

$\bar{k}_{e}=$ equivalent permeability, md

$k_{i}=$ grid block permeability, md

$\bar{k}_{w t}=$ well test permeability, md

$k_{w t i}=$ instantaneous well test permeability, md

$K\left(r_{D}, t_{D}\right)=$ Weighting or Kernel function

$m=$ semilog straight line slope

$N_{c}=$ number of conditioning data

$N_{d}=$ number of semi-variogram directions

$N_{f}=$ number of classes for distribution function

$N_{h}=$ number of semi-variogram lag distances

$N_{o}=$ number of constraints for a conditional simulation

$N_{p}=$ number of pairs in a semi-variogram

$N_{t}=$ total number of time points valid for Oliver's solution

$N_{w}=$ total number of well test constraints

$p_{w f}=$ well flowing pressure, psi

$p_{i}=$ initial reservoir pressure, $\mathrm{psi}$

$p_{w} d=$ dimensionless pressure

$q=$ rate, $\mathrm{STB} / \mathrm{D}$

$r_{D}=$ dimensionless radius

$r_{w}=$ wellbore radius

$s=$ skin

$t=$ time, days 


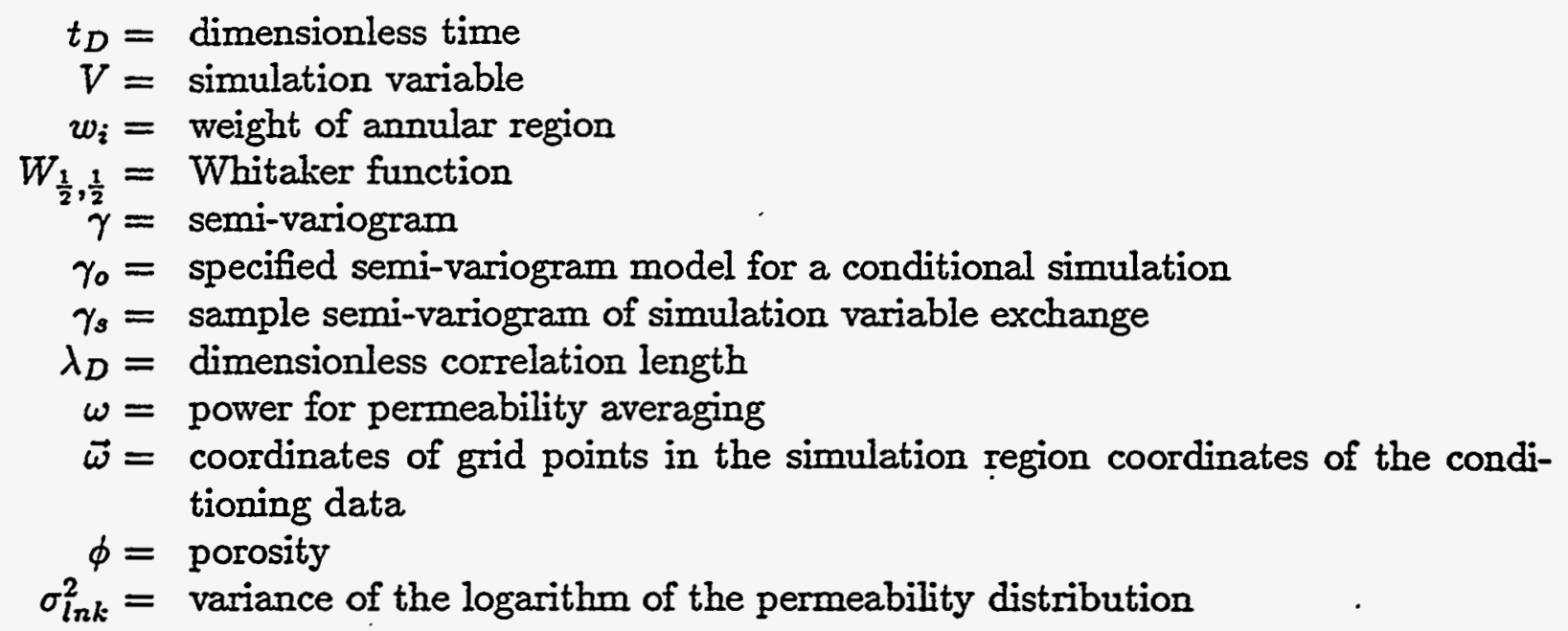

\section{References}

1. Pérez, G.: "Stochastic Conditional Simulation for Description of Reservoir Properties," Phd Dissertation, University of Tulsa, Tulsa, OK (1991).

2. Journel, A.G. and Huijbregts, C.J.: Mining Geostatistics, Academic Press, New York (1978).

3. Isaaks, E.H. and Srivastava, R.M.: Applied Geostatistics, Oxford University Press, New York (1989).

4. Ben-Rached, L.: "An Application of Geostatistical Techniques for Reservoir Performance Prediction" MS thesis, University of Tulsa, Tulsa, OK (1991).

5. De Costa e Silva, A.J.: "A New Approach to the Characterization of Reservoir Heterogeneity Based on the Geomathematical Model and Kriging Technique" paper SPE 14275 presented at the 1985 Annual Technical Conference and Exhibition, Las Vegas, Sept. 22-25.

6. Kelkar, M.: Application of Statistics to Reservoir Characterization, self -published, Tulsa, OK (1989).

7. Earlougher, R.C. Jr.:Advances in Well Test Analysis, Monograph Series, SPE, Richardson, TX (1977).

8. Van Poolen, H.K: "Radius of Drainage and Stabilization-Time Equations," Oil \&.Gas J. (Sept. 14, 1964) 138-146.

9. Lee, John: Well Testing, SPE, Dallas (1982).

10. Matthews, C.S. and Russell, D.G.: Pressure Buildup and Flow Tests in Wells, Monograph Series, SPE, Richardson, TX (1967).

11. Fasanino, G. and Molinard, J.E.: "Inverse Modelling of Gas Reservoirs," paper SPE 15592 presented at the 1986 Annual Technical Conference and Exhibition, New Orleans, LA, Oct. 5-8.

12. Alabert, F.G.: "Constraining Description of Randomly Heterogeneous Reservoirs to Pressure Test Data: A Monte Carlo Study," paper SPE 19600 presented at the 1989 Annual Technical Conference and Exhibition, San Antonio, TX, Oct. 8-11. 
13. Oliver, D.S.: " The Averaging Process in Permeability Estimation from WellTest Data," SPEFE (Sept. 1990) 319-324.

14. Jensen, J.L., Lake, L.W., and Hinkley, D.V.: " A Statistical Study of Reservoir Permeability: Distributions Correlations and Averages," paper SPE 14270 presented at the 1985 Annual Technical Conference and Exhibition, Las Vegas, NV, Sept 22-25.

15. Warren, J.E. and Price, H.S.: “Flow in Heterogeneous Porous Media," SPE Journal (Sept. 1961), 153-169.

16. Gómez-Hernández, J.J. and Gorelick, S.M.: "Effective Groundwater Model Parameter Values: Influence of Spatial Variability of Hydraulic Conductivity, Leakance and Recharge," Water Resources Research, 25(3), 405-419, 1989.

17. Oliver, D.S.: "Estimation of Radial Permeability Distribution from Well Test Data," paper SPE 20555 presented at the 1990 Annual Technical Conference and Exhibition, New Orleans, LA, Sept. 23-26.

18. Journal, A.G. and Alabert, F.G.: “ A New Method for Reservoir Mapping," JPT (Feb. 1990) 212-218.

19. Hewett, T.A. and Beherns, R.A.: "Conditional Simulation of Reservoir Heterogeneity with Fractals," SPEFE (Sept. 1990) 217-225.

20. Collins, N.E., Eglese, R.W. and Golden, B.L.:" Simulated Anealing - An Annotated Bibliography," Simulated Annealing (SA) \& Optimization: Modern Algorithms with VLSI, Optimal Design, and Missile Defense Applications, M.E. Johnson (editor), American Sciences Press, New York(1988) 209-307.

21. Farmer, C.L.: "Numerical Rocks, The Mathematical Generation of Reservoir Geology," paper presented at the 1989 Joint IMA/SPE European Conference Cambridge University, July 25-27.

22. Metropolis, N., Rosenbluth, A.W., Rosenbluth, M.N., Teller, A.G., and Teller, E. "Equation of State Calculations by Fast Computing Machines," The Journal of Chemical Physics (June 1953) 1087-92.

23. Mantoglou, A. and Wilson, J.L.:" The Turning Bands Method for Simulation of Random Fields Using Line Generation by a Spectral Method," Water Resources Research (Oct. 1982) 1379-94.

24. Yang, A.: "Program TBM - Turning Bands Method to Generate 2-D Random Fields with Autocorrelation," FORTRAN Source Code, University of Texas, Austin, TX (1987).

25. ECLIPSE 100 - Black Oil Simulator, ECL-Bergeson Petroleum Technologies, Inc., Oxfordshire, England (1990).

26. Peaceman, D.W.: "Interpretation of Well-Block Pressures in Numerical Reservoir Simulation," SPE of AIME (1978).

27. Feitosa, G.:" FORTRAN Program to Calculate the Kernel Function," U. of Tulsa, July 1991.

28. Mishra, S., Brigham, W.E., and Orr, F.M. Jr.: “Tracer and Pressure Test Analysis for Characterization of Areally Heterogeneous Reservoirs," paper SPE/DOE 17365 presented at the 1988 SPE/DOE Enhanced Oil Recovery Symposium, tulsa, OK, April 17-20. 
Table I - Variability of the Permebility Fields and the Differences in the Averaging Methods and the Well Test Permeability.

\begin{tabular}{|c|c|c|c|c|c|}
\hline$\sigma^{2} \ln k$ & $\begin{array}{c}\text { range } \\
(\mathrm{ft})\end{array}$ & HI & $\begin{array}{c}\text { Avg Diff } \\
\mathbf{k}_{\mathrm{h}}\end{array}$ & $\begin{array}{c}\text { Avg Diff } \\
\mathrm{kg}_{\mathrm{g}}\end{array}$ & $\begin{array}{c}\text { Avg Diff } \\
k_{a} \\
\end{array}$ \\
\hline $\begin{array}{l}0.50 \\
0.75 \\
0.50 \\
0.50 \\
0.50 \\
0.50 \\
0.75 \\
0.25 \\
0.75 \\
0.75 \\
0.50 \\
0.50 \\
0.75 \\
0.75 \\
0.50 \\
0.25 \\
0.50 \\
0.75 \\
0.25 \\
0.60 \\
0.40 \\
0.92 \\
1.61 \\
0.92 \\
1.61 \\
0.92 \\
1.61 \\
0.92 \\
1.61 \\
1.20 \\
1.20 \\
1.20\end{array}$ & $\begin{array}{c}500 \\
500 \\
1000 \\
1000 \\
500 \\
0 \\
0 \\
500 \\
2000 \\
1000 \\
1000 \\
3200 \\
1600 \\
3200 \\
2000 \\
3200 \\
1250 \\
1250 \\
1250 \\
1600 \\
2000 \\
1072 \\
348 \\
959 \\
311 \\
2144 \\
695 \\
595 \\
193 \\
221 \\
555 \\
1242 \\
\end{array}$ & $\begin{array}{l}0.0311 \\
0.0699 \\
0.0621 \\
0.0621 \\
0.0311 \\
0.0000 \\
0.0000 \\
0.0078 \\
0.2795 \\
0.1398 \\
0.0621 \\
0.1988 \\
0.2236 \\
0.4472 \\
0.1242 \\
0.0497 \\
0.0776 \\
0.1747 \\
0.0194 \\
0.1431 \\
0.0795 \\
0.2254 \\
0.2238 \\
0.2016 \\
0.2002 \\
0.4508 \\
0.4475 \\
0.1252 \\
0.1243 \\
0.0790 \\
0.1987 \\
0.4442 \\
\end{array}$ & $\begin{array}{l}-0.606 \\
-1.217 \\
-0.139 \\
-0.246 \\
-0.533 \\
-1.329 \\
-3.754 \\
0.015 \\
-5.977 \\
-3.361 \\
-0.392 \\
-2.523 \\
-1.373 \\
-0.108 \\
-0.902 \\
0.358 \\
-1.199 \\
-4.751 \\
-0.402 \\
-0.728 \\
-0.864 \\
-6.038 \\
-1.740 \\
-0.257 \\
-3.703 \\
2.211 \\
-3.047 \\
-3.891 \\
-4.315 \\
-3.433 \\
-3.613 \\
-14.519 \\
\end{array}$ & $\begin{array}{c}0.671 \\
1.572 \\
0.627 \\
0.586 \\
1.033 \\
0.601 \\
0.029 \\
0.465 \\
-2.204 \\
-0.685 \\
0.323 \\
-0.745 \\
0.047 \\
0.642 \\
0.670 \\
0.533 \\
0.532 \\
-1.784 \\
-0.153 \\
0.078 \\
-0.170 \\
-1.953 \\
1.310 \\
1.116 \\
0.211 \\
2.752 \\
0.798 \\
-0.165 \\
0.381 \\
1.154 \\
1.789 \\
-5.024 \\
\end{array}$ & $\begin{array}{c}2.076 \\
5.179 \\
1.398 \\
1.433 \\
2.792 \\
2.859 \\
5.042 . \\
0.931 \\
2.135 \\
2.201 \\
1.094 \\
1.479 \\
1.578 \\
1.564 \\
2.456 \\
0.732 \\
2.431 \\
1.879 \\
0.102 \\
0.904 \\
0.654 \\
4.152 \\
25.544 \\
2.854 \\
10.532 \\
3.319 \\
11.889 \\
5.431 \\
18.662 \\
13.193 \\
14.503 \\
8.622 \\
\end{array}$ \\
\hline & & Cotal AVG= & -2.262 & 0.157 & 4.988 \\
\hline
\end{tabular}




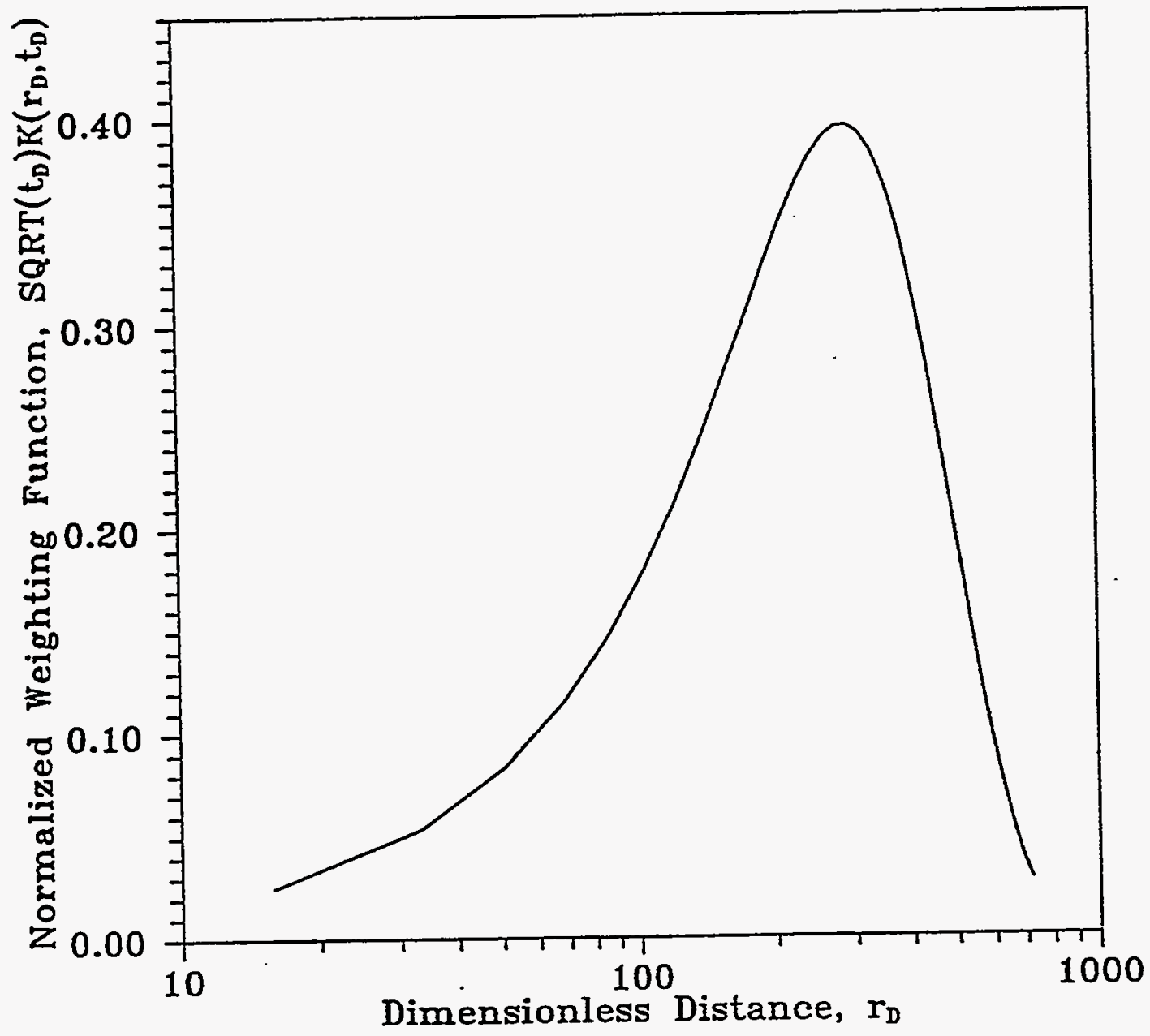

Figure 1 - Weighting function for the calculation of the pressure derivative in a reservoir with variable permeability ${ }^{13,27}\left(t_{D}=10^{5}\right)$. 


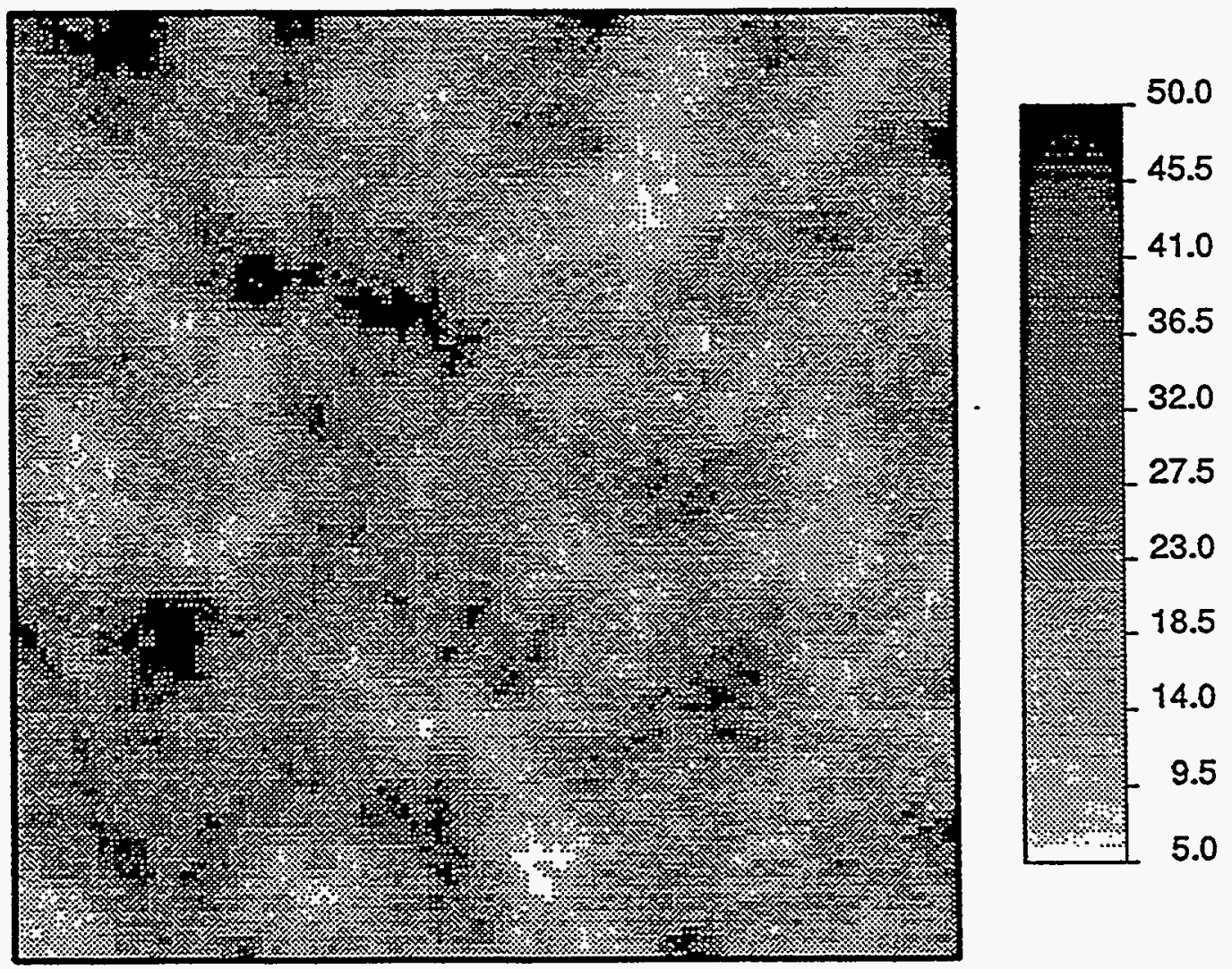

Figure 2 - Permeability field generated by $\mathrm{TBM}^{23,24}$ (seed $=-6$, scale $=500 \mathrm{ft}$, mean $=20$, $\left.\sigma_{l n k}^{2}=0: 5, \Delta X=\Delta Y=35 \mathrm{ft}, N X=N Y=115\right)$ 


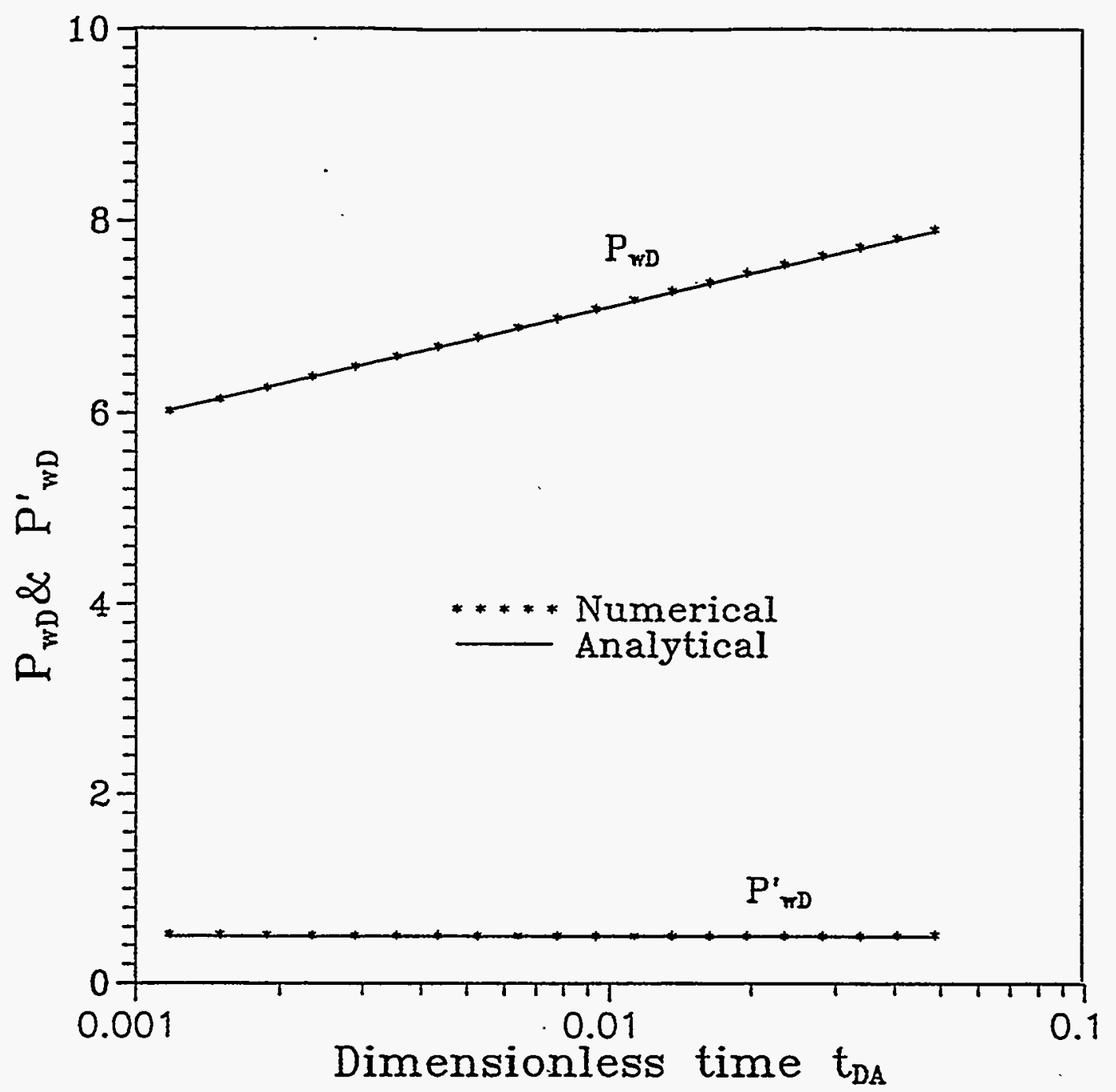

Figure 3 - Comparison between analytical ${ }^{7}$ and numerical ${ }^{25}$ drawdown solutions shows pressure differential and pressure derivative for a homogeneous reservoir.

Note:

$$
t_{D A}=0.0002637 k t /\left(\phi \mu c_{t} A r e a\right)
$$

for $t_{D A}<0.1$

$$
p_{w D}=2 \pi t_{D A}+0.5\left[\ln \left(A / r_{w}^{2}\right)+\ln \left(2.2458 / C_{A}\right)\right] .
$$



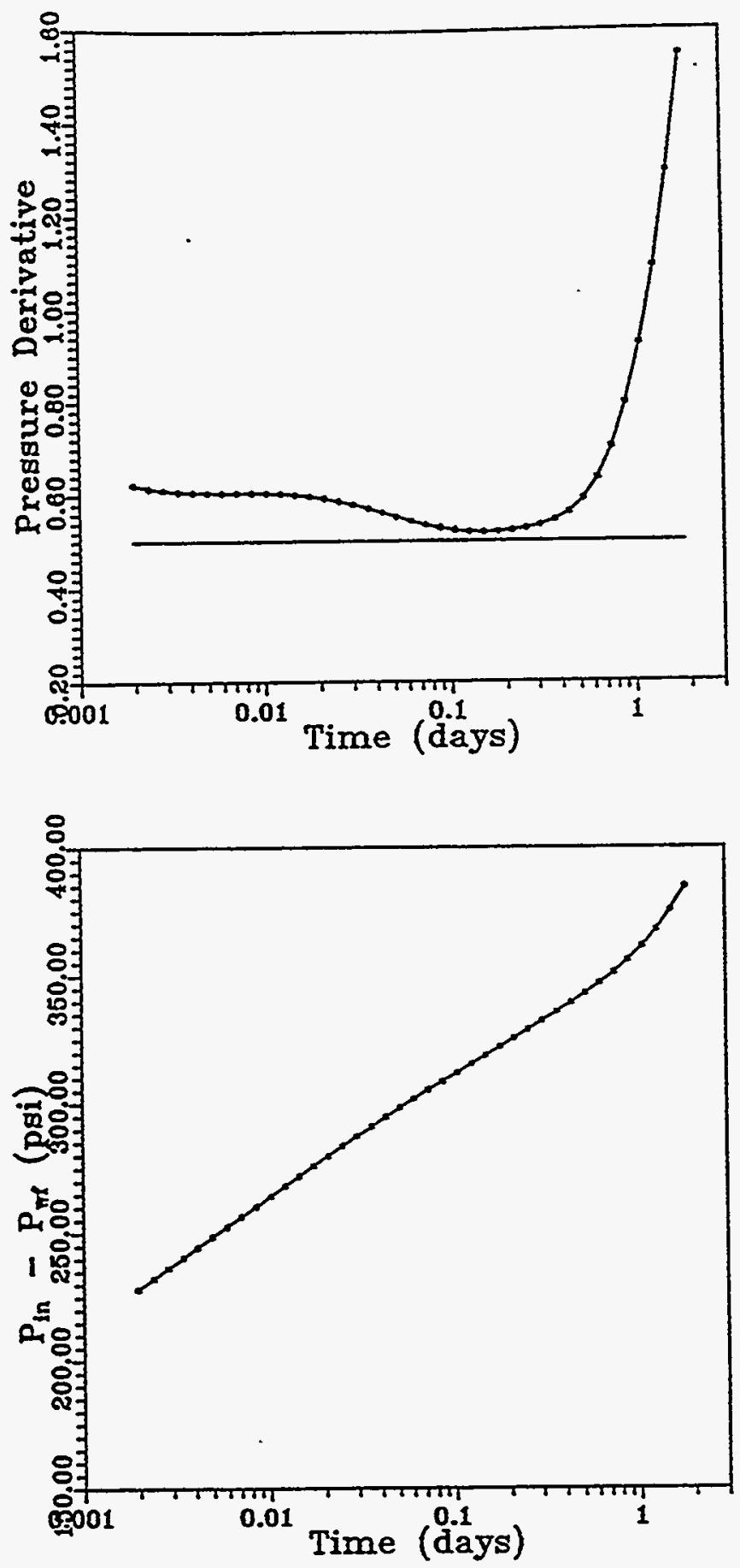

Figure 4 - Pressure differential and pressure derivative for a drawdown simulation of the permeability field shown in Figure 2. 


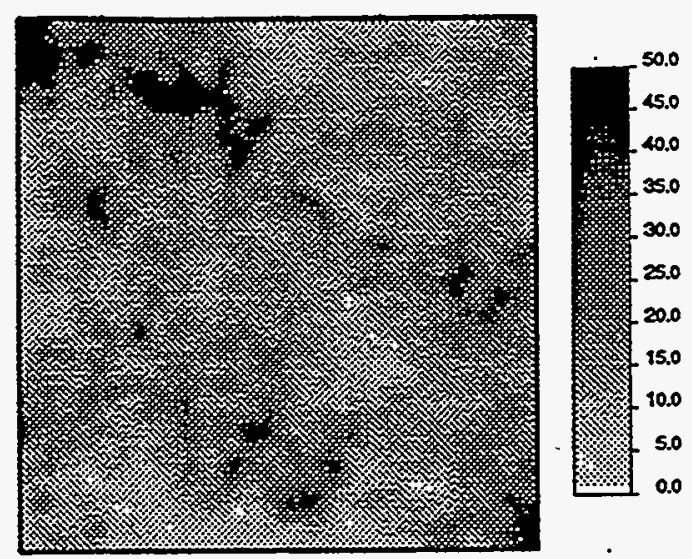

Figure 5 - Permeability Distribution $\left(\mathrm{HI}=0.06, \mathrm{a}=1000 \mathrm{ft}, \sigma_{l n k}^{2}=.5\right)$

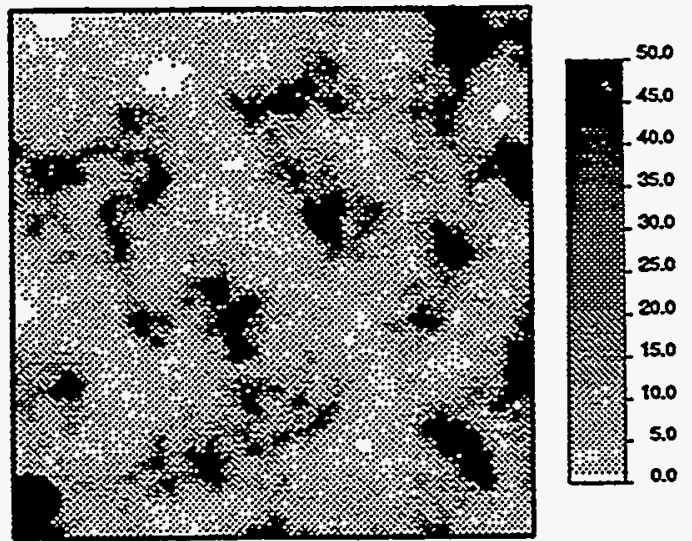

Figure 6 - Permeability Distribution $\left(\mathrm{HI}=0.45, \mathrm{a}=695 \mathrm{ft}, \sigma_{\operatorname{lnk}}^{2}=1.6\right)$ 

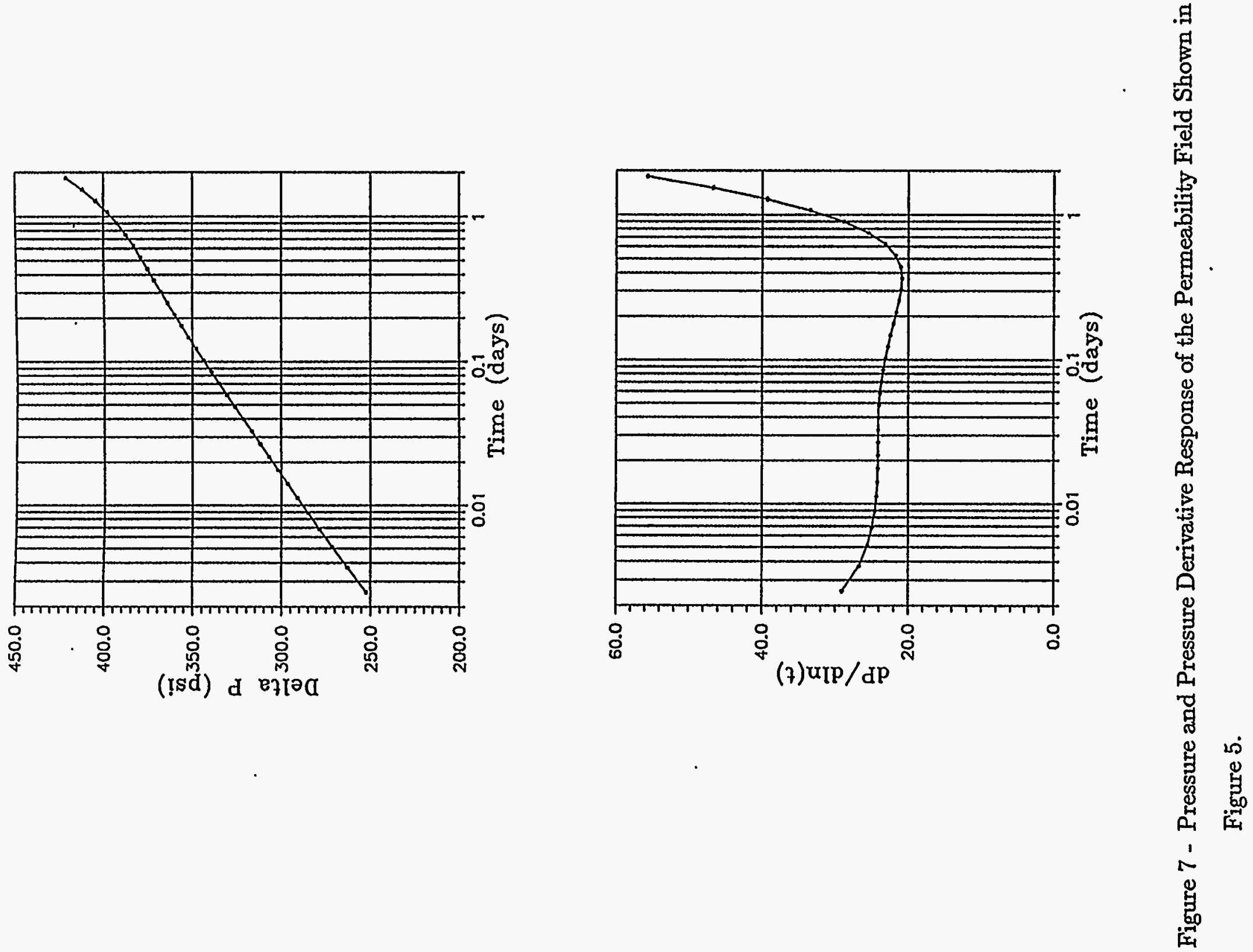

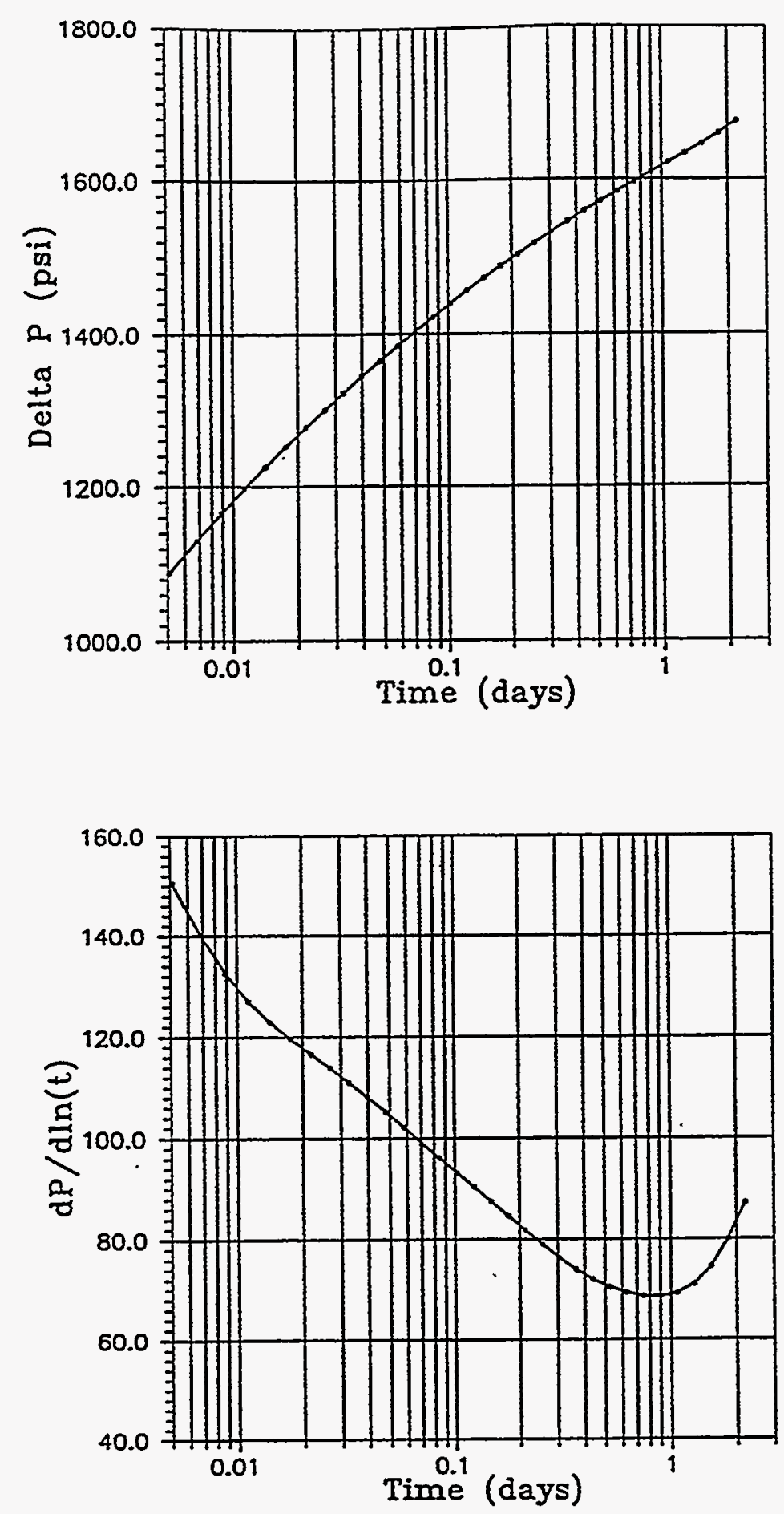

Figure 8 - Pressure and Pressure Derivative Response of the Permeability Field Shown in Figure 6. 


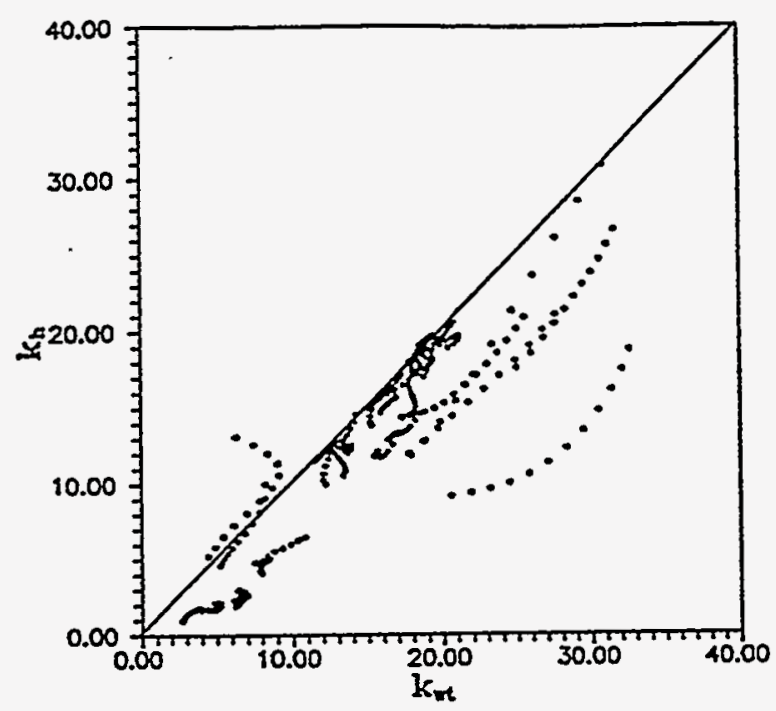

Figure 9 - Comparison between the Weighted Harmonic Average Permeability and the Instantaneous Well Test Permeability.

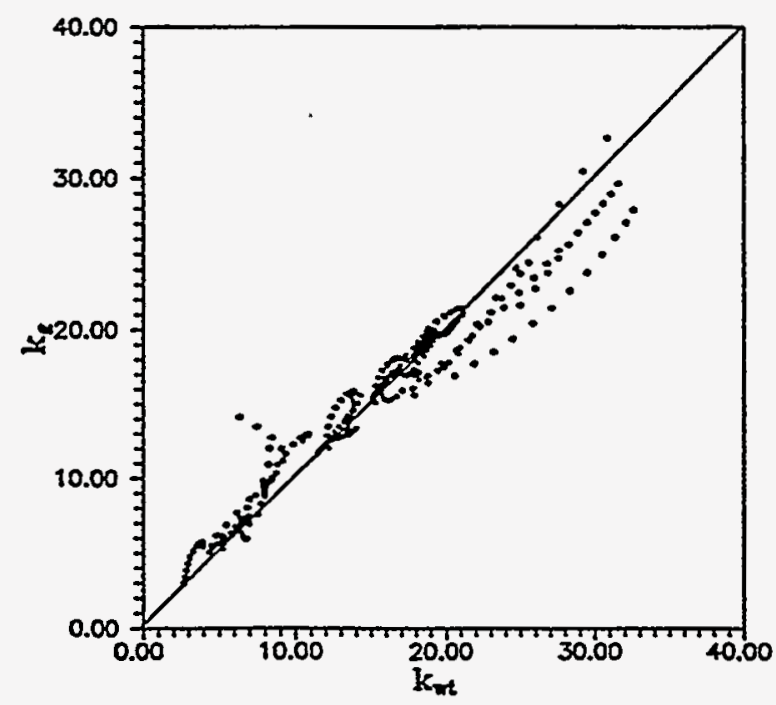

Figure 10 - Comparison between the Weighted Geometric Average Permeability and the Instantaneous Well Test Permeability. 


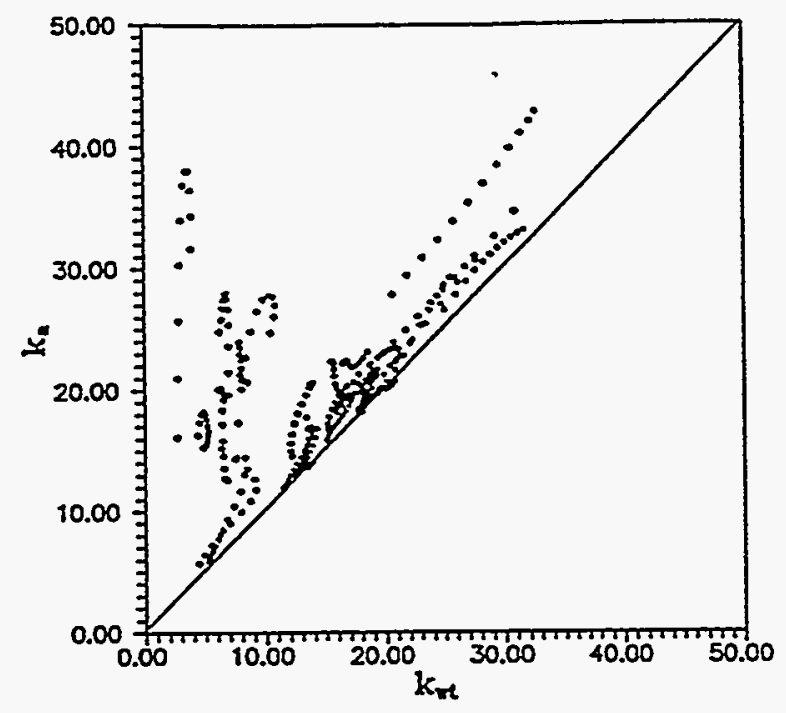

Figure 11 - Comparison between the Weighted Arithmetic Average Permeability and the Instantaneous Well Test Permeability. 


\title{
Section I
}

\section{Report III}

\section{Effective Properties of Reservoir Simulator Grid Blocks}

\author{
Y. Aasum, E. Kasap and M. Kelkar
}

\section{Summary}

Computational costs limit large scale reservoir simulations to coarse grid systems. Determination of an effective permeability for a simulation grid block requires a proper scale-up of small scale permeability heterogeneities within that grid block. Conventional scale-up techniques are limited to a diagonal tensor representation of effective permeability. Therefore, such techniques cannot handle general permeability anisotropy (full tensor) exemplified by cross-bedded permeability structures that may be present on a smaller scale.

An analytical method is developed to calculate an effective permeability tensor for a grid block by accounting for small scale heterogeneities within the grid block. The method honors both the location and the orientation of the small scale heterogeneities.

Most commercially available reservoir simulators are not equipped to handle general permeability anisotropy. This work shows in detail how transmissibility coefficients for cases of general permeability anisotropy are developed. These transmissibilities can be readily inserted into point-centered finite difference simulators which accommodate 9-point differencing.

Effective permeability tensors calculated using the analytical method and a numerical method show excellent agreement. Fluid flow simulations, both miscible displacements and waterfloods, show that the effective permeability tensor method outperforms conventional scale-up techniques when compared to the use of fine scale permeability distribution in cases of general permeability anisotropy.

This report is divided into two parts. The first part describes an analytical method for calculating the effective permeability as a full tensor. The second part describes how to incorporate full tensor permeability in a numerical simulator.

\section{PART 1 \\ ANALYTICAL UPSCALING OF SMALI SCALE PERMEABILITY USING A FULL TENSOR}

\section{Introduction}

Field development and reservoir management practices require large scale numerical simulations. Although more powerful computers and simulation techniques are continuously being developed, the size of the grid blocks used in field scale fluid 
flow simulations is too large to explicitly account for the effect of small scale heterogeneities. Such heterogeneities may be comprised of interwell laminations and cross-bedding structures as well as sand/shale sequences. For the large scale simulator grid blocks, the effect of small scale heterogeneities can only be accounted for by calculating an effective permeability. An effective permeability preserves the ratio of the fluid flux and the potential drop across a heterogeneous block and an equivalent homogeneous block. ${ }^{1}$

Several methods are presented in the literature for calculating effective permeability. These methods can, in general, be divided into numerical ${ }^{2-8}$ and analytical methods. ${ }^{1,9-21}$ Numerical (simulation) methods can handle complex heterogeneous systems, whereas analytical methods usually are restricted by simplifying assumptions. Analytical methods, however, tend to be less expensive than numerical methods in terms of computational cost.

In this study, an analytical effective permeability method is developed. This method combines the advantages of numerical and analytical methods. The proposed method is general in that it allows for full tensor representation of effective permeability. Both the location and orientation of permeability heterogeneities are considered. The method, in essence, captures the effect of the pressure distribution within the grid block. A directional search procedure in local areas (four quadrants of the grid block) identifies the principal axes of permeability and their orientation with respect to the simulation coordinate axes. Coordinate rotation yields effective permeability tensors. These tensors are combined into an effective permeability tensor for the entire grid block based on the coupling of cross-flow-averaged and no-cross-flow-averaged effective permeability tensors from the four quadrants. Darcy's law is manipulated throughout this process. Cross-flow-average refers to harmonic or series average of arithmetically averaged block permeabilities in parallel, whereas no-cross-flow-average refers to arithmetic average of harmonically averaged block permeabilities in series. In the homogenization process, transverse pressure gradients are calculated. These transverse pressure gradients result from local coordinate rotations and non-centered heterogeneities within the grid block. Non-zero transverse pressure gradients contribute to non-zero off-diagonal elements of the effective permeability tensor.

The material in this first part is presented in the following manner. First, a detailed description of the development of an analytical effective permeability tensor method is presented in a step-by-step procedure. A numerical method used to verify the analytical method is also presented. Second, comparisons between effective permeability tensors calculated using analytical and numerical methods are presented. Also illustrated in that section are flood front locations from miscible displacements using the initial permeability distribution and averaged permeability distributions using the proposed effective permeability tensor method and standard upscaling techniques. 


\section{Development of Effective Permeability Tensor}

Analytical Method

There are generally two situations in which a full permeability tensor is required to correctly account for the effect of the heterogeneities within a simulator grid block:

1. Heterogeneous or homogeneous permeability structures (layers) are anisotropic and oriented at angles other than those parallel and perpendicular to the simulation coordinate axes (general anisotropy). An example is shown in Figure 1.

2. Heterogeneities are not centered in the grid block. An example of this is shown in Figure 2.

The analytical method proposed here combines the advantages of numerical and analytical methods. Complex permeability heterogeneities are handled at a relatively high computational speed.

The location and size of small scale heterogeneities within a simulator grid block and the orientation of anisotropic permeability structures are accounted for using this method. The method estimates effective coarse scale (macroscale) permeability yielding a tensorial form of effective permeability.

Figures 1 and 2 are examples of cases where permeability heterogeneities generate a pressure drop transverse to the direction of applied pressure drop. In both of the cases shown, the applied pressure drop is along the horizontal axis.

The following procedure describes how cross-beddings and laminations (case 1) and location of permeability heterogeneities of high contrast, such as sand and shale (case 2), are considered in the development of an analytical method for calculating an effective permeability tensor.

The first step in this procedure is to divide the grid block containing small scale permeability heterogeneities into four quadrants or local blocks as shown in Figure 3. Dividing the grid block into four quadrants is done to capture the effect of the location of heterogeneities within the grid block (case 2). The next step will be to determine the effective permeability tensor in each of the four quadrants. The final step is to obtain the effective permeability tensor for the entire grid block by combining the effective permeability tensors from each of the four quadrants.

Steps 1 and 2 below are performed on each of the four quadrants for cases where permeability structures are anisotropic and not aligned with the simulation coordinate axes:

\section{Determine the permeabilities along the principal directions.}

The principal directions of permeability are the directions of maximum and minimum permeabilities, $k_{x x}^{\prime}$ and $k_{y y}^{\prime}$, respectively. In this study, it is assumed that, within each quadrant, the principal directions $\left(x^{\prime}\right.$ and $\left.y^{\prime}\right)$ are perpendicular to each other and that $k_{x y}^{\prime}$ and $k_{y x}^{\prime}$ are zero. Therefore, if anisotropy exists $\left(k_{x x}^{\prime} \neq k_{y y}^{\prime}\right)$, then the principal directions of permeability must be determined. 

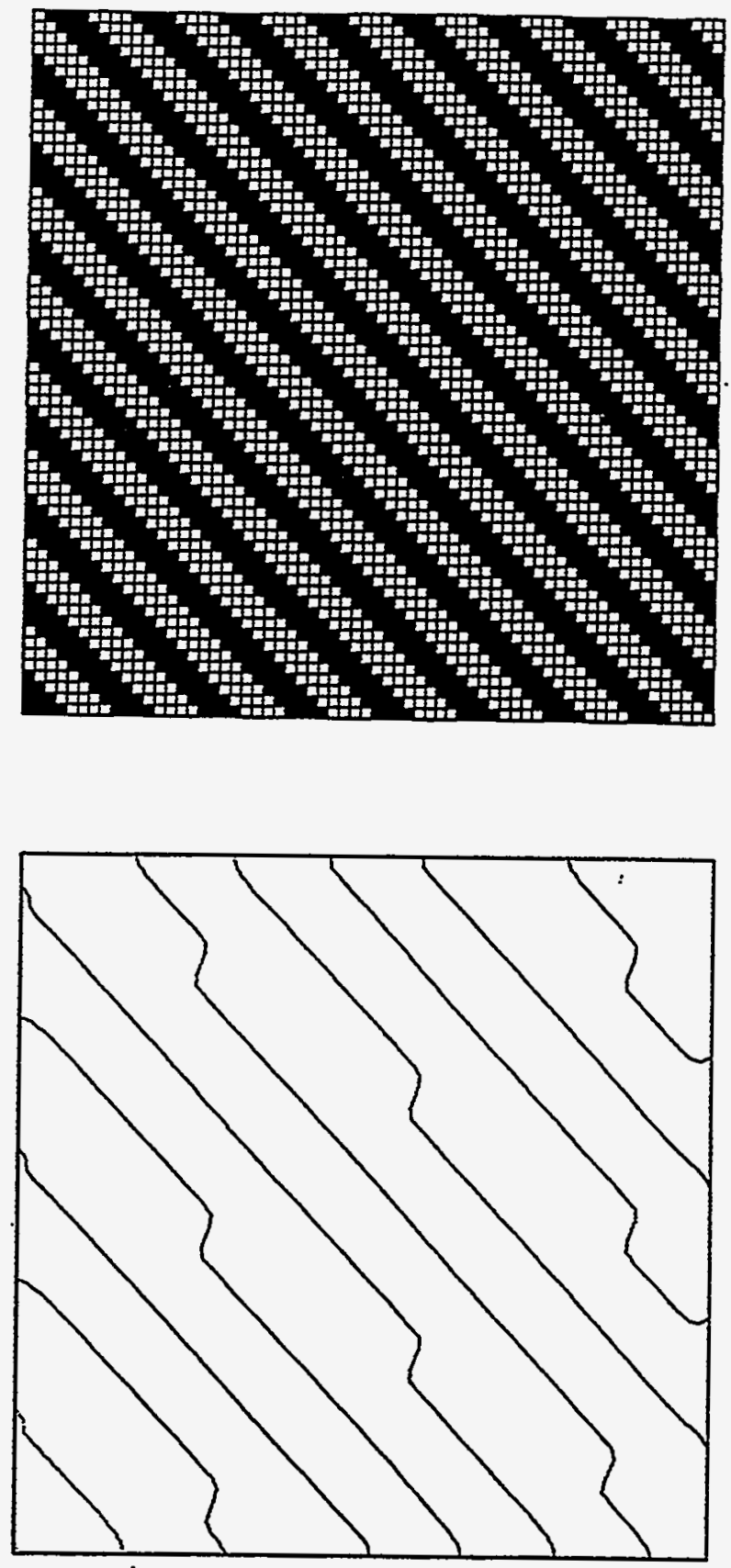

Fig. 1: Top: Cross-Bedded Permeability Distribution ( $-45^{\circ}$ Structure Orientation, Dark Grid Blocks: $20 \mathrm{md}$, Light Grid Blocks: $1000 \mathrm{md}$ ). Bottom: Pressure Map Indicating Non-Zero Transverse Pressure Gradient. Boundary Conditions: Closed Horizontal Boundaries; Ieft Face: Injection; Right Face: Production. 

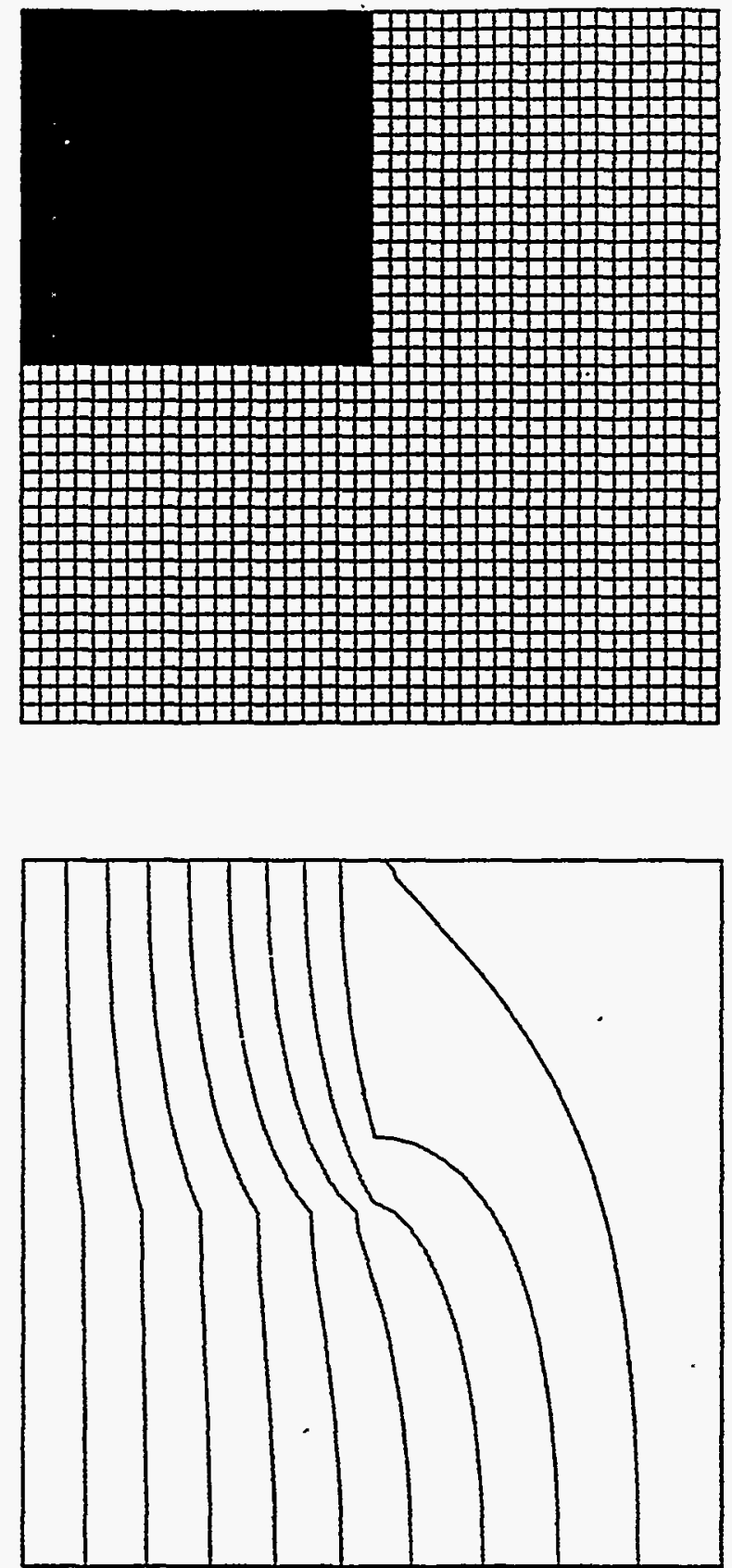

Fig. 2: Top: Permeability Field with Low Permeability Section (Dark Grid Blocks: $1 \mathrm{md}$, Light Grid Blocks: $1000 \mathrm{md}$ ).

Bottom: Pressure Map Indicating Non-Zero Transverse Pressure Gradient. Boundary Conditions: Closed Horizontal Boundaries; Left Face: Injection; Right Face: Production. 

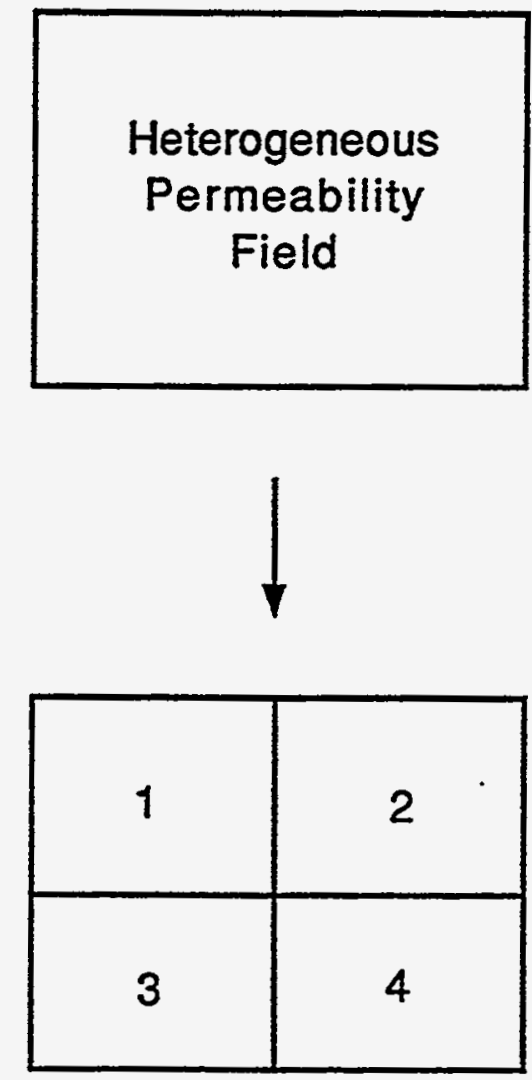

Fig. 3: Field of Small Scale Heterogeneities Divided into Four Quadrants. 
The method proposed to determine $k_{x x}^{\prime}$ and $k_{y y}^{\prime}$ is explained as follows. The objective is to find the direction which yields the maximum ratio of $k_{x x}^{\prime} / k_{y y}^{\prime}$. A "sweep" is performed in directions between $-90^{\circ}$ and $90^{\circ}$ relative to the horizontal $\mathrm{x}$-axis to determine in which direction $k_{x x}^{\prime} / k_{y y}^{\prime}$ is largest. As many as forty-nine directions are used to ensure accuracy to within a few degrees. Both $k_{x x}^{\prime}$ and $k_{y y}^{\prime}$ are calculated for each direction. $k_{y y}^{\prime}$ is the permeability in the direction perpendicular to that of $k_{x x}^{\prime}$. In addition to $k_{x x}^{\prime}$ and $k_{y y}^{\prime}$, the orientation angle, $\alpha$, in the direction of maximum $k_{x x}^{\prime} / k_{y y}^{\prime}$ is determined and used in Step 2. The orientation angle is defined with respect to the $\mathrm{x}$-axis and is positive in the anticlockwise direction.

Figure 4 shows the data points included in the calculation of $k_{x x}^{\prime}$ and $k_{y y}^{\prime}$ for the directions $45^{\circ}$ and $-45^{\circ}$ from the horizontal, respectively. $k_{x x}^{\prime}$ and $k_{y y}^{\prime}$ are determined by an analytical homogenization procedure of combining arithmetically averaged values in series and harmonically averaged values in parallel. For further details regarding these calculations, refer to Aasum. ${ }^{22}$

\section{Perform coordinate rotation to obtain the effective permeability tensor.}

$k_{x x}^{\prime}, k_{y y}^{\prime}$, and the orientation angle, $\alpha$, were determined in Step 1 above. These parameters are used in a coordinate rotation procedure which yields the following effective permeability tensor for the simulation coordinate system:

$$
\begin{gathered}
k_{x x}=(\cos \alpha)^{2} k_{x x}^{\prime}+(\sin \alpha)^{2} k_{y y}^{\prime} \\
k_{x y}=\sin \alpha \cos \alpha\left(k_{x x}^{\prime}-k_{y y}^{\prime}\right) \\
k_{y x}=\sin \alpha \cos \alpha\left(k_{x x}^{\prime}-k_{y y}^{\prime}\right) \\
k_{y y}=(\sin \alpha)^{2} k_{x x}^{\prime}+(\cos \alpha)^{2} k_{y y}^{\prime}
\end{gathered}
$$

where $k_{x x}, k_{x y}, k_{y x}$, and $k_{y y}$ are the effective permeability tensor elements in the $\mathrm{x}, \mathrm{y}$-coordinate system. $\alpha$ is the angle by which the $\mathrm{x}^{\prime}, \mathrm{y}^{\prime}$-coordinate system deviates from the $x, y$-coordinate system. The derivations of Eqs. (1)-(4) are shown in detail by Aasum. ${ }^{22}$

Rationale for dividing the permeability field into four blocks.

At this point an effective permeability tensor has been determined for each of the four quadrants or blocks in the permeability field as illustrated in Figure 5. The final step is to calculate the effective permeability tensor for the entire permeability field. Steps 1 and 2 could conceivably be performed on the complete permeability field.

However, the main reason for dividing the permeability field into four blocks is that transverse pressure gradients, yielding non-zero off-diagonal elements in the permeability tensor, are induced in cases such as the one illustrated in Figure 2. In this case, a perturbation (low permeability region, such as a shale) is located in the 


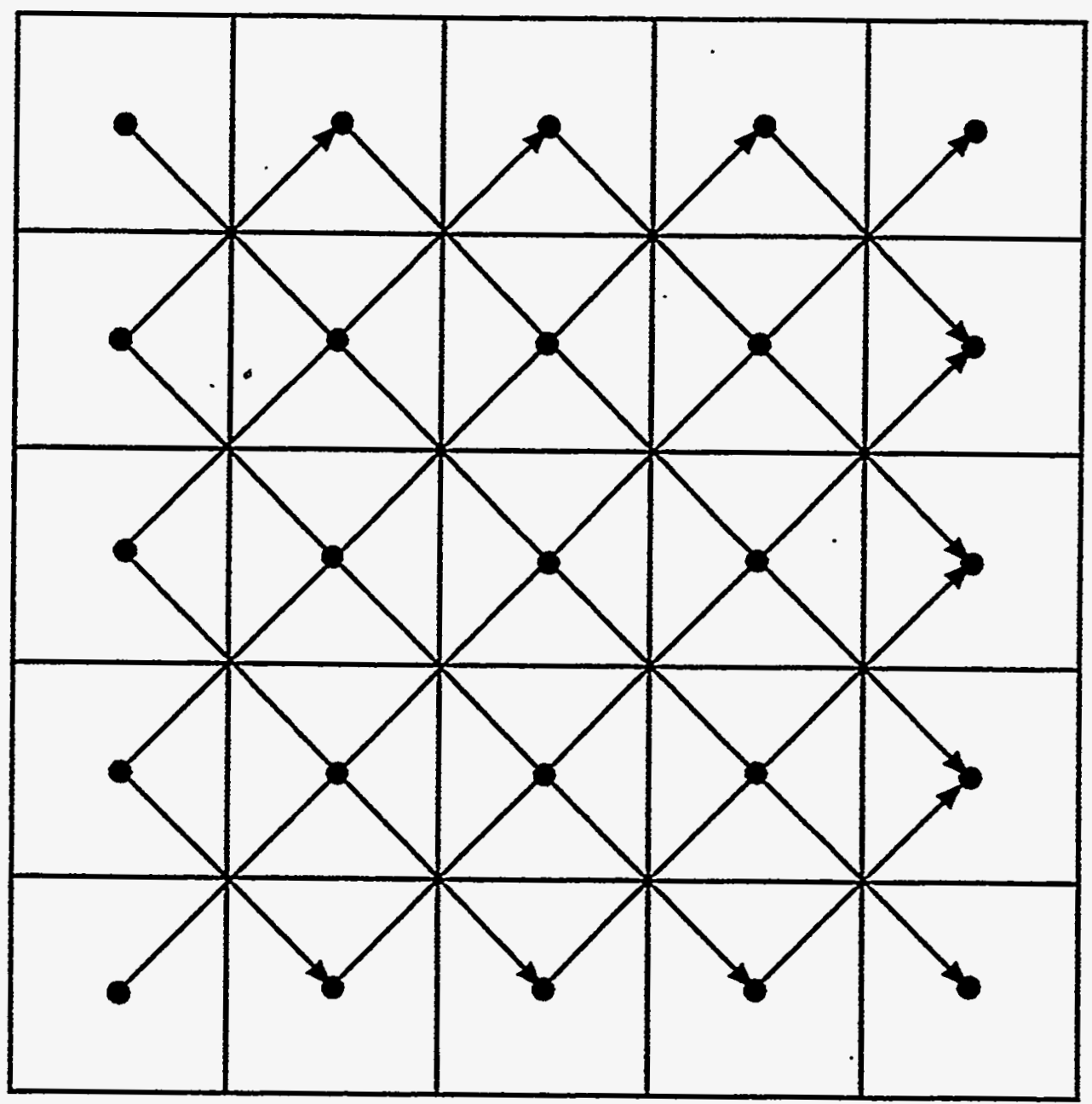

Fig. 4: Directions and Data Points to Consider in Calculating Maximum and Minimum Permeabilities Along $45^{\circ}$ and $-45^{\circ}$ From the Horizontal Axis. 


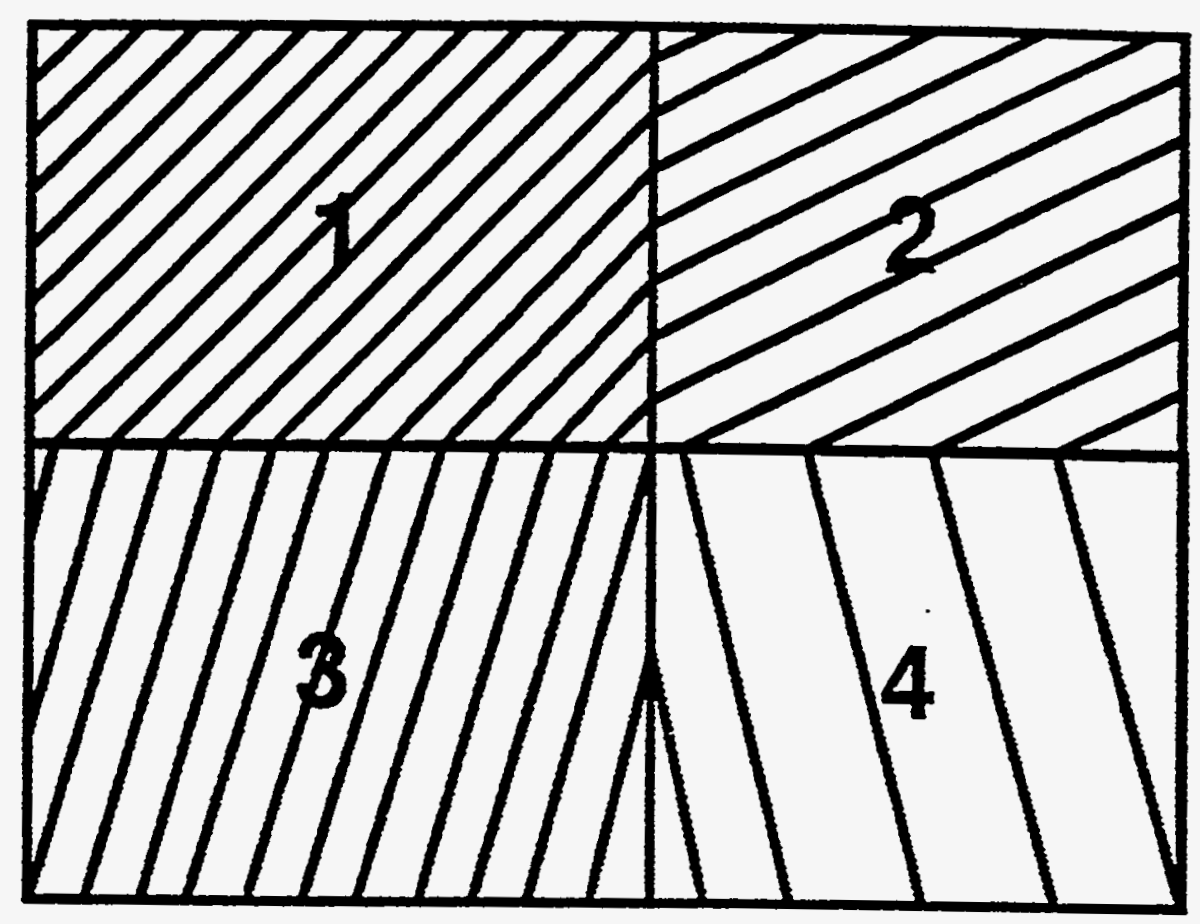

Fig. 5: System Containing Four Blocks and Four Permeability Tensors. 
upper left corner of the system. The location of this perturbation will control the sign of the off-diagonal elements in the effective permeability tensor. For example, the case illustrated in Figure 2 yields negative off-diagonal elements $\left(k_{x y}\right.$ and $\left.k_{y x}\right)$. If the perturbation were located in the bottom left corner of the permeability field, the off-diagonal elements would be positive. Therefore, the reason for dividing the system into four quadrants is to capture these "global" effects caused by such perturbations and their locations in the permeability field.

\section{Determine effective permeability tensor for the entire grid block.}

The physical system consists of four blocks or quadrants as shown in Figure 5. Each of the four blocks may have different, but possibly full $\left(k_{x y} \neq 0\right.$ and $k_{y x} \neq$ 0 ) permeability tensors obtained from the previous steps. Permeability anisotropy $\left(k_{x x} \neq k_{y y}\right)$ and varying block sizes are also permitted and accounted for in the determination of the elements of the effective permeability tensor.

The elements of the effective permeability tensor are obtained from the follow: ing expressions:

$$
\begin{gathered}
\tilde{k}_{x x}=\frac{\tilde{k}_{x_{a p p}}}{1-\left(\left[\left(\frac{\partial \tilde{p}}{\partial x}\right)_{Y} /\left(\frac{\partial \tilde{p}}{\partial y}\right)_{Y}\right] \times\left[\left(\frac{\partial \tilde{p}}{\partial y}\right)_{X} /\left(\frac{\partial \tilde{p}}{\partial x}\right)_{X}\right]\right)} \\
\tilde{k}_{y y}=\frac{\tilde{k}_{y_{a p p}}}{1-\left(\left[\left(\frac{\partial \tilde{p}}{\partial x}\right)_{Y} /\left(\frac{\partial \tilde{p}}{\partial y}\right)_{Y}\right] \times\left[\left(\frac{\partial \tilde{p}}{\partial y}\right)_{X} /\left(\frac{\partial \tilde{p}}{\partial x}\right)_{X}\right]\right)} \\
\tilde{k}_{x y}=-\tilde{k}_{x x}\left(\frac{\partial \tilde{p}}{\partial x}\right)_{Y} /\left(\frac{\partial \tilde{p}}{\partial y}\right)_{Y} \\
\tilde{k}_{y x}=-\tilde{k}_{y y}\left(\frac{\partial \tilde{p}}{\partial y}\right)_{X} /\left(\frac{\partial \tilde{p}}{\partial x}\right)_{X} .
\end{gathered}
$$

In Eqs. (5)-(8), $\tilde{k}_{x_{a p p}}$ and $\tilde{k}_{y_{a p p}}$ are the effective apparent permeabilities along the $\mathrm{x}$ - and $\mathrm{y}$-axis, respectively. Apparent permeabilities are used as effective permeabilities if the numerical simulator is not equipped to handle a tensor formulation of permeability. Clearly, $\tilde{k}_{x_{a p p}}$ reduces to $\tilde{k}_{x x}$ and $\tilde{k}_{y_{a p p}}$ reduces to $\tilde{k}_{y y}$ whenever the pressure gradient ratios in Eqs. (5) and (6) are zero. The pressure gradient ratio is defined as the ratio of transverse to longitudinal pressure gradients. $\left(\frac{\partial \tilde{p}}{\partial x}\right)_{Y} /\left(\frac{\partial \tilde{p}}{\partial y}\right)_{Y}$ is the pressure gradient ratio induced from injection along $\mathrm{y}$-axis whereas $\left(\frac{\partial \tilde{p}}{\partial y}\right)_{X} /\left(\frac{\partial \tilde{p}}{\partial x}\right)_{X}$ is the pressure gradient ratio induced from injection along $\mathrm{x}$-axis. In both cases, boundaries transverse to the principal direction of fluid injection are closed. Notice that the pressure gradient ratios are closely linked to the off-diagonal elements of the permeability tensor as can be seen from Eqs. (7) and (8). Aasum ${ }^{22}$ provides examples of the necessary equations for one- and two-block systems which are extended to a four-block system to obtain Eqs. (5)-(8). 
The apparent permeabilities and the pressure gradient ratios in Eqs. (5)-(8) must be determined to obtain the elements of the effective permeability tensor, $\tilde{k}_{x x}$, $\tilde{k}_{x y}, \tilde{k}_{y x}$, and $\tilde{k}_{y y}$.

$\tilde{k}_{x_{a p p}}$ and $\tilde{k}_{y_{a p p}}$ are determined from the following procedure. First, calculate $\hat{k}_{x_{a p p_{t} b}}$ which is the effective apparent $\mathrm{x}$-direction permeability assuming no crossflow between the top and the bottom layers (see Figure 6):

(i) Isolate the top layer (blocks 1 and 2) in Figure 5. Use Darcy's law with full permeability tensor to determine the apparent permeability in the $\mathrm{x}$-direction $\left(\hat{k}_{x_{a p p_{t}}}\right)$ of blocks 1 and 2 combined. Constant pressure drop is applied in the principal direction of flow ( $\mathrm{x}$-direction) with closed upper and lower boundaries.

(ii) Isolate the bottom laver (blocks 3 and 4) in Figure 5. Use Darcy's law with full permeability tensor to determine the apparent permeability in the $\mathrm{x}$-direction $\left(\hat{k}_{x_{a p p_{b}}}\right)$ of blocks 3 and 4 combined. Constant pressure drop is applied in the principal direction of flow ( $\mathrm{x}$-direction) with closed upper and lower boundaries.

(iii) The effective apparent permeability in the $\mathrm{x}$-direction of the top and bottom layers combined $\left(\hat{k}_{x_{a p p_{t}}}\right)$ is a weighted arithmetic average of $\hat{k}_{x_{a p p_{t}}}$ and $\hat{k}_{x_{a p p_{b}}}$ because these layers are in parallel with respect to the direction of flow.

Next, calculate $\hat{k}_{x_{a p p_{s}}}$ which is the effective apparent $\mathrm{x}$-direction permeability assuming cross-flow between top and bottom layers (see Figure 7):

(iv) Isolate the first column (blocks 1 and 3) in Figure 5. Use Darcy's law with full permeability tensor to determine the apparent permeability in the $\mathrm{x}$-direction $\left(\hat{k}_{x_{\text {app }}}\right)$ of blocks 1 and 3 combined. Constant pressure drop is applied in the principal direction of flow (x-direction) with closed upper and lower boundaries.

(v) Isolate the second column (blocks 2 and 4) in Figure 5. Use Darcy's law with full permeability tensor to determine the apparent permeability in the $\mathrm{x}$-direction $\left(\hat{k}_{x_{a p p_{s}}}\right)$ of blocks 2 and 4 combined. Constant pressure drop is applied in the principal direction of flow ( $x$-direction) with closed upper and lower boundaries.

(vi) The effective apparent permeability in the $x$-direction of the first and second columns combined $\left(\hat{k}_{x_{a p p_{p}}}\right)$ is a weighted harmonic average of $\hat{k}_{x_{\text {app }}}$ and $\hat{k}_{x_{\text {apps }}}$ because these columns are in series with respect to the direction of flow.

Steps (i)-(vi) are repeated to determine the effective apparent permeabilities for cross-flow $\left(\hat{k}_{y_{c p_{t b}}}\right)$ and no cross-flow $\left(\hat{k}_{y_{a p p_{j s}}}\right)$ cases along the y-axis. For these cases, injection of fluid is along the $y$-axis for each of the separate sections: top and bottom layers, and first and second columns. Consequently, the closed boundaries are the left and right vertical boundaries in Figure 5 for each case. Figure 8 corresponds to a cross-flow case for injection along the $y$-axis and the calculation of $\hat{k}_{y_{a p p_{t b}} \text {, whereas }}$ Figure 9 corresponds to a no cross-flow case for injection along the $y$-axis and the calculation of $\hat{k}_{y_{a p p_{s}} \text {. }}$.

Note that "no cross-flow" refers to no communication and "cross-flow" refers to maximum communication between blocks/layers in the direction transverse to the principal flow direction. 


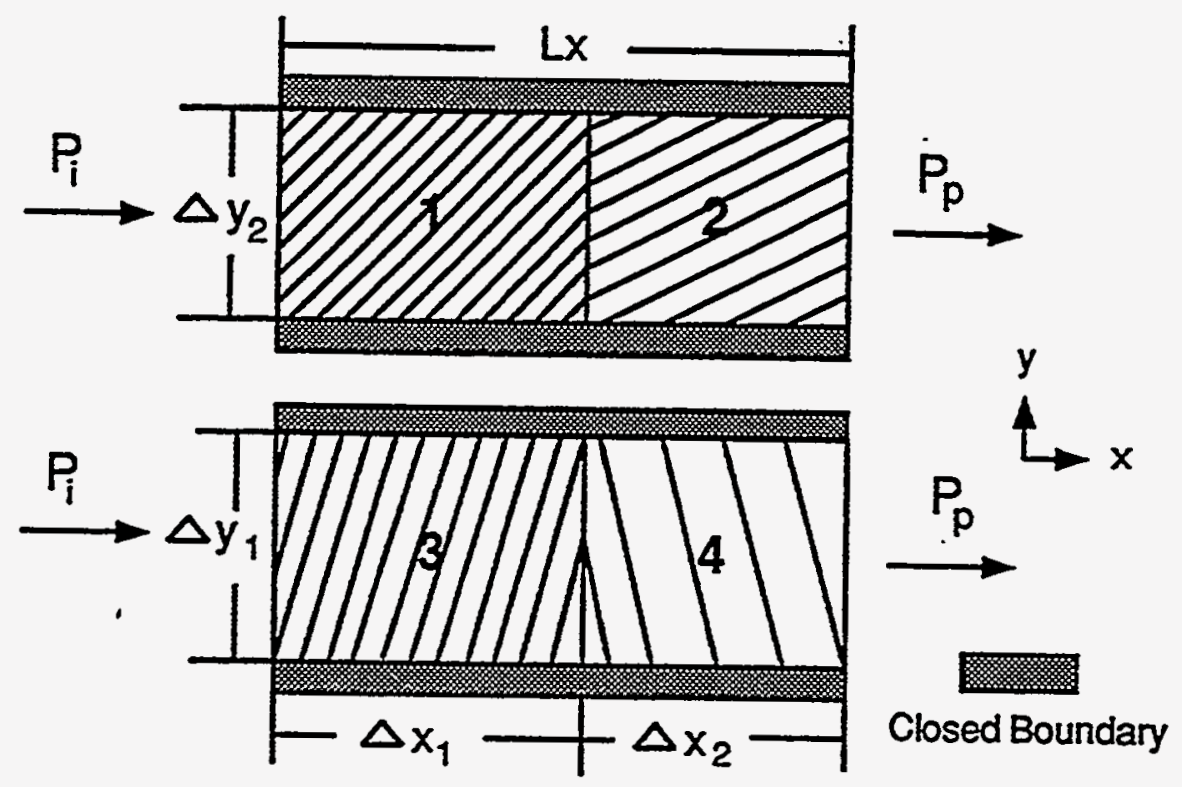

Fig. 6: Schematic for No Cross-Flow Case; $\hat{k}_{x_{a p p_{\mathrm{rb}}}}$ (Injection Along $x$-axis). 


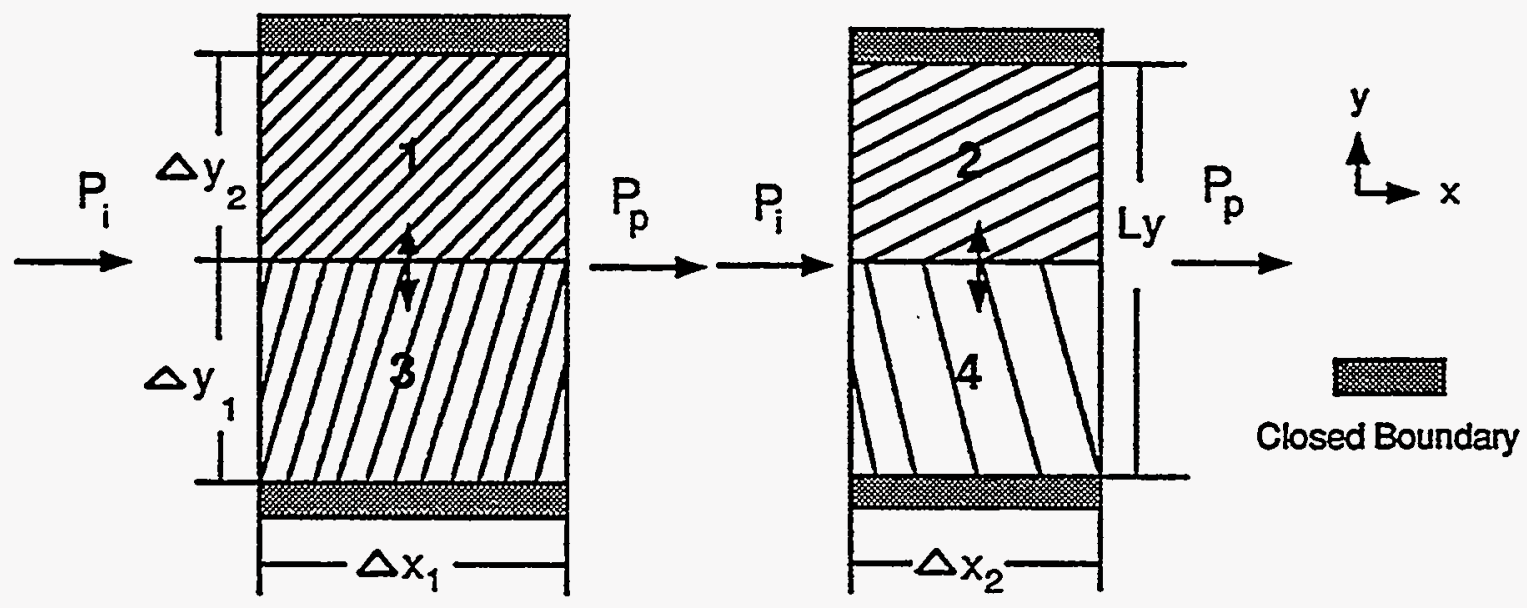

Fig. 7: Schematic for Cross-Flow Case; $\hat{k}_{x_{\text {cppss }}}$ (Injection Along $x$-axis). 


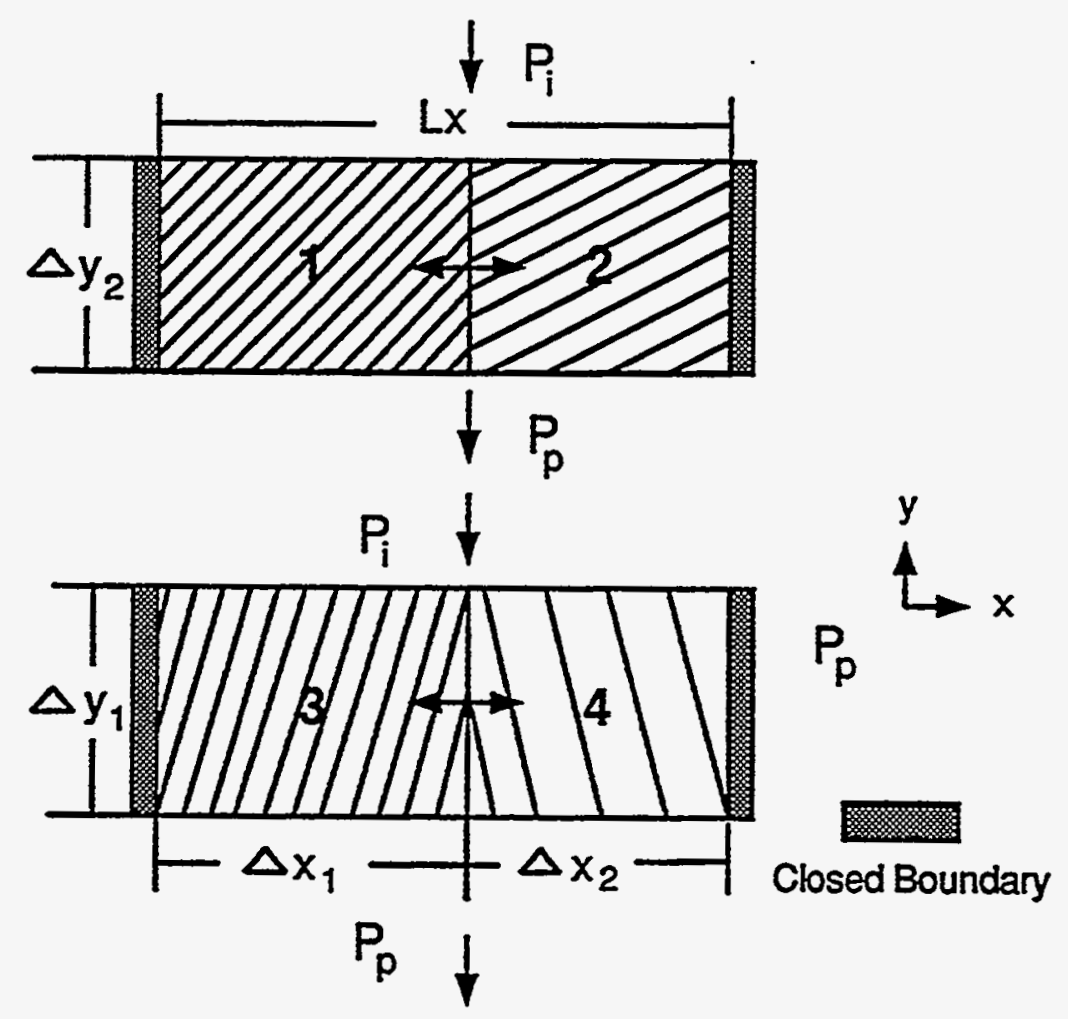

Fig. 8: Schematic for Cross-Flow Case; $\hat{k}_{y_{a p p t b}}$ (Injection Along y-axis). 

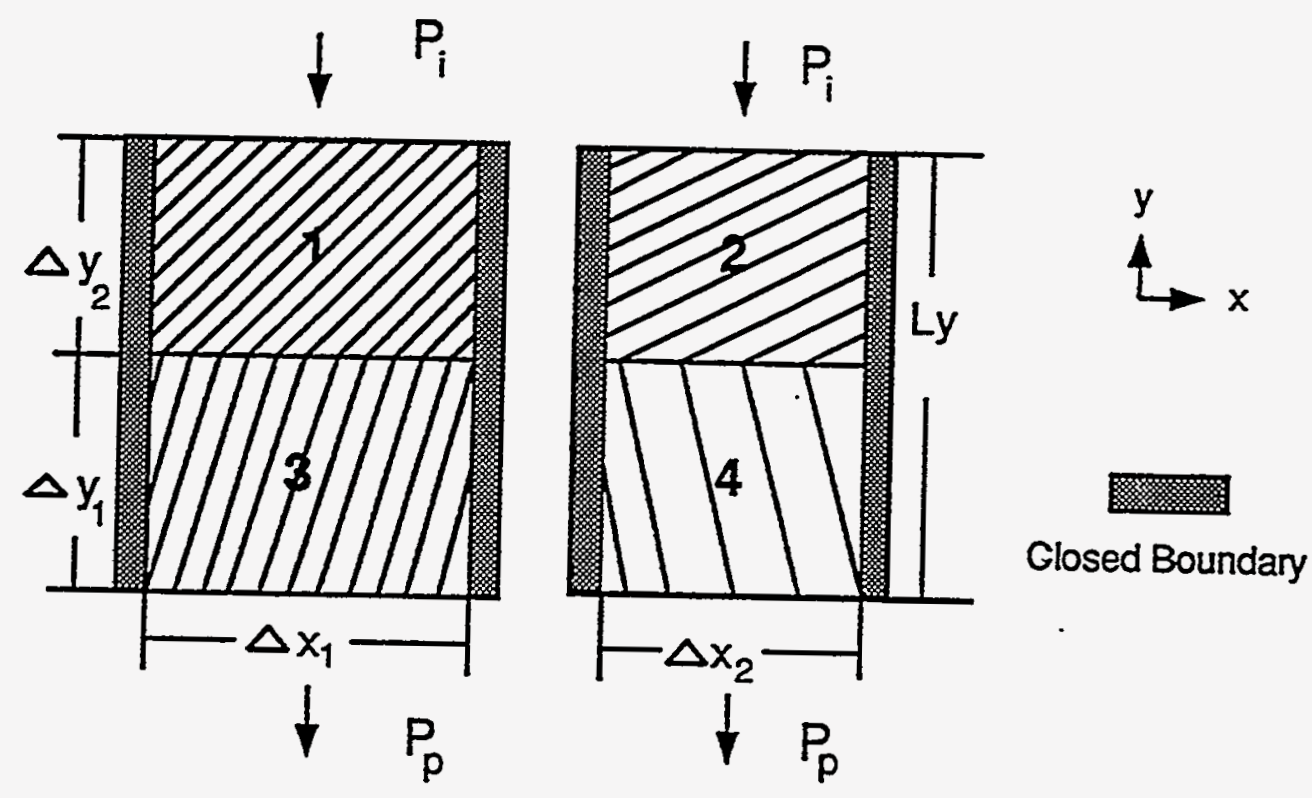

Fig. 9: Schematic for No Cross-Flow Case; $\hat{k}_{y_{c_{p p} p_{e}}}$ (Injection Along y-axis). 
For details regarding calculations of $\hat{k}_{x_{a p p_{t b}}}, \hat{k}_{x_{a p p_{f o}}}, \hat{k}_{y_{a p p_{t b}}}$, and $\hat{k}_{y_{a p p_{\rho}},}$, refer to Aasum. ${ }^{22}$. Their final expressions are as follows:

$$
\hat{k}_{x_{a p p_{t b}}}=\frac{\Delta y_{2}}{L_{y}} \hat{k}_{x_{a p p_{t}}}+\frac{\Delta y_{1}}{L_{y}} \hat{k}_{x_{a p p_{b}}}
$$

where

$$
\begin{aligned}
& \hat{k}_{x_{a p p_{t}}}=\frac{k_{x_{a p p_{1}}} k_{x_{a p p_{2}}} L_{x}}{k_{x_{a p p_{1}} \Delta x_{2}}+k_{x_{a p p_{2}}} \Delta x_{1}} \\
& \hat{k}_{x_{a p p_{b}}}=\frac{k_{x_{a p p_{3}}} k_{x_{a p p_{4}}} L_{x}}{k_{x_{a p p_{3}}} \Delta x_{2}+k_{x_{a p p_{4}}} \Delta x_{1}} .
\end{aligned}
$$

The local apparent permeabilities are defined as follows:

$$
\begin{aligned}
& k_{x_{a p p_{1}}}=k_{x x_{1}}-\frac{k_{x y_{1}} k_{y x_{2}}}{k_{y y_{1}}} \\
& k_{x_{a p p_{2}}}=k_{x x_{2}}-\frac{k_{x y_{2}} k_{y x_{2}}}{k_{y y_{2}}} \\
& k_{x_{a p p_{3}}}=k_{x x_{3}}-\frac{k_{x y_{3}} k_{y x_{3}}}{k_{y y_{3}}} \\
& k_{x_{a p p_{4}}}=k_{x x_{4}}-\frac{k_{x y_{4}} k_{y x_{4}}}{k_{y y_{4}}} \text {. } \\
& \hat{k}_{x_{a p p_{s} s}}=\frac{\hat{k}_{x_{a p p_{g}}} \hat{k}_{x_{a p p_{s}}} L_{x}}{\hat{k}_{x_{a p p_{f}}} \Delta x_{2}+\hat{k}_{x_{a p p_{s}}} \Delta x_{1}}
\end{aligned}
$$

where

$$
\begin{aligned}
& \hat{k}_{x_{a p p_{s}}}=\frac{\Delta y_{2}}{L_{y}} k_{x_{a p p_{1}}}+\frac{\Delta y_{1}}{L_{y}} k_{x_{a p p_{3}}} \\
& \hat{k}_{x_{a p p_{s}}}=\frac{\Delta y_{2}}{L_{y}} k_{x_{a p p_{2}}}+\frac{\Delta y_{1}}{L_{y}} k_{x_{a p p_{4}}} . \\
& \hat{k}_{y_{a p p_{t b}}}=\frac{\hat{k}_{y_{a p p_{t}}} \hat{k}_{y_{a p p_{b}}} L_{y}}{\hat{k}_{y_{a p p_{t}} \Delta y_{1}}+\hat{k}_{y_{a p p_{b}}} \Delta y_{2}}
\end{aligned}
$$

where 


$$
\begin{aligned}
& \hat{k}_{y_{a p p_{t}}}=\frac{\Delta x_{1}}{L_{x}} k_{y_{a p p_{1}}}+\frac{\Delta x_{2}}{L_{x}} k_{y_{a p p_{2}}} \\
& \hat{k}_{y_{a p p_{b}}}=\frac{\Delta x_{1}}{L_{x}} k_{y_{a p p_{3}}}+\frac{\Delta x_{2}}{L_{x}} k_{y_{a p p_{4}}} .
\end{aligned}
$$

The local apparent permeabilities are defined as follows:

$$
\begin{gathered}
k_{y_{a p p_{1}}}=k_{y y_{1}}-\frac{k_{x y_{1}} k_{y x_{2}}}{k_{x x_{1}}} \\
k_{y_{a p p_{2}}}=k_{y y_{2}}-\frac{k_{x y_{2}} k_{y x_{2}}}{k_{x x_{2}}} \\
k_{y_{a p p_{3}}}=k_{y y_{3}}-\frac{k_{x y_{3}} k_{y x_{3}}}{k_{x x_{3}}} \\
k_{y_{a p p_{4}}}=k_{y y_{4}}-\frac{k_{x y_{4}} k_{y x_{4}}}{k_{x x_{4}}} . \\
\hat{k}_{y_{a p p_{s}}}=\frac{\Delta x_{1}}{L_{x}} \hat{k}_{y_{a p p_{5}}}+\frac{\Delta x_{2}}{L_{x}} \hat{k}_{y_{a p p_{s}}}
\end{gathered}
$$

where

$$
\begin{aligned}
& \hat{k}_{y_{a p p_{s}}}=\frac{k_{y_{a p p_{1}}} k_{y_{a p p_{3}}} L_{y}}{k_{y_{a p_{1}}} \Delta y_{1}+k_{y_{a p p_{3}}} \Delta y_{2}} \\
& \hat{k}_{y_{a p p_{s}}}=\frac{k_{y_{a p p_{2}}} k_{y_{y_{a p p_{4}}} L_{y}}}{k_{y_{a p p_{2}} \Delta y_{1}}+k_{y_{a p p_{4}}} \Delta y_{2}} .
\end{aligned}
$$

Arithmetic, geometric, and harmonic means may be suggested as options to obtain effective four-block apparent permeabilities. However, it is not clear as to which of these averaging methods would yield the most physically correct result. Therefore, the decision was made to use neither of the above mentioned options and rather use the physical concept of cross-flow in determining effective apparent permeabilities along each of the two coordinate axes.

The apparent permeabilities are determined based on a weighting scheme using apparent permeability anisotropy. It is known ${ }^{23,24}$ that the permeability anisotropy to a large degree controls the amount of cross-flow in a system. Therefore, $\tilde{k}_{x_{a p p}}=$

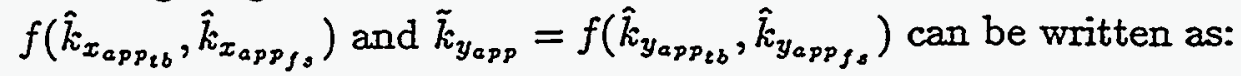

$$
\tilde{k}_{x_{a p p}}=\frac{\tilde{k}_{x_{a p p}} \hat{k}_{x_{a p p_{t b}}}+\tilde{k}_{y_{a p p}} \hat{k}_{x_{a p p} s s}}{\tilde{k}_{x_{a p p}}+\tilde{k}_{y_{a p p}}}
$$




$$
\tilde{k}_{y_{a p p}}=\frac{\tilde{k}_{x_{a p p}} \hat{k}_{y_{a p p} p}+\tilde{k}_{y_{a p p}} \hat{k}_{y_{a p p s s}}}{\tilde{k}_{x_{a p p}}+\tilde{k}_{y_{a p p}}} .
$$

Eqs. (29) and (30) are two equations which can be solved for $\tilde{k}_{x_{\text {app }}}$ and $\tilde{k}_{y_{a p p}}$ and subsequently the permeability anisotropy ratio $\frac{\bar{k}_{x_{a p p}}}{\tilde{k}_{y a p p}}$.

The next step is to determine the effective or overall ratios of transverse to longitudinal pressure gradients which are needed for determining the elements of the effective permeability tensor as seen in Eqs. (5)-(8). The generalized pressure gradient ratio $\left(\frac{\partial \bar{p}}{\partial t}\right)_{L} /\left(\frac{\partial \bar{p}}{\partial t}\right)_{L}$ consists of two components. It was decided to denote the pressure gradient ratio due to permeability contrasts between the four grid blocks the "global" pressure gradient ratio, whereas the "local" pressure gradient ratios evolve from local grid blocks (or quadrants) having non-zero off-diagonal elements of their effective permeability tensors. Figures 10 and 11 illustrate these concepts.

For details regarding the derivation of the "global" pressure gradient ratios, refer to Aasum. ${ }^{22}$ The basic idea for determining "global" pressure gradient ratios in the system is given in the following step-by-step procedure. This particular procedure represents injection along the $x$-axis:

(i) Isolate the top and bottom layers in Figure 5 and determine the magnitude of pressure at the points between blocks 1 and 2 , and 3 and 4, respectively, from applying Darcy's law in each layer separately.

(ii) Obtain the maximum transverse pressure by subtracting the expressions for the pressure values obtained in step (i).

(iii) Use the relationship between the total flow rate and the individual layer flow rates to eliminate flow rates from the expression obtained in step (ii).

(iv) Determine the average transverse pressure from geometric analysis as explained by Aasum.22

(v) Obtain the "global" pressure gradient ratio by multiplying the average transverse pressure by $\frac{L_{x}}{\left(\triangle p_{x} L_{y}\right)}$. This step eliminates pressure from the pressure gradient ratio expression.

The isotropic "global" pressure gradient ratio for injection along $\mathrm{x}$-axis has the following discrete form:

$$
\begin{aligned}
& {\left[\left(\frac{\partial \tilde{p}}{\partial y}\right)_{X} /\left(\frac{\partial \tilde{p}}{\partial x}\right)_{X}\right]_{i \text { so.global }}=\left(\frac{\Delta p_{y_{a v g}}}{\Delta p_{x}}\right)_{X} \frac{L_{x}}{L_{y}}} \\
& =\frac{1}{4 \Delta y_{2} I_{y}}\left(\hat{k}_{x_{a p p_{b}}} \Delta y_{1}-\hat{k}_{x_{a p p_{t} b}} L_{y}\right)\left(\frac{\Delta x_{2}}{k_{x_{a p p_{2}}}}-\frac{\Delta x_{1}}{k_{x_{a p p_{1}}}}\right) \\
& -\frac{1}{4 \Delta y_{1} L_{y}}\left(\hat{k}_{x_{a p p_{t}}} \Delta y_{2}-\hat{k}_{x_{a p p_{t b}}} L_{y}\right)\left(\frac{\Delta x_{2}}{k_{x_{a p p_{4}}}}-\frac{\Delta x_{1}}{k_{x_{a p p_{3}}}}\right) .
\end{aligned}
$$




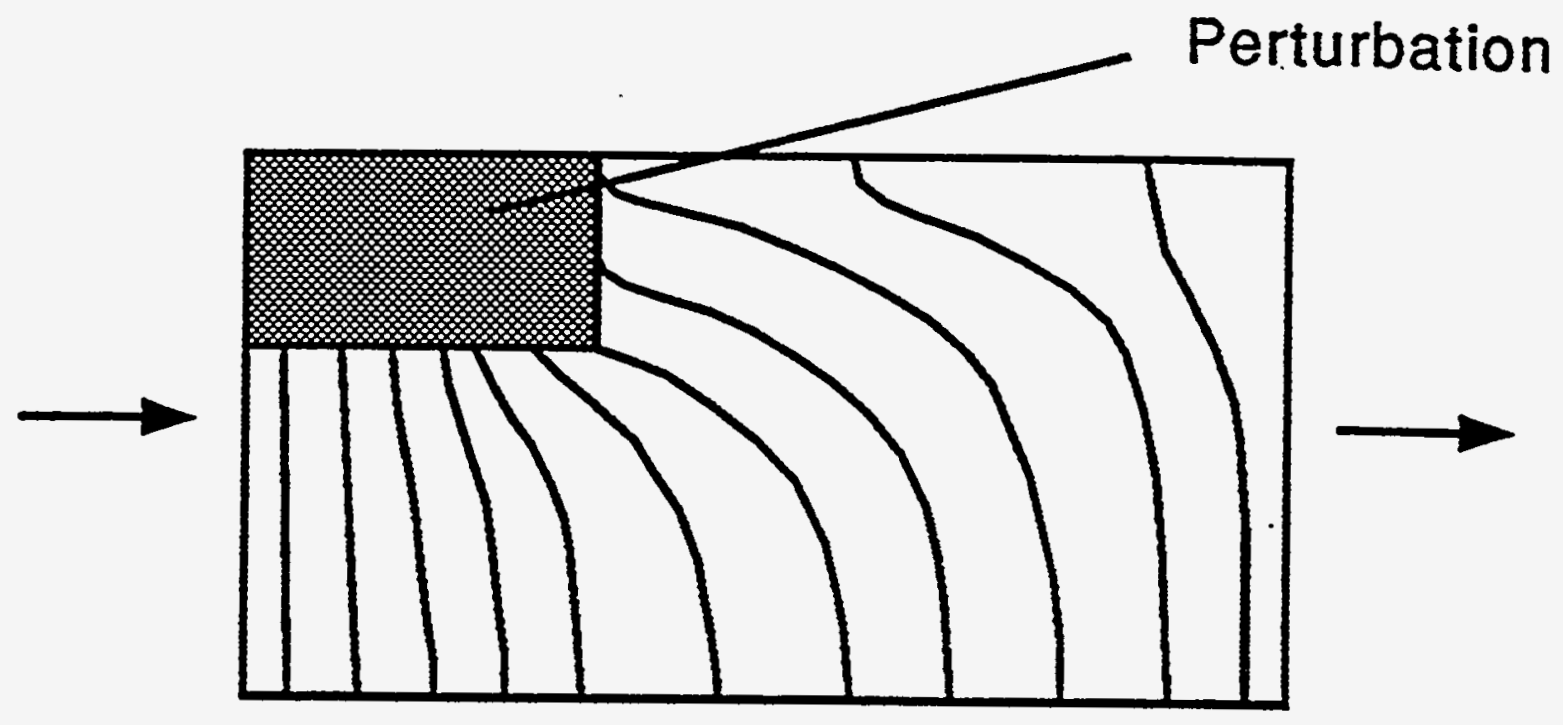

Fig. 10: Permeability Contrast Yielding a "Global" Non-Zero Transverse Pressure Gradient. 


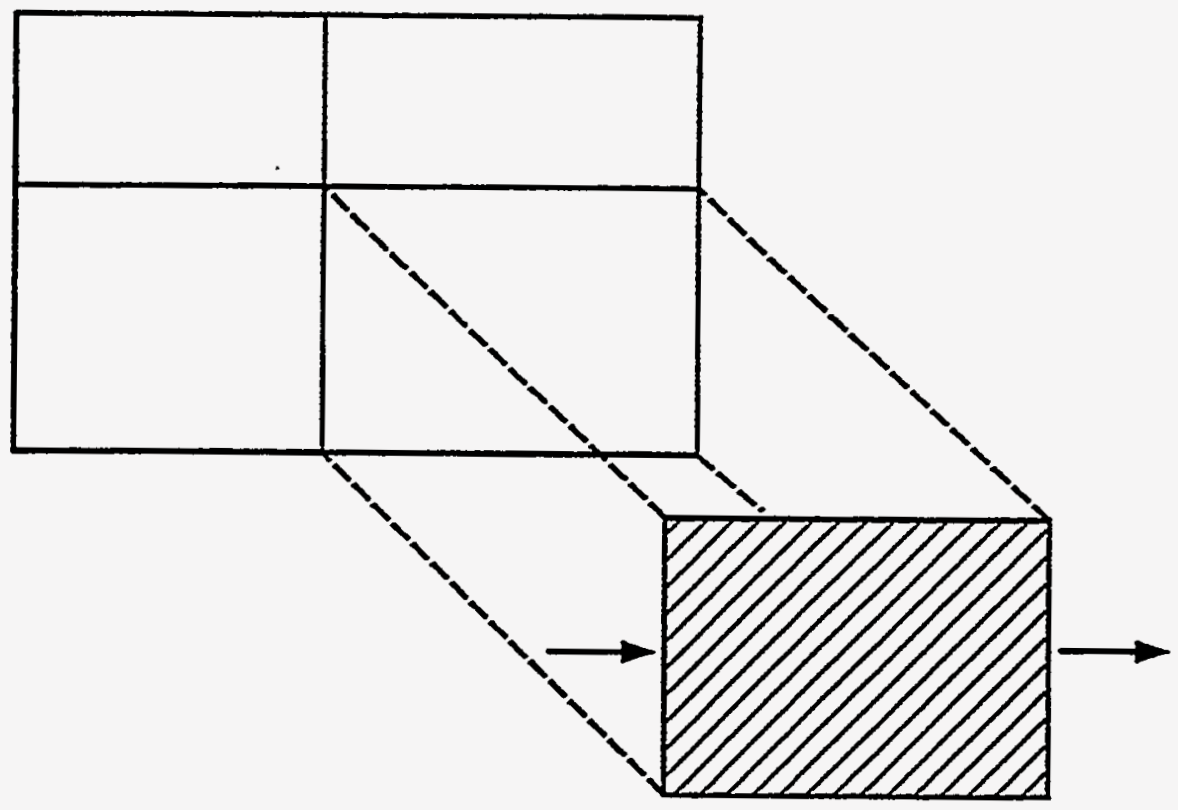

Fig. 11: Non-Zero Off-Diagonal Elements in Permeability Tensor of Local Grid Block Yielding "Local" Transverse Pressure Gradient. 
"Local" pressure gradient ratios are calculated for two-block systems in series and parallel which subsequently are combined into parallel and series four-block "local" pressure gradient ratios, respectively. Refer to Aasum ${ }^{22}$ for details.

The "global" and "local" pressure gradient ratios are also cross-flow corrected using the apparent permeability anisotropy. Once the corrected pressure gradient ratios are obtained, these "global" and "local" contributions are combined to yield the overall or effective pressure gradient ratio:

$$
\begin{aligned}
& \left(\frac{\partial \tilde{p}}{\partial y}\right)_{X} /\left(\frac{\partial \tilde{p}}{\partial x}\right)_{X}=\left[\left(\frac{\partial \tilde{p}}{\partial y}\right)_{X} /\left(\frac{\partial \tilde{p}}{\partial x}\right)_{X}\right]_{g l o b a l}+\left[\left(\frac{\partial \tilde{p}}{\partial y}\right)_{X} /\left(\frac{\partial \tilde{p}}{\partial x}\right)_{X}\right]_{\substack{\text { local } \\
(32)}} \\
& \left(\frac{\partial \tilde{p}}{\partial x}\right)_{Y} /\left(\frac{\partial \tilde{p}}{\partial y}\right)_{Y}=\left[\left(\frac{\partial \tilde{p}}{\partial x}\right)_{Y} /\left(\frac{\partial \tilde{p}}{\partial y}\right)_{Y}\right]_{\text {global }}+\left[\left(\frac{\partial \tilde{p}}{\partial x}\right)_{Y} /\left(\frac{\partial \tilde{p}}{\partial y}\right)_{Y}\right]_{\text {local }}
\end{aligned}
$$

Having determined the apparent permeabilities and the overall pressure gradient ratios, Eqs. (5)-(8) can, therefore, be solved for the elements of the effective permeability tensor.

To summarize, the analytical effective permeability tensor method consists of four basic steps:

0 . The grid block containing the small scale heterogeneous permeability field is divided into four quadrants or local blocks.

1. Determine the permeabilities along the principal directions and the orientation angle of $k_{x x}^{\prime}$ in each quadrant by calculating effective permeability along direction vectors between -90 and +90 degrees and deciding which direction results in the largest $k_{x x}^{\prime} / k_{y y}^{\prime}$ ratio.

2. Calculate the effective permeability tensor in each quadrant by coordinate rotation.

3. Calculate the effective permeability tensor for the entire permeability field within the grid block by combining the effective permeability tensors obtained from each quadrant.

\section{Numerical Method}

To validate the analytical method presented for obtaining an effective permeability tensor, a numerical method is developed. This method is based on the steady-state, single-phase, incompressible version of the continuity equation:

$$
\bar{\nabla} \cdot \bar{v}=0
$$

Darcy's law is the constitutive equation used: 


$$
\bar{v}=-\frac{\mathrm{K}}{\mu} \bar{\nabla} p .
$$

In Eqs. (34) and (35), $\bar{\nabla}$ is the divergence operator, $\bar{v}$ is the velocity vector, and $\mathrm{K}$ is the permeability tensor:

$$
\mathrm{K}=\left(\begin{array}{ll}
k_{x x} & k_{x y} \\
k_{y x} & k_{y y}
\end{array}\right)
$$

where $k_{x x}$ and $k_{y y}$ are the diagonal elements and $k_{x y}$ and $k_{y x}$ are the off-diagonal elements of the permeability tensor. $\mu$ is the fluid viscosity, and $p$ represents pressure.

A finite element simulator ${ }^{1}$ is used to solve Eqs. (34) and (35) for pressure and velocities. Two solutions are required to obtain all the elements of the effective permeability tensor. The first solution is obtained for a full face constant pressure gradient along the horizontal $x$-axis with closed upper and lower boundaries. The following version of Eq. (35), assuming fluid viscosity of $1.0 \mathrm{cp}$, represents two equations and four unknowns, $k_{x x}, k_{x y}, k_{y x}$, and $k_{y y}$ :

$$
\begin{aligned}
& v_{x_{X}}=-k_{x x}\left(\frac{\partial p}{\partial x}\right)_{X}-k_{x y}\left(\frac{\partial p}{\partial y}\right)_{X} \\
& v_{y_{X}}=-k_{y x}\left(\frac{\partial p}{\partial x}\right)_{X}-k_{y y}\left(\frac{\partial p}{\partial y}\right)_{X} .
\end{aligned}
$$

Two additional equations are required to obtain all the elements of the permeability tensor. Therefore, the second solution is obtained for a constant pressure gradient along the vertical $y$-axis with closed left and right boundaries:

$$
\begin{aligned}
& v_{x_{Y}=}-k_{x x}\left(\frac{\partial p}{\partial x}\right)_{Y}-k_{x y}\left(\frac{\partial p}{\partial y}\right)_{Y} \\
& v_{y Y}=-k_{y x}\left(\frac{\partial p}{\partial x}\right)_{Y}-k_{y y}\left(\frac{\partial p}{\partial y}\right)_{Y} .
\end{aligned}
$$

$v_{x_{X}}$ and $v_{x_{Y}}$ are velocity components in the $\mathrm{x}$-direction for injection along $\mathrm{x}$ and $y$-axis, respectively. $v_{y_{X}}$ and $v_{y Y}$ are the corresponding velocity components in the $y$-direction. $\left(\frac{\partial p}{\partial x}\right)_{X}$ and $\left(\frac{\partial p}{\partial x}\right)_{Y}$ are the pressure gradients in the $x$-direction for injection along $x$ - and $y$-axis, respectively, whereas $\left(\frac{\partial p}{\partial y}\right)_{X}$ and $\left(\frac{\partial p}{\partial y}\right)_{Y}$ are the corresponding gradients in the $y$-direction.

In summary, effective pressure gradients and velocities are obtained from two solutions on the same small scale permeability field. One solution is obtained from injection along the $\mathrm{x}$-axis and the other solution from injection along the $\mathrm{y}$-axis. Eqs. (37)-(40) are then solved for the unknowns, $k_{x x}, k_{x y}, k_{y x}$, and $k_{y y}$. 


\section{Results and Discussion}

Comparisons Between Analytical and Numerical Effective Permeabilities

The first case illustrates the effect on effective permeability of a low permeability region ("perturbation") located off-center in a permeability field. The next three cases include a well defined and regular cross-bedded permeability distribution, a simulated stochastic anisotropic permeability distribution, as well as minipermeameter data from the Algerita outcrop in New Mexico.

Case 1: The four local grid blocks contain contain anisotropic permeabilities with non-zero off-diagonal elements in their permeability tensors. Figure 12 shows the arrangement of the local permeability tensors.

Effective permeability tensors calculated using the analytical and numerical methods show excellent agreement. Notice that the off-diagonal elements of the effective permeability tensor are non-symmetric using both analytical and numerical methods. This phenomena is a result of anisotropic and full local permeability tensors. The two methods agree in both form and existence of non-symmetry of the effective permeability tensors, as shown in Figure 12.

Case 2: This case is based on the cross-bedded permeability distribution shown in Figure 13, where the light and the dark grid blocks initially have isotropic permeabilities of $1000 \mathrm{md}$ and $20 \mathrm{md}$, respectively.

Figure 13 also shows the effective permeability obtained from the numerical and analytical methods. The results agree well. Both methods predict isotropic diagonal elements of the effective permeability tensor, which is correct since the cross-beds are oriented at $a-45^{\circ}$ angle from the horizontal $x$-axis. The off-diagonal elements are correctly predicted to be negative and symmetric. For this particular permeability distribution, the exact effective permeability tensor can be obtained through simple coordinate rotation since the permeabilities along the principal axes are simple arithmetic and harmonic averages of the two permeability values in this distribution, $1000 \mathrm{md}$ and $20 \mathrm{md}$, and the angle of the cross-beds, $-45^{\circ}$, is known. The analytical method agrees exactly with the tensor obtained from simple coordinate rotation.

The results show that the directional search for $k_{x x}^{\prime} / k_{y y}^{\prime}$ is successful in determining the principal axes of permeability. Therefore, the directional search method may.also be a useful tool in detecting permeability structures in minipermeameter measured permeability data from outcrops.

Case 3: The permeability distribution used in this case contains a random component. A Turning Bands program ${ }^{25}$ is used to generate a permeability distribution with Dykstra-Parsons coefficient, $V$, of 0.7 and a dimensionless correlation length of 0.64 along the principal axis of maximum permeability which is at $-65^{\circ}$ from the horizontal $\mathrm{x}$-axis. The anisotropy ratio of the correlation lengths is 10 .

The results indicate that the analytical and the numerical methods agree well even for stochastic systems with cross-bedding structures. In terms of computer time, however, the analytical method is about two orders of magnitude faster than 


\begin{tabular}{|rc|rc|}
\hline 1 & 0.1 & 1000 & 100 \\
0.1 & 0.1 & 100 & 100 \\
1000 & 100 & 1000 & 100 \\
100 & 100 & 100 & 100 \\
\hline
\end{tabular}

\begin{tabular}{|cc|}
\hline \multicolumn{2}{|c|}{ Analytical Method: } \\
495 & 36 \\
48 & 62 \\
\hline Numerical Method: \\
501 & 34 \\
& 61 \\
44 & \\
\hline
\end{tabular}

Fig. 12: Effective Permeability Tensors Calculated by Analytical and Numerical Methods for Anisotropic and Full Local Permeability Tensors (Units: md). 
Light Blocks: 1000 md; Dark Blocks: 20 md

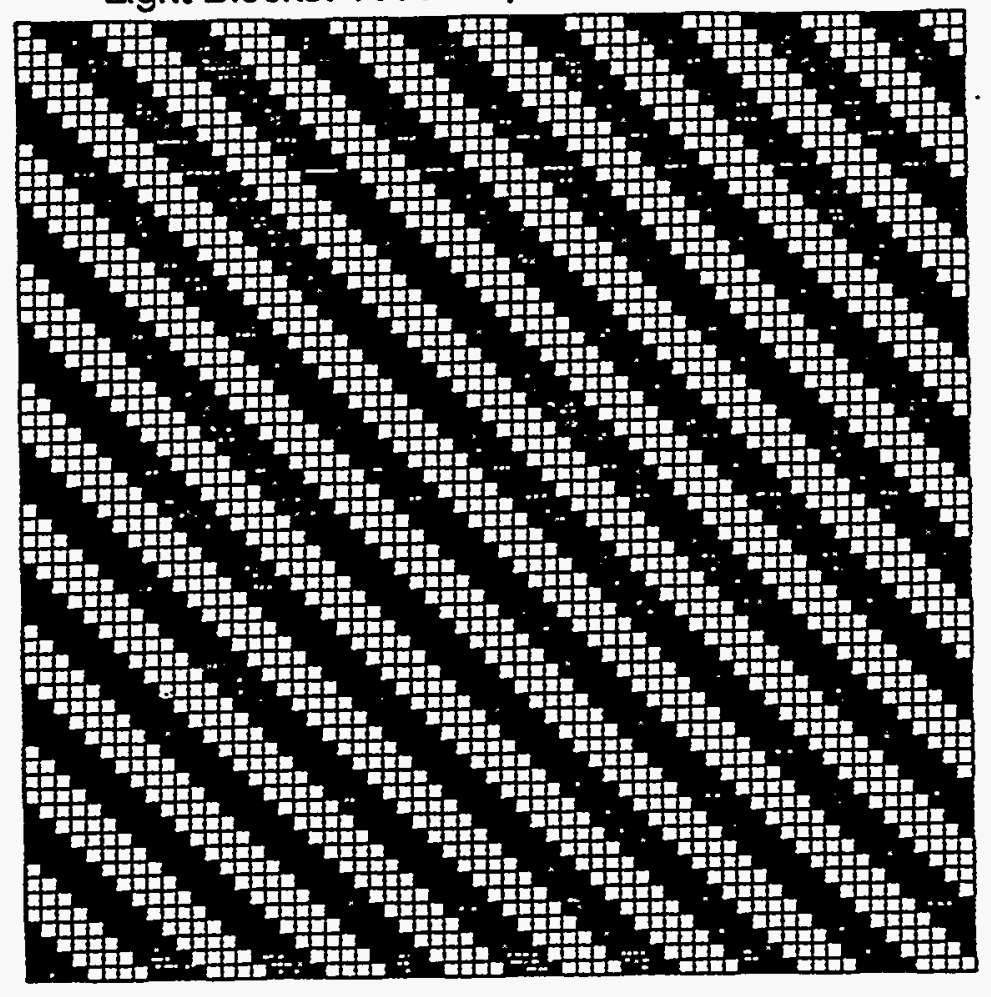

\begin{tabular}{|cr|}
\hline \multicolumn{2}{|c|}{ Analytical Method: } \\
\hline 275 & -235 \\
& \\
-235 & 275 \\
\hline Numerical Method: \\
271 & -224 \\
& 271 \\
\hline
\end{tabular}

Fig. 13: Effective Permeability Tensors Calculated by Analytical and Numerical Methods for Cross-Bedded Permeability Distribution (Units: md). 
the numerical method. As can be observed from Figure 14, both methods predict negative off-diagonal elements of the permeability tensor and $k_{y y}$ larger than $k_{x x}$. These results along with the results from Case 2 suggest that the analytical effective permeability method works well for both deterministic and stochastic permeability distributions. It will be shown next how this method works on "real" data.

Case 4: In this case, the effective permeability is determined from minipermeameter measured permeability data from a small section of a San Andres carbonate outcrop in Algerita, New Mexico. The distribution of these data is shown in Figure 15.

Comparing the permeability distributions of Figures 13 and 15, it is evident that the permeabilities in Figure 15 have less structure or correlation. In Figure 13 , there are complete high and low permeability layers whereas the outcrop data in Figure 15 appear to be more random in their distribution. This observation is supported by the analytical and numerical effective permeabilities calculated for the outcrop data. The off-diagonal elements in the permeability tensors are small compared to their respective diagonal elements. The ratios of $k_{x y} / k_{x x}$ and $k_{y x} / k_{y y}$ are indications of the sizes of the transverse to longitudinal pressure gradient ratios. Both numerical and analytical methods show ratios ranging from about 0.0 to 0.08 . In Figure 13 however, the ratios are -0.83 and -0.85 . In other words, the cross-bedded heterogeneities in Figure 13 induce much higher transverse pressure gradients than the more random heterogeneities in Figure 15 . The relatively small off-diagonal elements and the magnitudes of the diagonal elements indicate that the permeability heterogeneities for all practical purposes are oriented along the $\mathrm{x}$-axis.

There appears to be a difference in the prediction of $k_{x y}$ in Figure 15. Numerically $k_{x y}=0.9 \mathrm{md}$ and analy tically $k_{x y}=0.0 \mathrm{md}$. However, this difference is negligible considering the low pressure gradient ratios obtained both numerically and analytically. The diagonal elements show excellent agreement indicating anisotropy ratios of 7.0 and 6.9 for the analytical and numerical methods, respectively.

\section{Comparisons Between Results From Miscible Displacement Simulations}

To validate the method further, unit mobility ratio miscible displacements are simulated in heterogeneous systems at constant injection and production pressures. Both horizontal boundaries are closed to flow. The flood front locations using different methods are compared. Effects of numerical dispersion are eliminated by using the same number of grid blocks for initial permeability distributions and homogenized permeability distributions.

Case 1: Figure 16a shows the permeability distribution used in the simulation. It is essentially the same distribution as in Figure 13, except in Figure 16a the aspect ratio is 8 to 1 rather than 1 to 1 as in Figure 13.

Figures 16b-16e show 0.5 concentration contours of the flood fronts after 49.7 days of injection. Figure $16 \mathrm{~b}$ shows the location of the front using the initial permeability distribution. This figure is used as the base case. The analytical effective permeability tensor method (Figure 16c) yields the best prediction of the location of the flood front. The geometric mean predicts a flood front which is advancing too fast (Figure 16d). Thus, the geometric mean overpredicts the effective permeabil- 


\section{$T B M ; V=0.7$, Structure Angle:-65 Deg.}

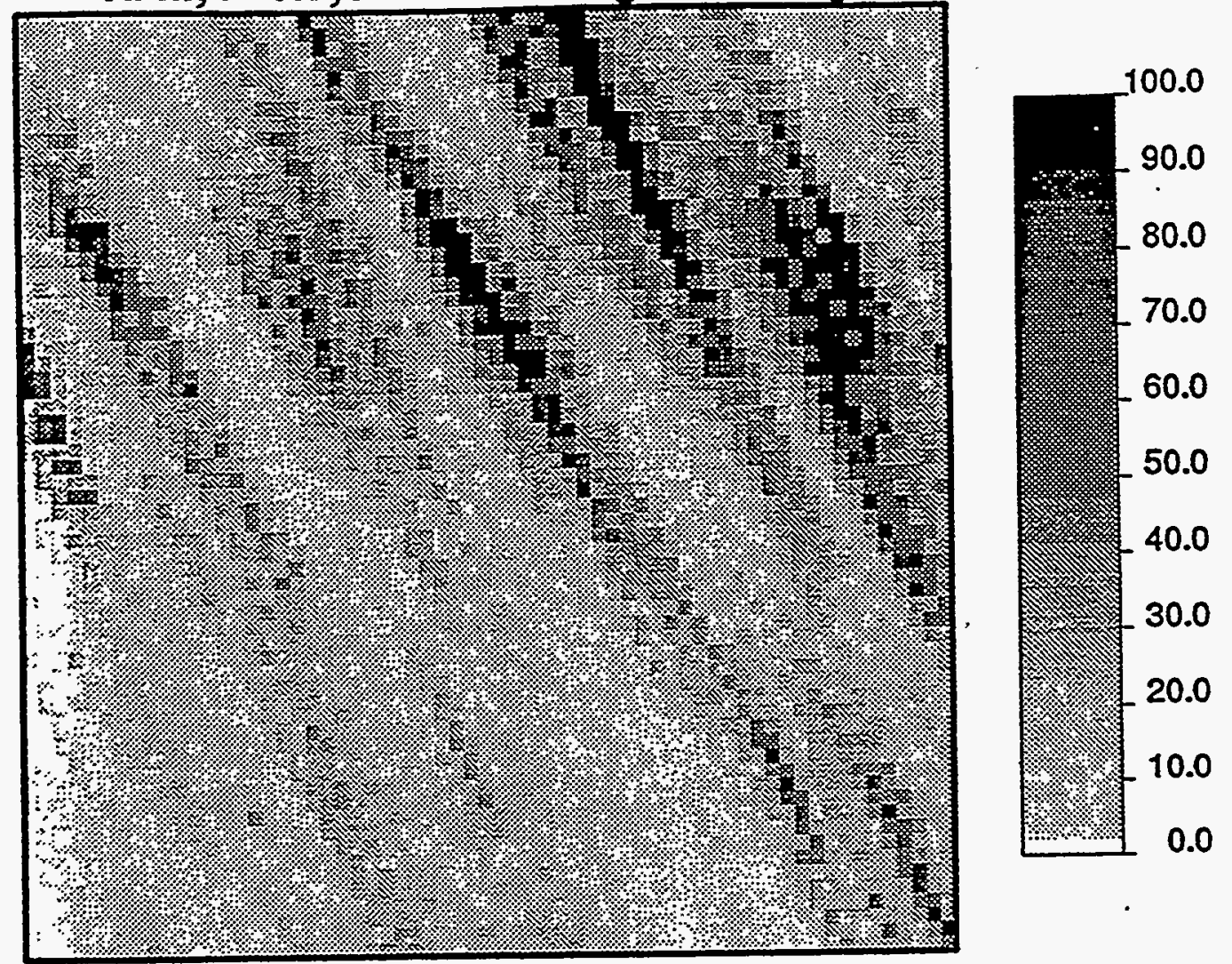

\begin{tabular}{|cr|}
\hline \multicolumn{2}{|c|}{ Analytical Method: } \\
10.3 & -2.4 \\
& \\
-2.5 & 12.6 \\
\hline & Numerical Method: \\
9.7 & -1.7 \\
& \\
-2.8 & 13.2 \\
\hline
\end{tabular}

Fig. 14: Effective Permeability Tensors Calculated by Analytical and Numerical Methods for Cross-Bedded Stochastic Permeability Distribution (Units: md). 


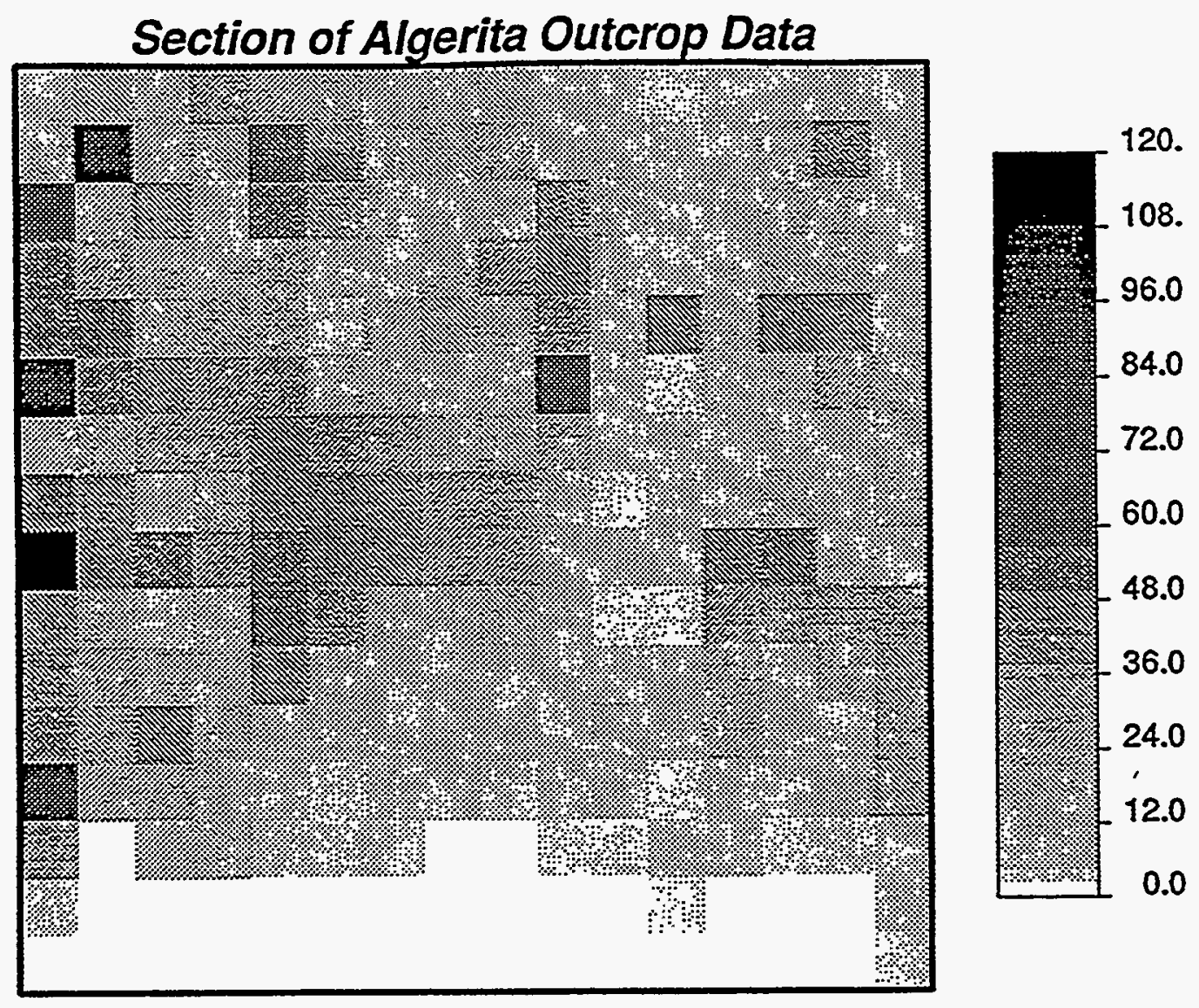

\begin{tabular}{|cc|}
\hline \multicolumn{2}{|c|}{ Analytical Method: } \\
11.9 & 0.0 \\
& \\
0.1 & 1.7 \\
\hline Numerical Method: & 0.9 \\
11.8 & \\
0.1 & 1.7 \\
\hline
\end{tabular}

Fig. 15: Effective Permeability Tensors Calculated by Analytical and Numerical Methods for Minipermeameter Measured Permeability Data From the Algerita Outcrop (Units: $\mathrm{md}$ ). 
Cross-bedded Permeability Distribution

a

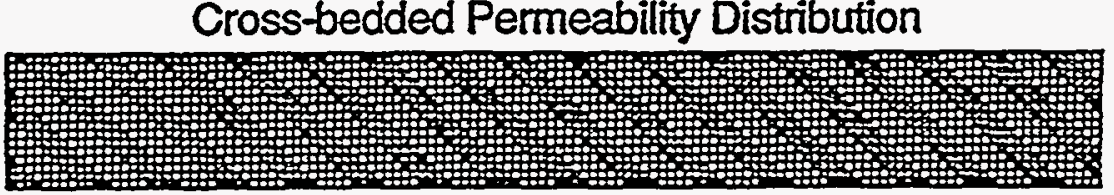

Using Initial Cross-bedded Permeability Distribution

b

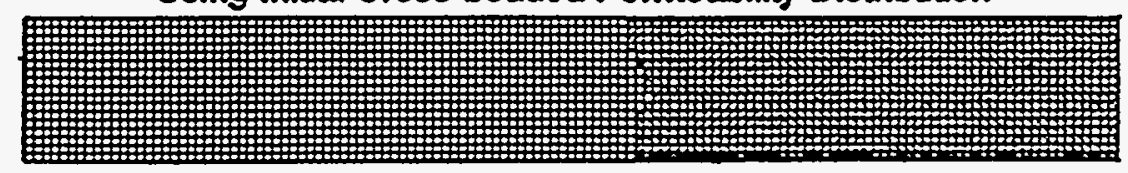

Using Effective Permeability Tensor

C

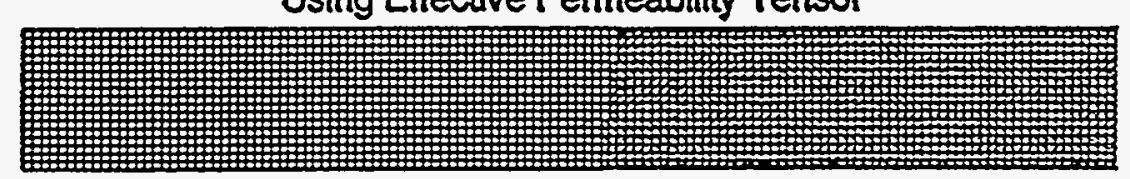

-...

Using Geometrically Averaged Permeability

d

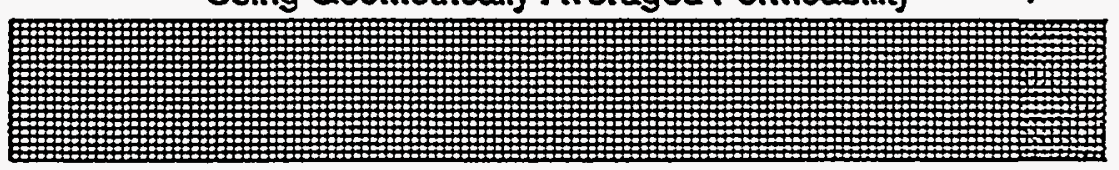

Using Renormalized Permeability

e

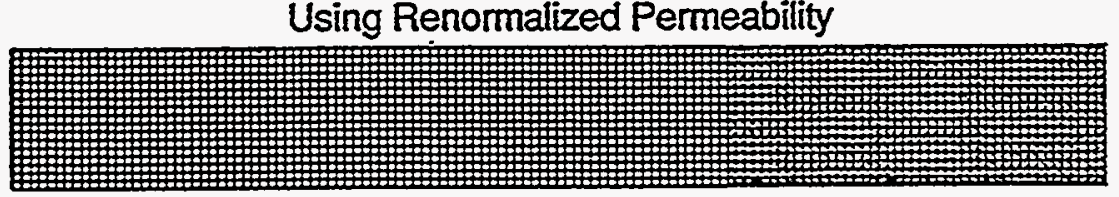

Fig. 16: Initial Permeability Distribution (Dark: $20 \mathrm{md}$; Light: $1000 \mathrm{md}$ ) and 0.5 Concentration Contours After 49.7 Days of Injection Using the Initial Permeability Distribution and the Permeability Distributions Obtained Using Various Upscaling Methods. 
ity. The renormalization method ${ }^{19}$ (Figure 16e) also predicts a flood front which is advancing too fast, although the overprediction of the effective permeability is not as severe as when using the geometric mean.

Case 2: This case involves a stochastic log-normal permeability distribution. The Dykstra-Parsons coefficient is $V=0.70$. The angle of the permeability structures is specified to be $-45^{\circ}$. The dimensionless correlation length along the oriented structures is $\lambda_{D_{l}}=0.5$ and the dimensionless correlation length transverse to the structures is $\lambda_{D_{t}}=0.05$. The permeability distribution is generated using the Turning Bands program. Figure 17a shows a map of this permeability distribution. The boundary conditions are identical to those in Case 1.

Figures $17 \mathrm{~b}-17 \mathrm{e}$ show 0.5 concentration contours of the flood fronts after 94.8 days of injection. Figure $17 \mathrm{~b}$ shows the location of the front using the initial stochastic permeability distribution. Both the analytical effective permeability tensor method (Figure 17c) and the geometric mean (Figure 17d) yield correct flood front matches. The renormalization method (Figure 17e), however, predicts a flood front which is advancing too slowly. Thus, the renormalization method underpredicts the effective permeability for this case.

Table 1 shows the flow rates obtained using the initial and upscaled permeability distributions for Cases 1 and 2.

In the above cases, the analytical effective permeability method has outperformed the other effective permeability methods. These results suggest that the analytical tensor method is more general than the other upscaling methods presented. 

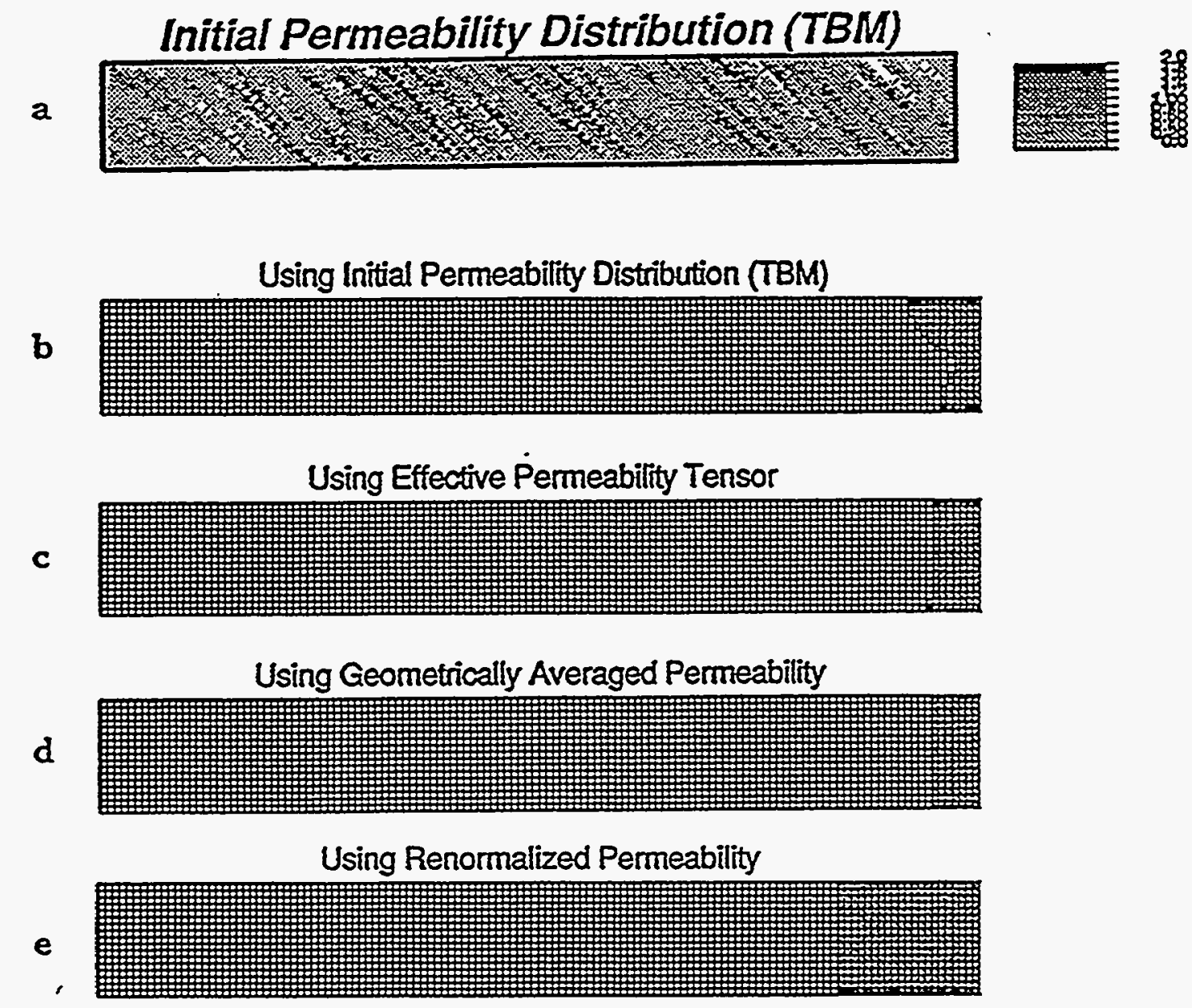

Fig. 17: Initial Permeability Distribution ( $V=0.7)$ and 0.5 Concentration Contours After 94.8 Days of Injection Using the Initial Permeability Distribution and the Permeability Distributions Obtained Using Various Upscaling Methods. 


\begin{tabular}{|c|c|}
\hline \multicolumn{2}{|c|}{ Table 1 } \\
\hline \multicolumn{2}{|c|}{ Flow Rates } \\
\hline Case 1 & $\mathrm{q}(\mathrm{cm} 3 / \mathrm{s}) \times 10^{-2}$ \\
\hline Using Initial Permeability Distribution: & 2.64 \\
\hline Using Effective Permeability Tensor: & 2.48 \\
\hline Using Geometric Average: & 4.40 \\
\hline Using Real-Space Renormalization: & 2.93 \\
\hline \hline Case 2 & $\mathrm{q}(\mathrm{cm} 3 / \mathrm{s}) \times 10^{-2}$ \\
\hline Using Initial Permeability Distribution: & 2.38 \\
\hline Using Effective Permeability Tensor: & 2.37 \\
\hline Using Geometric Average: & 2.35 \\
\hline \hline
\end{tabular}


PART 2

\title{
INCORPORATION OF FULL TENSOR PERMEABILITY IN A NUMERICAL SIMULATOR
}

\author{
Introduction
}

Most commercially available reservoir simulators are currently restricted to handle limited anisotropy, i.e., only the diagonal elements of the permeability tensor. However, some industry and research simulators have recently incorporated the general anisotropic model. . $^{1,3,4,26,27}$

Full tensor permeability can be incorporated in both finite difference and finite element simulators. A 9-point differencing scheme is required when using finite difference methods. The 9-point schemes are more computationally demanding, but reduce grid orientation effects compared to standard 5-point differencing schemes. ${ }^{26,27,28}$

This second part of the report will describe in some detail how to develop transmissibilities for point-centered 9-point schemes based on full tensor permeability. Finite element theory is used as a basis for this development. ${ }^{29,30}$ The material presented can, therefore, be useful in the development of both finite element and finite difference techniques that consider the full tensor form of absolute permeability.

Shiralkar ${ }^{27}$ has developed a method for calculating transmissibilities for generally anisotropic (full tensor) permeability. That method is based on satisfying Darcy's law and maximizing the range of positive transmissibilities. In this part of the report, it is shown how the proposed finite element based method and Shiralkar's method can be related. By selecting an appropriate value of a numerical integration parameter, Shiralkar's method is indeed a particular case of the proposed method for a rectangular element with homogeneous, but generally anisotropic permeability.

The general transmissibility expressions developed were used to calculate transmissibilities which were inserted in a point-centered finite difference simulator ${ }^{31}$ for waterflooding simulations. Results from these waterflood simulations are presented. Initial and upscaled permeability distributions are used. The upscaled distributions are obtained using both an effective permeability tensor method ${ }^{22}$ and standard upscaling techniques. Pressure maps, water saturation maps, cumulative recovery and fractional flow curves are compared. Finally, conclusions from this study are presented.

Development of General Transmissibilities From Finite Element Theory

The method presented in this section is based on Young's study ${ }^{29}$ which shows a relationship between finite element and finite difference methods. The development shown here extends Young's work to generally anisotropic permeability systems.

Single-phase, steady-state, incompressible flow is assumed as well as fluid viscosity of $I \mathrm{cp}$ to simplify derivations. The derivations are based on the continuity equation (pressure equation) without boundary conditions: 


$$
-\bar{\nabla} \cdot(\Lambda \bar{\nabla} p)=0
$$

where

$$
\Lambda=\left(\begin{array}{ll}
\lambda_{x x} & \lambda_{x y} \\
\lambda_{y x} & \lambda_{y y}
\end{array}\right)=\left(\begin{array}{ll}
k_{x x} & k_{x y} \\
k_{y x} & k_{y y}
\end{array}\right) h
$$

$\Lambda$ is the mobility tensor, $h$ is the thickness of the reservoir, $k_{x x}, k_{x y}, k_{y x}$, and $k_{y y}$ are the elements of the permeability tensor, and $p$ represents pressure.

For a two-dimensional areal Cartesian coordinate system, Eq. (4I) can be expanded as follows:

$$
-\frac{\partial}{\partial x}\left(\lambda_{x x} \frac{\partial p}{\partial x}+\lambda_{x y} \frac{\partial p}{\partial y}\right)-\frac{\partial}{\partial y}\left(\lambda_{y x} \frac{\partial p}{\partial x}+\lambda_{y y} \frac{\partial p}{\partial y}\right)=0 .
$$

Using finite element theory, pressure can be approximated by a piecewise continuous Lagrange interpolating polynomial:

$$
p \approx \sum_{i=1}^{n x} \sum_{j=1}^{n y} u_{i}(x) w_{j}(y) p_{i j}
$$

where $u_{i}(x)$ and $w_{j}(y)$ are trial functions. Linear trial functions are shown in Figure 18. $n x$ and $n y$ represent the number of nodes or grid points in $x$ - and $y$-direction, respectively. In the remaining derivations, the parentheses in Eq. (44) are dropped for notational convenience.

Differentiating pressure with respect to $x$ and $y$ in Eq. (44) yields:

$$
\begin{aligned}
& \frac{\partial p}{\partial x} \approx \sum_{i=1}^{n x} \sum_{j=1}^{n y} \frac{d u_{i}}{d x} w_{j} p_{i j} \\
& \frac{\partial p}{\partial y} \approx \sum_{i=1}^{n x} \sum_{j=1}^{n y} u_{i} \frac{d w_{j}}{d y} p_{i j} .
\end{aligned}
$$

The first step is to write the residual equation. The residual, $R$, is the difference between the left hand side and the right hand side of the given equation which in this case is Eq. (43). Combining Eqs. (43), (45), and (46), the residual, $R$, can be written as follows:

$$
R=\sum_{i=1}^{n x} \sum_{j=1}^{n y}\left[R_{x x}+R_{x y}+R_{y x}+R_{y y}\right] p_{i j}-0
$$

where 


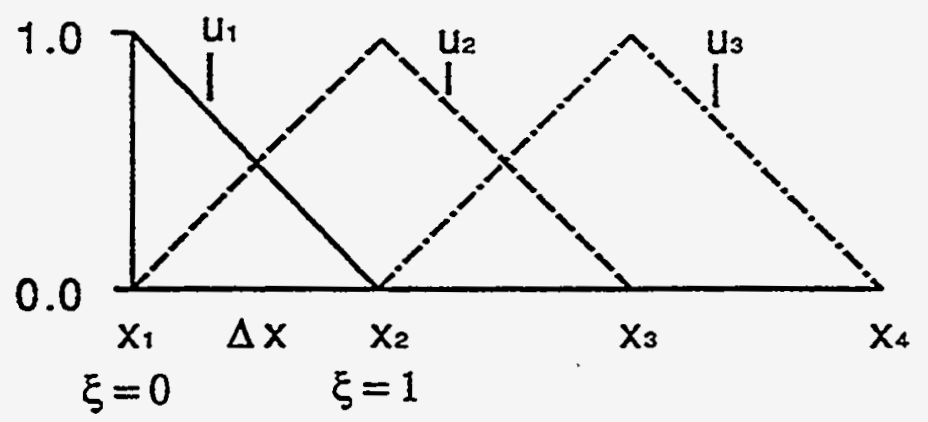

(a)

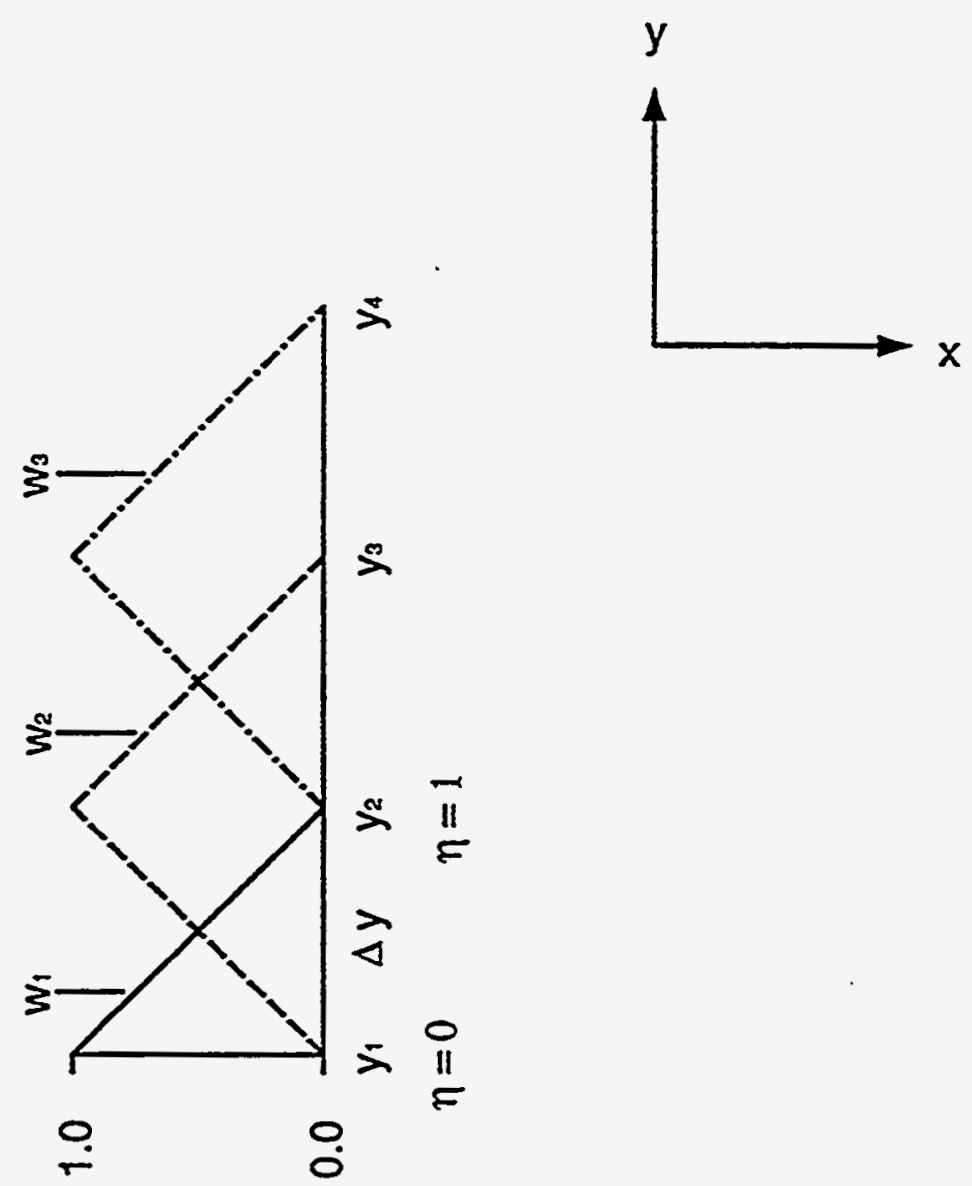

(b)

Fig. 18: Linear Trial Functions in (a) $x$-direction and (b) $y$-direction. 


$$
\begin{aligned}
& R_{x x}=-w_{j} \frac{\partial}{\partial x}\left(\lambda_{x x} \frac{\partial u_{i}}{\partial x}\right) \\
& R_{x y}=-\frac{d w_{j}}{d y} \frac{\partial}{\partial x}\left(\lambda_{x y} u_{i}\right) \\
& R_{y x}=-\frac{d u_{i}}{d x} \frac{\partial}{\partial y}\left(\lambda_{y x} w_{j}\right) \\
& R_{y y}=-u_{i} \frac{\partial}{\partial y}\left(\lambda_{y y} \frac{d w_{j}}{d y}\right) .
\end{aligned}
$$

The Galerkin optimizing criterion is then applied to make $R$ as small as possible for all values of $x$ and $y$ throughout the domain. Using the Galerkin criterion, a weighted average of $R$ over the entire domain is required to be zero. ${ }^{32}$ The weighting functions are the trial functions, $u_{k}$ and $w_{\ell}$ :

$$
\int_{0}^{L_{y}} \int_{0}^{L_{x}} u_{k} w_{\ell} R d x d y=0, \quad k=1, \ldots, n x ; \ell=1, \ldots, n y
$$

where $L_{x}$ and $L_{y}$ are system lengths in $\mathrm{x}$ - and y-directions, respectively. Eq. (47) is substituted into Eq. (48) to give the following expression:

$$
\begin{gathered}
\sum_{i=1}^{n x} \sum_{j=1}^{n y} \int_{0}^{L_{y}} \int_{0}^{L_{z}} u_{k} w_{\ell}\left[R_{x x}+R_{x y}+R_{y x}+R_{y y}\right] p_{i j} d x d y=0 \\
k=1, \ldots, n x ; \quad \ell=1, \ldots, n y .
\end{gathered}
$$

The next step is to integrate by parts. First, apply the "chain rule" for differentiating the product of two functions:

$$
\begin{gathered}
-\frac{\partial}{\partial x}\left(\lambda_{x x} \frac{d u_{i}}{d x}\right) u_{k}=-\left(\frac{\partial}{\partial x}\left(\lambda_{x x} \frac{d u_{i}}{d x} u_{k}\right)-\left(\lambda_{x x} \frac{d u_{i}}{d x}\right) \frac{d u_{k}}{d x}\right) \\
-\frac{\partial}{\partial x}\left(\lambda_{x y} u_{i}\right) u_{k}=-\left(\frac{\partial}{\partial x}\left(\lambda_{x y} u_{i} u_{k}\right)-\left(\lambda_{x y} u_{i}\right) \frac{d u_{k}}{d x}\right) \\
-\frac{\partial}{\partial y}\left(\lambda_{y x} w_{j}\right) w_{\ell}=-\left(\frac{\partial}{\partial y}\left(\lambda_{y x} w_{j} w_{\ell}\right)-\left(\lambda_{y x} w_{j}\right) \frac{d w_{\ell}}{d y}\right) \\
-\frac{\partial}{\partial y}\left(\lambda_{y y} \frac{d w_{j}}{d y}\right) w_{\ell}=-\left(\frac{\partial}{\partial y}\left(\lambda_{y y} \frac{d w_{j}}{d y} w_{\ell}\right)-\left(\lambda_{y y} \frac{d w_{j}}{d y}\right) \frac{d w_{\ell}}{d y}\right) .
\end{gathered}
$$


Substitute Eqs. (50)-(53) into Eq. (49):

$$
\begin{gathered}
\sum_{i=1}^{n x} \sum_{j=1}^{n y}\left(G_{x x}+G_{x y}+G_{y x}+G_{y y}\right) p_{i j} \\
-\sum_{i=1}^{n x} \sum_{j=1}^{n y}\left(G_{x x_{b}}+G_{x y_{b}}+G_{y x_{b}}+G_{y y_{b}}\right) p_{i j}=0, \\
k=1, \ldots, n x ; \quad \ell=1, \ldots, n y
\end{gathered}
$$

where

$$
\begin{gathered}
G_{x x}=\int_{0}^{L_{y}} \int_{0}^{L_{x}} \lambda_{x x} \frac{d u_{i}}{d x} w_{j} \frac{d u_{k}}{d x} w_{\ell} d x d y \\
G_{x y}=\int_{0}^{L_{y}} \int_{0}^{L_{x}} \lambda_{x y} u_{i} \frac{d w_{j}}{d y} \frac{d u_{k}}{d x} w_{\ell} d x d y \\
G_{y x}=\int_{0}^{L_{y}} \int_{0}^{L_{x}} \lambda_{y x} \frac{d u_{i}}{d x} w_{j} u_{k} \frac{d w_{\ell}}{d y} d x d y \\
G_{y y}=\int_{0}^{L_{y}} \int_{0}^{L_{x}} \lambda_{y y} u_{i} \frac{d w_{j}}{d y} u_{k} \frac{d w_{\ell}}{d y} d x d y \\
G_{x x_{b}}=\int_{0}^{L_{y}} \int_{0}^{L_{x}} w_{j} w_{\ell} \frac{\partial}{\partial x}\left(\lambda_{x x} \frac{d u_{i}}{d x} u_{k}\right) d x d y \\
G_{x y_{b}}=\int_{0}^{L_{y}} \int_{0}^{L_{x}} \frac{d w_{j}}{d y} w_{\ell} \frac{\partial}{\partial x}\left(\lambda_{x y} u_{i} u_{k}\right) d x d y \\
G_{y x_{b}}=\int_{0}^{L_{y}} \int_{0}^{L_{x}} \frac{d u_{i}}{d x} u_{k} \frac{\partial}{\partial y}\left(\lambda_{y x} w_{j} w_{\ell}\right) d x d y \\
L_{0}^{L_{y}} \int_{0}^{L_{x}} u_{i} u_{k} \frac{\partial}{\partial y} .\left(\lambda_{y y} \frac{d w_{j}}{d y} w_{\ell}\right) d x d y .
\end{gathered}
$$


The second part of Eq. (54) can be represented as a line integral around all external and internal (well) boundaries.

The reduction of the second part of Eq. (54) into a line integral is accomplished using the 2-D divergence theorem which is a corollary to Green's theorem for line integrals. ${ }^{32}$ The line integral can be expressed as follows: ${ }^{22}$

$$
-\oint_{\partial \Omega} u_{k} w_{\ell}(\Lambda \bar{\nabla} p) \cdot \bar{n} d \Omega
$$

where $\Omega$ is the two-dimensional domain and $\bar{n}$ is the outward unit normal vector.

Using Eq. (55), Eq. (54) has the following form:

$$
\begin{gathered}
\sum_{i=1}^{n x} \sum_{j=1}^{n y}\left(G_{x x}+G_{x y}+G_{y x}+G_{y y}\right) p_{i j} \\
=\oint_{\partial \Omega} u_{k} w_{\ell}(\Lambda \bar{\nabla} p) \cdot \bar{n} d \Omega, \\
k=1, \ldots, n x ; \ell=1, \ldots, n y .
\end{gathered}
$$

Eq. (56) is the basis for solving Eq. (41) using finite element analysis. The next step in the finite element approach would be to decide on specific expressions for the trial functions. These expressions are dependent on the choice of the finite element regarding shape (triangular or quadrilateral) and number of nodes (type of polynomial; linear, quadratic, etc.). For details about the general procedure of solving an expression like Eq. (56), refer to Burnett. ${ }^{32}$

The remainder of this section deals with the use of Eq. (56) in the development of transmissibilities for a 9-point finite difference scheme.

Figure 19 shows a quadrilateral element with a node in each corner. This element is labeled with the transmissibilities to be derived. $T_{x 1}, T_{x 2}, T_{y 1}$, and $T_{y 2}$ represent one-sided edge transmissibilities and are added to neighboring element transmissibilities to yield total transmissibility values. $T_{x y 1}$ and $T_{x y 2}$ are diagonal transmissibilities and represent total values. In Figure 20, it is shown how an element mesh is superimposed on a point-centered grid.

For simplicity in subsequent derivations, Eq. (44) will be written in terms of trial functions that are based on dimensionless coordinates. ${ }^{30}$ For a single element, Eq. (44) can be written as follows:

$$
p \approx \sum_{i=1}^{2} \sum_{j=1}^{2} U_{i}(\xi) W_{j}(\eta) p_{i j}
$$

where 


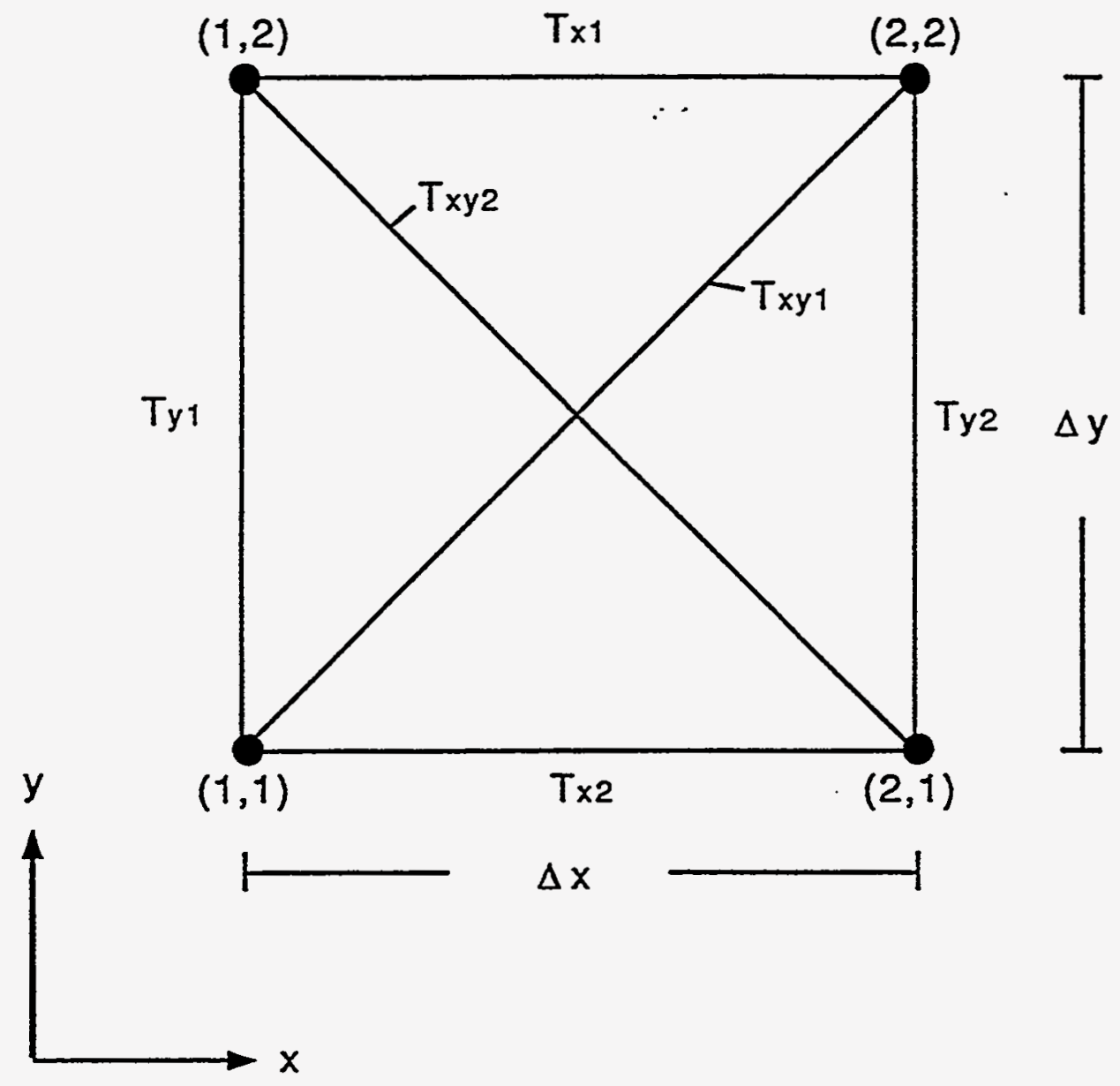

Fig. 19: Quadrilateral Element With Labeled. Transmissibilities. 
- Element Boundary

- Grid Block Boundary

- Grid Point

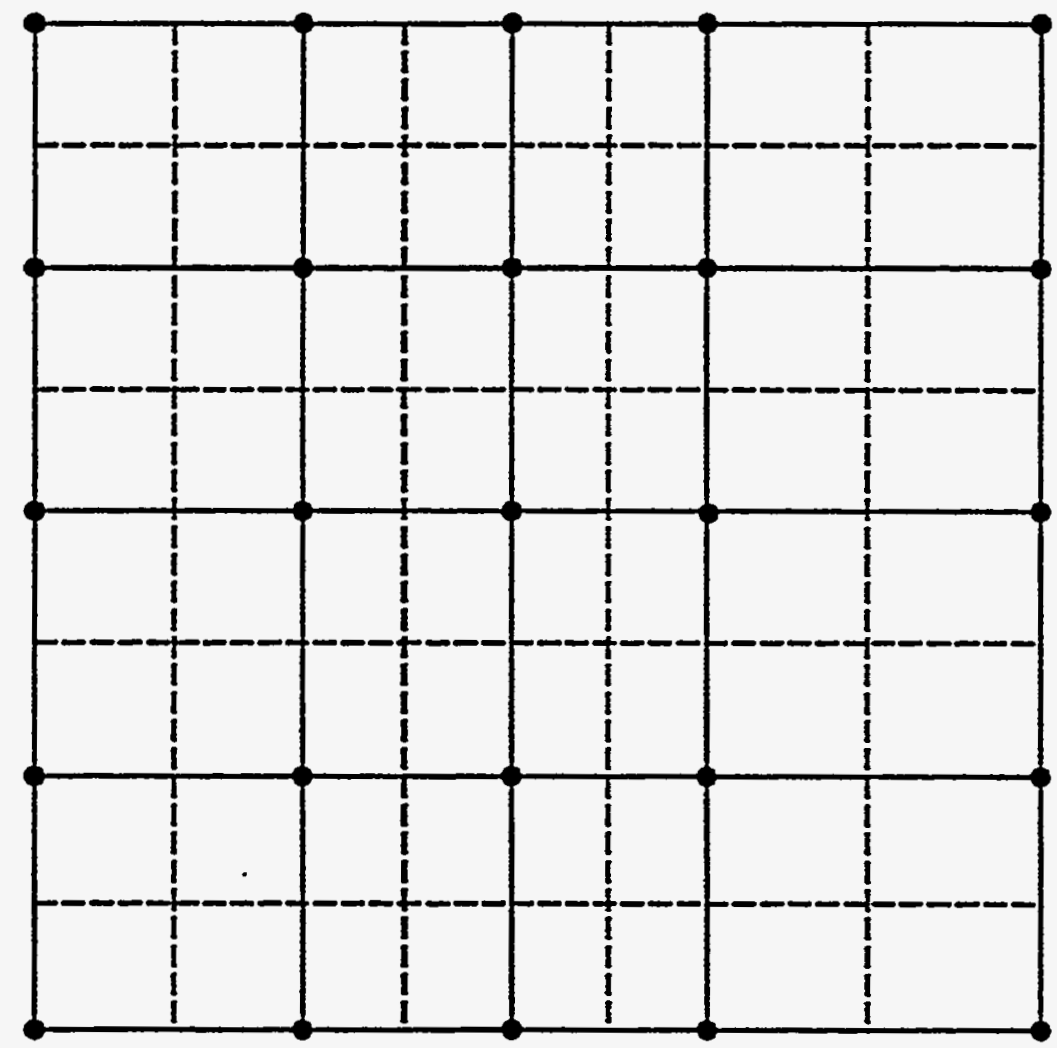

Fig. 20: Element Mesh Superimposed on Point-Centered Grid. ${ }^{30}$ 


$$
\begin{aligned}
\xi & =\frac{x-x_{1}}{x_{2}-x_{1}} \\
\eta & =\frac{y-y_{1}}{y_{2}-y_{1}} .
\end{aligned}
$$

Differentiating Eqs. (58) and (59) and rearranging gives the following expressions:

$$
\begin{aligned}
& d x=\left(x_{2}-x_{1}\right) d \xi=\Delta x d \xi \\
& d y=\left(y_{2}-y_{1}\right) d \eta=\Delta y d \eta .
\end{aligned}
$$

From analyzing the trial functions in Figure 18 for the first element, the corresponding dimensionless trial functions and their first derivatives can be expressed as follows:

$$
\begin{gathered}
U_{1}(\xi)=1-\xi \\
U_{2}(\xi)=\xi \\
W_{1}(\eta)=1-\eta \\
W_{2}(\eta)=\eta \\
U_{1}^{\prime}(\xi)=-1 \\
U_{2}^{\prime}(\xi)=1 \\
W_{1}^{\prime}(\eta)=-1 \\
W_{2}^{\prime}(\eta)=1 .
\end{gathered}
$$

Using Eqs. (60) and (61), the derivative of the trial functions can be written as:

$$
\begin{gathered}
\frac{d u_{i}}{d x}=\frac{d\left(U_{i}(\xi)\right)}{d x}=\frac{d\left(U_{i}(\xi)\right)}{\Delta x d \xi} \\
\frac{d w_{j}}{d y}=\frac{d\left(W_{j}(\eta)\right)}{d y}=\frac{d\left(W_{j}(\eta)\right)}{\Delta y d \eta}
\end{gathered}
$$


In Eq. (57), it is shown how pressure is represented using bilinear interpolation. The mobility elements $\left(\lambda_{x x}, \lambda_{x y}, \lambda_{y x}\right.$, and $\left.\lambda_{y y}\right)$ may each be represented in a similar manner:

$$
\begin{aligned}
\lambda(\xi, \eta) & \approx \sum_{i=1}^{2} \sum_{j=1}^{2} U_{i}(\xi) W_{j}(\eta) \lambda_{i j} \\
& =U_{1}(\xi) W_{1}(\eta) \lambda_{1,1}+U_{2}(\xi) W_{1}(\eta) \lambda_{2,1}+U_{1}(\xi) W_{2}(\eta) \lambda_{1,2}+U_{2}(\xi) W_{2}(\eta) \lambda_{2,2} \\
& =(1-\xi)(1-\eta) \lambda_{1,1}+\xi(1-\eta) \lambda_{2,1}+(1-\xi) \eta \lambda_{1,2}+\xi \eta \lambda_{2,2}
\end{aligned}
$$

In substituting Eqs. (60), (61), (70), and (71) into Eq. (56), the following expression can be obtained representing a general transmissibility coefficient:

$$
\begin{aligned}
B_{i j k \ell}= & \frac{\Delta y}{\Delta x} \int_{0}^{1} \int_{0}^{1} \lambda_{x x}(\xi, \eta) U_{i}^{\prime}(\xi) U_{k}^{\prime}(\xi) W_{j}(\eta) W_{\ell}(\eta) d \xi d \eta \\
& +\int_{0}^{1} \int_{0}^{1} \lambda_{x y}(\xi, \eta) U_{i}(\xi) U_{k}^{\prime}(\xi) W_{j}^{\prime}(\eta) W_{\ell}(\eta) d \xi d \eta \\
& +\int_{0}^{1} \int_{0}^{1} \lambda_{y x}(\xi, \eta) U_{i}^{\prime}(\xi) U_{k}(\xi) W_{j}(\eta) W_{\ell}^{\prime}(\eta) d \xi d \eta \\
& +\frac{\Delta x}{\Delta y} \int_{0}^{1} \int_{0}^{1} \lambda_{y y}(\xi, \eta) U_{i}(\xi) U_{k}(\xi) W_{j}^{\prime}(\eta) W_{\ell}^{\prime}(\eta) d \xi d \eta
\end{aligned}
$$

The following transmissibility coefficients can be written based on Figure 19 and Eq. (73):

$$
\begin{aligned}
& T_{x 1}=-B_{1222}=-B_{2212} \\
& T_{x 2}=-B_{1121}=-B_{2111} \\
& T_{y 1}=-B_{1112}=-B_{1211} \\
& T_{y 2}=-B_{2122}=-B_{2221} \\
& T_{x y 1}=-B_{1122}=-B_{2211}
\end{aligned}
$$




$$
T_{x y 2}=-B_{2112}=-B_{1221}
$$

Numerical integration is necessary to evaluate the integrals in Eq. (73). Details regarding the numerical integration procedure, the general expressions for the transmissibilities, and the treatment of non-symmetric permeability tensors are found in Appendix A.

\section{Relating the Proposed Method and Shiralkar's Method}

The proposed method is based on finite element theory and has a strong theoretical basis. A suitable choice of the integration parameter, $\omega$, of this method yields identical transmissibilities with Shiralkar's method ${ }^{27}$ for a rectangular finite element of homogeneous, but generally anisotropic permeability. Next, it is shown how to determine $\omega$ such that each of the individual transmissibilities of the two methods agree.

First, a value of $\omega$ is selected such that $T_{x y 2}$ is equal for both methods. $g_{x y_{a}}$ is positive for the following derivation. Based on Eq. (A20) in the Appendix, $\omega=1$ is used for all the off-diagonal elements of the mobility tensor. $T_{x y 2}$ of the proposed method [Eq. (A12)] is set equal to $T_{x y 2}$ of Shiralkar's method:

$$
\frac{1}{4} \omega\left(g_{x x}+g_{y y}\right)-\frac{1}{2} g_{x y_{a}}=\frac{1}{3} \frac{\left(g_{x x}-g_{x y_{a}}\right)\left(g_{y y}-g_{x y_{a}}\right)}{\left(g_{x x}-g_{x y_{a}}\right)+\left(g_{y y}-g_{x y_{a}}\right)}
$$

where $g_{x y_{a}}$ is defined by Eq. (A20) and $g_{x x}$ and $g_{y y}$ are defined as follows:

$$
\begin{aligned}
& g_{x x}=\frac{1}{4}\left[\left(\lambda_{x x}\right)_{1,1}+\left(\lambda_{x x}\right)_{2,1}+\left(\lambda_{x x}\right)_{1,2}+\left(\lambda_{x x}\right)_{2,2}\right] \frac{\Delta y}{\Delta x} \\
& g_{y y}=\frac{1}{4}\left[\left(\lambda_{y y}\right)_{1,1}+\left(\lambda_{y y}\right)_{2,1}+\left(\lambda_{y y}\right)_{1,2}+\left(\lambda_{y y}\right)_{2,2}\right] \frac{\Delta x}{\Delta y} .
\end{aligned}
$$

Eq. (80) can be solved for $\omega$ :

$$
\omega=\frac{4}{3} \frac{\left(g_{x x}-g_{x y_{a}}\right)\left(g_{y y}-g_{x y_{a}}\right)}{\left[\left(g_{x x}-g_{x y_{a}}\right)+\left(g_{y y}-g_{x y_{a}}\right)\right]\left(g_{x x}+g_{y y}\right)}+\frac{2 g_{x y_{a}}}{\left(g_{x x}+g_{y y}\right)} .
$$

Using the expression of $\omega$ shown in Eq. (83) for terms containing the diagonal elements of the mobility tensor and $\omega=1$ for the off-diagonal elements, the transmissibilities of the two methods become identical for a rectangular finite element with homogeneous, but generally anisotropic permeability.

When $g_{x y_{a}}$ is negative, a value of $\omega$ is selected such that $T_{x y 1}$ is equal for both methods. Again, $\omega=1$ is used for all the off-diagonal elements of the mobility tensor. $T_{x y 1}$ of the proposed method [Eq. (A11)] is set equal to $T_{x y 1}$ of Shiralkar's method: 


$$
\frac{1}{4} \omega\left(g_{x x}+g_{y y}\right)+\frac{1}{2} g_{x y_{a}}=\frac{1}{3} \frac{\left(g_{x x}+g_{x y_{a}}\right)\left(g_{y y}+g_{x y_{a}}\right)}{\left(g_{x x}+g_{x y_{a}}\right)+\left(g_{y y}+g_{x y_{a}}\right)} .
$$

Eq. (84) can be solved for $\omega$ :

$$
\omega=\frac{4}{3} \frac{\left(g_{x x}+g_{x y_{a}}\right)\left(g_{y y}+g_{x y_{a}}\right)}{\left[\left(g_{x x}+g_{x y_{a}}\right)+\left(g_{y y}+g_{x y_{a}}\right)\right]\left(g_{x x}+g_{y y}\right)}-\frac{2 g_{x y_{a}}}{\left(g_{x x}+g_{y y}\right)} .
$$

Comparisons Between Results From Waterflood Simulations

The cases presented involve comparisons of results from simulating waterfloods using initial permeability distributions and upscaled effective permeability distributions generated based on the analytical effective permeability method, ${ }^{22}$ the realspace renormalization technique, ${ }^{19}$ and the geometric average. Two-phase oil and water systems are simulated. The simulator used is a point-centered finite difference simulator which has the option of inserting separately computed transmissibilities. ${ }^{31}$ Therefore, the 9-point based transmissibility equations developed above are used to compute the transmissibility coefficients to be inserted into the simulator. The initial permeability distributions shown are all in Darcy units. Except for Case 1 , permeabilities are scaled (multiplied) by 0.03 before transmissibilities are calculated. Simulator input parameters are listed in Table 2. The relative permeability curves are shown in Figure 21 and are identical to those used by White and Horne. ${ }^{3}$

Case 1: The first case presented is based on the cross-bedded permeability distribution shown in Figure 22. The light grid cells have a permeability of $1000 \mathrm{md}$, whereas the dark grid cells represent permeability of $20 \mathrm{md}$. $32 \times 32$ grid points are used.

Water displaces the in-situ oil by full face injection at the left border, and full face fluid production takes place at the right border of the permeability distribution in Figure 22. Constant injection and production rates of $100 \mathrm{bbl} / \mathrm{d}$ are used in this case.

Waterflooding is performed using the initial permeability distribution, the analytical effective permeability tensor, and renormalized permeability. Results are compared in terms of pressure maps, water saturation maps and oil recovery curves.

The pressure maps after 0.5 days of injection are shown in Figure 23. The pressure map for the initial permeability distribution (Figure 23a) shows that there is clearly a transverse component of the pressure gradient since the isopotential lines have a general orientation of decreasing pressure from southwest to northeast. Notice, however, the effect of the high permeability layers, within which pressure is decreasing from northwest to southeast. Such detail can not be retained for pressure maps related to the upscaled permeability distribution. However, the effective permeability tensor produces a permeability map which preserves the general trend of decreasing pressure from southwest to northeast as can be observed from Figure 23b. Thus, the main effects of the cross-bedded permeability structures are 


\begin{tabular}{|c|c|}
\hline \multicolumn{2}{|c|}{ Table 2} \\
\hline \multicolumn{2}{|c|}{ Simulation Input Parameters } \\
\hline $\mathrm{mo}_{0}$ & $1.0 \mathrm{cp}$ \\
\hline $\mathrm{m}_{\mathbf{W}}$ & $1.0 \mathrm{cp}$ \\
\hline ro & $62.4 \mathrm{lbm} / \mathrm{ft}^{3}$ \\
\hline$r_{w}$ & $62.4 \mathrm{lbm} / \mathrm{ft}^{3}$ \\
\hline$c_{0}$ & $1.0 \times \mathrm{E}-10 \mathrm{psi}-1$ \\
\hline$c_{W}$ & $1.0 \times \mathrm{E}-6 \mathrm{psi}-1$ \\
\hline $\mathrm{I}_{\mathrm{X}}$ & $600 \mathrm{ft}$ \\
\hline Ly & $600 \mathrm{ft}$ \\
\hline h & $10 \mathrm{ft}$ \\
\hline$f$ & 0.20 \\
\hline Pinitial & $1000 \mathrm{psi}$ \\
\hline
\end{tabular}




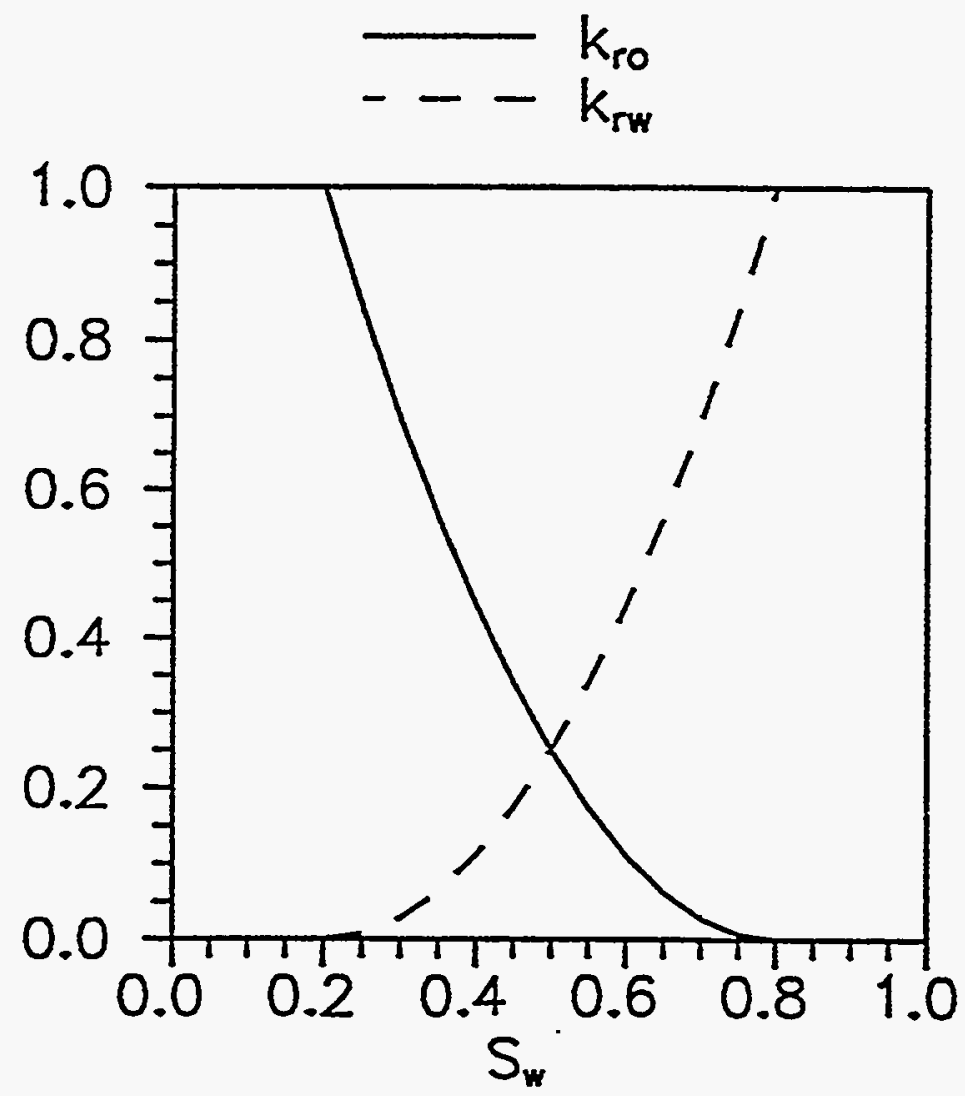

Fig. 21: Relative Permeability Curves. ${ }^{3}$ 
Light Blocks: $1000 \mathrm{md}$; Dark Blocks: $20 \mathrm{md}$

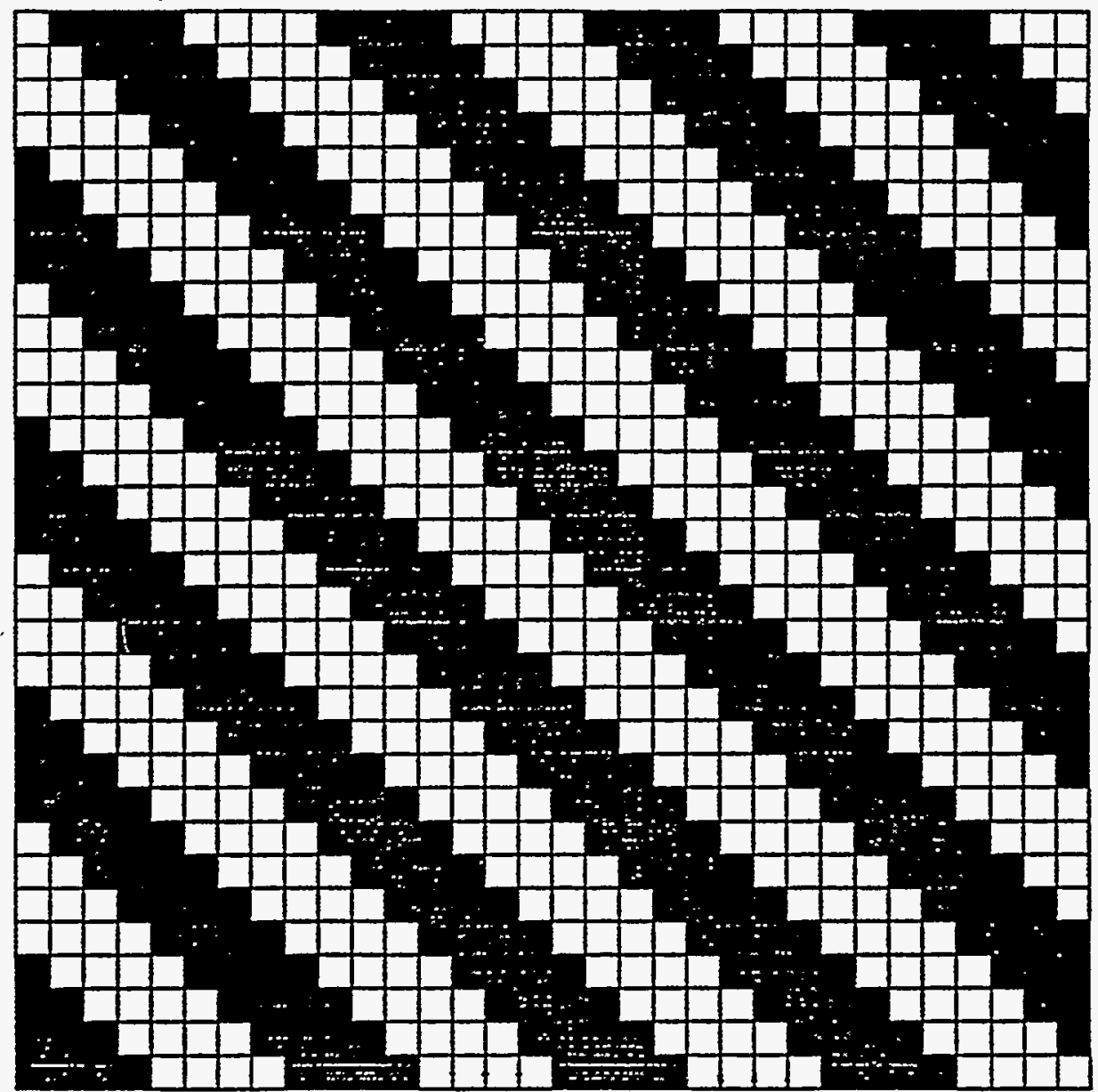

Fig. 22: Cross-Bedded Permeability Distribution. 

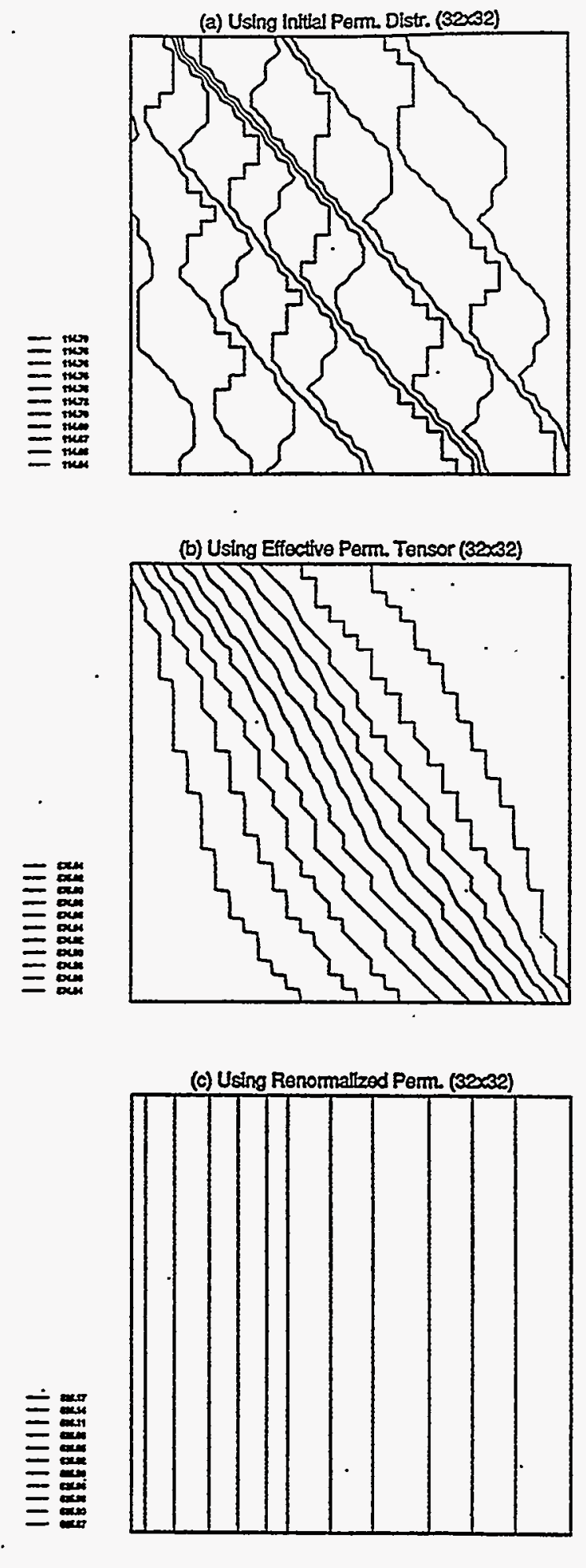

Fig. 23: Pressure Maps After 0.5 Days of Water Injection Using (a) the Initial Cross-Bedded Permeability Distribution (b) the Effective Permeability Tensor and (c) the Real-Space Renormalized Permeability (Units: psi). 
maintained through the upscaling process. Using real-space renormalization of permeability, however, the characteristics of the pressure map from using the initial permeability distribution are lost as can be observed from Figure 23c.

Water saturation maps after $0.5,1.0$, and 1.5 days of water injection are shown in Figures 24, 25, and 26, respectively. Figure 24a shows the water saturation map after 0.5 days of water injection using the initial cross-bedded permeability distribution. The injected water flows locally along the high permeability layers, but the non-uniform shape of the overall water saturation front is controlled by the net transverse pressure drop being almost perpendicular to the alternating high and low permeability layers as shown in Figure 23a. Even though the effects of some of the details of the initial permeability distribution are lost, the water saturation front after 0.5 days of injection using the effective permeability tensor is very similar in shape as can be seen in Figure 24b. However, using the renormalized permeability, the water saturation front after 0.5 days of injection (Figure 24c) does not show any of the characteristics of the water saturation front obtained using the initial permeability distribution. Water is uniformly sweeping the oil as can be seen in Figure 24c.

Figure 25a shows the water saturation map after 1.0 days of injection using the initial permeability distribution. More of the high permeability layers have been flooded, and water breakthrough is soon to occur in the lower portion of the east boundary. Using the effective permeability tensor, water breakthrough has just occurred in the lower portion of the east boundary as can be seen from Figure 25b. Loss of detail in upscaling the initial permeability distribution accounts for the slightly earlier water brealthrough obtained using the effective permeability tensor. The high permeability layers in the initial permeability distribution contribute to stabilizing the water saturation front somewhat and to some degree improving the sweep efficiency. Using the renormalized permeability, however, the water saturation front after 1.0 days of injection is far from breaking through and yields an overly optimistic sweep efficiency as can be observed from Figure 25c.

After 1.5 days of injection, water breakthrough has occurred using both the initial permeability distribution and the upscaled permeabilities (Figure 26). Both using the initial permeability distribution (Figure 26a) and the effective permeability tensor (Figure 26b) oil is left behind in the upper northeast corner of the field. Also, in both these cases, an incomplete sweep of the oil takes place in the extreme lower part of the field, but most clearly so using the effective permeability tensor. The water saturation front obtained using the renormalized permeability is uniform and show optimistic sweep efficiency as can be seen in Figure 26c.

The sweep efficiencies may be compared from the oil recovery curves shown in Figure 27 where cumulative oil recovery is plotted as a function of elapsed time of water injection. The effective permeability tensor underestimates the sweep effciency based on the lower recovery curve, whereas renormalized permeability clearly overestimates the sweep efficiency based on the higher recovery curve.

Case 2: The permeability distribution used in this case is shown in Figure 28. This isotropic permeability distribution is generated using the Turning Bands method. ${ }^{25}$ 

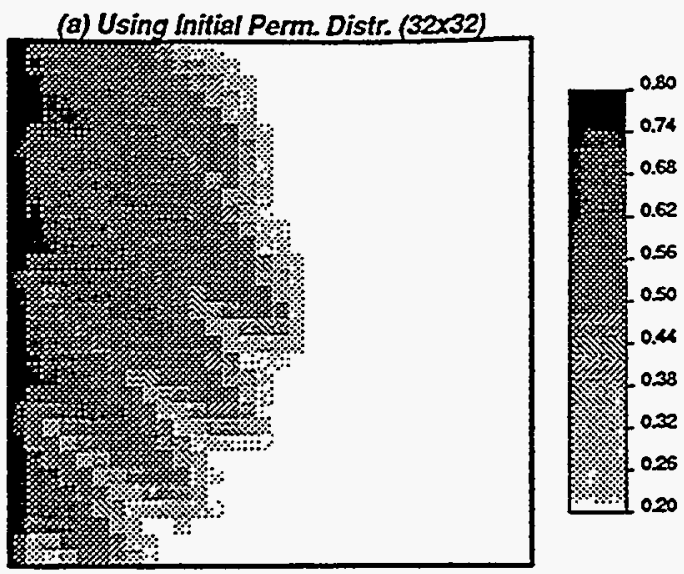

(b) Using Effective Perm. Tensor (32x32)
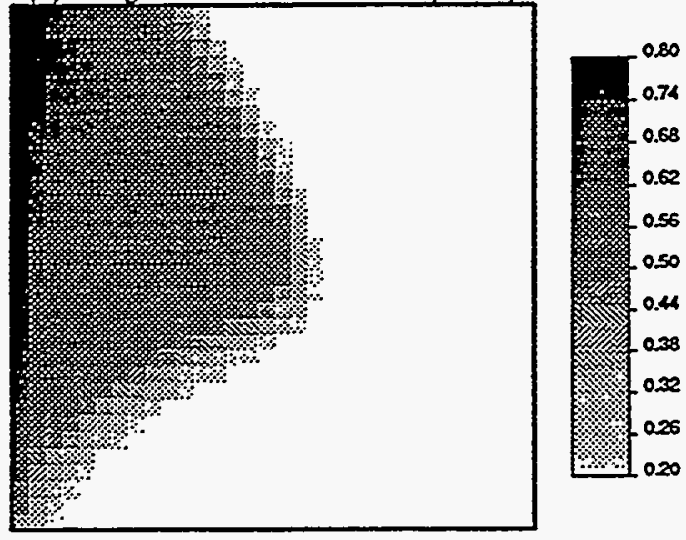

(c) Using Renormalized Perm. (32x32)
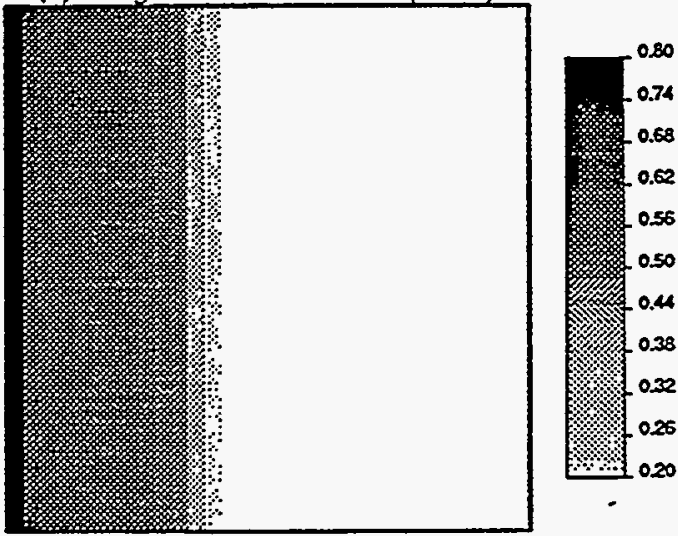

Fig. 24: Water Saturation Maps After 0.5 Days of Water Injection Using (a) the Initial Cross-Bedded Permeability Distribution (b) the Effective Permeability Tensor and (c) the Real-Space Renormalized Permeability. 
(a) Using Initial Perm. Distr. (32×32)

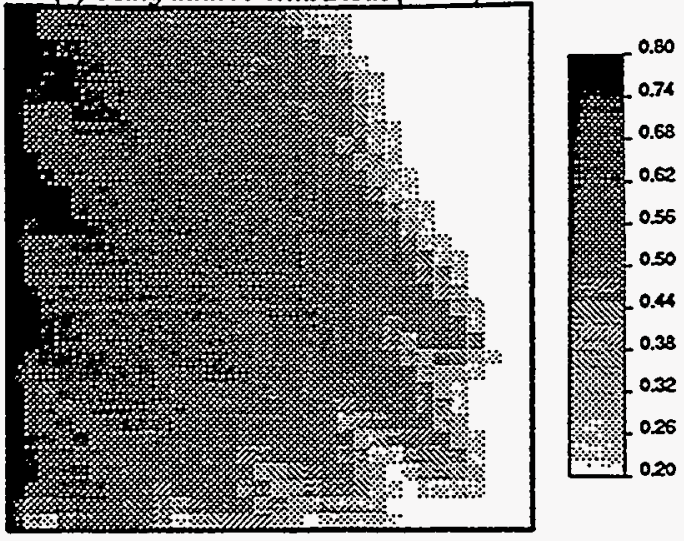

(b) Using Effective Perm Tensor (32x32)

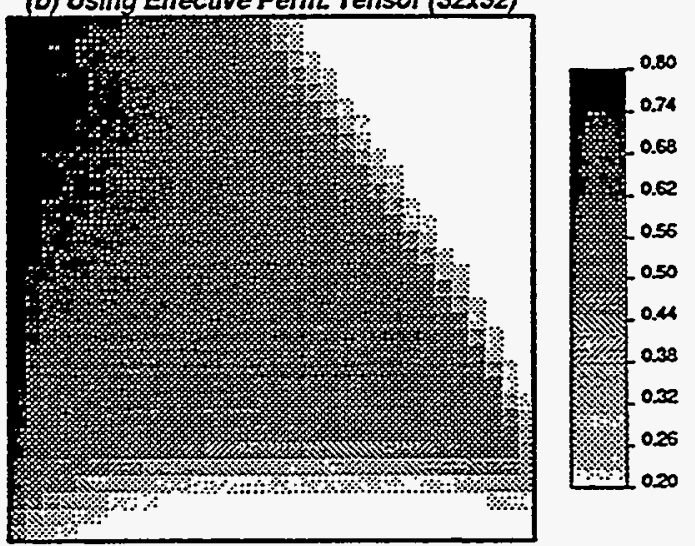

(c) Using Renormalized Perm. (32x32)

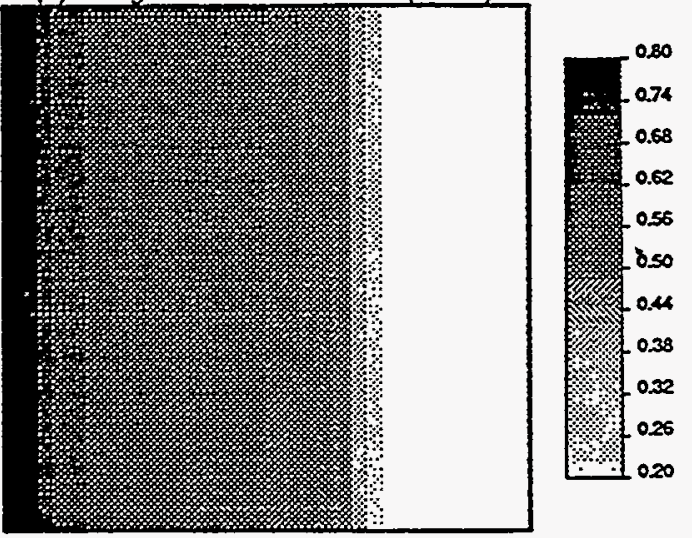

Fig. 25: Water Saturation Maps After 1.0 Days of Water Injection Using (a) the Initial Cross-Bedded Permeability Distribution (b) the Effective Permeability Tensor and (c) the Real-Space Renormalized Permeability. 

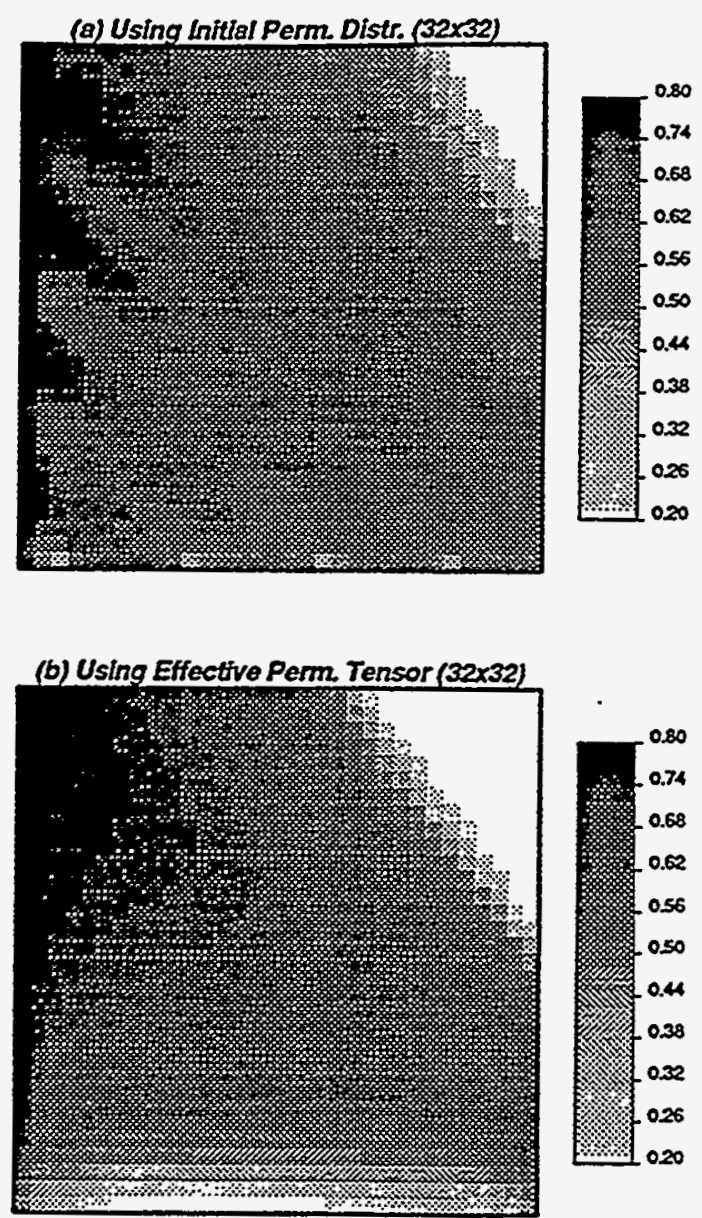

(c) Using Renomalized Pom. (32x32)

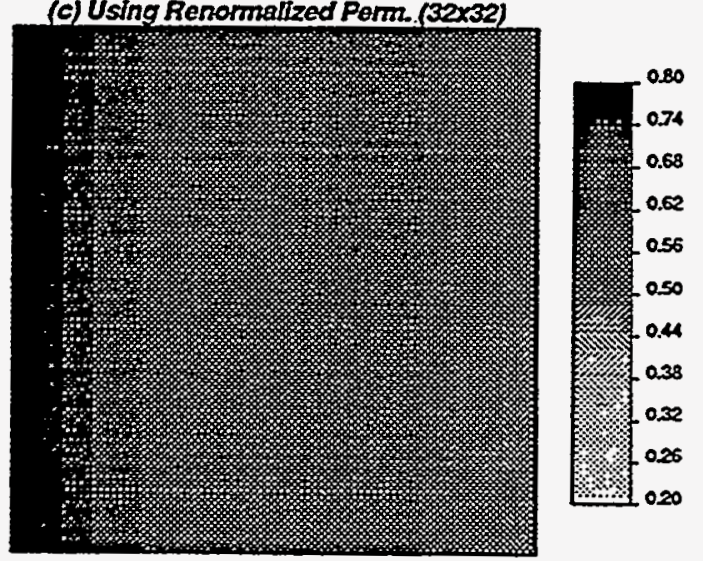

Fig. 26: Water Saturation Maps After 1.5 Days of Water Injection Using. (a) the Initial Cross-Bedded Permeability Distribution (b) the Effective Permeability Tensor and (c) the Real-Space Renormalized Permeability. 


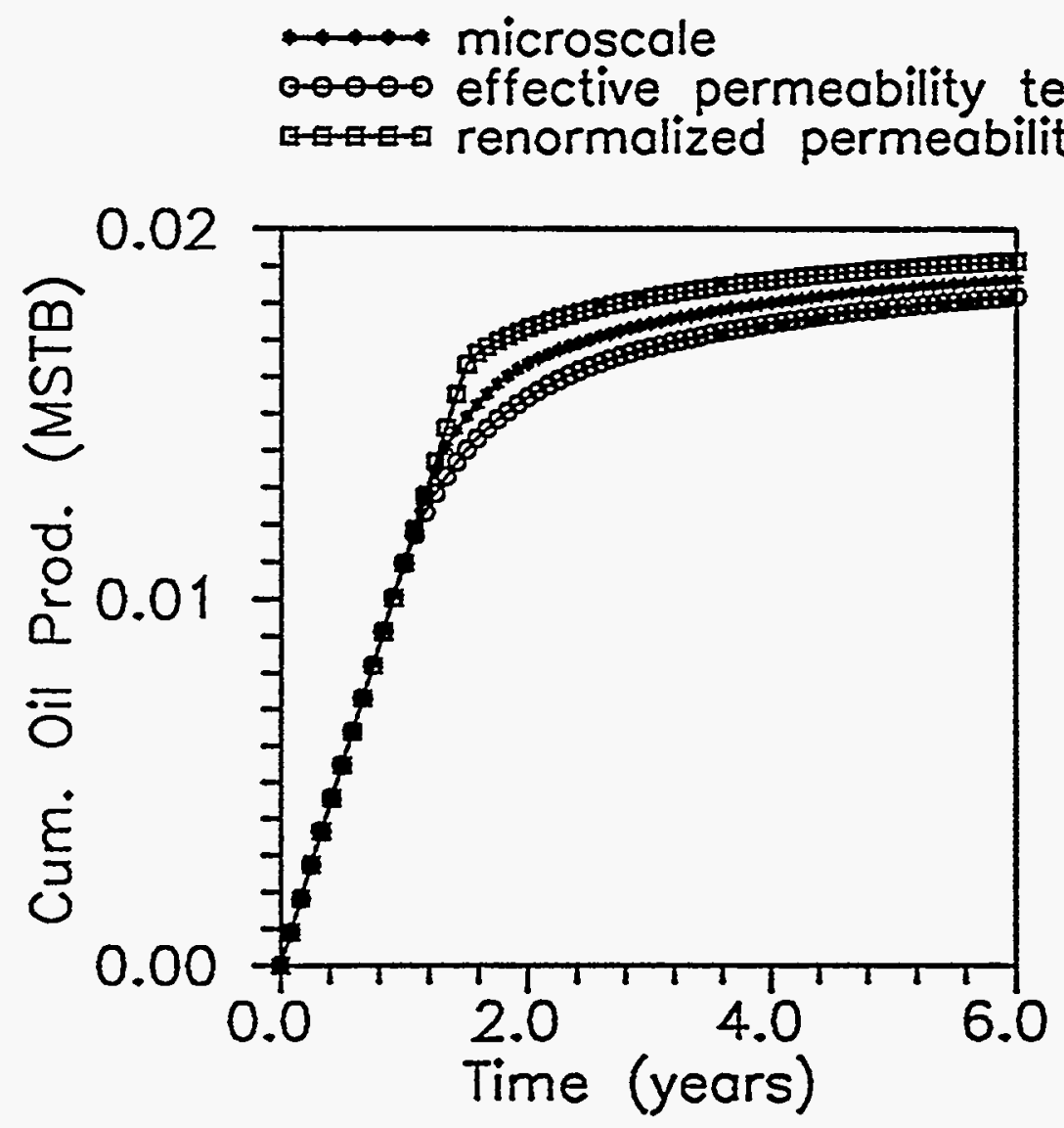

Fig. 27: Cumulative Oil Recovery Versus Time for Rectilinear Waterflood. Cross-Bedded Initial Permeability Distribution. Effect of Permeability Upscaling. 


\section{Isotropic Initial Permeability Distr.}
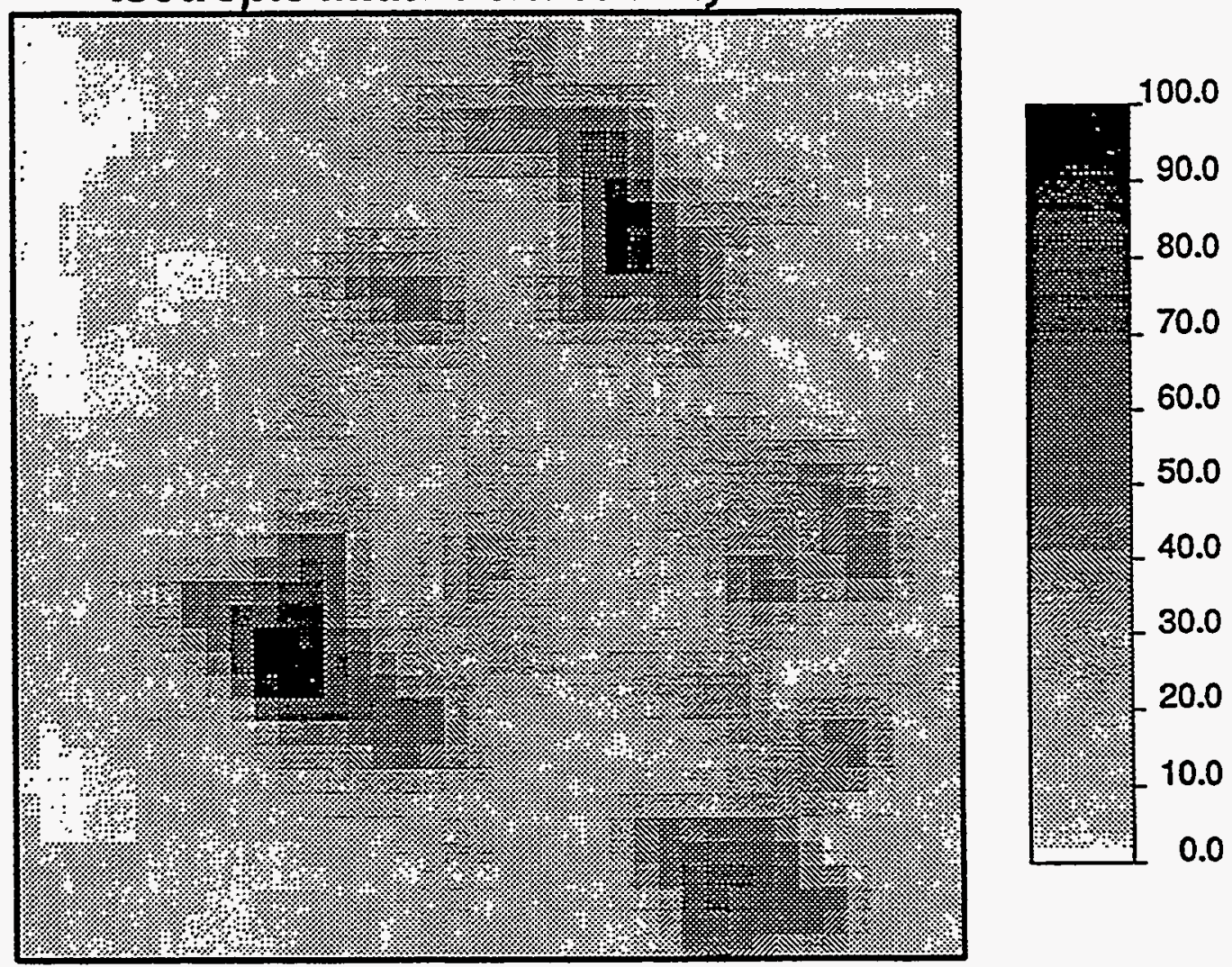

Fig. 28: Isotropic Stochastic Permeability Distribution.

( $\lambda_{D}=0.5, V=0.7,40 \times 40$ Values). 
Statistical input parameters are: isotropic dimensionless correlation length of 0.5 and Dykstra-Parsons coefficient, $V=0.7$. $40 \times 40$ grid points are used. Water is injected at $100 \mathrm{bbl} / \mathrm{d}$ from the southwest corner and fluid is produced ( $100 \mathrm{bbl} / \mathrm{d}$ ) at the northeast corner of the field in a quarter of a 5-spot pattern.

For this case and the remaining cases presented, the geometric average are used rather than the real-space renormalization technique.

Figure 29 shows cumulative oil produced as a function of injection time for the initial (microscale) permeability distribution, the effective permeability tensor, and the geometric average of the initial permeability distribution. The upscaled distributions are scaled from $40 \times 40$ to $5 \times 5$ permeability values. For this isotropic microscale permeability distribution, the effect of permeability upscaling is small and not sensitive to the upscaling technique used. The recovery curves obtained using upscaled permeability are indistinguishable from one another and do not differ much from the recovery curve using the initial permeability distribution.

Case 3: This case also represents waterflooding in a quarter of a 5-spot pattern. However, in this case, the permeability distribution is anisotropic and oriented at a $45^{\circ}$ angle from the horizontal $x$-axis. In other words, the orientation of the anisotropic permeability structures are parallel to the general flow direction from the injector in the southwest corner and the producer in the northeast corner of the field. The dimensionless correlation lengths are $\lambda_{D_{l}}=1.0$ and $\lambda_{D_{t}}=0.025$ along the layers and transverse to the layers, respectively. The Dykstra-Parsons coefficient is $V=0 . \tau$. There are $64 x 64$ permeability values in the initial permeability distribution shown in Figure 30. Injection pressure is 3000 psi and production pressure is 100 psi.

The water saturation fronts. after 20 days of injection are shown in Figure 31. Figure 31a shows the water saturation front using the initial, generally anisotropic permeability distribution. Water breakthrough has already occurred because of the preferential flow paths created by the cross-bedded arrangement of the permeability values in the field. The microscale permeability distribution is upscaled or homogenized from $64 x 64$ to $8 \times 8$ permeability values. Comparing Figures $31 \mathrm{~b}$ and $31 \mathrm{c}$, it is evident that using the effective permeability tensor, the water saturation front agrees better in terms of shape and amount of water broken through (Figure 31b) than using the geometric average of the permeability values (Figure 31c). The geometric average method is not able to capture the anisotropic nature of the initial permeability distribution.

In Figure 32, the cumulative oil recovery is plotted as a function of pore volumes of water injected for waterflooding through the microscale and the upscaled permeability distributions. From this figure, it can be observed that the recovery curve related to the effective permeability tensor shows excellent agreement with the recovery curve associated with the initial microscale permeability distribution. The recovery curve associated with the geometric average of the initial permeability distribution is too optimistic.

Case 4: The final case represents waterflooding in a 5-spot pattern. An injector is located in the middle of the field and injecting water at 3000 psi. Four producers, one 


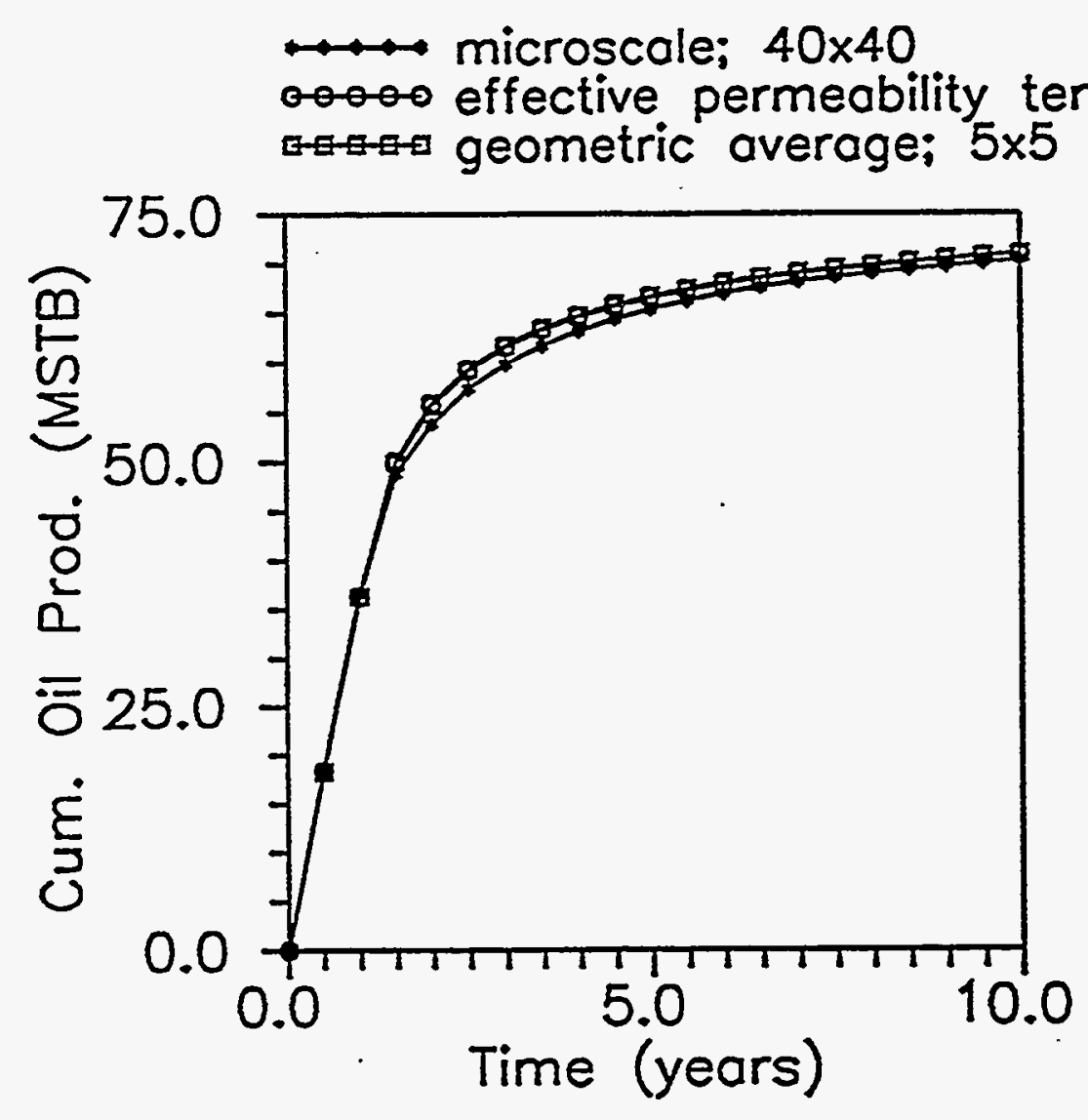

Fig. 29: Cumulative Oil Recovery Versus Time for Waterflood in a 1/4 of a 5-Spot. Isotropic Initial Permeability Distribution. Effect of Permeability Upscaling. 


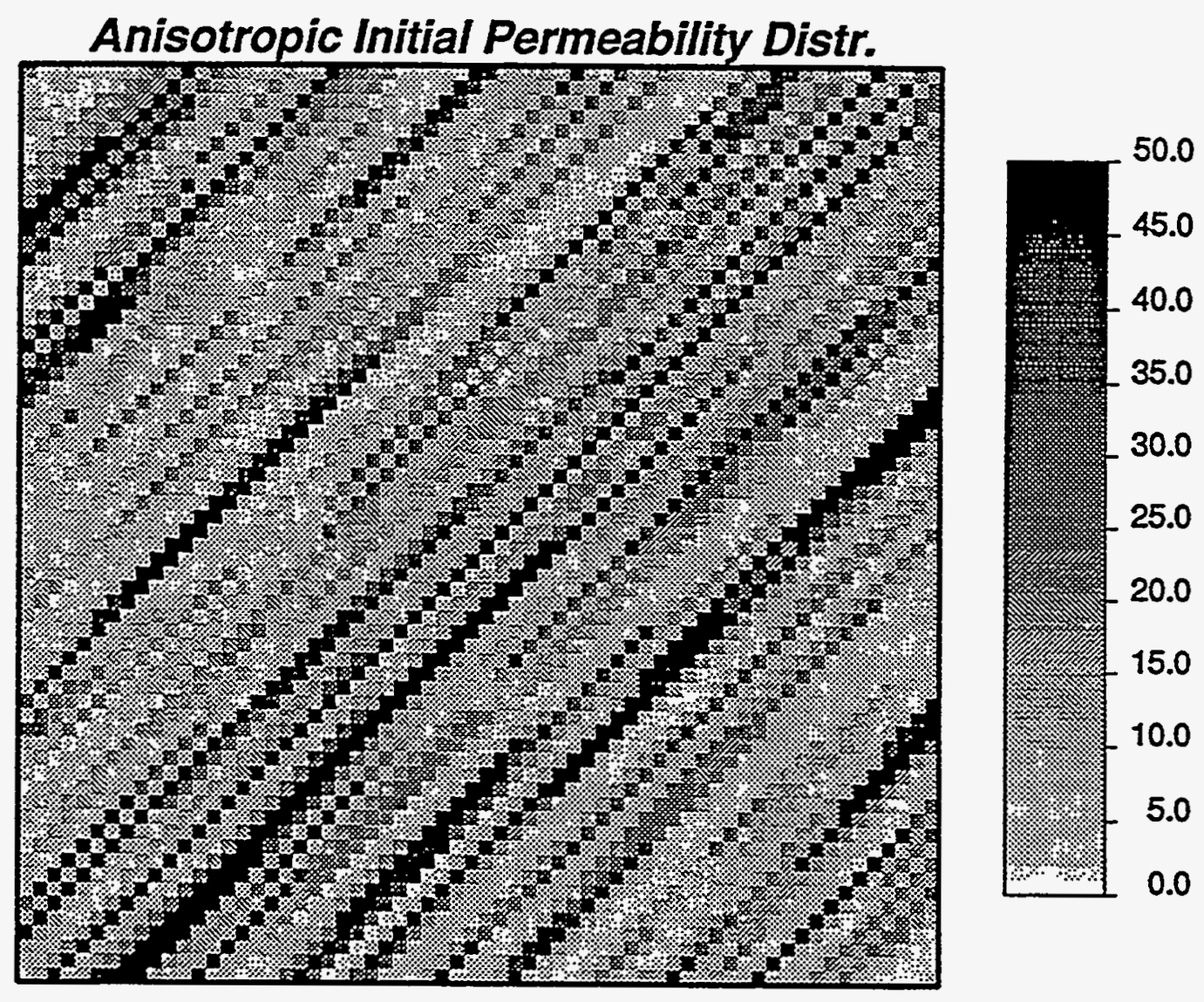

Fig. 30: Anisotropic (Cross-Bedded) Stochastic Permeability Distribution. $\left(\lambda_{D_{\ell}}=1.0, \lambda_{D_{t}}=0.025, V=0.7,45^{\circ}\right.$ Structure Orientation, $64 \times 64$ Values). 
(a) Using Initial Perm. Distr. $(64 \times 64)$

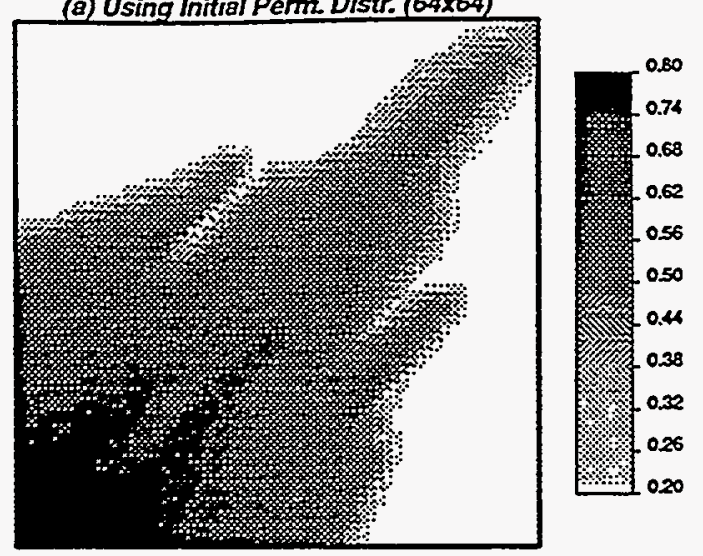

(b) Using Effective Perm Tensor (8x8)

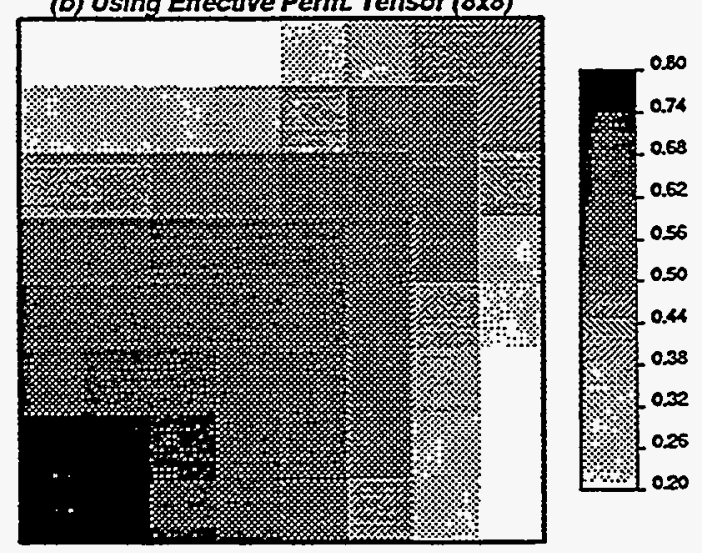

(c) Using Geometric Average ( $8 \times 8$ )

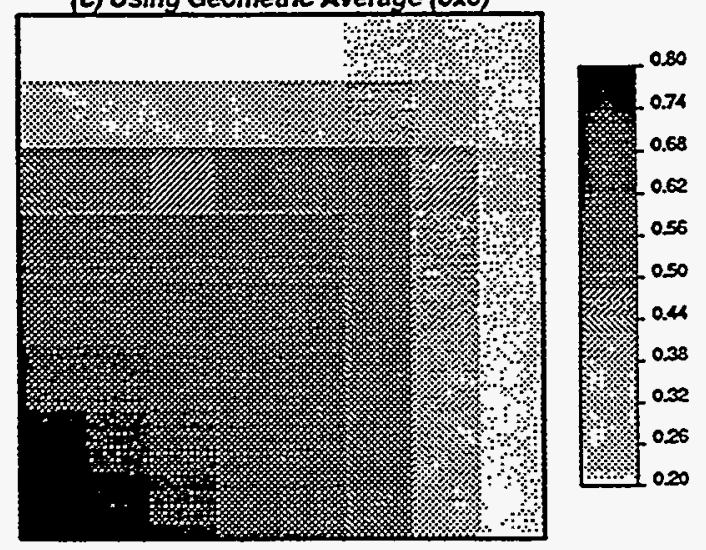

Fig. 31: Water Saturation Maps After 20 Days of Water Injection Using (a) the Initial Permeability Distribution (b) the Effective Permeability Tensor and (c) the Geometrically Averaged Permeability. 


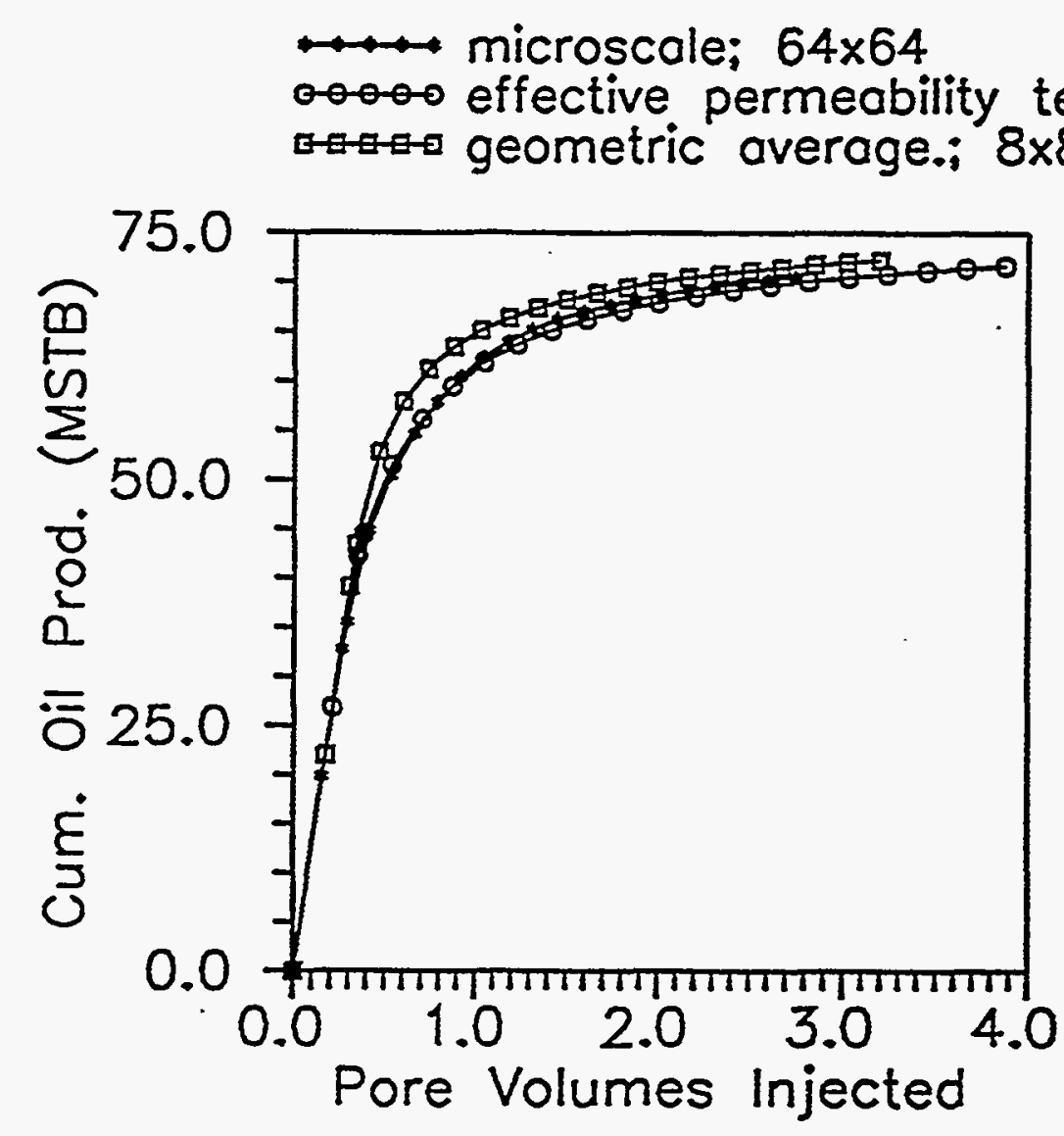

Fig. 32: Cumulative Oil Recovery Versus Pore Volumes Injected for Waterflood in a 1/4 of a 5-Spot. Anisotropic (Cross-Bedded) Initial Permeability Distribution. Effect of Permeability Upscaling. 
in each corner of the field, are producing fluid at $100 \mathrm{psi}$. The main difference from the permeability distribution used in Case 3 is the use of $63 \times 63$ permeability values. An odd number of grid points along each axis are necessary to place the injector in the very middle of the field. In addition, the dimensionless correlation length transverse to the layers is $\lambda_{D_{z}}=0.016$. Dykstra-Parsons coefficient is $V=0.7$. The distribution of the initial microscale permeability values are shown in Figure 33. The system is coarsened to $9 \times 9$ grid points using the effective permeability tensor and the geometric average. The simulations of the coarsened permeability distributions (9x9 grid points) are approximately 800 times faster than the simulation of the initial permeability distribution.

Figure 34 shows the water saturation maps after 8 days of injection. Using the initial permeability distribution, water has broken through in all of the four producing wells (Figure 34a). However, the wells with the highest water saturation are located in the southwest $(\mathrm{P}-3)$ and the northeast (P-2) corners of the field. This response agrees with the expected response based on the orientation of the permeability streaks. Figure $34 \mathrm{~b}$ shows the water saturation map which is based on the use of the effective permeability tensor. The water saturation distribution agrees well with that observed in Figure 34a in terms of the distribution of the major portion of the injected water along the southwest-northeast diagonal. The water saturation map based on using the geometric average of permeability does not indicate any particular direction of preferential fluid flow, as can be seen in Figure 34c. An approximately equal amount of water has broken through in each of the four producing wells after 8 days of water injection. Clearly, the geometric averaging process is not able to capture the effect of the anisotropic nature of the permeability distribution, whereas the effective permeability tensor is able to capture the existence of permeability anisotropy and the effect of the orientation of the permeability structures.

Figure 35 shows the cumulative oil recovered as a function of pore volumes of water injected for producer number one (P-1) located in the northwest corner of the field. Based on the recovery curves, there appears to be no distinct advantage of using the effective permeability tensor method in favor of the geometric average method since both recovery curves underpredicts the oil recovery to some degree. However, Figure 36 which shows the fractional flow of water as a function of pore volumes of water injected, indicates that water breakthrough is more accurately predicted using the effective permeability tensor.

Cumulative oil recoveries for producing well number two (P-2) are shown in Figure 37 as a function of pore volumes of water injected. A significantly better prediction of the oil recovery is realized using the effective permeability tensor compared to using the geometric average. This observation is closely linked to the water saturation map using the geometric average which shows a fairly uniform water distribution (Figure 34c). Consequently, oil recovery is too optimistic at well P-2 which receives a substantial portion of the injected water according to the waterfloods through the initial permeability distribution (Figure 34a) and using the effective permeability tensor (Figure $34 \mathrm{~b}$ ). Figure 38 supports this observation. 


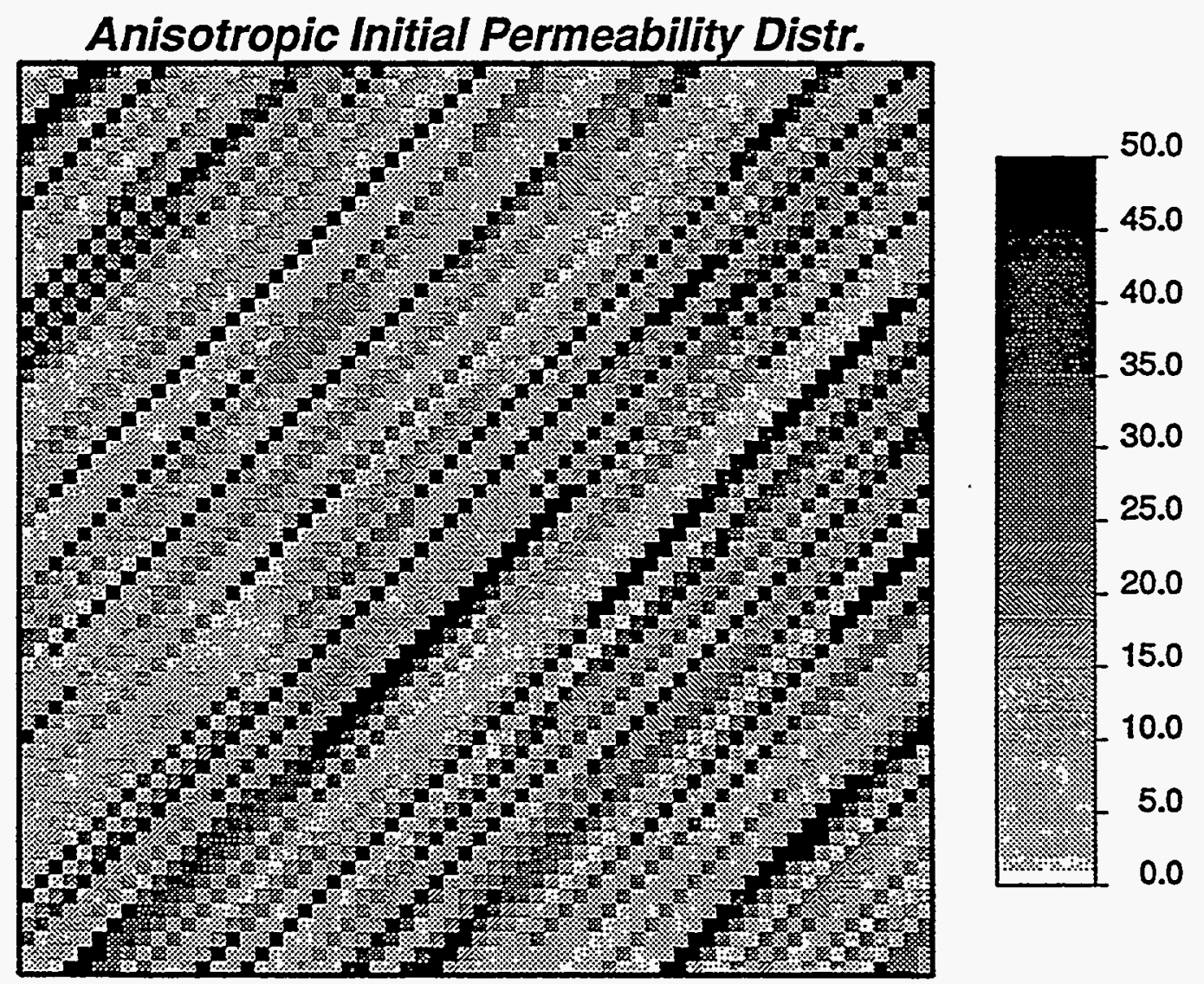

Fig. 33: Anisotropic (Cross-Bedded) Stochastic Permeability Distribution. $\left(\lambda_{D_{\ell}}=1.0, \lambda_{D_{t}}=0.016, V=0.7,45^{\circ}\right.$ Structure Orientation, $63 \times 63$ Values). 
(a) Using foitial Ferm. Distr. (63 6 63)

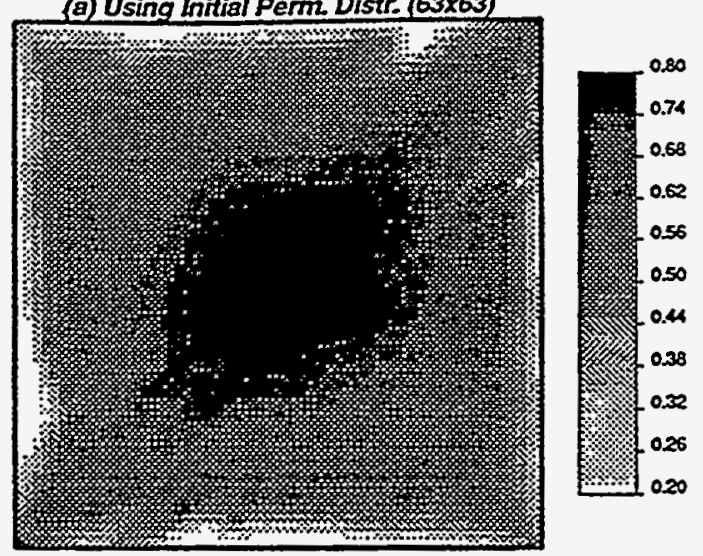

(b) Using Effective Perm. Tensor (9x9)
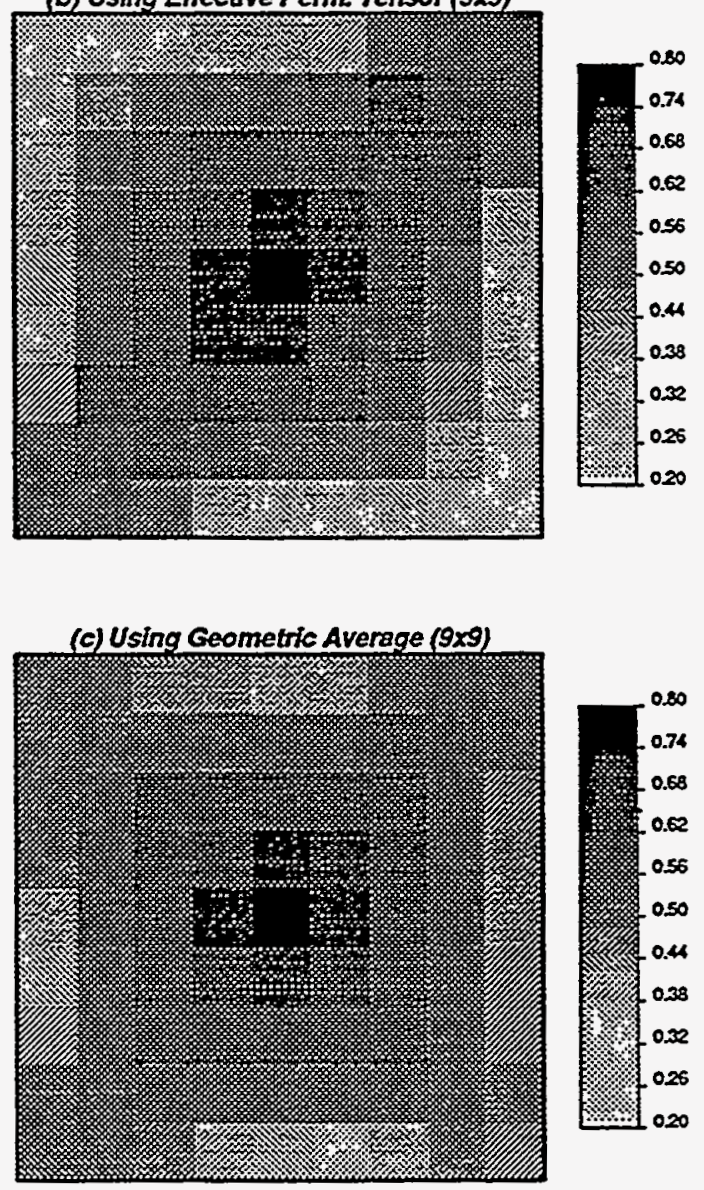

Fig. 34: Water Saturation Maps After 8 Days of Water Injection Using (a) the Initial Permeability Distribution (b) the Effective Permeability Tensor and (c) the Geometrically Averaged Permeability. 


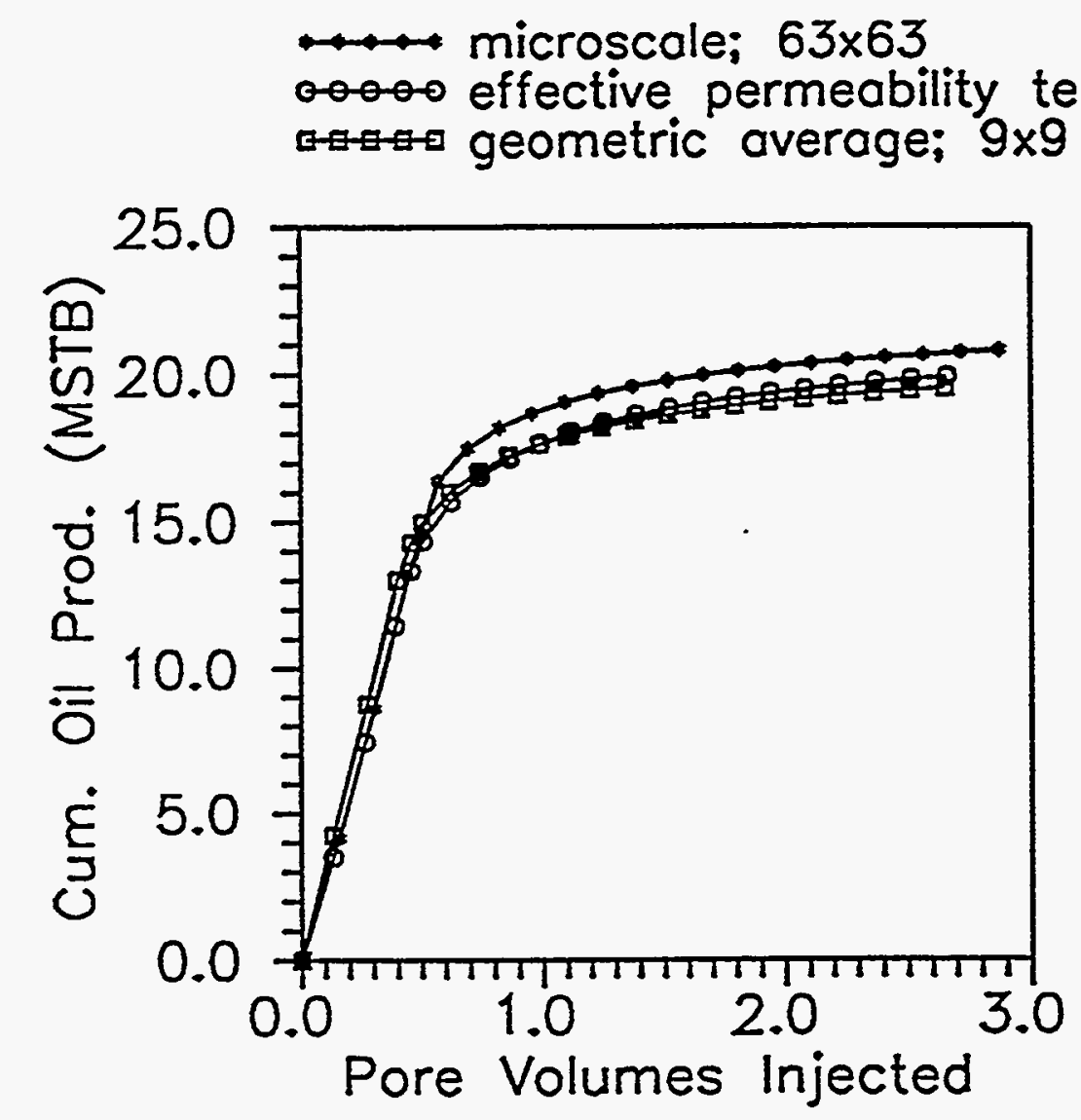

Fig. 35: Cumulative Oil Recovery Versus Pore Volumes Injected for Producing Well P-1 in a 5-Spot Waterflood. Anisotropic (Cross-Bedded) Initial Permeability Distribution. Effect of Permeability Upscaling. 


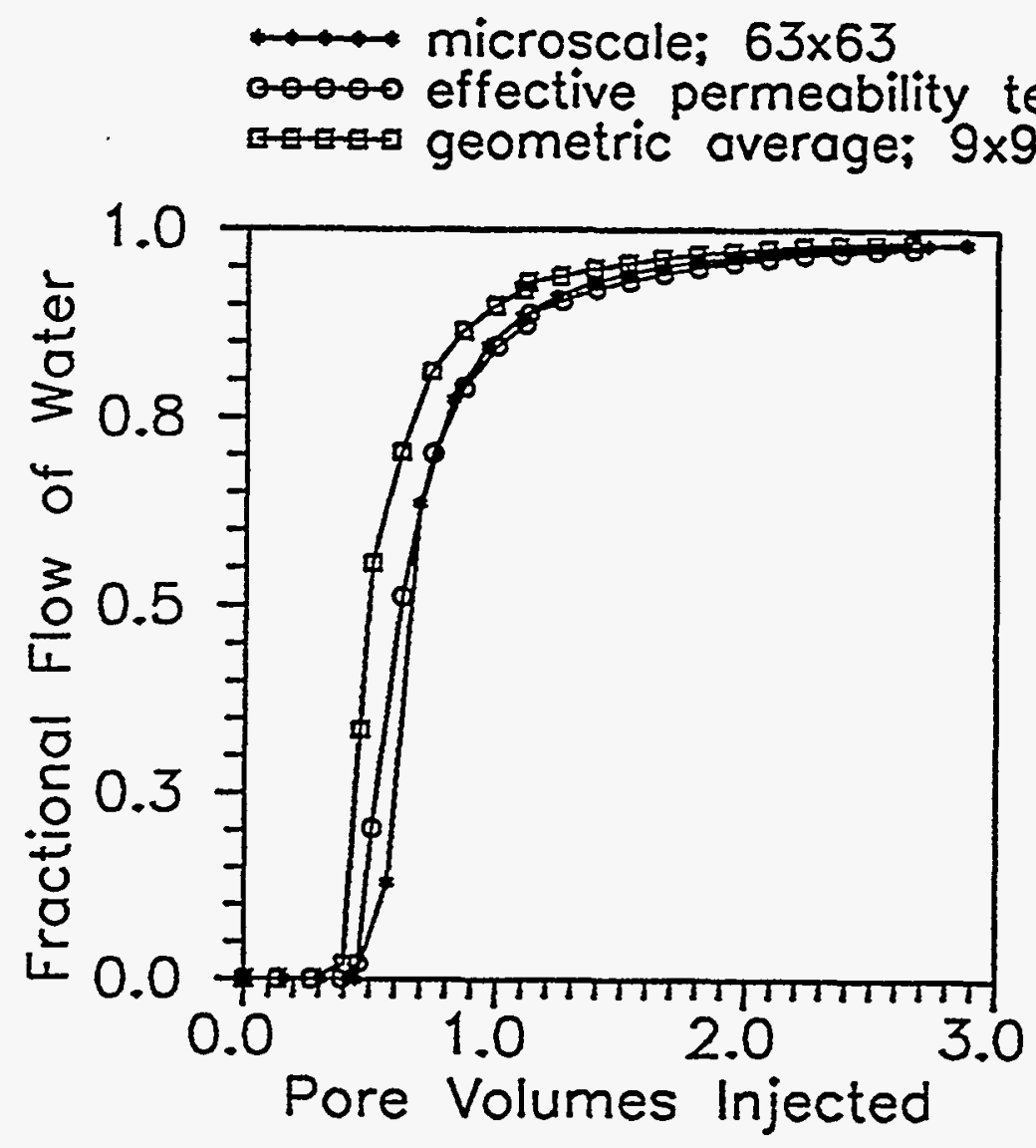

Fig. 36: Fractional Flow of Water Versus Pore Volumes Injected for Producing Well P-1 in a 5-Spot Waterflood. Anisotropic (Cross-Bedded) Initial Permeability Distribution. Effect of Permeability Upscaling. 


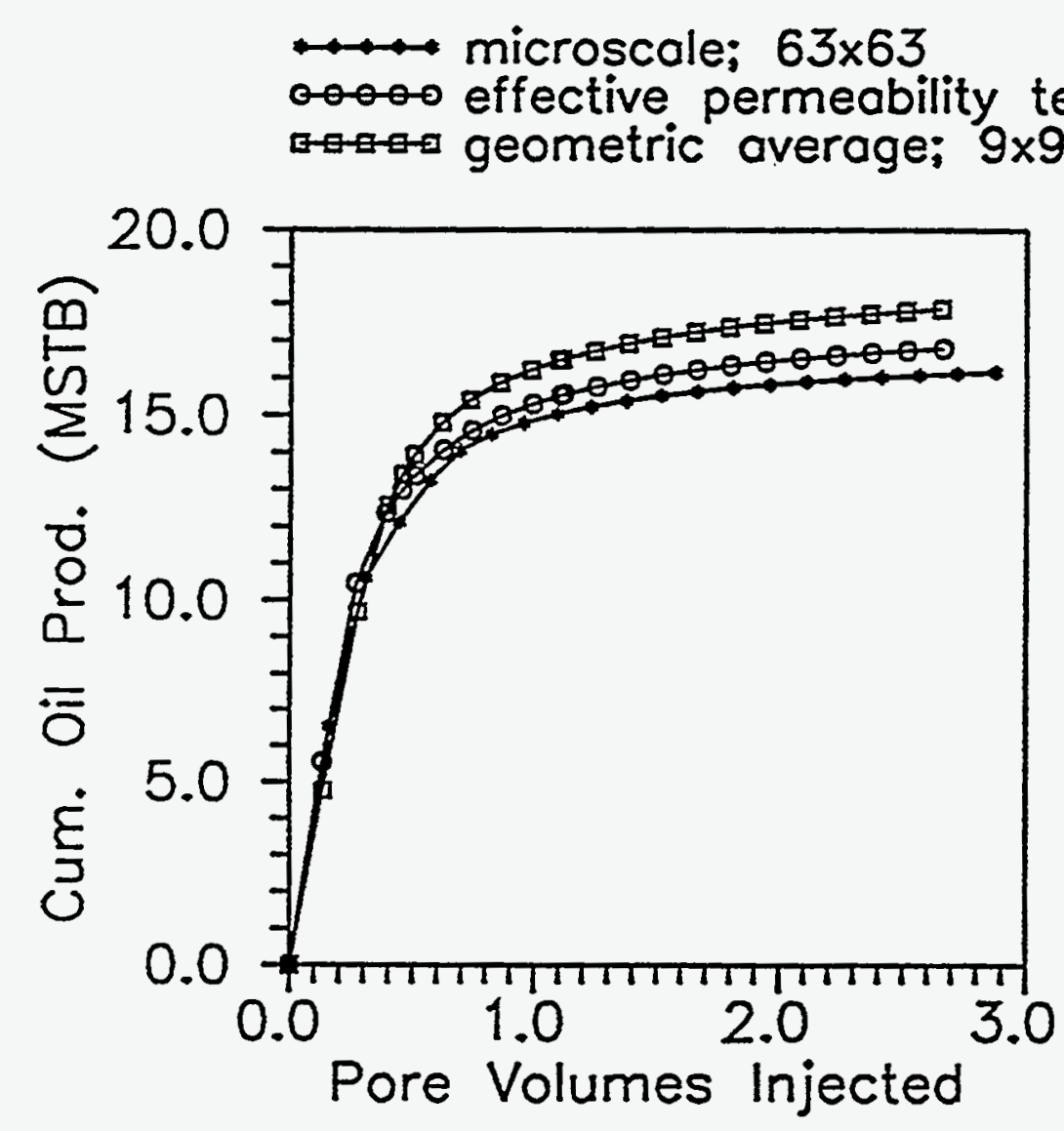

Fig. 37: Cumulative Oil Recovery Versus Pore Volumes Injected for Producing Well P-2 in a 5-Spot Waterflood. Anisotropic (Cross-Bedded) Initial Permeability Distribution. Effect of Permeability Upscaling. 


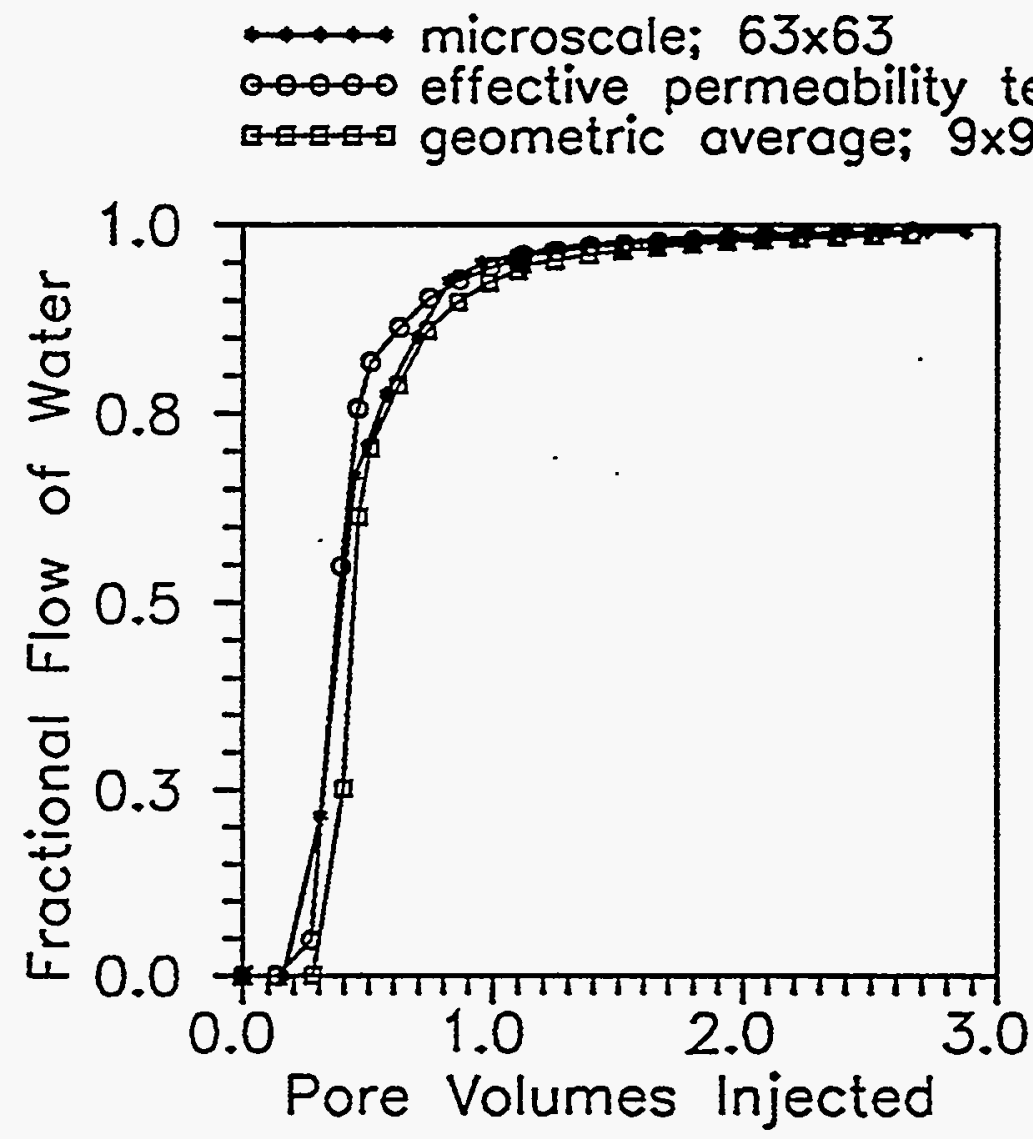

Fig. 38: Fractional Flow of Water Versus Pore Volumes Injected for Producing Well P-2 in a 5-Spot Waterflood. Anisotropic (Cross-Bedded) Initial Permeability Distribution. Effect of Permeability Upscaling. 
Water breaks through later in well P-2 using the geometric average of permeability than when using the microscale distribution and the effective permeability tensor.

\section{$\underline{\text { Summary and Conclusions }}$}

1. An analytical method for calculating effective permeability as a full tensor has been presented. This method can handle complex small scale heterogeneities; both the effect of location and orientation of these heterogeneities are included in the method.

2. Effective permeability tensors calculated by both the analytical method and a numerical simulation method show excellent agreement.

3. Miscible displacement simulations using initial small scale permeability distributions and upscaled permeability distributions are performed. Results indicate that using the proposed analytical effective permeability tensor method results in better prediction of flood front locations than using standard upscaling techniques for cross-bedded permeability distributions.

4. Transmissibilities for general permeability anisotropy have been developed from finite element theory. These transmissibilities can be inserted in point-centered 9-point finite difference simulators.

5. The proposed transmissibility method can be related to Shiralkar's method by a suitable choice of the numerical integration parameter.

6. Results from waterflood simulations show that the effective permeability tensor method outperforms standard permeability averaging methods for generally anisotropic permeability distributions.

$\underline{\text { Symbol }}$

\section{Nomenclature}

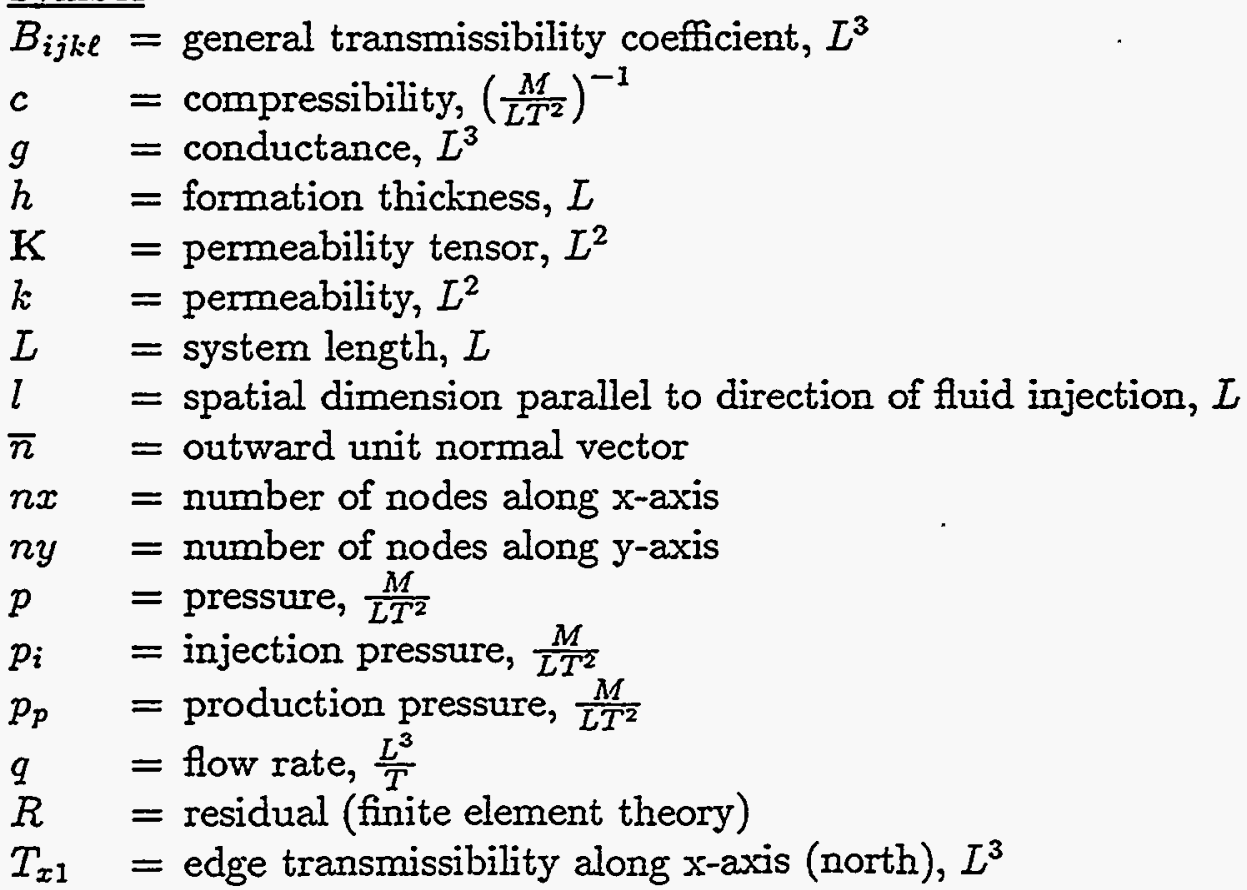



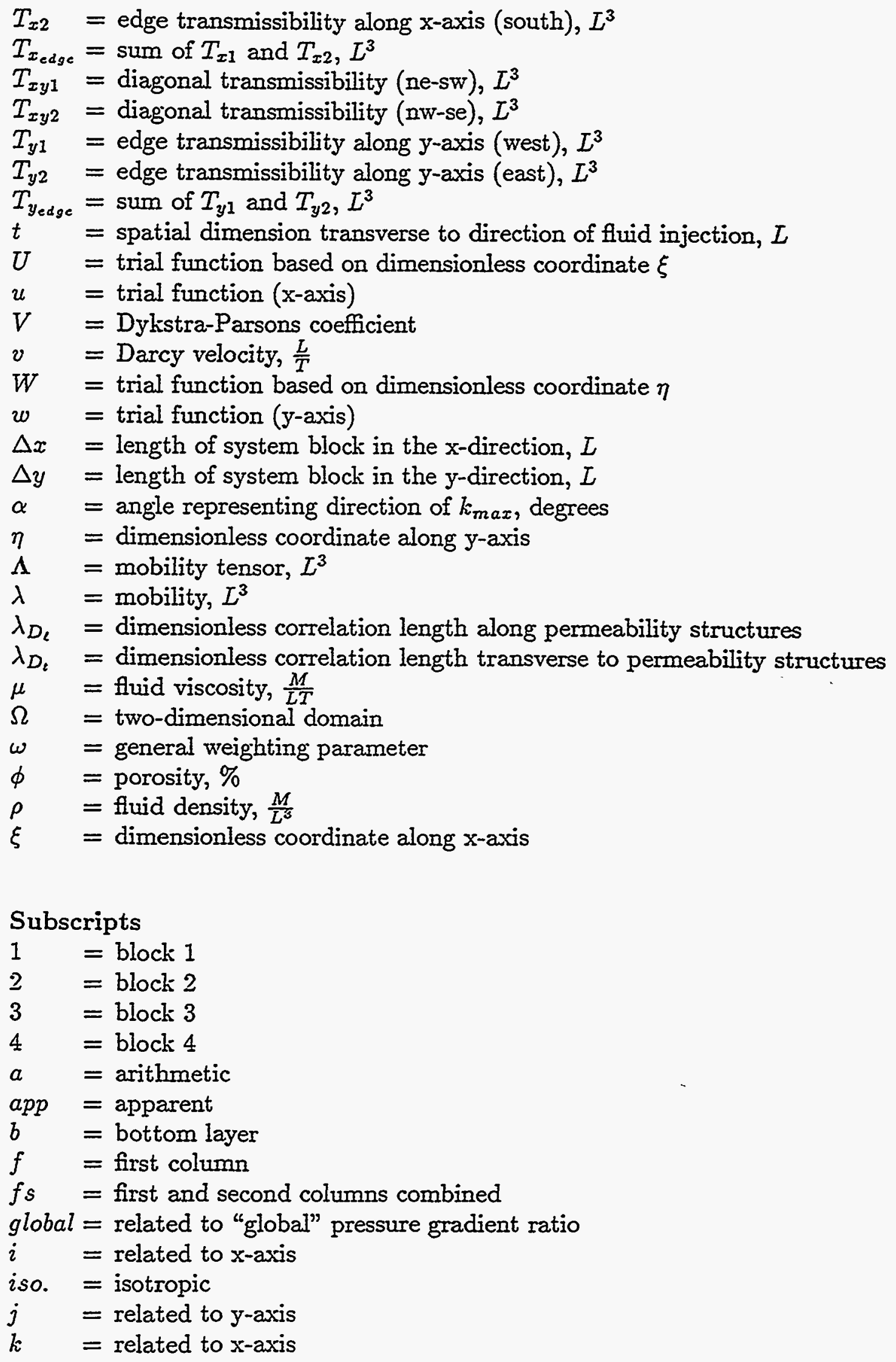


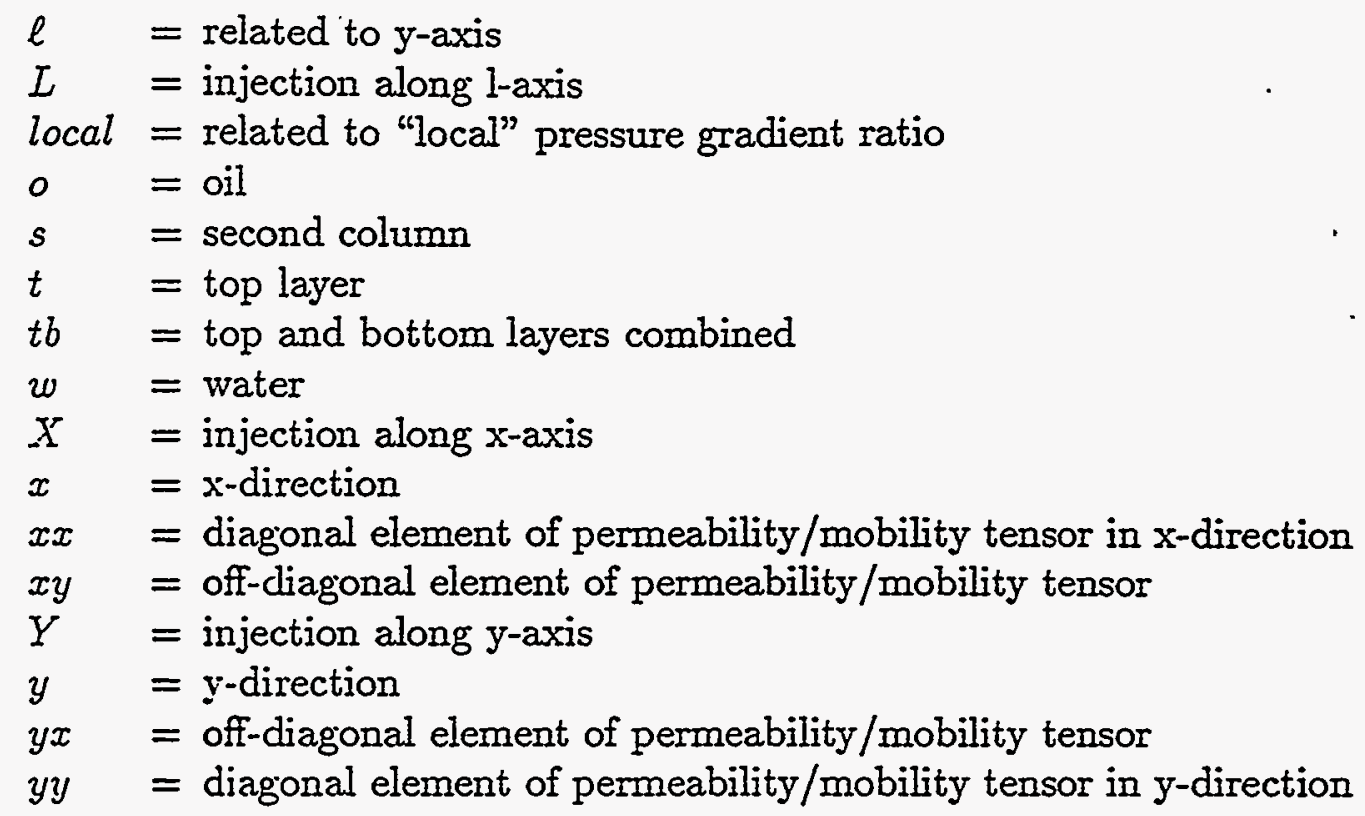

\section{Superscript}

1 = related to principal axes

- = indicates vector property

$\sim$ = effective system property

$\sim=$ effective property of part of system

\section{$\underline{\text { References }}$}

1. Kasap, E. and Lake, L.W.: "Calculating the Effective Permeability Tensor of a Gridblock," SPEFE (June 1990) 192-200.

2. Warren, J.E. and Price, H.S.: "Flow in Heterogeneous Porous Media," SPEJ (Sept. 1961) 153-169.

3. White, C.D. and Horne, R.N.: "Computing Absolute Transmissibility in the Presence of Fine-Scale Heterogeneity," paper SPE 16011 presented at the 1987 SPE Symposium on Reservoir Simulation, San Antonio, Feb. 1-4.

4. Samier, P.: "A Finite Element Method for Calculating Transmissibilities in Npoint Difference Equations Using a Non-Diagonal Permeability Tensor," paper presented at the 1990 2nd European Conference on the Mathematics of Oil

. Recovery, Arles, France, Sept. 11-14, 121-130.

5. Holden, L., Høiberg, J. and Lia, O.: "An Estimator for the Effective Permeability," paper presented at the 1990 2nd European Conference on the Mathematics 
of Oil Recovery, Arles, France, Sept. 11-14, 287-290.

6. Durlofsky, L.J. and Chung, E.Y.: "Effective Permeability of Heterogeneous Reservoir Regions," paper presented at the 1990 2nd European Conference on the Mathematics of Oil Recovery, Arles, France, Sept. 11-14, 57-64.

7. Gómez-Hernández, J.J. and Journel, A.G.: "Stochastic Characterization of Grid-Block Permeabilities: from Point Values to Block Tensors," paper presented at the 1990 2nd European Conference on the Mathematics of Oil Recovery, Arles, France, Sept. 11-14, 83-90.

8. Gallouët, T. and Guérillot, D.: "An Optimal Method for Averaging the Absolute Permeability," poster presented at the 1991 3rd International Reservoir Characterization Technical Conference, Tulsa, OK, Nov. 3-5.

9. Haldorsen, H.H. and Lake, L.W.: "A New Approach to Shale Management in Field-Scale Models," SPEJ (Aug. 1984) 447-457.

10. Begg, S.H. and King, P.R.: "Modelling the Effects of Shales on Reservoir Performance: Calculation of Effective Vertical Permeability," paper SPE 13529 presented at the 1985 SPE Symposium on Reservoir Simulation, Dallas, Feb. 10-13.

11. Begg, S.H., Chang, D.M. and Haldorsen, H.H.: "A Simple Statistical Method for Calculating the Effective Vertical Permeability of a Reservoir Containing Discontinuous Shales," paper SPE 14271 presented at the 1985 SPE Annual Technical Conference and Exhibition, Las Vegas, Sept. 22-25.

12. Begg, S.H., Carter, R.R. and Dranfield, P.: "Assigning Effective Values to Simulator Gridblock Parameters for Heterogeneous Reservoirs," SPERE (Nov. 1989) 455-463.

13. Abu-elbashar, O.B., Daltaban, T.S., Wall, C.G. and Archer, J.S.: "Statistical and Experimental Calculation of Effective Permeabilities in Presence of Oblique Shales," The Mathematics of Oil Recovery, King, P.R. (editor), Oxford University Press, New York (1992) 597-615.

14. King, P.R.: "The use of Field Theoretic Methods for the Study of Flow in a Heterogeneous Porous Medium," J. Phys. A: Math. Gen. 20 (1987) 3935-3947.

15. Otero, C., Sáez, A.E. and Rusinek, I.: "Effective Permeabilities for Model 
Heterogeneous Porous Media," In Situ (Sept. 1990) 229-244.

16. Rubin, Y. and Gómez-Hernández, J.J.: "A Stochastic Approach to the Problem of Upscaling of Conductivity in Disordered Media: Theory and Unconditional Numerical Simulations," Water Res. Res. (April 1990) 691-701.

17. Bernasconi, J.: "Conduction in Anisotropic Disordered Systems: EffectiveMedium Theory," Phys. Rev. B (May 1974) 4575-4579.

18. Harris, C.K.: "Application of Generalised Effective-Medium Theory To Transport in Porous Media," Transport in Porous Media (Oct. 1990) 517-542.

19. King, P.R.: "The use of Renormalization for Calculating Effective Permeability," Transport in Porous Media (Feb. 1989) 37-58.

20. Williams, J.K.: "Simple Renormalization Schemes for Calculating Effective Properties of Heterogeneous Reservoirs," The Mathematics of Oil Recovery, IKing, P.R. (editor), Oxford University Press, New York (1992) 281-298.

21. Poulisse, H.N.J.: "Effective Absolute Permeability in the Presence of Sub-Grid Heterogeneities: An Analytical Approach," The Mathematics of Oil Recovery, King, P.R. (editor), Oxford University Press, New York (1992) 699-720.

22 Aasum, Y.: "Effective Properties of Reservoir Simulator Grid Blocks," Ph.D. Dissertation, University of Tulsa (1992).

23. Goddin, C.S., Craig, F.F. Ir., Wilkes, J.O. and Tek, M.R.: "A Numerical Study of Waterflood Performance in a Stratified System With Crossflow," JPT (June 1966) 765-771.

24. Zapata, V.J. and Lake, L.W.: "A Theoretical Analysis of Viscous Crossflow," paper SPE 10111 presented at the 1981 SPE Annual Technical Conference and Exhibition, San Antonio, October 5-7.

25. Yang, A.-P.: "Turning Bands Method to Generate 2-D Random Field with Autocorrelation," University of Texas at Austin (April 1987).

26. Leung, W.F.: "A Tensor Model for Anisotropic and Heterogeneous Reservoirs With Variable Directional Permeabilities," paper SPE 15134 presented at the 1986 SPE California Regional Meeting, Oakland, April 2-4. 
27. Shiralkar, G.S.: "Reservoir Simulation of Generally Anisotropic Systems," SPERE (Aug. 1990) 409-414.

28. Yanosik, J.L. and McCracken, T.A.: "A Nine-Point, Finite-Difference Reservoir Simulator for Realistic Prediction of Adverse Mobility Ratio Displacements," SPEJ (Aug. 1979) 253-262.

29. Young, L.C.: "A Finite-Element Method for Reservoir Simulation," SPEJ (Feb. 1981) $115-128$.

30. Young, L.C.: Personal Communication, June 1992.

31. M.O.R.E. simulator, Black-Oil Version, Reservoir Simulation Research Corporation, Tulsa, May 1992.

32. Burnett, D.S.: Finite Element Analysis, From Concepts to Applications, Addison-Wesley Publishing Company, Reading, Massachusetts (1987).

33. Jain, M.K., Iyengar, S.R.K. and Jain, R.K.: Numerical Methods for Scientific and Engineering Computation, John Wiley \& Sons, Inc., New York (1985).

\section{Appendix A}

Numerical integration is necessary to evaluate the integrals in Eq. (73). The Lobatto integration method is used here. It reduces to the trapezoidal rule for two evaluation points and Simpson's rule for three evaluation points. ${ }^{29}$ Jain et al. ${ }^{33}$ show how the weights are determined. A general parameter, $\omega$, can be obtained based on the weights obtained from two- and three-point evaluations. The resulting general formula may be expressed as follows:

$$
\int_{0}^{1} f(x) d x=\omega f\left(\frac{1}{2}\right)+\frac{1}{2}(1-\omega)[f(0)+f(1)]
$$

where $\omega=0$ reduces Eq. (A1) to the trapezoidal rule, $\omega=\frac{2}{3}$ corresponds to Simpson's rule, and $\omega=1$ yields the midpoint rule. Using Eq. (A1) and Eqs. (62)-(69), Eq. (73) may be evaluated to obtain the desired transmissibilities. The expressions for these transmissibilities are presented next. The development of $T_{x 1}$ is shown in detail. In the following four equations, each integral in Eq. (73) is 
evaluated separately:

$$
B_{1222}^{x x}=\frac{\Delta y}{\Delta x} \int_{0}^{1} W_{2}(\eta) W_{2}(\eta)\left[\int_{0}^{1} \lambda_{x x}(\xi, \eta) U_{1}^{\prime}(\xi) U_{2}^{\prime}(\xi) d \xi\right] d \eta
$$

The integral within the square brackets in Eq. (A2) can be evaluated using Eqs. (72) and (AI) as follows:

$$
\begin{gathered}
\omega\left[\left(\frac{1}{2}(1-\eta) \lambda_{x x_{1,1}}+\frac{1}{2}(1-\eta) \lambda_{x x_{2,1}}+\frac{1}{2} \eta \lambda_{x x_{1,2}}+\frac{1}{2} \eta \lambda_{x x_{2,2}}\right)(-1)(1)\right] \\
+\frac{1}{2}(1-\omega)\left[\left((1-\eta) \lambda_{x x_{1,1}}+\eta \lambda_{x x_{1,2}}\right)(-1)(1)+\left((1-\eta) \lambda_{x x_{2,1}}+\eta \lambda_{x x_{2,2}}\right)(-1)(1)\right] \\
=-\frac{1}{2}(1-\eta) \lambda_{x x_{1,1}}-\frac{1}{2} \eta \lambda_{x x_{1,2}}-\frac{1}{2}(1-\eta) \lambda_{x x_{2,1}}-\frac{1}{2} \eta \lambda_{x x_{2,2}} .
\end{gathered}
$$

Substituting Eq. (A2b) into Eq. (A2) yields the following intermediate result:

$$
B_{1222}^{x x}=-\frac{\Delta y}{\Delta x} \int_{0}^{1} W_{2}(\eta) W_{2}(\eta)\left[\frac{1}{2}(1-\eta)\left(\lambda_{x x_{1,1}}+\lambda_{x x_{2,1}}\right)+\frac{1}{2} \eta\left(\lambda_{x x_{1,2}}+\lambda_{x x_{2,2}}\right)\right] d \eta .
$$

Numerically integrating the integral in Eq. (A2c) using Eq. (A1) produces the following expression:

$$
\begin{gathered}
\omega\left[\left(-\frac{1}{4} \lambda_{x x_{1,1}}-\frac{1}{4} \lambda_{x x_{2,1}}-\frac{1}{4} \lambda_{x x_{1,2}}-\frac{1}{4} \lambda_{x x_{2,2}}\right)\left(\frac{1}{2}\right)\left(\frac{1}{2}\right)\right] \\
+\frac{1}{2}(1-\omega)\left[\left(-\frac{1}{2} \lambda_{x x_{1,1}}-\frac{1}{2} \lambda_{x x_{2,1}}\right)(0)(0)+\left(-\frac{1}{2} \lambda_{x x_{1,2}}-\frac{1}{2} \lambda_{x x_{2,2}}\right)(1)(1)\right] .
\end{gathered}
$$

Substituting Eq. (A2d) into Eq. (A2c) yields the following expression for $B_{1222}^{x x}$ : 


$$
B_{1222}^{x x}=\frac{1}{4}(\omega-1)\left(\lambda_{x x_{1,2}}+\lambda_{x x_{2,2}}\right) \frac{\Delta y}{\Delta x}-\frac{1}{16} \omega\left(\lambda_{x x_{1,1}}+\lambda_{x x_{2,1}}+\lambda_{x x_{1,2}}+\lambda_{x x_{2,2}}\right) \frac{\Delta y}{\Delta x} .
$$

The other integrals in Eq. (73) are evaluated in a similar manner:

$$
\begin{aligned}
B_{1222}^{x y} & =\int_{0}^{1} W_{2}^{\prime}(\eta) W_{2}(\eta)\left[\int_{0}^{1} \lambda_{x y}(\xi, \eta) U_{1}(\xi) U_{2}^{\prime}(\xi) d \xi\right] d \eta \\
& =\frac{1}{4}\left(\frac{1}{2} \omega-1\right)^{2} \lambda_{x y_{1,2}}-\frac{1}{8}\left(\frac{1}{2} \omega-1\right) \omega\left(\lambda_{x y_{1,1}}+\lambda_{x y_{2,2}}\right)+\frac{1}{16} \omega^{2} \lambda_{x y_{2,1}} \\
B_{1222}^{y x} & =\int_{0}^{1} W_{2}(\eta) W_{2}^{\prime}(\eta)\left[\int_{0}^{1} \lambda_{y x}(\xi, \eta) U_{1}^{\prime}(\xi) U_{2}(\xi) d \xi\right] d \eta \\
= & -\frac{1}{4}\left(\frac{1}{2} \omega-1\right)^{2} \lambda_{y x_{2,2}}+\frac{1}{8}\left(\frac{1}{2} \omega-1\right) \omega\left(\lambda_{y x_{2,1}}+\lambda_{y x_{1,2}}\right)-\frac{1}{16} \omega^{2} \lambda_{y x_{1,1}}
\end{aligned}
$$

$$
\begin{aligned}
B_{1222}^{y y} & =\frac{\Delta x}{\Delta y} \int_{0}^{1} W_{2}^{\prime}(\eta) W_{2}^{\prime}(\eta)\left[\int_{0}^{1} \lambda_{y y}(\xi, \eta) U_{1}(\xi) U_{2}(\xi) d \xi\right] d \eta \\
& =\frac{1}{16} \omega\left(\lambda_{y y_{1,2}}+\lambda_{y y_{2,1}}+\lambda_{y y_{1,2}}+\lambda_{y y_{2,2}}\right) \frac{\Delta x}{\Delta y}
\end{aligned}
$$

Combining Eqs. (A2e)-(A5) yields:

$$
\begin{aligned}
B_{1222}= & B_{1222}^{x x}+B_{1222}^{x y}+B_{1222}^{y x}+B_{1222}^{y y} \\
= & \frac{1}{4}(\omega-1)\left(\lambda_{x x_{1,2}}+\lambda_{x x_{2,2}}\right) \frac{\Delta y}{\Delta x}-\frac{1}{16} \omega\left(\lambda_{x x_{1,1}}+\lambda_{x x_{2,2}}+\lambda_{x x_{1,2}}+\lambda_{x x_{2,2}}\right) \frac{\Delta y}{\Delta x} \\
& +\frac{1}{4}\left(\frac{1}{2} \omega-1\right)^{2} \lambda_{x y_{1,2}}-\frac{1}{8}\left(\frac{1}{2} \omega-1\right) \omega\left(\lambda_{x y_{1,2}}+\lambda_{x y_{2,2}}\right)+\frac{1}{16} \omega^{2} \lambda_{x y_{2,1}} \\
& -\frac{1}{4}\left(\frac{1}{2} \omega-1\right)^{2} \lambda_{y x_{2,2}}+\frac{1}{8}\left(\frac{1}{2} \omega-1\right) \omega\left(\lambda_{y x_{2,1}}+\lambda_{y x_{1,2}}\right)-\frac{1}{16} \omega^{2} \lambda_{y x_{1,1}} \\
& +\frac{1}{16} \omega\left(\lambda_{y y_{1,1}}+\lambda_{y y_{2,1}}+\lambda_{y y_{1,2}}+\lambda_{y y_{2,2}}\right) \frac{\Delta x}{\Delta y} .
\end{aligned}
$$


The transmissibility coefficient $T_{x 1}$ can, therefore, be expressed as follows:

$$
\begin{aligned}
T_{x 1}= & -B_{1222} \\
= & -\frac{1}{4}(\omega-1)\left(\lambda_{x x_{1,2}}+\lambda_{x x_{2,2}}\right) \frac{\Delta y}{\Delta x}+\frac{1}{16} \omega\left(\lambda_{x x_{1,1}}+\lambda_{x x_{2,1}}+\lambda_{x x_{1,2}}+\lambda_{x x_{2,2}}\right) \frac{\Delta y}{\Delta x} \\
& -\frac{1}{4}\left(\frac{1}{2} \omega-1\right)^{2} \lambda_{x y_{1,2}}+\frac{1}{8}\left(\frac{1}{2} \omega-1\right) \omega\left(\lambda_{x y_{1,1}}+\lambda_{x y_{2,2}}\right)-\frac{1}{16} \omega^{2} \lambda_{x y_{2,1}} \\
& +\frac{1}{4}\left(\frac{1}{2} \omega-1\right)^{2} \lambda_{y x_{2,2}}-\frac{1}{8}\left(\frac{1}{2} \omega-1\right) \omega\left(\lambda_{y x_{2,1}}+\lambda_{y x_{1,2}}\right)+\frac{1}{16} \omega^{2} \lambda_{y x_{1,1}} \\
& -\frac{1}{16} \omega\left(\lambda_{y y_{1,1}}+\lambda_{y y_{2,1}}+\lambda_{y y_{1,2}}+\lambda_{y y_{2,2}}\right) \frac{\Delta x}{\Delta y} .
\end{aligned}
$$

The other transmissibility coefficients are derived in a similar manner. their final expressions are as follows:

$$
\begin{aligned}
T_{x 2}= & -B_{1121} \\
= & -\frac{1}{4}(\omega-1)\left(\lambda_{x x_{1,1}}+\lambda_{x x_{2,1}}\right) \frac{\Delta y}{\Delta x}+\frac{1}{16} \omega\left(\lambda_{x x_{1,1}}+\lambda_{x x_{2,1}}+\lambda_{x x_{1,2}}+\lambda_{x x_{2,2}}\right) \frac{\Delta y}{\Delta x} \\
& +\frac{1}{4}\left(\frac{1}{2} \omega-1\right)^{2} \lambda_{x y_{1,1}}-\frac{1}{8}\left(\frac{1}{2} \omega-1\right) \omega\left(\lambda_{x y_{2,1}}+\lambda_{x y_{1,2}}\right)+\frac{1}{16} \omega^{2} \lambda_{x y_{2,2}} \\
& -\frac{1}{4}\left(\frac{1}{2} \omega-1\right)^{2} \lambda_{y x_{2,1}}+\frac{1}{8}\left(\frac{1}{2} \omega-1\right) \omega\left(\lambda_{y x_{1,1}}+\lambda_{y x_{2,2}}\right)-\frac{1}{16} \omega^{2} \lambda_{y x_{1,2}} \\
& -\frac{1}{16} \omega\left(\lambda_{y y_{1,1}}+\lambda_{y y_{2,1}}+\lambda_{y y_{1,2}}+\lambda_{y y_{2,2}}\right) \frac{\Delta x}{\Delta y} \\
T_{y 1}= & -B_{1112} \\
= & -\frac{1}{4}(\omega-1)\left(\lambda_{y y_{1,1}}+\lambda_{y y_{1,2}}\right) \frac{\Delta x}{\Delta y}+\frac{1}{16} \omega\left(\lambda_{y y_{1,1}}+\lambda_{y y_{2,1}}+\lambda_{y y_{1,2}}+\lambda_{y y_{2,2}}\right) \frac{\Delta x}{\Delta y} \\
& -\frac{1}{4}\left(\frac{1}{2} \omega-1\right)^{2} \lambda_{x y_{1,2}}+\frac{1}{8}\left(\frac{1}{2} \omega-1\right) \omega\left(\lambda_{x y_{1,1}}+\lambda_{x y_{2,2}}\right)-\frac{1}{16} \omega^{2} \lambda_{x y_{2,1}} \\
& +\frac{1}{4}\left(\frac{1}{2} \omega-1\right)^{2} \lambda_{y x_{1,1}}-\frac{1}{\delta}\left(\frac{1}{2} \omega-1\right) \omega\left(\lambda_{y x_{2,1}}+\lambda_{y x_{1,2}}\right)+\frac{1}{16} \omega^{2} \lambda_{y x_{2,2}} \\
& -\frac{1}{16} \omega\left(\lambda_{x x_{1,1}}+\lambda_{x x_{2,1}}+\lambda_{x x_{1,2}}+\lambda_{\left.x x_{2,2}\right)}\right) \frac{\Delta y}{\Delta x}
\end{aligned}
$$




$$
\begin{aligned}
T_{y 2}= & -B_{1122} \\
= & -\frac{1}{4}(\omega-1)\left(\lambda_{y y_{2,1}}+\lambda_{y y_{2,2}}\right) \frac{\Delta x}{\Delta y}+\frac{1}{16} \omega\left(\lambda_{y y_{1,1}}+\lambda_{y y_{2,1}}+\lambda_{y y_{1,2}}+\lambda_{y y_{2,2}}\right) \frac{\Delta x}{\Delta y} \\
& +\frac{1}{4}\left(\frac{1}{2} \omega-1\right)^{2} \lambda_{x y_{2,2}}-\frac{1}{8}\left(\frac{1}{2} \omega-1\right) \omega\left(\lambda_{x y_{2,1}}+\lambda_{x y_{1,2}}\right)+\frac{1}{16} \omega^{2} \lambda_{x y_{1,1}} \\
& -\frac{1}{4}\left(\frac{1}{2} \omega-1\right)^{2} \lambda_{y x_{2,1}}+\frac{1}{8}\left(\frac{1}{2} \omega-1\right) \omega\left(\lambda_{y x_{1,1}}+\lambda_{y x_{2,2}}\right)-\frac{1}{16} \omega^{2} \lambda_{y x_{1,2}} \\
& -\frac{1}{16} \omega\left(\lambda_{x x_{1,2}}+\lambda_{x x_{2,1}}+\lambda_{x x_{1,2}}+\lambda_{x x_{2,2}}\right) \frac{\Delta y}{\Delta x}
\end{aligned}
$$

$$
\begin{aligned}
T_{x y 1}= & -B_{1122} \\
= & \frac{1}{16} \omega\left(\lambda_{x x_{1,1}}+\lambda_{x x_{2,1}}+\lambda_{x x_{1,2}}+\lambda_{x x_{2,2}}\right) \frac{\Delta y}{\Delta x} \\
& +\frac{1}{4}\left(\frac{1}{2} \omega-1\right)^{2} \lambda_{x y_{1,2}}-\frac{1}{8}\left(\frac{1}{2} \omega-1\right) \omega\left(\lambda_{x y_{1,1}}+\lambda_{x y_{2,2}}\right)+\frac{1}{16} \omega^{2} \lambda_{x y_{2,1}} \\
& +\frac{1}{4}\left(\frac{1}{2} \omega-1\right)^{2} \lambda_{y x_{2,1}}-\frac{1}{8}\left(\frac{1}{2} \omega-1\right) \omega\left(\lambda_{y x_{1,1}}+\lambda_{y x_{2,2}}\right)+\frac{1}{16} \omega^{2} \lambda_{y x_{1,2}} \\
& +\frac{1}{16} \omega\left(\lambda_{y y_{1,1}}+\lambda_{y y_{2,1}}+\lambda_{y y_{1,2}}+\lambda_{y y_{2,2}}\right) \frac{\Delta x}{\Delta y}
\end{aligned}
$$

$$
\begin{aligned}
T_{x y 2}= & -B_{2112} \\
= & \frac{1}{16} \omega\left(\lambda_{x x_{1,1}}+\lambda_{x x_{2,1}}+\lambda_{x x_{1,2}}+\lambda_{x x_{2,2}}\right) \frac{\Delta y}{\Delta x} \\
& -\frac{1}{4}\left(\frac{1}{2} \omega-1\right)^{2} \lambda_{x y_{2,2}}+\frac{1}{8}\left(\frac{1}{2} \omega-1\right) \omega\left(\lambda_{x y_{2,1}}+\lambda_{x y_{1,2}}\right)-\frac{1}{16} \omega^{2} \lambda_{x y_{1,1}} \\
& -\frac{1}{4}\left(\frac{1}{2} \omega-1\right)^{2} \lambda_{y x_{1,1}}+\frac{1}{8}\left(\frac{1}{2} \omega-1\right) \omega\left(\lambda_{y x_{2,1}}+\lambda_{y x_{1,2}}\right)-\frac{1}{16} \omega^{2} \lambda_{y x_{2,2}} \\
& +\frac{1}{16} \omega\left(\lambda_{y y_{1,2}}+\lambda_{y y_{2,1}}+\lambda_{y y_{1,2}}+\lambda_{y y_{2,2}}\right) \frac{\Delta x}{\Delta y} .
\end{aligned}
$$

Transmissibilities calculated for the opposite directions to those listed above are as follows: 


$$
\begin{aligned}
T_{x 1}= & -B_{2212} \\
= & -\frac{1}{4}(\omega-1)\left(\lambda_{x x_{1,2}}+\lambda_{x x_{2,2}}\right) \frac{\Delta y}{\Delta x}+\frac{1}{16} \omega\left(\lambda_{x x_{1,1}}+\lambda_{x x_{2,1}}+\lambda_{x x_{1,2}}+\lambda_{x x_{2,2}}\right) \frac{\Delta y}{\Delta x} \\
& +\frac{1}{4}\left(\frac{1}{2} \omega-1\right)^{2} \lambda_{x y_{2,2}}-\frac{1}{8}\left(\frac{1}{2} \omega-1\right) \omega\left(\lambda_{x y_{2,1}}+\lambda_{x y_{1,2}}\right)+\frac{1}{16} \omega^{2} \lambda_{x y_{1,1}} \\
& -\frac{1}{4}\left(\frac{1}{2} \omega-1\right)^{2} \lambda_{y x_{1,2}}+\frac{1}{8}\left(\frac{1}{2} \omega-1\right) \omega\left(\lambda_{y x_{1,1}}+\lambda_{y x_{2,2}}\right)-\frac{1}{16} \omega^{2} \lambda_{y x_{2,1}} \\
& -\frac{1}{16} \omega\left(\lambda_{y y_{1,1}}+\lambda_{y y_{2,1}}+\lambda_{y y_{1,2}}+\lambda_{y y_{2,2}}\right) \frac{\Delta x}{\Delta y}
\end{aligned}
$$

$$
\begin{aligned}
T_{x 2}= & -B_{2111} \\
= & -\frac{1}{4}(\omega-1)\left(\lambda_{x x_{1,1}}+\lambda_{x x_{2,1}}\right) \frac{\Delta y}{\Delta x}+\frac{1}{16} \omega\left(\lambda_{x x_{1,1}}+\lambda_{x x_{2,1}}+\lambda_{x x_{1,2}}+\lambda_{x x_{2,2}}\right) \frac{\Delta y}{\Delta x} \\
& -\frac{1}{4}\left(\frac{1}{2} \omega-1\right)^{2} \lambda_{x y_{2,1}}+\frac{1}{8}\left(\frac{1}{2} \omega-1\right) \omega\left(\lambda_{x y_{1,1}}+\lambda_{x y_{2,2}}\right)-\frac{1}{16} \omega^{2} \lambda_{x y_{1,2}} \\
& +\frac{1}{4}\left(\frac{1}{2} \omega-1\right)^{2} \lambda_{y x_{1,1}}-\frac{1}{8}\left(\frac{1}{2} \omega-1\right) \omega\left(\lambda_{y x_{2,1}}+\lambda_{y x_{1,2}}\right)+\frac{1}{16} \omega^{2} \lambda_{y x_{2,2}} \\
& -\frac{1}{16} \omega\left(\lambda_{y y_{1,1}}+\lambda_{y y_{2,1}}+\lambda_{y y_{1,2}}+\lambda_{y y_{2,2}}\right) \frac{\Delta x}{\Delta y}
\end{aligned}
$$

$$
\begin{aligned}
T_{y 1}= & -B_{1211} \\
= & -\frac{1}{4}(\omega-1)\left(\lambda_{y y_{1,1}}+\lambda_{y y_{1,2}}\right) \frac{\Delta x}{\Delta y}+\frac{1}{16} \omega\left(\lambda_{y y_{1,1}}+\lambda_{y y_{2,1}}+\lambda_{y y_{1,2}}+\lambda_{y y_{2,2}}\right) \frac{\Delta x}{\Delta y} \\
& +\frac{1}{4}\left(\frac{1}{2} \omega-1\right)^{2} \lambda_{x y_{1,2}}-\frac{1}{8}\left(\frac{1}{2} \omega-1\right) \omega\left(\lambda_{x y_{2,1}}+\lambda_{x y_{1,2}}\right)+\frac{1}{16} \omega^{2} \lambda_{x y_{2,2}} \\
& -\frac{1}{4}\left(\frac{1}{2} \omega-1\right)^{2} \lambda_{y x_{1,2}}+\frac{1}{8}\left(\frac{1}{2} \omega-1\right) \omega\left(\lambda_{y x_{1,1}}+\lambda_{y x_{2,2}}\right)-\frac{1}{16} \omega^{2} \lambda_{y x_{2,1}} \\
& -\frac{1}{16} \omega\left(\lambda_{x x_{1,1}}+\lambda_{x x_{2,1}}+\lambda_{x x_{1,2}}+\lambda_{x x_{2,2}}\right) \frac{\Delta y}{\Delta x}
\end{aligned}
$$




$$
\begin{aligned}
T_{y 2}=- & B_{2221} \\
= & -\frac{1}{4}(\omega-1)\left(\lambda_{y y_{2,1}}+\lambda_{y y_{2,2}}\right) \frac{\Delta x}{\Delta y}+\frac{1}{16} \omega\left(\lambda_{y y_{1,1}}+\lambda_{y y_{2,2}}+\lambda_{y y_{1,2}}+\lambda_{y y_{2,2}}\right) \frac{\Delta x}{\Delta y} \\
- & \frac{1}{4}\left(\frac{1}{2} \omega-1\right)^{2} \lambda_{x y_{2,1}}+\frac{1}{8}\left(\frac{1}{2} \omega-1\right) \omega\left(\lambda_{x y_{1,1}}+\lambda_{x y_{2,2}}\right)-\frac{1}{16} \omega^{2} \lambda_{x y_{1,2}} \\
+ & \frac{1}{4}\left(\frac{1}{2} \omega-1\right)^{2} \lambda_{y x_{2,2}}-\frac{1}{8}\left(\frac{1}{2} \omega-1\right) \omega\left(\lambda_{y x_{2,1}}+\lambda_{y x_{1,2}}\right)+\frac{1}{16} \omega^{2} \lambda_{y x_{1,1}} \\
- & \frac{1}{16} \omega\left(\lambda_{x x_{1,1}}+\lambda_{x x_{2,1}}+\lambda_{x x_{1,2}}+\lambda_{\left.x x_{2,2}\right) \frac{\Delta y}{\Delta x}}\right. \\
T_{x y 1}= & -B_{2211} \\
= & \frac{1}{16} \omega\left(\lambda_{x x_{1,1}}+\lambda_{x x_{2,1}}+\lambda_{x x_{1,2}}+\lambda_{x x_{2,2}}\right) \frac{\Delta y}{\Delta x} \\
& +\frac{1}{4}\left(\frac{1}{2} \omega-1\right)^{2} \lambda_{x y_{2,1}}-\frac{1}{8}\left(\frac{1}{2} \omega-1\right) \omega\left(\lambda_{x y_{1,1}}+\lambda_{x y_{2,2}}\right)+\frac{1}{16} \omega^{2} \lambda_{x y_{1,2}} \\
& +\frac{1}{4}\left(\frac{1}{2} \omega-1\right)^{2} \lambda_{y x_{1,2}}-\frac{1}{8}\left(\frac{1}{2} \omega-1\right) \omega\left(\lambda_{y x_{1,1}}+\lambda_{y x_{2,2}}\right)+\frac{1}{16} \omega^{2} \lambda_{y x_{2,1}} \\
& +\frac{1}{16} \omega\left(\lambda_{y y_{1,1}}+\lambda_{y y_{2,1}}+\lambda_{y y_{1,2}}+\lambda_{y y_{2,2}}\right) \frac{\Delta x}{\Delta y} \\
& +\frac{1}{16} \omega\left(\lambda_{y y_{1,1}}+\lambda_{y y_{2,1}}+\lambda_{y y_{1,2}}+\lambda_{y y_{2,2}}\right) \frac{\Delta x}{\Delta y} \\
& -\frac{1}{4}\left(\frac{1}{2} \omega-1\right)^{2} \lambda_{y x_{2,2}}+\frac{1}{8}\left(\frac{1}{2} \omega-1\right) \omega\left(\lambda_{y x_{2,1}}+\lambda_{y x_{1,2}}\right)-\frac{1}{16} \omega^{2} \lambda_{y x_{1,1}} \\
T_{x y 2}= & -B_{1221} \\
= & \frac{1}{16} \omega\left(\lambda_{x x_{1,1}}+\lambda_{x x_{2,1}}+\lambda_{x x_{1,2}}+\lambda_{x x_{2,2}}\right) \frac{\Delta y}{\Delta x} \\
& \\
&
\end{aligned}
$$

For physically meaningful results in this finite difference framework, the transmissibilities calculated for opposite directions need to be identical. For example, 
Eqs. (A7) and (A13) must be equal. Equating these two equations yields the following equality:

$$
\begin{gathered}
{\left[\frac{1}{8}\left(\frac{1}{2} \omega-1\right) \omega-\frac{1}{16} \omega^{2}\right] \lambda_{x y_{1,1}}+\left[\frac{1}{8}\left(\frac{1}{2} \omega-1\right) \omega-\frac{1}{16} \omega^{2}\right] \lambda_{x y_{2,1}}} \\
+\left[\frac{1}{8}\left(\frac{1}{2} \omega-1\right) \omega-\frac{1}{4}\left(\frac{1}{2} \omega-1\right)^{2}\right] \lambda_{x y_{1,2}}+\left[\frac{1}{8}\left(\frac{1}{2} \omega-1\right) \omega-\frac{1}{4}\left(\frac{1}{2} \omega-1\right)^{2}\right] \lambda_{x y_{2,2}} \\
=\left[\frac{1}{8}\left(\frac{1}{2} \omega-1\right) \omega-\frac{1}{16} \omega^{2}\right] \lambda_{y x_{1,1}}+\left[\frac{1}{8}\left(\frac{1}{2} \omega-1\right) \omega-\frac{1}{16} \omega^{2}\right] \lambda_{y x_{2,1}} \\
+\left[\frac{1}{8}\left(\frac{1}{2} \omega-1\right) \omega-\frac{1}{4}\left(\frac{1}{2} \omega-1\right)^{2}\right] \lambda_{y x_{1,2}}+\left[\frac{1}{8}\left(\frac{1}{2} \omega-1\right) \omega-\frac{1}{4}\left(\frac{1}{2} \omega-1\right)^{2}\right] \lambda_{y x_{2,2}} .
\end{gathered}
$$

The equality in Eq. (A19) is satisfied if $\omega=1$ (midpoint rule) or if the offdiagonal elements of the tensor are equal (symmetric tensor). It has been suggested that non-symmetric permeability tensors can be made symmetric by arithmetically averaging the off-diagonal elements of the permeability tensor. ${ }^{8}$

Next, it is shown, based on Eqs. (A11) and (A12), that arithmetic averaging of the off-diagonal elements of the mobility tensor, $\Lambda$, is indeed appropriate in the context of 9-point finite differencing.

The difference between $T_{x y 1}$ and $T_{x y 2}$ translates into flow transverse to the direction of applied pressure gradient. This difference is determined here based on Eqs. (A11) and (A12) and using the midpoint rule $(\omega=1)$. The midpoint rule is appropriate based on the equality in Eq. (A19). Eq. (A12) subtracted from Eq. (A11) becomes:

$$
\begin{aligned}
T_{x y 1}-T_{x y 2} & =\frac{1}{8}\left(\lambda_{x y_{1,1}}+\lambda_{x y_{2,1}}+\lambda_{x y_{1,2}}+\lambda_{x y_{2,2}}+\lambda_{y x_{1,1}}+\lambda_{y x_{2,1}}+\lambda_{y x_{1,2}}+\lambda_{y x_{2,2}}\right) \\
& =\frac{1}{4}\left(\frac{\lambda_{x y_{1,1}}+\lambda_{y x_{1,1}}}{2}+\frac{\lambda_{x y_{2,1}}+\lambda_{y x_{2,1}}}{2}+\frac{\lambda_{x y_{1,2}}+\lambda_{y x_{1,2}}}{2}+\frac{\lambda_{x y_{2,2}}+\lambda_{y x_{2,2}}}{2}\right) \\
& =\frac{1}{4}\left(\lambda_{x y_{a 1,1}}+\lambda_{x y_{a 2,1}}+\lambda_{x y_{a 1,2}}+\lambda_{x y_{a 2,2}}\right) \\
& =\lambda_{y x_{a}}=\lambda_{x y_{a}}=g_{x y_{a}} .
\end{aligned}
$$

Thus, non-symmetric permeability tensors can be handled using this procedure. From Eq. (A20), it is clear that $g_{x y_{c}}$ is a simple arithmetic average of all the off- 
diagonal elements of the mobility tensor for the particular element (Figure 19) being considered. 


\title{
Section I
}

\author{
Report IV \\ Results for Closed Chamber Tests \\ Part I: Oil Wells
}

J. J. Xiao and A. C. Reynolds

SUMMARY

Closed chamber tests (CCT) are currently used in the oil industry as closed chamber drillstem tests, underbalanced perforation and backsurge perforation cleaning. Pressure responses in these tests are governed by a changing wellbore storage coefficient which results in a nonlinear mathematical problem with no known solution publicly available. Methods to analyze data from such a test include history matching through the use of numerical simulators, convolution methods utilizing flow rate directly measured or calculated from the measurement of the chamber gas pressure, and techniques developed from the short producing time concept.

In this study, a new inner boundary condition has been derived for a CCT problem, taking into account the changing wellbore storage. All results presented here assume that in the reservoir, we have single phase flow of a slightly compressible fluid of constant compressibility and viscosity. In a companion report, we consider single phase gas flow. A new pseudotime is applied to improve the correlation between a CCT and a conventional slug test, and to develop a new analysis procedure for closed chamber tests. Using pseudotime, pressure data from a CCT can be converted into an equivalent slug test response, and then type curve matching techniques can be used to deduce the reservoir flow capacity and well skin factor. The new analysis procedure was then used to analyze synthetic pressure data generated form a reservoir simulator. Parameters affecting pressure responses of closed chamber tests have also been studied.

The application of pseudotime concept was extended to slug tests which have a step change in the wellbore storage coefficient due to the change of wellstring diameter as the fluid level rises from the drill collar into the drill pipe during the test. It was shown that application of pseudotime can effectively correlate the resulting pressure response with the classical slug test solution for a constant wellbore storage coefficient. As a result, data from a slug test with a step change in the wellbore storage coefficient can be easily analyzed using methods developed for a slug test with a constant wellbore storage coefficient.

We have also studied the pressure response during the shut-in period of a closed chamber drillstem test. A rate convolution method was developed to analyze data 
measured during the shut-in period of a closed chamber drillstem test.

\section{INTRODUCTION}

Drillstem testing is well established as a primary technique for evaluating the commercial viability of newly discovered (virgin) reservoirs. A successful drillstem test (DST) gives a measurement of flow rates, an estimate of static reservoir pressure and pressure-time data corresponding to a short pressure transient test. Successful analysis of DST pressure data yields an estimate of the flow capacity, $k h$, and the skin factor, $s$. The flowing period of a DST corresponds to a slug test.

Slug tests originated in the field of groundwater hydrology, but interpretation methods used in petroleum industry were based on the work of Ramey et al. ${ }^{1}$. They derived detailed analytical solutions for slug tests including wellbore storage and skin effects for radial flow in a homogeneous reservoir, and developed type curves with $C_{D} e^{2 s}$ as a correlating factor. These type curves later become the industry standard for analyzing data from slug tests.

More recently, Peres et al. ${ }^{2}$ derived a general relationship between the slug test solution and the solution for the classical wellbore storage and skin constant surface rate production problem. This general relationship enabled them to develop new procedures to analyze slug tests. They showred that pressure data from slug tests can be conveniently converted to pressure data of equivalent constant surface rate production tests. Slug tests, therefore, can be analyzed using existing type curves for the classical wellbore storage and skin constant surface rate production problem, and slug test type curves are no longer needed.

Closed chamber drillstem tests were first introduced to the petroleum industry by Alexander ${ }^{3}$ as an improvement to conventional drillstem tests; the objective was to reduce operational and safety problems. As in a conventional drillstem test, a closed chamber DST is conducted to gain information on well potential and formation fluid type. The only major difference between a conventional DST and a closed chamber DST is that in a closed chamber DST, the well is closed in at the surface. Alexander developed methods to calculate flow rate when the well produced gas and/or liquid.

An extensive research was conducted by Erdle et al. ${ }^{4}$ for closed chamber drillstem testing. Calculation equations and test procedures for all commonly encountered combinations of cushion and fluid type were presented. Over one-hundred field closed chamber drillstem tests were conducted. They concluded that the advantages of closed chamber drillstem testing over conventional drillstem testing are improved safety, reduced test time and better test engineering at the wellsite.

The use of a closed chamber DST in low permeability gas reservoirs was reported by Reid ${ }^{5}$. Conventional drillstem tests are not feasible for this type of reservoirs. This is primarily due to 1) the presence of gas is often undetected when gas does not reach surface during the test, 2) when gas is detected, the very low 
rates are difficult to accurately monitor. Closed chamber drill stem tests were used to overcome these problems.

Kabir et al..$^{6}$ recently presented a new method to compute the individual phase flow rate of a two-phase gas-liquid mixture flow during a closed chamber DST. This method is based on a two-phase flow model, considering wellbore friction, gas and liquid compression and mass transfer effects. Field tests were also presented to illustrate their methods.

Although a closed chamber DST does offer several advantages over a conventional DST, the mathematical problem for a closed chamber DST is more complicated than for a conventional DST because the former has a nonlinear boundary condition. To the best of our knowledge, no analytical solution to the closed chamber DST problem has been presented in the literature. Quantitative data analysis of a closed chamber DST was not available until recently.

Saldana and Ramey ${ }^{7}$ presented detailed studies for closed chamber drillstem testing. A momentum balance including frictional and inertial effects was applied to the rising liquid level within the wellbore. This resulted in a nonlinear wellbore equation which was then coupled with the reservoir diffusivity equation through the wellbore storage coefficient. The solution was obtained numerically using a finite difference scheme.

A different approach was proposed by Simmons and $\mathrm{Grader}^{8}$ for closed chamber tests. Their method generates the pressure response of a closed chamber test using a numerical method based on superposition of the cumulative rate of the constant pressure production solution of the standard radial flow problem. Sensitivity studies were conducted for various tool and reservoir parameters. Methods to analyze field test data using their method were also outlined.

In the work of Simmons and Grader ${ }^{8}$, wellbore friction, liquid compression and inertial effects were neglected. As a follow-up study, Salas and Sageev ${ }^{9}$ adopted the approach developed by Simmons and Grader ${ }^{8}$, but included liquid compression and wellbore friction in their analysis. They found that the effects of wellbore friction and liquid compression can be significant. Parametric studies were also performed to analyze the influences of different test tools and reservoir parameters on closed chamber tests. An attempt was made to match field test data using their simulator.

An implicit numerical model has been developed for closed chamber tests by Mfonfu and Grader ${ }^{10}$. The wellbore flow and reservoir flow are combined through the use of Bernoulli's law. To regenerate field test data, variable skin factors were assumed.

Petak et al. ${ }^{11}$ presented another approach for closed chamber tests. In their work, a $I-\theta-z$ reservoir simulator was linked to the wellbore flow by using a wellbore storage term which is calculated at each time step by performing a mass balance on the wellbore fluids. In their study, however, only single phase flow in the wellbore is considered, and frictional and inertial effects are neglected.

In recent years, there has been a great interest in the interpretation of pressure 
data from underbalanced perforation and backsurge perforation cleaning. When a formation is perforated underbalanced, the reservoir sandface is suddenly exposed to a relatively low pressure and reservoir fluid may be produced at extremely high flow rates. Drilling fluid contamination around the wellbore, and gun debris and crushed rock in the perforation channels are washed out. As a result, an improved productivity is hopefully achieved. Although there are differences in operation, underbalanced perforation, backsurge perforation cleaning and closed chamber DST are the same from the transient well testing point of view. In this work, therefore, the term closed chamber test is generally used to refer to all of them.

During closed chamber tests, reservoir fluid flow rates continuously decline as the well bottomhole pressure increases resulting from the increase of the liquid level in the wellbore and the gas chamber pressure. If well flow rate is known, variable rate well test techniques can be used to interpret pressure data, and to allow reservoir flow capacity and skin factor to be obtained. Tariq and Ayestaran ${ }^{12}$ presented a convolution method for closed chamber tests. Flow rates are either directly measured from MWP (Measurement While Perforating) tools or calculated from the time derivative of the measured chamber gas pressure. They used their method to analyze pressure data from two field surge perforation tests. Simmons ${ }^{13}$ proposed a similar interpretation method which allows skin factors to be functions of time.

Viewing a closed chamber test as a short, high rate drawdown, Soliman et al. ${ }^{14}$ derived an approximate interpretation method applicable to tests with a small chamber volume. In their method, a log-log plot of pressure change (from original reservoir pressure) versus total test time yields a straight line with a slope of -1.0 during late time radial flow in a homogeneous reservoir. Reservoir flow capacity can then be calculated from any point of that straight line. Skin factor is determined by matching type curves generated from the calculated flow capacity. This method requires an estimate of the initial reservoir pressure.

Garrett et al. ${ }^{15}$ applied and compared interpretation techniques presented by Simmons ${ }^{13}$ and Soliman et al. ${ }^{14}$ to field underbalanced perforating pressure data. They also modified the Soliman et al. ${ }^{14}$ technique, and presented a Cartesian straight line method which can be used to obtain not only the reservoir flow capacity, but also the reservoir initial pressure.

Another interpretation method, Impulse ${ }^{T M}$ Testing, which also utilized the concept of short producing time, was developed by Ayoub et al. ${ }^{16}$. When this technique is applied to closed chamber tests, the product of the pressure change and the elapsed test time is plotted versus the elapsed time in a log-log scale, and matched with the classical wellbore storage and skin derivative type curves. Flow regimes may then be identified. Reservoir parameters are calculated in the same way as in the classical type curve matching techniques. As in the case of Soliman et al. ${ }^{14}$, this method also requires an estimate of the initial reservoir pressure. As reported by Manohar et al. ${ }^{17}$, Impulse Testing method has been applied to a number 
of field underbalanced perforating pressure data.

Despite the numerous attempts mentioned above, there has been only limited success in the interpretation of pressure data from closed chamber tests. More studies are needed in this area. In this study, we formulate a new inner boundary condition for closed chamber tests. Then, pseudotime is introduced to correlate closed chamber tests to slug tests. It is shown that, in many cases, pressure data from closed chamber tests can be analyzed using either slug test type curves or classical wellbore storage and skin type curves. We also propose a method to generate the pressure response, in the shut-in period of closed chamber tests and a rateconvolution method to analyze pressure data measured during the shut-in period of a closed chamber test.

- Throughout this study, pressure responses considered for closed chamber tests were generated using a computer simulator based on the superposition of the cumulative production solution for production at a constant bottomhole pressure ${ }^{8}$.

\section{DEFINITIONS}

In this section, definitions of the dimensionless variables used in this work are summarized. All definitions are presented in oil field units.

We consider slug tests and closed chamber tests conducted in a fullypenetrating well located in an infinite, homogeneous and isotropic reservoir of uniform thickness. Initially, the pressure is constant throughout the reservoir and equal to $p_{i}$ whereas, at the wellbore, the pressure is instantaneously set to $p_{o}$.

Dimensionless time, dimensionless radial distance and dimensionless wellbore storage coefficient are defined, respectively, by

$$
\begin{gathered}
t_{D}=\frac{2.637 \times 10^{-4} k t}{\phi \mu c_{t} r_{w}^{2}} \\
r_{D}=\frac{r}{r_{w}}
\end{gathered}
$$

and

$$
C_{D}=\frac{5.615 C}{2 \pi \phi c_{t} h r_{w}^{2}},
$$

where $C$ denotes the wellbore storage coefficient in $\mathrm{RB} / \mathrm{psi}$, due to the change of liquid level, the compression of a fluid in the wellbore, or the combined effects of both a changing liquid level and fluid compression. For a changing liquid level, the wellbore storage coefficient is given by

$$
C_{l}=\frac{144 \pi r_{p}^{2}}{(5.615 \rho g \sin \theta) / g_{c}},
$$


where $r_{p}$ is the radius of the wellstring, $\rho$ is'the density of the fluid being produced into the wellbore and $\theta$ represents the inclination of the wellstring from horizontal direction. Sometimes, slug tests are conducted with the wellbore closed in at the surface and filled with a slightly compressible liquid. In this case, the wellbore storage coefficient is given by

$$
C_{l}=V_{w} c_{l}
$$

where $V_{w}$ denotes the wellbore volume in $\mathrm{RB}$ and $c_{l}$ denotes the isothermal compressibility of the liquid.

In closed chamber tests, the chamber gas is compressed as the liquid level rises in the wellbore. The wellbore storage coefficient due to gas compression is defined as

$$
C_{g}=V_{g} c_{g}
$$

where $V_{g}$ is the wellbore volume in RB occupied by the chamber gas and $c_{g}$ is the isothermal gas compressibility.

In this study, several dimensionless pressure definitions are used. Throughout, $p_{D}$ denotes the dimensionless reservoir pressure for slug tests and closed chamber tests, $p_{w D}$ represents the corresponding dimensionless wellbore pressure, $p_{c D}$ denotes the dimensionless reservoir pressure for the classical wellbore storage and skin constant surface-rate problem and $p_{w c D}$ represents the corresponding dimensionless wellbore pressure. We define $p_{D}$ and $p_{w D}$, respectively, by

$$
p_{D}=\frac{p_{i}-p(r, t)}{p_{i}-p_{o}}
$$

and

$$
p_{w D}=\frac{p_{i}-p_{w f}(t)}{p_{i}-p_{o}}
$$

where $p(r, t)$ and $p_{w f}(t)$ are the reservoir pressure and wellbore pressure, respectively, in slug or closed chamber tests.

For production at a constant surface rate $q$, in STB/D, the dimensionless reservoir pressure and dimensionless wellbore pressure are defined by

$$
p_{c D}=\frac{k h\left[p_{i}-p(r, t)\right]}{141.2 q B \mu}
$$

and

$$
p_{w c D}=\frac{k h\left[p_{i}-p_{w f}(t)\right]}{141.2 q B \mu},
$$

where $p(r, t)$ and the $p_{w f}(t)$ are the corresponding dimensional pressure in the reservoir and at the wellbore, respectively, if the well produces at a constant surface rate. 
Based on the concept of an infinitesimally thin skin, the skin factor is defined by

$$
s=\frac{k h}{141.2 q_{s f}(t) \mu} \Delta p_{s k i n},
$$

where $q_{s f}$ denotes the sandface flow rate, in $\mathrm{RB} / \mathrm{D}$, and $\Delta p_{s k i n}$ denotes the additional pressure drop which occurs due to formation damage or improvement around the wellbore.

The dimensionless instantaneous sandface flow rate for a well produced at a constant bottomhole pressure, $p_{w f}$, is defined by

$$
q_{D}=\frac{141.2 q_{s f}(t) \mu}{k h\left(p_{i}-p_{w f}\right)} .
$$

The dimensionless cumulative production is defined as

$$
Q_{D}=\frac{5.615 Q_{s f}}{2 \pi \phi h c_{t} r_{w}^{2}\left(p_{i}-p_{w f}\right)},
$$

where $Q_{s f}$ denotes the dimensional sandface cumulative production, in $R B$, for a well which produces at a constant bottomhole pressure. $Q_{s f}(t)$ and $q_{s f}(t)$ are related by

$$
Q_{s f}(t)=\int_{0}^{t} q_{s f}(\tau) d \tau .
$$

\section{BACKGROUND AND THEORY}

In this section, we present a brief review of the most commonly used methods for analyzing slug tests, followed by an outline of the numerical procedure of Ref. 8 which was utilized to generate the pressure response for a closed chamber test. Then, we introduce new interpretation techniques for closed chamber tests and closed chamber DST buildup tests.

We consider slug tests and closed chamber tests conducted in a fullypenetrating well located in an infinite, homogeneous and isotropic reservoir of uniform thickness. Initially, the pressure is constant throughout the reservoir and equal to $p_{i}$ whereas, at the wellbore, the pressure is instantaneously set to $p_{o}$. In addition, it is assumed that in slug tests, friction and inertial effects in the wellbore can be neglected, and critical flow is not present. We also assume that the wellstring has a constant inner diameter and inclination angle.

\section{Slug Test Solution}

Under the stated assumptions and using the dimensionless variable defined previously, the slug test initial, boundary-value problem (IBVP) can be stated as

$$
\frac{1}{r_{D}} \frac{\partial}{\partial r_{D}}\left(r_{D} \frac{\partial p_{D}}{\partial r_{D}}\right)=\frac{\partial p_{D}}{\partial t_{D}}
$$




$$
\begin{gathered}
p_{D}\left(r_{D}, 0\right)=0 ; \quad 1<r_{D}<\infty \\
p_{w D}(0)=1 \\
\lim _{r_{D} \rightarrow \infty} p_{D}\left(r_{D}, t_{D}\right)=0 ; \quad t_{D}>0 \\
p_{w D}=\left[p_{D}-s\left(r_{D} \frac{\partial p_{D}}{\partial r_{D}}\right)\right]_{\tau_{D}=1}
\end{gathered}
$$

and

$$
\left(r_{D} \frac{\partial p_{D}}{\partial r_{D}}\right)_{r_{D}=1}=C_{D} \frac{\partial p_{w D}}{\partial t_{D}} .
$$

In Eq. 14.6, $C_{D}$ is the dimensionless wellbore storage coefficient during slug tests, due to either a rising liquid level or fluid compression. For slug tests conducted in a wellstring with a constant inner diameter and inclination angle, $C_{D}$ practically remains constant, if the well is filled with a slightly compressible liquid. This is true regardless of whether the well is opened at the surface while testing or closed in at the surface. Thus, the IBVP for a slug tests is linear, and can be solved by applying integral transform techniques. Ramey and Agarwal ${ }^{18}$ obtained the solution to Eqs. 14.1 through 14.6 by using the Laplace transform with respect to time.

Using the solution derived by Ramey and Agarwal ${ }^{18}$, Ramey et al. ${ }^{1}$ presented slug test type curves in which $p_{w D}$ is plotted versus $t_{D} / C_{D}$ with $C_{D} e^{2 s}$ as the correlating parameter. As pointed out by Ramey et al. ${ }^{1}$, although this approach is not rigorous and may not work for small values of $C_{D} e^{2 s}$, it is appropriate for the range of $C_{D} e^{2 s}$ normally encountered in a transient well test. For more than a decade, type-curve matching using these type curves remained the standard and primary technique for interpreting slug test pressure data.

Recently, Peres et al. ${ }^{2}$ developed a relationship relating the slug test solution and the solution for the classical wellbore storage and skin constant surface rate production problem. This relationship is valid for any well/reservoir geometry and holds at any position in the reservoir. At the wellbore, this relationship is described by

$$
p_{w c D}\left(t_{D}, C_{D}, s\right)=\frac{I\left(p_{w D}\right)}{C_{D}}
$$

and

$$
p_{w c D}^{\prime}=\frac{t_{D}}{C_{D}} p_{w D}
$$

In Eq. 15, $I\left(p_{w D}\right)$ denotes the pressure integral, given by

$$
I\left(p_{w D}\right)=\int_{0}^{t_{D}} p_{w D}\left(\tau, C_{D}, s\right) d \tau .
$$


In Eq. 16, $p_{w c D}^{\prime}$ denotes the logarithmic time derivative of the constant surface rate dimensionless wellbore pressure, defined as

$$
p_{w c D}^{\prime}=\frac{d p_{w c D}}{d \ln t_{D}}=t_{D} \frac{d p_{w c D}}{d t_{D}} .
$$

Eq. 15 shows that a simple integration of the slug test pressure data over the producing time will generate the equivalent pressure data that would be obtained if the well was produced at a constant surface flow rate. Moreover, as indicated in Eq. 16, the multiplication of the slug test data by. the producing time yields the equivalent constant surface rate pressure derivative data, without applying any numerical differentiation technique. Peres et al. $^{2}$, therefore, concluded that slug test pressure data can be analyzed using type curves constructed for the constant surface rate production problem, and type curves developed specifically for slug tests are no longer needed.

Numerical Solution for CCT

The configuration of a closed chamber test is schematically shown in Fig. 1. The primary difference between a closed chamber test and a slug test is that in a closed chamber test the well is always closed in at the surface when the test is being conducted. This difference, however, makes closed chamber tests mathematically more complicated. To the best of our knowledge, no analytical solution has been presented in the literature. In the present work, we use the numerical method proposed by Simmons and $\mathrm{Grader}^{8}$ to generate the pressure response during closed chamber tests. The generated pressure responses are then used to verify our new closed chamber test interpretation method, which is presented in a later section.

The numerical method developed by Simmons and $\mathrm{Grader}^{8}$ is based on the superposition of constant bottomhole pressure production solution of the radial diffusivity equation. The superposition principle is given by

$$
Q_{v D}\left(t_{D}\right)=\int_{0}^{t_{D}} Q_{c D}^{\prime}\left(t_{D}-\tau\right) p_{w v D}(\tau) d \tau
$$

where $Q_{v D}$ and $Q_{c D}$ are the dimensionless cumulative rate for variable and constant bottomhole pressure production problem, respectively. In Eq. $19, p_{w v D}$ denotes the variable dimensionless bottomhole pressure.

The closed chamber test response can be generated by producing the well at a sequence of constant bottomhole pressures, as indicated in Fig. 2, and then Eq. 19 can be approximated by

$$
\begin{aligned}
Q_{s f}\left(t_{N}\right) & =\beta\left(p_{i}-p_{o}\right) Q_{c D}\left(t_{D}\right) \\
& -\beta \sum_{j=1}^{N-1}\left(p_{j}-p_{j-1}\right) Q_{c D}\left(t_{D}-t_{D, j}\right)
\end{aligned}
$$


where $\beta=\left(2 \pi \phi h c_{t} r_{w}^{2}\right) / 5.615$ and $p_{j}=p_{w v}\left(t_{j}\right)$. For the well/reservoir geometry under consideration, when the well produces at a constant bottomhole pressure, the Laplace transform of the dimensionless cumulative rate is given by

$$
\bar{Q}_{c D}(u)=\frac{\sqrt{u} K_{1}(\sqrt{u})}{u^{2}\left[K_{0}(\sqrt{u})+s \sqrt{u} K_{1}(\sqrt{u})\right]}
$$

where $u$ is the Laplace transform variable, and $K_{0}$ and $K_{1}$ are modified Bessel functions of the second kind, of order zero and one, respectively. The Stehfest Algorithm is applied to Eq. 21 to obtain the cumulative rate in the real time domain.

Eq. 20 gives the cumulative production at time $t_{N}$. Then, the liquid level in the wellbore at this time can be calculated as

$$
X=L_{i}+\frac{5.615 Q_{s f}}{A_{c h}}
$$

where $L_{i}$ is the initial height of the liquid in the wellbore (drillstring) and $A_{c h}=\pi r_{p}^{2}$ is the cross sectional area of the drillpipe. In Eq. 22, we implicitly assume that the liquid in the wellbore is incompressible. Assuming that the chamber gas behaves as an ideal gas and the temperature and pressure gradients in the gas column are negligible, the chamber gas pressure can obtained from

$$
p_{c h}=\frac{p_{c h i}\left[L_{c}-L_{i}\right]}{\left[L_{c}-X\right]}
$$

where $L_{c}$ is the length of the chamber and $p_{c h i}$ is the initial pressure in the gas cushion. The bottomhole pressure is the summation of the chamber gas pressure and the hydrostatic pressure caused by the liquid column. Therefore, at any time $t_{N}$, the bottomhole pressure is given by

$$
p_{w f}=p_{c h}+\frac{\rho g}{144 g_{c}} X
$$

Starting at $t=0$ and $p_{w f}(t=0)=p_{o}$, we can use the procedure outlined above and generate the pressure response for a closed chamber test for a given set of tool and reservoir parameters. The specific steps of the solution are as follows:

1. Choose $t_{m a x}$, the test duration one wants to investigate, and $\Delta t$, the uniform time step. Calculate the dimensionless time step, $\Delta t_{D}$.

2. Invert $\bar{Q}_{c D}(u)$ (see Eq. 21) for $t_{D}=i \Delta t_{D}$, for $i=1,2, \ldots, M=t_{\max } / \Delta t$ and store values for later use.

3. Begin calculation, at $N=1$. Assume that from $t_{N-1}$ to $t_{N}$, the wellbore pressure remains constant and calculate the cumulative production at $t_{N}$ from Eq. 20. 
4. Calculate the liquid level at $t_{N}$ from Eq. 22 .

5. Calculate the chamber gas pressure at $t_{N}$ from Eq. 23.

6. Calculate the wellbore pressure at $t_{N}$ from Eq. 24 .

7. Update $N$ and repeat steps from 3 through 6 until $N=M$.

Theoretically, one can use variable time steps in the numerical simulator, but in order to speed-up the calculation a uniform time step is more desirable such that inversion of $\bar{Q}_{c D}(u)$ for $t_{D}=i \Delta t_{D}(i=1,2, \ldots, M)$ can be carried out outside the loop of the pressure calculation. To insure numerical stability, a small time step is usually required. Numerical calculations indicated that the size of the time step that can be used primarily depends on the length of the chamber. For $L_{c}=1000 \mathrm{ft}$, $\Delta t=0.01$ second was used, while for $L_{c}=10,000 \mathrm{ft}, \Delta t=2$ seconds can be used. In principle, the time step is chosen such that the pressure change during each time step is relatively small.

In our work, we also implemented the methods of Salas and Sageev ${ }^{9}$ to take into account the effects of the wellbore friction and liquid compression. Salas and Sageev developed methods for three cases: 1) a model with frictional effect and no compressional effects, 2) a model without frictional effect but including compressional effects and 3) a model with frictional and with compressional effects. To simplify the numerical calculation, we consider the effects of wellbore friction and liquid compression separately, i.e., only case 1 and case 2 .

The cumulative production is calculated in exactly the same way as previously presented. The average liquid flow rate during each time step is approximated by

$$
q_{s f}=\frac{Q_{s f}\left(t_{N}\right)-Q_{s f}\left(t_{N-1}\right)}{\Delta t / 86400}
$$

where $q_{s f}$ is in RB/D and $\Delta t$ is in seconds. From Eq. 25 , the average liquid velocity in the drillstring can be obtained

$$
v_{l}=\frac{5.615 q_{s f}}{86400 A_{c h}},
$$

where $v_{l}$ is in $\mathrm{ft} / \mathrm{s}$. Thus, the frictional pressure drop along the liquid column can be evaluated

$$
\Delta p_{f}=\frac{1}{144} \frac{f \rho v_{l}^{2}}{2 d_{p} g_{c}} X
$$

where $f$ is the friction factor calculated from the liquid Reynolds number and the roughness of the drillpipe and $d_{p}$ is the diameter of the drillpipe. Therefore, when wellbore friction is considered in the CCT numerical model, the wellbore pressure will be obtained by the following equation:

$$
p_{w f}=p_{c h}+\frac{\rho g}{144 g_{c}} X+\frac{1}{144} \frac{f \rho v_{l}^{2}}{2 d_{p} g_{c}} X .
$$


One can see that the previously outlined calculation procedure can be followed for the CCT model with the effect of wellbore friction included. The only modification is that, during each time step, we calculate the liquid flow rate and add the frictional pressure drop along the liquid column to the wellbore pressure calculation.

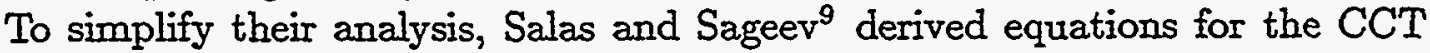
problem with the effect of liquid compression included only for cases with a zero initial liquid level. The hydrostatic pressure drop along the liquid column at time $t_{N}$ is given by

$$
\Delta p=\frac{5.615 \rho Q_{s f}}{144 A_{c h}} \frac{g}{g_{c}} \text {. }
$$

The chamber gas pressure depends on the liquid level as shown by Eq. 23. On the other hand, the liquid level, which is a function of the liquid compressibility, also depends on the chamber gas pressure, as shown by Salas and Sageev ${ }^{9}$. Their relation -is

$$
X=\frac{144}{c_{l} \rho} \frac{g_{c}}{g} \ln \left[1+\frac{c_{l} \Delta p}{1+c_{l}\left(p_{c h}-p_{i}\right)}\right]
$$

Hence, at each time step, an iteration process is needed to determine the liquid level and the chamber gas pressure in order to calculate the wellbore pressure. Specifically, at each time step the following procedure is adopted:

1. Calculate the cumulative production in the same way as for the case when the effect of liquid compression is neglected.

2. Calculate the hydrostatic pressure drop along the liquid column using Eq. 29.

3. Estimate the liquid level using Eq. 22.

4. Calculate the chamber gas pressure from Eq. 23.

5. Update the liquid level using Eq. 30.

6. Compare the current liquid level with the value of the previous iteration step. Repeat steps 4 and 5 using the current liquid level if $\left|X^{k}-X^{k-1}\right| \leq \epsilon$ is not satisfied ( $k$ is the index of the iteration and $\epsilon$ is usually set to $0.001 \mathrm{ft}$ ). Stop iteration if convergence is reached.

Once convergence is achieved, the wellbore pressure can be obtained as

$$
p_{w f}=p_{c h}+\Delta p
$$

New Interpretation Method for CCT

For a CCT conducted with the same well/reservoir geometry as assumed in the beginning of this section, Eqs. 14.1 - 14.5 still hold. The inner boundary condition, Eq. 14.6, however, is no longer appropriate since the wellbore storage coefficient is influenced by the compression of the wellbore gas and is not constant.

In Appendix A, we derive the effective wellbore storage coefficient for a CCT, under the assumptions considered in this work. The effective wellbore storage is given by

$$
C_{a}=\frac{C_{g} C_{l}}{C_{g}+C_{l}}
$$


where $C_{l}$ and $C_{g}$, respectively, denote the wellbore storage coefficients due to a rising liquid level and gas compression, given by Eq. 4.1 and Eq. 5 respectively. Eq. 32 can be written in a dimensionless form as

$$
C_{a D}=\frac{C_{g D} C_{l D}}{C_{g D}+C_{l D}}
$$

Eqs. 32 and 33 suggest that both the rising liquid level and gas compression influence the test simultaneously. $C_{a}$ is a function of pressure (and hence a function of time) because of the pressure-dependence of $C_{g}$ (caused by the change of gas volume and gas compressibility during the test); however $C_{l}$ can be considered to be a constant. Hence, a closed chamber test is a variable wellbore storage problem.

Corresponding to Eq. 14.6, the new inner boundary condition for a CCT becomes

$$
\left(r_{D} \frac{\partial p_{D}}{\partial r_{D}}\right)_{r_{D}=1}=C_{a D} \frac{\partial p_{w D}}{\partial t_{D}} .
$$

Although Eq. 34 appears to be the same as Eq. 14.6, the two equations differ significantly. In Eq. 34, because of the dependence of $C_{a D}$ on $p_{w D}$, the inner boundary condition is non-linear. Therefore, the Laplace Transform is not applicable to the IBVP for the closed chamber test.

The application of pseudotime $\mathrm{e}^{19}$ has been widely accepted to improve the analysis of gas well buildup tests. This is primarily due to the fact that the use of pseudotime can effectively linearize the inner boundary condition of the problem. As discussed above, the inner boundary condition is nonlinear in a closed chamber test due to a changing wellbore storage coefficient. Motivated by the use of pseudotime in gas well buildup tests, we consider its application to the analysis of closed chamber tests.

We define dimensionless pseudotime as

$$
t_{a D}=\int_{0}^{t_{D}} \frac{C_{a D i}}{C_{a D}} d t_{D}^{\prime},
$$

where $C_{a D i}$ is the dimensionless effective wellbore storage coefficient at the beginning of a closed chamber test. Using Eq. 35, it follows that we can rewrite Eq. 34 to obtain the following linearized inner boundary condition for closed chamber tests:

$$
\left(r_{D} \frac{\partial p_{D}}{\partial r_{D}}\right)_{r_{D}=1}=C_{a D i} \frac{\partial p_{w D}}{\partial t_{a D}} .
$$

Comparing Eqs. 14.6 and 36 suggests that if we replace time by pseudotime in analyzing closed chamber test data, we may be able to correlate the closed chamber test with an equivalent slug test solution with a constant wellbore storage equal 
to the initial effective wellbore storage of the closed chamber test, $C_{a D i}$. Consequently, interpretation methods developed for slug tests can be easily applied to closed chamber tests.

The change of variable completely linearizes the inner boundary condition of our problem, but corresponding changes have to be made to the original flow equation. With the definition given by Eq. 35, Eq. 14.1 becomes

$$
\frac{1}{r_{D}} \frac{\partial}{\partial r_{D}}\left(r_{D} \frac{\partial p_{D}}{\partial r_{D}}\right)=\frac{C_{a D i}}{C_{a D}} \frac{\partial p_{D}}{\partial t_{a D}} .
$$

Eq. 37 is nonlinear based on the same argument given to Eq. 34. Therefore, the use of pseudotime has transferred the non-linearity of our problem from the inner boundary condition to the original flow equation. The magnitude of the $C_{a D i} / C_{a D}$ will affect how much the solution of the CCT IBVP deviates from the solution of the corresponding slug test IBVP. Thus, $C_{a D i} / C_{a D}$ is the critical factor that controls the applicability of pseudotime to correlate closed chamber test solutions with slug test solutions.

Investigation of the applicability of the pseudotime concept to closed chamber tests is given in a later section. Eq. 35 is used to convert dimensionless time to dimensionless pseudotime. Then a plot of $p_{w D}$ versus $t_{a D}$ from a CCT is compared with the analytical slug test solution with a wellbore storage coefficient equal to $C_{a D i}$.

The effectiveness of using pseudotime to correlate a closed chamber test solution with the solution of a slug tests is also considered by using the classical solution of the wellbore storage and skin constant surface-rate production problem. This is accomplished by using the procedure developed by Peres et al. ${ }^{2}$. We first calculate the integral of the closed-chamber-test dimensionless pressure solution by

$$
I\left(p_{w D}\right)=\int_{0}^{t_{a D}} p_{w D}\left(\tau, C_{a D}, s\right) d \tau .
$$

Then, the equivalent dimensionless pressure, and its logarithmic derivative, for the constant surface-rate production problem are calculated by

$$
p_{w c D}\left(t_{a D}, C_{a D i}, s\right)=\frac{I\left(p_{w D}\right)}{C_{a D i}}
$$

and

$$
p_{w c D}^{\prime}=\frac{t_{a D}}{C_{a D i}} p_{w D},
$$

respectively. Eqs. 38, 39 and 40 indicate that the Peres-Onur-Reynolds procedure can be applied to field closed chamber test data provided one converts time to pseudotime. 
The dimensional form of Eq. 35 is

$$
t_{a}=\int_{0}^{t} \frac{C_{a i}}{C_{a}} d t^{\prime}
$$

Numerical integration techniques can be used to obtain pseudotime from Eq. 41 . The wellbore storage coefficient due to a rising liquid level, $C_{l}$, is given by Eq. 4.1 and is assumed to be a constant. Therefore, according to Eq. 32, the calculation of the effective wellbore storage coefficient will require the values for the wellbore storage coefficient due to gas compression. With the assumption that the chamber gas behaves as an ideal gas, the following equation can be derived from Eq. 5

$$
C_{g}=\frac{p_{c h i}\left(L_{c}-L_{i}\right) A_{c h}}{5.615 p_{c h}^{2}} .
$$

It follows that the measurement of the chamber gas pressure during a closed chamber test can be used to give the wellbore storage coefficient due to gas compression and, as a result, time can be converted to pseudotime and field closed chamber test data can be analyzed as in the analysis of slug test data using the wellbore pressure versus pseudotime data.

\section{Closed Chamber DST Buildup Analysis}

As in a conventional DST, a number of cycles of flow and shut-in may also be possible for a closed chamber DST. A new interpretation method for the flow period of a closed chamber DST has been presented in the preceding sub-section. In this sub-section, we focus on the shut-in period of a closed chamber DST.

Since we consider the same well/reservoir geometry as stated previously, Eqs. 14.1 - 14.5 are still valid. When the well is shut-in for pressure buildup, however, the wellbore storage coefficient changes from the one due a combination of rising liquid level and gas compression to the one due to liquid compression. This results in a new inner boundary condition for our problem, which is given by

$$
\left(r_{D} \frac{\partial p_{D}}{\partial r_{D}}\right)_{r_{D}=1}= \begin{cases}C_{a D} \frac{d p_{w D}}{d t_{D}}, & t_{D}<t_{p D} \\ C_{s D} \frac{d p_{w D}}{d t_{D}}, & t_{D}>t_{p D}\end{cases}
$$

In Eq. $43, C_{s D}$ is the dimensionless wellbore storage coefficient for the shut-in period of a closed chamber DST, and can be treated as a constant. The combined wellbore storage coefficient, $C_{a D}$, on the other hand, depends on the dimensionless wellbore pressure, as in the case of a closed chamber test. Therefore, the inner boundary condition given by Eq. 43 is nonlinear. To the best of our knowledge, no analytical solution is presented in the literature.

In the present study, we extend the numerical method developed by Simmons and Grader $^{8}$ for closed chamber tests to the shut-in period of a closed chamber DST. 
The isothermal compressibility of a fluid is defined by

$$
c_{l}=-\frac{1}{V} \frac{d V}{d p} .
$$

Since we assume that the well produces a slightly compressible liquid of constant compressibility, Eq. 44 can be integrated to give

$$
p=p_{r}-\frac{1}{c_{l}} \ln \frac{V}{V_{T}}
$$

where $p_{r}$ is a reference pressure and $V_{r}$ is the liquid volume at the reference pressure.

The superposition principle, given in Eq. 20, can be applied to the shut-in period of a closed chamber DST. Let $Q_{s f}(\Delta t=0)$ and $p_{w s}(\Delta t=0)$, respectively, denote the cumulative production and the wellbore pressure at the instant of shutin. For $t_{N}>t_{p}$, the flowing time, the cumulative production is calculated from Eq. 20. Then applying the equation of state, Eq. 45, we obtain the bottomhole pressure at the current time $t_{N}$ :

$$
p_{w s}=p_{w s}(\Delta t=0)-\frac{1}{c_{l}} \ln \left(\frac{V_{w}-\left(Q_{s f}(\Delta t)-Q_{s f}(\Delta t=0)\right)}{V_{w}}\right),
$$

where $\Delta t$ is the shut-in time, and $V_{w}$ is the wellbore volume between the shut-in valve and the sandface of a formation being tested. Eqs. 20 and $46 \mathrm{can}$ be used to generate the pressure responses for the shut-in period of a closed chamber DST for a given set of tool and reservoir parameters. Then, reservoir flow capacity and skin factor can be deduced by history matching techniques, as suggested by Simmons and Grader ${ }^{8}$.

Rate convolution methods for the interpretation of drillstem buildup pressure data have been developed by Peres et al. ${ }^{20}$. A similar approach is adopted here for analyzing pressure data from the shut-in period of a closed chamber DST. A detailed derivation can be found in Appendix B. The convolution method, in dimensionless form, is given by

$$
\frac{I\left(p_{s D}\right)}{C_{a D i} \tilde{p}_{s D}}-\frac{C_{s D}}{C_{a D i}} \tilde{s}_{D}=t_{M R D}
$$

In Eq. $47, I\left(p_{s D}\right)$ is the integral of the dimensionless buildup wellbore pressure, i.e.,

$$
I\left(p_{s D}\right)=\int_{0}^{\Delta t_{D}} p_{s D}(\tau) d \tau
$$

$C_{a D i}$ is the initial dimensionless effective wellbore storage coefficient, $\tilde{p}_{s D}$ denotes the dimensionless buildup pressure rise given by

$$
\tilde{p}_{s D}=p_{w D}\left(t_{p D}\right)-p_{s D}\left(\Delta t_{D}\right),
$$


$\tilde{s}_{D}$ is a constant given by

$$
\tilde{s}_{D}=\frac{1}{2} \ln \left(\frac{4}{e^{\gamma}}\right)+s,
$$

and $t_{M R D}$ is the multirate dimensionless time, defined by

$$
\begin{aligned}
t_{M R D}=- & \frac{1}{2 \tilde{p}_{s D}}\left[\sum_{j=0}^{n} \ln \left(\frac{t_{D}-\hat{t}_{j+1, p D}}{t_{p D}-\hat{t}_{j+1, p D}}\right) \frac{C_{a D}\left(\hat{t}_{j+1, p D}\right)}{C_{a D i}}\left[p_{w D}\left(t_{j+1, p D}\right)-p_{w D}\left(t_{j, p}\right)\right]\right. \\
& \left.+\frac{C_{s D}}{C_{a D i}} \sum_{i=0}^{m} \ln \left(\Delta t_{D}-\Delta \hat{t}_{i+1, D}\right)\left[p_{s D}\left(\Delta t_{i+1, D}\right)-p_{s D}\left(\Delta t_{i, D}\right)\right]\right]
\end{aligned}
$$

A corresponding dimensional form of Eq. 47 which can be applied to interpret field pressure data from the shut-in period of a closed chamber DST is presented in Appendix B.

\section{VALIDATION OF NUMERICAL MODELS}

In the previous section, we reviewed the numerical model for closed chamber tests proposed by Simmons and Grader and extended the model to compute the pressure response during the shut-in period of a closed chamber drillstem test. Computer simulators were developed to implement these numerical methods. In this section, we verify these numerical models by comparing pressure responses generated by computer simulators with solutions found in the literature.

\section{Duplication of Slug Test Solutions}

The analytical solution for slug tests derived by Ramey and Agarwal ${ }^{18}$ is

$$
\bar{p}_{w D}=C_{D} \frac{K_{0}(\sqrt{u})+s \sqrt{u} K_{1}(\sqrt{u})}{\sqrt{u} K_{1}(\sqrt{u})+C_{D} u\left[K_{0}(\sqrt{u})+s \sqrt{u} K_{1}(\sqrt{u})\right]} .
$$

When the dimensionless wellbore storage coefficient and the skin factor are known, the Stehfest algorithm can be used to invert Eq. 52 to obtain the dimensionless wellbore pressure in the real time domain for a given dimensionless time.

Conventional slug tests are special cases of closed chamber tests. Specifically, if we hold the chamber pressure constant, then compression of chamber gas does not occur and the closed chamber test is equivalent to the classical slug test. This suggests a method to verify our numerical simulator.

Parameters given in Table 1 are used in our simulator. Note that the total chamber length, initial liquid height and initial chamber gas pressure are not listed in this table, because the dimensionless wellbore pressure generated by the simulator is independent of these parameters as long as the chamber gas pressure is held constant. With these parameters, the dimensionless wellbore storage coefficient due 
to a rising liquid level was found to be 1127. Using $C_{D}=1127$ and $s=0$, analytical dimensionless wellbore pressures are obtained by inverting Eq. 52. Numerical and analytical solutions are listed in Table 2. Note that good agreement was obtained between the two solutions for the wide range of dimensionless time investigated.

\section{Comparison with Simmons and Grader Solutions}

Simmons and Grader presented results for a base case CCT, and also conducted sensitivity analysis for various tool and reservoir parameters. Table 3 gives the parameters for the base case CCT used by Simmons and Grader. In their numerical model, the chamber gas was treated as a real gas and, thus, the gas compressibility factor was allowed to change as a function of pressure and temperature. However, they found out that the effect of assuming chamber gas as an ideal gas is not large. Our simulator was run with the parameters listed in Table 3 without considering the change of gas compressibility. Results are compared in Table 4.

From Table 4, we see that the two solutions agree very well except for the period of 15-20 seconds. This may be attributed to the effect of the change of gas compressibility, but the difference between the two solutions did not exceed $5 \%$.

\section{Comparison with Salas and Sageev Solutions}

We also compare our solutions with the solutions obtained by Salas and Sageev when a compressible liquid is considered. It was assumed that the initial liquid height $L_{i}=0 \mathrm{ft}$ and liquid compressibility $c_{l}=1 \times 10^{-6}$. All other parameters are exactly the same as given in Table 3. Our simulator solutions are compared with the solutions given by Salas and Sageev in Table 5. The two solutions give similar results. Again, except during a short period of time, the deviation is small and may be attributed to assuming ideal gas behavior in our model.

Duplication of Drillstem Buildup Test Solutions

A conventional DST buildup test is a special case of a closed chamber DST buildup test since by holding the chamber gas pressure as a constant in the flowing period of a closed chamber DST, a closed chamber DST buildup test degenerates to a conventional DST buildup test. Correa and Ramey ${ }^{21}$ derived analytical solutions for conventional DST buildup tests. Therefore, the numerical method proposed in this study for the shut-in period of a closed chamber DST can be verified by duplicating the known analytical solution.

The dimensionless wellbore pressure for the shut-in period of a drillstem test is $^{21}$

$$
p_{w D}=C_{s D} p_{D}^{\prime}\left(S, C_{s D}, t_{D}\right)+\left(C_{s D}-C_{f D}\right) \int_{0}^{t_{p D}} p_{D}^{\prime}\left(S, C_{s D}, t_{D}-\tau_{D}\right) p_{w D}^{\prime}\left(\tau_{D}\right) d \tau_{D}
$$

where $p_{D}^{\prime}$ is the time derivative of the dimensionless wellbore pressure for the classical wellbore storage and skin constant-surface-rate problem, and $p_{w D}^{\prime}$ is the time derivative of the dimensionless wellbore pressure solution for slug tests. For given 
values of $C_{f D}, C_{s D}, s$ and $t_{p D}$, Eq. 53 can be applied to generate dimensionless wellbore pressure responses for the shut-in period of a drillstem test.

Parameters used in this comparison study are the same as in Table 1 , and thus the dimensionless wellbore storage coefficient for the flowing period caused by the rising liquid level is $C_{f D}=1127$. Additional input data which are necessary for the comparison run are the height between the shut-in valve and the formation sandface and the compressibility of the produced liquid. Assuming the height between the shut-in valve and the formation sandface is $100 \mathrm{ft}$ and the compressibility of the produced liquid is $c_{l}=2.28 \times 10^{-4}$, we obtain the dimensionless wellbore storage coefficient for the shut-in period equal to $C_{s}=10$. With these parameters and the assumption that the dimensionless production time is $t_{p D}=10,000$, the dimensionless wellbore pressure for the shut-in period of a drillstem test was obtained from the simulator. The analytical solution and computer generated results are given in Table 6. Note that the two solutions are in close agreement.

The results discussed previously suggest that our computer simulator can be used to generate CCT solutions.

\section{RESULTS AND DISCUSSIONS}

In this section, we illustrate the application of new interpretation methods for closed chamber tests and closed chamber DST buildup tests, using pressure data generated from computer simulators. Further verification is planned if and when field test data become available.

\section{Closed Chamber Test Analysis}

We first establish a base case closed chamber test in which typical values are assigned to test tool and reservoir parameters, and investigate typical pressure responses from such a test. A sensitivity study is then conducted to evaluate the influence of various parameters on the performance of our new interpretation methods.

Parameters for the base case closed chamber test are presented in Table 7. Results for the base case closed chamber test are given in Figs. 3-7.

Fig. 3 is a Cartesian plot of the bottomhole pressure and the gas chamber pressure versus test time. As shown, both pressures increase rather rapidly until about 600 seconds and then the solutions become almost flat. The liquid level changes in a similar fashion, as shown in Fig. 4

A CCT is characterized by a wellbore storage coefficient which varies with time due to gas compression. Fig. 5 is a log-log plot of the dimensionless wellbore storage coefficients versus dimensionless time. As suggested in this figure, the wellbore storage coefficient due to gas compression changes significantly during the test and finally approaches a constant value as the flow becomes very small. On the other hand, the wellbore storage coefficient due to rising liquid level remains constant 
during the test. For $t_{D} \leq 1000$ (approximately $t \leq 100$ seconds), the combined wellbore storage coefficient is dominated by the wellbore storage due to the rising liquid level and remains almost equal to its initial value of $C_{a D}=994$. During the intermediate flow period the combined wellbore storage is controlled by both rising liquid level and gas compression. However, as the bottomhole pressure approaches the static reservoir pressure, the combined wellbore storage coefficient tends to approach another constant value, $C_{a D}=362$.

The changing wellbore storage coefficient during a CCT causes the pressure response to differ significantly from a slug test. This is shown in Fig. 6 . Three curves are shown in Fig. 6. Two of them represent the slug test solutions with constant wellbore storage coefficients equal to the initial $\left(C_{a D}=994\right)$ and final $\left(C_{a D}=362\right)$ wellbore storage coefficients during the CCT, respectively. The response of the CCT, plotted as a solid line, does not follow either of the slug test responses, but falls between them. This implies that parameters obtained may not be reliable if one analyzes the CCT data using the method developed in Refs. 1 and 2.

Fig. 7 shows the use of pseudotime for the base case CCT. In Fig. 7, the dashed line represents a plot of the slug test solution (with a constant wellbore storage coefficient equal to the initial wellbore storage coefficient of the CCT) versus $t_{D}$, and the solid line is the pressure response for the CCT plotted versus $t_{D}$. Also shown in Fig. 7 as circular data points is a plot of $p_{w D}$ versus $t_{a D}$ calculated from Eq. 35. As shown, when plotted versus dimensionless pseudotime, the CCT dimensionless pressure solution correlates almost perfectly with the slug test solution for $t_{D} \leq 10^{4}$ $\left(t_{D} / C_{a D i} \leq 10\right.$ and $t \leq 0.22$ hour). Therefore, the base case CCT can be analyzed using methods developed for slug tests.

Effect of Skin Factor. No near wellbore damage is assumed in our base case $\mathrm{CCT}$, i.e., we have used $s=0$. Drilling operations and later perforation very often contaminate the formation near the wellbore and create higher skin factors. Thus, it is important to identify the effect of the skin factor on the pressure response of a CCT. In Fig. 8, pressure responses from closed chamber tests with $s=5$ and $s=10$ are compared to the base case. (Except for the variation in the skin factor, all other parameters used to generate the results of Figs. 8-11 are the same as those given for the base case, Table 1.) It is observed that shin factors significantly influence the pressure response of a CCT. It is also found that a higher $p_{w D}$ is obtained for a CCT with a larger skin factor. This is obvious because a larger skin factor represents a higher resistance to flow near wellbore, and thus results in a slower pressure recovery.

The application of pseudotime for the $s=5$ case is shown in Fig. 9. Comparing these results with the base case of Fig. 7, we note that the CCT solution plotted versus pseudotime correlates with the corresponding slug test solution $\left(C_{D}\right.$ for slug test equal to the initial combined wellbore storage coefficient for CCT and same $s$ value) for a longer period of time for $s=5$ case than for $s=0$ case. The application of pseudotime for the $s=10$ case is shown in Fig. 10. The period of time for which 
the correlation applies increases further as compared with $s=0$ and $s=5$ cases.

It has been pointed out that introduction of pseudotime transfers the nonlinearity of the CCT problem from its inner boundary condition to the original flow equation and $C_{a D i} / C_{a D}$ is the controlling factor that influences the application of the correlation using pseudotime. Fig. 11 presents plots of $C_{a D_{i}} / C_{a D}$ versus $t_{D}$ for the $s=0,5$ and 10 cases of Fig. 8. The results of Fig. 11 indicate that as the skin factor increases, the increase in $C_{a D i} / C_{a D}$ not only is delayed but also becomes more gradual. Therefore, pseudotime can be applied for a longer period of time for cases with higher skin factors. This explanation confirms the results shown in Figs. 9 and 10.

Effect of Initial Chamber Gas Pressure. In a CCT, to effectively wash out drilling mud around the wellbore and debris in the perforation channels, it is desirable to impose a higher pressure differential on the sandface of a formation being tested. On the other hand, to avoid casing collapses, formation failure and severe sand production, a lower pressure differential is often required. Therefore, an optimum initial chamber gas pressure in a CCT is chosen to carefully balance the two opposing factors. Here, a parametric study is conducted to investigate the effect of the initial chamber gas pressure. Pressure responses are generated for a wide range of initial gas chamber pressures, specifically, for $p_{c h i}=0,100,1000$, 2000, 3000, and 4000 (psig). Results are given in Figs. 12 - 18.

- Different initial chamber gas pressures give different values of the initial combined wellbore storage coefficient, $C_{a D i}$, and therefore, we have to compare a CCT with a slug test having a constant wellbore storage coefficient equal to the initial wellbore storage of the corresponding CCT. Except for the variation in $p_{c h i}$ and $C_{a} D_{i}$, all parameters are the same as in the base case.

The effect of the initial chamber gas pressure on the change in $C_{a D i} / C_{a D}$ is shown in Fig. 12. It is shown that for $t_{D}<8 \times 10^{3}, C_{a D i} / C_{a D}$ increases only slightly for all cases considered. When, $t_{D}>8 \times 10^{3}$, a higher initial gas chamber pressure gives a smaller magnitude change in the combined wellbore storage coefficient relative to its initial value. Hence, pseudotime is expected to work more effectively for a test with a higher initial chamber gas pressure. This is confirmed in Figs. 13-18. From $p_{c h i}=0$ (psig) to $p_{c h i}=4000$ (psig), a continuous improvement in the effectiveness of pseudotime is noticed. And, in fact, when $p_{c h i}=4000$ (psig), pseudotime completely correlates the CCT to a slug test. In this case, however, the difference between $t_{a D}$ and $t_{D}$ is small. Moreover, an initial chamber gas pressure of 4000 (psig) would be very atypical.

Effect of Initial Liquid Level. The differential pressure imposed on the sandface of a formation is controlled by the initial chamber gas pressure and the initial liquid level. The effect of the initial liquid level on closed chamber tests is considered. This is accomplished by allowing the initial liquid level to change from $L_{i}=100 \mathrm{ft}$ for the base case to $L_{i}=2500$ and $5000 \mathrm{ft}$. Fig. 19 compares the changes of $C_{a D i} / C_{a D}$ for cases with $L_{i}=2500$ and $5000 \mathrm{ft}$ with the values for the 
base case. Fig. 20 and Fig. 21 illustrate the application of pseudotime for cases with $L_{i}=2500(\mathrm{ft})$ and $L_{i}=5000(\mathrm{ft})$, respectively.

Fig. 19 reveals that a case with a higher liquid level results in a higher magnitude change in $C_{a D i} / C_{a D}$, but overall the difference in $C_{a D i} / C_{a D}$ caused by the different liquid level is small. Comparing Figs. 7, 20 and 21, we found that the influence of the initial liquid level on the duration in which pseudotime applies is insignificant. This is consistent with the results shown in Fig. 19.

Effect of Total Chamber Length. Safety concerns and reservoir formation depth dictate the total chamber length to be used in conducting a CCT. To evaluate the effect of total chamber length, the base case length of $10,000 \mathrm{ft}$ is reduced to $5000 \mathrm{ft}$ and $2500 \mathrm{ft}$. Results are plotted in Figs. 22, 23 and 24.

Fig. 22 shows that a longer total chamber length gives a smaller increase in the magnitude of $C_{a D i} / C_{a D}$. It is then expected that when the length of the chamber becomes infinite $C_{a D_{i}} / C_{a D}$ will remain equal to 1 and a closed chamber test will degenerate to a conventional slug test. Hence, a longer total chamber length will cause the CCT solution, $p_{w D}$, plotted versus pseudotime to correlate for a longer period of time with the corresponding slug test $p_{w D}$ plotted versus $t_{D}$. This is reflected in the results given in Figs. 7, 23 and 24.

Effect of Initial Reservoir Pressure. It is anticipated that pseudotime can be used to correlate a CCT solution with the corresponding slug test solution more effectively as the initial reservoir pressure is decreased if all other tool and reservoir parameters remain unchanged. This occurs as a result of the lower magnitude change in the combined wellbore storage coefficient for a lower initial reservoir pressure. This argument is supported by Fig. 25, where the initial reservoir pressure is reduced from the base case of $5000 \mathrm{psig}$ to $4000 \mathrm{psig}$. As a matter of fact, for a case with initial reservoir pressure smaller than 4000 psig; the pressure response for a CCT was found to be quite close to a slug test even without applying pseudotime.

Correspondents have indicated that, when analyzing pressure data obtained after perforating underbalanced, the slug test analysis techniques of Peres et al. ${ }^{2}$ yielded less reliable results when pressure changes during the tests were large (greater than $1000 \mathrm{psi}$ ). This observation seems to indicate that the controlling factor is the pressure differential imposed upon the formation sandface. However, the current study shows that the controlling factor should be $C_{a D i} / C_{a D}$. This is further supported by the example given in Figs. 26 and 27. Here, two cases with the same pressure differential $\left(p_{i}-p_{c h i}=5000 \mathrm{psi}\right)$ are considered; one case has $p_{i}=5000$ psig and $p_{c h i}=0.0 \mathrm{psig}$, and the other case has $p_{i}=6000 \mathrm{psig}$ and $p_{c h i}=1000$ psig. Fig. 26 reveals the dramatic difference in $C_{a D i} / C_{a D}$ between the two cases. This difference affects the application of pseudotime, as can be seen by comparing the results shown in Figs. 13 and 27.

Effect of Liquid Compression and Wellbore Friction. So far, we have neglected the effect of liquid compression and wellbore friction to a CCT. Salas and Sageev ${ }^{9}$ showed that liquid compression and wellbore friction can be significant. To 
investigate these effects, the numerical method by Salas and Sageev ${ }^{9}$ is implemented in this study. Also, we change $L_{i}$ from $100 \mathrm{ft}$ in the base case to $0 \mathrm{ft}$, since the numerical method of Salas and Sageev was derived by assuming zero initial liquid level. All other parameters are the same as in the base case. When wellbore friction is considered, we use a value of 0.00025 for the absolute roughness of the drillpipe.

Fig. 28 illustrates the effect of liquid compression for $s=0$ and $s=5$. Cases with $c_{l}=1.0 \times 10^{-6}$ are equivalent to neglecting the liquid compressibility, as pointed out by Salas and Sageev. The results of Fig. 28 indicate that liquid compression has only a small effect on the CCT solution.

Fig. 29 shows the effect of wellbore friction on the pressure response of closed chamber tests for cases with $s=2$ and $s=10$. We were not able to run the case with $s=0$ because of pressure oscillation due to the neglect of the inertial effect. A similar problem was reported by Salas and Sageev ${ }^{9}$. Like the effect of liquid compression, the effect of wellbore friction is also small. Moreover, wellbore friction only affects the tests in the early time, as suggested by Fig. 30, and usually pressure recorded during the first 5 minutes of field tests are not useful because of oscillations in pressure. For the results of Fig. $30, t=1000$ seconds corresponding to $t_{D}=12000$.

Figs. 28 and 29 indicate that, at least for the situation studied, pseudotime can be used without introducing significant errors by neglecting liquid compression and wellbore friction.

Peres et al. ${ }^{2}$ reported that slug tests can be analyzed using type curves developed for the classical wellbore storage and skin surface constant rate production problem. The same idea can be also applied to interpret closed chamber tests, if pressure data are plotted versus pseudotime (see Eqs. 38-40). Results for cases with $p_{c h i}=0,100,500,1000,2000,3000$, and 4000 (psig) are given in Figs. 31-37.

In Figs. 31-37, three sets of results are plotted. Throughout, $p_{w c D}$ and $p_{w c D}^{\prime}$, respectively, represent the dimensionless pressure solution and its logarithmic derivative for the classical wellbore storage and skin problem. They are generated using $s=0$ and a dimensionless wellbore storage coefficient equal to the initial wellbore storage coefficient of the closed chamber test. The second set of curves are the converted data obtained from the procedure developed by Peres et al. ${ }^{2}$ using dimensionless time. The third set of results are plots of the converted data obtained using dimensionless pseudotime (see Eqs. 38-40). These figures show that the use of pseudotime can improve the data analysis and this improvement becomes more significant as the initial chamber gas pressure increases.

\section{Step Change in Wellbore Storage Coefficient: Slug Tests}

Examples presented above clearly demonstrate the utility of the pseudotime concept. Pseudotime can improve our analysis because pseudotime accounts for the variation in the wellbore storage coefficient, regardless of what causes this variation. In slug tests, as the liquid level rises from drill collar into drill pipe, there is usually a change in the inner diameter of the wellstring, and this translates into a step change 
in the wellbore storage coefficient. Correa and Ramey ${ }^{21}$ developed an analytical solution for this problem. In Fig. 38, we explore the applicability of pseudotime in the analysis of data from such a test. In Fig. 38, we consider a slug test with a step change in the dimensionless wellbore storage coefficient from $C_{D}=500$ to $C_{D}=1000$ at $t_{D}=1000$. As shown in this figure, pseudotime almost completely correlates the test to a test with a single constant wellbore storage coefficient. As a result, corresponding field data can be easily analyzed.

It is interesting to note that a conventional DST buildup test also represents a step change in wellbore storage coefficient as in the case of a slug test conducted in a wellstring with changing inner diameter. However, during a conventional DST buildup test, the wellbore storage coefficient usually changes two orders of magnitude, and this large change makes the use of pseudotime ineffective. Although we completely linearized the inner boundary condition of a CCT with pseudotime, the non-linearity is transferred to our original flow equation. The non-linear term grows as the magnitude of the change of the wellbore storage coefficient increases. When the wellbore storage coefficient changes beyond a certain limit, the non-linear term becomes dominant and that is where the use of pseudotime breaks down.

\section{Closed Chamber DST Buildup Analysis}

Thus far, we have concentrated on the analysis of closed chamber tests. These results are equally applicable to the flowing period of a closed chamber DST since it is one of the various forms of closed chamber tests. In this sub-section, we present results for the shut-in period of a closed chamber DST.

Typical Response of Closed Chamber DST Buildup Tests. Fig. 39 illustrates the pressure responses of closed chamber DST buildup tests with $p_{c h i}=0$, 500 and 3000 (psig). All three cases have the same producing time, $t_{p D}=2.25 \times 10^{4}$ (corresponding to $t_{p}=30 \mathrm{~min}$.) and $t_{D}$ denotes the total dimensionless time. For all cases, it is assumed that the height between the shut-in valve and the formation sandface is $100 \mathrm{ft}$ and the compressibility of the produced liquid is $2.28 \times 10^{-4}$.

Fig. 40 shows the effect of skin factor on the pressure responses of closed chamber DST buildup tests. In the flowing period, the skin factor strongly influences the pressure response, while in the shut-in period, pressure responses for all three cases tend to collapses into each other, which implies that the effect of skin factor is not significant. This is expected since the wellbore storage coefficient is so small for the shut-in period, flow quickly ceases.

Rate Convolution Method. The rate-convolution method proposed in this study is given by Eq. 47. When Eq. 47 applies, a Cartesian plot of the left hand side of Eq. 47, which is referred to as the correlated dimensionless group, versus dimensionless multirate time, $t_{M R D}$ (see Eq. 51 ), will be a straight line with unit slope and intercept equal to zero.

Figs. 41-43 represent the results obtained by applying the rate convolution method to closed chamber DST buildup tests with $p_{c h i}=0,500$ and 3000 (psig) respectively ( $s=0$ for all three cases). As expected, straight lines with unit slope 
and zero intercept are obtained for all the cases.

Figs. 44 and 45 represent results for $s=5$ and 10 respectively $\left(p_{c h i}=500\right.$ psig for all two cases). Again, the results confirm the applicability of the rate convolution method.

\section{CONCLUSIONS}

We have presented new interpretation methods for closed chamber tests and closed chamber DST buildup tests. For closed chamber tests, a new inner boundary condition has been formulated. It has been shown that pseudotime can be applied to correlate CCT data with corresponding slug test data. In general, this correlation applies for a sufficiently long period of time so that slug test analysis techniques can be applied to CCT pressure data.

A successful application of pseudotime largely depends on the magnitude of the change in the combined wellbore storage coefficient.

Among the various parameters studied, skin factor, chamber length, initial chamber gas pressure and initial reservoir pressure were found to have the most influence on the length of time for which the correlation based on pseudotime applies. In general, the length of the correlation time period increases as the skin factor increases, the chamber length increases, the initial chamber gas pressure increases or the initial reservoir pressure decreases.

Pseudotime can also be used to analyze data from slug tests with a changing wellbore storage coefficient due to the change of wellstring diameter or inclination angle.

A rate-convolution method has been proposed and shown to be useful for analyzing data from the shut-in period of a closed chamber DST. 


\section{NOMENCLATURE}

Symbol

$A_{c h}=$ cross sectional area of the wellstring, $\mathrm{ft}^{2}$.

$B=$ formation volume factor, RB/STB.

$C=$ wellbore storage coefficient, $\mathrm{RB} / \mathrm{psi}$.

$C_{a}=$ combined wellbore storage coefficient, $\mathrm{RB} / \mathrm{psi}$.

$C_{a i}=$ initial combined wellbore storage coefficient, $\mathrm{RB} / \mathrm{psi}$.

$C_{a D}=$ combined dimensionless wellbore storage coefficient.

$C_{C D i}=$ initial combined dimensionless wellbore storage coefficient.

$C_{D} \quad=$ dimensionless wellbore storage coefficient.

$C_{f D} \quad=$ flowing period dimensionless wellbore storage coefficient.

$C_{g} \quad=$ wellbore storage coefficient due to gas compression, $\mathrm{RB} / \mathrm{psi}$.

$C_{g D}=$ dimensionless wellbore storage coefficient due to gas compression.

$C_{l} \quad=$ wellbore storage coefficient due to rising liquid level or liquid compression, $\mathrm{RB} / \mathrm{psi}$.

$C_{l D}=$ dimensionless wellbore storage coefficient due to rising liquid level, or liquid compression.

$C_{s} \quad=$ buildup wellbore storage coefficient, $\mathrm{RB} / \mathrm{psi}$.

$C_{s D}=$ dimensionless buildup wellbore storage coefficient.

$c_{g} \quad=$ gas isothermal compressibility, $\mathrm{psi}^{-1}$.

$c_{l} \quad=$ liquid isothermal compressibility, $\mathrm{psi}^{-1}$.

$c_{t} \quad=$ total system compressibility, $\mathrm{psi}^{-1}$.

$d_{p} \quad=$ diameter of the drillpipe, $\mathrm{ft}$.

$f \quad=$ friction factor, dimensionless.

$g \quad=$ acceleration of gravity, $\mathrm{ft} / \mathrm{sec}^{2}$.

$g_{c} \quad=$ unit conversion factor, $32.17 \mathrm{lbm}-\mathrm{ft}^{\prime} /\left(\mathrm{lbf}_{-} \mathrm{sec}^{2}\right)$.

$h \quad=$ formation thickness, $\mathrm{ft}$.

$i \quad=$ initial or index.

$k=$ absolute permeability, md or index.

$L_{c} \quad=$ total chamber length, $\mathrm{ft}$.

$L_{i} \quad=$ initial liquid height, $\mathrm{ft}$.

$M \quad=$ total number of time steps.

$N \quad=$ index for time step.

$p(r, t) \quad=$ pressure at $\mathrm{r}$ and $\mathrm{t}$, psia.

$p_{c h} \quad=$ chamber gas pressure, psia.

$p_{c h i}=$ initial chamber gas pressure, psia.

$p_{g} \quad=$ gas pressure, psia.

$p_{i} \quad=$ initial reservoir pressure, psia.

$p_{0} \quad=$ initial wellbore pressure, psia. 


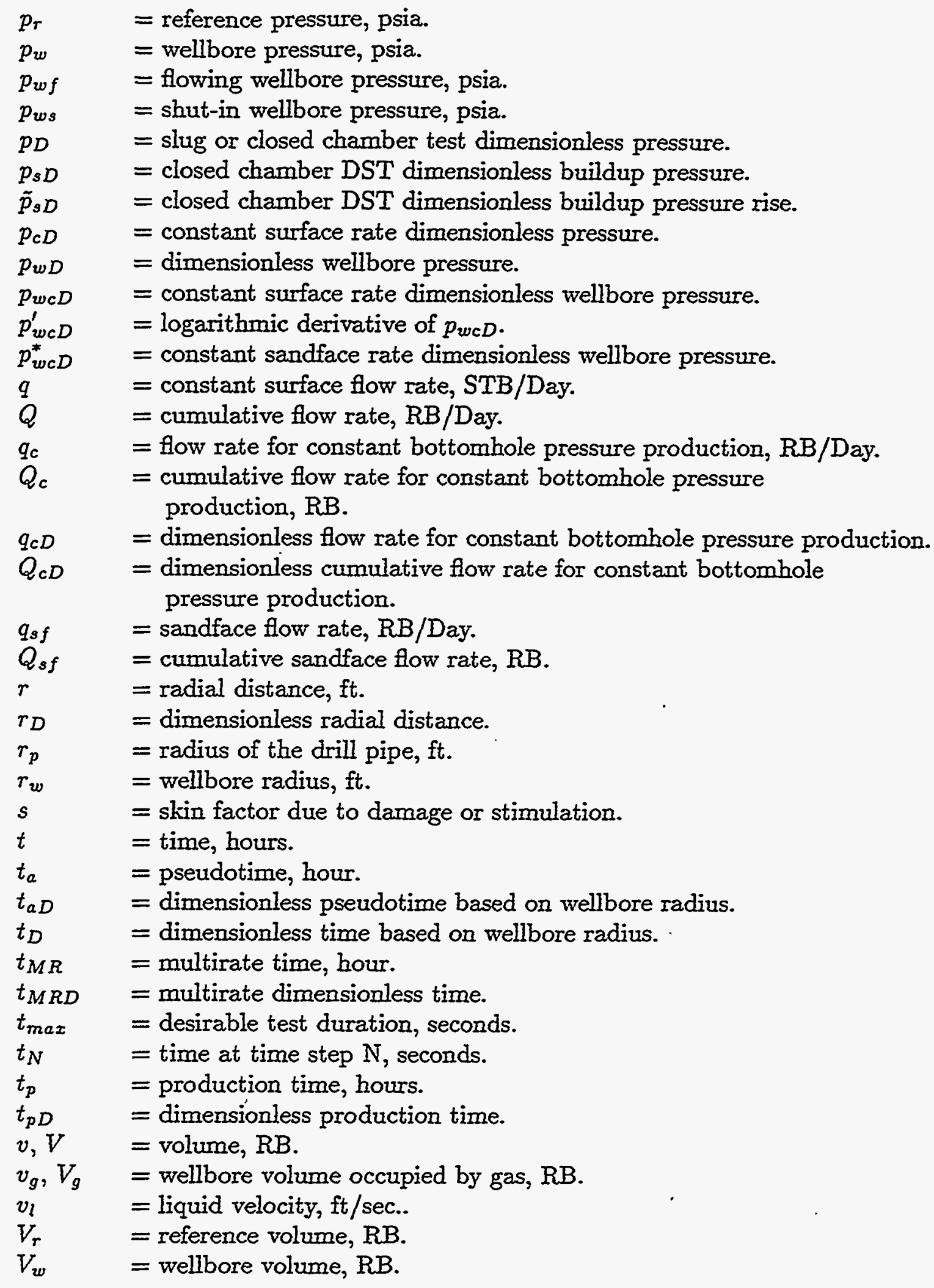




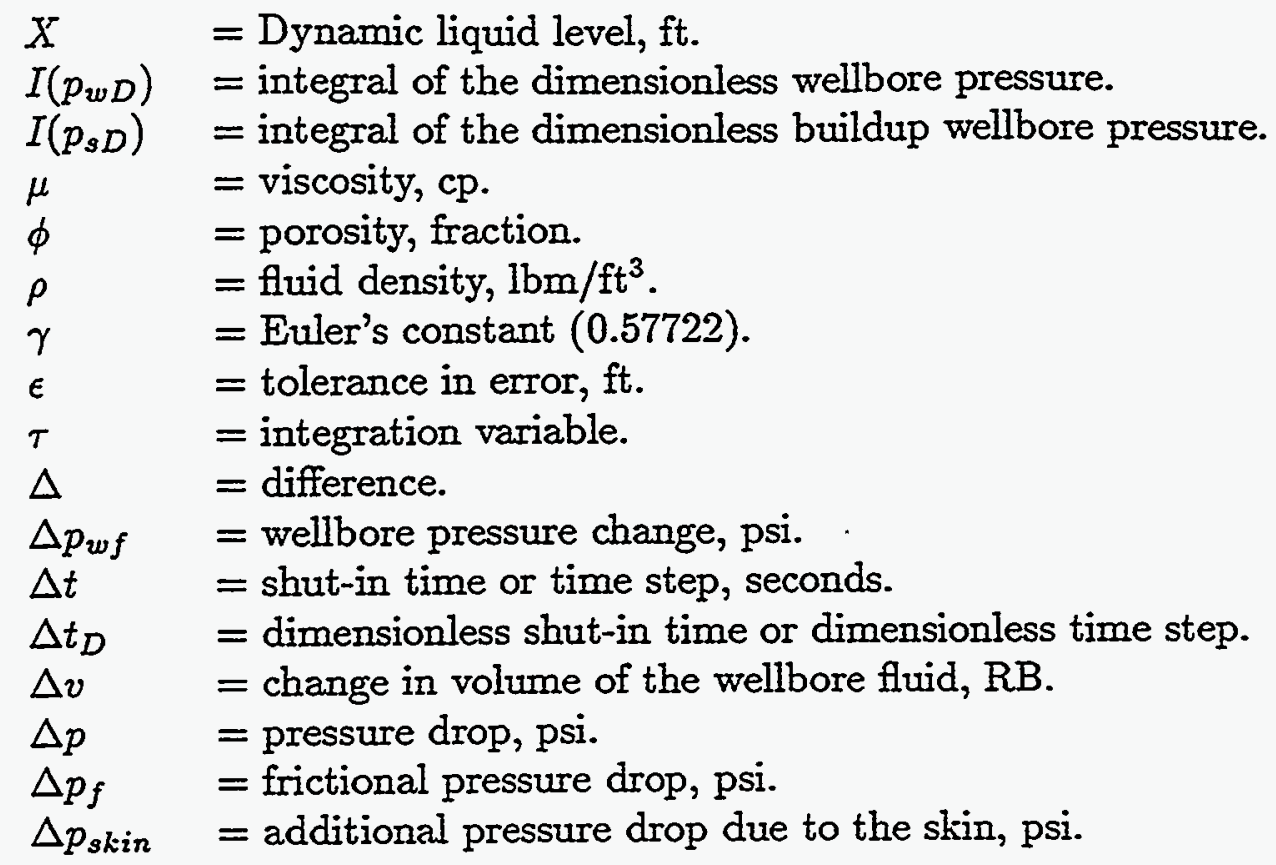

\section{REFERENCES}

1. Ramey, H. J. Jr., Agarwal, R. G. and Martin, I.: "Analysis of 'Slug Test' or DST Flow Period Data,". J. Can. Pet. Tech., 14 (July-Sept. 1975) 37-47.

2. Peres, A., Onur, M. and Reynolds, A.: "A New General Pressure Analysis for Slug Tests," paper SPE 18801 presented at the 1989 SPE California Regional meeting, Bakersfield, CA, April 5-7.

3. Alexander, L. G.: "Theory and Practice of the Closed-Chamber Drillstem Test Method," J. Pet. Tech., (December 1977) 1539-1544.

4. Erdle, J. C., Upchurch, J. M. and Warren, D. A.:"Early Fluid Entry Determination; Key to Safe, Optimum Drill Stem Testing,"paper SPE 6884 presented at the 52nd Annual Fall Technical Conference and Exhibition of SPE of AIME, Oct. 9-12, 1977, Denver, Colorado.

5. Reid, H. W.: "Evaluation of Low Permeability Gas Reservoirs Utilizing Closed Chamber Drillstem Test Techniques,"paper SPE 10184 presented at the 56th Annual Fall Technical Conference and Exhibition of SPE of AIME, Oct. 5-7, 1981, San Antonio, Texas.

6. Kabir, C. S., Badry, R. A. and Hasan, A. R.:"Two-Phase Gas/Liquid Flow Rate Estimation During Closed-Chamber Testing," paper no. 89-40-7 presented at the 40th Annual Technical Meeting of the Petroleum Society of CIM, May 28-31, 1989, Banff, Canada.

7. Saldana-C, M. A. and Ramey, H. J. Jr.: "Slug Test and Drillstem Test Flow Phenomena Including Wellbore Inertial and Frictional Effects," paper SPE 15118 presented at the 56th SPE California Regional Meeting, Oakland, CA, 
April 2-4, 1986.

8. Simmons, J. F. and Grader, A. S.:"Application of Closed-Chamber Theory to Backsurge Completion testing," SPE Production Engineering, (November 1988) 527-535.

9. Salas, B. and Sageev, A.: "Closed Chamber Testing: The Effects of Wellbore Friction and Fluids Compression," paper SPE 16800 presented at the 62nd Annual Technical Conference and Exhibition of SPE, Sept. 27-30, 1987, Dallas, Texas.

10. Mfonfu, G. B. S. and Grader, A. S.:"An Implicit Numerical Model for the Closed Chamber Test," paper SPE 19832 presented at the 64th Annual Technical Conference and Exhibition of SPE, Oct. 8-11, 1989, San Antonio, Texas.

11. Petak, K. R., Prasad, R. K. and Coble, L. E.: "Surge Test Simulation," paper SPE 21832 presented at Rocky Mountain Regional Meeting and Low Permeability Reservoir Symposium, April 15-17, 1991, Denver, Colorado.

12. Tariq, S. M. and Ayestaran, L.:"Analyses and Application of Pressure, Flowrate, and Temperature Measurements During a Perforation Run," paper SPE 15475 presented at the 61st Annual Technical Conference and Exhibition of SPE, Oct. 5-8, 1986, New Orleans, LA.

13. Simmons, J. F.: "Interpretation of Underbalanced Surge Pressure Data by RateTime Convolution," paper SPE 15477 presented at the 61st Annual Technical Conference and Exhibition of SPE, Oct. 5-8, 1986, New Orleans, LA.

14. Soliman, M. Y., Petak, K. and Christensen, J.:"Analysis of Sequential Formation Testing and Surge Tests Using New Techniques," paper no. 88-39-53 presented at the 39th Annual Technical Meeting of the Petroleum Society of CIM, June 12-16, 1988, Calgray, Canada.

15. Garrett, J. C., Shealy, R. A., Centanni, R. J., Ruiz, S. J. and Chacon, E. A.:“ The Analysis of Pressure Transient Data from Underbalanced Perforating," paper SPE 18878 presented at SPE Production Operation Symposium, March 13-14, 1989, Oklahoma, OK.

16. Ayoub, J. A., Bourdet, D. P. and Chauvel, Y. L.:"Impulse Testing," SPE Formation Evaluation, (September 1988) 534-546.

17. Manohar, M. M., Morris, C. W., Brunner, S. R. and Hill, D. D.:"Evaluation of Underbalanced Through-Tubing Perforating and Closed Chamber test Interpretation techniques," paper SPE 21657 presented at SPE Production Operation Symposium, April 7-9, 1991, Oklahoma, OK.

18. Ramey, H. J. Ir. and Agarwal, R. G.: "Annulus Unloading Rates as Influenced by Wellbore Storage and Skin Effect," Trans. AIME, 253 (1972) 453-463.

19. Agarwal, R.: "Real Gas Pseudotime' - A New Function for Pressure Buildup Analysis of MHF Gas Wells," paper SPE 8279 presented at the 54th SPE Annual Technical Conference and Exhibition, Las Vegas, Nevada, Sept. 23-26, 1979

20. Peres, A., Onur, M. and Reynolds, A.: "New Methods for the Analysis of 
Drillstem Test Data," paper SPE 19843 presented at the 1989 SPE Annual Technical Conference and Exhibition, Oct. 8-11, San Antonio.

21. Correa, A. C. and Ramey, H. J. Jr.: "A Method for Pressure Buildup Analysis of Drillstem Tests," paper SPE 16802 presented at the 1987 SPE Annual Technical Conference and Exhibition, Dallas, Sept. 27-30.

22. Earlougher, R. C. Jr.:"Advances in Well Test Analysis," SPE Monograph No. $5,1977$.

23. Peres, A: "Analvsis of Slug and Drillstem Test," $\mathrm{Ph} . \mathrm{D}$. dissertation, University of Tulsa, Tulsa, OK (July 1989).

\section{Appendix A}

\section{Wellbore Storage Coefficient}

Once a closed chamber test is initialized and the reservoir begins to produce, the liquid level moves upward in the wellstring and, as a result, compresses the chamber gas. It is rather obvious that two types of wellbore storage phenomena simultaneously govern the test: one is due to rising liquid level and the other one is due to gas compression. Here, we derive the proper equation for the wellbore storage coefficient in a closed chamber test. Our derivation utilizes the basic definition of the wellbore storage coefficient ${ }^{22}$ as a starting point, however, a material balance on the wellbore fluid could be used to obtain the same results.

In order to simplify our analysis, we assume the following: 1) the reservoir produces a single phase liquid; 2) there is no mass transfer between chamber gas and produced formation fluids; 3 ) liquid in the wellstring is incompressible and chamber gas behaves as an ideal gas; 4) there are no temperature and pressure gradients in the gas column; 5) friction and momentum effects are neglected; and 6) critical flow is not present. We also assume that the wellstring has a constant inner diameter and inclination angle.

The wellbore storage coefficient is defined as the change of the total volume of wellbore fluids per unit change in bottomhole pressure ${ }^{22}$,

$$
C_{a}=\frac{\Delta v}{\Delta p_{w f}}
$$

where

$C_{a}=$ Wellbore storage coefficient, $\mathrm{bbl} / \mathrm{psi}$

$\Delta v=$ Change in volume of fluid in the wellbore at wellbore conditions, bbl

$\Delta p_{w f}=$ Change in bottomhole pressure, psi.

During a closed chamber test the bottomhole pressure continuously increases until it reaches the reservoir static pressure. Assuming that the liquid level is $h=h(t)$ at some time during the test, the bottomhole pressure is the summation of 
the chamber gas pressure and the hydrostatic pressure caused by the liquid column, that is,

$$
p_{w f}=p_{g}+\frac{\rho g}{144 g_{c}} h
$$

Therefore, the change in bottomhole pressure can be written as

$$
\Delta p_{w f}=\Delta p_{g}+\frac{\rho g}{144 g_{c}} \Delta h
$$

For an ideal gas under isothermal condition, the equation of state can be stated as

$$
p_{g} v_{g}=\text { constant. }
$$

From Eq. A-4, the change of $\left(p_{g} v_{g}\right)$ will be

$$
\Delta\left(p_{g} v_{g}\right)=0
$$

or

$$
\left(\Delta p_{g}\right) v_{g}+\left(\Delta v_{g}\right) p_{g}=0 .
$$

Note that the change of gas volume is related to the change of liquid volume by

$$
\Delta v_{g}=-\Delta v \text {. }
$$

Combining Eqs. $A-6$ and $A-7$ leads to the following expression for the change of chamber gas pressure,

$$
\Delta p_{g}=p_{g} \frac{\Delta v}{v_{g}} .
$$

Substituting Eq. $A-8$ into Eq. $A-3$, we obtain the change of bottomhole pressure

$$
\Delta p_{w f}=p_{g} \frac{\Delta v}{v_{g}}+\frac{\rho g}{144 g_{c}} \Delta h .
$$

Combing Eqs. $A-1$ and $A-9$ and using $\Delta v=A \Delta h / 5.615$, we have

$$
\frac{1}{C_{a}}=\frac{p_{g}}{v_{g}}+\frac{5.615 \rho g}{144 g_{c} A} .
$$

Note the isothermal compressibility of an ideal gas is the inverse of its pressure, that is,

$$
c_{g}=\frac{1}{p_{g}} .
$$

Substituting Eq. $A-11$ into Eq. $A-10$ and rearranging, we find

$$
\frac{1}{C_{a}}=\frac{1}{C_{g}}+\frac{1}{C_{l}}
$$


or

$$
C_{a}=\frac{C_{g} C_{l}}{C_{g}+C_{l}}
$$

where $C_{l}$ denotes the wellbore storage coefficient due to rising liquid level and $C_{g}$ denotes the wellbore storage coefficient due to gas compression.

Eq. $A-13$ suggests that the effective wellbore storage coefficient for a closed chamber test is a combination of wellbore storage due to changing liquid level and gas compression. Note that during the test $C_{l}$ remains constant while $C_{g}$ continuously decreases, and thus a closed chamber test is controlled by a variable wellbore storage. Also note that if the gas volume remains relatively large throughout the test the combined wellbore storage given in Eq. $A-13$ approaches $C_{l}$, and in this case the closed chamber test becomes a conventional slug test.

With the wellbore storage coefficient derived above, we define the inner boundary condition for closed chamber tests. A material balance yields

$$
1.12 \tau \times 10^{-3}(2 \pi) \frac{k h}{\mu}\left(r \frac{\partial p}{\partial r}\right)_{r=r_{w}}=C_{a} \frac{\partial p_{w f}}{\partial t}
$$

In terms of dimensionless variables, Eq. $A-14$ can be written as

$$
\left(r_{D} \frac{\partial p_{D}}{\partial r_{D}}\right)_{r_{D}=1}=C_{a D} \frac{\partial p_{w D}}{\partial t_{D}}
$$

where $C_{a D}$ is the combined dimensionless wellbore storage coefficient; see Eq. 33 .

Eq. $A-15$ represents the inner boundary condition for a closed chamber test. This equation is similar to the inner boundary condition for the conventional slug test IBVP except that, in the CCT case, the wellbore storage coefficient is a function of the bottomhole pressure.

\section{Appendix B}

Closed Chamber Drillstem Buildup Analysis

In this appendix, we derive a convolution method used to analyze pressure buildup data from the shut-in period of a closed chamber drillstem test. Peres ${ }^{23}$ developed a similar method for the shut-in period of a conventional drillstem test. Here, Peres' procedure is followed.

From Duhamel's principle, we have

$$
p_{w D}\left(t_{D}\right)=\int_{0}^{t_{D}} q_{D}(\tau) \frac{d p_{w C D}^{*}}{d t_{D}}\left(t_{D}-\tau\right) d \tau
$$


where $p_{w c D}^{*}$ is the constant sandface rate dimensionless wellbore pressure solution. From Leibnitz's Rule, it follows that we can rewrite Eq. $B-1$ as

$$
p_{w D}\left(t_{D}\right)=\frac{d}{d t_{D}} \int_{0}^{t_{D}} q_{D}(\tau) p_{w c D}^{*}\left(t_{D}-\tau\right) d \tau
$$

Integrating both sides of Eq. $B-2$ gives

$$
\int_{0}^{t_{D}} p_{w D}(\tau) d \tau=\int_{0}^{t_{D}} q_{D}(\tau) p_{w c D}^{*}\left(t_{D}-\tau\right) d \tau
$$

For the shut-in period of closed chamber drillstem testing, $t_{D}>t_{p D}$, Eq. $B-3$ becomes

$$
\int_{0}^{t_{D}} p_{w D}(\tau) d \tau=\int_{0}^{t_{p} D} q_{D}(\tau) p_{w c D}^{*}\left(t_{D}-\tau\right) d \tau+\int_{t_{p D}}^{t_{D}} q_{D}(\tau) p_{w C D}^{*}\left(t_{D}-\tau\right) d \tau .(B-4)
$$

Applying Eq. $B-4$ at the instant of shut-in, i.e., when $t_{D}=t_{p D}$, we obtain

$$
\int_{0}^{t_{p D}} p_{w D}(\tau) d \tau=\int_{0}^{t_{p D}} q_{D}(\tau) p_{w c D}^{*}\left(t_{p D}-\tau\right) d \tau
$$

Subtracting Eq. $B-5$ from Eq. $B-4$ yields

$$
\begin{aligned}
I\left(p_{s}\right) & =\int_{0}^{t_{p D}} q_{D}(\tau)\left[p_{w c D}^{*}\left(t_{D}-\tau\right)-p_{w c D}^{*}\left(t_{p D}-\tau\right)\right] d \tau \\
& +\int_{0}^{\Delta t_{D}} q_{D}\left(t_{p D}+\tau\right) p_{w c D}^{*}\left(\Delta t_{D}-\tau\right) d \tau,
\end{aligned}
$$

where $p_{s} D$ denotes the dimensionless pressure in the shut-in period, and $I\left(p_{s} D\right)$ denotes the integration of shut-in period dimensionless bottomhole pressure, i.e.,

$$
I\left(p_{s D}\right)=\int_{0}^{\Delta t_{D}} p_{s D}(\tau) d \tau
$$

We consider partitions for the intervals $\left[0, t_{p D}\right]$ and $\left[0, \Delta t_{D}\right]$ given, respectively, by

$$
0=t_{0, p D}<t_{1, p D}<\ldots<t_{n, p D}<t_{n+1, p D}=t_{p D}
$$

and

$$
0=\Delta t_{0, D}<\Delta t_{1, D}<\ldots<\Delta t_{m, D}<\Delta t_{m+1, D}=t_{p D}
$$


Then, numerical integration can be applied to approximate the right hand side of Eq. $B-6$ to obtain

$$
\begin{aligned}
I\left(p_{s} D\right) & =\sum_{j=0}^{n}\left[p_{w c D}^{*}\left(t_{D}-\hat{t}_{j+1, p D}\right)-p_{w c D}^{*}\left(t_{p D}-\hat{t}_{j+1, p D}\right)\right] \int_{t_{j, p D}}^{t_{j+1, p D}} q D(\tau) d \tau \\
& +\sum_{i=0}^{m} p_{w c D}^{*}\left(\Delta t_{D}-\Delta \hat{t}_{i+1, D}\right) \int_{\Delta t_{i, D}}^{\Delta t_{i+1, D}} q D\left(t_{p D}+\tau\right) d \tau,
\end{aligned}
$$

where

$$
\begin{aligned}
\hat{t}_{j+1, p D} & =\theta_{j+1} t_{j+1, p D}+\left(1-\theta_{j+1}\right) t_{j, p D} \\
\Delta \hat{t}_{i+1, D} & =\theta_{i+1} \Delta t_{i+1, D}+\left(1-\theta_{i+1}\right) \Delta t_{i, D}
\end{aligned}
$$

with

$$
0 \leq \theta_{j+1} \leq 1 ; \quad 0 \leq \theta_{i+1} \leq 1 .
$$

The dimensionless sandface flow rate for closed chamber drillstem testing is given by

$$
q_{D}\left(t_{D}\right)= \begin{cases}-C_{a D} \frac{d p_{w D}}{d t_{D}}, & t_{D}<t_{p D} ; \\ -C_{s D} \frac{d p_{s D}}{d t_{D}}, & t_{D}>t_{p D} .\end{cases}
$$

Substituting Eq. $B-14$ into Eq. $B-10$ and using numerical integration, we obtain

$$
\begin{gathered}
I\left(p_{s D}\right)=-\sum_{j=0}^{n}\left[p_{w c D}^{*}\left(t_{D}-\hat{t}_{j+1, p D}\right)-p_{w c D}^{*}\left(t_{p D}-\hat{t}_{j+1, p D}\right)\right] \\
C_{a D}\left(\hat{t}_{j+1, p D}\right)\left[p_{w D}\left(t_{j+1, p D}\right)-p_{w D}\left(t_{j, p}\right)\right] \\
-C_{s D} \sum_{i=0}^{m} p_{w c D}^{*}\left(\Delta t_{D}-\Delta \hat{t}_{i+1, D}\right)\left[p_{s D}\left(\Delta t_{i+1, D}\right)-p_{s D}\left(\Delta t_{i, D}\right)\right] .
\end{gathered}
$$

We assume that the logarithmic approximation is valid for the constant sandface rate dimensionless wellbore pressure solution, i.e.,

$$
p_{w c D}^{*}\left(t_{D}\right)=\frac{1}{2} \ln \left(t_{D}\right)+\tilde{s}_{D}
$$

where

$$
\tilde{s}_{D}=\frac{1}{2} \ln \left(\frac{4}{e^{\gamma}}\right)+s .
$$

Throughout, $\gamma=0.57722$ represents Euler's constant. Substituting Eq. $B-16$ into Eq. $B-15$ and dividing both sides of the resulting equation by $C_{a D_{i}}$, we have

$$
\frac{I\left(p_{s D}\right)}{C_{a D i}}=-\sum_{j=0}^{n} \frac{1}{2} \ln \left(\frac{t_{D}-\hat{t}_{j+1, p D}}{t_{p D}-\hat{t}_{j+1, p D}}\right) \frac{C_{a D}\left(\hat{t}_{j+1, p D}\right)}{C_{a D i}}\left[p_{w D}\left(t_{j+1, p D}\right)-p_{w D}\left(t_{j, p D}\right)\right]
$$




$$
-\frac{C_{s D}}{C_{a D i}} \sum_{i=0}^{m}\left[\frac{1}{2} \ln \left(\Delta t_{D}-\Delta \hat{t}_{i+1, D}\right)+\tilde{s}_{D}\right]\left[p_{s D}\left(\Delta t_{i+1, D}\right)-p_{s D}\left(\Delta t_{i, D}\right)\right]
$$

or equivalently,

$$
\begin{gathered}
\frac{I\left(p_{s D}\right)}{C_{a D i}}=-\frac{1}{2} \sum_{j=0}^{n} \ln \left(\frac{t_{D}-\hat{t}_{j+1, p D}}{t_{p D}-\hat{t}_{j+1, p D}}\right) \frac{C_{a D}\left(\hat{t}_{j+1, p}\right)}{C_{a D i}}\left[p_{w D}\left(t_{j+1, p D}\right)-p_{w D}\left(t_{j, p D}\right)\right] \\
-\frac{1}{2} \frac{C_{s D}}{C_{a D i}} \sum_{i=0}^{m} \ln \left(\Delta t_{D}-\Delta \hat{t}_{i+1, D}\right)\left[p_{s D}\left(\Delta t_{i+1, D}\right)-p_{s}\left(\Delta t_{i, D}\right)\right] \\
-\frac{C_{s D}}{C_{a D i}} \sum_{i=0}^{m} \tilde{s}_{D}\left[p_{s}\left(\Delta t_{i+1, D}\right)-p_{s D}\left(\Delta t_{i, D}\right)\right] .
\end{gathered}
$$

The last term in the RHS of Eq. $B-19$ can be simplified as

$$
-\frac{C_{s D}}{C_{a D i}} \sum_{i=0}^{m} \tilde{s}_{D}\left[p_{s} D\left(\Delta t_{i+1, D}\right)-p_{s D}\left(\Delta t_{i, D}\right)\right]=\frac{C_{s D}}{C_{a D i}} \tilde{s}_{D} \tilde{p}_{s D}\left(\Delta t_{D}\right), \quad(B-20)
$$

where $\tilde{p}_{s D}$ is the buildup pressure rise in the shut-in period of closed chamber drillstem testing defined by

$$
\tilde{p}_{s D}=p_{w D}\left(t_{p D}\right)-p_{s D}\left(\Delta t_{D}\right)
$$

Combining Eqs. $B-19$ and $B-20$ and rearranging, we obtain

$$
\frac{I\left(p_{s} D\right)}{C_{a D i} \tilde{p}_{s D}}-\frac{C_{s D}}{C_{a D i}} \tilde{s}_{D}=t_{M R D}
$$

where $t_{M R D}$ is the multirate dimensionless time, given by

$$
\begin{aligned}
t_{M R D} & =-\frac{1}{2 \tilde{p}_{s} D}\left[\sum_{j=0}^{n} \ln \left(\frac{t_{D}-\hat{t}_{j+1, p D}}{t_{p D}-\hat{t}_{j+1, p D}}\right) \frac{C_{a D}\left(\hat{t}_{j+1, p D}\right)}{C_{a D i}}\left[p_{w D}\left(t_{j+1, p D}\right)-p_{w D}\left(t_{j, p D}\right)\right]\right. \\
& \left.+\frac{C_{s D}}{C_{a D i}} \sum_{i=0}^{m} \ln \left(\Delta t_{D}-\Delta \hat{t}_{i+1, D}\right)\left[p_{s} D\left(\Delta t_{i+1, D}\right)-p_{s D}\left(\Delta t_{i, D}\right)\right]\right] . \quad(B-23)
\end{aligned}
$$

Eq. $B-22$ is the convolution method for closed chamber drillstem testing buildup analysis.

A corresponding dimensional form of Eq. $B-22$ which can be used for application in the analysis of field data is

$$
\frac{2.9508 \times 10^{-4} k h}{\mu C_{a i}} \frac{I\left(p_{i}-p_{w s}\right)}{p_{w s}(\Delta t)-p_{w f}\left(t_{p}\right)}=1.151 t_{M R}+\tilde{s} \frac{C_{s}}{C_{a i}},
$$


where $I\left(p_{i}-p_{w s}\right), t_{M R}$ and $\tilde{s}$ are given, respectively, by

$$
\begin{gathered}
I\left(p_{i}-p_{w s}\right)=\int_{0}^{\Delta t}\left(p_{i}-p_{w s}(\tau)\right) d \tau \\
t_{M R}=\sum_{j=0}^{n} \log \left(\frac{t-\hat{t}_{j+1}}{t_{p}-\hat{t}_{j+1}}\right) \frac{C_{a}\left(\hat{t}_{j+1}\right)}{C_{a i}} \frac{p_{w f}\left(t_{j+1}\right)-p_{w f}\left(t_{j}\right)}{p_{w s}(\Delta t)-p_{w f}\left(t_{p}\right)} \\
+\frac{C_{s}}{C_{a i}} \sum_{i=0}^{m} \log \left(\Delta t-\Delta \hat{t}_{i+1}\right) \frac{p_{w s}\left(\Delta t_{i+1}\right)-p_{w s}\left(\Delta t_{i}\right)}{p_{w s}(\Delta t)-p_{w f}\left(t_{p}\right)}
\end{gathered}
$$

and

$$
\tilde{s}=1.151\left[\log \left(\frac{k}{\phi c_{t} \mu r_{w}^{2}}\right)-3.23+0.87 s\right] . \quad(B-27)
$$


Table 1: Parameters Used for Slug Test Comparison

\begin{tabular}{|l|rl|}
\hline \multicolumn{1}{|c|}{ Parameter } & Value & Unit \\
\hline Initial Reservoir Pressure & 5000.0 & psig \\
\hline Fluid Gravity & 25.0 & API \\
\hline Fluid Viscosity & 1.25 & $\mathrm{cp}$ \\
\hline Formation Porosity & 0.27 & - \\
\hline Formation Permeability & 100.0 & $\mathrm{md}$ \\
\hline Skin Factor & 0.0 & - \\
\hline Total System Compressibility & 0.00001 & 1/psi \\
\hline Reservoir Thickness & 25.0 & $\mathrm{ft}$ \\
\hline Well Diameter & 10.0 & in. \\
\hline Chamber ID & 2.441 & in. \\
\hline Initial Wellbore Storage Coefficient & 1127.0 & - \\
\hline
\end{tabular}


Table 2: Comparison with Analytical Slug Test Solutions

\begin{tabular}{|c|c|c|}
\hline$t_{D}$ & $\begin{array}{c}p_{w D} \\
\text { (Numerical) }\end{array}$ & $\begin{array}{c}p_{w D} \\
\text { (Analytical) }\end{array}$ \\
\hline 10.0 & 0.993430 & 0.993461 \\
\hline 20.0 & 0.989107 & 0.989145 \\
\hline 30.0 & 0.985240 & 0.985286 \\
\hline 40.0 & 0.981632 & 0.981685 \\
\hline 50.0 & 0.978201 & 0.978260 \\
\hline 60.0 & 0.974903 & 0.974969 \\
\hline 70.0 & 0.971712 & 0.971783 \\
\hline 80.0 & 0.968608 & 0.968685 \\
\hline 90.0 & 0.965579 & 0.965662 \\
\hline 100.0 & 0.962616 & 0.962703 \\
\hline 200.0 & 0.935430 & 0.935561 \\
\hline 300.0 & 0.911064 & 0.911231 \\
\hline 400.0 & 0.888508 & 0.888706 \\
\hline 500.0 & 0.867314 & 0.867540 \\
\hline 600.0 & 0.847228 & 0.847478 \\
\hline 700.0 & 0.828082 & 0.828355 \\
\hline 800.0 & 0.809759 & 0.810052 \\
\hline 900.0 & 0.792170 & 0.792481 \\
\hline 1000.0 & 0.775245 & 0.775573 \\
\hline 2000.0 & 0.633533 & 0.633967 \\
\hline 3000.0 & 0.526575 & 0.527043 \\
\hline 4000.0 & 0.442757 & 0.443227 \\
\hline 5000.0 & 0.375714 & 0.376179 \\
\hline 6000.0 & 0.321339 & 0.321801 \\
\hline 7000.0 & 0.276774 & 0.277235 \\
\hline 8000.0 & 0.239933 & 0.240397 \\
\hline 9000.0 & 0.209256 & 0.209722 \\
\hline 10000.0 & 0.183546 & 0.184011 \\
\hline 20000.0 & 0.064649 & 0.064837 \\
\hline 30000.0 & 0.032962 & 0.032864 \\
\hline 40000.0 & 0.021090 & 0.020935 \\
\hline 50000.0 & 0.015361 & 0.015252 \\
\hline 60000.0 & 0.012067 & 0.012019 \\
\hline 70000.0 & 0.009938 & 0.009937 \\
\hline 80000.0 & 0.008451 & 0.008478 \\
\hline 90000.0 & 0.007351 & 0.007395 \\
\hline 100000.0 & 0.006506 & 0.006556 \\
\hline
\end{tabular}


Table 3: Parameters Used for Comparison with Simmons \& Grader Solutions

\begin{tabular}{|l|rl|}
\hline \multicolumn{1}{|c|}{ Parameter } & Value & Unit \\
\hline Initial Reservoir Pressure & 5000.0 & psig \\
\hline Fluid Gravity & 25.0 & API \\
\hline Fluid Viscosity & 1.25 & $\mathrm{cp}$ \\
\hline Formation Porosity & 0.27 & - \\
\hline Formation Permeability & 100.0 & $\mathrm{md}$ \\
\hline Skin Factor & 0.0 & - \\
\hline Total System Compressibility & 0.00001 & $1 / \mathrm{psi}$ \\
\hline Reservoir Thickness & 25.0 & $\mathrm{ft}$ \\
\hline Well Diameter & 10.0 & in. \\
\hline Initial Fluid Height & 100.0 & $\mathrm{ft}$ \\
\hline Chamber ID & 2.441 & in. \\
\hline Total Chamber Length & 1000. & $\mathrm{ft}$ \\
\hline Initial Chamber Gas Pressure & 30.0 & $\mathrm{psig}$ \\
\hline Initial Wellbore Storage Coefficient & 999.8 & - \\
\hline
\end{tabular}


Table 4: Comparison with Simmons and Grader Solutions

\begin{tabular}{|c|c|c|c|c|c|}
\hline $\begin{array}{c}\text { time } \\
\text { (sec.) }\end{array}$ & $\begin{array}{c}p_{w f} \\
(\mathrm{psia})\end{array}$ & $\begin{array}{c}\text { S-G } \\
p_{w f} \\
(\mathrm{psia})\end{array}$ & $\begin{array}{c}\text { time } \\
(\mathrm{sec} .)\end{array}$ & $\begin{array}{c}p_{w f} \\
(\mathrm{psia})\end{array}$ & $\begin{array}{c}\text { S-G } \\
p_{w f} \\
(\mathrm{psia})\end{array}$ \\
\hline 1.000 & 127.13 & 127.11 & 26.00 & 4019.27 & 4023.00 \\
\hline 2.000 & 156.83 & 156.78 & 27.00 & 4086.91 & 4090.22 \\
\hline 3.000 & 184.12 & 184.05 & 28.00 & 4144.87 & 4147.84 \\
\hline 4.000 & 210.36 & 210.25 & 29.00 & 4195.28 & 4197.95 \\
\hline 5.000 & 236.17 & 236.02 & 30.00 & 4239.64 & 4242.06 \\
\hline 6.000 & 261.99 & 261.79 & 31.00 & 4279.04 & 4281.25 \\
\hline 7.000 & 288.21 & 287.94 & 32.00 & 4314.35 & 4316.37 \\
\hline 8.000 & 315.25 & 314.87 & 33.00 & 4346.19 & 4348.05 \\
\hline 9.000 & 343.59 & 343.09 & 34.00 & 4375.10 & 4376.81 \\
\hline 10.00 & 373.92 & 373.24 & 35.00 & 4401.46 & 4403.05 \\
\hline 11.00 & 407.22 & 406.27 & 36.00 & 4425.64 & 4427.10 \\
\hline 12.00 & 444.96 & 443.61 & 37.00 & 4447.88 & 4449.25 \\
\hline 13.00 & 489.61 & 487.62 & 38.00 & 4468.44 & 4469.72 \\
\hline 14.00 & 545.51 & 542.43 & 39.00 & 4487.50 & 4488.69 \\
\hline 15.00 & 621.08 & 615.96 & 40.00 & 4505.22 & 4506.34 \\
\hline 16.00 & 734.56 & 725.16 & 41.00 & 4521.75 & 4522.80 \\
\hline 17.00 & 931.26 & 911.59 & 42.00 & 4537.21 & 4538.20 \\
\hline 18.00 & 1333.31 & 1287.28 & 43.00 & 4551.70 & 4552.63 \\
\hline 19.00 & 2120.57 & 2055.68 & 44.00 & 4565.31 & 4566.19 \\
\hline 20.00 & 2891.78 & 2879.14 & 45.00 & 4578.12 & 4578.95 \\
\hline 21.00 & 3308.51 & 3312.87 & 46.00 & 4590.20 & 4590.99 \\
\hline 22.00 & 3551.17 & 3557.09 & 47.00 & 4601.62 & 4602.36 \\
\hline 23.00 & 3716.76 & 3722.23 & 48.00 & 4612.42 & 4613.13 \\
\hline 24.00 & 3840.75 & 3845.56 & 49.00 & 4622.66 & 4623.33 \\
\hline 25.00 & 3938.84 & 3943.06 & 50.00 & 4632.38 & 4633.02 \\
\hline
\end{tabular}


Table 4: Comparison with Simmons and Grader Solutions (cont'd)

\begin{tabular}{|c|c|c|c|c|c|}
\hline $\begin{array}{c}\text { time } \\
\text { (sec.) }\end{array}$ & $\begin{array}{c}p_{w f} \\
(\mathrm{psia})\end{array}$ & $\begin{array}{c}\text { S-G } \\
p_{w f} \\
(\mathrm{psia})\end{array}$ & $\begin{array}{c}\text { time } \\
(\mathrm{sec} .)\end{array}$ & $\begin{array}{c}p_{w f} \\
(\mathrm{psia})\end{array}$ & $\begin{array}{c}\text { S-G } \\
p_{w f} \\
(\mathrm{psia})\end{array}$ \\
\hline 51.00 & 4641.62 & 4642.23 & 76.00 & 4781.48 & 4781.71 \\
\hline 52.00 & 4650.42 & 4651.00 & 77.00 & 4784.91 & 4785.13 \\
\hline 53.00 & 4658.81 & 4659.36 & 78.00 & 4788.24 & 4788.45 \\
\hline 54.00 & 4666.81 & 4667.34 & 79.00 & 4791.47 & 4791.68 \\
\hline 55.00 & 4674.45 & 4674.96 & 80.00 & 4794.61 & 4794.81 \\
\hline 56.00 & 4681.76 & 4682.25 & 81.00 & 4797.67 & 4797.86 \\
\hline 57.00 & 4688.76 & 4689.22 & 82.00 & 4800.64 & 4800.83 \\
\hline 58.00 & 4695.46 & 4695.91 & 83.00 & 4803.53 & 4803.71 \\
\hline 59.00 & 4701.89 & 4702.32 & 84.00 & 4806.34 & 4806.52 \\
\hline 60.00 & 4708.07 & 4708.48 & 85.00 & 4809.08 & 4809.25 \\
\hline 61.00 & 4714.00 & 4714.40 & 86.00 & 4811.74 & 4811.91 \\
\hline 62.00 & 4719.71 & 4720.08 & 87.00 & 4814.34 & 4814.51 \\
\hline 63.00 & 4725.19 & 4725.56 & 88.00 & 4816.87 & 4817.03 \\
\hline 64.00 & 4730.48 & 4730.83 & 89.00 & 4819.34 & 4819.50 \\
\hline 65.00 & 4735.58 & 4735.91 & 90.00 & 4821.75 & 4821.90 \\
\hline 66.00 & 4740.49 & 4740.82 & 91.00 & 4824.10 & 4824.24 \\
\hline 67.00 & 4745.23 & 4745.55 & 92.00 & 4826.39 & 4826.53 \\
\hline 68.00 & 4749.81 & 4750.11 & 93.00 & 4828.63 & 4828.76 \\
\hline 69.00 & 4754.24 & 4754.53 & 94.00 & 4830.81 & 4830.94 \\
\hline 70.00 & 4758.51 & 4758.80 & 95.00 & 4832.94 & 4833.07 \\
\hline 71.00 & 4762.65 & 4762.93 & 96.00 & 4835.03 & 4835.15 \\
\hline 72.00 & 4766.66 & 4766.92 & 97.00 & 4837.06 & 4837.19 \\
\hline 73.00 & 4770.54 & 4770.79 & 98.00 & 4839.05 & 4839.18 \\
\hline 74.00 & 4774.30 & 4774.54 & 99.00 & 4841.00 & 4841.12 \\
\hline 75.00 & 4777.94 & 4778.18 & 100.00 & 4842.90 & 4843.02 \\
\hline
\end{tabular}


Table 5: Comparison with Salas and Sageev Solutions

\begin{tabular}{|c|c|c|c|c|c|}
\hline $\begin{array}{c}\text { time } \\
(\mathrm{sec} .)\end{array}$ & $\begin{array}{c}p_{w f} \\
(\mathrm{psia})\end{array}$ & $\begin{array}{c}\mathrm{S}-\mathrm{S} \\
p_{w f} \\
(\mathrm{psia})\end{array}$ & $\begin{array}{c}\text { time } \\
(\mathrm{sec} .)\end{array}$ & $\begin{array}{c}p_{w f} \\
(\mathrm{psia})\end{array}$ & $\begin{array}{c}\mathrm{S}-\mathrm{S} \\
p_{w f} \\
(\mathrm{psia})\end{array}$ \\
\hline 1.000 & 87.73 & 87.69 & 23.00 & 3083.47 & 3110.79 \\
\hline 2.000 & 117.13 & 117.06 & 24.00 & 3386.93 & 3410.80 \\
\hline 3.000 & 144.01 & 143.91 & 25.00 & 3584.75 & 3601.05 \\
\hline 4.000 & 169.68 & 169.55 & 26.00 & 3728.00 & 3740.95 \\
\hline 5.000 & 194.74 & 194.56 & 27.00 & 3838.92 & 3850.56 \\
\hline 6.000 & 219.55 & 219.32 & 28.00 & 3928.66 & 3939.98 \\
\hline 7.000 & 244.41 & 244.13 & 29.00 & 4003.43 & 4011.74 \\
\hline 8.000 & 269.61 & 269.25 & 30.00 & 4067.10 & 4074.60 \\
\hline 9.000 & 295.44 & 294.97 & 31.00 & 4122.22 & 4129.56 \\
\hline 10.00 & 322.26 & 321.67 & 32.00 & 4170.56 & 4178.25 \\
\hline 11.00 & 350.55 & 349.83 & 33.10 & 4217.43 & 4220.78 \\
\hline 12.00 & 380.95 & 380.05 & 34.00 & 4251.70 & 4257.87 \\
\hline 13.00 & 414.37 & 413.15 & 35.10 & 4289.51 & 4294.17 \\
\hline 14.00 & 452.22 & 450.58 & 36.00 & 4317.55 & 4322.41 \\
\hline 15.00 & 496.76 & 494.48 & 37.20 & 4351.51 & 4354.56 \\
\hline 16.00 & 551.85 & 548.55 & 38.00 & 4372.25 & 4375.42 \\
\hline 17.00 & 624.61 & 619.43 & 39.00 & 4396.32 & 4403.23 \\
\hline 18.00 & 729.50 & 720.79 & 40.00 & 4418.55 & 4424.58 \\
\hline 19.00 & 898.97 & 883.07 & 41.00 & 4443.10 & 4445.03 \\
\hline 20.00 & 1210.98 & 1180.74 & 42.00 & 4458.33 & 4464.65 \\
\hline 21.00 & 1803.28 & 1764.72 & 43.00 & 4476.21 & 4484.56 \\
\hline 22.00 & 2562.09 & 2570.56 & 44.00 & 4492.93 & 4499.46 \\
\hline
\end{tabular}


Table 5: Comparison with Salas and Sageev Solutions (cont'd)

\begin{tabular}{|c|c|c|c|c|c|}
\hline $\begin{array}{c}\text { time } \\
(\text { sec. })\end{array}$ & $\begin{array}{c}p_{w f} \\
(\mathrm{psia})\end{array}$ & $\begin{array}{c}\text { S-S } \\
p_{w f} \\
(\mathrm{psia})\end{array}$ & $\begin{array}{c}\text { time } \\
(\text { sec. }\end{array}$ & $\begin{array}{c}p_{w f} \\
(\mathrm{psia})\end{array}$ & $\begin{array}{c}\text { S-S } \\
p_{w f} \\
(\mathrm{psia})\end{array}$ \\
\hline 44.90 & 4505.55 & 4511.49 & 67.00 & 4708.06 & 4711.04 \\
\hline 46.10 & 4524.75 & 4529.90 & 67.90 & 4712.88 & 4715.77 \\
\hline 47.10 & 4538.54 & 4540.73 & 68.80 & 4717.04 & 4720.38 \\
\hline 49.10 & 4563.84 & 4564.59 & 69.70 & 4722.08 & 4723.37 \\
\hline 50.20 & 4576.61 & 4579.36 & 70.20 & 4724.53 & 4725.38 \\
\hline 50.10 & 4586.52 & 4595.88 & 71.80 & 4731.66 & 4733.81 \\
\hline 52.40 & 4600.04 & 4596.88 & 72.80 & 4736.21 & 4742.03 \\
\hline 53.40 & 4609.87 & 4611.81 & 74.80 & 4744.89 & 4745.36 \\
\hline 54.10 & 4616.47 & 4620.12 & 76.70 & 4753.04 & 4753.90 \\
\hline 55.10 & 4625.52 & 4629.03 & 77.30 & 4755.00 & 4756.45 \\
\hline 56.00 & 4633.32 & 4637.85 & 77.90 & 4757.70 & 4759.34 \\
\hline 57.40 & 4644.79 & 4647.54 & 79.30 & 4762.56 & 4768.78 \\
\hline 58.00 & 4649.53 & 4652.39 & 84.70 & 4781.22 & 4771.64 \\
\hline 59.20 & 4658.62 & 4661.29 & 85.00 & 4782.16 & 4778.98 \\
\hline 60.20 & 4665.85 & 4667.63 & 86.00 & 4785.23 & 4788.12 \\
\hline 61.00 & 4671.42 & 4673.51 & 87.10 & 4788.52 & 4797.78 \\
\hline 62.30 & 4680.09 & 4682.39 & 97.00 & 4814.33 & 4805.95 \\
\hline 63.30 & 4686.47 & 4689.23 & 98.20 & 4817.06 & 4810.06 \\
\hline 64.00 & 4690.78 & 4694.34 & 99.20 & 4819.28 & 4815.83 \\
\hline 65.30 & 4697.92 & 4699.60 & 99.70 & 4820.37 & 4818.93 \\
\hline 66.60 & 4705.31 & 4706.50 & 100.20 & 4821.02 & 4819.90 \\
\hline
\end{tabular}


Table 6: Comparison with Analytical DST Buildup Solutions

\begin{tabular}{|c|c|c|}
\hline$\Delta t_{D}$ & $\begin{array}{c}p_{s D} \\
\text { (Numerical) }\end{array}$ & $\begin{array}{c}p_{s} D \\
\text { (Analytical) }\end{array}$ \\
\hline 10.0 & 0.156856 & 0.153972 \\
\hline 20.0 & 0.149803 & 0.146502 \\
\hline 30.0 & 0.141169 & 0.140537 \\
\hline 40.0 & 0.135854 & 0.135655 \\
\hline 50.0 & 0.131189 & 0.131577 \\
\hline 60.0 & 0.127514 & 0.128111 \\
\hline 70.0 & 0.124367 & 0.125119 \\
\hline 80.0 & 0.121684 & 0.122502 \\
\hline 90.0 & 0.119336 & 0.120187 \\
\hline 100.0 & 0.117260 & 0.118118 \\
\hline 200.0 & 0.104145 & 0.104807 \\
\hline 300.0 & 0.096781 & 0.097292 \\
\hline 400.0 & 0.091586 & 0.092003 \\
\hline 500.0 & 0.087531 & 0.087885 \\
\hline 600.0 & 0.084183 & 0.084491 \\
\hline 700.0 & 0.081319 & 0.081591 \\
\hline 800.0 & 0.078809 & 0.079053 \\
\hline 900.0 & 0.076570 & 0.076790 \\
\hline 1000.0 & 0.074545 & 0.074746 \\
\hline 2000.0 & 0.060697 & 0.060806 \\
\hline 3000.0 & 0.052252 & 0.052328 \\
\hline 4000.0 & 0.046221 & 0.046279 \\
\hline 5000.0 & 0.041593 & 0.041640 \\
\hline 6000.0 & 0.037886 & 0.037926 \\
\hline 7000.0 & 0.034832 & 0.034865 \\
\hline 8000.0 & 0.032260 & 0.032290 \\
\hline 9000.0 & 0.030060 & 0.030086 \\
\hline 10000.0 & 0.028153 & 0.028176 \\
\hline 20000.0 & 0.017341 & 0.017351 \\
\hline 30000.0 & 0.012571 & 0.012577 \\
\hline 40000.0 & 0.009867 & 0.009871 \\
\hline 50000.0 & 0.008122 & 0.008125 \\
\hline 60000.0 & 0.006903 & 0.006905 \\
\hline 70000.0 & 0.006002 & 0.006004 \\
\hline 80000.0 & 0.005310 & 0.005311 \\
\hline 90000.0 & 0.004761 & 0.004762 \\
\hline & & \\
\hline & & \\
\hline & & \\
\hline & & \\
\hline
\end{tabular}


Table 7: Parameters for Base Case Closed Chamber Test

\begin{tabular}{|l|rl|}
\hline \multicolumn{1}{|c|}{ Parameter } & Value & Unit \\
\hline Initial Reservoir Pressure & 5000.0 & psig \\
\hline Fluid Gravity & 25.0 & API \\
\hline Fluid Viscosity & 1.25 & $\mathrm{cp}$ \\
\hline Formation Porosity & 0.27 & - \\
\hline Formation Permeability & 100.0 & $\mathrm{md}$ \\
\hline Skin Factor & 0.0 & - \\
\hline Total System Compressibility & 0.00001 & I/psi \\
\hline Reservoir Thickness & 25.0 & $\mathrm{ft}$ \\
\hline Well Diameter & 10.0 & in. \\
\hline Initial Fluid Height & 100.0 & $\mathrm{ft}$ \\
\hline Chamber ID & 2.441 & in. \\
\hline Total Chamber Length & $10,000.0$ & $\mathrm{ft}$ \\
\hline Initial Chamber Gas Pressure & 500.0 & psig \\
\hline Initial Wellbore Storage Coefficient & 994.0 & - \\
\hline
\end{tabular}




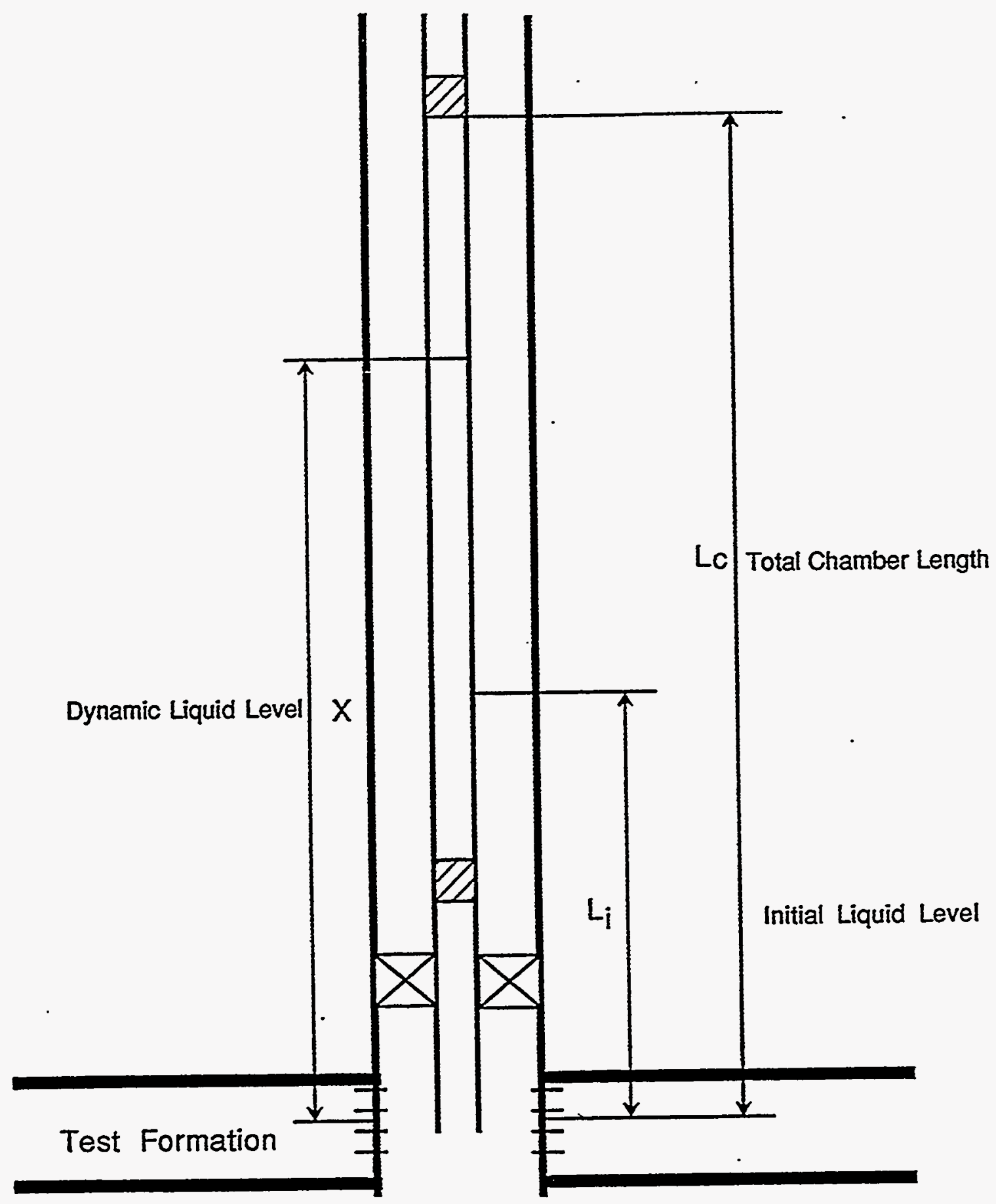

Fig. 1 - Schematic of Closed Chamber Test 


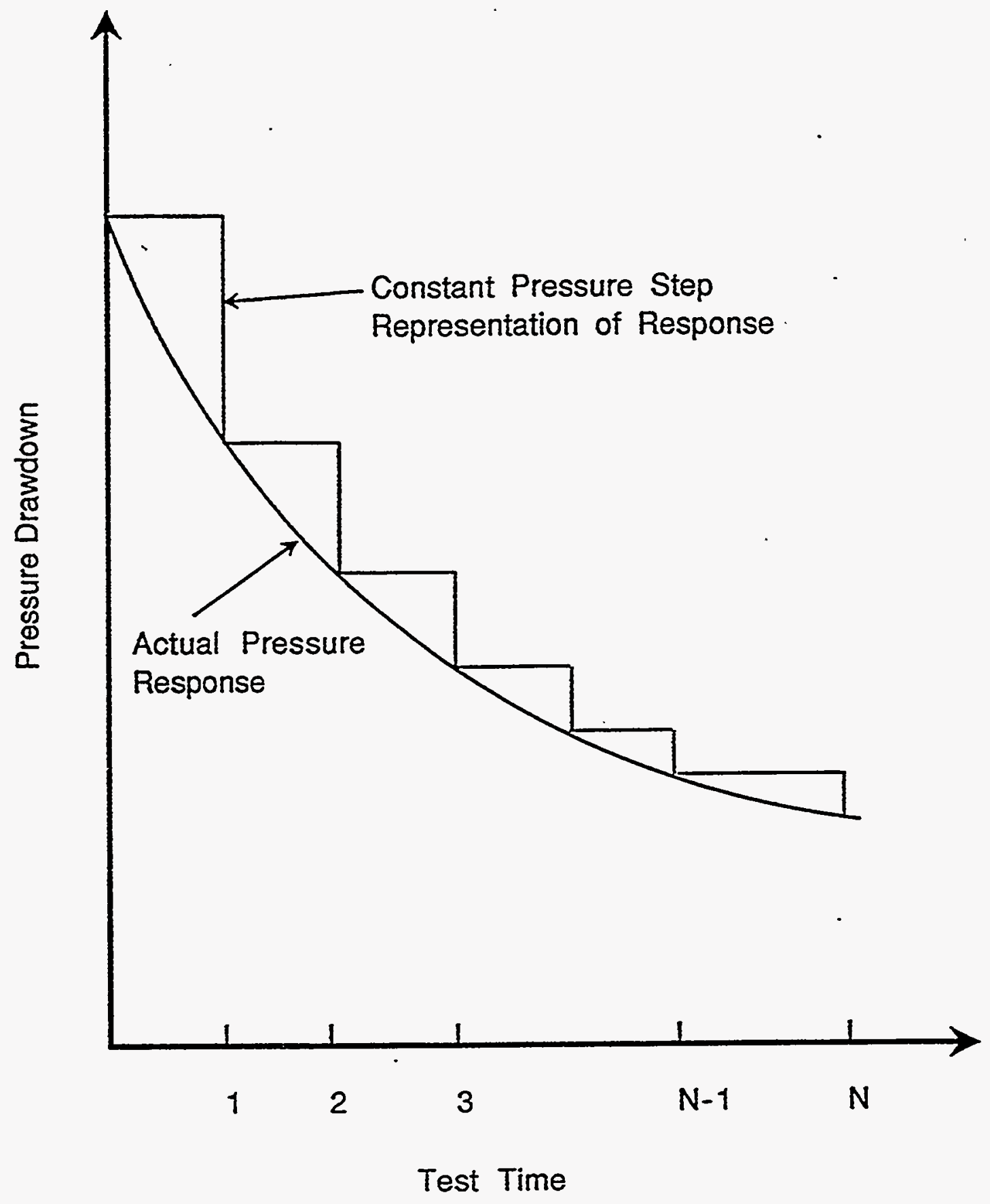

Fig. 2 - Discrete Representation of the Pressure Response of a Closed Chamber Test 


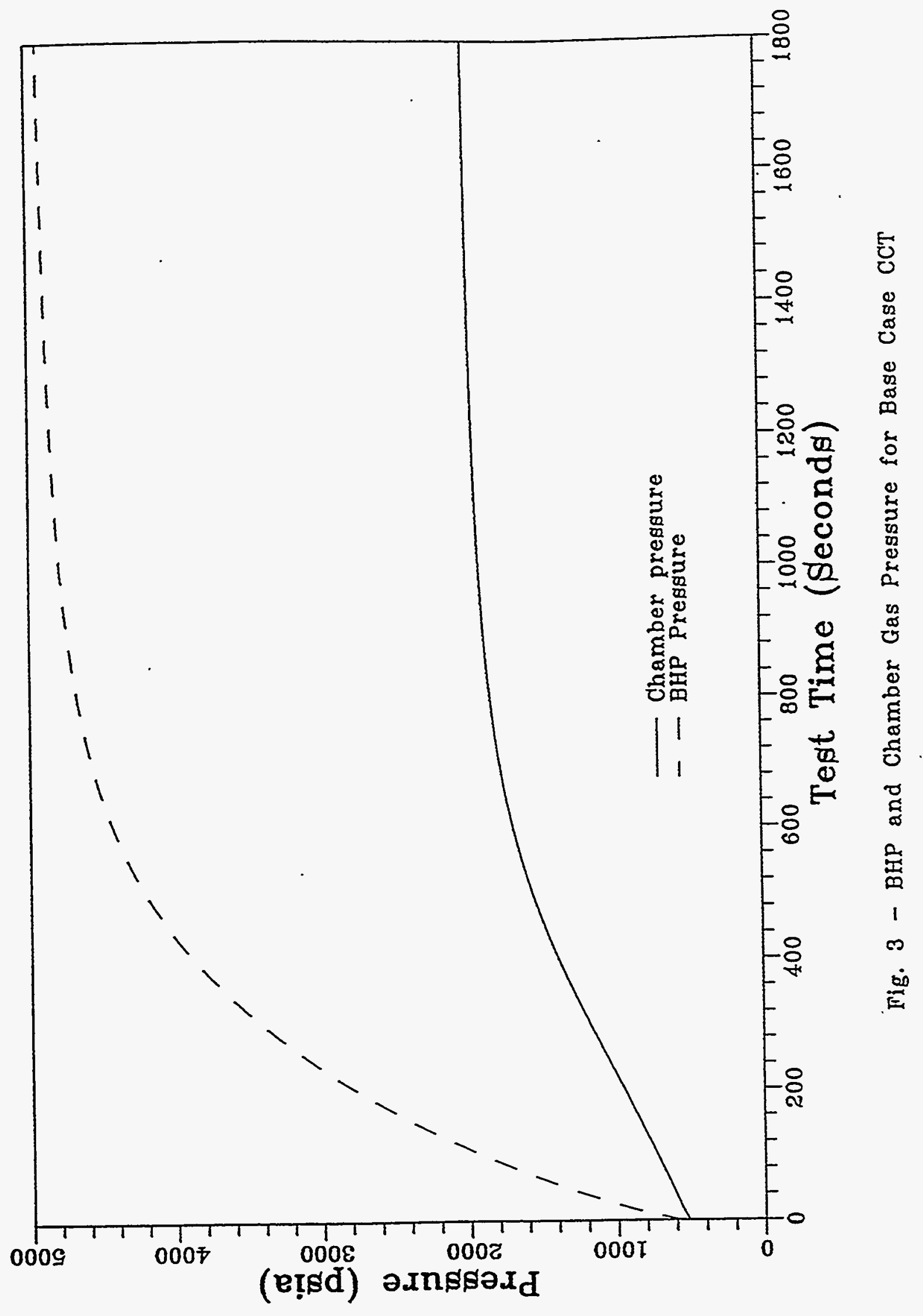




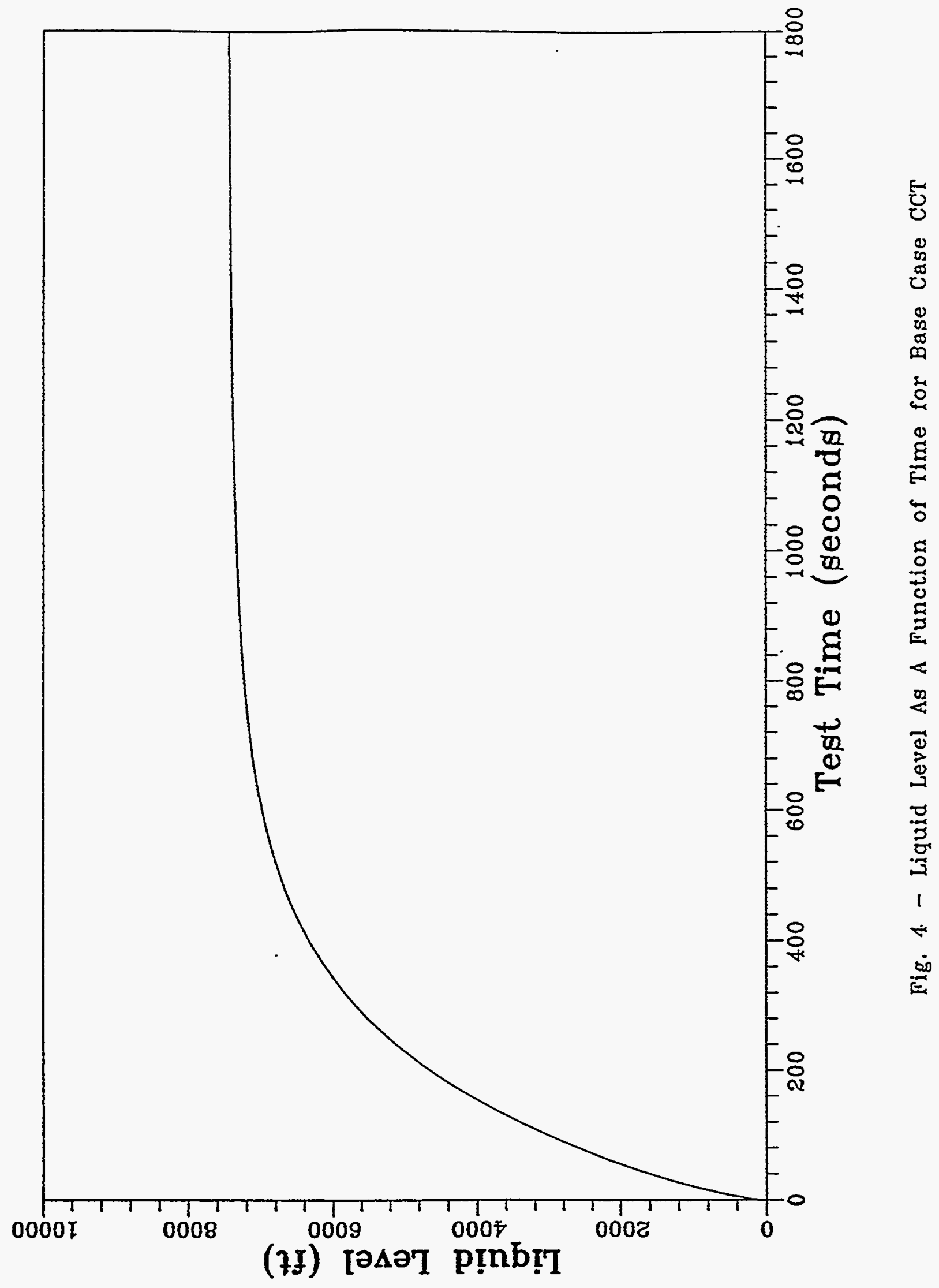




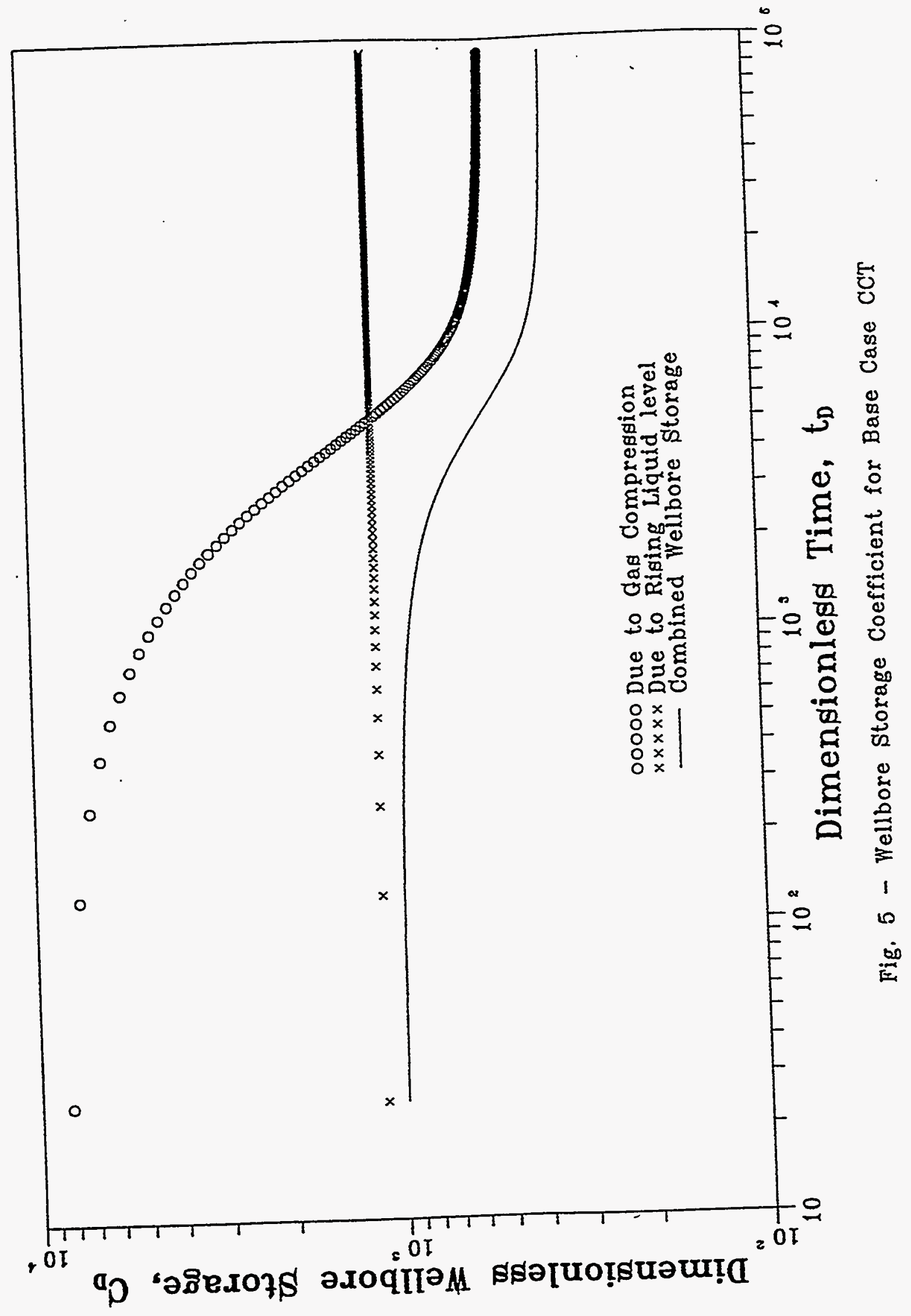




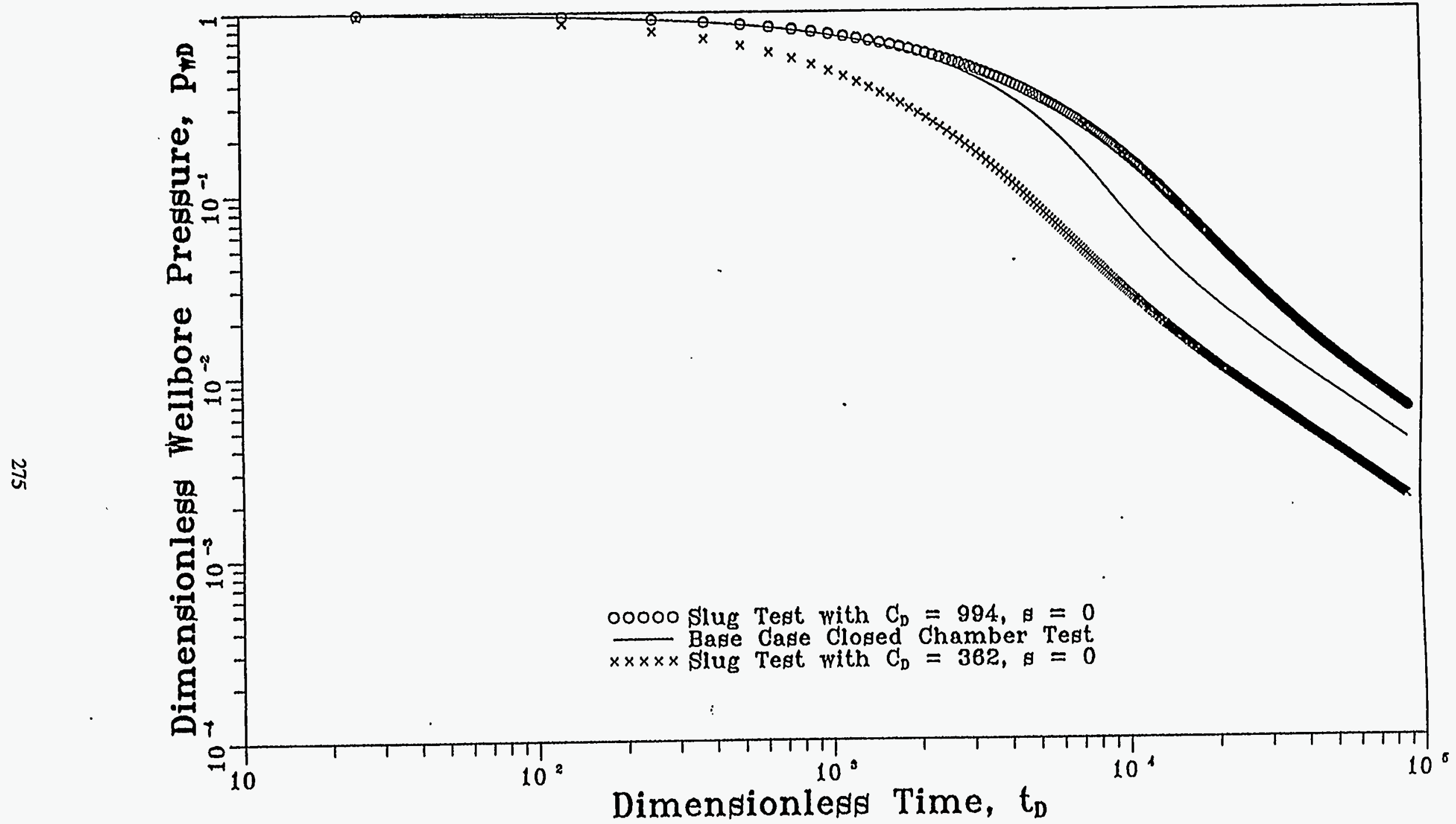

Fig. 6 - Comparison of Slug Test and Base Case Closed Chamber Test Solutions 


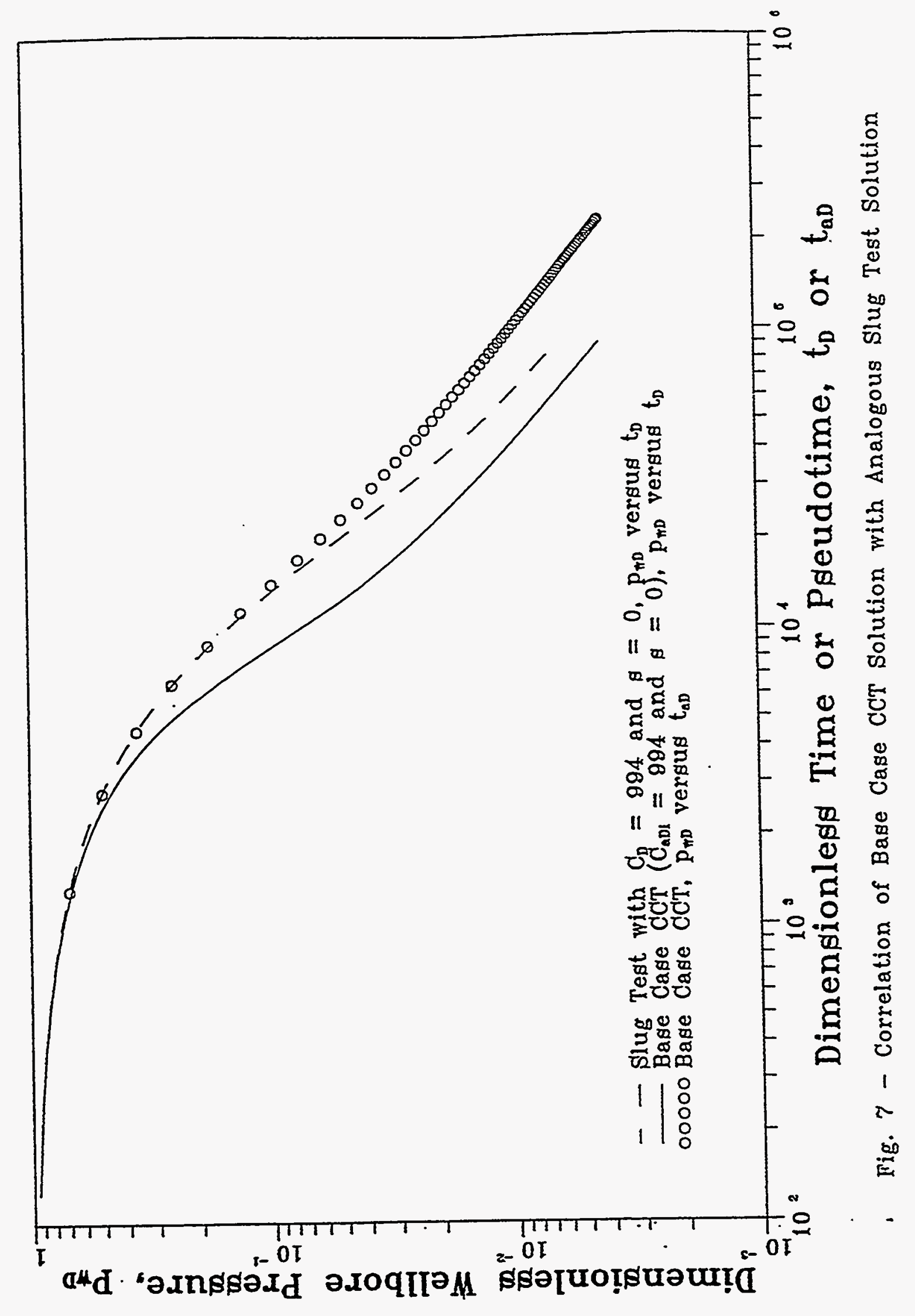




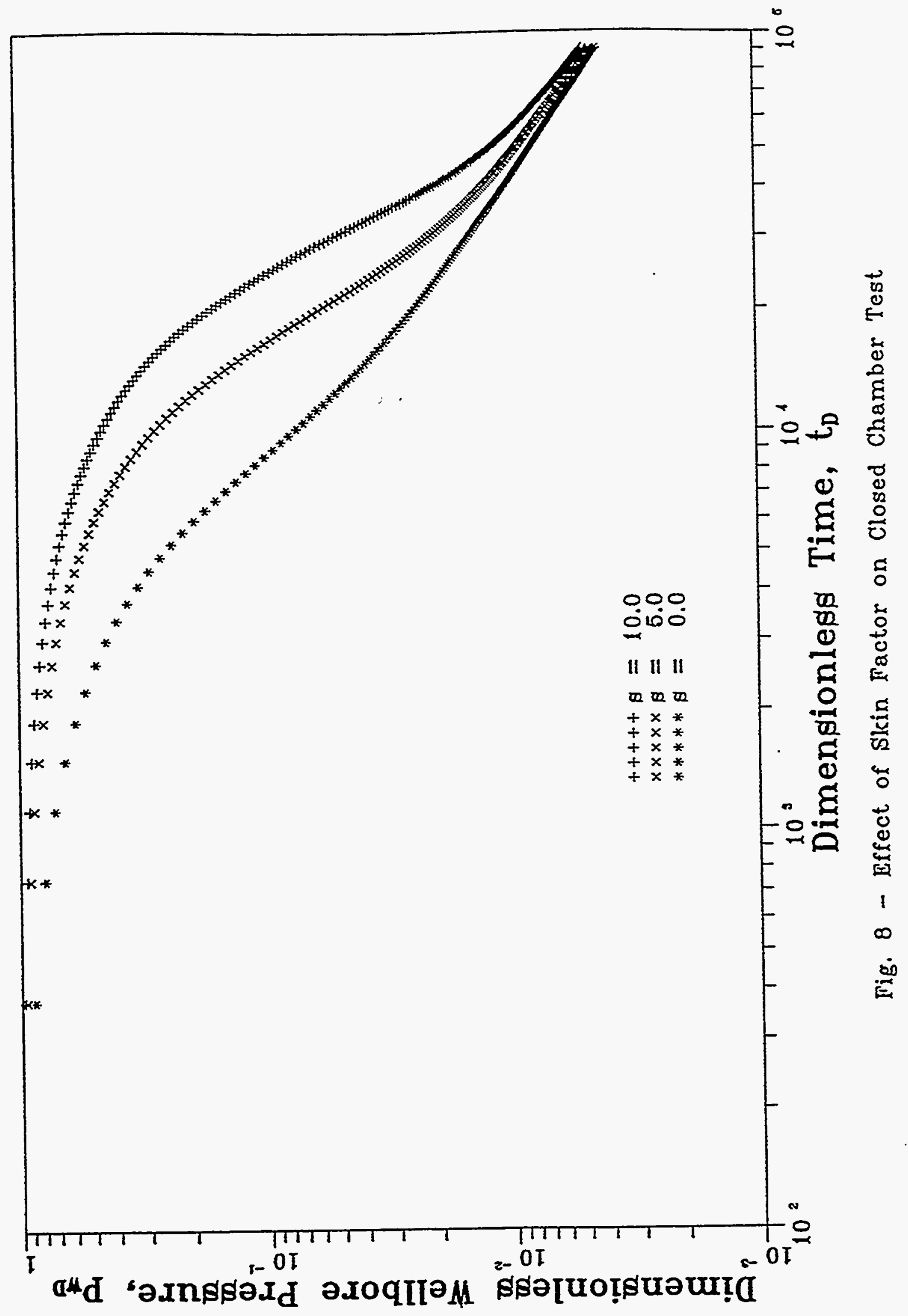




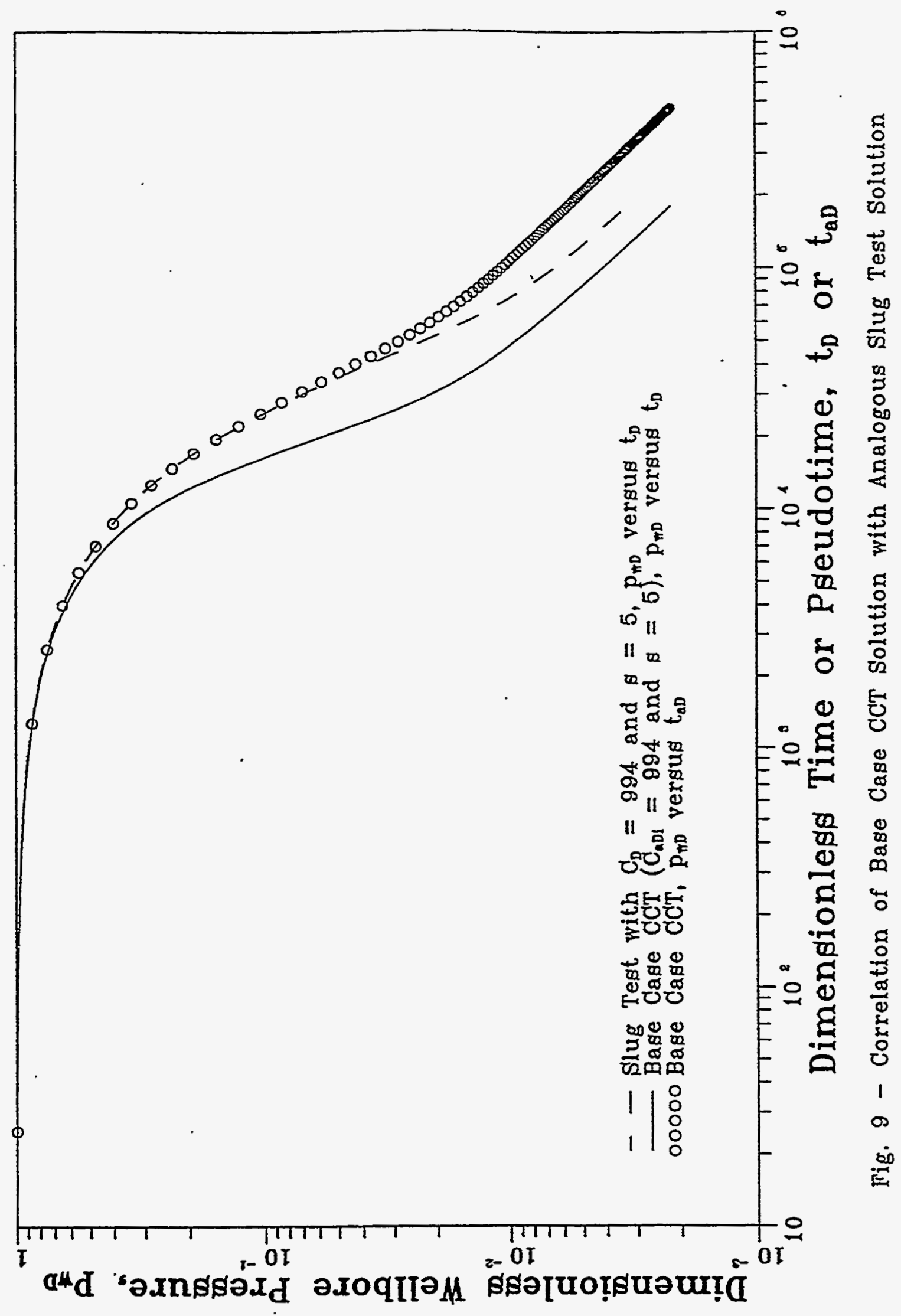




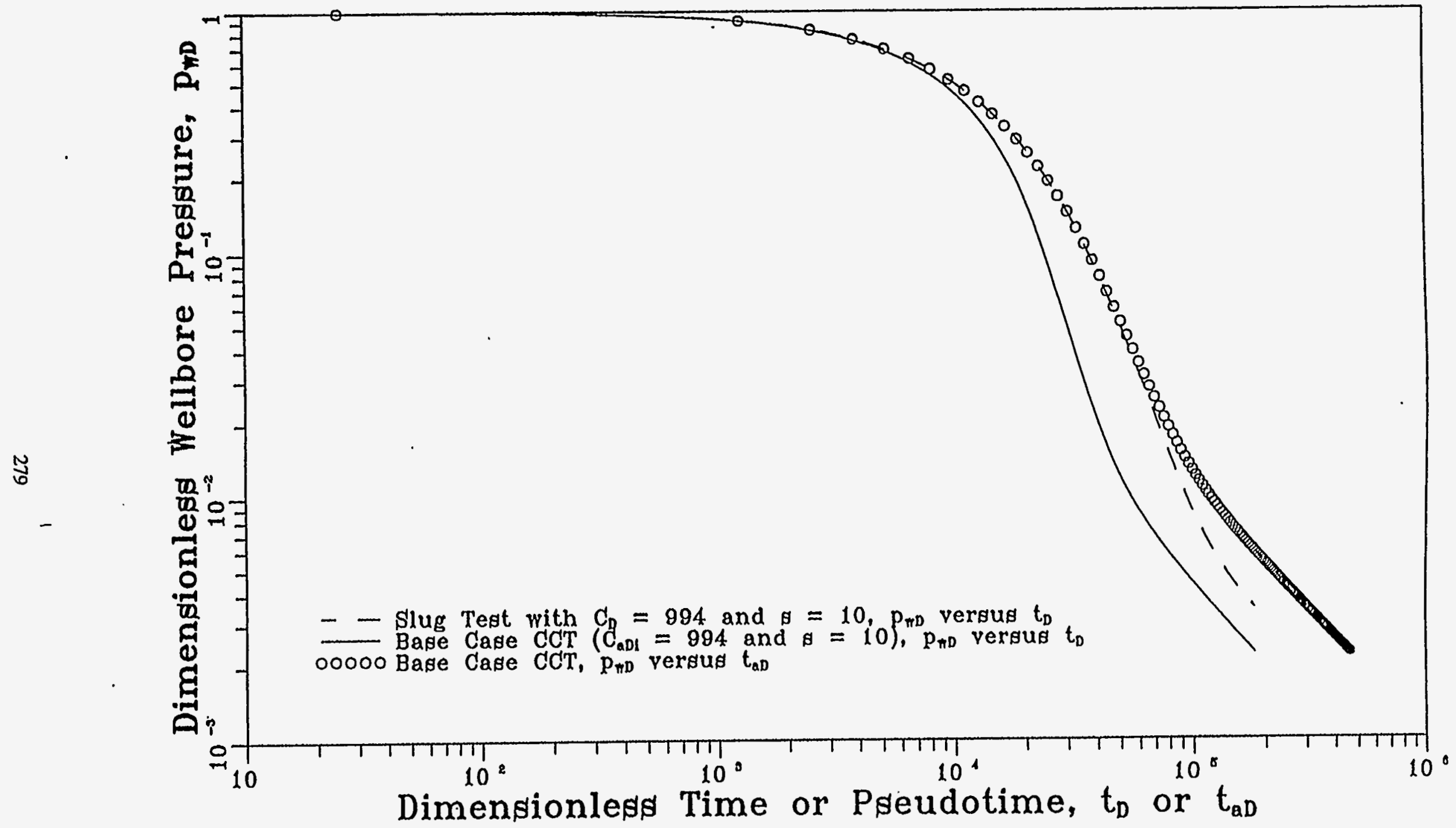

Fig. 10 - Correlation of Base Case CCT Solution with Analogous Slug Test Soluti 


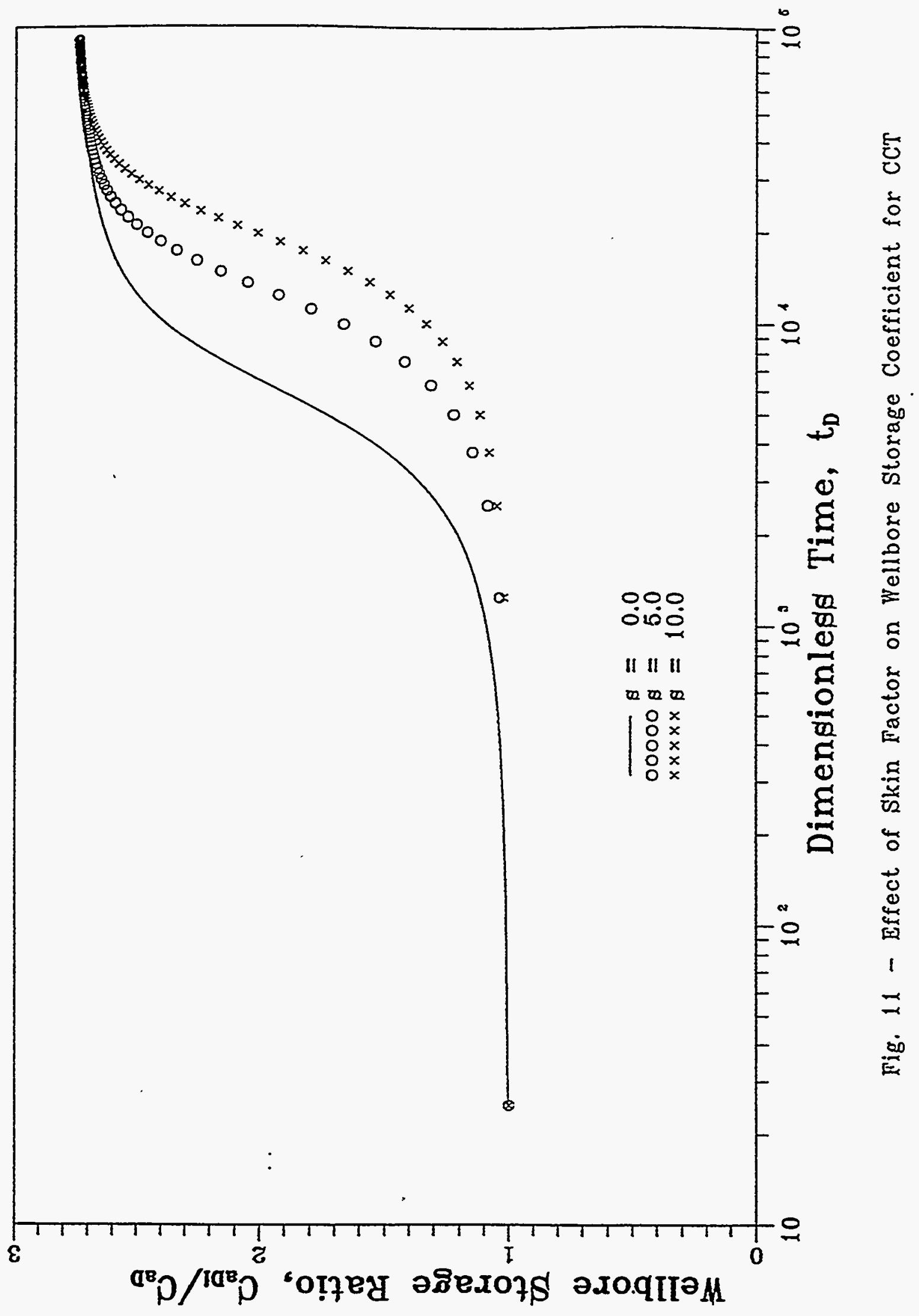




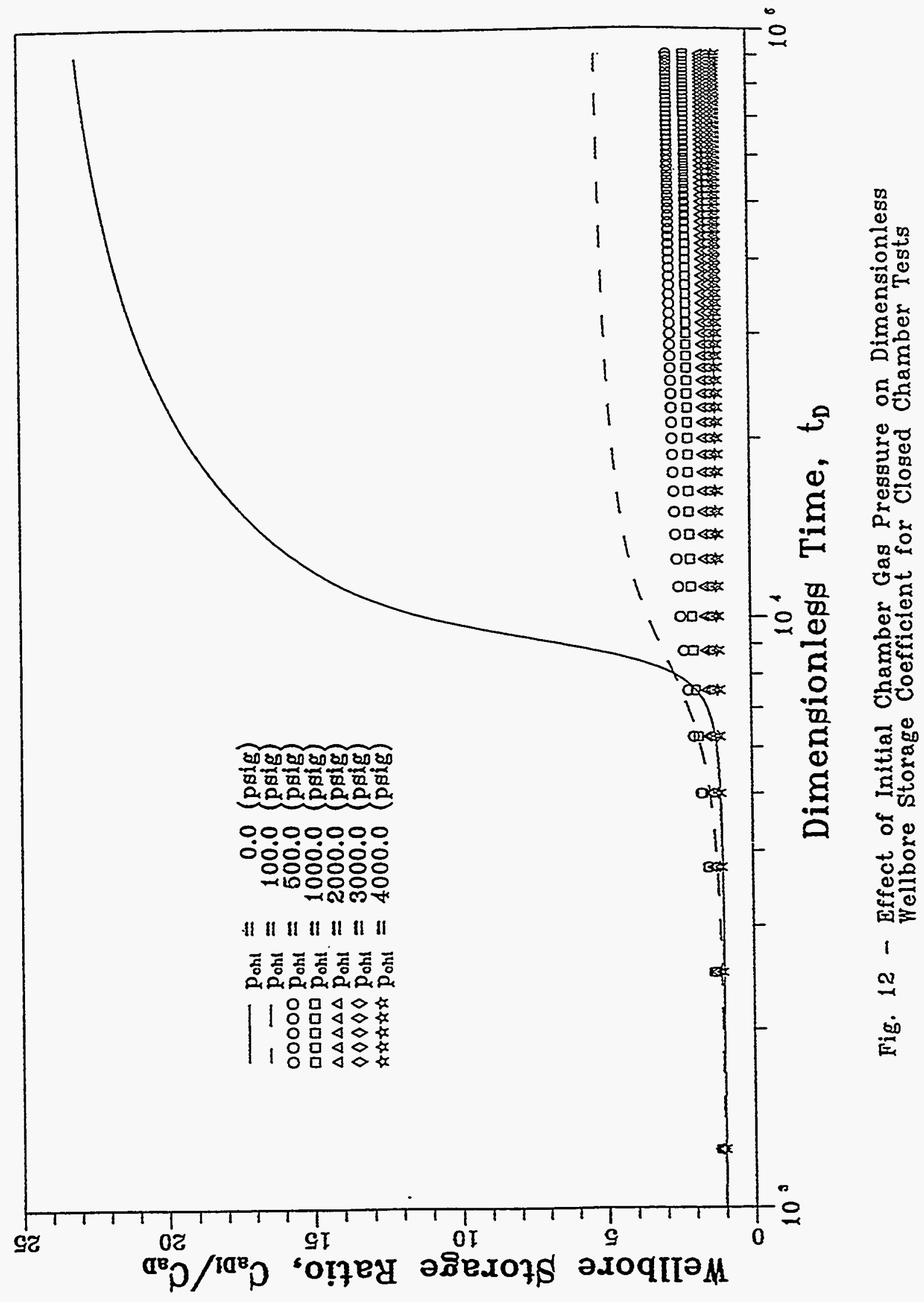




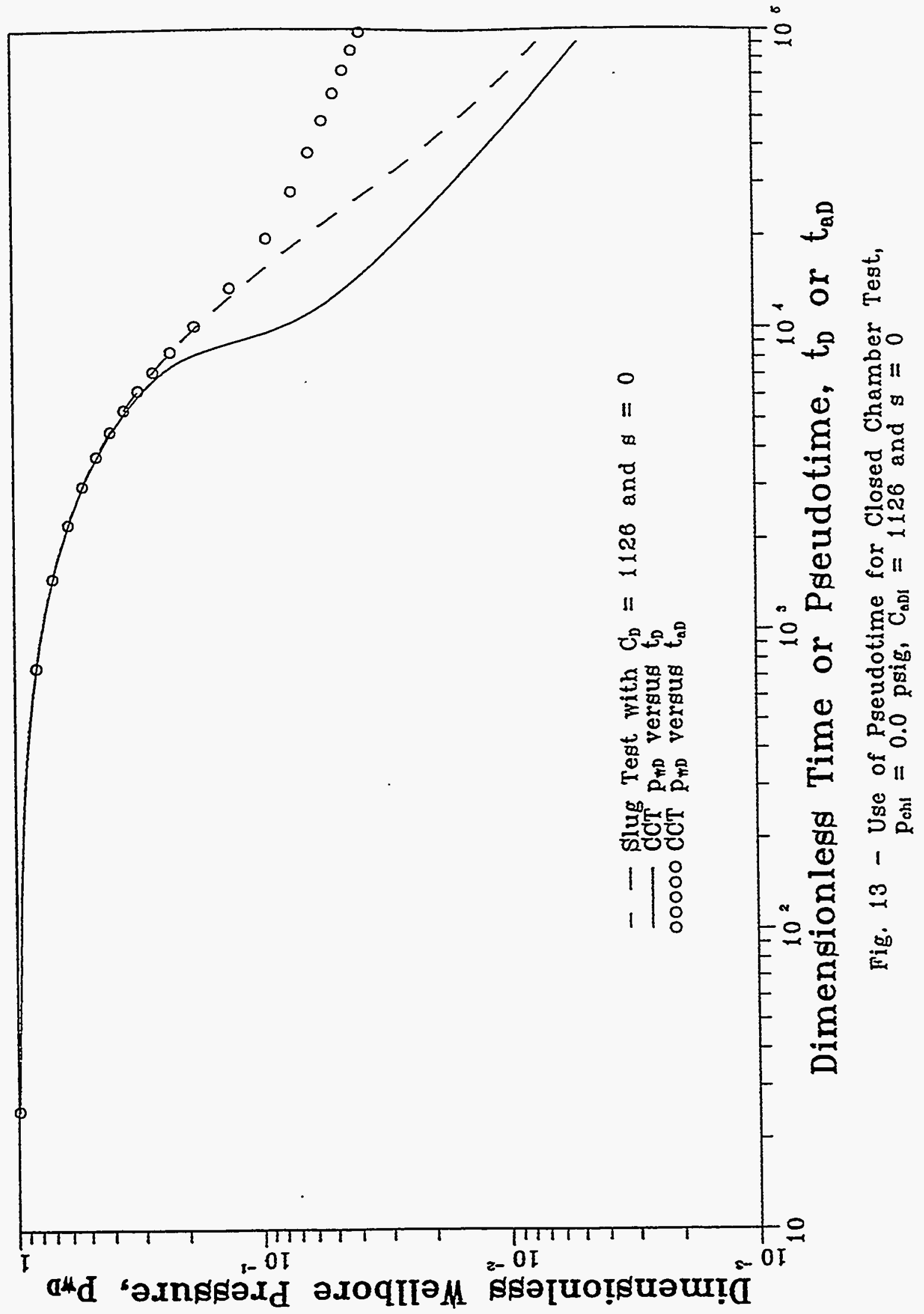




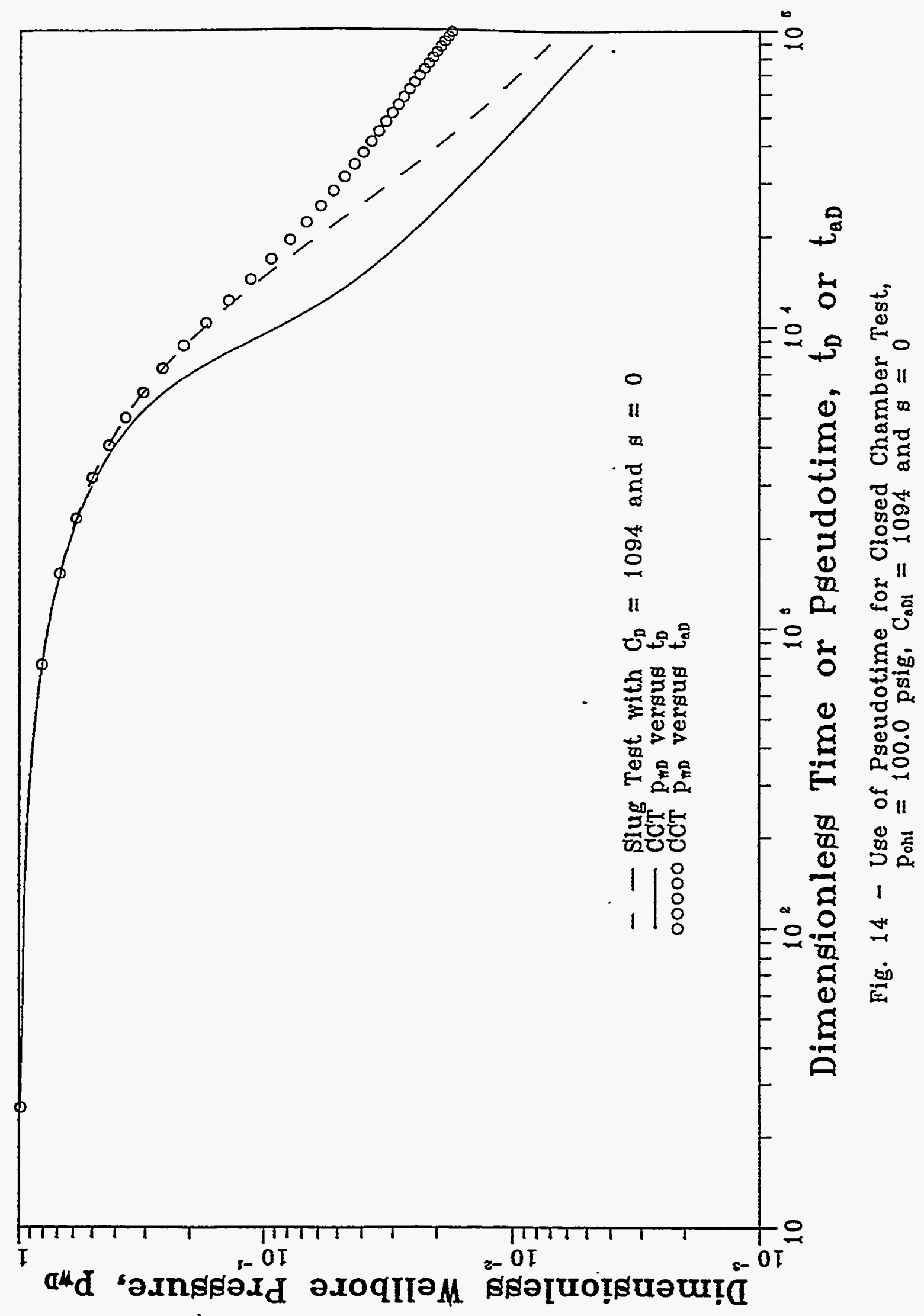




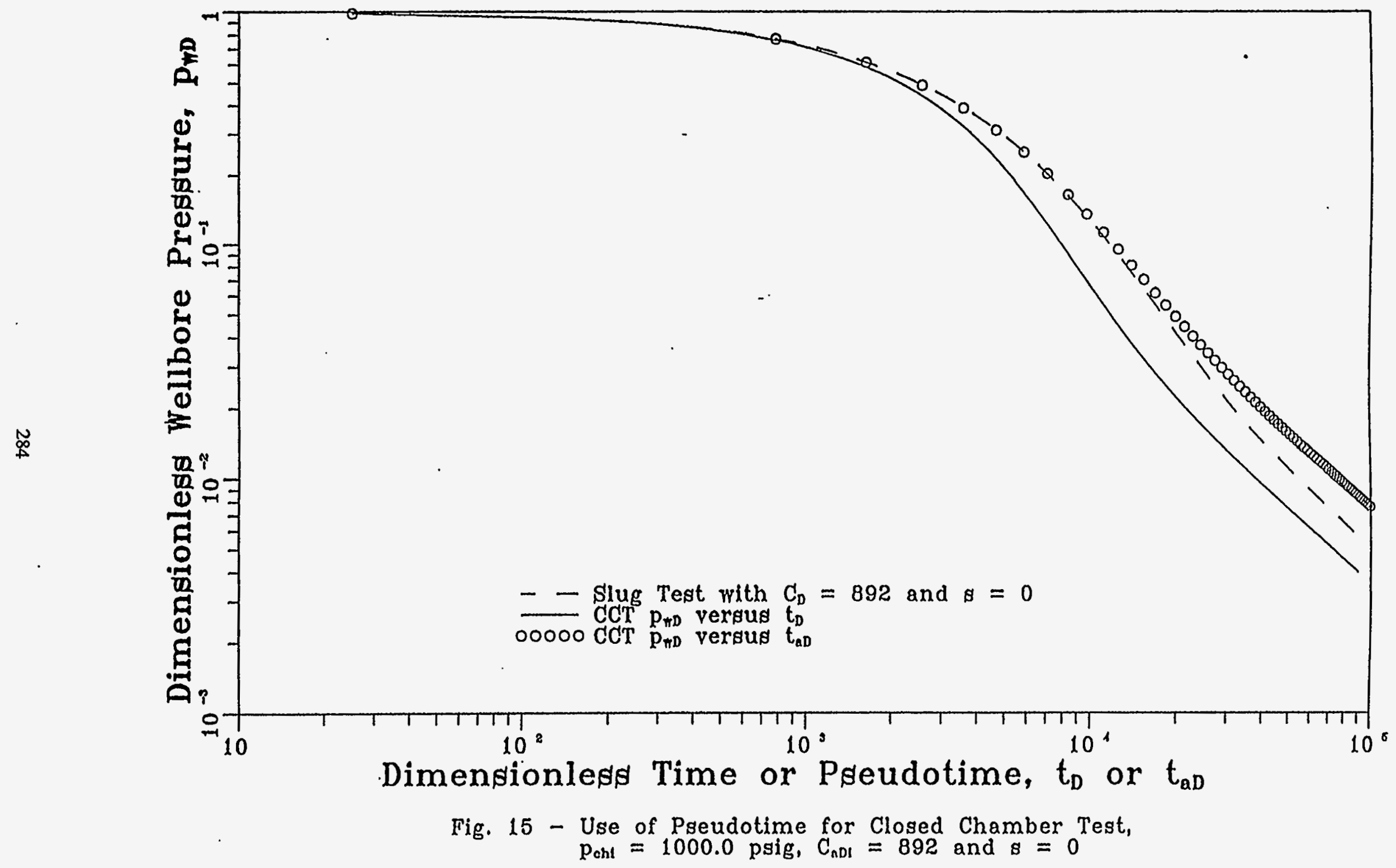




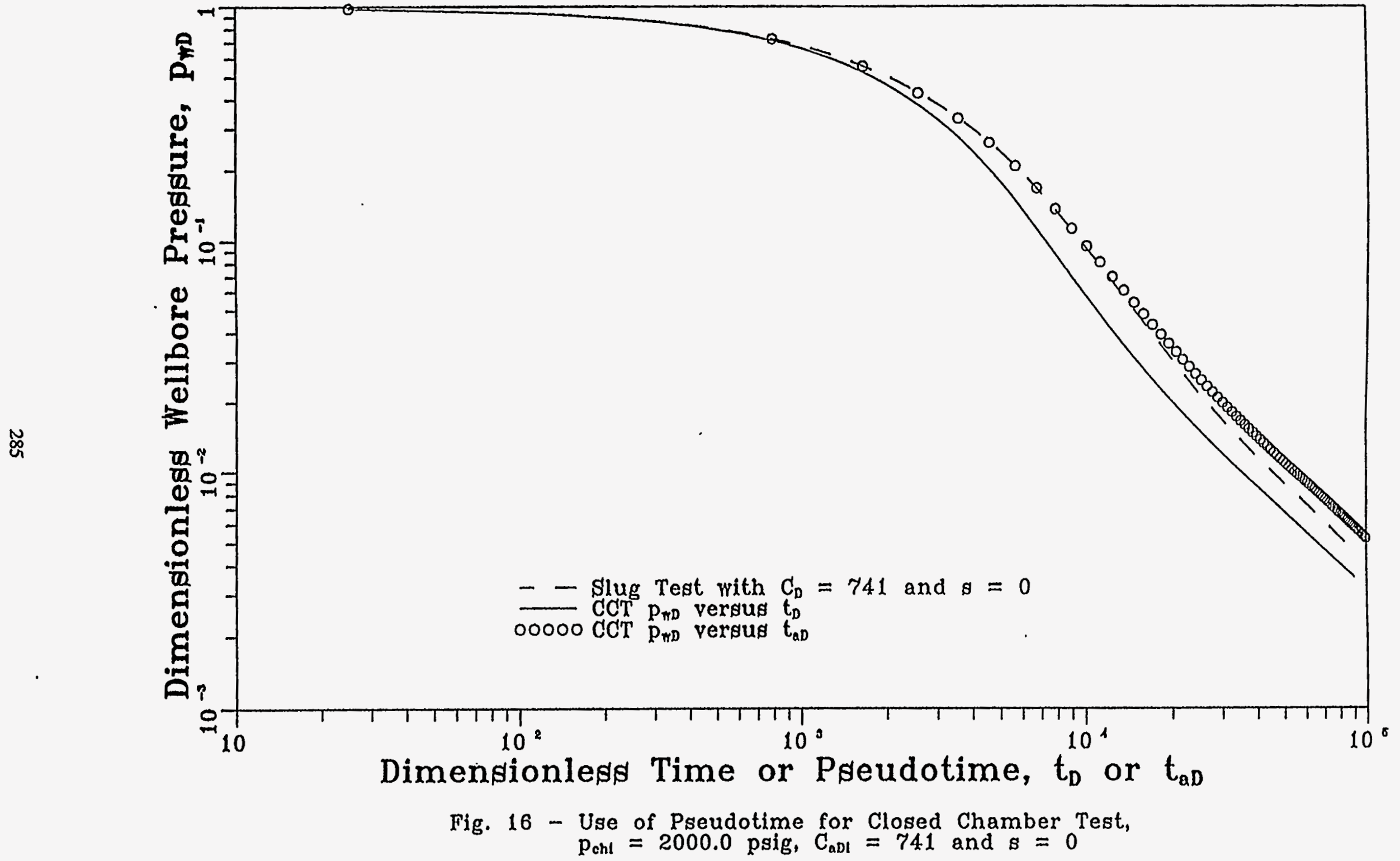




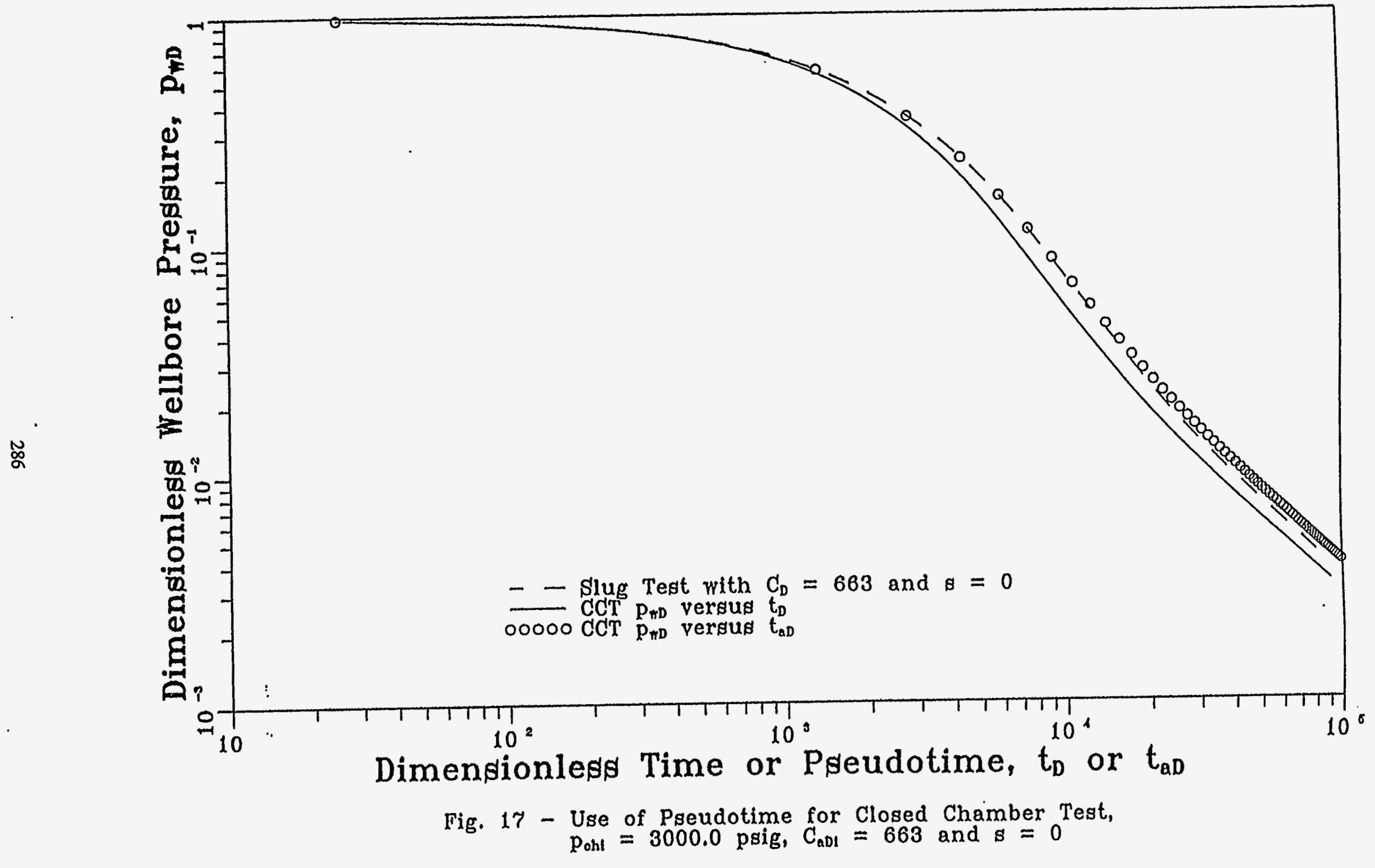




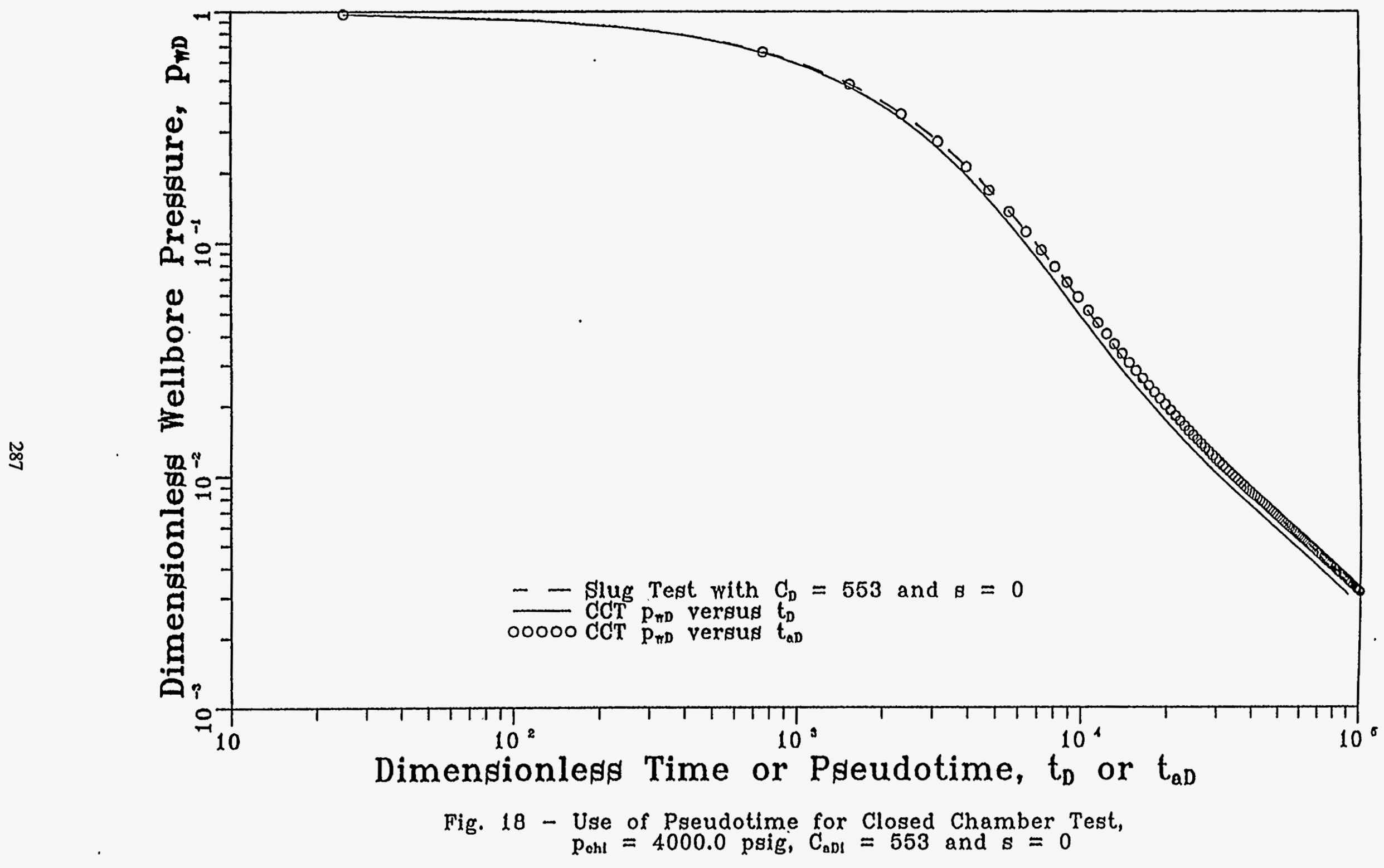




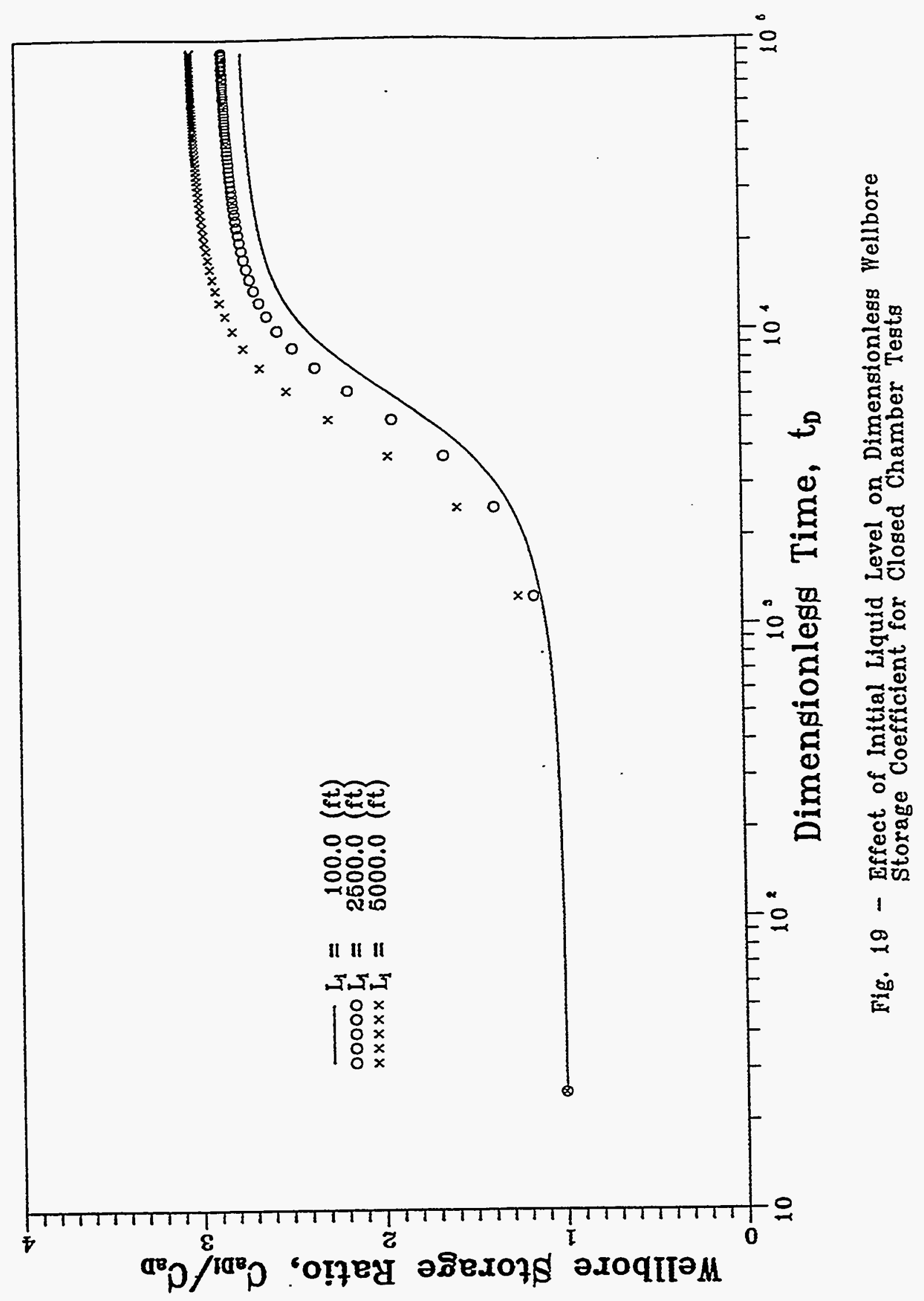




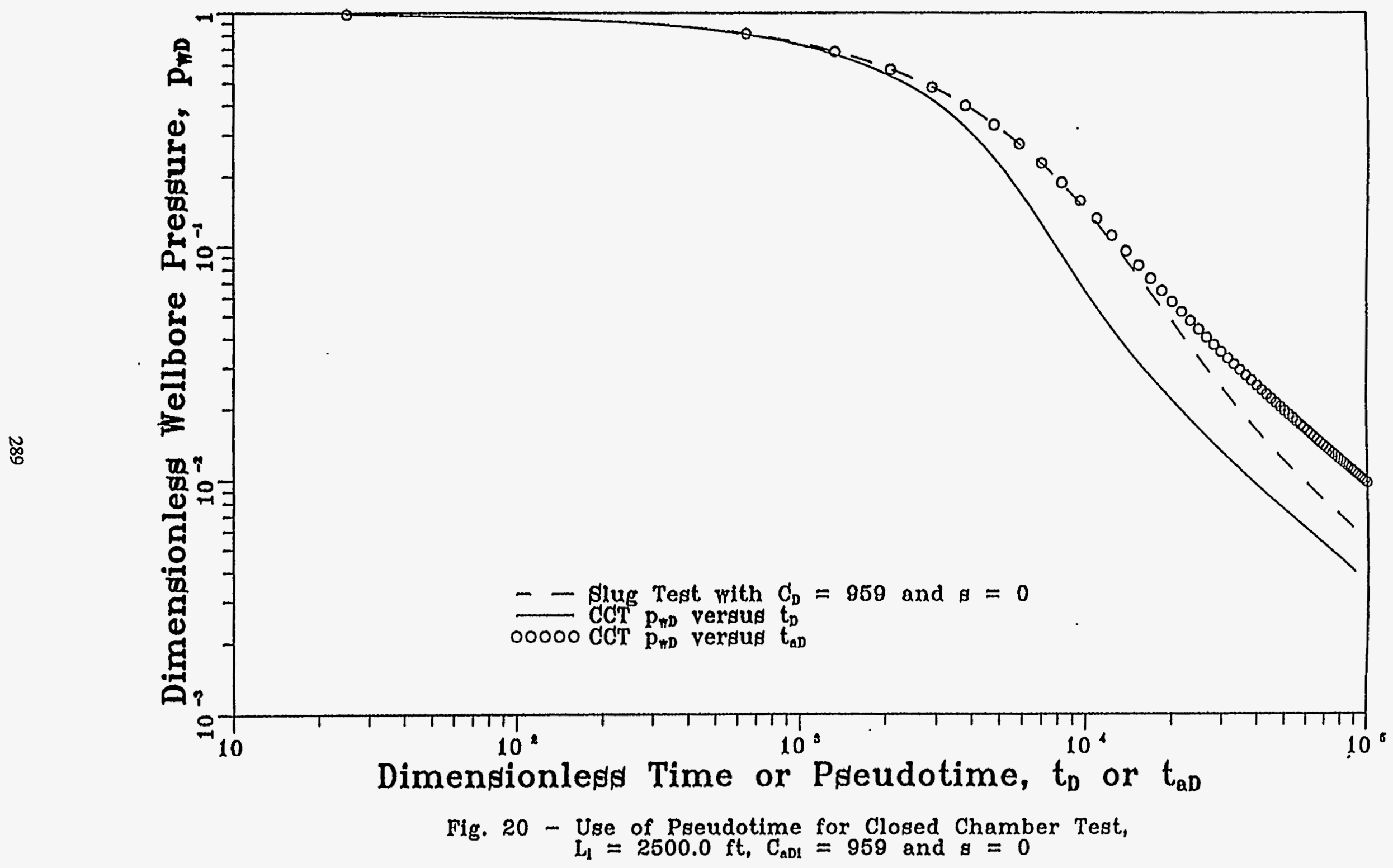




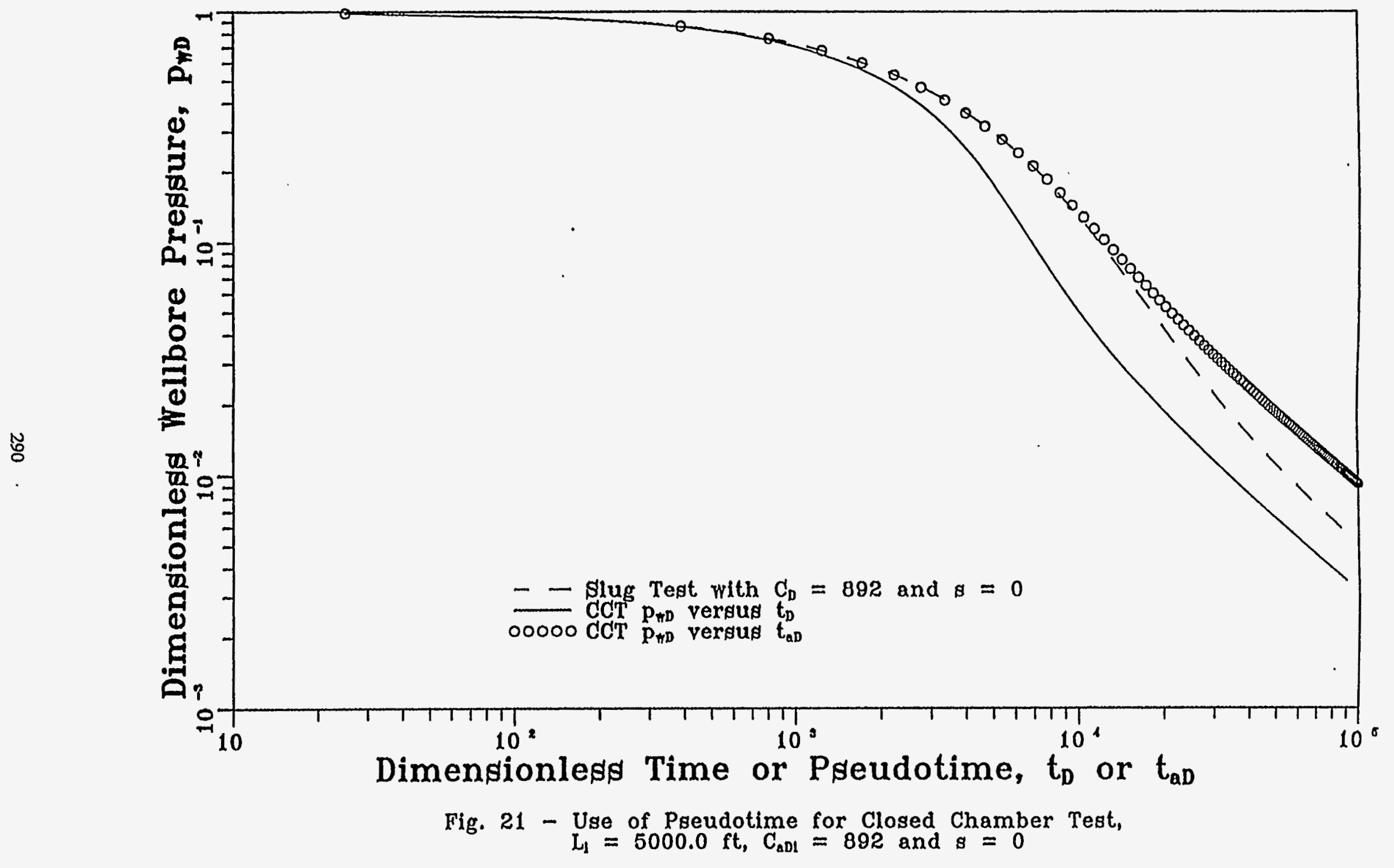




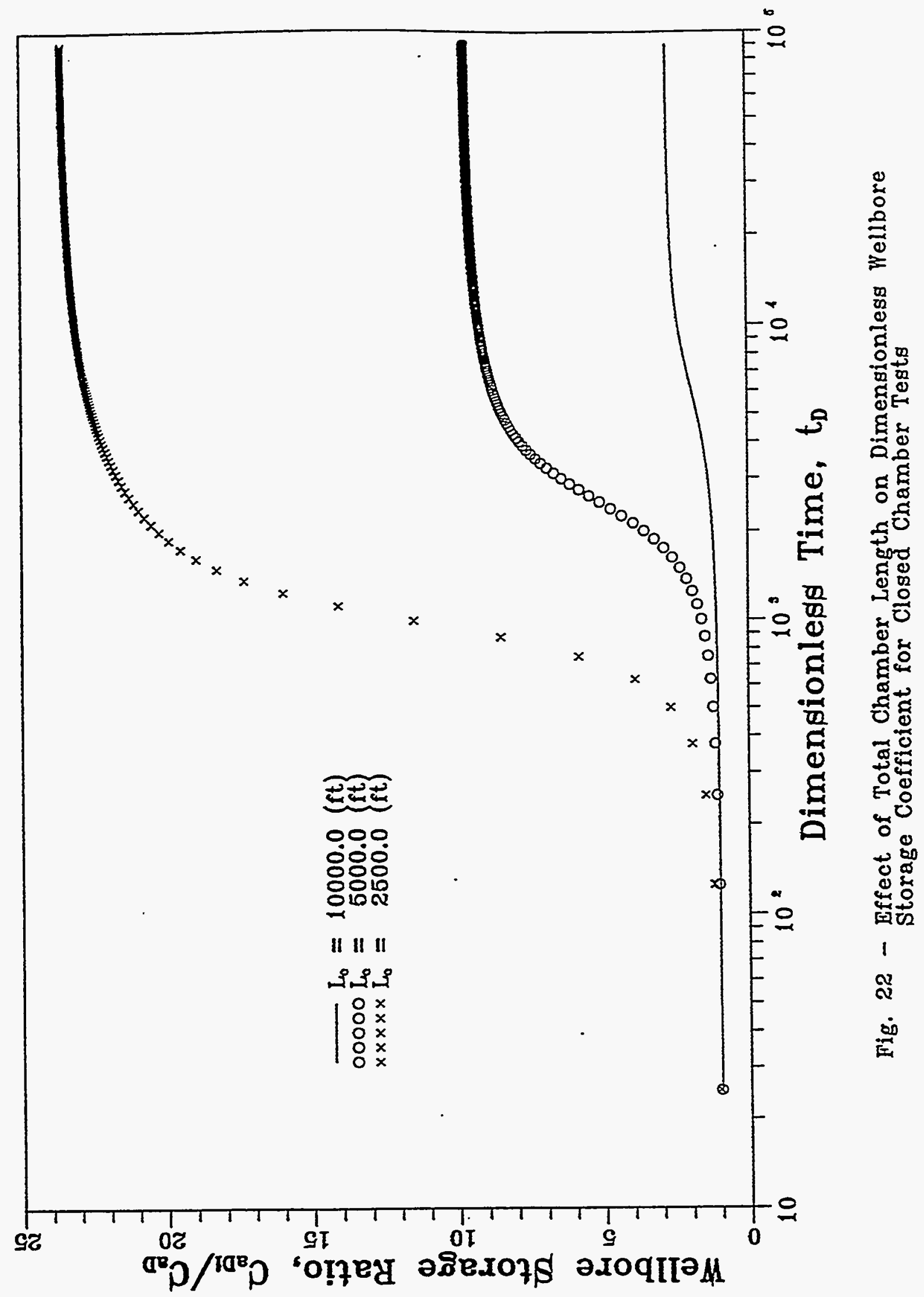




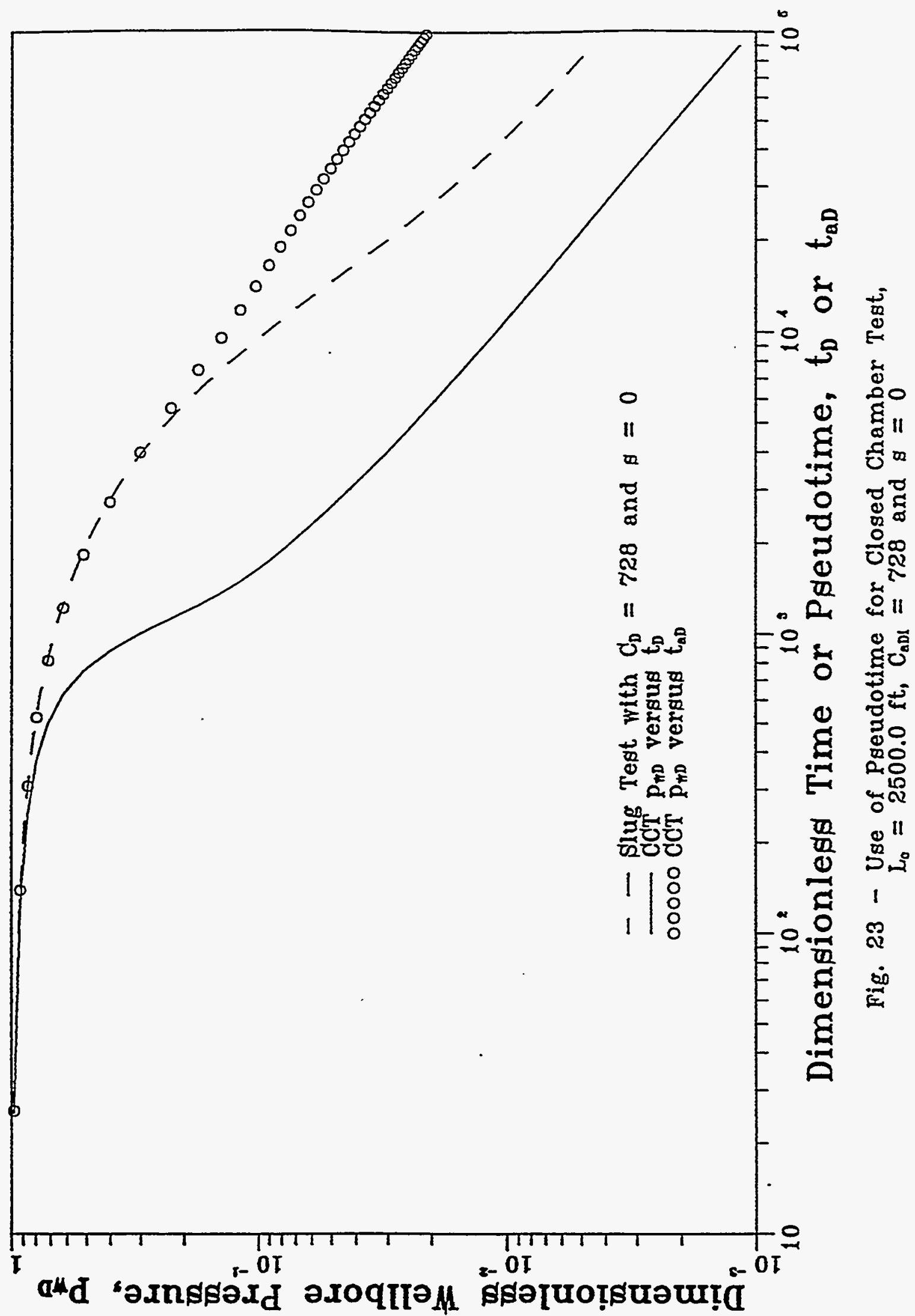




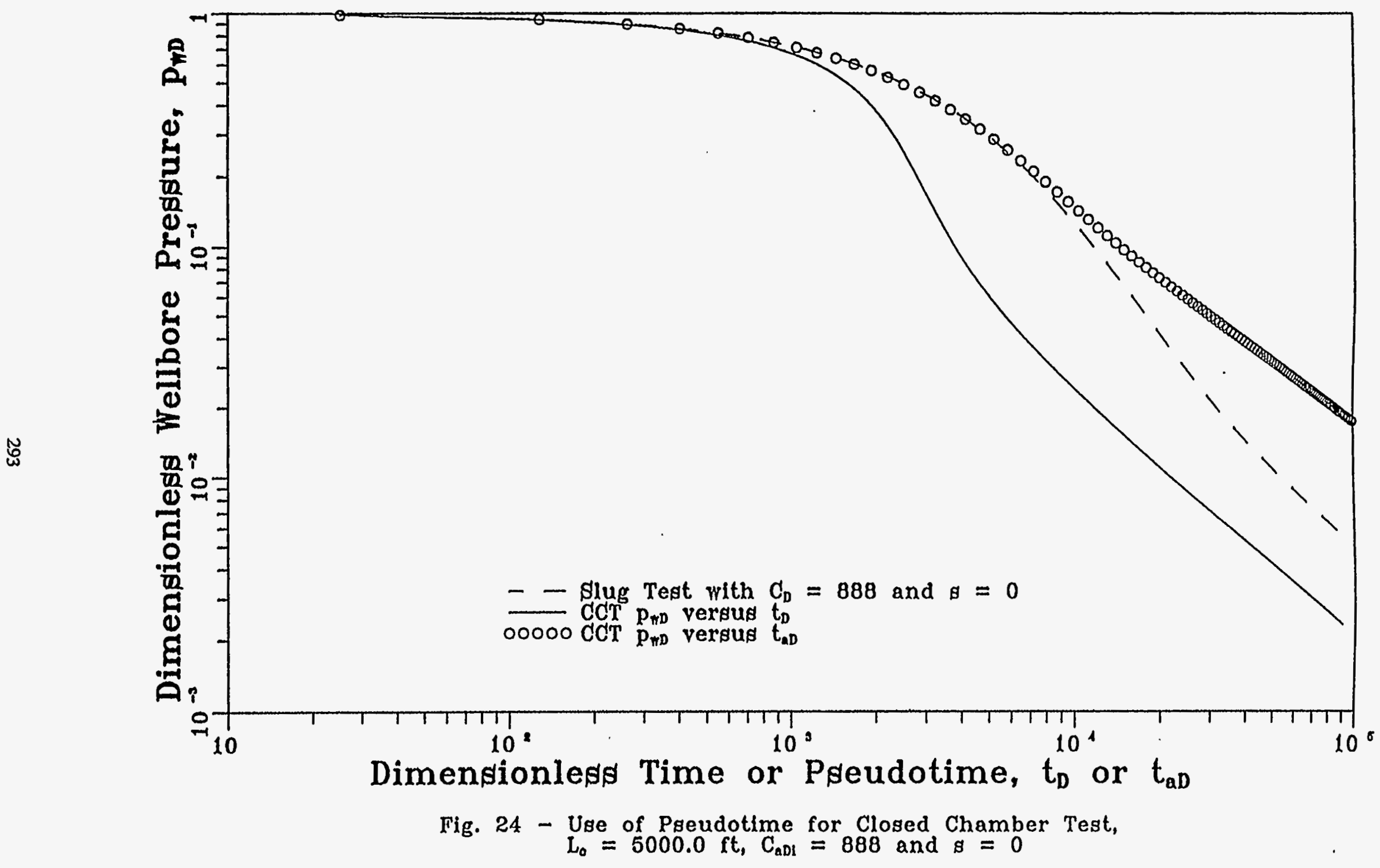




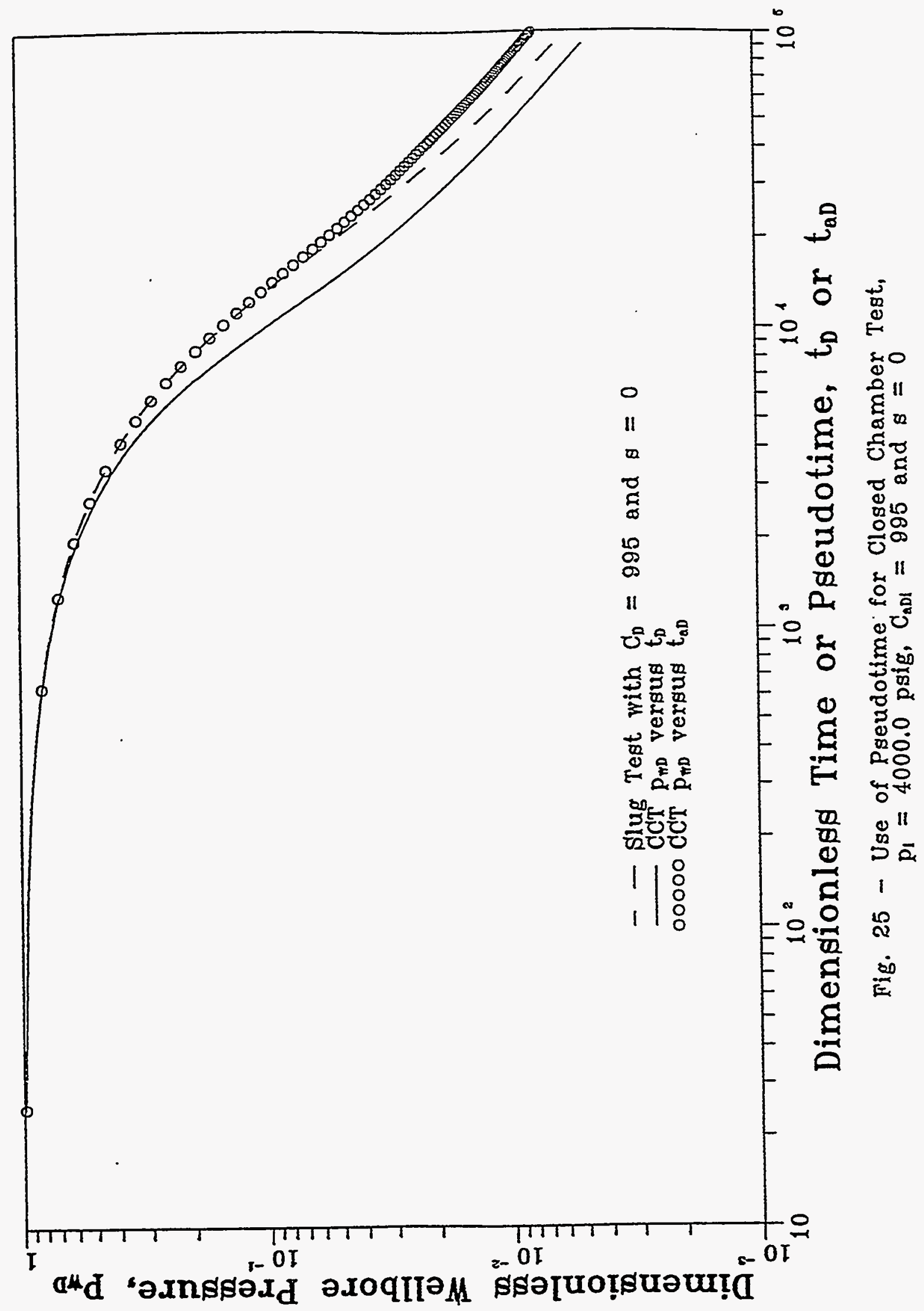




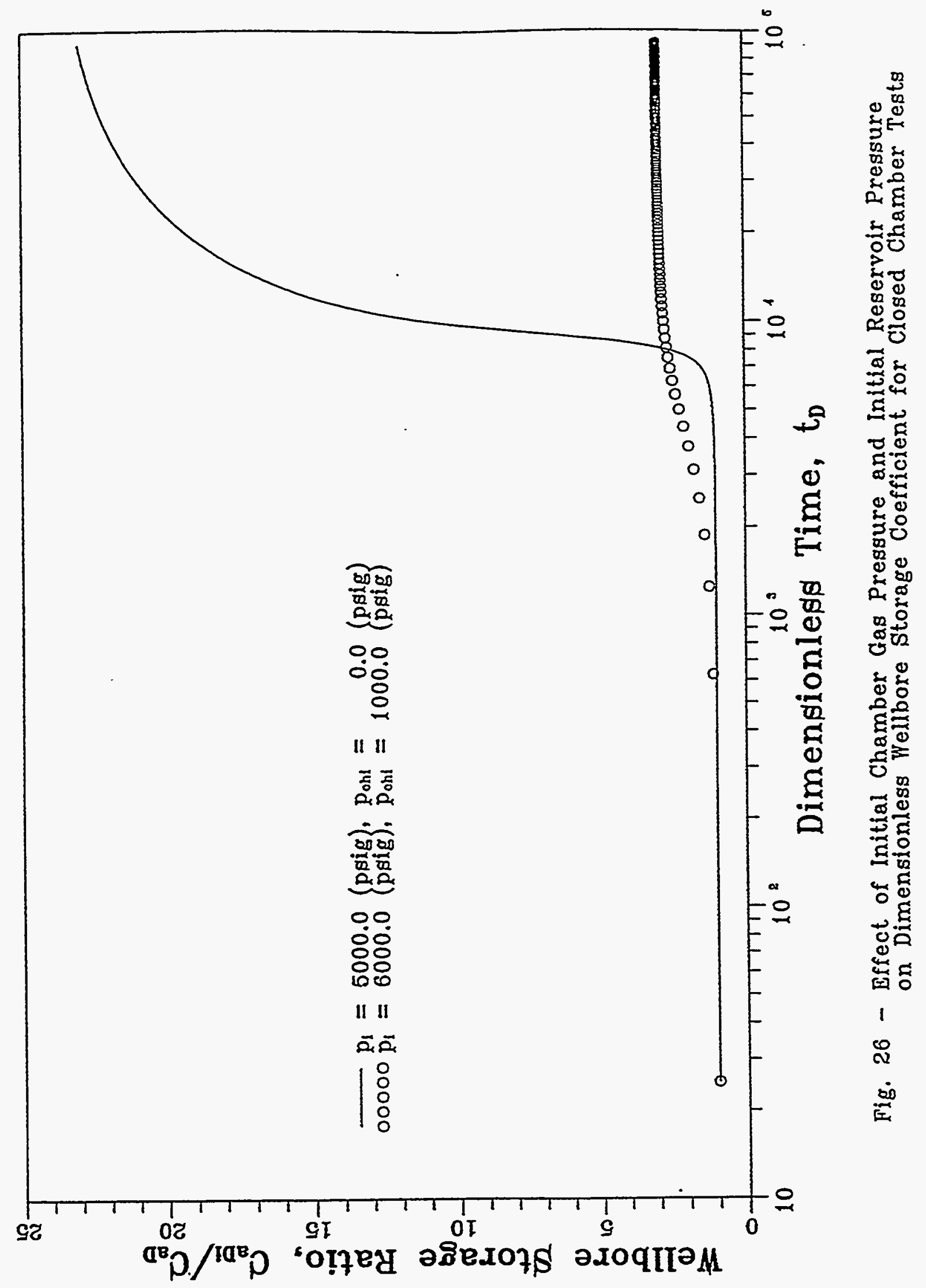




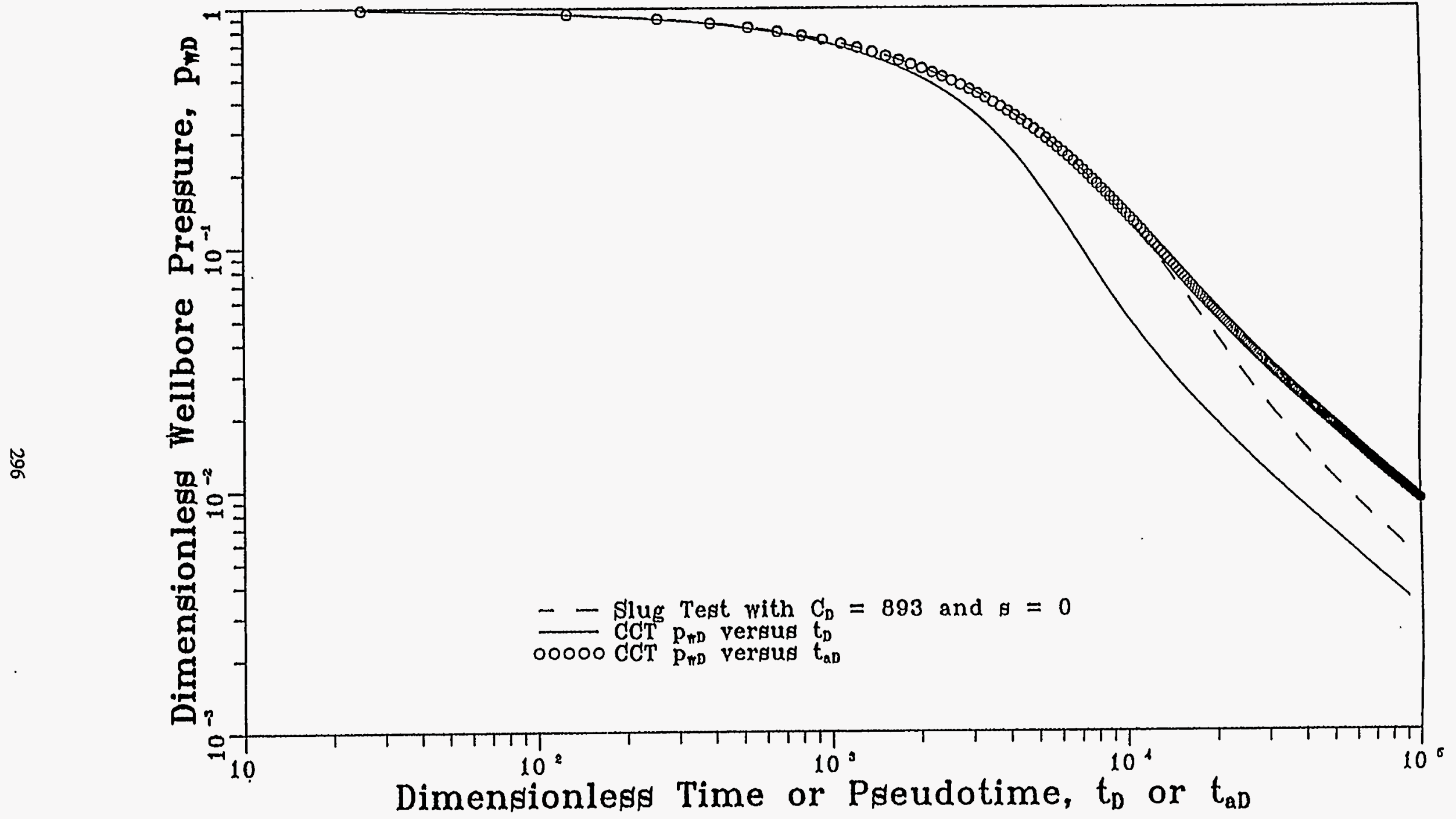
Fig. 27 - Use of Pseudotime for Closed Chamber Test, $p_{1}=6000.0$ psig, 


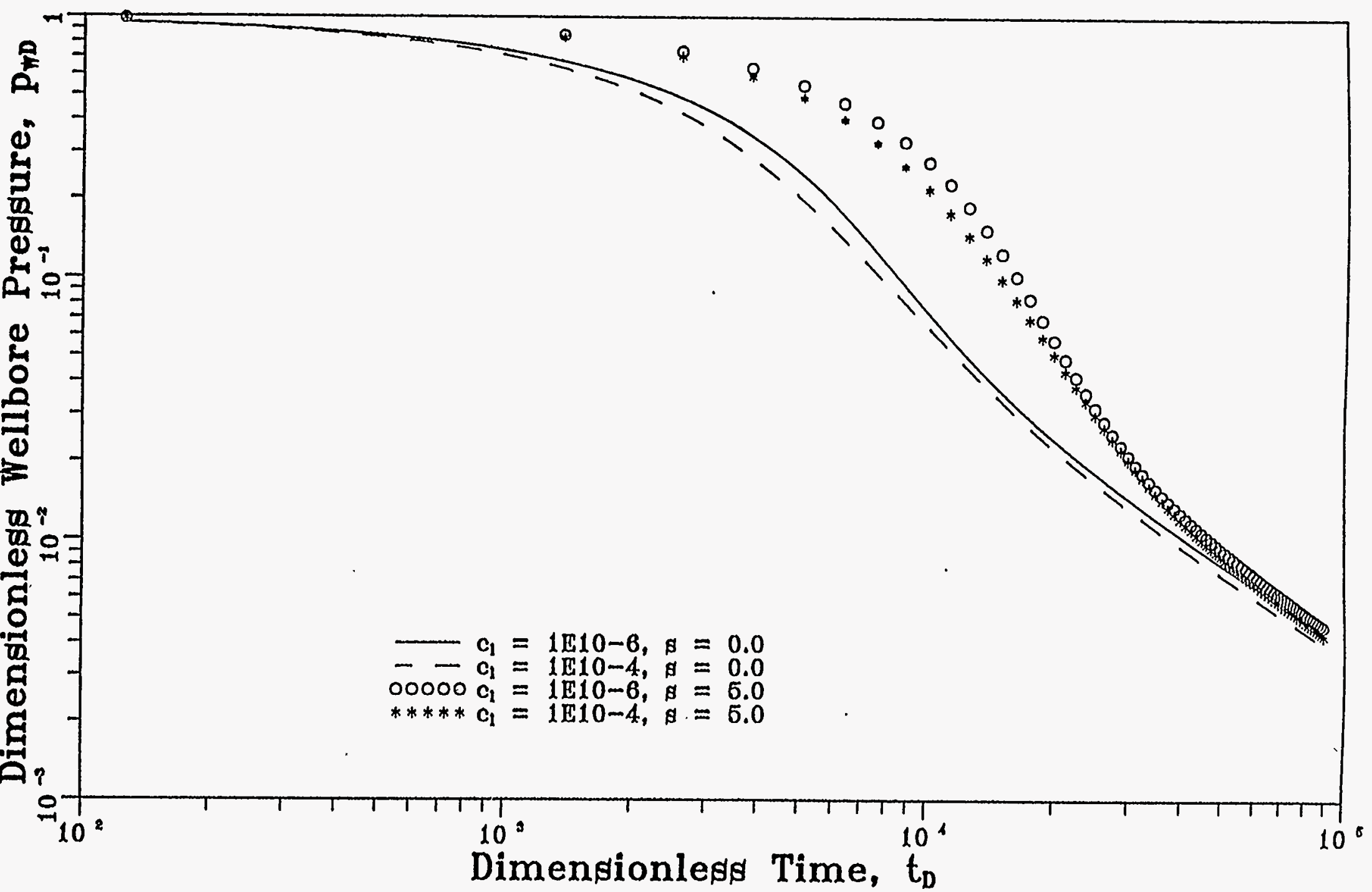

Fig. 28 - Effect of Liquid Compressibility on Closed Chamber Test $\left(L_{l}=0 \mathrm{ft}\right)$ 


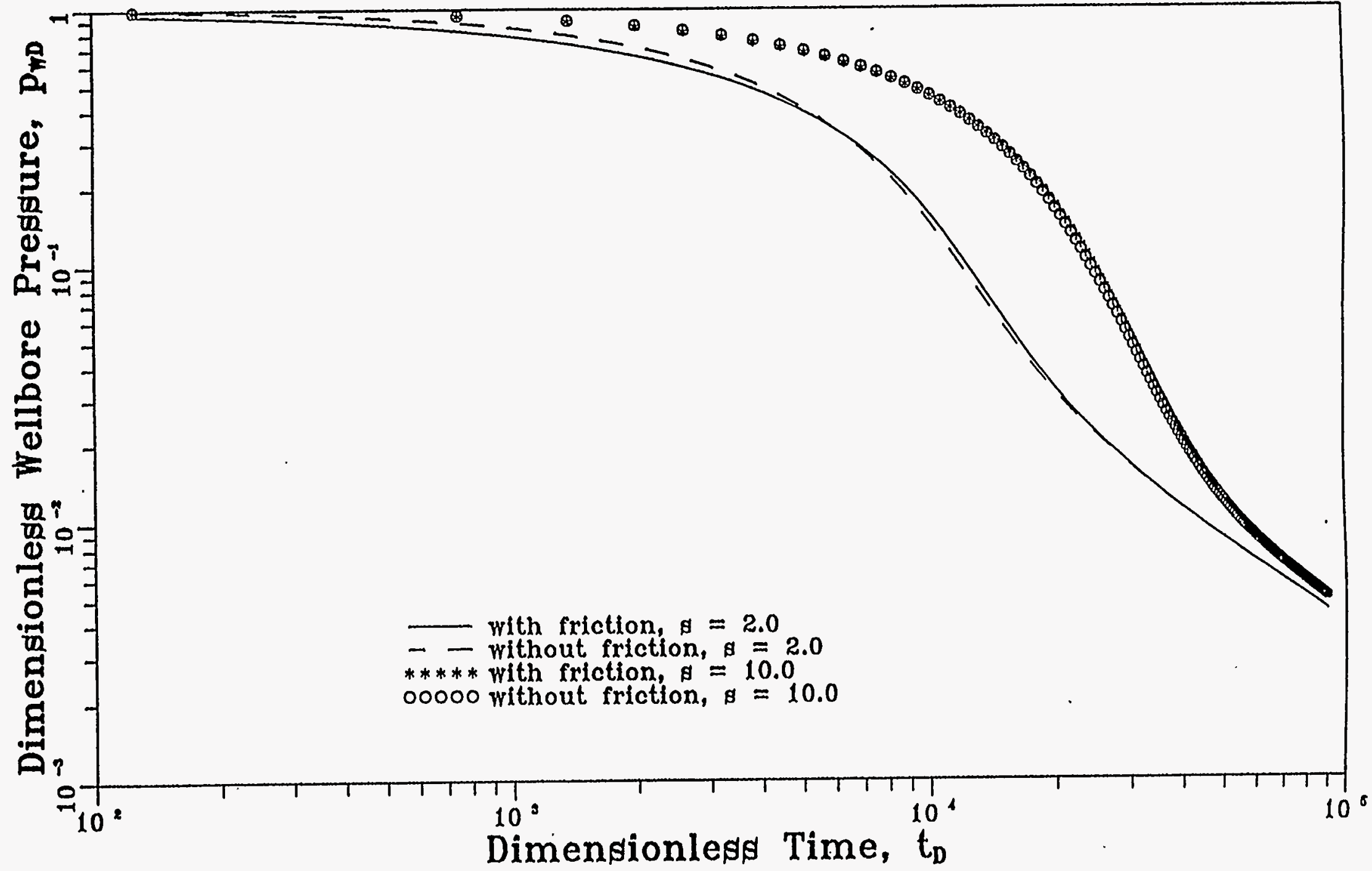

Fig. 29 - Effect of Wellbore Friction on Closed Chamber Test $\left(L_{1}=0 \mathrm{ft}\right)$ 


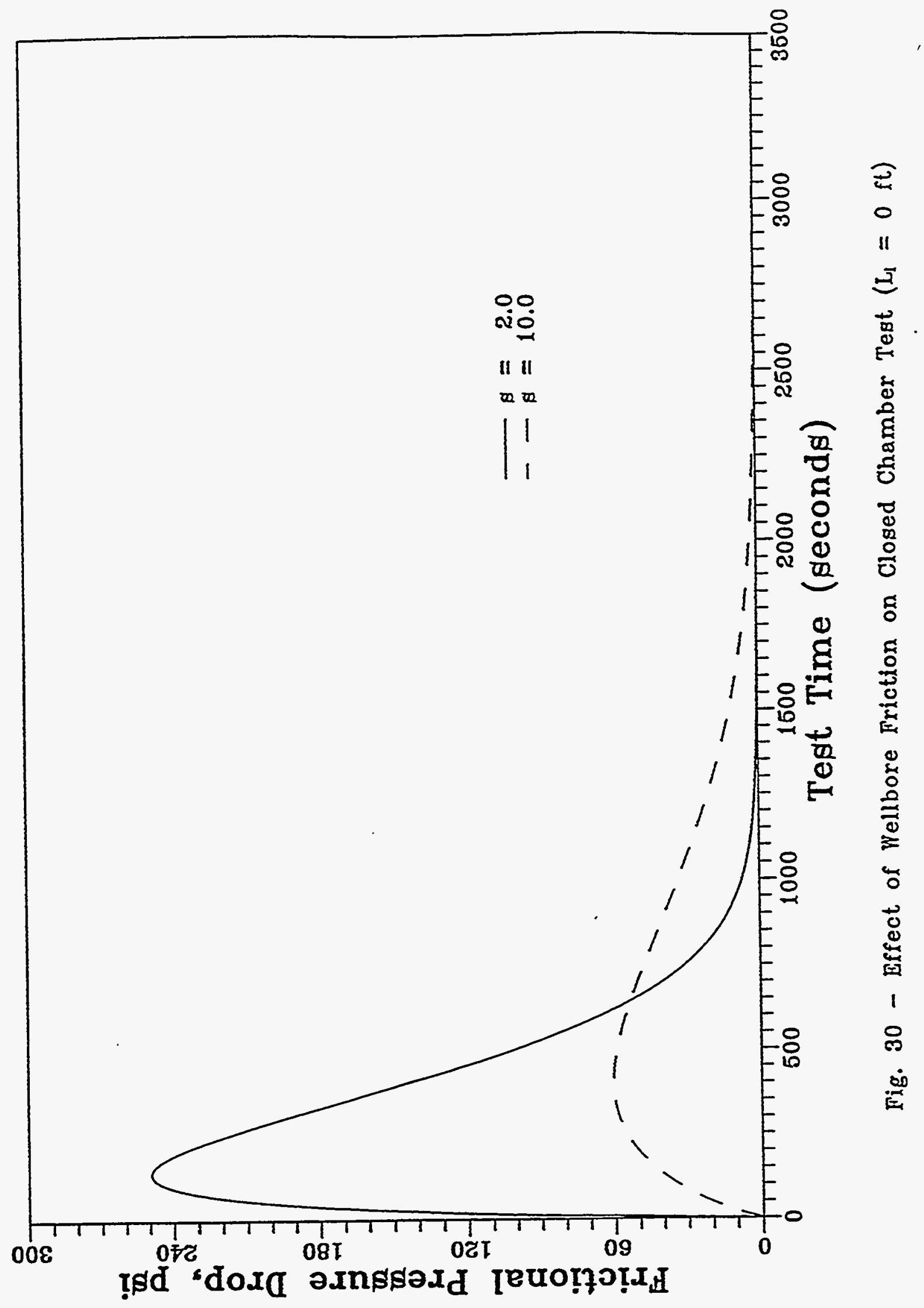




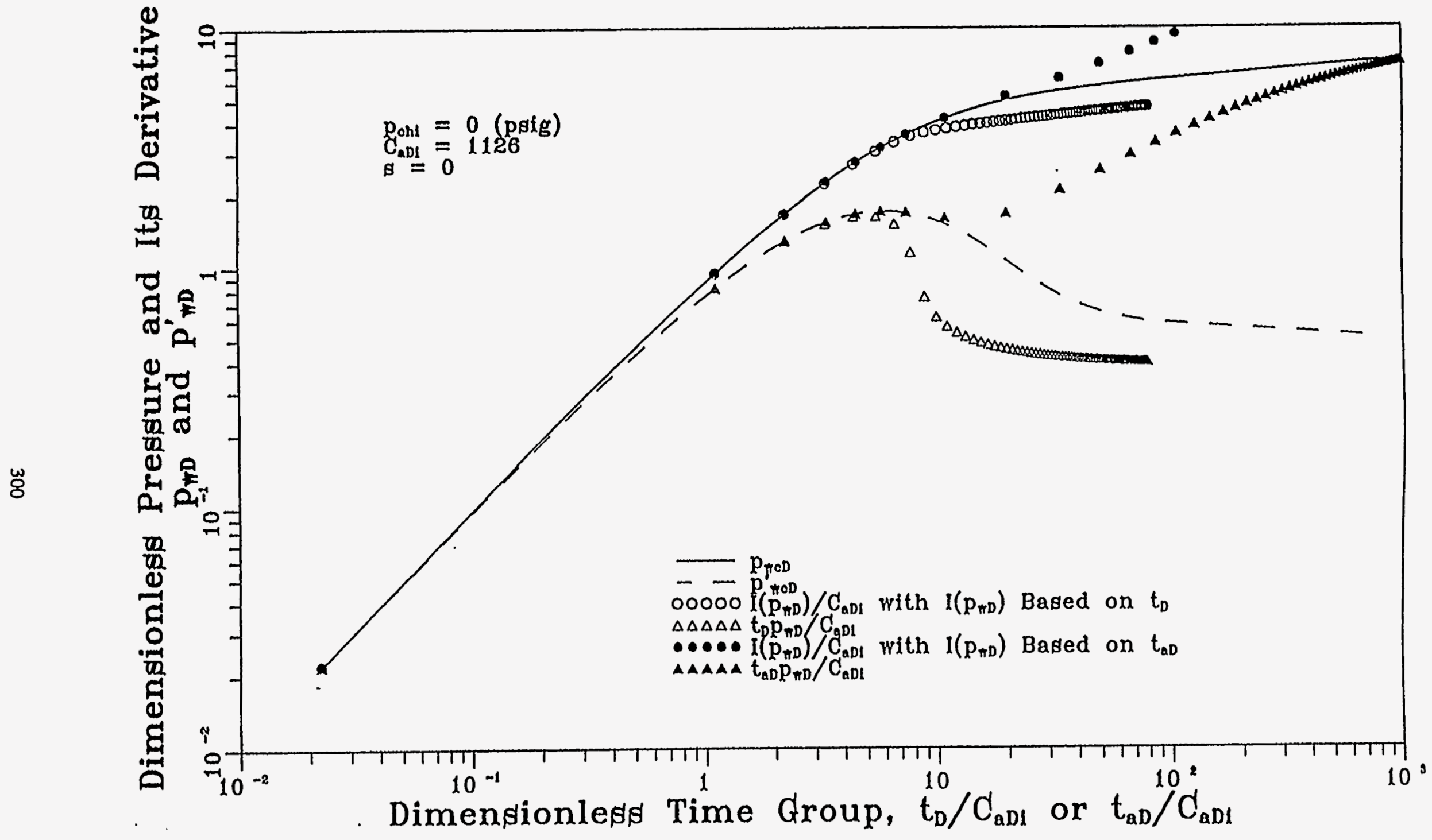

Fig. 31 - Comparison of Converted Closed Chamber Test Response with Wellbore Storage and Skin Constant Surface Rate Solution 


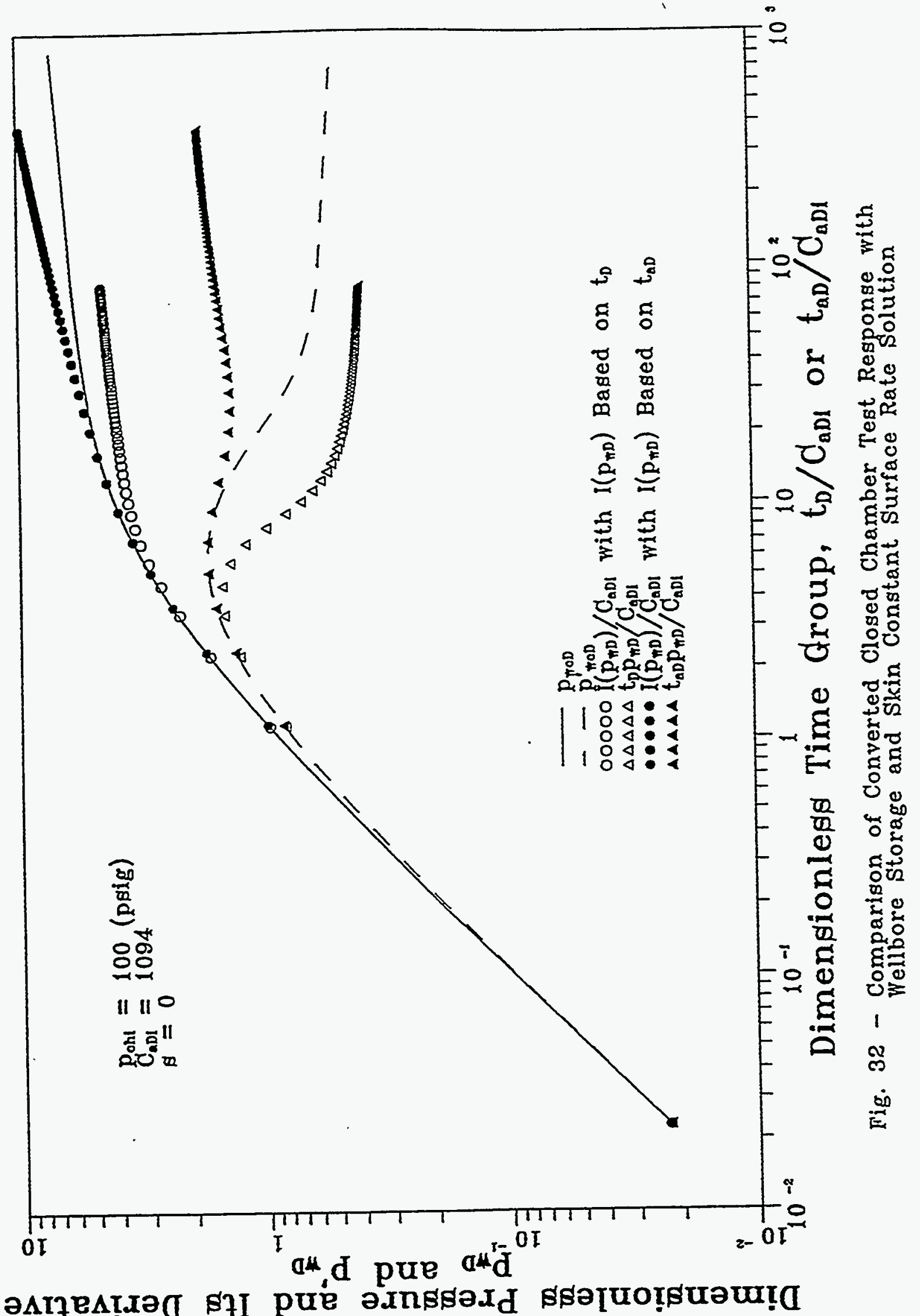




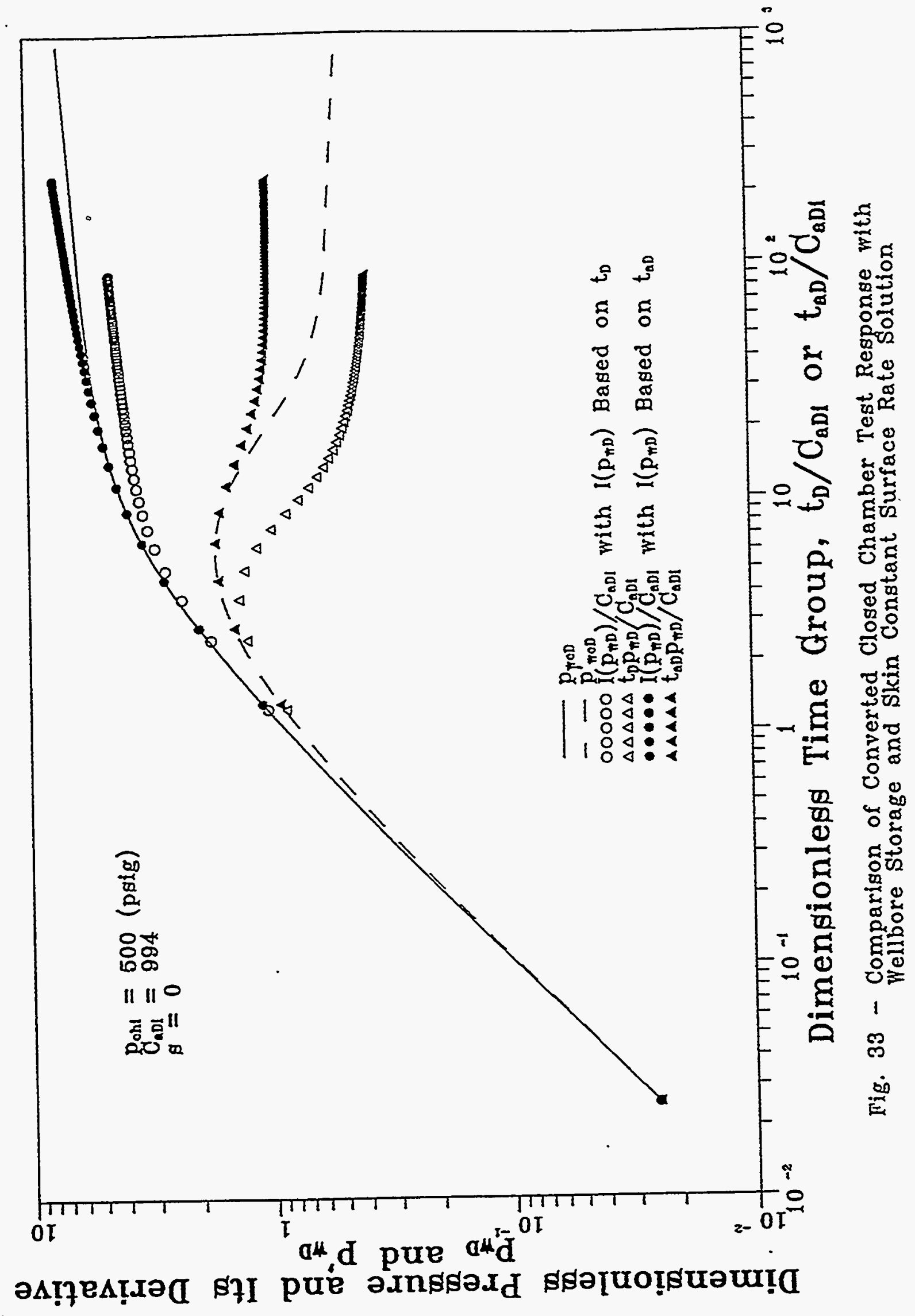




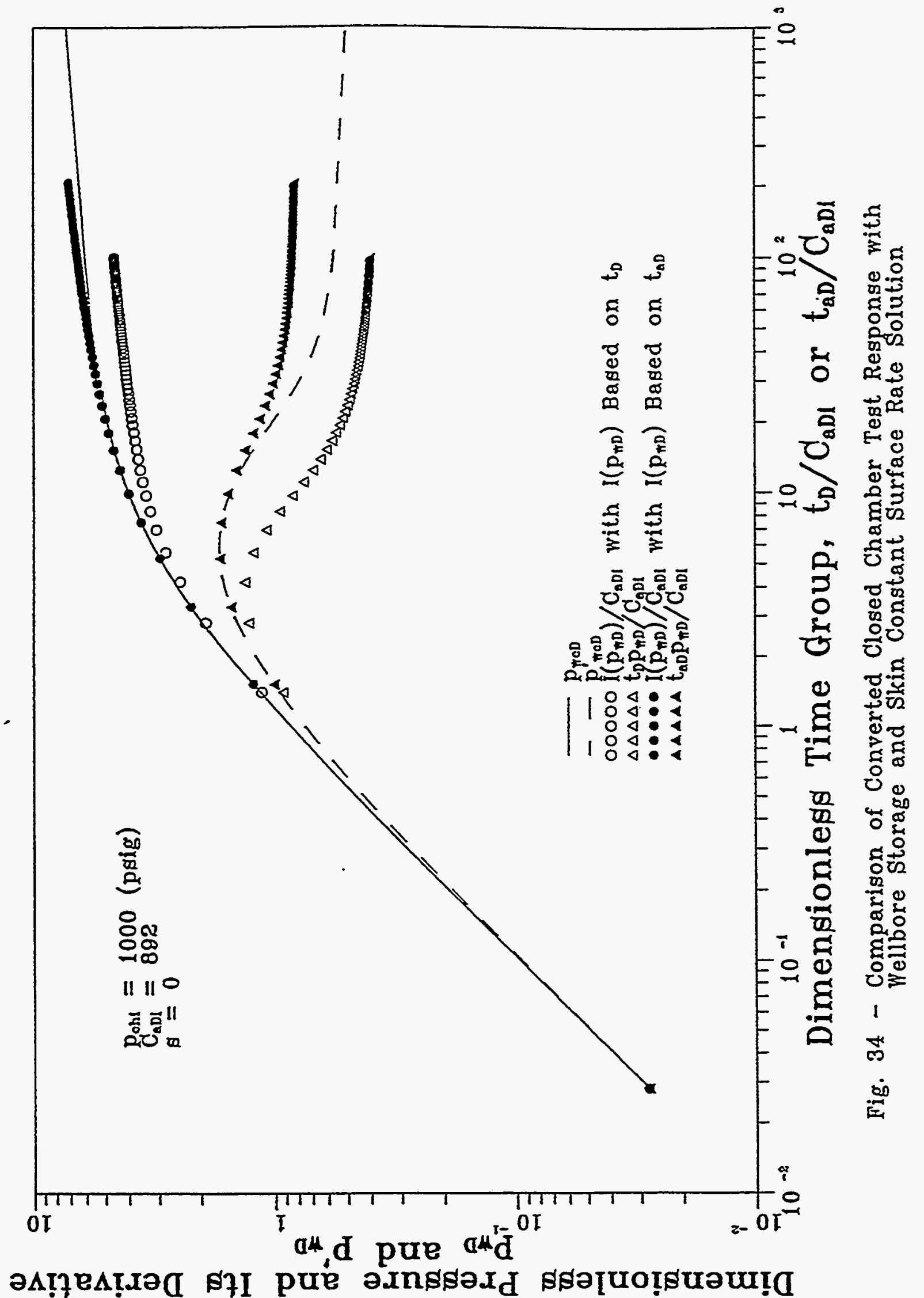




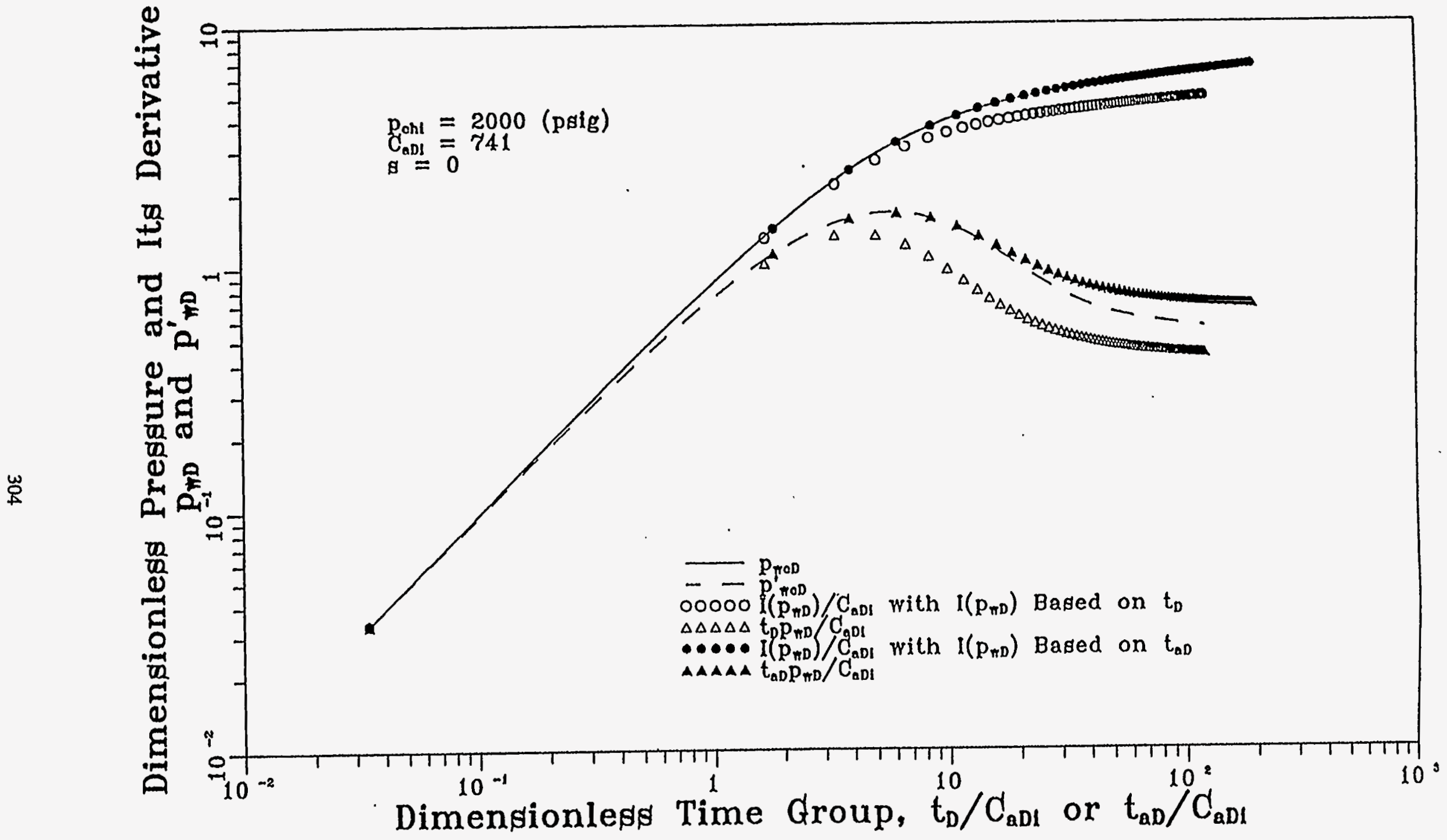

Fig. 35 - Comparison of Converted Closed Chamber Test Response with Wellbore Storage and Skin Constant Surface Rate Solution 


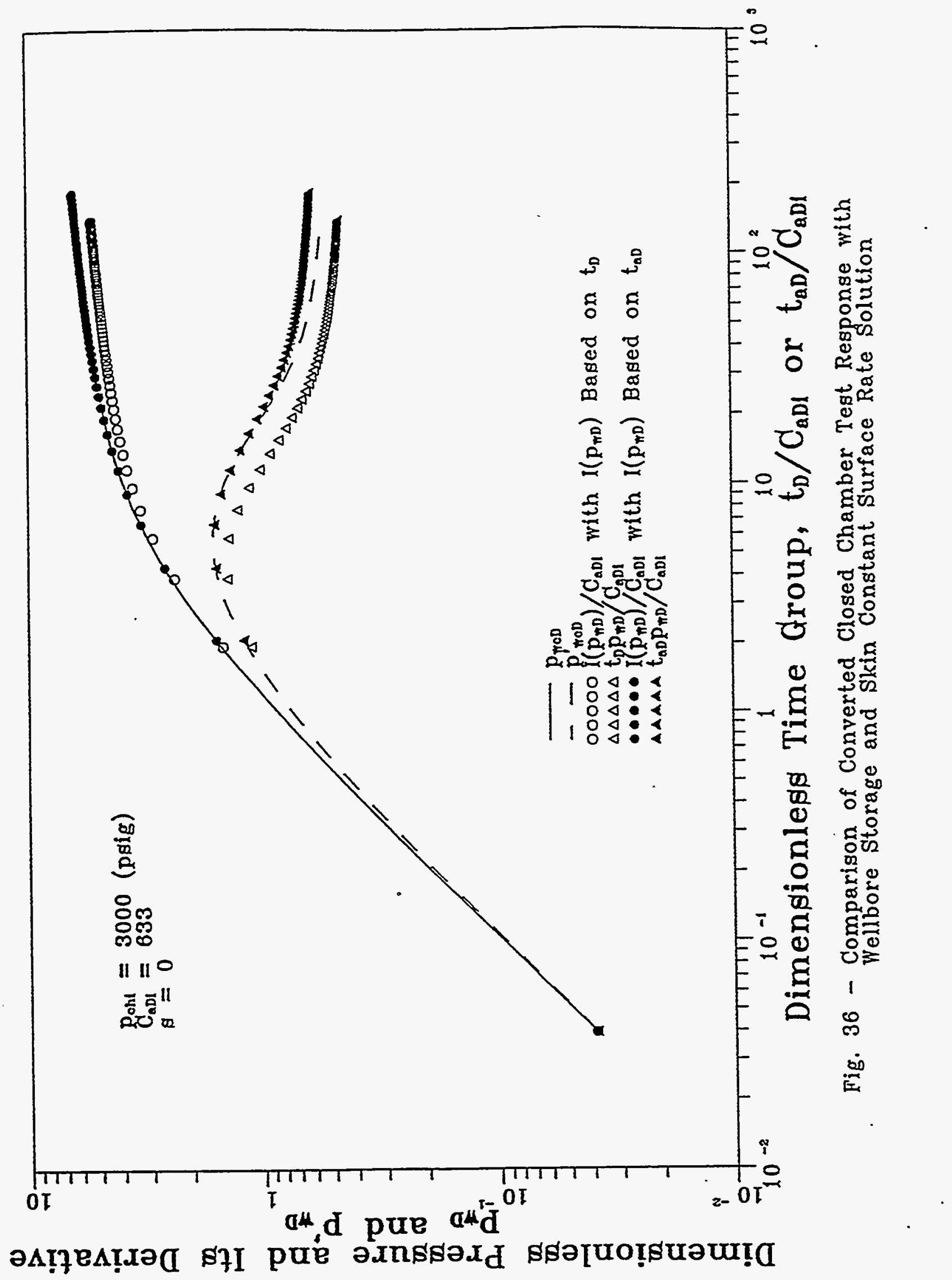




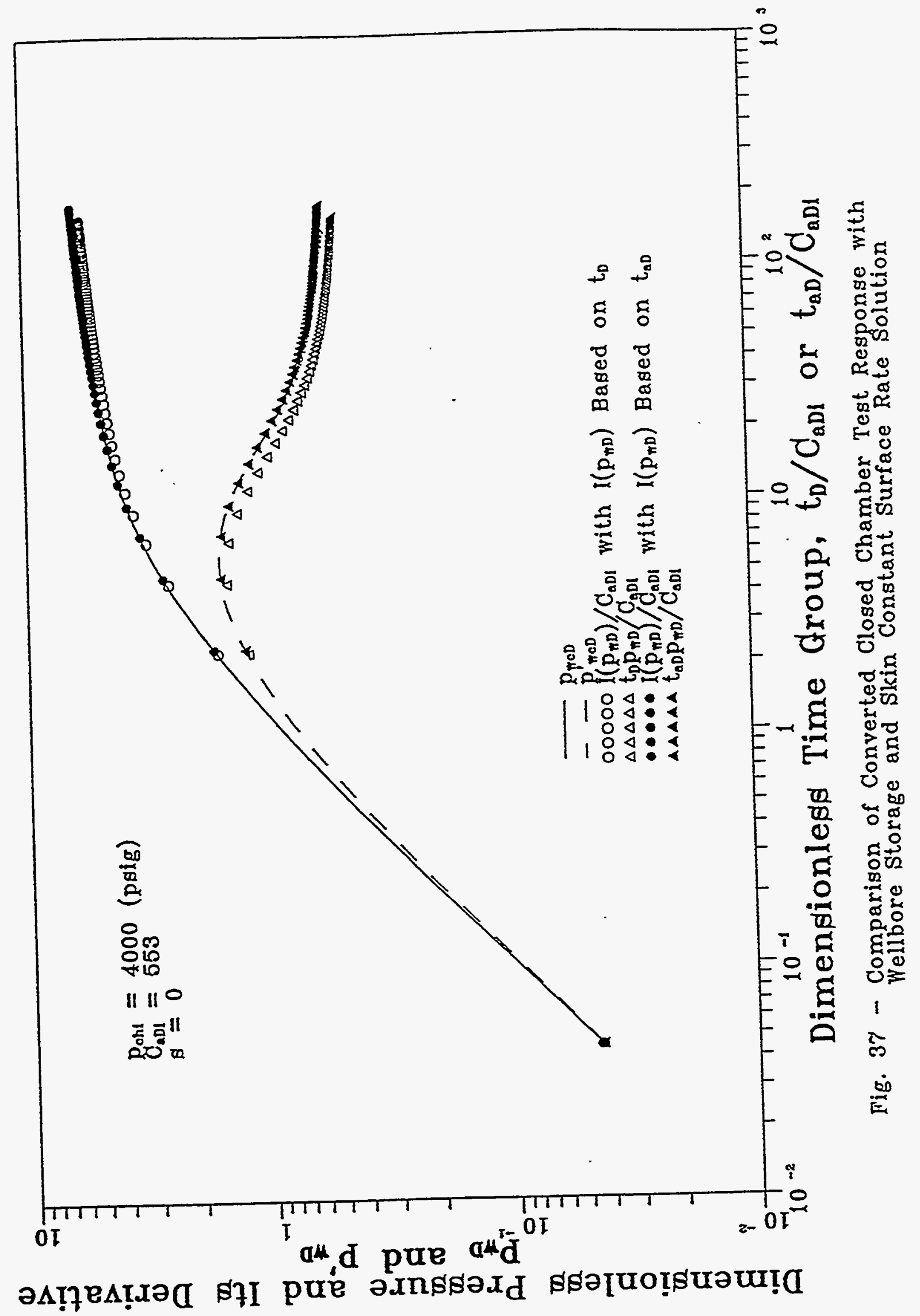




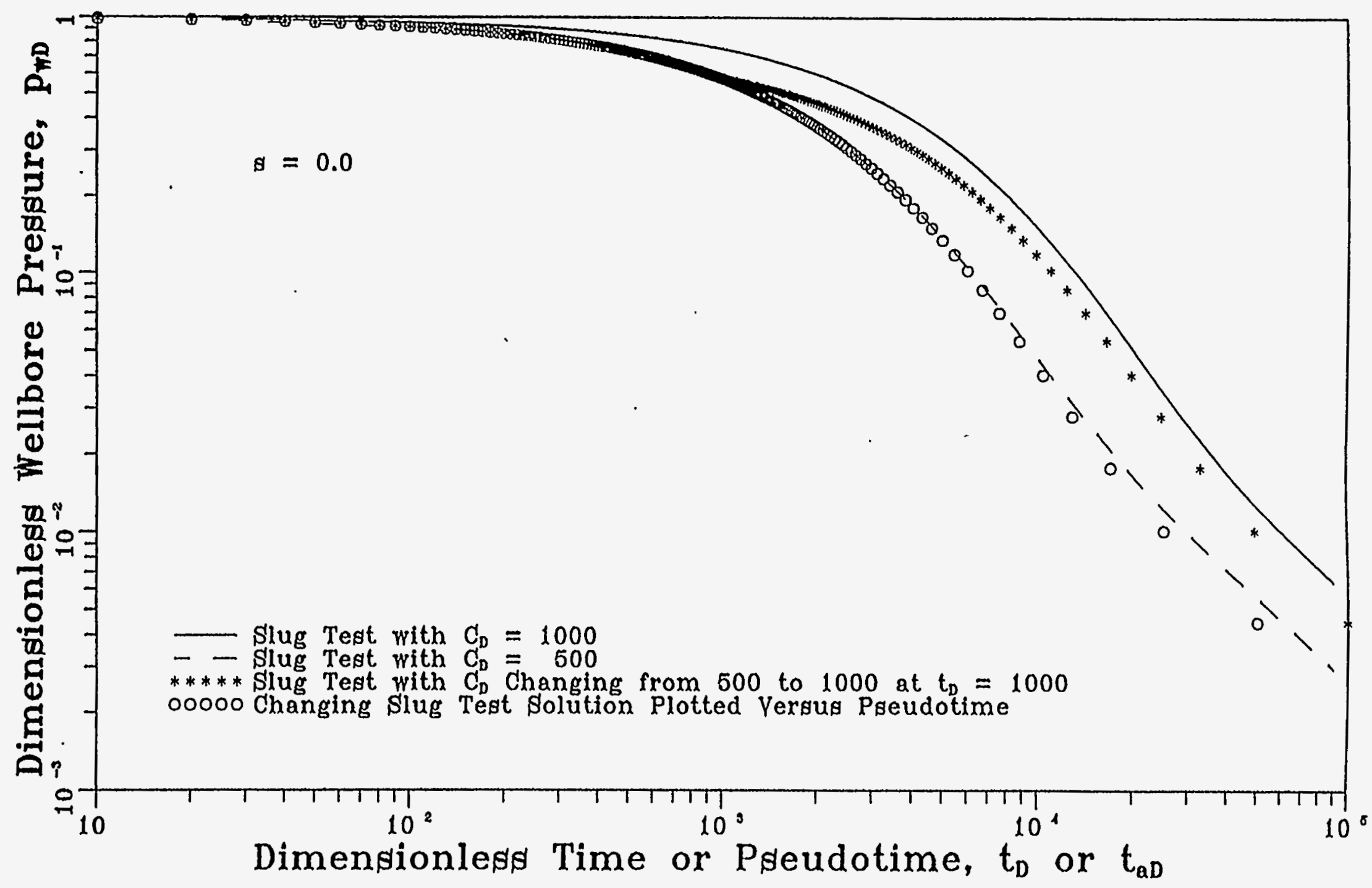

Fig. 38 - Use of Pseudotime for Slug Test with Step Change in Wellbore Storage 


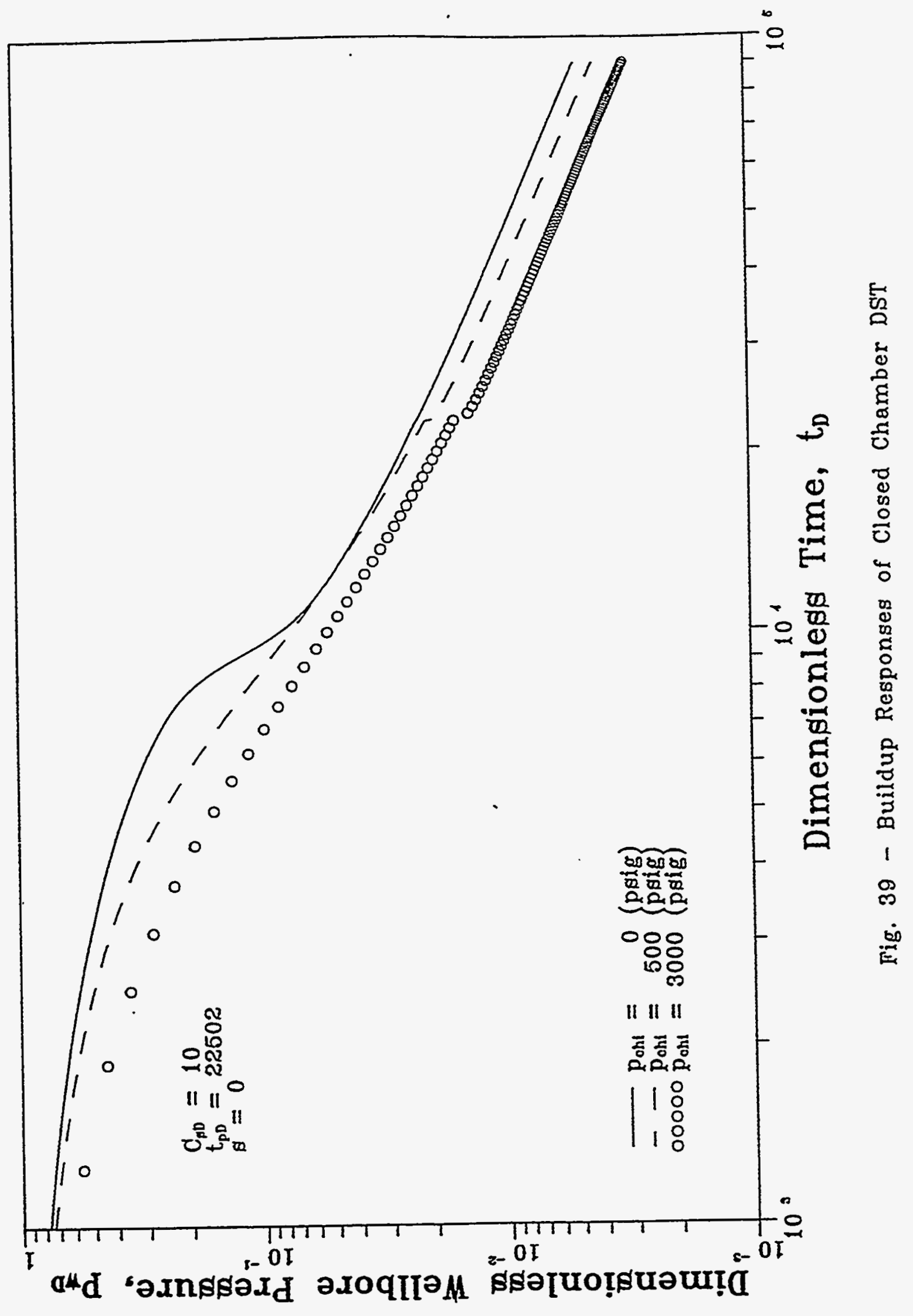




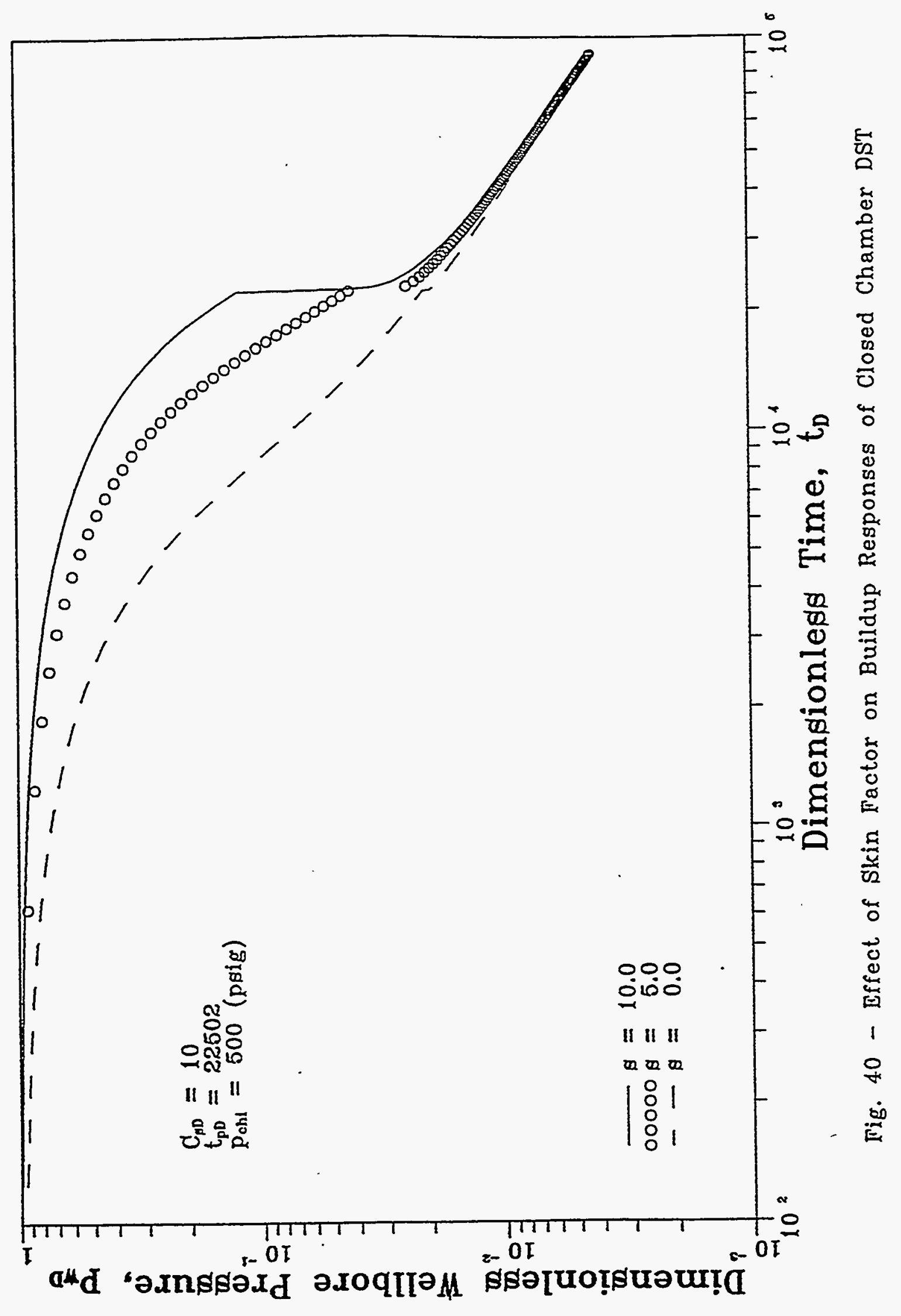




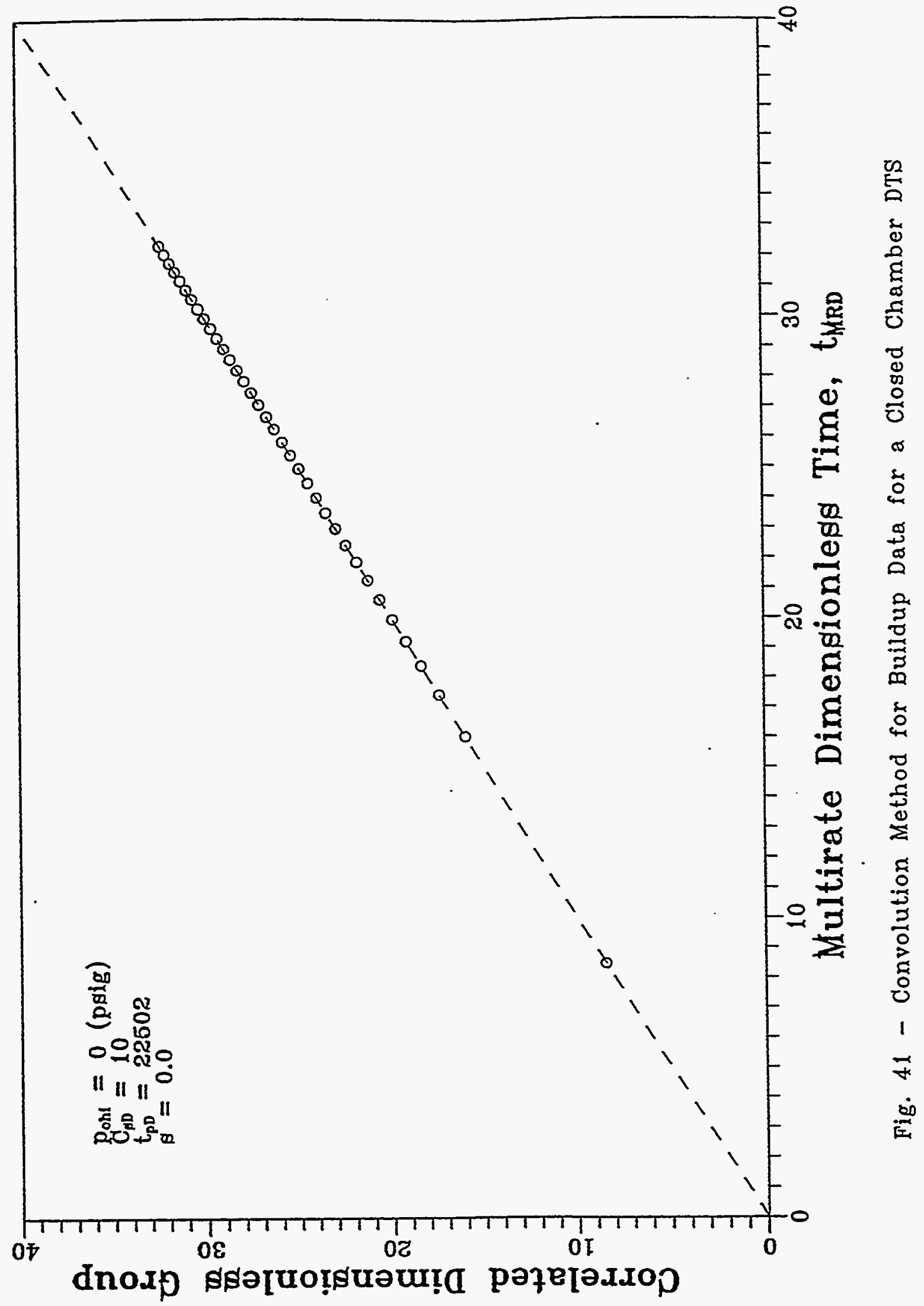




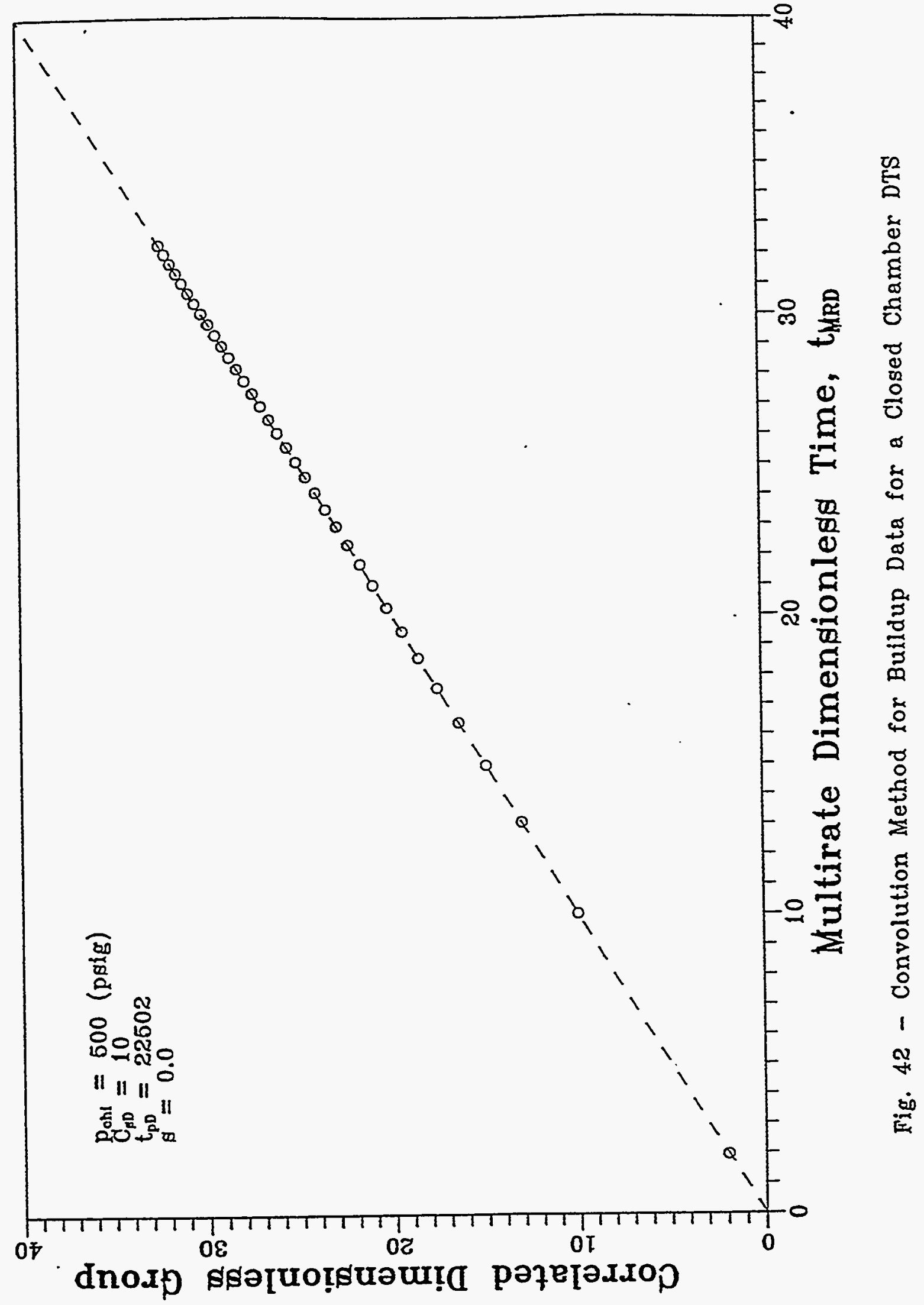




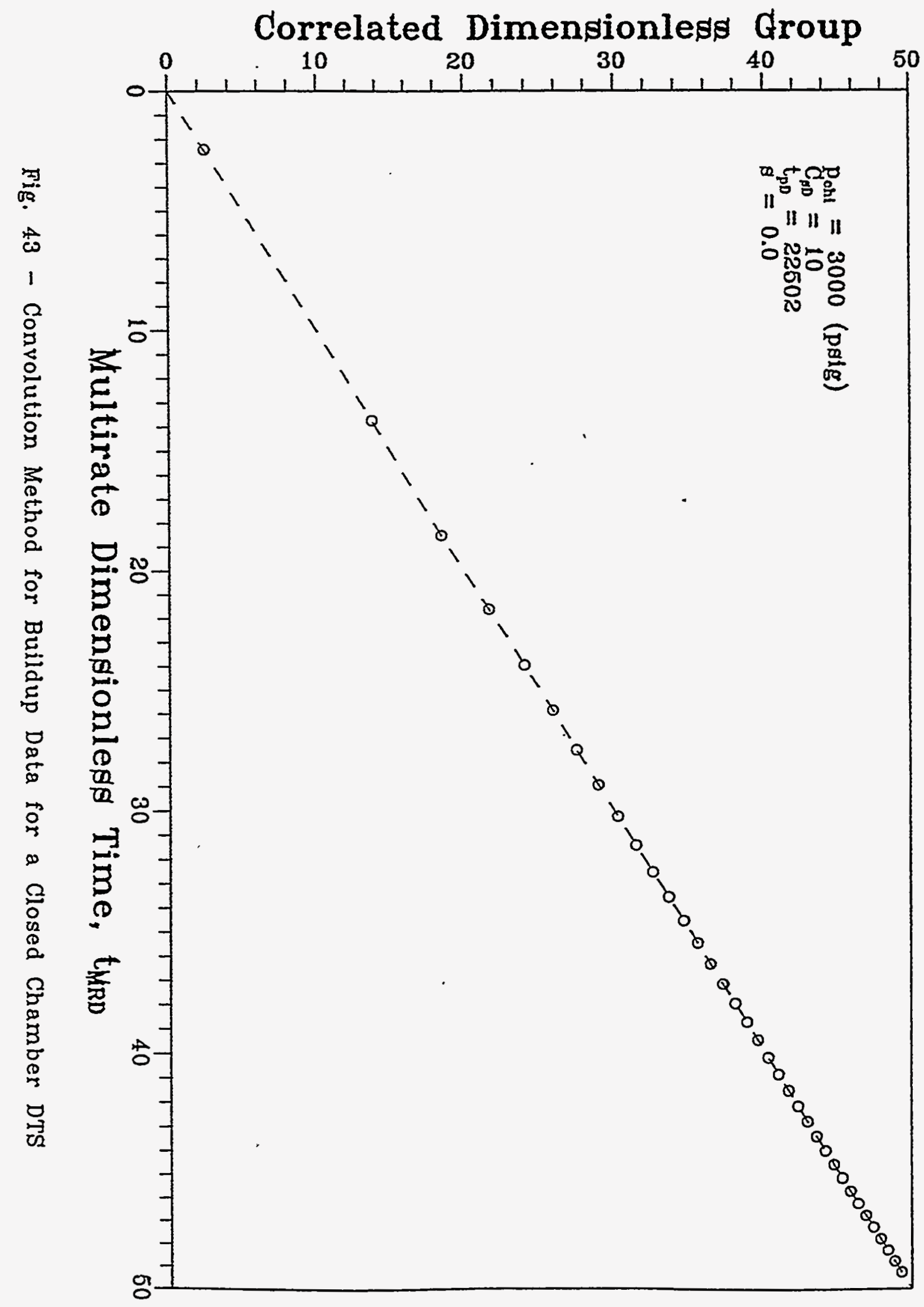




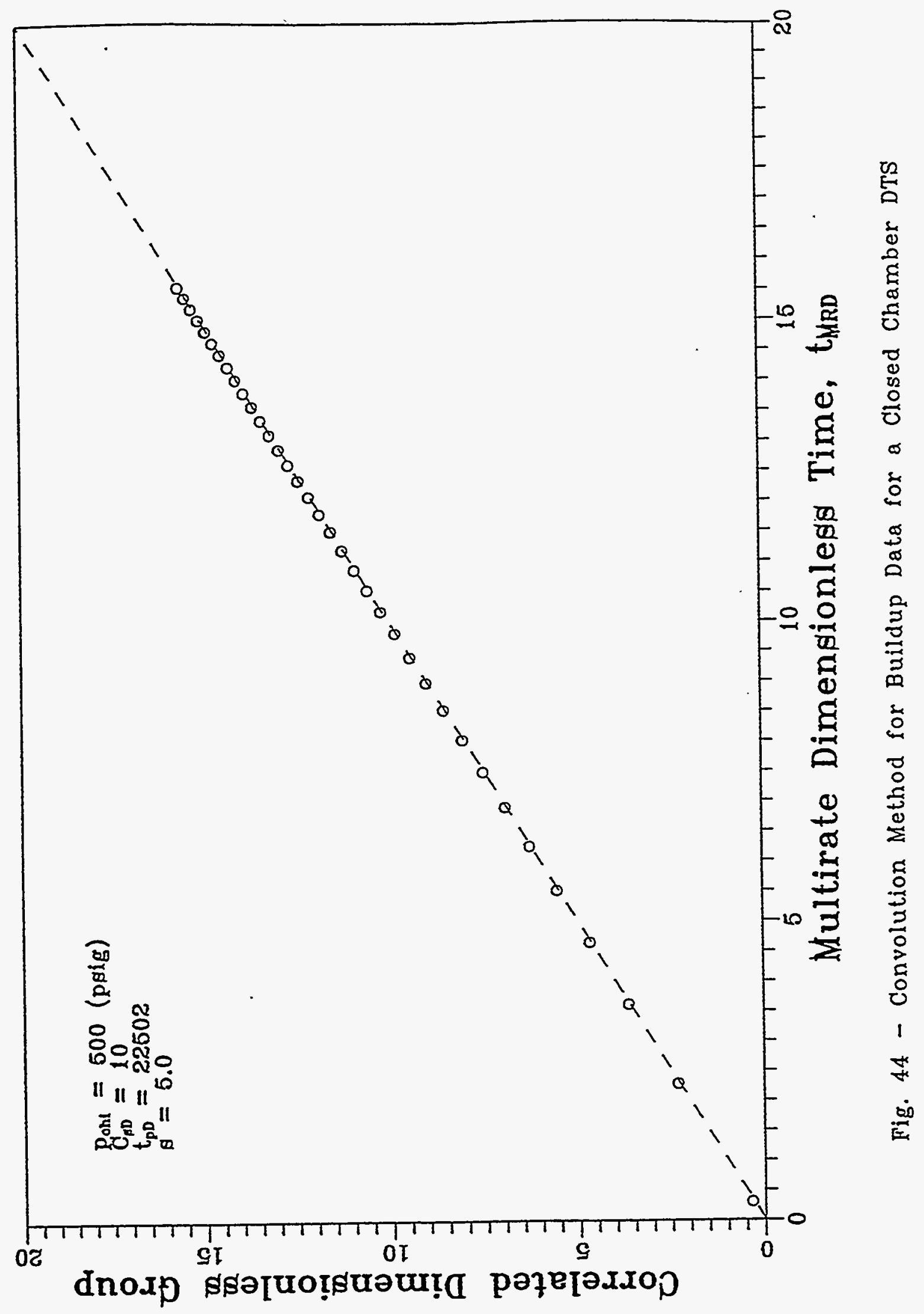




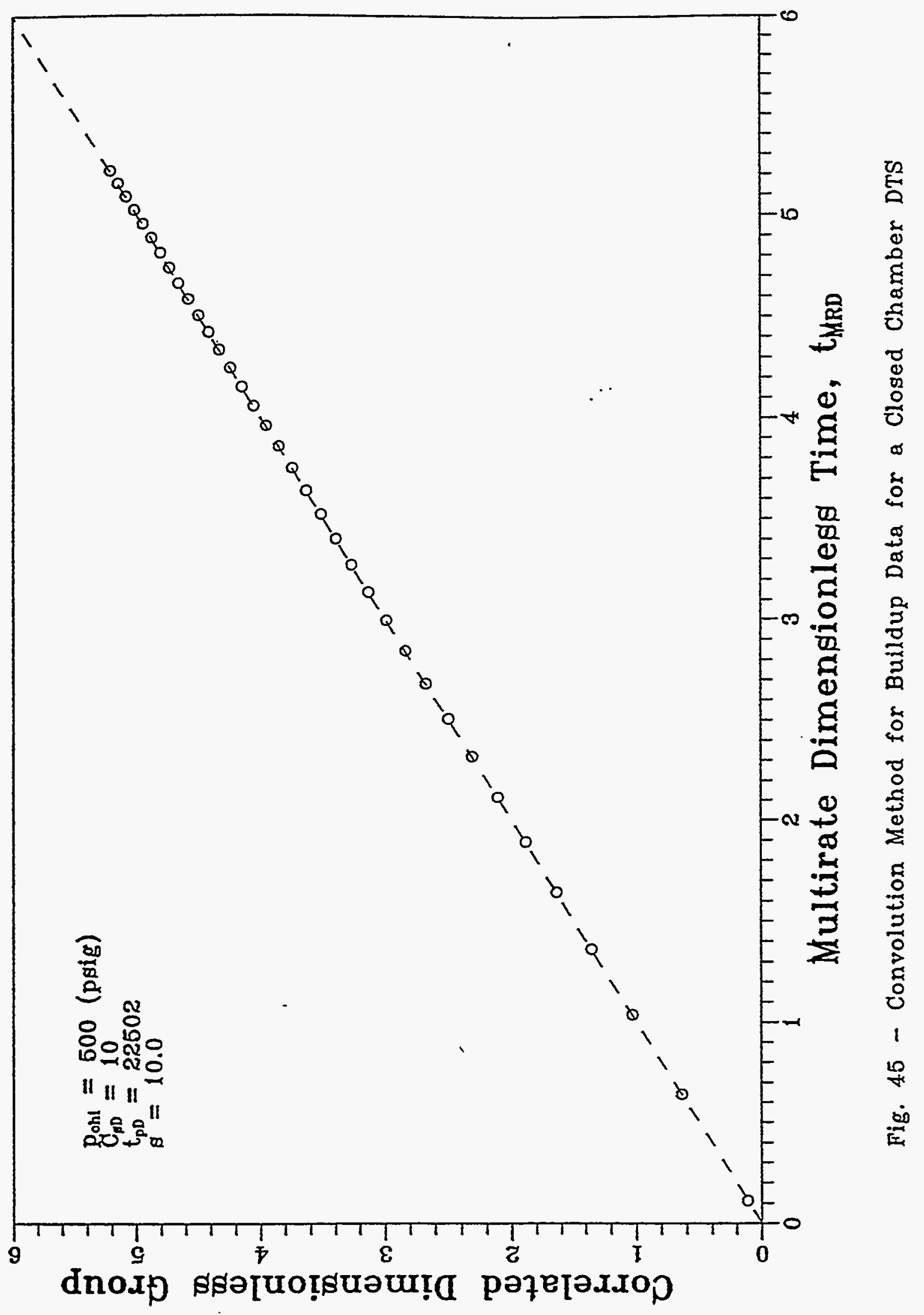




\title{
Section I
}

\section{Report IV}

\section{Results for Closed Chamber Tests Part II: Gas Wells}

\author{
J. J. Xiao and A. C. Reynolds
}

SUMMARY

This report presents methods for the analysis of gas well closed chamber tests. It is shown that, in most cases, pseudopressure and pseudotime can be used to correlate gas-well closed chamber test results with the analogous liquid slug test solutions. This allows data from gas well closed chamber tests to be analyzed using slug test analysis procedures that assume single phase flow of a slightly compressible liquid of constant compressibility and viscosity within the reservoir. Multirate analysis techniques were found to be applicable to gas well closed chamber tests in terms of pseudopressure and time, not pseudotime.

\section{INTRODUCTION}

In a companion report, we presented new techniques for the analysis of closed chamber tests (CCT) in reservoirs containing a single-phase slightly compressible fluid of constant compressibility and viscosity. It was demonstrated that a new form of pseudotime can be used to correlate closed chamber test results with slug test solutions, and thus, data from closed chamber tests can be analyzed using slug test type curves and, after simple data transformation, the wellbore storage and skin pressure and pressure derivative type curves. Here, we extend the previous work to gas well closed chamber tests.

Drillstem tests are commonly used in the petroleum industry to sample formation fluids, determine initial or average reservoir pressure, deduce formation flow capacity and assess near wellbore formation damage. Closed chamber drillstem tests were introduced to the petroleum industry by Alexander ${ }^{1}$ at about the same time as conventional drillstem tests. Closed chamber drillstem tests are designed to reduce operational and safety problems associated with conventional drillstem tests. In addition, as reported by Reid $^{2}$, for low permeability gas reservoirs, closed chamber drillstem tests are more suitable than conventional drillstem tests.

Agarwal ${ }^{3}$ introduced pseudotime to analyze pressure buildup data from vertically fractured wells in tight gas reservoirs with finite conductivity fractured well 
type curves. Lee and Holditch ${ }^{4}$ later showed that the improvement in type curve matching obtained by using pseudotime comes from the fact that pseudotime can effectively linearize the inner boundary condition of the problem. They used the buildup period of a gas well closed chamber drillstem test as a field example to illustrate that pseudotime is necessary for type curve matching to be accurate.

In this study, we focus on the flowing period of closed chamber drillstem tests. We use a one-dimensional numerical reservoir simulator developed previously to generate synthetic data for a gas well closed chamber test (CCT). With simulator results, it is demonstrated that pseudopressure and pseudotime can be used to correlate data from gas well closed chamber tests with liquid slug test solutions. This correlation is usually valid for a sufficient period of time to render analysis tractable and, therefore, slug test type curves can be used for gas well closed chamber test data analysis. As shown by Peres et al. ${ }^{5}$, data can also be analyzed by using wellbore storage and skin pressure and pressure derivative type curves.

We also conducted parametric studies to investigate the effect of various parameters on the correlation. Our results show that the correlation improves as the skin factor increases, initial wellbore pressure increases, initial reservoir pressure decreases and chamber volume increases.

A gas well closed chamber test problem is a variable rate problem. We show that multirate analysis techniques can be applied to gas well closed chamber tests by analyzing data in terms of pseudopressure and time (not pseudotime).

\section{MATHEMATICAL DEVELOPMENT}

We consider a gas well closed chamber test conducted in a fully penetrating well located in an infinite, homogeneous and isotropic cylindrical reservoir of uniform thickness. Initially, the pressure is constant throughout the reservoir and equal to $p_{i}$ whereas, at the wellbore, the pressure is instantaneously set to $p_{o}$. We neglect temperature effects. In addition, it is assumed that in the tests, friction and inertial effects in the wellbore can be neglected, and critical flow is not present. We also assume that rock compressibility is negligible compared to gas compressibility.

Under the stated assumptions, the initial boundary value problem (IBVP) for gas well closed chamber tests can be stated as

$$
\begin{gathered}
1.127 \times 10^{-3} \frac{1}{r} \frac{\partial}{\partial r}\left[\frac{p}{\mu_{g} z} r \frac{\partial p}{\partial r}\right]=\frac{1}{5.615} \frac{\phi c_{g}}{k} \frac{p}{z} \frac{\partial p}{\partial t}, \\
\lim _{r \rightarrow \infty} p(r, t)=p_{i}, \\
p(r, 0)=p_{i}, \quad r>r_{w}, \\
p_{w f}(0)=p_{o}
\end{gathered}
$$


and

$$
1.127 \times 10^{-3}(2 \pi) \frac{k}{\mu_{g}} h\left(r \frac{\partial p}{\partial r}\right)_{r=r_{w}}=C \frac{\mu_{g i} c_{g w}}{\left(\mu_{g} c_{g}\right)_{0}} \frac{\partial p_{w f}}{\partial t},
$$

where $\mu_{g i}$ is the gas viscosity at $p_{i}, c_{g w}$ is the gas compressibility at $p_{w f}$, and $\left(\mu_{g} c_{g}\right)_{0}$ is the gas viscosity and isothermal compressibility product at $p_{o}$. In Eq. $1.5, C$ is the wellbore storage coefficient, defined as

$$
C=V_{w} c_{g o} \frac{\mu_{g \circ}}{\mu_{g i}}
$$

where, $V_{w}$ is the wellbore volume in $\mathrm{bbl}$ and $\mu_{g o}$ is the gas viscosity at $p_{o}$. Note that the right hand side of Eq. $1.5 \mathrm{can}$ actually be reduced to $V_{w} c_{g w}\left(\partial p_{w f} / \partial t\right)$, but, as seen later, it is convenient to use the form given in Eq. 1.5 and the definition of $C$ given in Eq. 2.

The real gas pseudopressure defined by Al-Hussainy et al. ${ }^{6}$ is normalized by choosing variables at the initial reservoir pressure, i.e., we define real gas pseudopressure as

$$
m(p)=\int_{p_{r}}^{p} \frac{\left(2 p^{\prime} / \mu_{g} z\right)}{\left(2 p / \mu_{g} z\right)_{i}} d p
$$

where $p_{r}$ is a base pressure or reference pressure. In terms of $m(p)$, the IBVP given by Eqs. 1.1 - 1.5 becomes

$$
\begin{gathered}
1.127 \times 10^{-3} \frac{1}{r} \frac{\partial}{\partial r}\left[r \frac{\partial m}{\partial r}\right]=\frac{1}{5.615} \frac{\phi c_{g} \mu_{g}}{k} \frac{\partial m}{\partial t} \\
\lim _{r \rightarrow \infty} m(r, t)=m\left(p_{i}\right) \\
m(r, 0)=m\left(p_{i}\right), \quad r>r_{w} \\
m\left(p_{w f}\right)=m\left(p_{o}\right), \quad t=0
\end{gathered}
$$

and

$$
1.127 \times 10^{-3}(2 \pi) \frac{k}{\mu_{g}} h\left(r \frac{\partial m}{\partial r}\right)_{r=r_{w}}=C \frac{\mu_{g i} c_{g w}}{\left(\mu_{g} c_{g}\right)_{o}} \frac{\partial m\left(p_{w f}\right)}{\partial t} .
$$

We define dimensionless variables as

$$
\begin{gathered}
m_{D}=\frac{m\left(p_{i}\right)-m(p)}{m\left(p_{i}\right)-m\left(p_{o}\right)} \\
t_{D}=\frac{0.006328 k t}{\phi\left(\mu_{g} c_{g}\right)_{i} r_{w}^{2}}
\end{gathered}
$$

where $t$ is in days,

$$
r_{D}=\frac{r}{r_{w}}
$$


and

$$
C_{D}=\frac{5.615 C}{2 \pi \phi c_{g i} h r_{w}^{2}}
$$

In Eq. $6,\left(\mu_{g} c_{g}\right)_{i}$ is the compressibility and viscosity product evaluated at $p_{i}$. The IBVP in dimensionless form is

$$
\begin{gathered}
\frac{1}{r_{D}} \frac{\partial}{\partial r_{D}}\left(r_{D} \frac{\partial m_{D}}{\partial r_{D}}\right)=\frac{\left(\mu_{g} c_{g}\right)}{\left(\mu_{g} c_{g}\right)_{i}} \frac{\partial m_{D}}{\partial t_{D}} \\
\lim _{r_{D} \rightarrow \infty} m_{D}\left(r_{D}, t_{D}\right)=0 \\
m_{D}\left(r_{D}, 0\right)=0, \quad r_{D}>1 \\
m_{w D}(0)=1
\end{gathered}
$$

and

$$
\left(r_{D} \frac{\partial m_{D}}{\partial r_{D}}\right)_{r_{D}=1}=\frac{\left(\mu_{g} c_{g}\right)_{w}}{\left(\mu_{g} c_{g}\right)_{o}} C_{D} \frac{\partial m_{w D}}{\partial t_{D}} .
$$

In Eq. 9.5, $\left(\mu_{g} c_{g}\right)_{w}$ denotes the compressibility-viscosity product evaluated at $p_{w f}$. Note that the inner boundary condition given by Eq. 9.5 is non-linear because $\left(\mu_{g} c_{g}\right)_{u}$ is a function of the wellbore pressure, $p_{w f}$. To linearize Eq. 9.5, we define dimensionless pseudotime as

$$
t_{a D}=\int_{0}^{t_{D}} \frac{\left(\mu_{g} c_{g}\right)_{o}}{\left(\mu_{g} c_{g}\right)_{w}} d \tau
$$

Then, the IBVP in terms of dimensionless normalized pseudopressure and pseudotime can be written as

$$
\begin{gathered}
\frac{1}{r_{D}} \frac{\partial}{\partial r_{D}}\left(r_{D} \frac{\partial m_{D}}{\partial r_{D}}\right)=a_{D} \frac{\partial m_{D}}{\partial t_{a D}} \\
\lim _{r_{D} \rightarrow \infty} m_{D}\left(r_{D}, t_{a D}\right)=0, \\
m_{D}\left(r_{D}, 0\right)=0, \quad r_{D}>1, \\
m_{w D}(0)=1,
\end{gathered}
$$

and

$$
\left(r_{D} \frac{\partial m_{D}}{\partial r_{D}}\right)_{r_{D}=1}=C_{D} \frac{\partial m_{w D}}{\partial t_{a D}}
$$

where in Eq. 11.1, $a_{D}$ is given by

$$
a_{D}=\frac{\left(\mu_{g} c_{g}\right)}{\left(\mu_{g} c_{g}\right)_{w}} \frac{\left(\mu_{g} c_{g}\right)_{o}}{\left(\mu_{g} c_{g}\right)_{i}}
$$




\section{Type Curve Matching}

Eqs. 11.1 - 11.5 for gas well closed chamber tests reduce to the standard equations for the liquid slug test case if $a_{D}$ is equal to 1 . Note that $a_{D}$ is a function of time, as well as position, and, for any $r_{D}>1, a_{D}$ has the minimum value of $a_{D}=1.0$ at $t_{D}=0.0$ and the maximum value of $a_{D}=\left(\mu_{g} c_{g}\right)_{o} /\left(\mu_{g} c_{g}\right)_{i}$ as $t_{D} \rightarrow \infty$. Intuitively, we expect that one can correlate data from gas well closed chamber tests with slightly compressible liquid slug test solution when the deviation of $a_{D}$ from unity is small. This implies that data from gas well closed chamber tests can be analyzed using methods developed for slug tests after one transforms pressure versus time data into pseudopressure versus pseudotime data.

In order to use wellbore storage and skin pressure and pressure derivative type curves, further data transformation is necessary. According to Peres et al. ${ }^{5}$, the relevant equations are

$$
p_{w c D}\left(t_{a D}, C_{D}, s\right)=\frac{I\left(m_{w D}\right)}{C_{D}}
$$

and

$$
p_{w c D}^{\prime}=\frac{t_{a D}}{C_{D}} m_{w D}
$$

In Eq. $12, I\left(m_{w D}\right)$ is given by

$$
I\left(m_{w D}\right)=\int_{0}^{t_{a D}} m_{w D}\left(\tau, C_{D}, s\right) d \tau .
$$

Multirate Analysis

From Darcy's law, we have

$$
1.127 \times 10^{-3}(2 \pi) \frac{k}{\mu_{g}} h\left(r \frac{\partial p}{\partial r}\right)_{r=r_{w}}=q
$$

where $q$ is the gas sandface volumetric flow rate in RB/D. According to Eq. 1.5, $q$ can be approximated by

$$
q=V_{w} c_{g w} \frac{d p_{w f}}{d t}
$$

We let $Q$ denote the gas sandface volumetric flow rate in $\mathrm{RB} / \mathrm{D}$ at $p_{i}$, given by

$$
Q=q \frac{(p / z)_{w}}{(p / z)_{i}}
$$

and let $Q_{D}$ denote the dimensionless converted sandface flow rate defined by

$$
Q_{D}=\frac{141.2 Q \mu_{g i}}{k h\left[m\left(p_{i}\right)-m\left(p_{o}\right)\right]} .
$$


With our definitions of dimensionless variables, the inner boundary condition given by Eq. 15 becomes

$$
\left(r_{D} \frac{\partial m_{D}}{\partial r_{D}}\right)_{r_{D}=1}=-Q_{D}
$$

The computational study by Reynolds et al. ${ }^{7}$ and the theoretical work of Finjord ${ }^{8}$ and Aanonsen ${ }^{9}$ indicate that we should not use pseudotime to analyze gas well drawdown tests. Thus, we expect that we should apply Duhamel's principle in terms of $t_{D}$ rather than $t_{a D}$. Then, Duhamel's principle for gas well closed chamber tests can written as

$$
m_{w D}\left(t_{D}\right)=\int_{0}^{t_{D}} Q_{D}(\tau) p_{w c D}^{* \prime}\left(t_{D}-\tau\right) d \tau+s Q_{D}\left(t_{D}\right)
$$

where $p_{w c D}^{* \prime}$ is the derivative of the dimensionless wellbore pressure solution for the constant sandface rate problem. For the standard radial flow problem, when $t_{D} \geq 100, p_{w c D}^{*}$ is given by

$$
p_{w c D}^{*}=\frac{1}{2} \ln \left(\frac{4 t_{D}}{e^{\gamma}}\right)
$$

where $\gamma=0.57722$ is Euler's constant.

In gas well closed chamber tests, although the sandface flow rate continuously declines, we approximate the flow rate profile as a series of discrete constant rates, i.e., from $t_{0 D}$ to $t_{1 D}$ the flow rate is assumed to be $Q_{1 D}$, from $t_{1 D}$ to $t_{2 D}$ the flow rate is assumed to be $Q_{2 D}$, and so on. Using the approximate flow rate profile, Duhamel's principle can be written as

$$
m_{w D}\left(t_{D}\right)=\sum_{i=1}^{n}\left[Q_{i D}-Q_{(i-1) D}\right] p_{w c D}^{*}\left[t_{D}-t_{(i-1) D}\right]+s Q_{n D}
$$

Note that in deriving Eq. 22, we have used $p_{w c D}^{*}(0)=0$ and the notation $Q_{0 D}=0$. Using the definitions for dimensionless variables and Eq. 21 in Eq. 22, we obtain

$$
\frac{m\left(p_{i}\right)-m\left(p_{w f}\right)}{Q_{n}}=m^{\prime} \sum_{i=1}^{n}\left[\frac{\left(Q_{i}-Q_{i-1}\right)}{Q_{n}} \log \left(t-t_{i-1}\right)\right]+b^{\prime}
$$

where

$$
m^{\prime}=\frac{162.6 \mu_{g i}}{k h}
$$

and

$$
b^{\prime}=m^{\prime}\left[\log \left(\frac{k}{\phi\left(\mu_{g} c_{g}\right)_{i} r_{w}^{2}}\right)-3.2275+0.86859 s\right]
$$


In Eq. 23.1, $t$ is in hours. Eq. 23.1, which was first presented by Odeh and Jones ${ }^{10}$, is the working equation for multirate test analysis. Eq. 23.1 suggests that a plot of rate-normalized pseudopressure drop versus the multirate time function, i.e.,

$$
\frac{m\left(p_{i}\right)-m\left(p_{w f}\right)}{Q_{n}} \text { vs } \sum_{i=1}^{n}\left[\frac{\left(Q_{i}-Q_{i-1}\right)}{Q_{n}} \log \left(t-t_{i-1}\right)\right]
$$

will be a straight line with slope $m^{\prime}$ and intercept $b^{\prime}$. The formation flow capacity and skin factor can be obtained form the values of $m^{\prime}$ and $b^{t}$ by the obvious rearrangement of Eqs. 23.2 and 23.3.

In applying the convolution method, the sandface flow rate must be obtained either from direct measurement or calculation using Eqs. 16 and 17.

\section{RESULTS AND DISCUSSIONS}

An implicit one-dimensional radial flow numerical simulator was used to simulate gas well closed chamber tests. In the simulator, the skin factor is modeled as an inner zone of different permeability, using Hawkins formula.

Gas physical properties input into the simulator are presented in Table 1. These properties were used by Fraim and Wattenbarger ${ }^{11}$ in gas reservoir decline curve analysis. We begin with a base case gas well closed chamber test and then present results obtained from the sensitivity study. Parameters for the base case are listed in Table 2.

\section{Tvpe Curve Matching}

Fig. 1 presents type curve matching of base case gas well closed chamber test with liquid slug test solutions. In Fig. 1, the solid line represents the Ramey, Agarwal and Martin ${ }^{12}$ slug test solution with wellbore storage and skin equal to the corresponding gas well CCT wellbore storage and skin, the dashed line represents the wellbore dimensionless pressure plotted versus dimensionless time, and the plot designated as circles represents the wellbore dimensionless pseudopressure plotted versus dimensionless pseudotime. The same notation applies to all subsequent slug test type curve matching plots. (Note that dimensionless wellbore pressure is defined as $p_{w D}=\left(p_{i}-p_{w f}\right) /\left(p_{i}-p_{o}\right)$.) As shown in Fig. 1, gas well closed chamber test data plotted as dimensionless pseudopressure versus dimensionless pseudotime correlate well with the analogous liquid slug test solution. However, this correlation becomes less accurate as dimensionless time increases. Also shown in Fig. 1 is a plot of $m_{w D}$ versus $t_{D}$. This plot differs significantly from the liquid slug test solutions, indicating that to correlate the gas well CCT data with the liquid slug test solution, one has to use pseudopressure and pseudotime.

Fig. 2 shows $a_{D}$ as a function of time for three locations within the reservoir. As expected, $a_{D}$ for all the three locations has a minimum value of $a_{D}=1.0$ at $t_{D}=0.0$, and at late times, $a_{D}$ reaches the maximum value of $\left(\mu_{g} c_{g}\right)_{o} /\left(\mu_{g} c_{g}\right)_{i} \approx 9.39$. 
Effect of Skin Factor: For the results considered in this subsection, only the skin factor is varied and all other parameters are the same as in the base case. Figs. 3 and 4 present results for $s=5$ and $s=-2$, respectively. Comparing Figs. 1, 3 and 4 , one can see that the proposed correlation is improved for a case with a larger skin factor. The effect of skin factor on the variation of $a_{D}$ for a fixed point in the reservoir ( $r_{D}=15.22$ ) is shown in Fig. 5. As illustrated in this figure, a case with a larger skin factor has a lower $a_{D}$ value for a given time. Thus, intuitively, one would expect that the correlation will work for a longer period of time for cases with a higher skin factor.

Effect of Initial Wellbore Pressure: Figs. 6 and 7 present results for cases with $p_{o}=2000$ psig and $p_{o}=4000$ psig, respectively. These plots demonstrated that as one increases the initial wellbore pressure the correlation between gas well closed chamber test data and liquid slug test solutions becomes better. The variations of $a_{D}$ for cases with $p_{0}=500,2000$ and 4000 psig are compared in Fig. 8. As one increases the wellbore initial pressure, the change in $a_{D}$ becomes small, and thus, one expects that the correlation will work better for a case with a higher wellbore initial pressure than for a case with a lowrer wellbore initial pressure.

Effect of Initial Reservoir Pressure: The base case initial reservoir pressure $p_{i}=$ 5000 psig was reduced to $p_{i}=4000$ psig and $p_{i}=2500$ psig. The results are shown in Figs. 9 and 10, respectively. Unlike the effect of $p_{o}$, as the initial reservoir pressure decreases, the proposed correlation works for a longer period of time and for larger values of $p_{w f}$. This can also be explained from the variations of $a_{D}$, as indicated in Fig. 11.

Effect of Chamber Volume: The proposed correlation is also affected by the size of the chamber volume. This is shown in Figs. 12 and 13. These plots illustrate that the correlation becomes better if a larger chamber is used in conducting the test. The effect of chamber volume on $a_{D}$ is illustrated in Fig. 14. As the chamber volume increases, the increase in $a_{D}$ is delayed and also occurs more gradually. Hence, intuitively, we expect that a larger chamber will make the correlation work better. Naturally, a longer period of time is needed for the wellbore pressure to buildup when a larger chamber is used.

Peres-Onur-Reynolds (POR) Method: Figs. 15 and 16 show that one can also use wellbore storage and shin pressure and pressure derivative type curves by converting data using the generalization of the Peres et al..$^{5}$ procedure given by Eqs. 12 and 13. A unique match should be possible for both cases since the correlation applies well until the pressure derivative has passed its maximum point. Estimates of $k h$ and $s$ obtained from such a type curve match could be refined using a numerical model such as the one developed by Mfonfu and Grader ${ }^{13}$.

\section{Multirate Analysis}

Sandface flow rates output from the simulator were used in the convolution analysis. Rate-normalized pseudopressure drop and the multirate time function calculated for the base case and the case with $s=5$ are plotted in Figs. 17 and 18, 
respectively. Fig. 17 shows a perfect straight line with $m^{\prime}=1.490$ and $b^{\prime}=5.292$. From Eqs. 23.2 and 23.3, we obtain $k h=24.64$ and $s=-0.06$. These estimated parameters agree well with the input values of $k h=25.0$ and $s=0.0$. The accuracy of these results indicates that Eq. 20 is valid, and thus, deconvolution methods can also be used.

In Fig. 18, the plot first seems to reflect the skin zone flow capacity and then undergoes a transitional period. After a short period of time, a straight line develops. From the slope and the intercept of the straight line we obtain $k h=24.53$ and $s=4.86$. Again, theses values match well with input values of $k h=25.0$ and $s=5.0$. The results shown in Figs. 17 and 18 illustrate our conclusion that in convolution analysis, one should use pseudopressure and time, not pseudopressure and pseudotime.

In the literature, convolution techniques based on a piecewise linear approximation of the flow rate profile have been developed and used for analysis of afterflow data and data from underbalanced perforations ${ }^{14-16}$. Although not presented here, it was found out that these techniques yield similar results to those given in Figs. 17 and 18.

\section{CONCLUSIONS}

1. In many cases, data from gas well closed chamber tests can be correlated with the liquid slug test solutions by using an approximate pseudopressure and pseudotime transformation. This correlation usually works sufficiently long so that methods developed for slug test analysis such as type curve matching can be applied to gas well closed chamber tests to obtain estimates of formation flow capacity and skin factor.

2. The proposed correlation works for a longer period of time and up to a higher magnitude of pressure drop recovery for cases with a larger skin factor, higher initial wellbore pressure, lower initial reservoir pressure and a larger gas chamber.

3. Convolution analysis techniques in terms of pseudopressure and time can always be used for gas well closed chamber tests if an accurate measurement of sandface flow rate is available, or one can accurately determine the sandface flow rate from the measurement of chamber gas pressure.

\section{NOMENCLATURE}

Symbol

$a_{D} \quad=$ parameter defined in Eq. 11.6.

$C \quad=$ wellbore storage coefficient, $\mathrm{RB} / \mathrm{psi}$.

$C_{D} \quad=$ dimensionless wellbore storage coefficient.

$c_{g} \quad=$ gas isothermal compressibility, $\mathrm{psi}^{-1}$.

$c_{g i} \quad=$ gas isothermal compressibility evaluated at $p_{i}, \mathrm{psi}^{-1}$. 


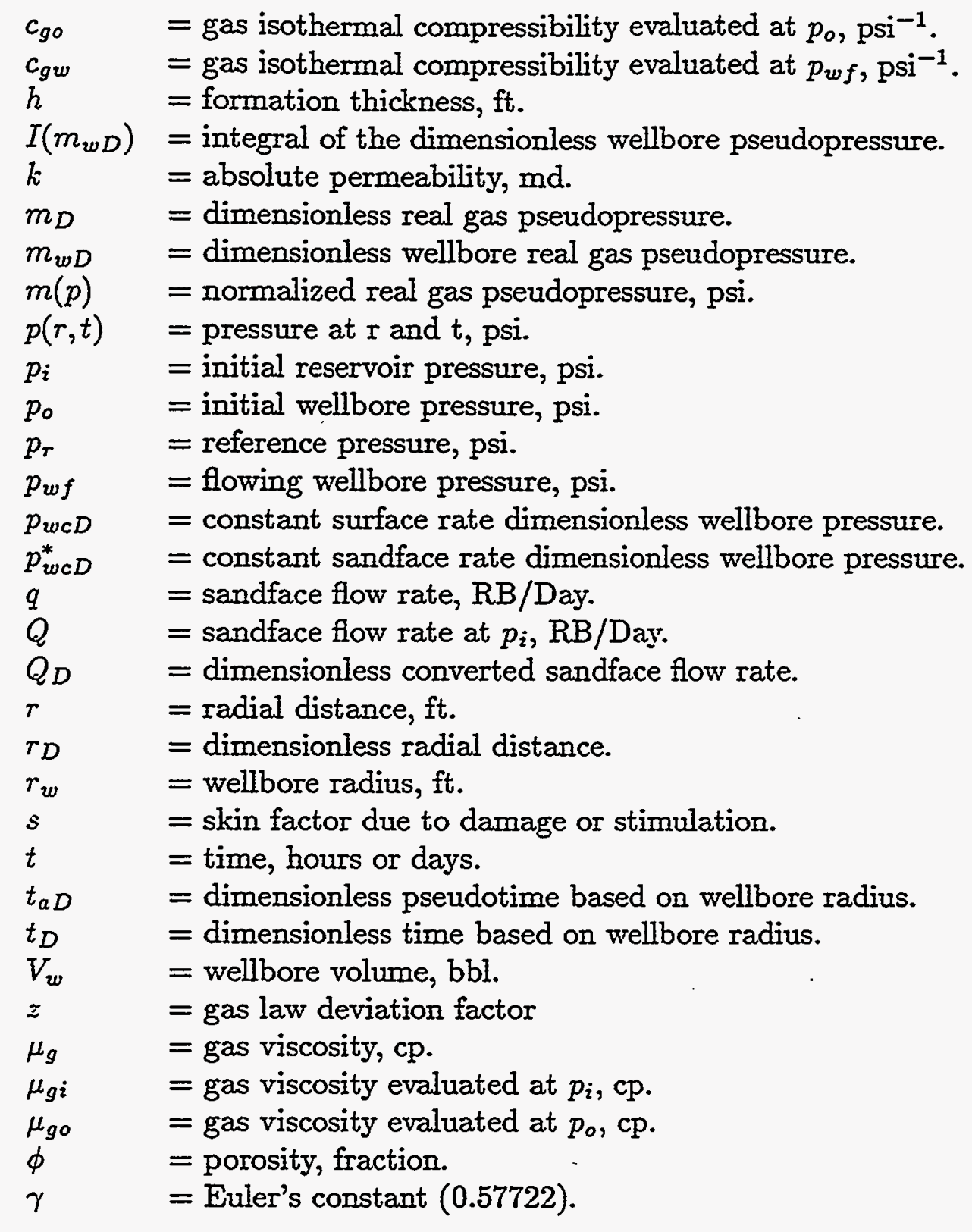

\section{REFERENCES}

1. Alexander, L. G.: "Theory and Practice of the Closed-Chamber Drillstem Test Method," J. Pet. Tech., (December 1977) 1539-1544.

2. Reid, H. W.: "Evaluation of Low Permeability Gas Reservoirs Utilizing Closed Chamber Drillstem Test Techniques,"paper SPE 10184 presented at the 56th Annual Fall Technical Conference and Exhibition of SPE of AIME, Oct. 5-7, 1981, San Antonio, Texas.

3. Agarwal, R.: "'Real Gas Pseudotime' - A New Function for Pressure Buildup 
Analysis of MHF Gas Wells," paper SPE 8279 presented at the 54th SPE Annual Technical Conference and Exhibition, Las Vegas, Nevada, Sept. 23-26, 1979.

4. Lee, W. J. and Holditch, S. A.: "Application of Pseudotime to Buildup Test Analysis of Low-Permeability Gas Wells With Long-Duration Wellbore Storage Distortion," J. Pet. Tech., (December 1982) 2877-2887.

5. Peres, A., Onur, M. and Reynolds, A.: "A New General Pressure Analysis for Slug Tests," paper SPE 18801 presented at the 1989 SPE California Regional meeting, Bakersfield, CA, April 5-7.

6. Al-Hussainy, R., Ramey, H. J. Jr. and Crawford, P. B.:"The Flow of Real Gases Through Porous Media," J. Pet. Tech., (May 1966) 624-636.

7. Reynolds, A. C., Bratvold, R. B. and Ding, W.: "Semilog Analysis of Gas Well Drawdown and Buildup Data," SPE Formation Evaluation, (December 1987) $657-670$.

8. Finjord, J.:"A Study of Pseudotime," paper SPE 12577, SPE Book Order Department, Richardson, TX.

9. Aanonsen, S.:"Nonlinear Effects During Transient Fluid Flow in Reservoirs as Encountered in Well Test Analysis," Dr. Scient. Dissertation, U. of Bergen, Norway, (1985).

10. Odeh, A. S. and Jones, L. G.:"Pressure Drawdown Analysis, Variable-Rate Case," J. Pet. Tech., (August 1965) 960-964.

11. Fraim, M. L. and Wattenbarger, R. A.: "Gas Reservoir Decline-Curve Analysis Using Type Curves With Real Gas Pseudopressure and Normalized Time," SPE Formation Evaluation, (December 1987) 671-682.

12. Ramey, H. J. Jr., Agarwal, R. G. and Martin, I.: "Analysis of 'Slug Test' or DST Flow Period Data," J. Can. Pet. Tech., 14 (July-Sept. 1975) 37-47.

13. Mfonfu, G. B. S. and Grader, A. S.:"An Implicit Numerical Model for the Closed Chamber Test," paper SPE 19832 presented at the 64th Annual Technical Conference and Exhibition of SPE, Oct. 8-11, 1989, San Antonio, TX.

14. Meunier, D., Wittmann, M. J. and Stewart, G.:"Interpretation of Pressure Build-up Test Using In-Situ Measurement of Afterflow," J. Pet. Tech., (January 1985) 143-152.

15. Tariq, S. M. and Ayestaran, L.:"Analyses and Application of Pressure, Flowrate, and Temperature Measurements During a Perforation Run," paper SPE 15475 presented at the 61st Annual Technical Conference and Exhibition of SPE, Oct. 5-8, 1986, New Orleans, LA.

16. Ablewhite, P. K.: "Formation Damage and Flow Capacity Estimation Using Pressure Data Recorded While Perforating," paper OSEA 90168 presented at the Eighth Offshore South East Asia Conference in Singapore, 4-7 December 1990. 
Table 1: Gas Physical Properties as Functions of Pressure

\begin{tabular}{|cccc|}
\hline Pressure $(\mathrm{psia})$ & $\mu_{g}(p)(\mathrm{cp})$ & $z(p)(-)$ & $c_{g}(p)\left(\mathrm{psi}^{-1}\right)$ \\
\hline 514.7 & 0.1272 & 0.9693 & $2.2870 \mathrm{E}-03$ \\
1014.7 & 0.1373 & 0.9388 & $1.0500 \mathrm{E}-03$ \\
1514.7 & 0.1479 & 0.9184 & $0.6743 \mathrm{E}-03$ \\
2014.7 & 0.1570 & 0.9102 & $0.5299 \mathrm{E}-03$ \\
2514.7 & 0.1676 & 0.9105 & $0.3890 \mathrm{E}-03$ \\
3014.7 & 0.1786 & 0.9198 & $0.3046 \mathrm{E}-03$ \\
3514.7 & 0.1899 & 0.9364 & $0.2433 \mathrm{E}-03$ \\
4014.7 & 0.2018 & 0.9590 & $0.1978 \mathrm{E}-03$ \\
4514.7 & 0.2136 & 0.9861 & $0.1635 \mathrm{E}-03$ \\
5014.7 & 0.2258 & 1.0165 & $0.1372 \mathrm{E}-03$ \\
\hline
\end{tabular}

Table 2: Parameters for Base Case Gas Well Closed Chamber Test

\begin{tabular}{|l|rl|}
\hline \multicolumn{1}{|c|}{ Parameter } & Value & Unit \\
\hline Initial Reservoir Pressure & 5000.0 & psig \\
Formation Porosity & 0.075 & - \\
Formation Permeability & 1.0 & $\mathrm{md}$ \\
Skin Factor & 0.0 & - \\
Reservoir Thickness & 25.0 & $\mathrm{ft}$ \\
Well Radius & 0.25 & $\mathrm{ft}$ \\
Chamber Volume & 130.0 & $\mathrm{bbl}$ \\
Initial Chamber Gas Pressure & 500.0 & psig \\
Initial Wellbore Storage Coefficient & 9309.0 & - \\
\hline
\end{tabular}




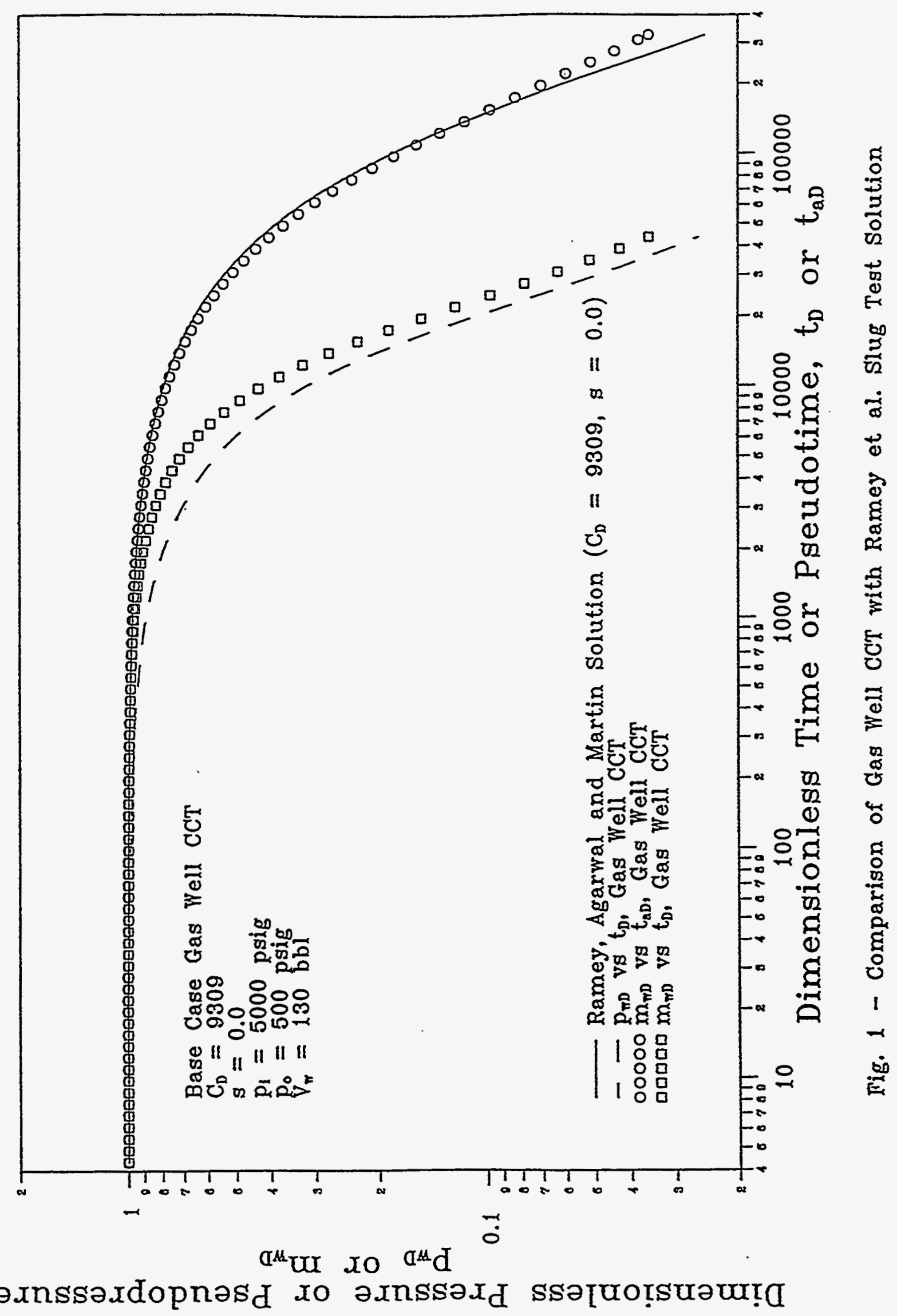




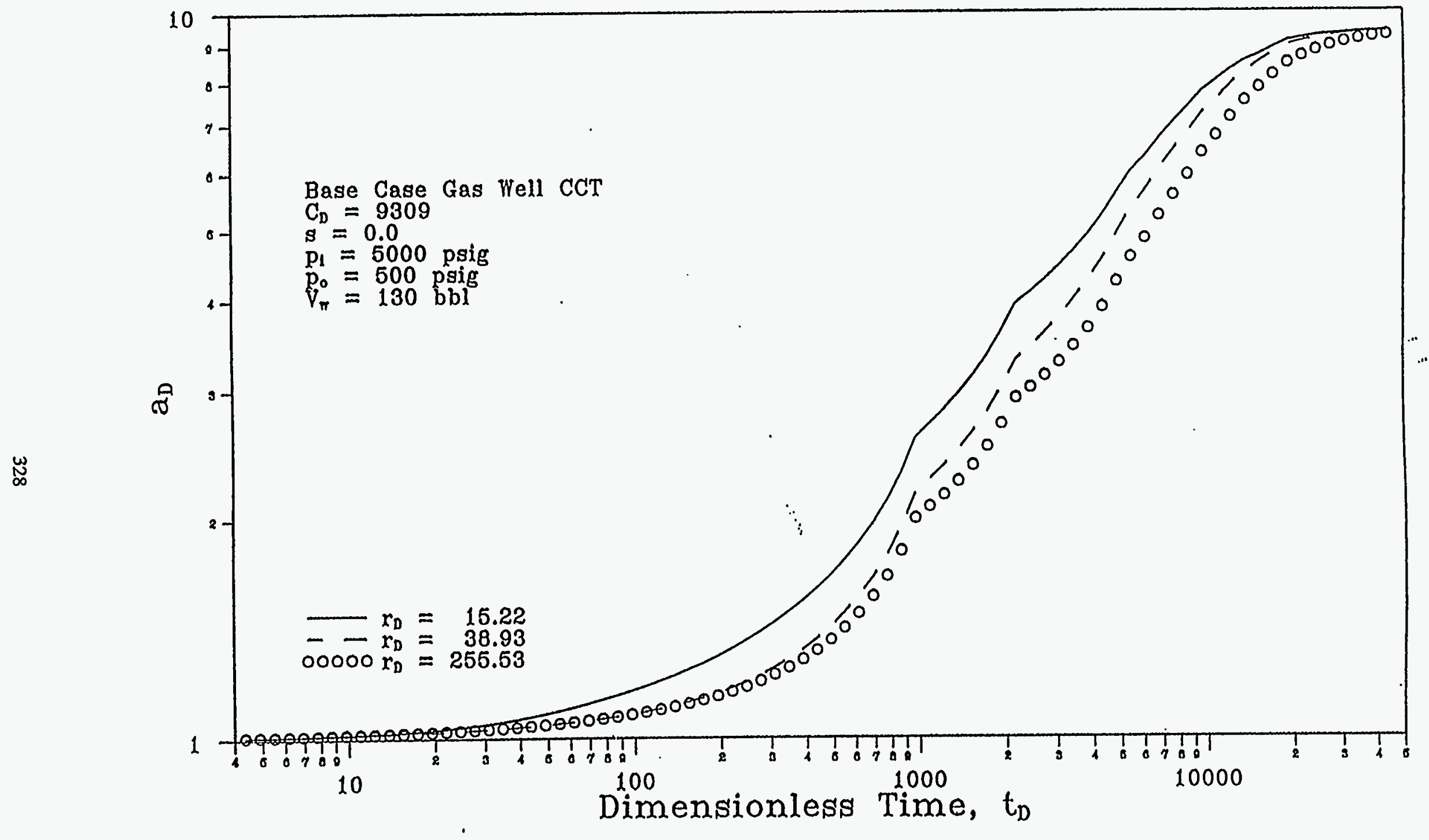

Fig. 2 - as as a function of time and position, base case 


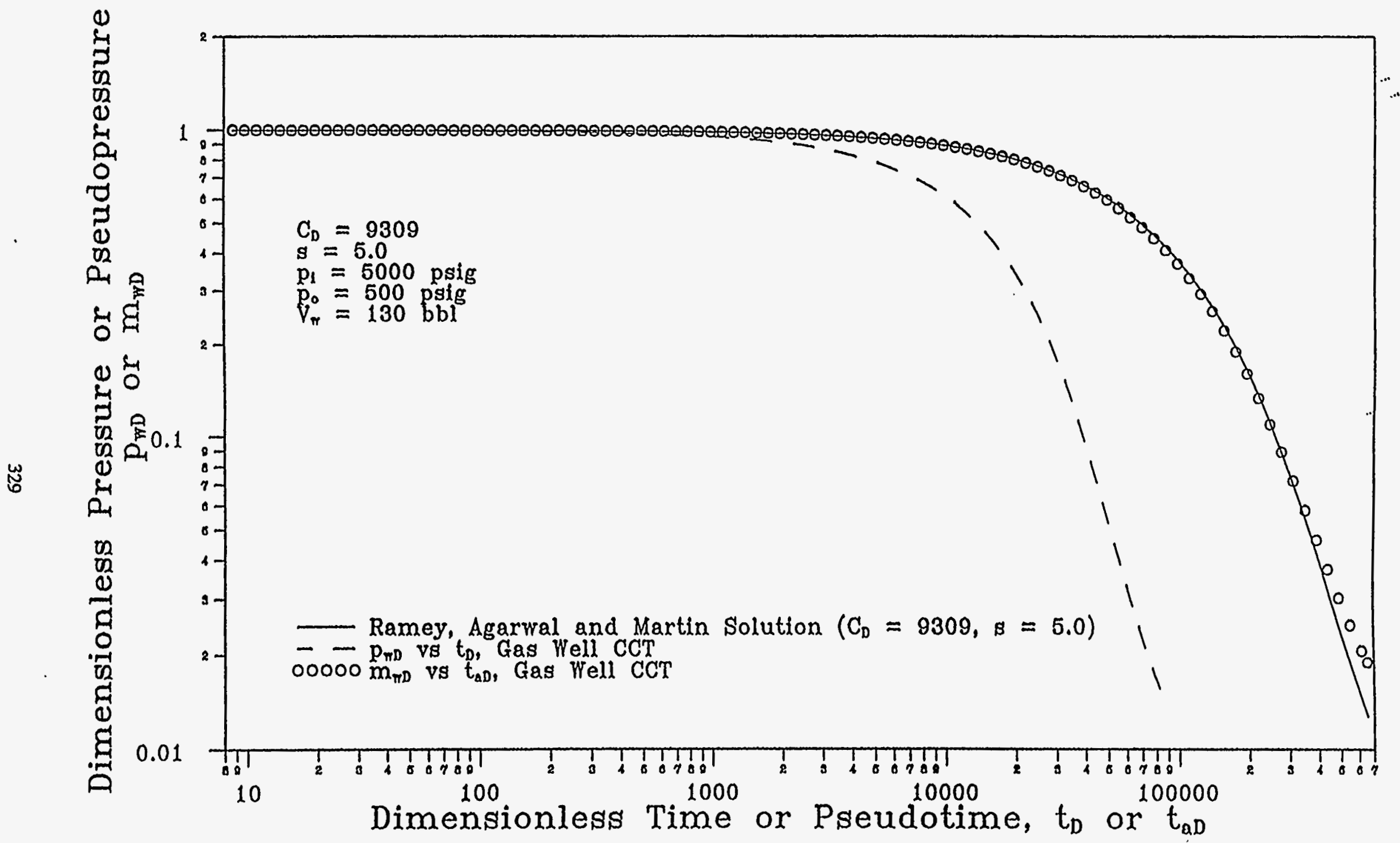

Fig. 3 - Effect of Skin Factor on Gas Well CCT $(s=5.0)$ 


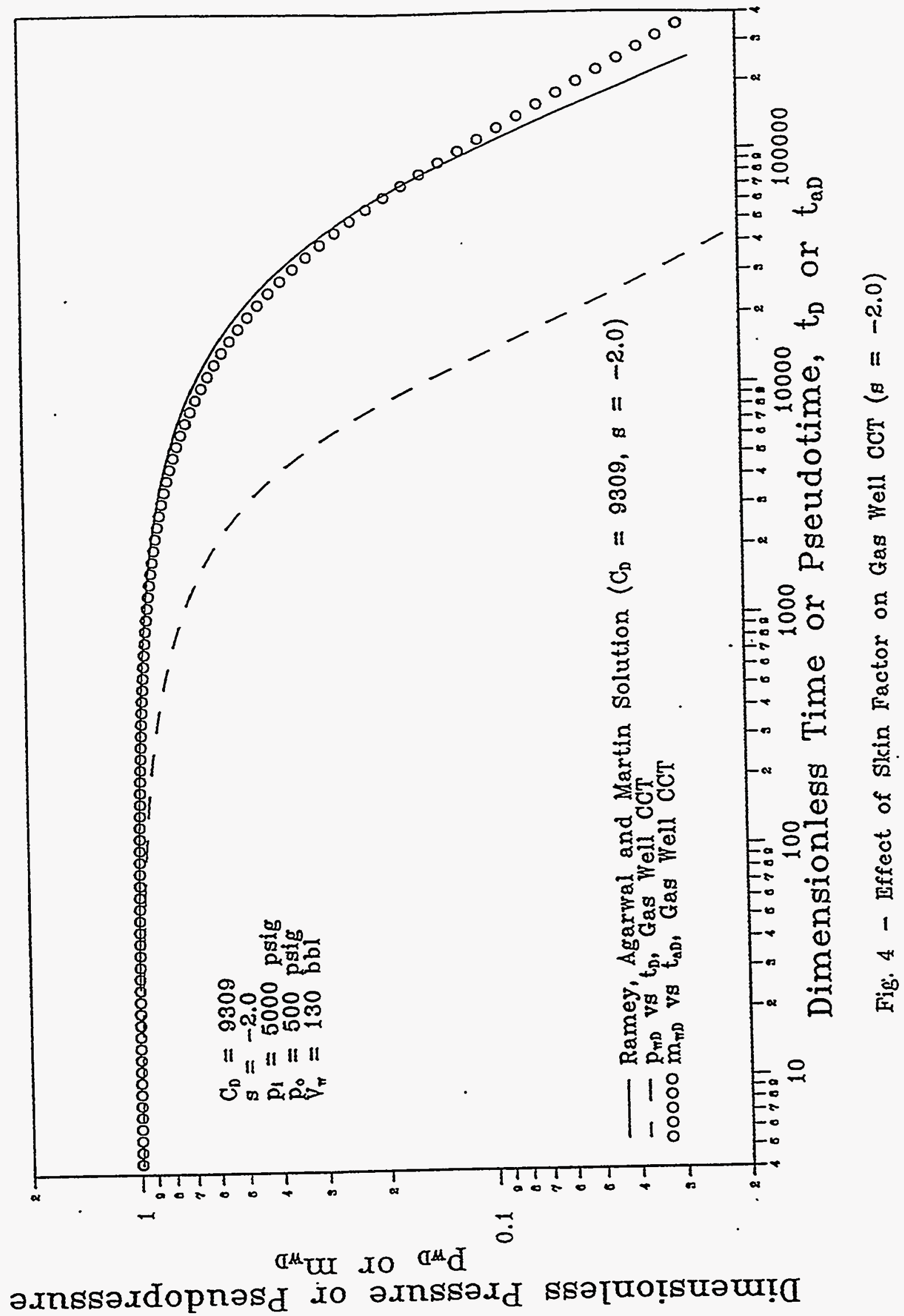




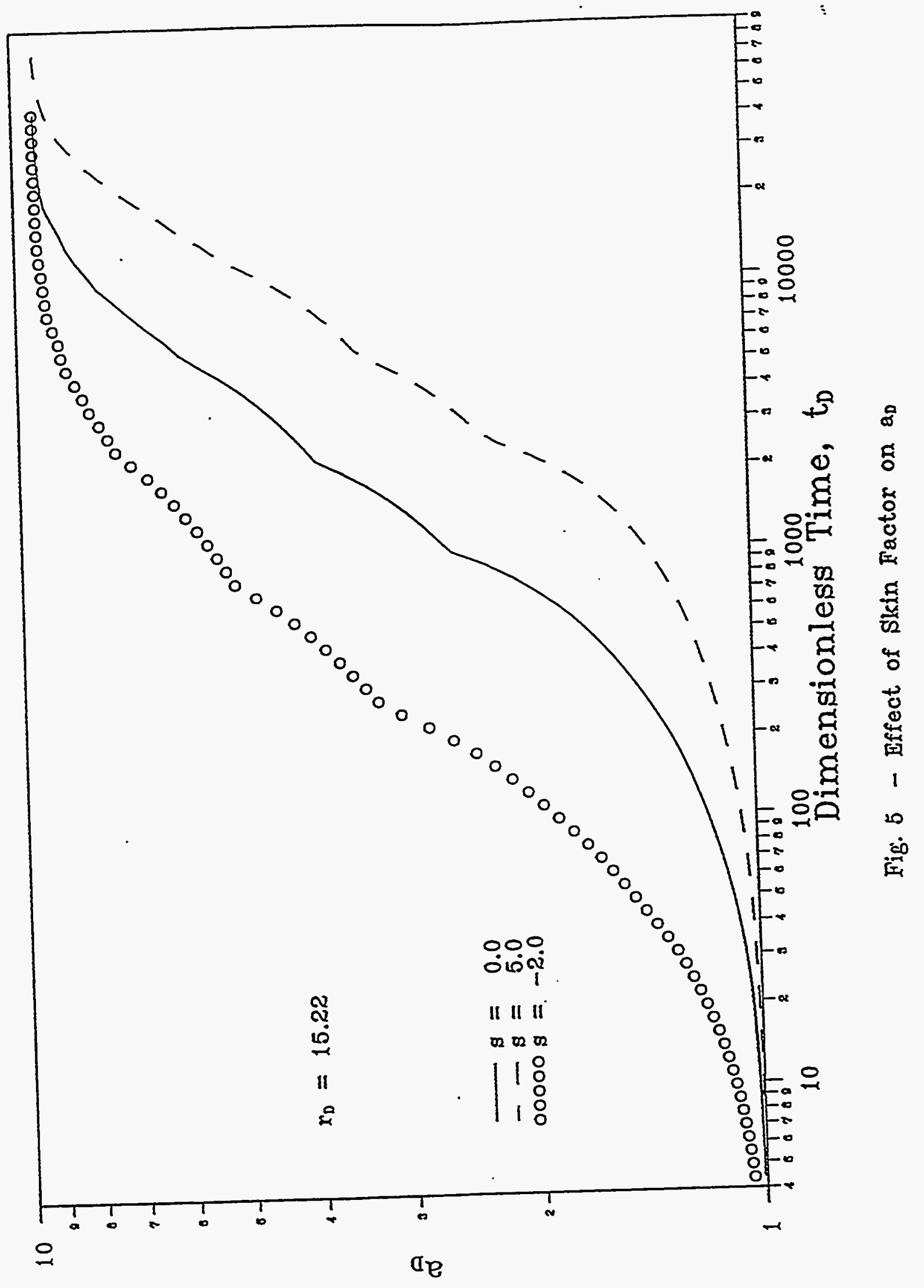




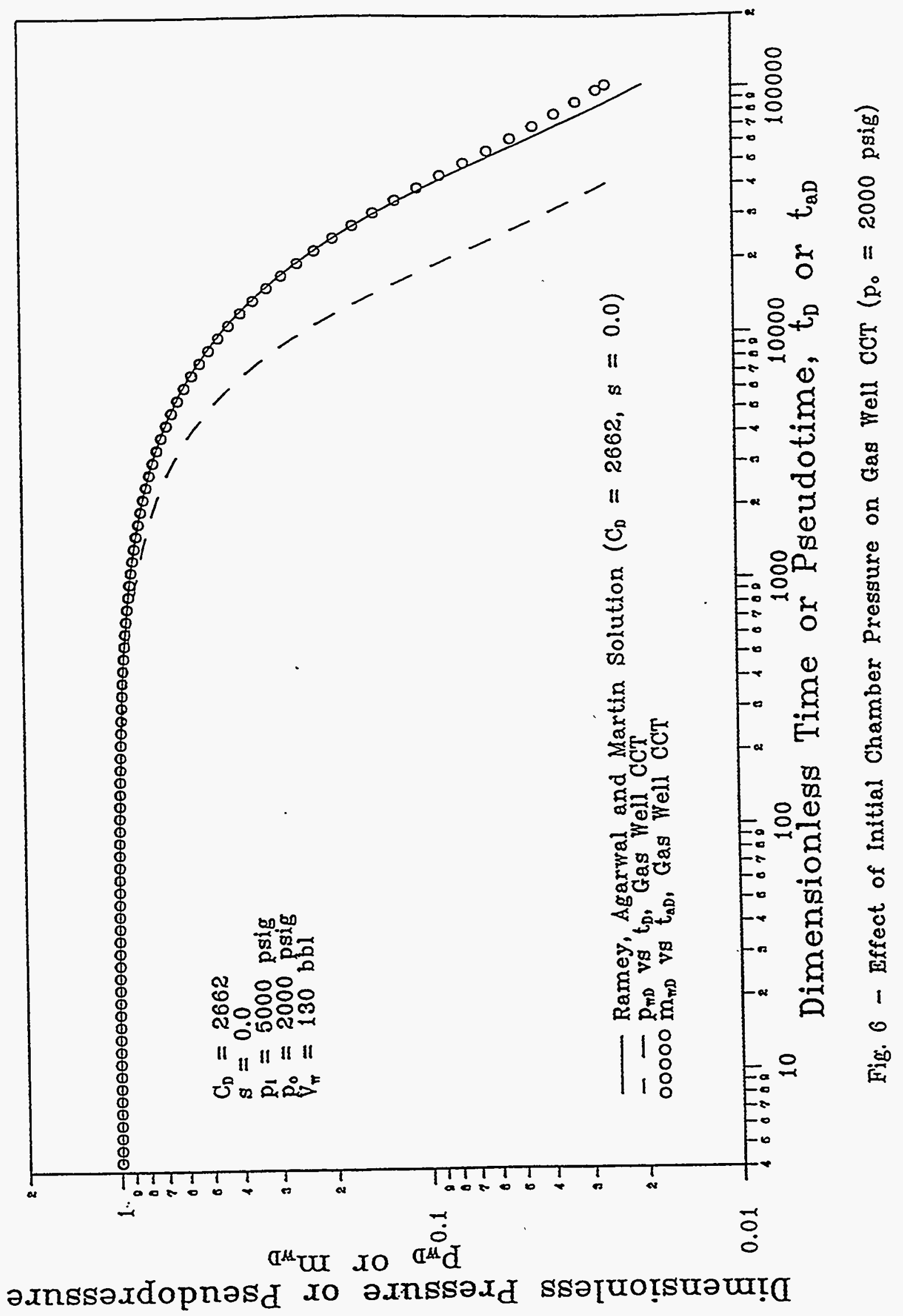




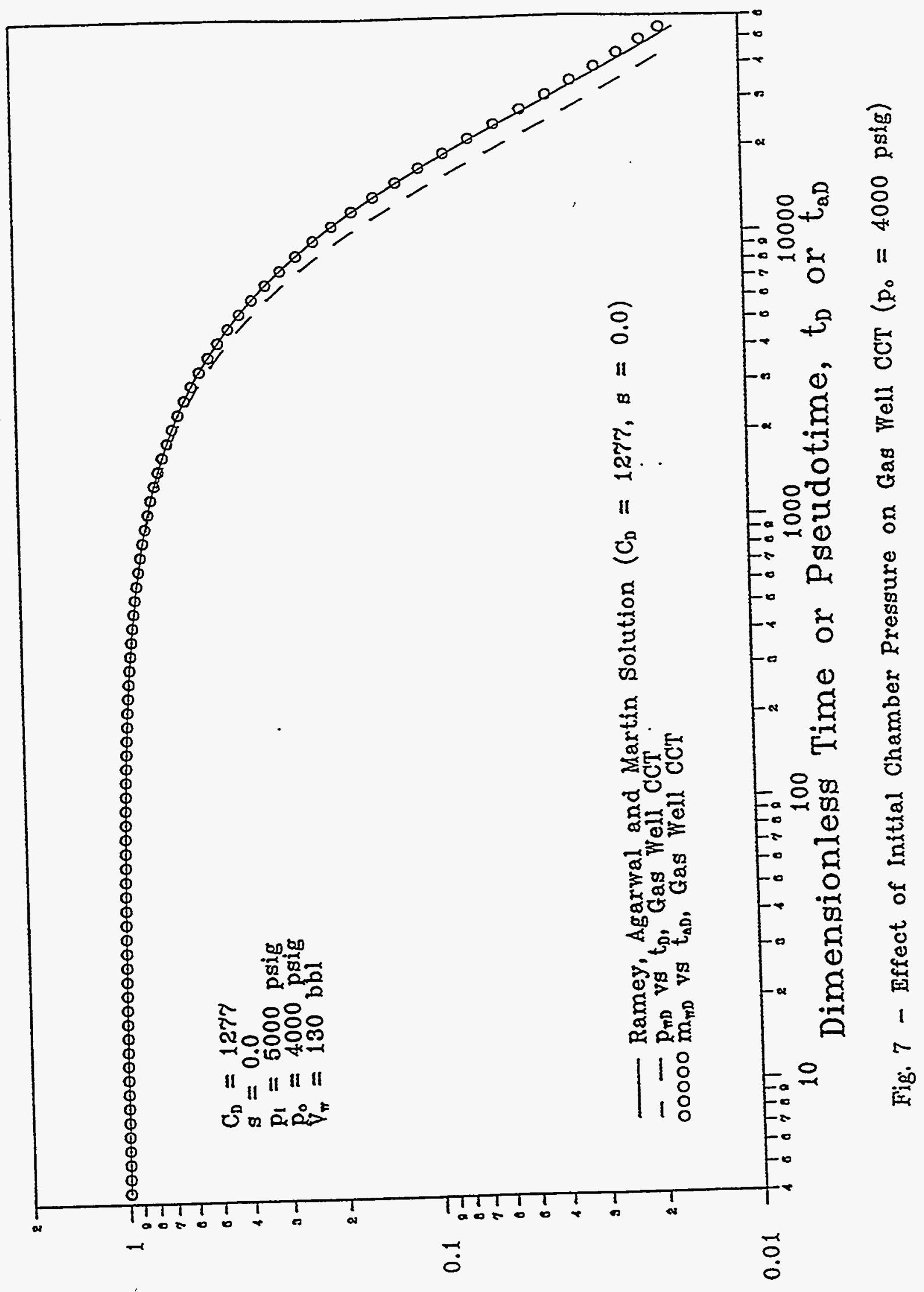

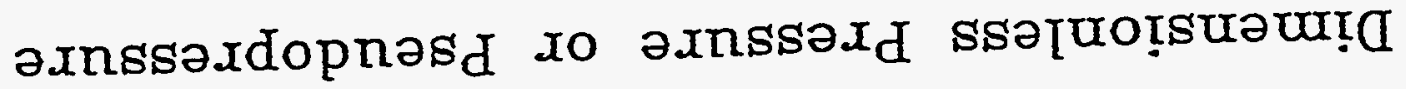




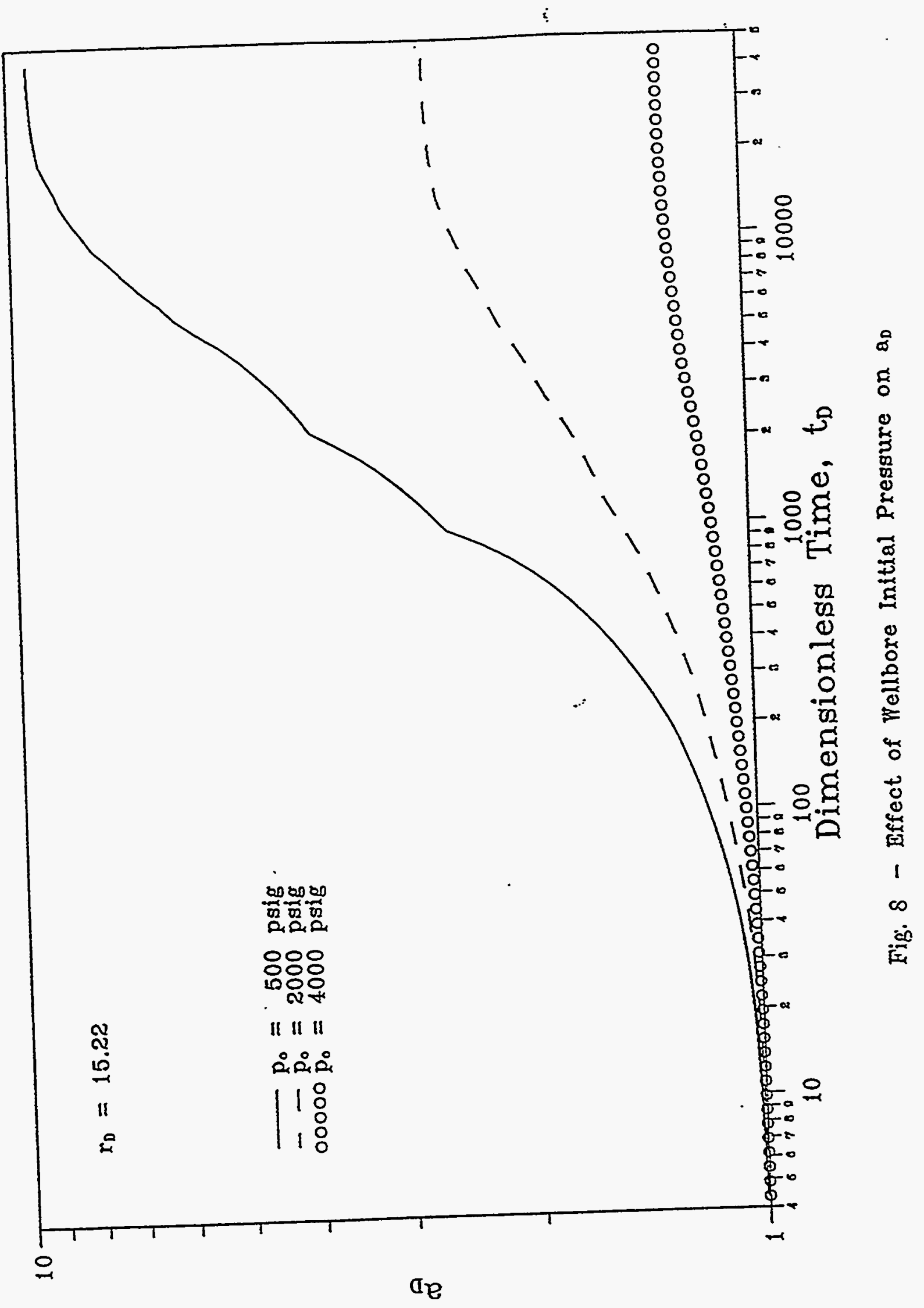




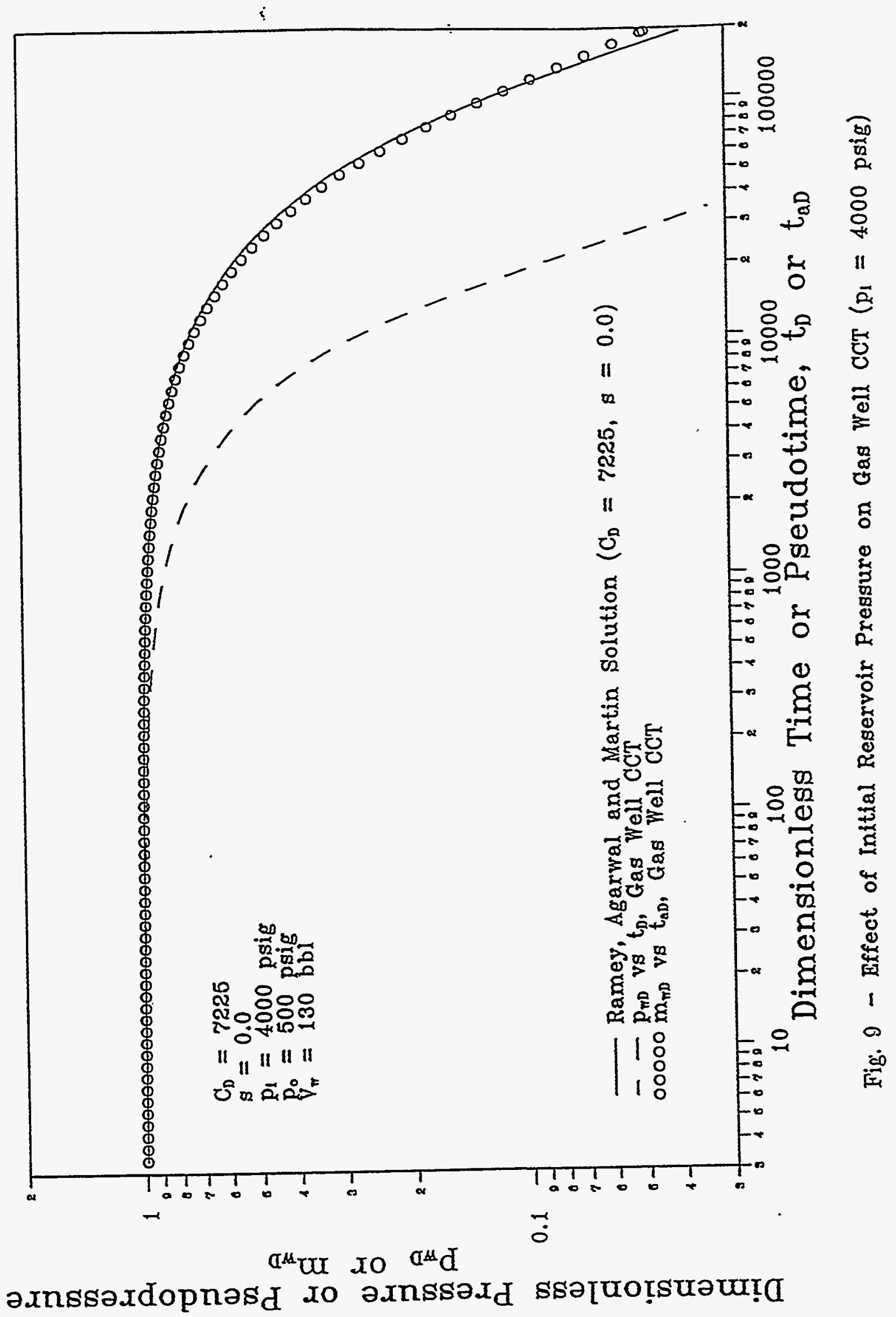




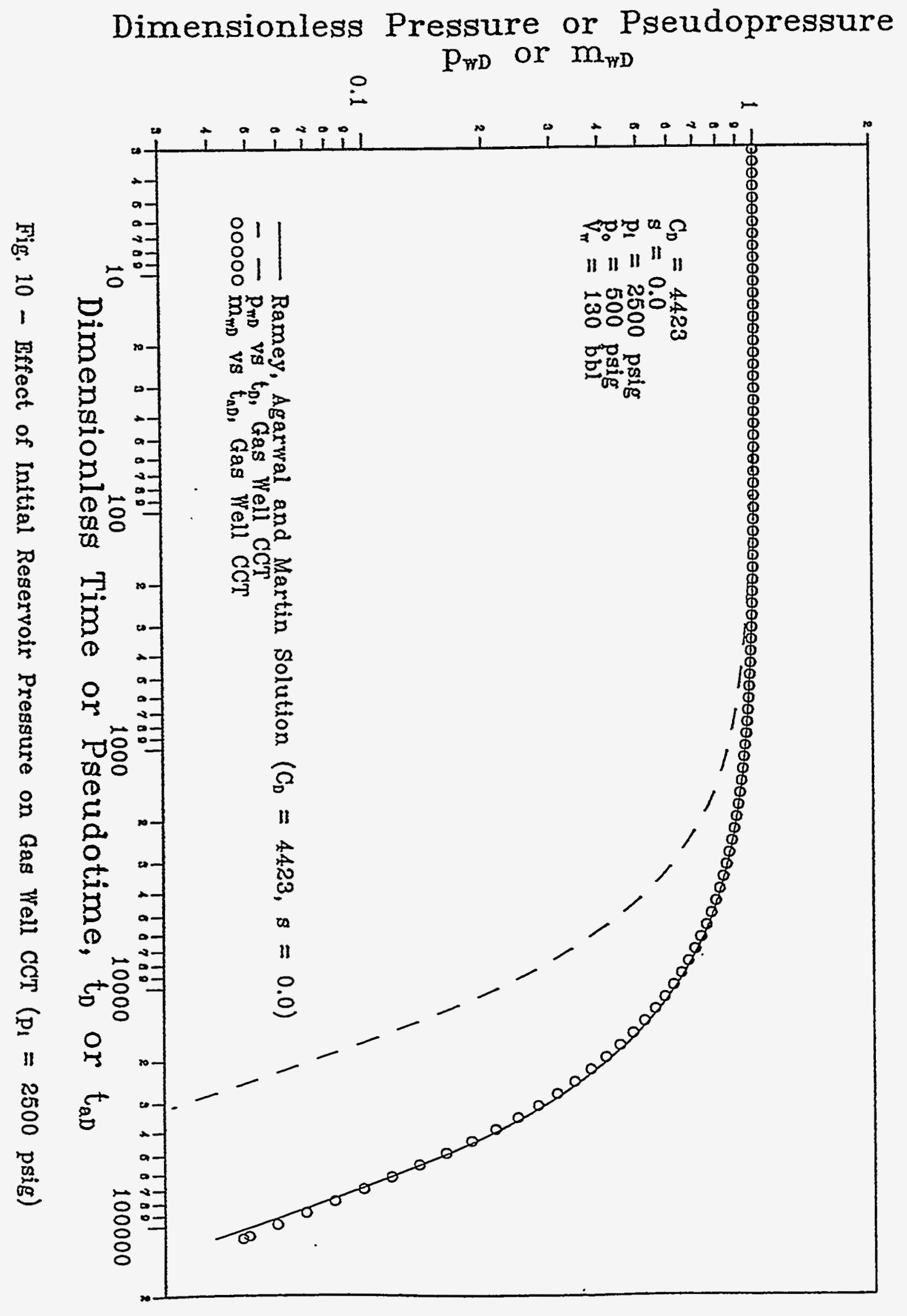




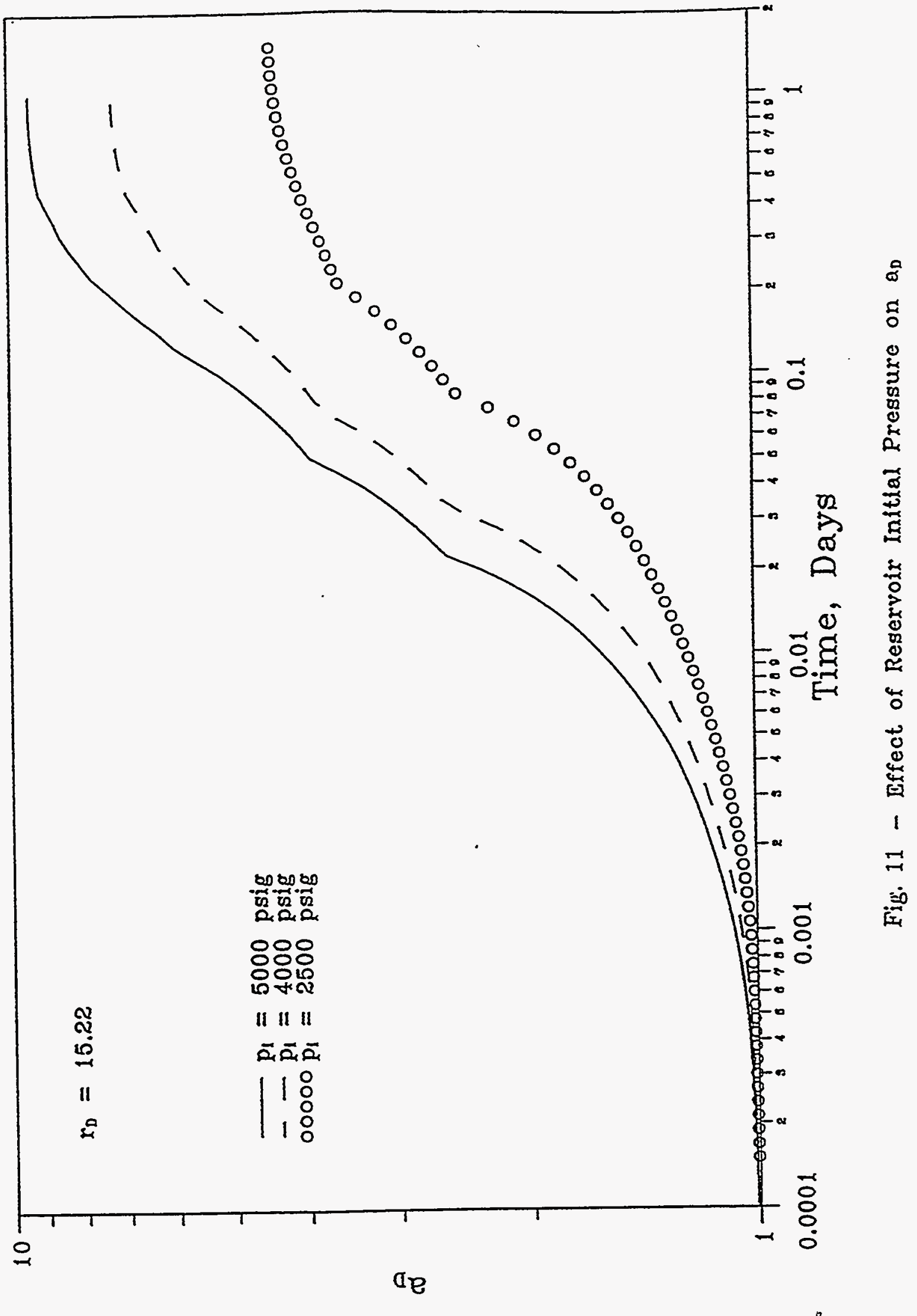




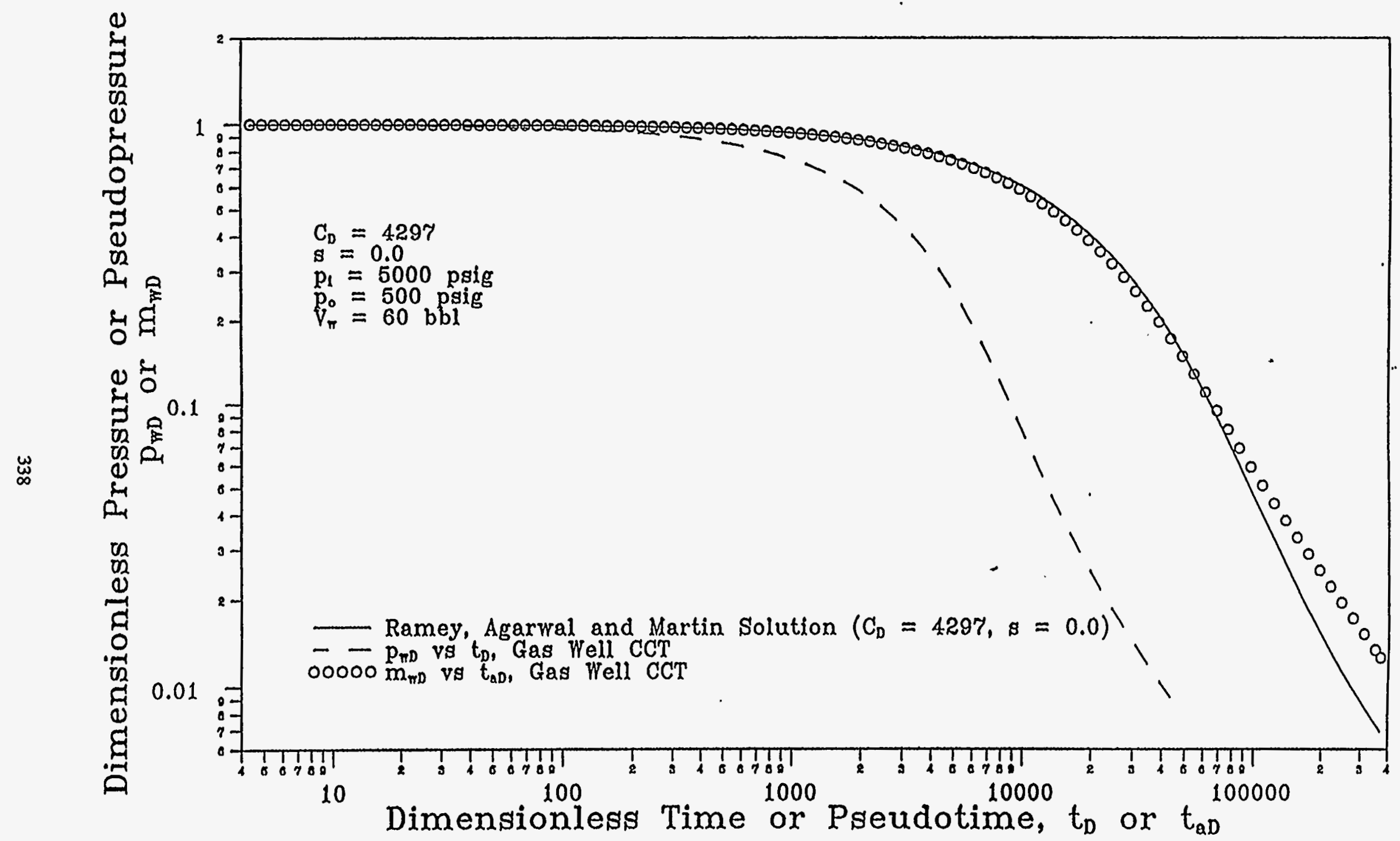

Fig. 12 - Effect of Chamber Volume on Gas Well CCT $\left(V_{\pi}=60 \mathrm{bbl}\right)$ 


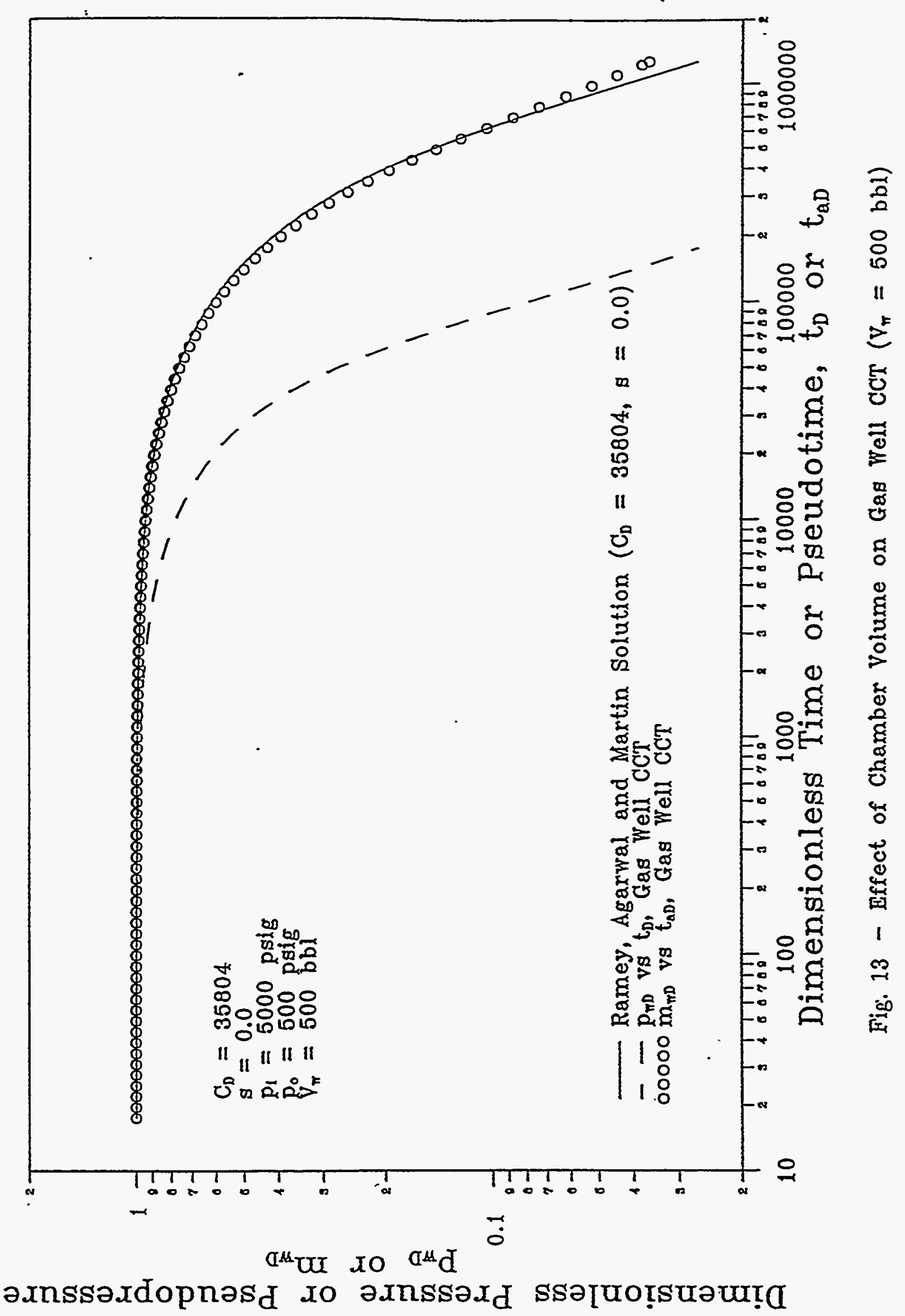




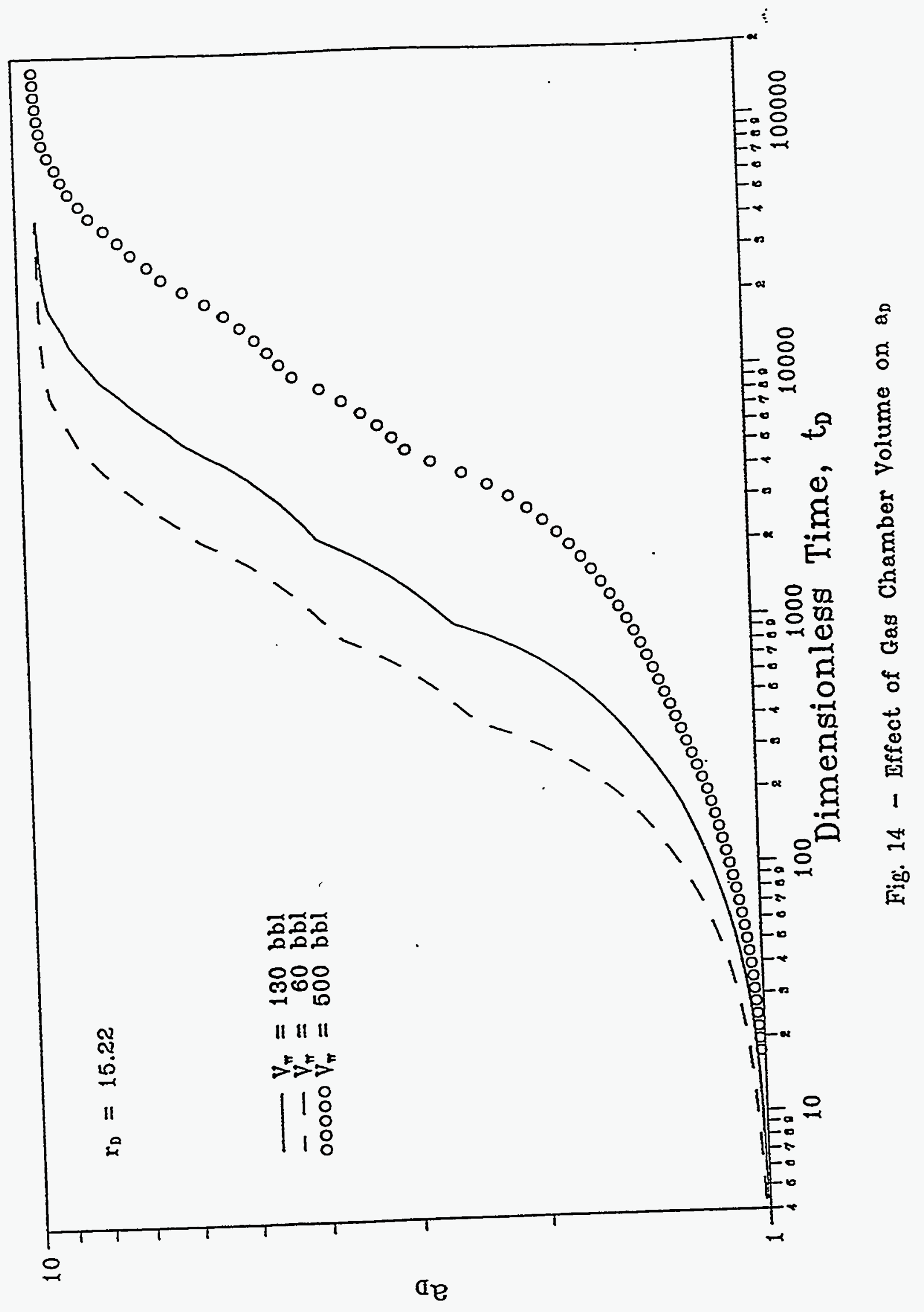




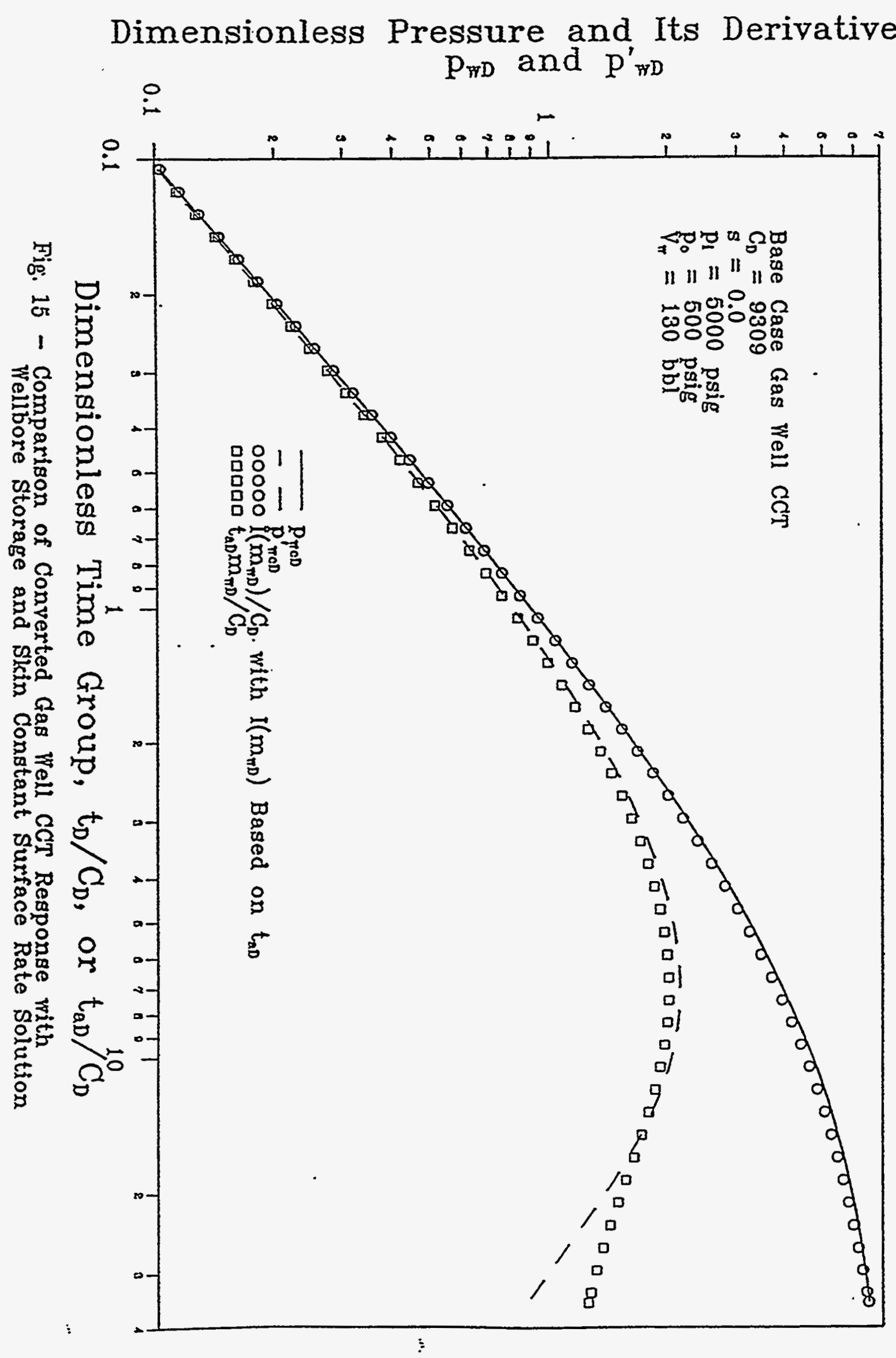




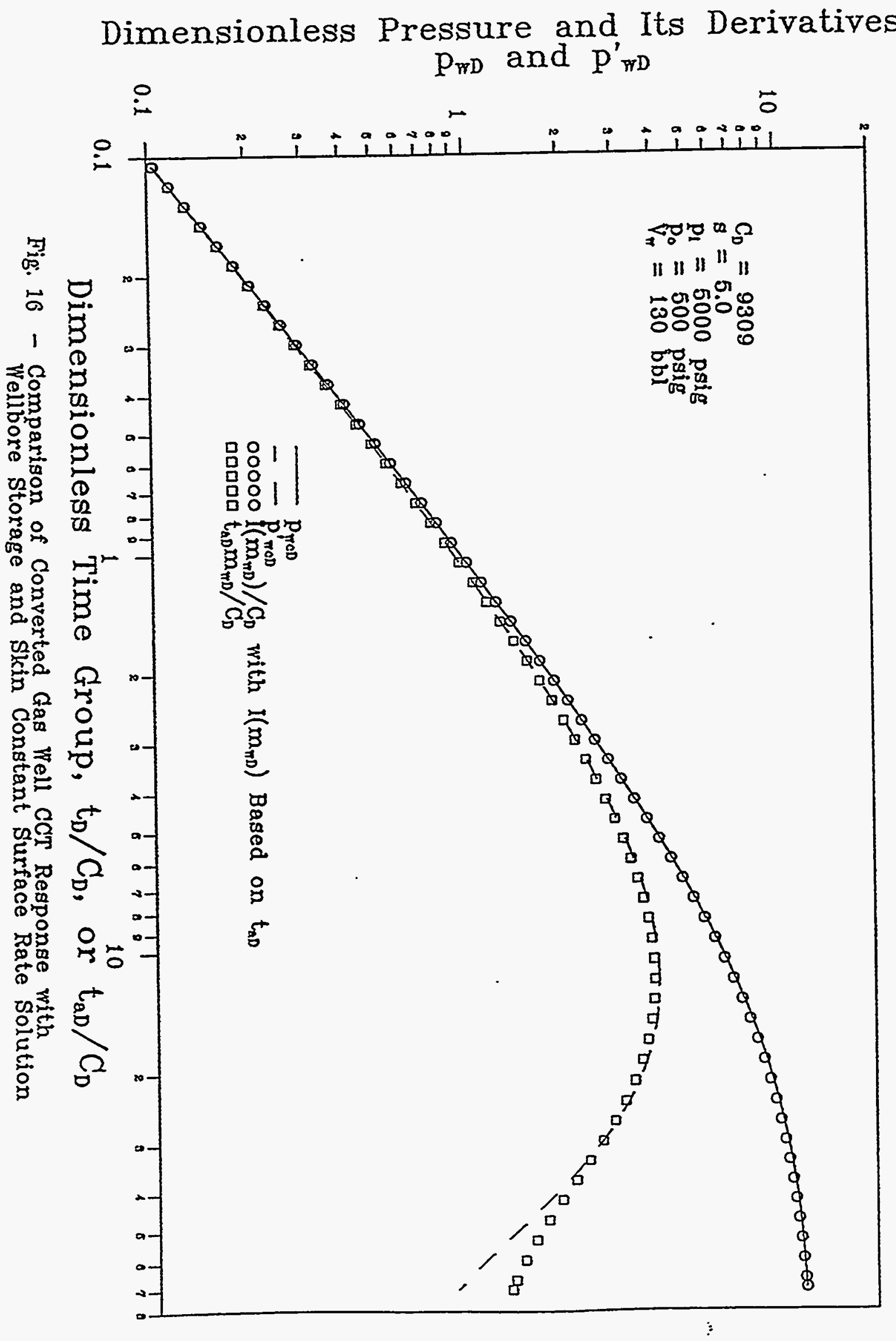




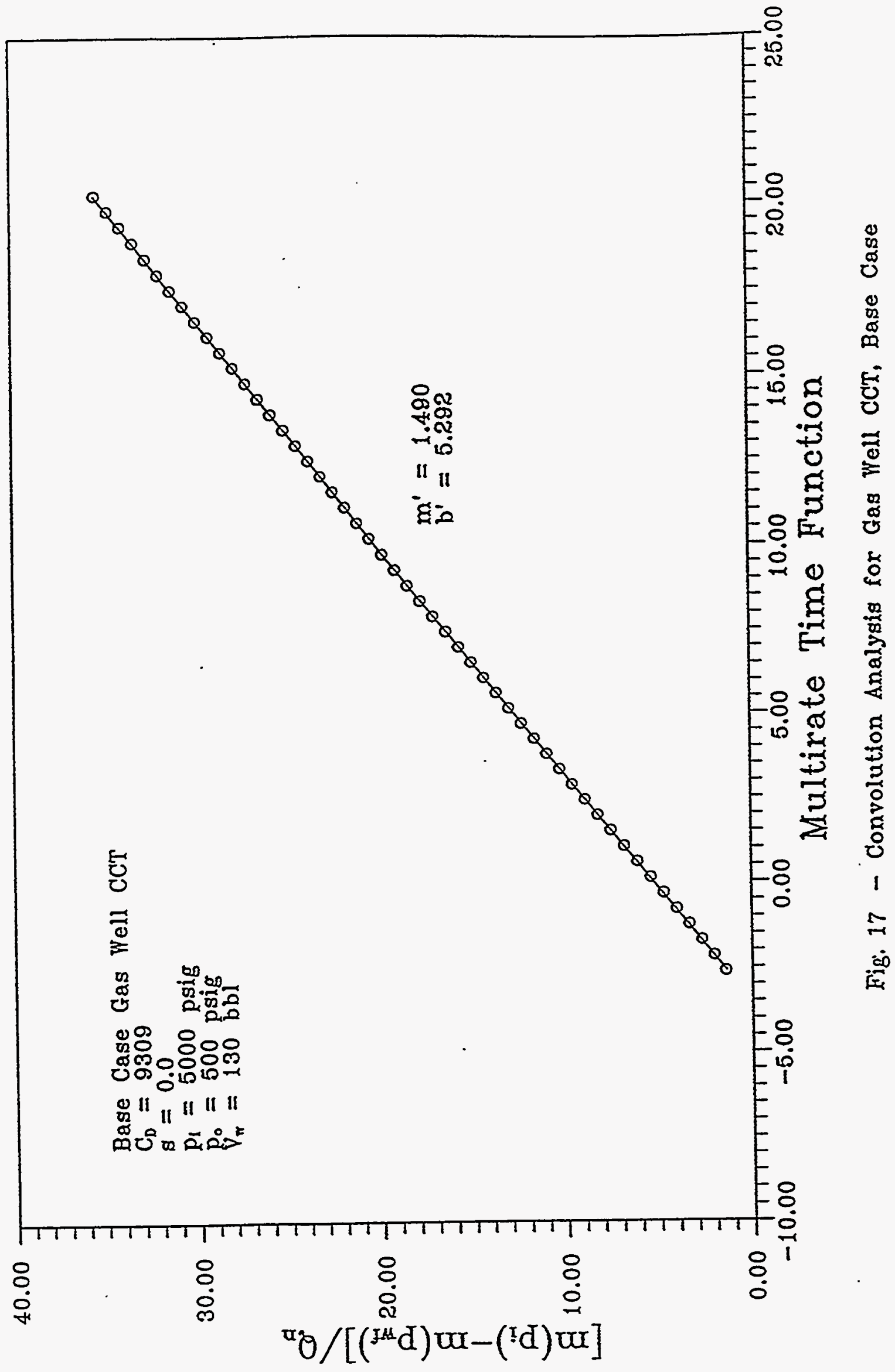




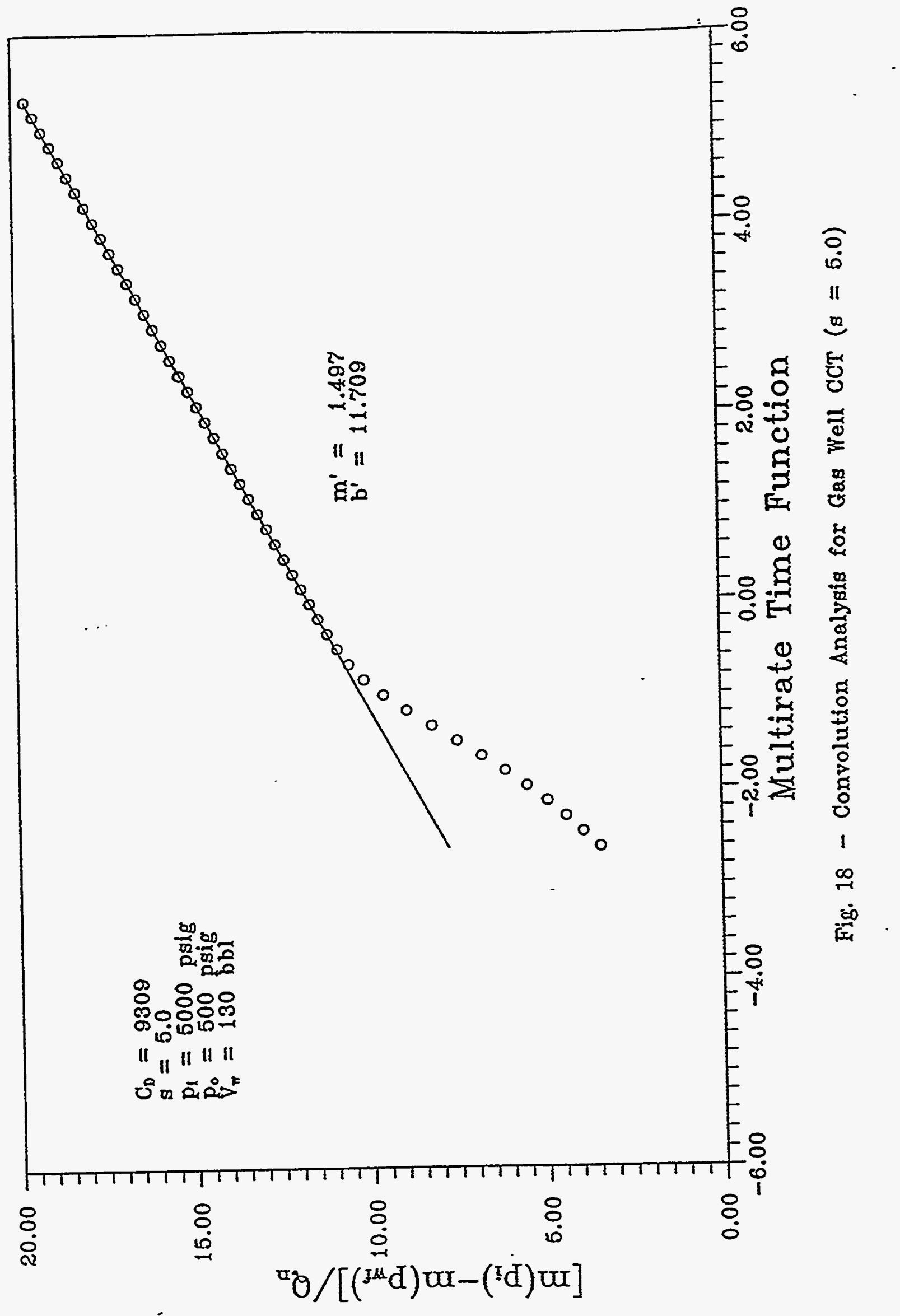




\author{
Section II \\ Outcrop Studies. \\ Geologic and Engineering Report \\ I. Azof, G. Martinez, M. Kelkar, D. R. Kerr
}

\begin{abstract}
Summary
This report summarizes the activities performed on the Bartlesville sandstone Outcrop located near Pryor. This outcrop is an analog of some of the most prolific producing fields in Oklahoma including the Glen Pool field. During the last year, Twelve well were drilled behind the outcrop to asses the three dimensional characteristic of the outcrop. The wells were cored and logged. In one of the wells, FMS (formation micro scanner Survey) log was run to obtain detailed small scale varaibilities. The cores from wells were slabbed and photographed. In addition, horizontal permeability measurements were made using minipermeameter and were verified by comparing the values with the core plugs. Simultaneously, geologic description of two wells as well as few vertical transects on the outcrop is also completed.
\end{abstract}

\title{
Introduction
}

Reservoir description involves combining various source of quantitative and qualitative data to describe reservoir characteristics. These data may involve well log and core data as well as production information. Using this information, we may be able to construct reservoir description which can be used to simulate the reservoir performance. Unfortunately, in many instances, the information regarding small scale variabilities of the reservoir properties may not be easily available. These properties are critical in describing inter well distributions of the reservoir properties.

One way to obtain information on the small scale variabilities is to use the outcrop analog of the producing reservoir. Outcrop, being easily accessible, can be studied in much more detail with respect to petrophysical properties can be quantified by detailed measurements and can be correlated to geologic depositional environment. This information, which quantifies the properties in reservoir description.

in the following sections, we detail the current progress on describing the small scale variabilities for a Bartlesville sandstone outcrop. The first section describes the data so far. The next section presents the preliminary results based on the data analysis. The last section discusses the work planned in the future. 


\section{Data Collection}

\section{Well Drilling}

During October and November 1991, twelve of the thirteen attempted wells were successfully drilled, cored and logged behind the outcrop face. Due to technical difficulties, the Holeman \# 9 well was not completed and only $7.5 \mathrm{ft}$ of core were recovered from Brown \# 2 well.

Figure 1 shows a base map of the study area, behind the outcrop face, where the 13 wells are located. Table 1 summarizes the work performed for each well. Of the total 820 $\mathrm{ft}$ of mostly 3-inch diameter cores cut in the wells, the total footage of recovered sandstone was $641 \mathrm{ft}$ and the total footage of siltstone and shale was $178.5 \mathrm{ft}$. In Brown \# 1 well, $6 \mathrm{ft}$ of core were cut with a 2-inch diameter tool. All these cores have been stored in cardboard boxes in sections of approximately $5 \mathrm{ft}$ per box.

\section{Core Processing and Manipulation}

For the processing and manipulation of the cores, $\mathrm{K} \& \mathrm{~A}$ Energy Consultants and the Amoco Production Company Research Center, both of Tulsa, have generously provided the use of their facilities.

All $641 \mathrm{ft}$ of sandstone and about $70 \mathrm{ft}$ of shale were slabbed at the $\mathrm{K} \& \mathrm{~A}$ and Amoco core facilities. The slabbing divided the cores along their lengths into two parts of 2-inch and 1-inch thickness. The rest of the examination has been done at the Amoco facility. The flat surface of the 2-inch-thick portion of each core was photographed using a 35-mm camera, mounted for this purpose on a special photographing device. The photographs were taken on dry and water-wetted surfaces (Figure 2). Also on this flat surface, permeability readings were taken approximately every 6 inches for all wells, using a portable minipermeameter. A total of 1388 permeability measurements were taken.

Staying as close as possible from the points where the minipermeameter readings were taken, 200 plugs, of 1 -inch diameter, have been planned. Because of the wider range of permeability values measured by the minipermeameter, the Holeman \# 3 well was chosen for the cutting of 119 core plugs. The remaining 81 core plugs are to be distributed among the rest of the wells, including some vertical plugs, whose location will depend on the geologic description. Vertical plugs will be selected to investigate the impact of sedimentologic features on petrophysical properties. Permeability and porosity measurements were taken on these 119 plugs in the Amoco Research Center Lab.

The geologic description of the cores is under way. The Holeman \# 3 core has been completely described and the Holeman \# 4 core is currently undergoing description. It is anticipated that all core descriptions will be completed by late October, 1992. 


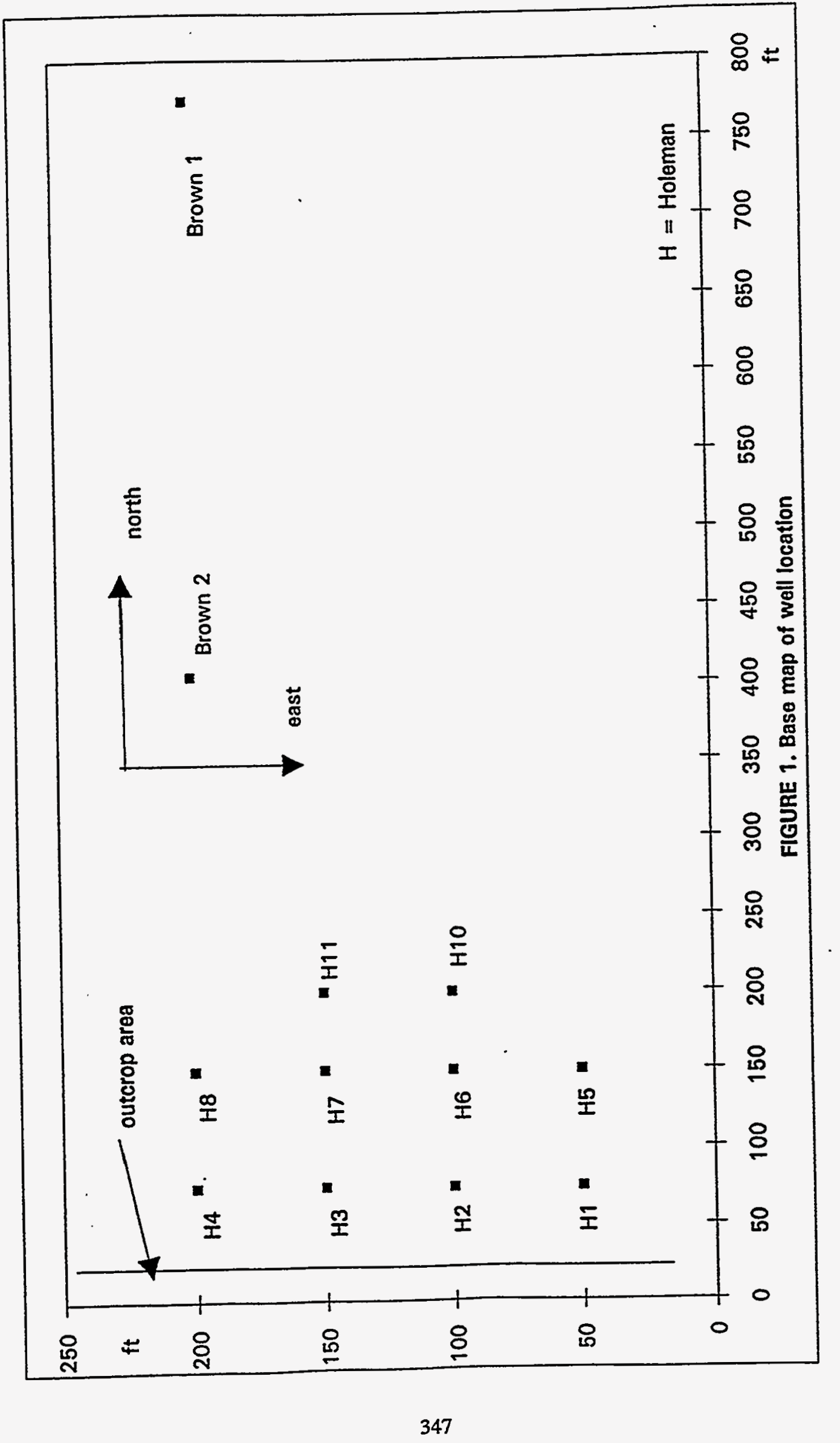




\begin{tabular}{|c|c|c|c|c|c|c|}
\hline \multirow[b]{2}{*}{ WELL } & \multicolumn{2}{|c|}{ CORED INTERVAL } & \multicolumn{3}{|c|}{ LOGGED INTERVAL } & \multirow[b]{2}{*}{ TD } \\
\hline & SANDSTONE & SLT \& CLAY & DIL & LDT/CNL & FMS & \\
\hline HOLEMAN\# 1 & $6^{\prime}-62^{\prime}$ & $62^{\prime}-73^{\prime}$ & $0^{\prime}-71^{\prime}$ & $0^{\prime} \cdot 74^{\prime}$ & $-\cdot$ & $77^{\circ}$ \\
\hline HOLEMAN\# 2 & $6^{\prime}-61.5^{\prime}$ & - & $0^{\prime}-70^{\prime}$ & $0^{*}-73^{\prime}$ & - & $75^{\circ}$ \\
\hline HOLEMAN \# 3 & $6^{t}-62^{\prime}$ & $62^{\prime}-73^{\prime}$ & $0^{\circ}-70^{\circ}$ & $0^{\prime}-73^{\prime}$ & $\cdots$ & $76^{\prime}$ \\
\hline HOLEMAN \# 4 & $6^{\prime}-63^{\prime}$ & $63^{\prime}-72^{\prime}$ & $0^{\prime}-70^{\prime}$ & $0^{\prime}-72^{\prime}$ & - & $75^{\prime}$ \\
\hline HOLEMAN \# 5 & $6^{\prime}-63^{\prime}$ & $63^{\circ}-73^{\circ}$ & - & $\cdots$ & $\therefore$ & -. \\
\hline HOLEMAN \# 6 & $6^{\prime}-63^{\prime}$ & $63^{\prime}-103^{\prime}$ & $0^{\prime}-106^{\circ}$ & $\begin{array}{ll}0 & -109 \\
\end{array}$ & $\cdots$ & $112^{\prime}$ \\
\hline HOLEMAN \# 7 & $6^{\prime}-56.5^{\prime}$ & $56.5^{\prime}-73^{\prime}$ & $\cdots$ & $\cdots$ & $\cdots$ & $\cdots$ \\
\hline HOLEMAN \# 8 & $6^{\prime}-56^{\prime}$ & $56^{\prime}-63^{\prime}$ & $\cdots$ & -- & $\cdots$ & $\cdots$ \\
\hline HOLEMAN \# 10 & $6^{\prime}-62^{\prime}$ & $62^{\prime}-72^{\prime}$ & - & $\cdots$ & $\cdots$ & $\ldots$ \\
\hline HOLEMAN \# 11 & $6^{\prime}-53^{\prime}$ & $53^{\prime}-67^{\prime}$ & $\cdots$ & $\therefore$ & $\cdots$ & $\cdots$ \\
\hline BROWN \# 1 & $0^{\prime}-92^{\prime}$ & $92^{\prime}-142^{\prime}$ & $0^{\prime}-138^{\circ}$ & $0^{\prime}-142^{\prime}$ & $0^{\prime} 138^{\prime}$ & $144^{\prime}$ \\
\hline BROWN \#2 & $\begin{array}{l}53^{\prime}-57 \\
67.5^{\prime}-71\end{array}$ & $\cdots$ & $0^{\prime}-93^{\prime}$ & $5^{2}-96^{4}$ & $\cdots$ & $99^{\prime}$ \\
\hline
\end{tabular}

Table 1. Summary of coring and logging activity 


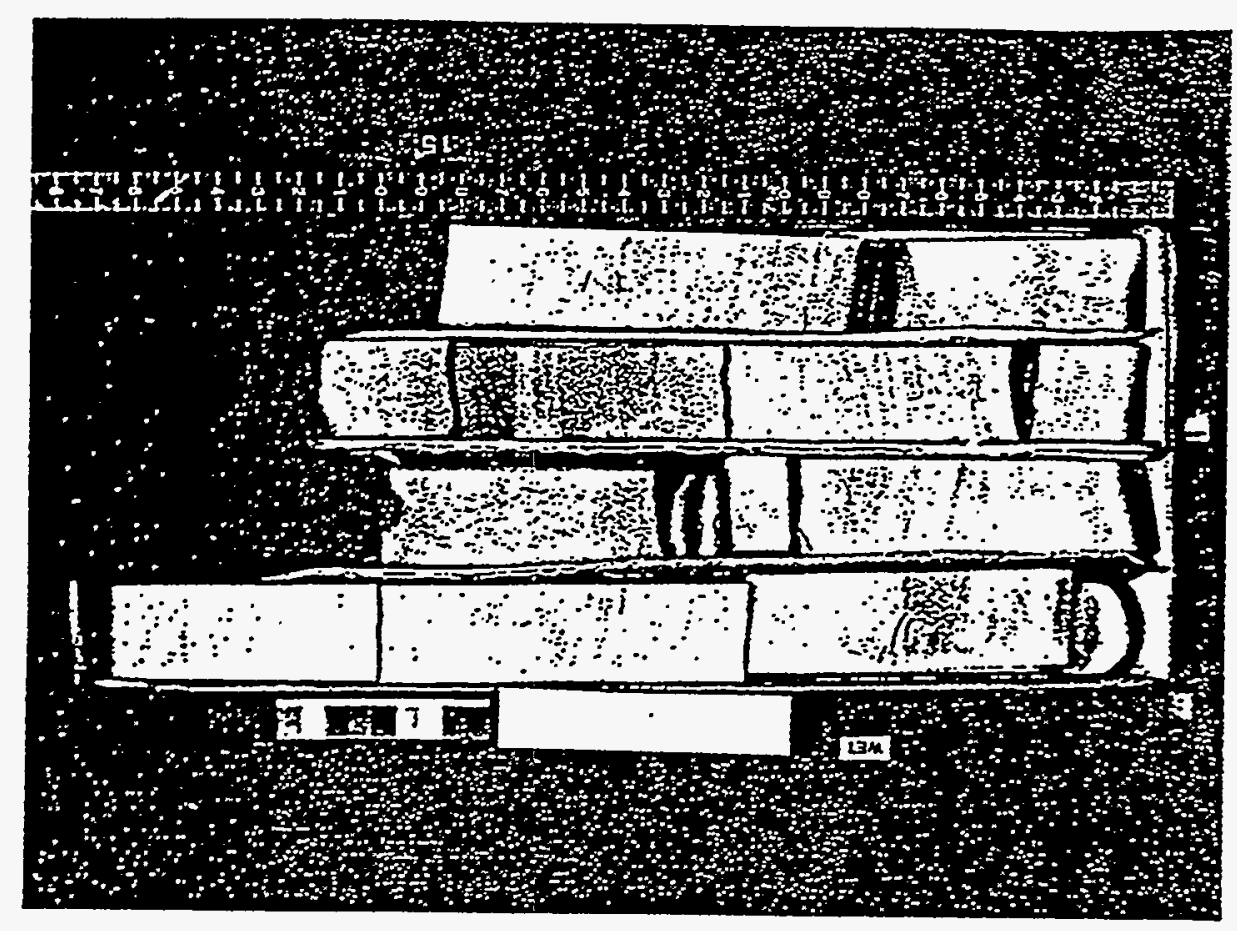

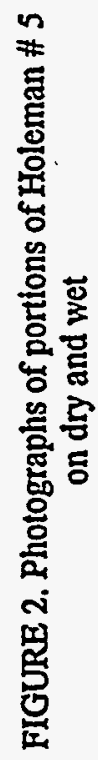

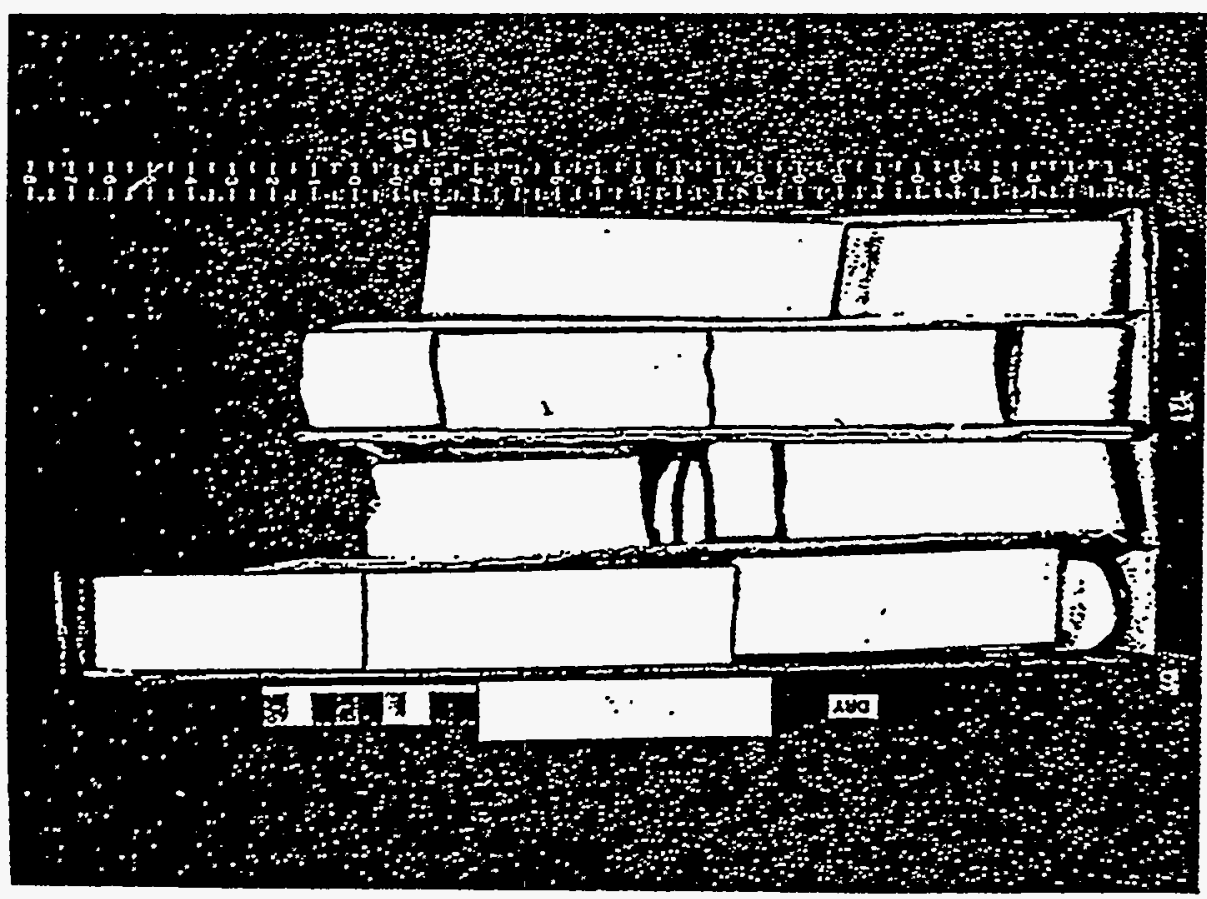




\section{Outcrop Surface}

The grid surveyed on the outcrop face is shown in Figure 3. It contains 16 vertical transects. Each vertical transect will be geologically described and measured for permeability, using the minipermeameter. The geological description consists of a measured section of each vertical transect. At present, the description is mainly restricted to aerially exposed sections. Part of the section is covered with soil and vegetation. Selected areas will be cleared for description.

So far, transects 1, 2, 3,13, and A have been completed; the rest are expected to be completed by late October, 1992. The results of these descriptions are to be used for mapping genetic units and associated facies changes on the photo panorama of the outcrop face.

Safety is maintained by using technical rock climbing gear. The field investigator ascends a statically anchored climbing rope along a transect and describes the exposed section. Height and thickness of each section are measured and recorded with the geologic description.

\section{Well Log Analysis}

Well log analysis has not been started yet. Well log character will be compared to core and outcrop descriptions, and it is expected to be aided by the interpretation of the FMS log. This tool was run only in the Brown \# 1 well, however it creates a picture of the borehole, based on an array of resistivity measurements around it. It can yield valuable information for the interpretation and characterization of formation properties, sedimentologic features, and permeability paths and barriers.

\section{Preliminary Results}

The Bartlesville Sandstone, in the study area, is composed of multiple stories of channel-fill deposits. In this study, each channel-fill story and its contiguous facies is referred to as a discrete genetic interval (contiguous, genetically related facies deposited during a short, discrete increment of time). At outcrop and in the Holeman \#3 well, up to 4 discrete genetic intervals have been recognized (Figures 4 and 5).

The Bartlesville Sandstone is dominated by channel-fill facies. The channel-fill facies of each discrete genetic interval is 15 to $20 \mathrm{ft}$ thick; some wedge out laterally. Erosion surfaces of variable relief typically separate either the upper, middle, or lower part of the underlying channel-fill deposits from the base of the overlying channel-fill deposits (Figure 5). Marked changes in texture and sedimentary structures occur across these erosional contacts. The basal few feet of fill consists of abundant mudstone ripup clasts in medium to thickly bedded structureless, medium sandstone (Figure 5). The succeeding upward portion of the channel fill consists medium-scale trough cross-stratified, medium 


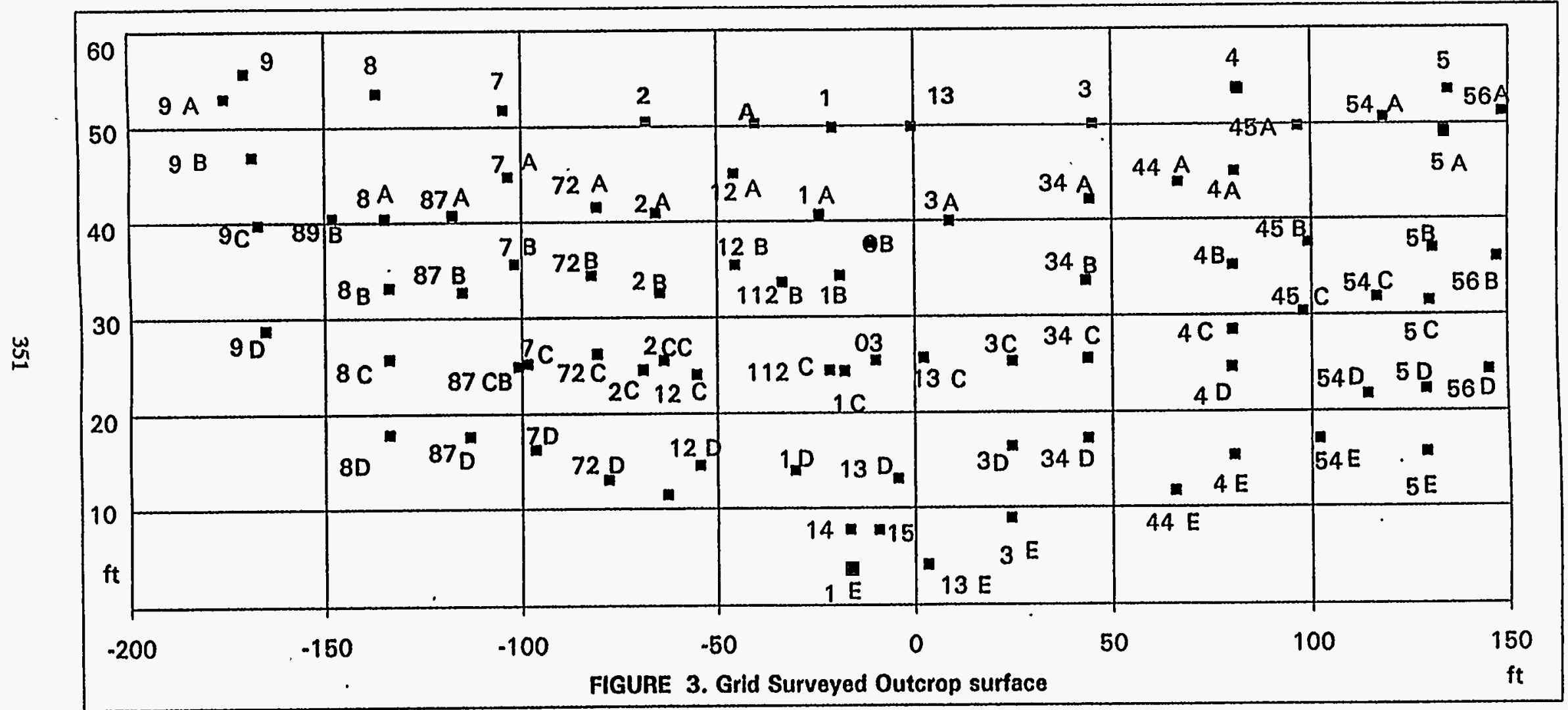




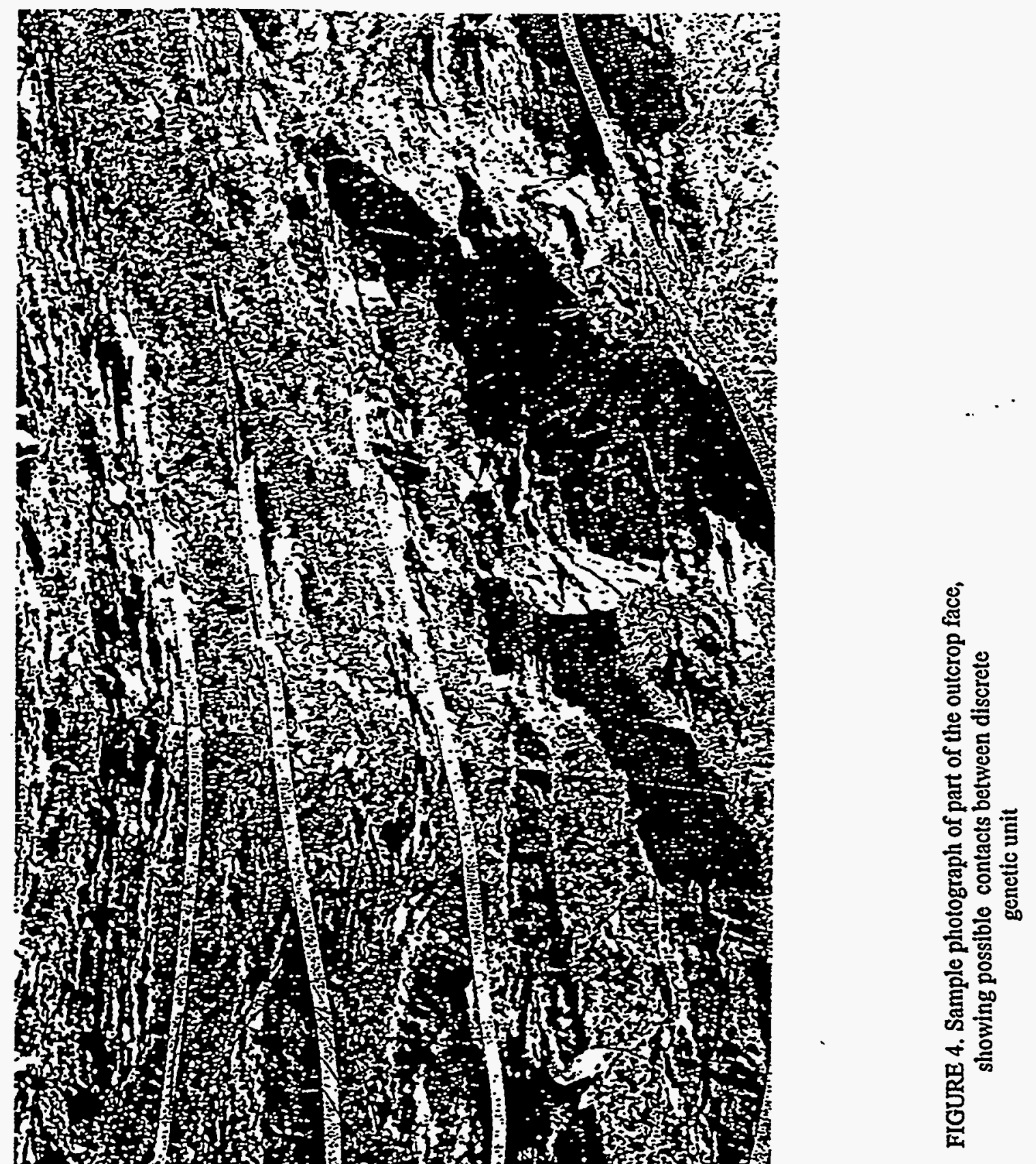




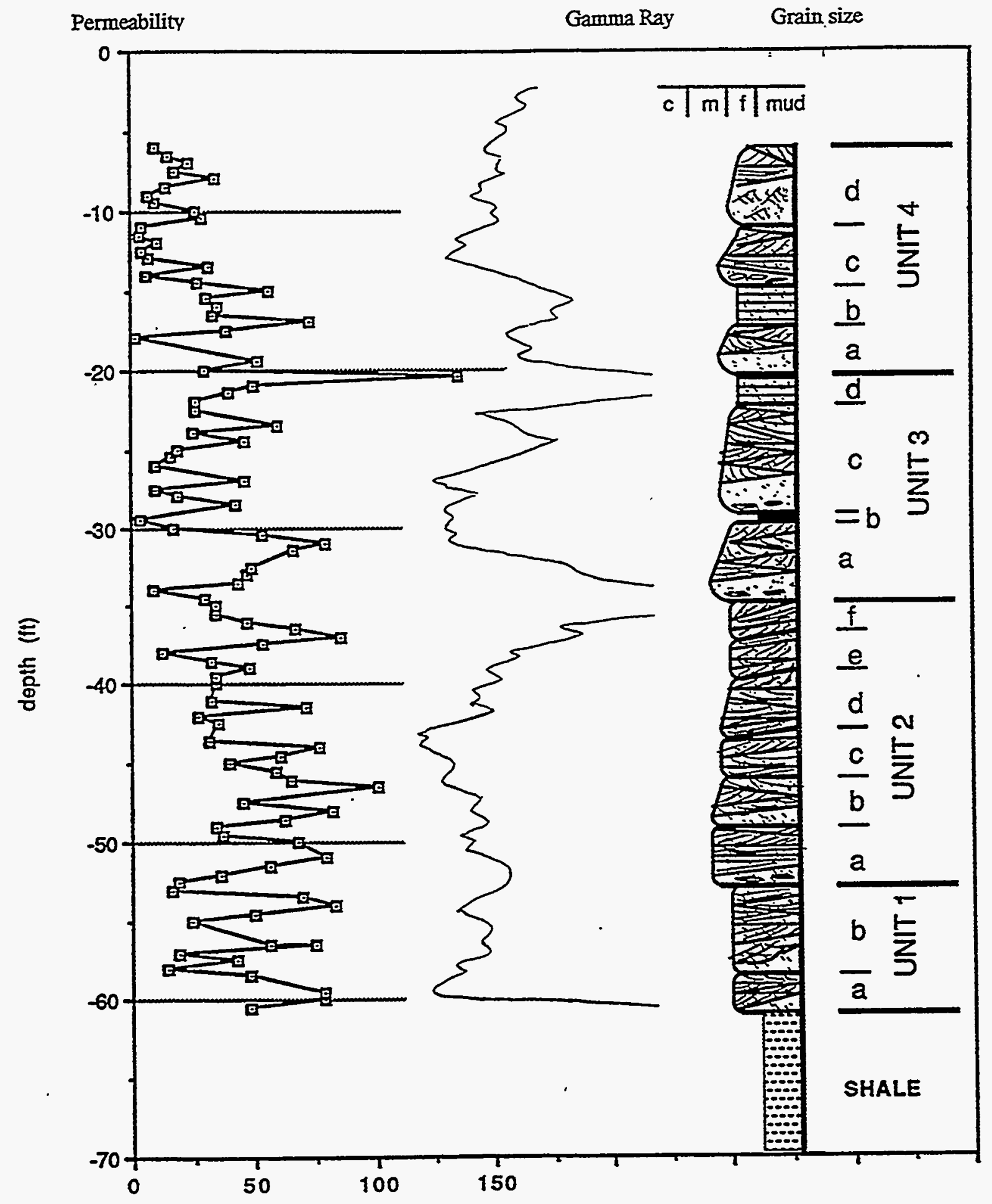

FIGURE S. Permeability and Gamma Ray profiles and geologic of Holeman \# 3. Discrete genetic unit are represented by number.Associated facies are identified by small letters 
to fine sandstone; intraclast lags are common at the base of cross-strata sets and within cross-strata foresets. The middle portion of the channel fill is characterized by low-angle parallel stratified and ripple laminated fine to very fine sandstone; however, ripup clasts and mud drapes are common throughout, some of which may be associated with lateral accretion surfaces (Table 2, unit 2). Isolated sets or cosets of low-angle medium-scale trough cross-stratification is also developed in the middle part of the channel fill. The upper fill, not well represented in core or outcrop because of the deep cutting of successive channel, is comprised of very fine sandstone and mudstone with evidence of early diagenesis related to paleosol formation and plant bioturbation. As a preliminary interpretation, the channel-fill facies is regarded as having been deposited by subaqueous crescentic dunes under or near the thalweg (lower part of channel fill) and chute-modified, lateral accretion bars (middle part of channel fill) with modification and mud sedimentation following bar migration and channel abandonment (upper part of fill).

The upper $6 \mathrm{ft}$. or so of sandstone exposed in the roadcut is tentatively regarded as crevasse splay facies. Other facies common to fluvial systems (e.g., levee and floodplain) have not been recognized at this time. This may be because such fine-grained facies are readily eroded at the surface; with continued study of cores cut behind the outcrop face and study of outcrops away from the road cut, such facies may be recognized. Alternatively, the Bartlesville Sandstone may have been deposited in an incised valley in which case the finer-grained facies may not be present or at least would be restricted in their distribution.

In the Holeman \# 3 well, permeability and porosity profiles along with log curves, were used in the recognition of contacts between genetic units. As seen in Figure 5, contacts tend to correlate with sharp deflections in the gamma ray curve responding to the higher clay content (intraclasts and abandonment fill) of deposits underlying and overlying these contacts. The contact between Units 1 and 2 does not seem to affect the gamma ray behavior as sharply as the other contacts because grain size does not show significant changes across this contact. In addition, a 2 -inch thick clay plug (abandonment fill), at a depth of $29 \mathrm{ft}$, does not correspond to a prominent gamma ray deflection; the thickness of the clay plug may be below the minimum resolvable by the gamma ray tool.

Lithologies associated with discrete genetic interval contacts and textural plus fabric variations within the channel-fill facies appear to affect pore system configuration. Although further assessment is required, general trends in permeability and lithology appear to correspond to each other (Figures 5 and 7).

The permeability measurements conducted on all cores show a range from $0.223 \mathrm{md}$ to 213 md ( Tables 3 and 4). The Holeman \# 3 core shows good correlation between minipermeameter and lab permeabilities, and between density log and lab porosities (Table 5 and Figure 6). The values that show higher disagreement will be validated. Figure 7 shows the variogram for the gross sand thickness Holeman \# 3 well. The variogram shows the fractional gaussian noise (fgn). Interpretation of variograms is in progress. 
TABLE 2. Core description, Holeman \# 3 well.

\section{Unit \# Depth Thickness Geologic Description/Interpretation}

4

$6^{\prime}-22^{\prime}$

$16^{\prime}$

3

$34^{\prime}-22^{\prime}$

$12^{\prime}$

2

$53^{\prime}-34^{\prime}$

$19^{\prime}$

1

$62^{\prime}-53^{\prime}$

$9^{\prime}$ d-) Poorly sorted, subrounded, lower very fine Ss. Low angle and climbing ripple, small scale stratification. Upper chute channel.

c-) Moderately to well sorted, rounded, upper fine Ss. Little stratification. Root-like structures and mud intraclasts at the base Lower chute channel.

b-) Moderately sorted, rounded, lower very fine to silty Ss. laminae intercalated with mud laminae. Highly contorted, complex stratification. Upper point bar.

a-) Moderately sorted, subrounded, upper fine Ss. Few ripple and tabular stratification. Mud intraclasts at the base. Lower point bar.

d-) Moderately sorted, subrounded, lower fine Ss. Low scale climbing ripple and trough cross stratification with mud drapes. Upper chute channel.

c-) Moderately sorted, subrounded, lower medium Ss. Few tabular, medium scale stratifications. Lower chute channel.

b-) One to two inch-thick laminated mud layer with concretions. Mud plug.

a-) Well sorted, rounded, upper fine Ss. Structureless at bottom. Low scale cross sets separated by mud drapes. Point bar.

Well sorted, rounded, medium to fine, Ss. Mud drapes separate large scale trough cross stratification sets $(a, b, c, d, f)$, probably representing accretionary surfaces. Point bar

b-) Well sorted, rounded, upper to lower fine Ss. Little stratification at the base with mud intraclasts. Becomes more parallel to low angle stratified to the top. Oblique fractures, filled with coal at the top are truncated by overlying unit. Chute channel.

a-) Well sorted, rounded, upper to lower fine Ss. Few stratification at base. Cross stratification increases to the middle arid becomes more abundant and complex to the top, Where contorted mud drapes separate mid-scale cross strata sets. Point bar. 
Table 3. Summary of permeability values for all wells.

\begin{tabular}{|c|c|c|c|c|c|}
\hline Core Well & Depch & Mrinioneren & Mescionsea & Meara & Variance \\
\hline & (fi) & $\mathrm{K}_{2}$ (md) & $K_{2}(n d)$ & & \\
\hline Holeman 1 & $6.5-61.5$ & 15.665 & 76.522 & 55.527 & 2513 \\
\hline Holeconen 2 & $6 .-61$ & 4.708 & 213.425 & 45.616 & 1113.242 \\
\hline Holeonan 3 & $6 .-62$ & 1.435 & 125.295 & 38326 & 591342 \\
\hline Holemen 4 & $6 .-625$ & 0.223 & 178.413 & 45.746 & 1222169 \\
\hline Holemans & $6 .-50.5$ & 1.465 & 12934 & 52553 & 992518 \\
\hline Holeman 6 & $6.5-63$ & 2511 & 158.034 & 53.805 & 982.498 \\
\hline Holeman? & $6-56.5$ & 1.526 & 122975 & 43.042 & 769.477 \\
\hline Holeming 8 & $6 .-55$ & 1.729 & 114.132 & 47.289 & 734.535 \\
\hline Holemen 10 & $6 .-625$ & 5 & 178.637 & 53.13 & 997.015 \\
\hline Holeman 11 & $6 .-53$ & 4.468 & 115.062 & 50.427 & 961.16 \\
\hline Brown 1 & $32-92$ & 0.517 & 138.663 & 36.617 & 816.021 \\
\hline Brown 2 & $55 .-71$. & 10.656 & 81.029 & 41.157 & 422662 \\
\hline
\end{tabular}


Table 4. Measured core data

\begin{tabular}{|c|c|c|c|c|c|c|c|}
\hline Depot(a) & Fow trie (oofsec) & Prestin) & $K=(\infty \infty)$ & $D=p t(\theta)$ & Fow date (cotsox) & Prescure(pis) & $\operatorname{Ke}(\infty)$ \\
\hline 65 & 2330 & st.4 & 67.15007 & 34 & 2390 & 547 & 7652168 \\
\hline 7 & 1650 & sect & 4755263 & 345 & 2300 & $\$ 47$ & 53.68151 \\
\hline 75 & 2050 & se. & 59.00054 & 35 & 2000 & $\$ 47$ & 185014 \\
\hline 8 & 200 & 54.4 & 63.4035 & 355 & 1960 & $\operatorname{set}$ & 6159009 \\
\hline 9 & 1500 & 54.4 & 022065 & 36 & 2300 & set & 63.15876 \\
\hline 9.5 & $2<50$ & sed & 70.0080 & 37 & 1000 & $\operatorname{set}$ & 2201109 \\
\hline 10 & 2050 & 54,4 & 70.6080 & 375 & 2380 & set & 7287999 \\
\hline II & 2400 & 523 & 7652168 & 38 & 2380 & 54.7 & 2583604 \\
\hline I1s & 1870 & ses & \$3.68156 & 385 & 1920 & $\operatorname{ses}$ & $63.15 \sqrt{76}$ \\
\hline 12 & 1700 & ses & 180014 & 39 & 1300 & $\operatorname{ses}$ & 20.09469 \\
\hline 125 & 200 & ses & 6159009 & 395 & 2350 & 518 & 725813 \\
\hline 13 & 2000 & ses & $63.15 \times 76$ & 40 & 2370 & 547 & 5224521 \\
\hline 14 & 2400 & 53.8 & 7201109 & us & 230 & 54.7 & $39328: 9$ \\
\hline ies & $2<0$ & 535 & 7257999 & 41 & 2370 & $5 \leqslant 7$ & 33.01271 \\
\hline is & 900 & ses & 2583604 & ats & 2370 & $\sec 7$ & 33.7413 \\
\hline Iss & 2200 & 545 & 63.15476 & 0 & 2370 & 547 & 0.06006 \\
\hline 165 & 700 & ses & 20.02459 & as & 2370 & 547 & 3202584 \\
\hline 17 & 2030 & 53.5 & 728813 & 0 & 2370 & set & 620075 \\
\hline 175 & 1850 & ses & 5224521 & os & 140 & 51.7 & 69.19825 \\
\hline 18 & 1370 & ses & 3932819 & $\mu$ & 2370 & 51.7 & 2esent1 \\
\hline 185 & 1150 & Ses & 3301271 & us & 1700 & 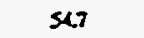 & 50.04006 \\
\hline 19 & 1180 & 54.6 & 33.7413 & is & 1930 & 547 & 68.62636 \\
\hline 195 & $15 \infty$ & $545^{\circ}$ & 0.06006 & es & 2360 & 587 & 30.02403 \\
\hline 20 & 1120 & 54.6 & 3202564 & $4 s$ & 2030 & 54.6 & 15.25734 \\
\hline 205 & 2000 & se. & 620075 & 455 & 1180 & $\sec 7$ & 47.18053 \\
\hline $2 I$ & 200 & 51.6 & 69.19805 & $Q T$ & 1150 & 547 & 41.010s \\
\hline 215 & 800 & $\$ 46$ & 2459111 & 175 & 2350 & $\mathrm{set}$ & 68.6007 \\
\hline 2 & 1750 & 51.6 & 50.04005 & $<$ & 230 & sus & 6550999 \\
\hline 225 & 2000 & se6 & 280505 & 085 & 2360 & $\sec$ & 6891231 \\
\hline $\mathbf{2 3}$ & 1050 & 546 & 30.02403 & $\theta$ & 2360 & $\operatorname{set}$ & 5781908 \\
\hline 235 & 1500 & set & 1528734 & os & 860 & $\operatorname{set}$ & $\$ 981367$ \\
\hline 24 & 1650 & 51.6 & 47,18003 & 50 & 2370 & 547 & 0835825 \\
\hline 205 & 1400 & 54.7 & dLotes & sos & 2350 & 547 & 25.73 .089 \\
\hline 25s & 2410 & $S C T$ & 086007 & SI & 2350 & 547 & 50.69903 \\
\hline 265 & 2300 & $5 \times 7$ & 6550999 & sis & 2000 & Set & 67.15007 \\
\hline 27 & 2410 & sic & 6891231 & $s 2$ & 2350 & ses & 47.55263 \\
\hline 275 & 2030 & $\sec$ & 5781968 & ses & 2350 & $\operatorname{set}$ & 59.00054 \\
\hline 285 & 2100 & 547 & 5981317 & $\$ 3$ & 2350 & 547 & 63.0035 \\
\hline 20 & 2400 & 547 & 6835825 & 535 & 2350 & 547 & 022566 \\
\hline 295 & 900 & set & 25.73089 & sr & 2350 & Set & 20.0000 \\
\hline 30 & 1780 & $\mathrm{set}$ & 50.69903 & ses & 2350 & 54.7 & 70.60845 \\
\hline 305 & 1230 & 546 & 67.15007 & 35 & 2350 & Set & $76 \Omega 2168$ \\
\hline 31 & 2000 & 58.7 & 1755263 & ss.s & 2100 & 547 & 53.68154 \\
\hline 315 & sso & $\operatorname{set}$ & sosososi & 56 & 2300 & SAT & 485014 \\
\hline 3 & 2030 & Set & 0.4035 & 565 & 1670 & $\operatorname{set}$ & $6 e s 9009$ \\
\hline 325 & 2250 & ser & 02066 & 57 & 2350 & $5<.7$ & 03.15775 \\
\hline 33 & 200 & $\operatorname{set}$ & 70.00815 & 58 & 1600 & 548 & 7201109 \\
\hline \multirow[t]{3}{*}{335} & 2390 & 54.7 & 70,0080 & $\cos$ & 2350 & 547 & 2287999 \\
\hline & & & & 61 & $\infty 0$ & 547 & 2583604 \\
\hline & & & & 615 & 750 & set & $63.15 \times 76$ \\
\hline
\end{tabular}


Table 4.(continued)

Holeman 2

\begin{tabular}{|c|c|c|c|c|c|c|c|}
\hline Depex(a) & Flow note $(x)$ & Prescin) (P) & $\operatorname{Ka}(\infty)$ & Depers (a) & Frow sete $(\infty)$ & Prosere (Bsia) & $K=(m \infty)$ \\
\hline 6 & 52 & 262 & 200502 & 305 & $3 \pi 2$ & 259 & 622304 \\
\hline 65 & $\$ 8$ & 262 & 9.39020 & 35 & 1100 & 269 & 165.6524 \\
\hline 7 & 200 & 25.4 & $S 127402$ & $35 s$ & $\infty$ & 255 & 85.65788 \\
\hline 75 & 345 & 252 & O2S50606 & 36 & 204 & 26.4 & 3235035 \\
\hline 8 & 90 & 26.2 & $1<5 \pi / 4$ & 365 & 381 & 258 & 6l.3535 \\
\hline 8.5 & 30 & 255 & (2707811 & 37 & 410 & 258 & 69.33988 \\
\hline 9 & 40 & 263 & 6.410088 & 375 & 723 & 25 & 1338174 \\
\hline 95 & so & 263 & 8.013008 & 38 & $\infty$ & 25.6 & 8258509 \\
\hline 10 & 115 & 259 & 1923789 & 385 & 105 & 268 & IS974 \\
\hline 105 & 64 & 252 & 1036516 & 39 & 105 & 268 & 159746 \\
\hline II & 30 & 263 & 1807800 & 395 & 200 & 26.4 & 31.71603 \\
\hline its & 390 & 25 & 7218367 & $\infty$ & 370 & 263 & S9206ss \\
\hline 12 & 300 & $25 s$ & 6293966 & 405 & $\$$ & 25.7 & 83.4044 \\
\hline 125 & 350 & 255 & 61.19134 & 41 & CS & 258 & 735572 \\
\hline 13 & 200 & 258 & 09.04528 & A1S & sso & 25.6 & 95.09157 \\
\hline 135 & 320 & 25.6 & 5532606 & 2 & 153 & 26.6 & 23.7615 \\
\hline 14 & 280 & 258 & .735400 & as & 330 & 262 & 53.450 .96 \\
\hline 145 & 170 & 26.1 & 2783097 & $B$ & 330 & 262 & 53.150 .06 \\
\hline 15 & 280 & 258 & 0735406 & CS & 620 & 25.4 & 109.6203 \\
\hline iss & 95 & 26.4 & 1506sit & $\mu$ & 325 & 263 & $52 \cos c$ \\
\hline 16 & 140 & 26.6 & 21.70055 & us & 368 & 253 & 6580525 \\
\hline 165 & 206 & 258 & 3483506 & $\alpha s$ & 200 & 252 & 4535193 \\
\hline 17 & 215 & 258 & 3636115 & 15s & $2 \pi 0$ & 263 & 1327046 \\
\hline 175 & 135 & 262 & 2186611 & 46 & 310 & 263 & 8.0000 \\
\hline 18 & 280 & 258 & 4735006 & 465 & 360 & 26.2 & 5830563 \\
\hline 185 & 356 & 25.6 & 61.20046 & 8 & $\infty$ & 26 & ogsonses \\
\hline 19 & 350 & 25.6 & 650897 & 85 & 99 & 273 & 1432191 \\
\hline 195 & 210 & 26 & 39.71591 & 18 & 134 & 2688 & 203807 \\
\hline 20 & 200 & 26 & 3300659 & 085 & 208 & 26.4 & $20 \cos$ \\
\hline 205 & 175 & 262 & 283406 & $\Leftrightarrow$ & 160 & 268 & 2034154 \\
\hline 21 & 320 & 258 & sct11893 & os & 395 & 252 & 6397862 \\
\hline $2 ! 5$ & 360 & 25.7 & $\$ 813683$ & so & 350 & 26.4 & SSSO30S \\
\hline 2 & 300 & 8 & 50.18881 & sos & 300 & 265 & 87.07841 \\
\hline 225 & 200 & 25.1 & 3270032 & SI & 130 & 272 & 180940 \\
\hline 23 & 300 & 258 & 50.7365 & Sts & 166 & 268 & $2525 \times 76$ \\
\hline 235 & 190 & 263 & 30.44958 & 52 & 191 & 26.7 & 2935807 \\
\hline 24 & 122 & 26.4 & 1936578 & 525 & 189 & 258 & 28.75392 \\
\hline 205 & 175 & 262 & 2833006 & 53 & 395 & 262 & 0397862 \\
\hline 25 & 160 & 263 & 2564175 & 535 & 76 & 27.4 & 10888608 \\
\hline 255 & 140 & 265 & 2196992 & st & 235 & 26.6 & 36.06642 \\
\hline 26 & 100 & 263 & 2564175 & ses & 340 & 25.4 & 5391725 \\
\hline 26.5 & 250 & 263 & $2 \cos 14$ & ss & 1180 & 252 & 213008 \\
\hline 27 & 6 & 268 & 1000105 & s5s & $\infty$ & 262 & 7936580 \\
\hline 275 & 65 & 258 & 9588513 & $\$ 5$ & 121 & 27.4 & 173320 \\
\hline 28 & 140 & 26.4 & 220122 & 565 & 460 & 253 & 73.720004 \\
\hline 285 & 85 & 268 & 1293166 & 57 & 103 & 263 & $\pi \operatorname{ses} 21$ \\
\hline 20 & 682 & 269 & 127.7091 & 575 & $w / \mathbf{a}$ & $\mathbf{z} / \mathbf{z}$ & $n / 2$ \\
\hline 205 & $460 \ldots$ & 255 & 80.000 & 58 & 25 & $\pi / 2$ & $x / 2$ \\
\hline 30 & $250 \quad:$ & 252 & $\leqslant 21 \times 00$ & 585 & Dx & a/z & $\boldsymbol{n} / \mathbf{z}$ \\
\hline 305 & 135 & 263 & 21.03523 & $\$$ & 100 & 259 & 24.050 \\
\hline 32 & 210 & 26.6 & 17.0836 & S9S & 240 & 26.5 & 37.66273 \\
\hline 31.5 & 130 & 265 & 20.40054 & 60 & 20 & 26.7 & 3735085 \\
\hline 32 & 236 & 26.1 & 3303594 & $\cos$ & 100 & 272 & 2337783 \\
\hline 325 & 400 & 255 & 80.020 & 61 & 240 & 268 & 3651291 \\
\hline 33 & 550 & 25.4 & 9720383 & & & & \\
\hline 335 & 382 & 252 & 69.09172 & & & & \\
\hline 34 & 236 & 25.6 & 36.65173 & & & & \\
\hline
\end{tabular}


Table 4.(continued)

Holeman 3

\begin{tabular}{|c|c|c|c|c|c|c|c|}
\hline Depth ( t ) & Sownte $(\infty)$ & Presure (Pii) & $K a(z x d)$ & Depth (A) & Gow rate $(\infty)$ & Presente (PÁ) & $\operatorname{Ka}(\operatorname{rod})$ \\
\hline 6 & 200 & 38.4 & 124073 & $3 e s$ & 340 & 392 & 20.1856 \\
\hline 65 & 90 & 388 & 5.470413 & 35 & 830 & 383 & 51.57782 \\
\hline 7 & 200 & 382 & 17.64075 & 35.5 & 740 & 38.4 & 46.051 \\
\hline 75 & 270 & 383 & I600ess & 36 & 1040 & 382 & 655227 \\
\hline 8 & 590 & 37.7 & 3832110 & 365 & 1070 & 382. & 67.41285 \\
\hline 85 & 260 & 38.4 & 16.18455 & 37 & 2500 & 40.4 & 83.13732 \\
\hline 9 & 145 & 38.8 & 881340 & 37.5 & os & 22 & 2137785 \\
\hline 95 & 175 & 38.7 & 、 10.70017 & 38 & 350 & 123 & 17.0870 \\
\hline 10 & 145 & 38 & 28.37813 & 385 & $\infty$ & 2 & 2132305 \\
\hline 105 & 376 & 38.1 & 23.83278 & 39 & 850 & 412 & 45.0601 \\
\hline 11 & 70 & 393 & 4131693 & 395 & 230 & 43 & 11.0174 \\
\hline its & 180 & 38.6 & 11.071ss & $\infty$ & 85 & 02 & $\angle .051<51$ \\
\hline 12 & 66 & 393 & 3595597 & $\omega s$ & $\infty 0$ & 0 & 21.32305 \\
\hline 125 & 174 & 38.8 & 1057613 & 4 & $\infty$ & as & 21.90576 \\
\hline 13 & 200 & 38.6 & . $\quad 1230173$ & 415 & 980 & 40.7 & 53.41248 \\
\hline 13.5 & 640 & 37.7 & $\angle 156901$ & 0 & 1000 & 40.5 & S9S2392 \\
\hline 14 & 950 & 373 & 6325072 & QS & 750 & 412 & 39.76315 \\
\hline ies & 28 & 38.1 & 27.1288 & 0 & 260 & Q.5 & 1285397 \\
\hline 15 & $\mu 0$ & 38.1 & 27.8894 & c.s & 500 & 415 & 3025081 \\
\hline 155 & 408 & 382 & 28.22519 & $\mu$ & 1320 & 403 & 7357353 \\
\hline 16 & 530 & 37.7 & 34001 & us & 1090 & 40.5 & 60.07507 \\
\hline 16.5 & 470 & 38 & 299724 & es & 820 & 49 & «1987 \\
\hline 17 & 980 & 373 & 6524811 & ess & 1460 & 42 & 8183794 \\
\hline 17.5 & 360 & 38.5 & 2103815 & 45 & 980 & $\omega 8$ & 53.11638 \\
\hline 18 & $\infty$ & 395 & 3500586 & 465 & 1000 & 399 & 95.78889 \\
\hline 18.5 & 80 & 39.6 & 1600571 & 48 & 620 & 418 & 3181664 \\
\hline 19 & 710 & 378 & 2583356 & 45 & 1060 & 4.7 & 57.77268 \\
\hline 19.5 & 700 & 348 & S186061 & $\infty$ & 1300 & 405 & 71.6017 \\
\hline 20 & ब10 & 38 & 3890036 & ess & 1100 & 408 & $\$ 9.02013$ \\
\hline 20.5 & 840 & 37.6 & $s(0) 28$ & $\theta$ & 800 & Ats & 270830 \\
\hline 21 & 730 & 378 & 47.12451 & os & 880 & 41.6 & 053701 \\
\hline 21.5 & 620 & 38 & 3953808 & so & 1320 & 405 & 7275146 \\
\hline 22 & 440 & 383 & 27SSASI & sos & 2160 & 39.6 & 1252054 \\
\hline 25 & 350 & 38.6 & 2152203 & st & 1360 & 40.6 & 7453793 \\
\hline 23 & 600 & 378 & 41.31476 & sis & 800 & 418 & 0.67208 \\
\hline 235 & 810 & 37.5 & 5326377 & $\Omega$ & 830 & Ats & 020595 \\
\hline 24 & 335 & 388 & 2030200 & sis & 380 & 24 & 1888005 \\
\hline 205 & 570 & 38.1 & 36.12088 & $\$ 3$ & 780 & is & 40.08212 \\
\hline 25 & 330 & 388 & 20.05818 & 535 & 1000 & 412 & 53.0175x \\
\hline 255 & 281 & 39 & 168795 & st & 1120 & 41.1 & $\$ 9.70659$ \\
\hline 26 & 230 & 29.6 & 15.19003 & ses & 720 & -41.6 & 373057 \\
\hline 265 & $w / 2$ & $w / 2$ & $x / 2$ & ss & 250 & B2 & 1191007 \\
\hline 27 & 730 & 379 & 46.8374 & sss & 1360 & 408 & 7262852 \\
\hline 275 & 460 & 39.4 & 2699376 & so & 1330 & 408 & 7208652 \\
\hline 23 & 520 & 38,4 & 323003 & 565 & 1130 & 112 & 5990982 \\
\hline 285 & 540 & 38.4 & 336107 & 57 & 177 & 02 & 8.0657 \\
\hline 29 & $\pi / a$ & $2 \sqrt{2}$ & $D / 2$ & 575 & 910 & 41.4 & 47.7210 \\
\hline 295 & 107 & 39.8 & 6.135832 & $\$ 8$ & 600 & 418 & 3489567 \\
\hline 30 & 640 & 382 & 4032171 & 585 & 1000 & $\infty .6$ & 89.3359 \\
\hline 305 & 810 & 38.1 & $51.3418 \theta$ & $S 9$ & 386 & 08 & 18.2805 \\
\hline 31 & 230 & 402 & 1280228 & s9s & ISSO & 48 & $8<0105$ \\
\hline 315 & 10080 & 378 & 09.71855 & $\infty$ & 1360 & 112 & 7.003 \\
\hline 32 & $\pi 0$ & 382 & 451205 & 605 & 1120 & 41.6 & 5800934 \\
\hline 325 & 640 & 38.6 & 3936555 & $6 \mathbf{l}$ & $\infty \infty$ & 0.4 & 2082008 \\
\hline 33 & 750 & 38.4 & 46.0060 & 612 & 32 & 44 & 1.055023 \\
\hline 335 & 360 & 392 & 213729 & as & 1360 & 412 & 7210385 \\
\hline 36 & 140 & 399 & 7980408 & $Q 2$ & 750 & 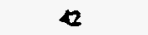 & 38.07088 \\
\hline
\end{tabular}


Table 4.(continued)

Holeman 4

\begin{tabular}{|c|c|c|c|c|c|c|c|}
\hline Deptin (if) & Flow tate $(\infty)$ & Presare (Pin) & $K_{2}(m d)$ & Depth (A) & Flow tate $(\infty)$ & Presure (Pis) & $\mathrm{Ke}(m d)$ \\
\hline 6 & 35 & 253 & 6258652 & 305 & 82 & 25.6 & $1<1 T B$ \\
\hline 6.5 & 30 & 253 & 5.361559 & 35 & 136 & 258 & 2300054 \\
\hline 7 & 17 & 255 & 2072151 & 355 & 205 & 24.6 & 39.77572 \\
\hline 75 & 26 & 255 & esissoe & 36 & 366 & 23.7 & 7808081 \\
\hline 8 & 36 & 253 & 6.078 & 365 & 270 & 243 & $s c 32821$ \\
\hline 8.5 & $7 x$ & 248 & 1402076 & 37 & 75 & 258 & $12 \operatorname{sen} 112$ \\
\hline 9 & 28 & 255 & 1895307 & 375 & 40 & 233 & 1029176 \\
\hline 9.5 & 90 & 248 & 17.05227 & 38 & 85 & 258.8 & 1037534 \\
\hline 10 & $\infty$ & 218 & 25.15758 & 385 & $\infty$ & 232 & Il3 5818 \\
\hline 105 & so & 25.1 & 9.178582 & 39 & 297 & 263 & 59.76103 \\
\hline 11 & 107 & 262 & 299054 & 395 & sos & 23.1 & 118.6569 \\
\hline IIS & 188 & 263 & 27.76775 & 10 & 360 & 239 & 7191452 \\
\hline 12 & DS & 238 & 503005 & 405 & 202 & 243 & 587505 \\
\hline 125 & 122 & 248 & 2025212 & 41 & 200 & 248 & 3789394 \\
\hline 13 & 71 & 252 & 1284165 & dis.s & 371 & 238 & 79.47775 \\
\hline 135 & 303 & 23 & 9123167 & 42 & $i \pi$ & 252 & 320137 \\
\hline 11 & 353 & 23 & secoss & es & 185 & 262 & 37.08544 \\
\hline 145 & 333 & 232 & 77.65286 & 13 & 362 & 238 & 775407 \\
\hline is & $\infty 0$ & 221 & 1518140 & B.s & 100 & 233 & 1029176 \\
\hline 155 & 37 & 28 & 9282032 & $\mu$ & 241 & 24.6 & 45.70073 \\
\hline 16 & $m$ & 21 & 1239513 & 45 & 165 & 246 & 3220553 \\
\hline 165 & 150 & 24.6 & 20.10019 & 45 & 360 & 238 & 77.12126 \\
\hline 17 & $\mathbf{3 1 3}$ & 23.2 & 7.18526 & 15s & 678 & 223 & 178.4135 \\
\hline 175 & $\pi$ & 25.2 & 12.1818 & 45 & 199 & 25.1 & 36.40840 \\
\hline 18 & 121 & 208 & 2225583 & 465 & 331 & 202 & 67.0517 \\
\hline 185 & 19 & 265 & 2881633 & $\$ 7$ & 250 & 266 & 1850598 \\
\hline 19 & 10 & 268 & 3007266 & 175 & 174 & 268 & 3295773 \\
\hline 19.5 & 139 & 252 & 25.1407 & 18 & 78 & 258 & 13.19140 \\
\hline 20 & 19 & 226 & 3764141 & 65 & 300 & 262 & 6.11314 \\
\hline 205 & 205 & 24 & 4699209 & $\Leftrightarrow$ & 360 & 238 & 77.12125 \\
\hline 21 & 275 & 238 & $\$ 891208$ & os & 208 & 208 & 39.4097 \\
\hline 215 & 300 & 236 & 6590053 & so & 300 & 212 & GLI1314 \\
\hline 22 & 288 & 238 & 5527024 & sos & 160 & 252 & 2853594 \\
\hline 225 & $\infty$ & 232 & 92.71983 & si & 110 & 255 & 1923156 \\
\hline 23 & 05 & 229 & $102.6 \mathrm{css}$ & sis & 200 & 28 & ICII3OS \\
\hline 23.5 & 70 & 259 & 11.7002 & 52 & 860 & 413 & $\mu 2161$ \\
\hline 24 & 141 & 269 & 26403211 & 525 & 690 & 418 & 35.40084 \\
\hline 205 & ss & 25.7 & 9.404866 & 33 & 5 & «s & 0.223106 \\
\hline 25 & IIS & 252 & 20.6899 & 535 & $\otimes$ & 418 & 3.00507 \\
\hline 255 & $\pi / 2$ & $z / 2$ & $n / 2$ & ss & 800 & 41.6 & 1352613 \\
\hline 26 & 156 & 205 & 30.6360 & ses & 1120 & 40.8 & 60.7040 \\
\hline 265 & 135 & 246 & 25.19377 & ss & 1060 & 408 & 563686 \\
\hline 27 & 156 & 266 & 30.20835 & sss & 360 & 028 & 1654533 \\
\hline 27.5 & 80 & 253 & 143050 & $\$ 6$ & $6 \infty 0$ & 419 & 33.08774 \\
\hline 28 & 96 & 252 & 1730335 & 565 & 640 & 0 & 320227 \\
\hline 285 & 113 & 25 & 2096176 & 57 & 700 & 41.6 & 399020 \\
\hline 20 & 146 & 268 & 27.65258 & 575 & 190 & 3.4 & 20035 \\
\hline 205 & 15s & 246 & 30.0703 & 58 & $11 \omega$ & 108 & Cos7245 \\
\hline 30 & 302 & 232 & 8391144 & 585 & 1130 & $\infty 9$ & 0090396 \\
\hline 305 & 250 & 239 & 5257832 & 9 & 730 & 11.7 & 3766607 \\
\hline 31 & 250 & 239 & 5287832 & Sg.s & $\infty$ & 26 & 1957167 \\
\hline 3ts & 10 & 2018 & $270 \operatorname{so1} 17$ & $\infty$ & 331 & 132 & 15.77088 \\
\hline 32 & 205 & 242 & 1583086 & 605 & 1270 & 108 & $\cos 40$ \\
\hline 325 & $153 \quad \therefore$ & 248 & 2358386 & 61 & 1920 & 398 & 110.1009 \\
\hline 33 & 185 & 245 & 3632051 & 61.5 & 82 & $\mu 7$ & 3.02718 \\
\hline 335 & $32 !$ & 23.7 & 60.55598 & 2 & 1380 & 408 & TLTOSS3 \\
\hline 36 & $<0$ & 23 & 207.191 & 625 & 2000 & $\infty$ & $113.385 t$ \\
\hline
\end{tabular}


Table 4.(continued)

Holeman 5

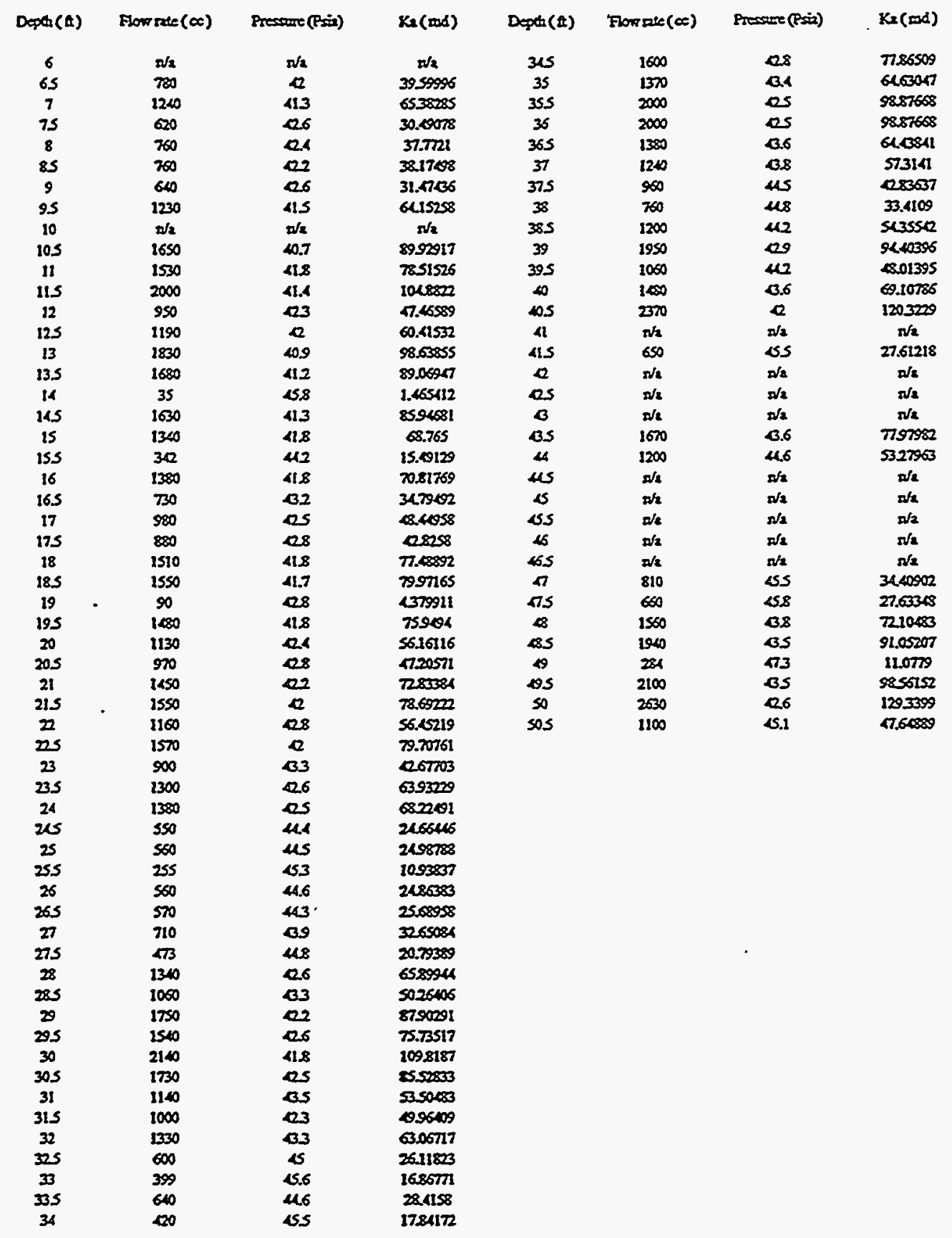


Table 4.(continued)

Holeman 6

\begin{tabular}{|c|c|c|c|c|c|c|c|}
\hline$D$ Datin(a) & Flow rute $(\infty)$ & Preare (Psi) & $\mathrm{Ka}$ (md) & Depu(A) & Flow rate (c) & Prescere (Pii) & $K_{x}(\operatorname{and})$ \\
\hline 65 & 530 & 28 & 25.79281 & 345 & 1250 & 48 & 64.14645 \\
\hline 7 & 1200 & 108 & 65.04047 & 35 & 100 & 42.4 & 76.03963 \\
\hline 75 & 850 & $\infty$ & 0.1538 & 355 & 980 & 26 & $\$ .19511$ \\
\hline 8 & 1200 & $\infty 9$ & $6<\cos 102$ & 36 & 1350 & 41.6 & To.0300 \\
\hline es & 750 & Q. & 372751 & 365 & 2400 & 39.4 & 140.837 \\
\hline 9 & 1090 & 118 & $55935 \pi$ & 37 & 100 & as & 2400233 \\
\hline 9.5 & 1230 & 41.5 & 64.15258 & 375 & 2600 & 388 & 1580031 \\
\hline 10 & $\omega 0$ & os & 20.65099 & 38 & 1000 & 0.7 & 1024615 \\
\hline 105 & 530 & 83 & 25.13203 & 385 & 1320 & $\$ 1.7$ & 68.10059 \\
\hline 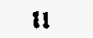 & 40 & BS & 21.12033 & 39 & 30 & 0.6 & 3408699 \\
\hline iss & 600 & 28 & 31.14003 & 395 & 50 & 28 & $28.712 \pi$ \\
\hline 12 & 1360 & +18 & 73.71253 & 4 & 590 & 32 & 28.12192 \\
\hline 125 & 1070 & 4.7 & 5520524 & us & 1410 & $\infty .7$ & 76.84897 \\
\hline 13 & 890 & 02 & 40001 & 4 & 1410 & 40.7 & 7684557 \\
\hline 135 & 940 & 2.1 & 47.40857 & as & 1560 & $\omega 3$ & 8695059 \\
\hline 14 & 830 & 2.3 & 41.6702 & 12 & 840 & 22 & 0.1934 \\
\hline ins & 980 & 2.1 & 4.40852 & as & ws & 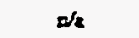 & ist \\
\hline 15 & 900 & 23 & 496769 & $B$ & 125 & 255 & 2185405 \\
\hline iss & 1500 & $\omega 8$ & 8130058 & cs. & 560 & 242 & 1140779 \\
\hline 16 & $2 / 2$ & $\pi / 2$ & $2 / 2$ & $\mu$ & 278 & 252 & 5028141 \\
\hline 16.5 & 1500 & 108 & 8130058 & us & 410 & 24.6 & 79ssi44 \\
\hline 17 & 1600 & 43 & 89.1804 & is & 200 & 252 & $\mathbf{S 2 \Delta 1 8 3}$ \\
\hline 175 & 404 & 42 & 1870956 & uss & 560 & 203 & 1086564 \\
\hline 18 & 710 & 133 & 3366744 & 45 & 383 & 24.8 & $\pi 5669$ \\
\hline 185 & 1200 & 41.6 & 022020 & 465 & 265 & 25,4 & 46.85385 \\
\hline 19 & 180 & 456 & 7.60803 & 47 & 500 & 24.4 & 9938558 \\
\hline 195 & 850 & 26 & 420188 & 475 & 281 & 252 & 5082401 \\
\hline 20 & 1550 & 108 & scolos & 48 & s5o & 24.4 & 1093241 \\
\hline 20.5 & 2180 & 39.7 & 125,729 & 185 & 300 & 253 & 53.64559 \\
\hline 21 & 660 & c.5 & 3097648 & $\theta$ & 460 & 248 & 8715606 \\
\hline 215 & 12000 & 41.7 & 66.04111 & os & 160 & 258 & 23.57703 \\
\hline$n$ & 2450 & $\infty$ & 138898 & so & 360 & 252 & 65.11261 \\
\hline 25 & 1730 & 408 & 93.75567 & sos & 410 & 25 & 752854 \\
\hline 23 & 1630 & 08 & 7932506 & SI & 2700 & 253 & 4828103 \\
\hline 235 & 960 & 28 & 46.71905 & sis & 210 & 25.6 & 3630773 \\
\hline 24 & 1050 & es & 5191026 & 52 & 230 & 255 & $40211<$ \\
\hline 2es & 1270 & 119 & 6482337 & s2s & 210 & 255 & 36.718 \\
\hline 25 & 1750 & 41.4 & 9220538 & 53 & 230 & 25.4 & 40.6656 \\
\hline 255 & 000 & 36 & 31.75226 & s3s & 135 & 258 & 228310 \\
\hline 26 & 60 & Q.J & 3159065 & si & 300 & 25.2 & 5126051 \\
\hline 265 & 1200 & 22 & 6027628 & ses & 91 & 25 & IS.0S\$S \\
\hline 27 & 530 & 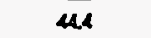 & 2376757 & ss & 310 & 252 & 5600019 \\
\hline 275 & 820 & 0.4 & 3868393 & SSS & 305 & 252 & 58.78022 \\
\hline 28 & 820 & 0.4 & 38.08393 & 56 & 440 & 248 & 8336657 \\
\hline 285 & 1220 & 24 & 60.63417 & sos & 280 & 255 & 4895307 \\
\hline 20 & 1390 & 21 & 70.19304 & 57 & 280 & 255 & 4855307 \\
\hline 205 & 1620 & 41.6 & $8 c 035 s 4$ & 575 & 220 & 25.6 & 3803667 \\
\hline 30 & 1000 & 41.6 & 2009005 & 58 & 165 & 258 & 2790507 \\
\hline 30.5 & 630 & 138 & 20.11926 & s8s & $\omega$ & 262 & 6.478848 \\
\hline 31 & 670 & 08 & 309581 & $\$ 9$ & 500 & 247 & 9585316 \\
\hline 315 & 1000 & as & 0.0834 & 595 & 125 & 259 & 2091075 \\
\hline 32 & 520 & 42 & 2355102 & $\infty$ & 31 & 265 & 1854769 \\
\hline 325 & 405 & 4.7 & 1789284 & 605 & 18 & 26.6 & 2795871 \\
\hline 33 & $8 \infty$ & 28 & 02058 & $\infty$ & D/2 & $\pi / 2$ & $\mathbf{D} / \mathbf{a}$ \\
\hline 335 & 1170 & 02 & 58.70038 & 615 & is & 263 & 2403914 \\
\hline \multirow[t]{3}{*}{34} & 990 & 26 & $\$ 0809$ & 62 & s/e & $2 / 2$ & $8 / \mathrm{s}$ \\
\hline & & & & 625 & 14 & 265 & 2190992 \\
\hline & & & & 63 & 16 & 265 & 2510848 \\
\hline
\end{tabular}


Table 4. (continued)

Holeman 7

\begin{tabular}{|c|c|c|c|c|c|c|c|}
\hline Depth (ft) & Flow rate $(\propto)$ & Pressure (Psia) & $K_{3}$ (md) & Depth (ti) & Flow rate $(\propto)$ & Pressure (Psin) & $\mathrm{K}_{2}$ (md) \\
\hline 6 & 43 & 49.4 & 1.525629 & 35 & 1100 & 38 & 70.1482 \\
\hline 6.5 & 373 & 48.8 & 1359048 & 35.5 & 1060 & 38 & 67.59735 \\
\hline 7 & 250 & 49.2 & 8948506 & 36 & 670 & 38.6 & 41.21081 \\
\hline 75 & 1100 & 47.6 & 42.31758 & 36.5 & 600 & 38.8 & 36.46942 \\
\hline 8 & 1150 & .479 & 43.63751 & 37 & 920 & 38.3 & 57.61397 \\
\hline 8.5 & 1200 & 47.8 & 45.74326 & 37.5 & 410 & 39.6 & 23.78293 \\
\hline 9 & 1410 & 47.6 & 5424344 & 38 & 1670 & 40.2 & 93.60915 \\
\hline 9.5 & 1600 & 47.5 & 61.83675 & 38.5 & 970 & 38.8 & 58.95889 \\
\hline 10 & 342 & 49 & 12.35052 & 39 . & 265 & 40.1 & 1493853 \\
\hline 10.5 & 1600 & 47.2 & 62.70073 & 39.5 & 282 & 40.2 & 15.80705 \\
\hline II & 950 & 48.2 & 35.56017 & 40 & 660 & 39.4 & 38.73018 \\
\hline 11.5 & 680 & 48.5 & 25.11138 & 40.5 & 450 & 39.9 & 25.65774 \\
\hline 12 & 690 & 48.5 & 25.48057 & 41 & 230 & 40.5 & 12.67639 \\
\hline 12.5 & 1180 & 47.8 & 44.98087 & 41.5 & 560 & 399 & 31.92953 \\
\hline 13 & 1130 & 48 & 42.68366 & 42 & 1030 & 39.4 & 60.41256 \\
\hline 13.5 & 1710 & 47.5 & 66.08803 & 42.5 & 460 & 40.4 & 25.49545 \\
\hline 14 & 2520 & 46.7 & 101.0878 & 43 & 790 & 39.7 & 45.56261 \\
\hline 145 & 1810 & 47.5 & 69.95283 & 43.5 & 840 & 39.8 & 48.16915 \\
\hline 15 & 620 & 48.7 & 22.69124 & 44 & 1280 & 39.5 & 74.67916 \\
\hline 15.5 & 126 & 493 & 4.490179 & 445 & 1150 & 39.7 & 66.32532 \\
\hline 16 & 1050 & 48.2 & 3930334 & 45 & 1660 & 39.3 & 9758016 \\
\hline 16.5 & 770 & 48.2 & 2882245 & 45.5 & 1720 & 39.3 & 1015216 \\
\hline 17 & 1220 & 479 & 46.2937 & 46 & 1720 & 39.3 & 1015216 \\
\hline 17.5 & 1300 & 47.8 & 49.55519 & 46.5 & 1210 & 40 & 0859852 \\
\hline 18 & 1560 & 47.6 & 60.01402 & 47 & 1600 & 39.7 & 9227871 \\
\hline 18.5 & 1336 & 48.8 & 48.67798 & 47.5 & 1890 & 39.6 & 109.6335 \\
\hline 19 & 890 & 478 & 3392525 & 48 & 710 & 41.2 & 37.64245 \\
\hline 19.5 & 305 & 48.5 & 1126319 & 48.5 & 520 & 41.6 & 2697469 \\
\hline 20 & 680 & 48 & 25.68575 & 49 & 740 & 413 & 39.0188 \\
\hline 20.5 & 740 & 36.7 & 51.16721 & 49.5 & 2120 & 39.6 & 1229751 \\
\hline 21 & 600 & 369 & 4096369 & so & 1540 & 40.4 & 85.35 .432 \\
\hline 21.5 & 430 & 37.3 & 28.62227 & sos & 1170 & 40.6 & 64.12455 \\
\hline 22 & 440 & 37.4 & 29.11336 & 51 & 167 & 42.5 & 8.256203 \\
\hline 225 & 590 & 37.1 & 39.77657 & sl.s & 580 & 41.9 & 29.60438 \\
\hline 23 & 560 & 37.2 & 3751818 & $S 2$ & 1400 & 40.8 & 75.88054 \\
\hline 23.5 & 320 & 37.5 & 2498794 & 52.5 & 450 & 422 & 2260361 \\
\hline 24 & 650 & 37 & 44.08811 & 53 & 660 & 42 & 3350766 \\
\hline 245 & 360 & 37.5 & 23.67279 & 53.5 & 560 & 42 & 28.12893 \\
\hline 25 & 380 & 37.5 & 24,98794 & 54 & 1030 & 41.5 & 53.72126 \\
\hline 255 & 200 & 37.9 & 1283216 & S45 & 560 & 423 & 27.97989 \\
\hline 26 & 135 & 38 & 8.609097 & ss & 1580 & $4 !$ & 8469413 \\
\hline 26.5 & 350 & 37.6 & 2287345 & 55.5 & 1300 & 41.1 & 6930200 \\
\hline 27 & 392 & 37.6 & 25.61826 & 56 & 58 & 432 & 2.764528 \\
\hline 27.5 & 82 & 383 & 5.135158 & 56.5 & 200 & 43.7 & 9221368 \\
\hline 28 & 1000 & 36.8 & 68.70668 & & & & \\
\hline 28.5 & 1500 & 362 & 107.105 & & & & \\
\hline 29 & 1100 & 36.7 & 76.05937 & & & & \\
\hline 29.5 & 650 & 37.6 & 44.43984 & & & & \\
\hline 30 & 350 & 382 & 2205093 & & & & \\
\hline 30.5 & 260 & 38.4 & 16.18455 & & & & \\
\hline 31 & 380 & 38.2 & 2394101 & & & & \\
\hline 315 & 250 & 38.6 & 1537717 & & & & \\
\hline 32 & 260 & 38.5 & 16.088 & & & & \\
\hline 32.5 & $n / a$ & $2 / 8$ & $2 / 2$ & & & & \\
\hline 33 & 430 & 38.7 & 2629185 & & & & \\
\hline 33.5 & 250 & 39.1 & 1492945 & & & & \\
\hline 34 & 860 & 37.4 & 56.90338 & & & & \\
\hline 34.5 & 470 & 38.7 & 28.7376 & & & & \\
\hline
\end{tabular}


Table 4.(continued)

Holeman 8

\begin{tabular}{|c|c|c|c|c|c|c|c|}
\hline Deph (A) & Flow ate $(\infty)$ & Presene (Pis) & $b=(\operatorname{lnd})$ & Depen (t) & Flow tute $(\infty)$ & Presere (Pis) & $b(m)$ \\
\hline 6 & 100 & 228 & $24 \cos s$ & 345 & 210 & 232 & 486791 \\
\hline 65 & SS & 23.6 & 1209056 & 35 & 318 & 22.4 & 8246334 \\
\hline 7 & $\pi / 2$ & $\mathbf{n} / 2$ & $n / 2$ & 355 & 286 & 22.6 & $7205 n \pi 1$ \\
\hline 75 & 85 & 232 & 1993676 & 36 & 300 & 225 & 76.67522 \\
\hline 8 & 93 & 228 & 227760 & 365 & 255 & 220 & 61.58562 \\
\hline 85 & 84 & 23 & 271008 & 37 & 123 & 243 & 247052 \\
\hline 9 & 119 & 227 & 20.55746 & 375 & 305 & 22.6 & 7684156 \\
\hline 95 & 176 & 22 & 88.0979 & 38 & 164 & 238 & 35.13302 \\
\hline 10 & 171 & 22.1 & 4635754 & 385 & 328 & 25 & 8383157 \\
\hline 105 & 78 & 232 & 18.08037 & 39 & 24 & 232 & 5655909 \\
\hline 11 & 100 & 232 & 23.17996 & 395 & 260 & 229 & 62720 \\
\hline its & 34 & 241 & 6.75822 & 40 & $3 i$ & 25.4 & 5.481016 \\
\hline 12 & $\pi$ & 235 & 17.14963 & ws & 210 & 23.4 & 4739299 \\
\hline 125 & 268 & 21.4 & 81.05046 & 41 & 308 & 228 & 75.0189 \\
\hline 13 & 27 & 21.6 & 7233836 & us & 187 & 242 & 29044 \\
\hline 13.5 & 276 & 215 & 8213258 & 0 & 200 & 23.5 & 14sesto \\
\hline Is & 248 & 21.7 & 71.0344 & os & 105 & 245 & 20.61945 \\
\hline $1<3$ & 18 & 25 & 3332554 & $\bar{B}$ & 305 & 228 & 7<6971 \\
\hline 145 & 285 & $2 t .4$ & 85.19172 & 13.5 & 263 & 232 & 6095320 \\
\hline 15 & 112 & 233 & 25.61506 & 4 & 298 & 228 & 7258274 \\
\hline 15s & 285 & 21.6 & 83.46733 & us & $x$ & 25.1 & 1280701 \\
\hline 16 & 187 & 2.1 & 08.0259 & 4 & 330 & 228 & 80.81981 \\
\hline 165 & 192 & 24 & 4.78919 & ess & 10 & 243 & 28.77383 \\
\hline 17 & 312 & 215 & 9281553 & 46 & 316 & 228 & 7739109 \\
\hline 175 & 150 & 228 & 36.73628 & 465 & 168 & 24 & 350081 \\
\hline 18 & 187 & 225 & 0.7902 & 47 & 01 & 22.1 & I141317 \\
\hline 185 & 60 & 245 & 11.72051 & 675 & 192 & 23.8 & (1)23134 \\
\hline 19 & 173 & 25 & L21604 & 48 & 390 & 226 & 582560 \\
\hline 195 & 186 & 25 & 4753864 & 185 & 300 & 228 & 8325859 \\
\hline 20 & 190 & 205 & 8.50097 & $\theta$ & 110 & 248 & 2084167 \\
\hline 205 & 201 & 205 & S213915 & os & 310 & 232 & $\pi 85787$ \\
\hline 21 & 256 & 27 & 70.05788 & so & 252 & 232 & 6536748 \\
\hline 21.5 & 205 & 2.1 & 65.4187 & sos & 34 & 228 & 8424853 \\
\hline 22 & 250 & 221 & 67.77418 & $s i$ & 278 & 23.4 & 6273929 \\
\hline 225 & 214 & 223 & 57.1533 & sis & $\pi / 2$ & $2 / 2$ & $2 / 2$ \\
\hline 23 & 100 & 23 & 38.11236 & $s 2$ & in & $2 / 2$ & $g / 2$ \\
\hline 235 & 92 & 232 & 21.32556 & ses & $2 / 2$ & $d / 2$ & $n / 2$ \\
\hline 24 & 95 & 238 & 203524 & 53 & 108 & 228 & 109.719 \\
\hline 215 & Its & 23.6 & 25200027 & 535 & 382 & 228 & 9355505 \\
\hline 25 & 10 & 25.6 & 1.728939 & si & 133 & 248 & 25.19947 \\
\hline 255 & 20 & 22 & 646313 & ses & 230 & 238 & 027192 \\
\hline 26 & 165 & 232 & 3824003 & ss & 152 & 245 & 2084II \\
\hline 265 & 245 & 22.6 & 61.72519 & & & & \\
\hline 27 & 120 & 238 & 25.70709 & & & & \\
\hline 275 & 182 & 232 & 218752 & & & & \\
\hline 28 & 240 & 226 & 00.4550 & & & & \\
\hline 28.5 & 177 & 233 & 40.48094 & & & & \\
\hline 29 & 195 & 23.1 & 4581803 & & & & \\
\hline 295 & 208 & 23 & 75.78625 & & & & \\
\hline 30 & 263 & 26 & 662001 & & & & \\
\hline 305 & 124 & 24 & 2585836 & & & & \\
\hline 31 & 230 & 23 & seress2 & & & & \\
\hline 31.5 & $2 / 2$ & $2 / 2$ & sfa & & & & \\
\hline 32 & she & $w$ & $w_{2}$ & & & & \\
\hline 325 & $2 / x$ & $\mathrm{I} / \mathrm{a}$ & $\mathrm{r} / \mathrm{a}$ & & & & \\
\hline 33 & $\pi / 2$ & $2 / a$ & $w$ & & & & \\
\hline 335 & $\pi / 2$ & $2 \sqrt{2}$ & $2 / 2$ & & & & \\
\hline 34 & 169 & 235 & 37.64009 & & & & \\
\hline
\end{tabular}


Table 4.(continued)

Holeman 10

\begin{tabular}{|c|c|c|c|c|c|c|c|}
\hline $\operatorname{Depta}(n)$ & Flow ate $(\infty)$ & Presure (Pis) & $K=(m d)$ & Depth ( $(\Omega)$ & Fow tate(c) & Presener (Psia) & $\mathrm{K}=(\mathrm{md})$ \\
\hline 6 & 600 & $\$ 9.4$ & 24.12622 & 345 & 1200 & 388 & 7293854 \\
\hline 65 & $\pi / 2$ & n/a & $D / 2$ & 35 & 810 & 40 & 1592139 \\
\hline 7 & 600 & os & 2331396 & 355 & 132 & 413 & 2277354 \\
\hline 75 & 640 & 0.6 & 250859 & 36 & 850 & 39.8 & 4.7059 \\
\hline 8 & 750 & 0.1 & 26.6098 & 365 & 630 & 383 & 39.15304 \\
\hline 8.5 & 1040 & 485 & 38.40565 & 37 & 1930 & 383 & 120.8641 \\
\hline 9 & 1100 & ess & 40.02136 & 37.5 & $\mathrm{n} / \mathrm{s}$ & $w / 2$ & $n / 2$ \\
\hline 95 & 870 & $\$ 2$ & 31.3408 & 38 & 680 & 40.4 & 37.68592 \\
\hline 10 & 740 & 9.6 & 26.02556 & 38.5 & 1500 & 38 & 95.65853 \\
\hline 105 & 3150 & 485 & Q 24578 & 39 & 1620 & 38 & 1033092 \\
\hline II & 370 & 50.6 & 1245166 & 39.5 & 268 & 419 & 13.67926 \\
\hline 115 & $\Delta 1$ & 49 & 25.65527 & 40 & 332 & 41.6 & 172023 \\
\hline 12 & 900 & $\mu$ & 3250136 & L.S.S & 740 & 203 & $41,2<593$ \\
\hline 12.5 & 1080 & 48.7 & 3952568 & 4 & 121 & 06 & S9s02021 \\
\hline 13 & 1820 & $\$ 8$ & 53.63788 & Ais & 1050 & 39.4 & 62.20302 \\
\hline 13.5 & 1230 & 175 & 47537 & $a$ & 213 & 123 & 10.60235 \\
\hline$u$ & 1510 & 978 & 5756026 & 25 & $\infty$ & ais & 23.47046 \\
\hline 125 & 970 & 48.8 & 3530256 & $B$ & 224 & 0.3 & 11.19196 \\
\hline 15 & 810 & 193 & 2886544 & B.S & 1750 & 38 & 111.5994 \\
\hline 155 & 1030 & 48.7 & 37.09574 & $\mu$ & 1650 & 38.2 & $10 \mathrm{css}$ \\
\hline 16 & 1190 & 482 & $\mu s 0379$ & us & . $\quad 800$ & 40.4 & 43399 \\
\hline 165 & 1530 & 87.7 & 5858033 & 45 & 1830 & 379 & 117.4113 \\
\hline 17 & 2090 & 465 & 86,63283 & ess & 1920 & 378 & 123940 \\
\hline 175 & 1550 & 87.6 & 60.01402 & 45 & 1190 & 39.6 & 69.0280 \\
\hline 18 & 1800 & 458 & 74,6021 & 465 & 100 & 389 & 85.80241 \\
\hline 185 & 2000 & 46.7 & 8020358 & 47 & 2600 & 368 & 178.6374 \\
\hline 19 & 175 & so & 6.003338 & 17.5 & 1350 & 39.4 & 7922003 \\
\hline 195 & $n / z$ & $\pi / 2$ & w/a & 18 & 1090 & 398 & 0250521 \\
\hline 20 & 780 & 28.1 & 2032015 & 485 & 1600 & 38.6 & 98.41387 \\
\hline 20.5 & 2000 & 453 & 81.75951 & $\theta$ & 1240 & 39.6 & 7192858 \\
\hline 21 & 1500 & 17.1 & 59.05582 & QS & 1510 & 388 & 93.60084 \\
\hline 215 & 1850 & 45.4 & 7526988 & so & 383 & 2.3 & 19.13025 \\
\hline 22 & 720 & 176 & 27.69878 & sos & 880 & 108 & «cs8532 \\
\hline 225 & 900 & 078 & 3430744 & si & 1250 & 39.6 & 702014 \\
\hline 23 & 2300 & 45 & 9537836 & sis & 700 & Ais. & 365056 \\
\hline 235 & 2350 & 45.8 & 9839193 & 52 & 520 & 0 & 2639997 \\
\hline 24 & 750 & 872 & 2939097 & 525 & 1300 & 39.5 & 75.40928 \\
\hline 265 & 790 & 459 & 31.3905 & 53 & 1440 & 393 & 800503 \\
\hline 25 & 1150 & 45.6 & 463002 & 53.5 & 1200 & $\$ 0.1$ & 6764517 \\
\hline 255 & 1150 & 46.1 & 46.7892 & 54 & $10<0$ & 40.6 & 569996 \\
\hline 26 & 750 & 15.7 & 31.55338 & ses & 1800 & 39.6 & 8527000 \\
\hline 265 & 920 & 415 & 1798604 & SS & sso & 023 & 27.45025 \\
\hline 27 & 970 & 39.2 & 5758533 & sss & 393 & 027 & 19.22601 \\
\hline 275 & 890 & 395 & 5192535 & 36 & 1000 & $\infty 0.5$ & 580163 \\
\hline 28 & 60 & $\$ 0.1$ & 38.89655 & 585 & 800 & 41.4 & $\mu \cos 0 s \alpha$ \\
\hline 285 & 34 & 43 & 1233838 & 57 & 1000 & 408 & $5 \times 30039$ \\
\hline 20 & 520 & $\$ .6$ & 28,4998 & 575 & 530 & Q. & $263 \times 107$ \\
\hline 205 & 500 & 40.2 & 3138989 & $\$ 8$ & 800 & 41.4 & 15.62378 \\
\hline 30 & 920 & 398 & 5275699 & 585 & 12000 & 405 & 65.13769 \\
\hline 305 & 800 & 398 & Sigris & $s g$ & 454 & 27 & 20984 \\
\hline 31 & 720 & 402 & 403880 & 592 & 1130 & $\$ .6$ & 6LO3255 \\
\hline 31.5 & 700 & $\$ .1$ & 0,40020 & 59.5 & 1380 & 399 & 7208373 \\
\hline 32 & 500 & $\$ 0.6$ & 30.09209 & 60 & $1<0$ & 399 & 820704 \\
\hline 325 & 830 & 39.7 & 4786958 & $\cos$ & 2210 & 38.1 & 140.081 \\
\hline 33 & 1230 & 38.7 & 7520091 & 6 & 900 & $\$ 1.6$ & 51.35586 \\
\hline 335 & 1310 & 38.4 & 81.56573 & 6.5 & 11so & 408 & 0233045 \\
\hline \multirow[t]{2}{*}{34} & 900 & 395 & $\$ 250878$ & 62 & $\pi 0$ & 0 & 3905227 \\
\hline & & & & 625 & 1930 & 42 & 87.0163 \\
\hline
\end{tabular}


Table 4.(continued)

Holeman II

\begin{tabular}{|c|c|c|c|c|c|c|c|}
\hline Deph (a) & Flow bate $(x)$ & Presore (Pis) & $K_{1}(\operatorname{tad})$ & Depth (A) & Flow pate $(x)$ & Presene (Pin) & $\mathrm{K}_{2}(\infty+\infty)$ \\
\hline 6 & 980 & 285 & 35.45137 & 3es & 2150 & 523 & 67.42689 \\
\hline 65 & 1300 & 48.4 & 820362 & 35 & 3000 & 523 & 9408403 \\
\hline 7 & $\$ 90$ & $\$ 2$ & 21.11847 & 35s & 2400 & 53.1 & 7284952 \\
\hline 75 & 1130 & $\$ 8.6$ & 415027 & 36 & 2000 & 526 & 8983522 \\
\hline 8 & whe & $\pi / 2$ & $n k$ & 36.5 & $30 \%$ & 533 & 920788 \\
\hline 85 & 1720 & $\Delta 8$ & 6496983 & 37 & 2400 & 533 & 73.46803 \\
\hline 9 & 1000 & 03 & 35.03634 & 375 & 780 & 548 & 221281 \\
\hline 9.5 & 1200 & 48.7 & 46.11445 & 38 & 1880 & 528 & S53065s \\
\hline 10 & 1720 & 4.4 & 63200356 & 385 & 1000 & Sut & 45.11164 \\
\hline 105 & 980 & $\otimes .6$ & 34,45008 & 39 & $\mathrm{~A} / \mathrm{L}$ & $\pi / 2$ & $D / 2$ \\
\hline I! & 800 & 99 & 27.70987 & 39.5 & $2 / 2$ & $\mathbf{D a}$ & $2 / 2$ \\
\hline Its & 1300 & 0.4 & 46.12365 & $\omega$ & $D / 2$ & sin & $2 / 2$ \\
\hline 12 & 2000 & $\$ 8.6$ & 7352614 & ws & she & $D / 2$ & $d / 2$ \\
\hline 125 & 2300 & 28.6 & ses5\$07 & 41 & $n / 2$ & $\pi / 2$ & $n / a$ \\
\hline 13 & 3060 & $<8.1$ & 115.0517 & 41.5 & $n / 2$ & $D / 2$ & s/8 \\
\hline 13.5 & 2800 & 183 & 1043359 & $Q$ & n/a & $n / 2$ & $n / 2$ \\
\hline 11 & 2000 & 0.6 & 2033934 & os & $8 / 2$ & $3 / 2$ & $\pi / 2$ \\
\hline ins & 2400 & $\$ .4$ & 85.15136 & 13 & 3100 & $\theta$ & 111901 \\
\hline is & 2350 & 4.5 & 83.01182 & os & 1410 & 522 & 4140212 \\
\hline iss & 2140 & 0.7 & $7<93008$ & 4 & 2560 & sis & 8209761 \\
\hline 16 & 1100 & sos & 2735458 & 4as & 3070 & 50.4 & locesss \\
\hline 165 & 910 & 512 & 298776 & $15^{\circ}$ & 3000 & 50.6 & 103.7369 \\
\hline 17 & $D / 2$ & ns & $n / a$ & 4s.5 & 3000 & 50.6 & 103.730 \\
\hline 175 & $\mathrm{n} / \mathrm{a}$ & $\mathrm{n} / \mathrm{z}$ & $\pi / 2$ & 46 & 240 & 51.6 & 78.77654 \\
\hline 18 & $\pi / 2$ & $\pi / 2$ & $2 / 2$ & 465 & 1120 & 524 & 3458056 \\
\hline 18.5 & $w / 2$ & $n / 2$ & $\pi / 2$ & 87 & 1500 & 522 & 4723520 \\
\hline 19 & $2 / 2$ & $=/ 2$ & $2 / 2$ & as & 1600 & 521 & $\$ 0 \$ 9393$ \\
\hline 195 & $\pi / 2$ & $n \sqrt{2}$ & $z / 2$ & $\infty$ & 9800 & 389 & 5921575 \\
\hline 20 & $\mathrm{~d} / \mathrm{a}$ & D/a & $2 / 2$ & 185 & $\pi 0$ & 39.4 & 0.18521 \\
\hline 205 & $20 / 2$ & $n / a$ & $2 / 2$ & 4 & 780 & 395 & $\angle 50761$ \\
\hline 21 & n/e & $w / s$ & $\sqrt{2}$ & os & 250 & 405 & 13.77269 \\
\hline 215 & 570 & 522 & 1794979 & so & $\pi / 2$ & $w / a$ & $\boldsymbol{I} / \mathbf{2}$ \\
\hline 22 & 2100 & sos & 7103188 & sos & 1270 & 386 & 78.11601 \\
\hline 20 & 1000 & 52 & 31.75237 & SI & 1000 & 39 & 60.06939 \\
\hline 23 & 1700 & si & 5628936 & sis & 1100 & 38 & 0.1452 \\
\hline 23.5 & 230 & 53.4 & 6.897474 & $s 2$ & 980 & 39.1 & 5852344 \\
\hline 24 & $\pi$ he & $=12$ & she & s2s & 560 & 399 & 3152053 \\
\hline 205 & 1510 & 51.7 & $4 s 1245$ & 53 & 375 & 403 & 2090165 \\
\hline 25 & 2300 & 50.8 & 768536 & & & & \\
\hline 255 & 2550 & 50.8 & 85.15408 & & & & \\
\hline 26 & 152 & 539 & $<408113$ & & & & \\
\hline 265 & 1950 & $s 2$ & 6191713 & & & & \\
\hline 27 & 1750 & 522 & 55.10001 & & & & \\
\hline 27.5 & 1120 & 527 & 3055353 & & & & \\
\hline 28 & $\infty \infty$ & 535 & 1792131 & & & & \\
\hline 285 & 2250 & 51.7 & 7239002 & & & & \\
\hline 29 & 1100 & 53 & 335201 & & & & \\
\hline 29 & 1800 & 522 & 56.60355 & & & & \\
\hline 30 & 2100 & 522 & 66.13081 & & & $=$ & \\
\hline 305 & 1000 & 528 & 30.72885 & & & & \\
\hline $3 !$ & 3060 & 509 & 101.7517 & & & & \\
\hline 315 & 1900 & 53.1 & 5767202 & & & & \\
\hline 32 & 2000 & 526 & 6195532 & & & & \\
\hline 325 & .2000 & 532 & 60.40318 & & & & \\
\hline 33 & 1700 & 533 & 51.18088 & & & & \\
\hline 335 & 2150 & 527 & 6533044 & & & & \\
\hline 34 & 520 & 54.7 & 1481005 & & & & \\
\hline
\end{tabular}


Table 4.(continued)

Brown I

\begin{tabular}{|c|c|c|c|c|c|c|c|}
\hline$D_{\text {epth }}(\theta)$ & Fow rate $(\infty)$ & Presolere (Psi) & $K_{1}(\mathrm{md})$ & Deth (A) & Flow nte $(\infty)$ & (Pن) (Pia) & $K=(\infty \infty)$ \\
\hline 32 & 12 & 452 & 0.517267 & 61 & 960 & 47 & 37.18118 \\
\hline 325 & 150 & 47 & 6.626979 & $6 ! 5$ & 950 & 47 & 35.76336 \\
\hline 33 & 13 & $\Delta 3$ & 055764 & 62 & 920 & 87.1 & 362209 \\
\hline 33.5 & so & 153 & 2.14778 & 625 & 1760 & 46.1 & 7263702 \\
\hline 36 & 500 & us & 21.98005 & 63 & $5 s 0$ & 8.7 & 21.06188 \\
\hline 35 & $\pi / a$ & $x / 2$ & $\pi / 2$ & 635 & 1700 & 159 & 20.83583 \\
\hline 35 & 21 & 45.7 & 028005 & 6 & 650 & 45 & 25.12118 \\
\hline 355 & 36 & 45.7 & 1.51452 & $6 \times 5$ & 160 & 13 & 5.216797 \\
\hline 36 & 1200 & $\mu .6$ & $\$ 3.27953$ & 65 & 1250 & 468 & 1090782 \\
\hline 365 & 206 & 45.6 & 8.020092 & 655 & 1150 & 46.9 & $\$ 5.70063$ \\
\hline 37 & 112 & 158 & 2689318 & 66 & 60 & 87.7 & 2450037 \\
\hline 375 & $D / 4$ & $w / a$ & $2 / 2$ & 605 & s/e & $\pi / 2$ & Ife \\
\hline 38 & ah & $2 \sqrt{2}$ & $\pi / 2$ & 67 & 1130 & 47.7 & 288565 \\
\hline 385 & 500 & 452 & 2457017 & 675 & 1500 & 473 & s8stoot \\
\hline 39 & 67 & 452 & 2752003 & 68 & sso & 48 & 20.77524 \\
\hline 39.5 & $\pi$ & 463 & 2002453 & 68.5 & 1100 & 172 & B.10575 \\
\hline 40 & 200 & QSB & 10.0485 & 69 & 1260 & 172 & 4937083 \\
\hline los & $\pi$ & $\$ 2$ & 33.62234 & 69.5 & 1830 & 46.6 & 73.75539 \\
\hline 11 & 650 & ess & 27.61218 & 70 & $\infty$ & 48 & 162046 \\
\hline 41.5 & 970 & 45 & 4022479 & 70.5 & 150 & 17.7 & 5.7415 \\
\hline 0 & 200 & 463 & 8.175951 & $\pi$ & 850 & 87.1 & 33.4506 \\
\hline os & 220 & 462 & 9.407175 & ins & 1200 & 87.4 & 46.59202 \\
\hline$B$ & 132 & 465 & 530231 & $\pi$ & 2400 & 462 & 9857922 \\
\hline os & 265 & 463 & 10.83314 & 725 & 3000 & 46.3 & 122.6393 \\
\hline 4 & 220 & 46.4 & 935784 & 73 & 920 & 87.7 & 3523078 \\
\hline 4.5 & 300 & 45 & 15.78816 & 735 & $1<\infty$ & 172 & 5486314 \\
\hline 15 & 650 & 159 & 27.0800 & 74 & 2600 & 46.4 & 105.7813 \\
\hline uss & 200 & 45.5 & 11.7031 & 745 & 600 & 679 & 25.04413 \\
\hline 16 & $\pi$ & 458 & $3265 \mathrm{~ms}$ & 75 & 800 & 478 & 30.4555 \\
\hline 465 & 1050 & us & 40000 & $75 S$ & 1850 & 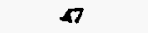 & 7357127 \\
\hline 87 & 1800 & $\mu 7$ & 80.40734 & 76 & 600 & 878 & 2592118 \\
\hline 875 & 160 & 46.4 & 1871568 & 765 & $n / 2$ & s/2 & $n$ \\
\hline 18 & 70 & 46.1 & 3095328 & $\pi$ & 2100 & 46.8 & 8381514 \\
\hline 485 & $\mathbf{w} / \mathbf{a}$ & $2 / 2$ & $8 / 2$ & 75 & 300 & $\$ 7.8$ & 11.0381 \\
\hline 4 & $M$ & $\pi / x$ & $\mathbf{D}$ & 78 & sso & 176 & 21.15879 \\
\hline so & wa & $n / 8$ & ara & 785 & 1700 & 46.8 & 6787464 \\
\hline sos & wa & $3 / 2$ & $a / 2$ & $x$ & 860 & 473 & 3351574 \\
\hline$S 1$ & $w / 2$ & $n / 2$ & D/a & 795 & 2000 & 45 & 829377 \\
\hline sis & 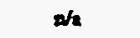 & ale & $\nabla / 2$ & 80 & 720 & 15.6 & 30.0797 \\
\hline 52 & 1350 & 152 & $\$ 819252$ & 805 & 1200 & 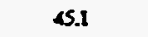 & 5587915 \\
\hline sos & 3000 & 138 & 1386631 & 81 & 570 & 46 & 23.63725 \\
\hline 53 & 1000 & 152 & 0890891 & 815 & 2050 & 42 & 9285717 \\
\hline 535 & 40 & 469 & 17.48546 & 82 & 870 & 15.7 & 3660192 \\
\hline si & 1020 & 469 & 6437828 & 825 & 850 & 159 & 35.41792 \\
\hline ses & 1400 & 15.7 & 5889965 & 80 & 900 & 459 & 3750132 \\
\hline ss & 2110 & 49 & $923021 !$ & 83.5 & 483 & 465 & $195 \$ 869$ \\
\hline SSS & 900 & 463 & 3924457 & 84 & $w / 2$ & w/a & I/ \\
\hline 56 & 1720 & $\Delta .1$ & $74 \operatorname{sos} 53$ & sus & $\sin$ & 465 & 23.48652 \\
\hline 565 & 1180 & 46.3 & 1823811 & 85 & $\infty$ & 46.8 & 1796082 \\
\hline 57 & 1120 & 463 & 15.78533 & 855 & 1000 & 159 & $\$ .00159$ \\
\hline 575 & 620 & 469 & 246386 & 86 & 1780 & 45.4 & $75 S 8311$ \\
\hline $\mathbf{8}$ & 40 & 48.1 & 1504074 & 86.5 & 1830 & 43 & 780888 \\
\hline 585 & 1000 & 46.5 & 40.018 & 87 & 1960 & $\Delta s$ & 8326134 \\
\hline sq & 1820 & $\$ 5.7$ & $76 \operatorname{ses} 4$ & 875 & 1000 & 46.6 & $10303 \theta$ \\
\hline s9s & 730 & 47.1 & 28.7405 & 88 & sso & 473 & 21.4557 \\
\hline 60 & 540 & 8.4 & 2096641 & 885 & 1210 & 46.4 & 022038 \\
\hline \multirow[t]{7}{*}{605} & 1400 & 465 & 56.69185 & .89 & 850 & 46.8 & 3353732 \\
\hline & & & & 895 & 1100 & 46.7 & 46.12561 \\
\hline & & & & 90 & 1300 & 465 & 52600 \\
\hline & & & & 905 & 1180 & 46.6 & 755812 \\
\hline & & & & 91 & 1000 & 46.8 & 3992626 \\
\hline & & & & 915 & sio & 479 & 193520 \\
\hline & & & & 92 & 350 & $\$ 1.1$ & 13.16065 \\
\hline
\end{tabular}


Table 4.(continued)

Brown 2

\begin{tabular}{|c|c|c|c|}
\hline Deptan(n) & Fion nete $(\infty / 5 x)$ & Presore (Psin) & $\mathrm{N}_{2}(\mathrm{md})$ \\
\hline ss & 700 & 23 & 3697486611 \\
\hline sss & 620 & 27 & 30.33110466 \\
\hline 56 & 1400 & 113 & 7351930762 \\
\hline s8.5 & 530 & $B$ & 2552529731 \\
\hline 57 & 1120 & 122 & $\$ 625786871$ \\
\hline 575 & 1240 & 03 & 619s5nitil \\
\hline 58 & 850 & 26 & 4120185065 \\
\hline$\$ 8.5$ & 2800 & $\$ 3.7$ & 13.00791478 \\
\hline 59 & 980 & 2.6 & $\$ .19510045$ \\
\hline 675 & 960 & 13 & 2523450078 \\
\hline$B Q$ & $s \infty 0$ & 4 & $225 \pi 0583$ \\
\hline 685 & 630 & 0.8 & 20.11925871 \\
\hline 69 & 1200 & 0 & 57.79312598 \\
\hline 69.5 & 240 & 4.6 & 10.6559251 \\
\hline 70 & 1700 & 132 & 81.0292744 \\
\hline 205 & 1000 & 0.7 & 46.4508385 \\
\hline$\pi$ & 40 & 445 & 19.63333583 \\
\hline
\end{tabular}


Table 5. Porosity and permeability data, Holeman:3.

\begin{tabular}{|c|c|c|c|c|c|c|c|c|}
\hline Deth (t) & 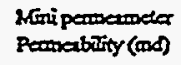 & 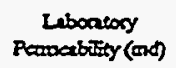 & $\begin{array}{l}\text { well Log } \\
\text { Pocosity }\end{array}$ & $\begin{array}{l}\text { Luboctiony } \\
\text { Porocity es }\end{array}$ & Deph (B) & 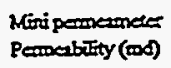 & $\begin{array}{c}\text { Leboretory } \\
\text { Permenstity (10) }\end{array}$ & $\begin{array}{l}\text { well Log } \\
\text { Porosity (S) }\end{array}$ \\
\hline 6 & 12.45 & 20.01 & 22 & 185 & us & 20.1 & 33 & \\
\hline 6.5 & 5.47 & 15.6 & & 205 & 35 & S:.97 & 372 & \\
\hline 7 & 17.61 & 239 & & 192 & 35.5 & 46.06 & 37 & \\
\hline 75 & 16.9 & 18.5 & & 19.3 & 36 & 655 & 50.6 & 215 \\
\hline 8 & 3832 & 34 & 23 & 19.4 & 36.5 & 67.41 & 79 & \\
\hline 85 & 162 & 14.6 & & 19.2 & 37 & 83.14 & 881 & \\
\hline 9 & 8.81 & 697 & & 17.1 & 37.5 & $2 i 3$ & 58.1 & \\
\hline 95 & 10.7 & 957 & & 179 & 38 & 175 & $1<7$ & $\mathbf{D} / \mathbf{2}$ \\
\hline 10 & 2837 & 255 & 22 & 192 & 385 & 2132 & 325 & \\
\hline 10.5 & 23.83 & 28.9 & & 19.7 & 39 & 15 & 52 & \\
\hline 11 & 413 & 447 & & 16 & 395 & IIs & 38.1 & \\
\hline 115 & 11.07 & 357 & & 165 & $\infty$ & 2132 & 38.1 & 19 \\
\hline 12 & 389 & 10.6 & 19.5 & 19.6 & 405 & 21.9 & 0.7 & \\
\hline 125 & 1057 & 492 & & 17.6 & 41 & 21.9 & 324 & \\
\hline 13 & 123 & 639 & & 16.6 & 415 & 53.4 & 71.8 & \\
\hline 13.5 & Als & 321 & & 19.6 & $Q$ & sos & 27 & 19 \\
\hline 11 & 63.25 & 925 & 225 & 17.6 & 425 & 39.7 & $\pi / 2$ & \\
\hline is & 27.12 & 275 & & 19.4 & $B$ & $n / 2$ & $n / 2$ & \\
\hline 15 & 27888 & 558 & & 19.6 & 435 & 302 & 31.4 & \\
\hline Iss & 282 & 30.5 & & 19 & 4 & 73.6 & $\pi 3$ & 205 \\
\hline 16 & 34.4 & 35.4 & 21 & 198 & ks & 60 & 64.2 & \\
\hline 16.5 & 299 & 33.7 & & 19.2 & 45 & $\mu 2$ & 399 & \\
\hline 17 & 652 & 73.4 & & 19.6 & ess & 81.8 & 582 & \\
\hline 175 & 21.63 & 388 & & 18.7 & 46 & $\$ 3.11$ & 65.4 & 21 \\
\hline 18 & 3.5 & 1.72 & 16 & 18.7 & 465 & 95.78 & 100.9 & \\
\hline 185 & 464 & $\mathrm{a} / \mathrm{R}$ & & $8 / 2$ & 47 & 31.8 & $n / 2$ & \\
\hline 19 & vs & $2 / 2$ & & $n / 2$ & ars & 57.7 & 45 & \\
\hline 195 & 54.8 & 51.2 & & $n / 2$ & 48 & 71.64 & 823 & 19 \\
\hline 20 & 38.9 & 20.4 & 21 & 19.4 & 485 & 59.6 & 626 & \\
\hline 205 & 548 & 136 & & 20.6 & $\theta$ & 27 & 34.1 & \\
\hline 21 & 8.12 & Q.4. & & 18.6 & os & os & 375 & \\
\hline 215 & 395 & 39.4 & & 28.6 & so & 125 & $n / 2$ & \\
\hline 2 & 275 & 26 & 20 & 189 & 505 & 125 & $8 / 2$ & \\
\hline 205 & 215 & 263 & & 182 & SI & 745 & 80 & \\
\hline 23 & 113 & $n \sqrt{2}$ & & $2 / 2$ & sis & 156 & 56 & \\
\hline 235 & 5326 & 593 & & 19.4 & 52 & 03 & 36 & \\
\hline 24 & 2036 & 896 & 195 & 17 & s2s & 189 & 19.1 & \\
\hline 215 & 36.12 & 46.01 & & 19.4 & 53 & 10.6 & 162 & \\
\hline 25 & 20.06 & 18.7 & & 17.7 & 535 & 53 & 70.06 & \\
\hline $25 s$ & 1687 & 16.6 & & 179 & 54 & Sي.7 & 70.06 & \\
\hline 26 & 152 & 9.63 & 195 & 162 & ses & $\$ 9.7$ & 835 & \\
\hline 265 & $w / 2$ & $n / 2$ & & $\mathbf{w} / \mathbf{z}$ & s5 & 119 & 245 & \\
\hline 27 & 45.8 & css & & 19 & ss.s & 726 & 75 & \\
\hline 275 & 259 & 1002 & & 269 & 58 & 721 & 93.4 & 16 \\
\hline 28 & 324 & 189 & 18 & 18.7 & 555 & $\$ 99$ & 565 & \\
\hline 285 & 33.6 & 03 & & 18.4 & 57 & 8.4 & 18.6 & \\
\hline 20 & $\mathrm{~s} / \mathrm{h}$ & $\overline{w / 2}$ & & $\mathrm{n} / 2$ & 57.5 & R.7 & 26 & \\
\hline 205 & 6.1 & 336 & & 119 & 88 & 349 & 140 & \\
\hline 30 & 4032 & 172 & 205 & 168 & 585 & 893 & $\Delta$ & \\
\hline 305 & 5134 & 53.1 & & 18.6 & $s$ & 18.7 & I/ & \\
\hline $3 t$ & 1259 & 793 & & 19.4 & 595 & 81 & 841 & \\
\hline 315 & 6.7 & 65.7 & & 198 & 60 & 71 & 79 & \\
\hline 32 & $n / 2$ & $n / 2$ & Dfe & n'a & 60.5 & 58.1 & 48 & \\
\hline 325 & 39.4 & $\$ .1$ & & 19 & 61 & 298 & & \\
\hline 33 & 46.6 & 172 & & 18.6 & 61.5 & 72.1 & & \\
\hline 335 & 21.4 & 18.1 & & 182 & 62 & 38 & & \\
\hline 34 & 79 & 11.t & 205 & 17.6 & & & & \\
\hline
\end{tabular}




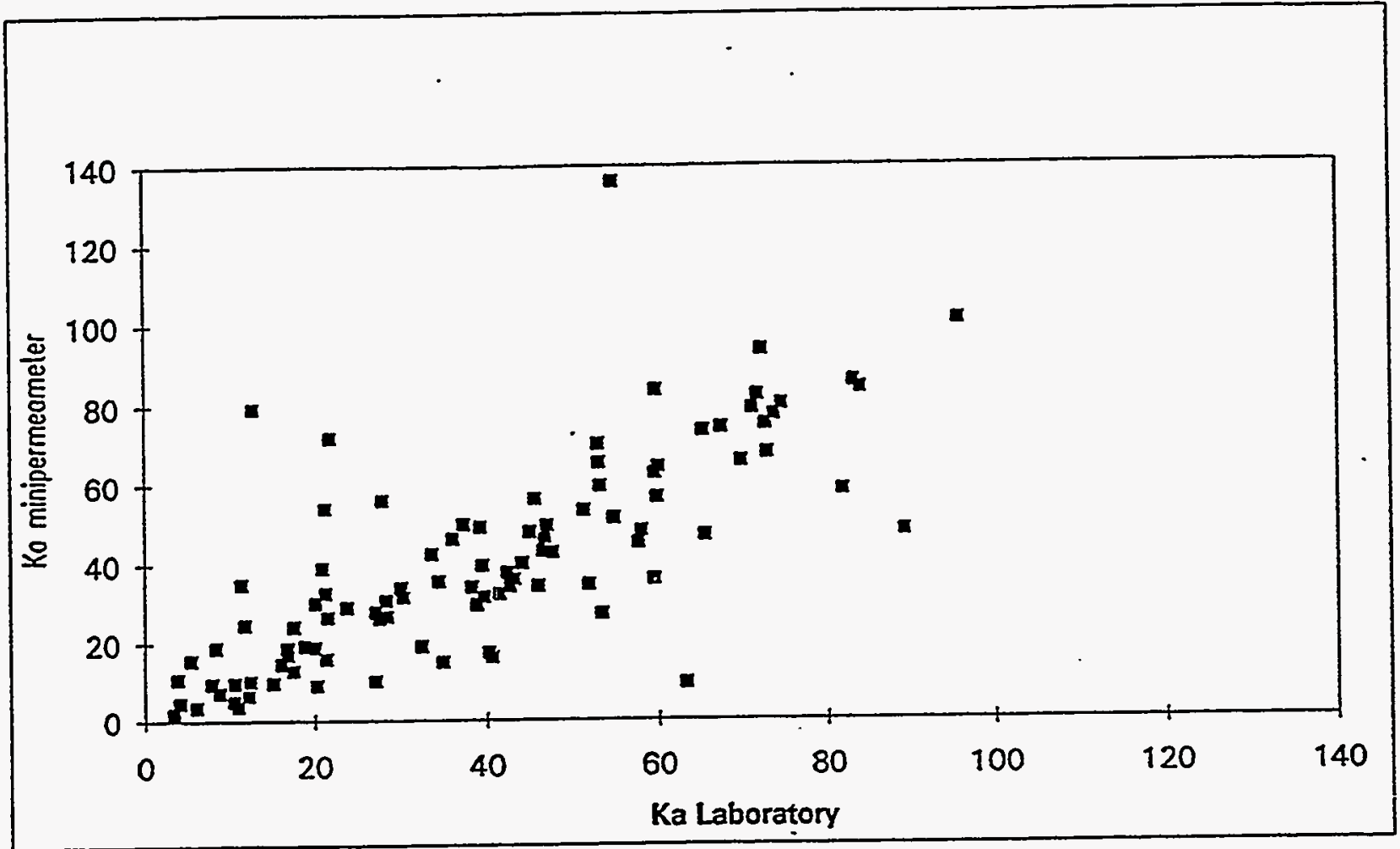

FIGURE 6. Graph Ka minipermeameter Vs Ka Laboratory 


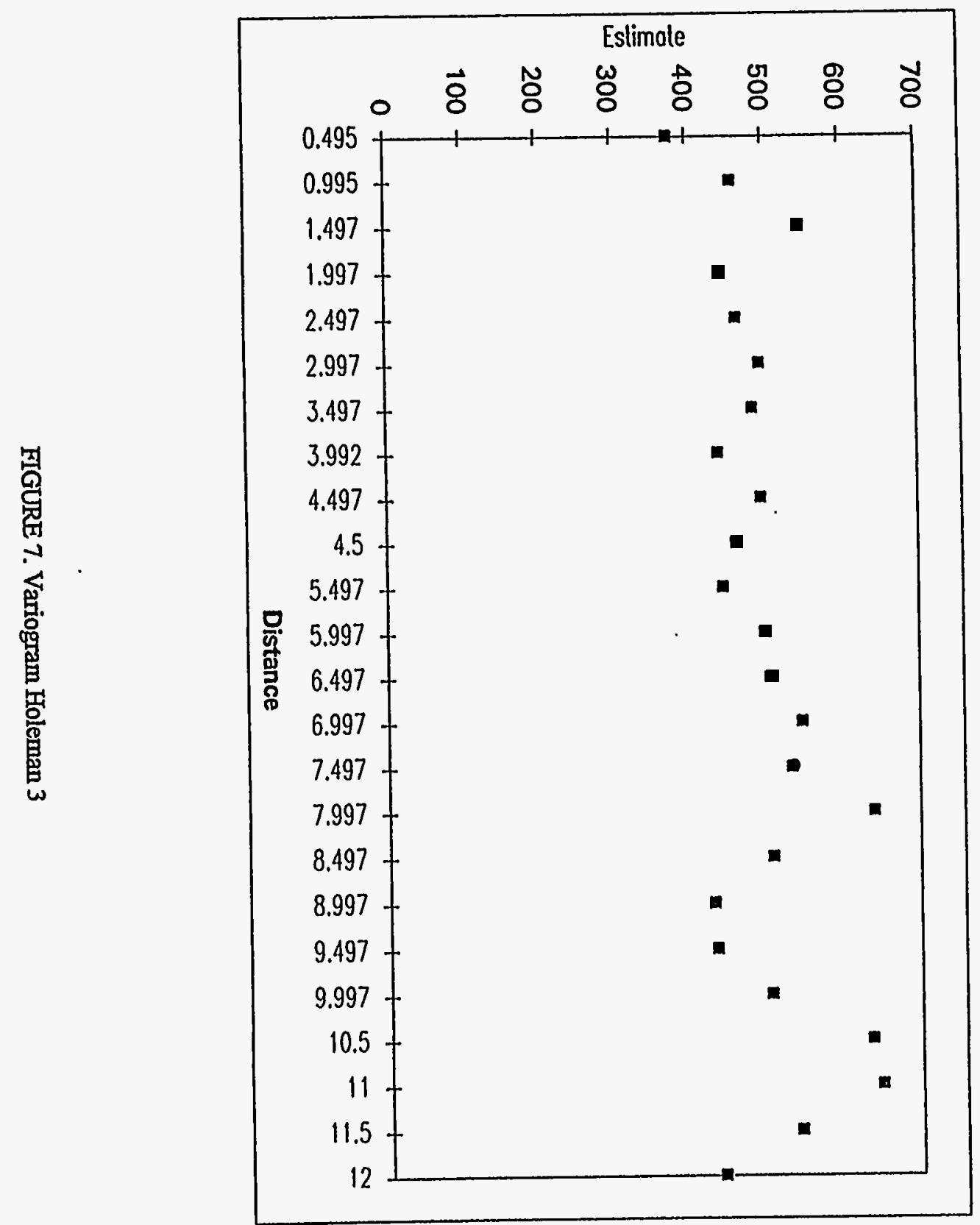




\section{Current and Future Study}

A lot of activities are still to be accomplished and their results integrated into a final interpretation. The planning of the remaining 80 core plugs is almost finished. Probably 10 of these 81 plugs will be vertically drilled across key surfaces, in order to test vertical permeability.

The remaining outcrop transects and cores will be described and interpreted to validate and complement the preliminary results presented in this report. Among additional source of information, thin sections have also been considered. Interpretation of FMS log will also be used.

Permeability and porosity disagreements will be validated. So far there has been little disagreement. Emphasis is being placed on the correlation of every information that is generated from the engineering or geologic approach.

At the end of this project, it is expected that the model generated for the study area can be extrapolated to the prolific section of the Bartlesville sandstone. in the Glenn Pool field. 


\title{
STATUS REPORT
}

\section{Application of Geostatistical Techniques in the Evaluation of Infill Drilling Prospects}

By

\author{
K. Hird and M. Kelkar
}

\section{Abstract}

Infill drilling programs are typically initiated because of low hydrocarbon recoveries due to reservoir heterogeneities. The ability to properly assess infill drilling potential is greatly dependent on the accuracy of the reservoir description. Stochastic conditional simulation techniques have been developed which attempt to realistically reproduce the heterogeneous nature of hydrocarbon reservoirs. These techniques are currently limited by their inability to constrain reservoir descriptions to well performance. This study attempts to address this limitation by developing a conditional simulation technique which constrains areal permeability images to conventional statistical information and waterflood well performance. Reservoir connectivity and effective permeability variance parameters are used to constrain well performance. The simulated annealing algorithm is used to generate the permeability fields. The types of engineering and field data necessary to reproduce historical waterflood performance is investigated. Flow pattern statistical parameters are defined and incorporated within the objective function of the simulated annealing algorithm. The correlation between these parameters and waterflood performance is demonstrated. 


\section{Previous Results}

The previous status report ${ }^{1}$ investigated the feasibility of using connectivity to condition simulated images of permeability to well performance data. Waterflood response in a quarter of a 5-spot pattern was simulated for various areal distributions of permeability. The simulated annealing algorithm ${ }^{2,3}$ was used to constrain images of permeability to conventional statistical information (permeability univariate distribution and variogram model), well data and reservoir connectivity. Reservoir connectivity was shown to correlate directly with waterflood performance data, i.e., water breakthrough time, initial injectivity and initial productivity. Reservoir connectivity was defined using a fractional connectivity function, which represents the fractional area connected between the injector and producer as a function of permeability cutoffs. These permeability cutoffs were of two kinds--high values (connectivity of high permeabilities) and low values (connectivity of low permeabilities). For the cases considered, the connectivity of high permeability values was found to have a much greater influence on waterflood performance than the connectivity of low values. Additionally, the most important connectivity parameter was found to be the permeability threshold at which the connectivity of high values was reduced to zero.

The determination of fractional connectivity is computationally intensive; cpu time is increased by a factor of 40 compared to conventional simulated annealing. The computing time requirement must be reduced significantly if the algorithm is to be of any practical benefit. Although water and oil rates were reasonably constrained for the cases considered, waterflood efficiency based on water-oil ratio (WOR) trends was not adequately reproduced. It was concluded that future work should be directed towards the identification of a parameter which adequately constrains WOR trends. The results of the synthetic case study were based on an extremely heterogeneous permeabilty field. 
Additional testing was needed for less heterogeneous cases.

\section{Connectivity}

\section{Connectivity Algorithm Improvements}

The controlling connectivity parameter had been determined to be the permeability threshold for the connectivity of high values $\left(k_{L F}\right)$. This value represents the limiting permeability along the most conductive path between the injection well and the producer. The determination of $k_{\mathrm{EH}}$ requires considerably fewer computations than finding the fractional area connected $\left(\mathrm{C}_{\mathrm{H}}\right)$; only one path rather than all possible paths needs to be identified. It is more convenient, and more general, to determine the permeability percentile threshold of high values $\left(\mathrm{p}_{\mathrm{HH}}\right)$ rather than $\mathrm{k}_{\mathrm{LH}}$. It was found to be more efficient to determine a connected path systematically rather than by using a random walk type of method. Movement from grid block to grid block is best performed by tracing the perimeter of the connected area, beginning at one of the well blocks. Movement to an adjacent grid block is considered acceptable only when the permeability percentile $\left(p_{k}\right)$ of the neighboring block is greater than or equal to $\mathrm{p}_{\mathbb{L}}$. Diagonal movement is not permitted. The perimeter of the connected area is traced beginning at one well until the other well is visited or the first well is revisited. If closure of the perimeter occurs prior to visiting the second well, $\mathrm{P}_{\mathrm{ra}}$ has been exceeded and a lower threshold exists.

The general procedure implemented is as follows:

1. Using conventional simulated annealing, swap pairs of values until the objective function has been reduced to 0.4 . A value of 0.4 was selected because permeability trends begin to form at approximately this objective value. 
2. During subsequent swaps, randomly define a connected path between the two wells. As advancement along the path progresses, swapping of values on the path are allowed only if the grid block $p_{k}$ equals or exceeds $p_{t r}$. If a path is not forced in this manner, there is no assurance that connectivity will ever be attained. It is best to define this path early so that neighboring values can be selected which are consistent with the desired spatial correlation.

3. Once a connected path has been developed, it is highly probable that the actual $\mathrm{p}_{\mathrm{rH}}$ exceeds the desired, or model $\mathrm{p}_{\mathrm{rH}}$. Swapping is continued until an acceptable tolerance between the actual $\mathrm{p}_{\mathrm{LA}}$ and the model $\mathrm{p}_{\mathrm{LA}}$ is achieved (and any other constraints are met, e.g., the variogram model).

As a result of employing this technique, computing time was decreased by an order of magnitude; the timing ratio between simulated annealing with connectivity and conventional simulated annealing was reduced from 40 to four.

\section{Testing of New Connectivity Algorithm}

\section{Approach and Base Case Properties}

Two synthetic base cases were designed to test the new connectivity algorithm. The same approach used in the previous report ${ }^{1}$ was applied. Waterflood response in a quarter of a 5-spot pattern was simulated for various areal distributions of permeability. The system dimensions were $1320 \mathrm{ft}$ by $1320 \mathrm{ft}$, equivalent to 80 acre well spacing in a 160 acre 5-spot pattern. The reservoir was assumed to have a uniform pressure of 300 psi and a constant gas saturation of $20 \%$. The black oil properties used in the simulation are listed in Table 1. The injector and producer were pressure-constrained at $2000 \mathrm{psi}$ 
and $300 \mathrm{psi}$, respectively. A slightly favorable mobility ratio of 0.96 was used. Reservoir thickness and porosity were assumed to be uniform and equal to $30 \mathrm{ft}$ and $25 \%$, respectively. Grid block dimensions were defined as $40 \mathrm{ft}$ by $40 \mathrm{ft}$. The flow simulation data are summarized in Table 2. A normalized, isotropic spherical variogram model (sill $=1.0$ ) having a nugget value of 0.2 and a range of $1000 \mathrm{ft}$ was assumed to appropriately describe the $\log (k)$ spatial correlation.

The primary difference between the two base cases is their permeability distributions. The permeability distribution for the first base case was assumed to be log normally distributed, with a mean $\log (\mathrm{k})$ of $2.0(100 \mathrm{md})$ and a standard deviation, $\sigma_{\log (\Delta)}$, of 0.4 . For this mean and $\sigma_{\text {bog( }(2)}, 68 \%$ of the values lie between $1.6(40 \mathrm{md})$ and $2.4(250$ $\mathrm{md})$. The well block $\log (\mathrm{k})$ values were assumed to be known and equal to 2.1 (125 md) for the injection well and $2.4(250 \mathrm{md})$ for the producer. A larger mean $\log (\mathrm{k})$ of 2.5 $(316 \mathrm{md})$ and a smaller $\sigma_{\text {log(t) }}$ of 0.2 was used for the second base case. For this second case, $68 \%$ of the values lie between $2.3(200 \mathrm{md})$ and $2.7(500 \mathrm{md})$. Injector and producer grid block permeabilities were assumed to be 2.55 ( $355 \mathrm{md})$ and 2.40 (250 $\mathrm{md})$, respectively. A more detailed description of each base case and associated simulations follows.

Base Case No. 1 Results

A total of 21 permeability realizations were generated using the conventional simulated annealing algorithm and the variogram model described previously. Greyscale maps of six of the permeability images are shown in Figure 1. The permeability fields were used to simulate waterflood performance over a 20 year period. Performance for the 21 flow simulations, based on water injection rates, oil producing rates, water producing rates and WOR's, is presented in Figure 2. Waterflood performance varies considerably. For example, initial water injection rates vary from 410 to 1200 STB/D, . 
water breakthrough times range from 1.8 years to 5.0 years and peak oil responses vary from 300 to 600 STB/D. At the end of the 20 year simulation period, WOR values range from 19 to $50 \mathrm{STB} / \mathrm{STB}$. It is obvious that additional constraints are required prior to considering these reservoir descriptions as being "equally probable". Note that the performance of one of the flow simulations has been highlighted. The permeability image corresponding to this flow simulation (Figure 1, top left image) was used as the first "truth case" for testing of the connectivity algorithm.

This particular permeability realization was selected as the first truth case because it has somewhat of an anomalous WOR trend. A large increase in the WOR occurs approximately two years after water breakthrough. Also, the average permeability surrounding the injection well is significantly greater than that surrounding the producer. However, as previously stated, the injection well grid block permeability is greater than the producer grid block value. This discrepancy is apparent in the performance data, with the initial injection rate for the first truth case being greater than that of any other run. The $\mathrm{p}_{\mathrm{LA}}$ for the first truth case was determined to be 0.319 , i.e., the limiting permeability value along the most conductive path between the injector and producer is greater than or equal to $31.9 \%$ of all permeability values.

Synthetic Case Study No. 1

The simulated annealing with connectivity algorithm was used to generate 20 permeabiity realizations, all having a $\mathrm{p}_{\mathrm{LH}}$ of 0.319 . Figure 3 contains greyscale maps for the truth case realization and five of the permeability images. Although all permeability images have the same interwell connectivity, the locations of the high/low permeability regions vary from image to image. Waterflood performance for the first truth case and the 20 flow simulations are included in Figure 4. A comparison of Figures 2 and 4 indicates that although the inclusion of the connectivity constraint reduced the spread in 
waterflood performance, the results appear to be biased. None of the 20 flow simulations reproduced the WOR trend exhibited by the first truth case. This biasedness also is evident in the producing oil rate history.

\section{Base Case No. 2 Results}

Base case waterflood performance was also generated for the more homogeneous permeability distribution described above. Figure 5 contains six of the 20 greyscale images of permeability created using simulated annealing conditional simulation; the first permeability image is the truth case selected for the second case study. For the second truth case, the permeability surrounding the injection well is greater than the permeability surrounding the producer. This is consistent with the well block permeability relationships. Figure 6 illustrates the flow rates and WOR trends based on flow simulation using the 20 permeability realizations. The variation in waterflood performance is less than that observed for the first base case runs because of the smaller degree of heterogeneity. The differences between the flow simulation results are still much greater than desirable. Note that the initial injectivity of the second truth case is greater than any of the others.

\section{Synthetic Case Study No. 2}

The $\mathrm{p}_{\mathrm{uf}}$ of the second truth case is $\mathbf{0 . 2 9 8}$. This value was used to generate 20 additional permeability realizations using the simulated annealing with connectivity algorithm. Five of these realizations and the second truth case realization are displayed in Figure 7. Once again, although the connectivity is preserved, the location of high and low permeability areas change from image to image. Many of the images have areas of high permeability surrounding the injector because of its high well block permeability. Inspection of the corresponding performance plots (Figure 8) show that the resulting trends are unbiased with respect to the second truth case. Also, the spread in 
performance has been reduced somewhat in comparison to the base case results (Figure 6). The variation in performance could be reduced if the initial injectivity and productivity were better constrained.

\section{Conclusions}

Simulated annealing is a relatively slow conditional simulation method in comparison to other methods. The inclusion of connectivity as an additional constraint increases simulation time by a factor of four. Synthetic Study Case No. 1 demonstrated that simulated annealing with connectivity can give biased results. Although the results of Synthetic Case Study No. 2 were unbiased, the reduction in the spread in performance due to the additional constraint of connectivity was only marginal. This spread in performance could be reduced if the near well effective permeability could be better preserved.

\section{Near Well Permeability Conditioning}

The results from the two previously discussed synthetic case studies indicate that knowledge of near well permeability $\left(\mathrm{k}_{\mathrm{N} w}\right)$ is required as an additional simulated annealing constraint; knowing the well block permeability is not sufficient. The most likely source of this information is from early production/injection data and/or pressure transient data. Others have investigated using pressure data to obtain a description of the permeability field ${ }^{4-7}$. Additional work in this area is being done within TUPREP ${ }^{8}$. For this present study, it will be assumed that $\mathrm{k}_{\mathrm{Nw}}$ is available. A geometric mean will be used to determine this value. A rather arbitrary radius of $240 \mathrm{ft}$, or 6 by 6 grid blocks, will be considered as the "near well" area. Note that the near well constraint is the mean permeability for a $240 \mathrm{ft}$ by $240 \mathrm{ft}$ - not the actual grid block permeability values. The 
added assumption that $\mathrm{k}_{\mathrm{N} w}$ can be estimated should not be considered a major limitation. As was shown in the previous two case studies, variations in the initial rates are directly related to $\mathrm{k}_{\mathrm{NW}}$. These results can be used to estimate $\mathrm{k}_{\mathrm{NW}}$ required to match historical initial rates. In effect, this is a history matching technique using stochastic conditional simulation.

The simulated annealing with connectivity algorithm was extended to include the additional constraint of $k_{\mathrm{NW}}$. Two more synthetic case studies were performed, using properties of the two previousiy discussed truth cases as the specified constraints.

\section{Synthetic Case Study No. 3}

The geometric means of the $k_{\mathrm{Nw}}$ 's for the first truth case are 370 and $190 \mathrm{md}$ for the injection well and producer, respectively. These values are equivalent to $\log (\mathrm{k})$ values of 2.57 and 2.28. As before, 20 permeability realizations were generated and five of the resulting greyscale images (plus the first truth case greyscale image) are depicted in Figure 9. Flow performance plots of the corresponding flow simulations are illustrated in Figure 10. The improvement resulting from including $\mathbf{k}_{\mathrm{NW}}$ as a constraint can be observed by comparing Figure 10 performance curves with the base case curves (Figure 2) and the results obtained without inclusion of $k_{\mathrm{NW}}$ as a constraint (Figure 4). It can be seen that the spread in waterflood performance is significantly reduced, especially during early waterflood performance. However, the results are still biased when compared to the truth case performance. The location of the low permeability areas in Figure 9 are mostly in the diagonal corners of the pattern, as far as possible from the high permeability areas surrounding each well. This is not true of the first truth case. Although there is a region of high permeability surrounding the producer, this quickly degrades into a low permeability region. This is the reason for the conflicting performance trends between the truth case and all attempts to reproduce it. 


\section{Synthetic Case Study No. 4}

The near well permeabilities of the second, less heterogeneous second truth case were used as additional constraints to generate 20 permeability realizations. The values of $k_{\mathrm{NWi}}$ and $\mathrm{k}_{\mathrm{NWp}}$ for the second truth case are $2.76(575 \mathrm{md})$ and 2.53 (339 $\left.\mathrm{md}\right)$. The truth case greyscale image and five example greyscale images are shown in Figure 11. Flow simulation waterflood performance plots are illustrated in Figure 12. An extremely good comparison is obtained between the second truth case and the other 20 flow simulations. Not only is performance from the second truth case reproduced very well, but the scatter between the 20 simulated waterflood performances is very small. This is a significant improvement over the results obtained in Case Study No. 2. Unlike the first truth case, the regions of low permeability for the second, less heterogeneous truth case are located in the diagonal corners. This is a much more likely outcome of the conditional simulation process and therefore is easier to reproduce.

\section{Conclusions}

The results from Case Study No. 3 and Case Study No. 4 show that the additional constraint of near well permeability reduces the uncertainty, or spread, in simulated waterflood performance. However, the simulated results can be biased so that the actual waterflood performance does not fall within the range of uncertainty. Biasedness occurs when there is low connectivity between the two wells. Another constraint is required to eliminate this biasedness.

\section{Flow Pattern Permeability Variance}

The above results show that the misrepresentation of low permeability regions via conditional simulation results in anomalous, or biased WOR trends. The WOR trend 
supplies a significant amount of information regarding waterflood efficiency. Statistical information related to the permeability images and their corresponding WOR trends were used to identify two parameters which impact waterflood efficiency. These two parameters will be referred to as the dimensionless mid-pattern permeability $\left(k_{m D}\right)$ and the flow pattern permeability coefficient of variance $\left(\mathrm{CV}_{\mathrm{K}^{*}}\right)$. The parameters were developed using equal rate, unit mobility ratio flow pattern segments, or stream tubes. Stream tubes were used because they represent fluid flow patterns better than other geometries. The first parameter, $k_{\operatorname{mb}}$, represents the ratio of the average permeability of the mid-pattern area normalized by the overall average permeability of the quarter of a 5-spot pattern. This ratio was found to be indirectly related to waterflood recovery; as $k_{m b}$ increases, waterflood sweep efficiency decreases. The second parameter, $\mathbf{C V}_{\mathbf{k}}$, represents the coefficient of variation (standard deviation divided by the mean) of a distance-normalized flow pattern segment permeability. Since the coefficient of variation is a measure of variability, large variations in flow pattern segment permeabilities are characterized by

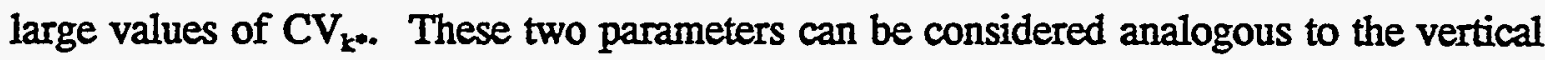
and horizontal lithofacies proportion curves ${ }^{9-13}$. Whereas the proportion curves are statistical geological information, the flow pattern parameter values statistically characterize flow pattern sweep efficiency. More information regarding the definition of the two flow pattern parameters is included in Appendix A.

\section{Synthetic Case Study No. 5 - Parameter Sensitivity}

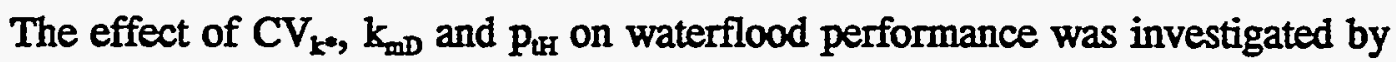
performing a sensitivity study. The parameters were varied one at a time, using the first truth case as the base case. The parameters were varied such that the parameter values for the first truth case were approximately in the middie of the range of values. Table 3 lists the parameter values used for the conditional simulations. Five realizations were 
generated for each condition so that the variability in performance under constant parameter conditions could be estimated.

Figure 13 contains waterflood performance plots for the $\mathrm{CV}_{\mathbf{k}}$ sensitivity flow simulations. Several significant points are worth mentioning. The effect of varying $\mathrm{CV}_{\mathbf{k}}$ * results in a systematic change in flow performance. For instance, the variation in water breakthrough time indicates that an increase in $\mathrm{CV}_{\mathbf{k}}$ results in a decrease in water breakthrough time. The flow simulations having the same value of $\mathrm{CV}_{\mathbf{k}}$ have similar waterflood performance behavior. The performance of the base case (highlighted) falls about in the middle on each of the four plots, as would be expected since its parameters are mid-range values.

It is rather difficult to visualize waterflood performance effects in Figure 13 since the results from all 21 realizations are plotted. Waterflood performance from only one realization per sensitivity has been included in Figure 14 for clarity. Figure 15 better demonstrates the correlation between water breakthrough time and $\mathrm{CV}_{\mathbf{k}^{*}}$. Data from all 21 sensitivity flow simulations have been plotted in Figure 15 . Note that as $\mathbf{C V}_{\mathbf{k}}$ is increased, the spread in breakthrough times for the same $\mathrm{CV}_{\mathrm{k}}$, is reduced. Larger values of $\mathrm{CV}_{\mathrm{k}^{*}}$ reflect stronger trends between the two well. These results demonstrate that $\mathrm{CV}_{\mathrm{k}}$ does have a strong effect of waterflood performance and its value can be estimated by performing a sensitivity study.

$A$ better understanding of the effect of $\mathrm{CV}_{\mathrm{r}}$ on waterflood performance can be obtained by comparing the permeability images. A permeability image representing each value of $\mathbf{C V}_{\mathbf{k}^{*}}$ investigated is contained in Figure 16. It can be seen that as $\mathbf{C V}_{\mathbf{k}^{*}}$ is increased, the high and low permeability trends become more aligned with the direction of fluid flow. Thus, higher values of $\mathrm{CV}_{\mathrm{k}}$ result in more continuous conductive paths, decrease water breakthrough time and increase WOR trends, thereby decreasing 
waterflood efficiency.

Waterflood performance plots for the $\mathrm{k}_{\mathrm{mb}}$ sensitivity simulations are shown in Figure 17. These results are for variations in $k_{m b}$, maintaining all other constraining variables constant. For these cases, the impact of varying $k_{m D}$ is not very significant. Also, the base case does not represent the average performance as would be expected. Therefore, $k_{m b}$ does not appear to be a very good constraining parameter.

The sensitivity of waterflood performance to $p_{u H}$ is illustrated in Figure 18. Although $\mathrm{p}_{\mathrm{tu}}$ was varied over a large range $(0.1-0.5)$, there is only a fairly small variation in waterflood performance. This is probably due to the fact that $\mathrm{CV}_{\mathrm{k}^{*}}$ is not only constant for all of the flow simulations, but is fixed at a fairly small value of 0.186 (based on the base case value). A small value of $\mathrm{CV}_{\mathrm{k}}$ implies that each flow pattern segment (or stream tube) has very similar average permeabilities. This inhibits large variations in effective connectivity. Synthetic Case Study No. 6

Case Study No. 6 is analogous to Case Study Nos. 1 and 3, with the more heterogeneous permeability distribution being used as the truth case. Once again 20 realizations were generated using all previously investigated parameters $\left(\mathrm{p}_{\mathrm{LA}}=0.319\right.$, $\mathrm{k}_{\mathrm{NWi}}=2.57, \mathrm{k}_{\mathrm{NWp}}=2.28, \mathrm{k}_{\mathrm{mD}}=0.610$ and $\mathrm{CV}_{\mathrm{k}^{*}}=0.186$ ) as well as the conventional constraints (univariate distribution, well block values and variogram model). Greyscale maps of these permeability images are depicted in Figure 19. When compared to the truth case permeability image, it is seen that the location of high and low permeability regions are consistent among the 20 realizations. More importantly, the location of the high and low permeability regions are consistent with that of the truth case. The ability to locate permeability trends is especially important for selecting infill well locations. The similar spatial variations in permeability exhibited by the 20 realizations and the first 
truth case result in very similar waterflood performance, as shown in Figure 20. Note that the anomalous WOR trend characteristics of the first truth case are better represented by the realizations, unlike the previous case studies which did not include $k_{m D}$ and $C_{k^{*}}$ as constraints.

\section{Synthetic Case Study No. 7}

This study is similar to Case Study Nos. 2 and 4 in that parameter values characteristic of the less heterogeneous second truth case were used as constraints. Similar to Case Study No. 6, the parameters $\mathrm{k}_{\mathrm{mD}}$ and $\mathrm{CV}_{\mathrm{k}^{*}}$ were used as constraints. The values of the parameters are: $\mathrm{p}_{\mathrm{tH}}=0.298, \mathrm{k}_{\mathrm{NWWi}}=2.76, \mathrm{k}_{\mathrm{NW}}=2.53, \mathrm{k}_{\mathrm{md}}=1.023$ and $\mathrm{CV}_{\mathrm{K}^{*}}=0.300$. Five of the 20 permeability images are shown in Figure 21 and are compared to the second truth case. Waterflood performance is depicted in Figure 22. As in Case Study No. 6, the inclusion of $\mathrm{k}_{\mathrm{mb}}$ and $\mathrm{CV}_{\mathrm{k}}$ as additional constraints significantly reduces the variation in waterflood performance and improves the reproducibility of the truth case performance. Virtually all variations in waterflood performance have been eliminated.

\section{Synthetic Case Study No. $8-\mathbf{C V}_{\mathbf{k}^{*}}$ Sensitivity Without $\mathrm{p}_{\mathrm{tB}}$ Constraint}

Another sensitivity study was performed to determine how well simulated annealing performs using $\mathrm{CV}_{\mathrm{x}^{*}}$ as a constraint without constraining $\mathrm{P}_{\mathrm{LH}}$. Other constraints used included the conventional ones and $\mathrm{k}_{\mathrm{Nw}}$. Similar to the first sensitivity study, the values used for $\mathrm{CV}_{\mathrm{k}=}$ ranged from 0.05 to 0.40 . Five realizations were generated for each value of $\mathrm{CV}_{\mathrm{k}}$. The resulting performance plots are shown in Figure 23. The elimination of $\mathrm{p}_{\mathrm{H}}$ as a constraint does not appear to reduce the effect of $\mathrm{CV}_{\mathbf{k}}$ on breakthrough time and WOR trends. Additional testing will be performed to see if $\mathrm{CV}_{\mathbf{k}}$ * can be used effectively without the inclusion of $\mathrm{p}_{\mathrm{tH}}$ and $\mathrm{k}_{\mathrm{mD}}$ as additional constraints. 


\section{Conclusions}

Waterflood performance appears to be more strongly affected by the flow pattern permeability variance parameter $\mathrm{CV}_{\mathbf{k}^{*}}$ than the two other parameters investigated, $\mathrm{p}_{\mathrm{u}}$ and $\mathrm{k}_{\mathrm{mD}}$. It has been shown that $\mathrm{CV}_{\mathbf{k}}$ is related to both waterflood efficiency and reservoir connectivity. Realizations generated using $\mathrm{CV}_{\mathbf{k}}$ as a constraint exhibit unbiased waterflood performance. One of the major benefits of using $\mathrm{CV}_{\mathbf{k}^{*}}$ is that, unlike $\mathrm{p}_{\mathrm{tH}}$, its value can be updated very easily. As a result, there is only a slight increase in computing time as compared to conventional simulated annealing.

\section{Recommendations for Future Work}

This work has demonstrated the effectiveness of a new conditional simulation technique in constraining areal permeability realizations to waterflood performance for a quarter of a 5-spot pattern. Additional work is required to determine if the new technique can be applied to a full 5-spot pattern as well as multiple waterflood patterns. The variables $\mathrm{p}_{\mathrm{tH}}$ and $\mathrm{CV}_{\mathrm{k}^{*}}$ have been shown to effectively characterize areal reservoir connectivity. It is desirable to investigate vertical cross-section connectivity characteristics and how they can be related to waterflood performance. So far, the focus using primary production data as conditional simulation constraints should also be investigated. The goal is to be able to generate reservoir descriptions which are consistent with all available geological, engineering and production data so that the risks associated with infill drilling can be minimized. 


\section{Nomenclature}

\section{Symbols}

$$
\begin{array}{ll}
\mathrm{C} & =\text { fractional area connected } \\
\mathrm{CV} & =\text { coefficient of variation } \\
\mathrm{k} & =\text { grid block or flow pattern segment permeability } \\
1 & =\text { flow pattern segment length } \\
\mathrm{p} & =\text { percentile } \\
\sigma & =\text { standard deviation } \\
\mu & =\text { mean }
\end{array}
$$

Subscripts

$$
\begin{array}{ll}
\mathrm{D} & =\text { dimensionless } \\
\mathrm{H} & =\text { high permeability values } \\
\mathrm{i} & =\text { injection well } \\
\mathbf{k} & =\text { permeability } \\
\mathrm{k}^{*} & =\text { flow pattern segment normalized permeability } \\
\mathrm{m} & =\text { mid-pattern area } \\
\mathrm{p} & =\text { producer } \\
\mathrm{seg} & =\text { flow pattern segment } \\
\mathrm{t} & =\text { threshold }
\end{array}
$$




\section{References}

1. Hird, K.B. and Kelkar, M.G.: "Application of Geostatistical Techniques in the Evaluation of Infill Drilling Prospects," TUPREP Research Report 6, presented at the 1991 TUPREP meeting, Tulsa, OK, Nov. 12.

2. Farmer, C.L.: "The mathematical generation of reservoir geology," presented at the joint IMA/SPE European Conference on the Mathematics of Oil Recovery, Robinson College, Cambridge University, July 25-27, 1989.

3. Perez, G.: "Stochastic Conditional Simulation for Description of Reservoir Properties," PhD dissertation, U. of Tulsa, Tulsa, OK, 1991.

4. Chang, J. and Yortsos, Y.C.: "Pressure Transient Analysis of Fractal Reservoirs," SPE 18170, presented at the 1988 SPE Annual Technical Conference and Exhibition, Houston, TX, Oct. 2-5.

5. Oliver, D.S.: "The Averaging Process in Permeability Estimation From Well Test Data," SPE 19845, presented at the 1989 SPE Annual Technical Conference and Exhibition, San Antonio, TX, Oct. 8-11.

6. Alabert, F.G.: "Constraining Description of Randomly Heterogeneous Reservoirs to Pressure Test Data: A Monte Carlo Study, "SPE 19600, presented at the 1989 SPE Annual Technical Conference and Exhibition, San Antonio, TX, Oct. 8-11.

7. Oliver, D.S.: "Estimation of Radial Permeability Distribution From Well Test Data," SPE 20555, presented at the 1990 SPE Annual Technical Conference and Exhibition, New Orleans, LA, Sept. 23-26, 1990.

8. Sagar, R.: "Reservoir Characterization for Waterfloods," TUPREP Research Report 7, presented at the 1992 TUPREP meeting, Tulsa, Oklahoma, May 12. 
Table 1

Fluid Properties Used in Flow Simulation Runs

\begin{tabular}{|c|c|c|c|c|c|}
\hline $\begin{array}{c}\text { pressure, } \\
\text { psi }\end{array}$ & $\begin{array}{c}\text { oil res. } \\
\text { vol. fac., } \\
\text { RB/STB }\end{array}$ & $\begin{array}{c}\text { oil } \\
\text { visc., } \\
\text { cp }\end{array}$ & $\begin{array}{c}\text { gas res. } \\
\text { vol. fac., } \\
\text { RB/Mscf }\end{array}$ & $\begin{array}{c}\text { gas } \\
\text { visc., } \\
\text { cp }\end{array}$ & $\begin{array}{c}\text { solution } \\
\text { GOR, } \\
\text { Mscf/STB }\end{array}$ \\
\hline \hline 14.7 & 1.0249 & 2.486 & 195.3693 & 0.0086 & 0.000 \\
50.0 & 1.0363 & 2.299 & 56.5568 & 0.0099 & 0.022 \\
100.0 & 1.0386 & 2.236 & 28.1765 & 0.0108 & 0.031 \\
150.0 & 1.0409 & 2.178 & 18.7435 & 0.0112 & 0.039 \\
250.0 & 1.0471 & 2.059 & 11.1524 & 0.0116 & 0.057 \\
350.0 & 1.0543 & 1.942 & 7.8864 & 0.0118 & 0.078 \\
450.0 & 1.0617 & 1.837 & 6.0781 & 0.0119 & 0.099 \\
700.0 & 1.0849 & 1.589 & 3.7942 & 0.0124 & 0.160 \\
950.0 & 1.1128 & 1.380 & 2.7078 & 0.0129 & 0.229 \\
1024.0 & 1.1215 & 1.326 & 2.4887 & 0.0131 & 0.250 \\
1200.0 & 1.1197 & 1.341 & 2.0158 & 0.0136 & 0.250 \\
1250.0 & 1.1192 & 1.345 & 1.9608 & 0.0143 & 0.250 \\
1500.0 & 1.1168 & 1.366 & 1.6858 & 0.0150 & 0.250 \\
1750.0 & 1.1146 & 1.386 & 1.4108 & 0.0157 & 0.250 \\
2000.0 & 1.1124 & 1.407 & 1.1358 & 0.0164 & 0.250 \\
\hline
\end{tabular}




\section{Table 2}

Pertinent Data Used in Flow Simulation Runs

\begin{tabular}{|c|c|}
\hline Type of simulation & 2D black oil \\
\hline Waterflood pattern type & $1 / 45$-spot \\
\hline Pattern size & $160 \mathrm{Ac}$ \\
\hline Well spacing & $80 \mathrm{Ac}$ \\
\hline Areal grid dimensions & $40 \mathrm{ft} \times 40 \mathrm{ft}$ \\
\hline Areal grid density & $33 \times 33$ \\
\hline Sand thickness (uniform) & $30 \mathrm{ft}$ \\
\hline porosity (uniform) & 0.25 \\
\hline initial pressure (uniform) & $300 \mathrm{psi}$ \\
\hline bubble point pressure & $1024 \mathrm{psi}$ \\
\hline initial gas saturation (uniform) & $20 \%$ \\
\hline mobility ratio & $2000 \mathrm{psi}$ \\
\hline producing pressure & 0.96 \\
\hline
\end{tabular}




\section{Table 3}

Values of Parameters Used in Sensitivity Study

\begin{tabular}{|c|c|c|c|c|c|}
\hline Type of Sensitivity & $\mathrm{p}_{\mathrm{trH}}$ & $\mathrm{CV}_{\mathbf{r}}$ & $\mathbf{k}_{\mathrm{mD}}$ & $\mathbf{k}_{\mathrm{NW}}$ & $\mathrm{k}_{\mathrm{NWP}}$ \\
\hline (Base Case) & 0.3186 & 0.186 & 0.610 & 2.57 & 2.28 \\
\hline $\mathrm{CV}_{\mathrm{k}^{*}}$ & 0.3186 & 0.050 & 0.610 & 2.57 & 2.28 \\
\hline $\mathrm{CV}_{\mathrm{k}^{*}}$ & 0.3186 & 0.100 & 0.610 & 2.57 & 2.28 \\
\hline $\mathrm{CV}_{\mathrm{r}}$ & 0.3186 & 0.300 & 0.610 & 2.57 & 2.28 \\
\hline $\mathrm{CV}_{\mathrm{r}^{*}}$ & 0.3186 & 0.400 & 0.610 & 2.57 & 2.28 \\
\hline $\mathrm{k}_{\mathrm{mD}}$ & 0.3186 & 0.186 & 0.500 & 2.57 & 2.28 \\
\hline$k_{m D}$ & 0.3186 & 0.186 & 0.650 & 2.57 & 2.28 \\
\hline$k_{\text {mo }}$ & 0.3186 & 0.186 & 0.800 & 2.57 & 2.28 \\
\hline$k_{m b}$ & 0.3186 & 0.186 & 1.00 & 2.57 & 2.28 \\
\hline $\mathrm{P}_{\mathrm{HH}}$ & 0.10 & 0.186 & 0.610 & 2.57 & 2.28 \\
\hline $\mathbf{p}_{\mathrm{EH}}$ & 0.20 & 0.186 & 0.610 & 2.57 & 2.28 \\
\hline $\mathrm{P}_{\mathrm{uI}}$ & 0.40 & 0.186 & 0.610 & 2.57 & 2.28 \\
\hline $\mathrm{p}_{\mathrm{HH}}$ & 0.50 & 0.186 & 0.610 & 2.57 & 2.28 \\
\hline
\end{tabular}




\section{Appendix A}

\section{Definitions of Flow Pattern Permeability Variance Parameters}

The two statistical flow parameters defined in this study, $\mathrm{CV}_{\mathrm{k}^{*}}$ and $\mathrm{k}_{\mathrm{mb}}$, were developed using flow pattern geometry rather than linear geometry. An average flow pattern geometry was assumed, i.e., it is assumed that the streamlines for an equal rate, unit mobility 5-spot pattern are applicable for heterogeneous permeability fields. Another alternative would be to calculate the flow pattern geometry after each swap of grid block permeability values during the simulated annealing procedure. This would most likely be too time-consuming; therefore, it was concluded that the advantages of using an average flow pattern geometry for computing flow pattern statistics outweighed the disadvantages. The goal was to determine a single-value statistical parameter which captures important waterflood performance characteristics. In addition, this parameter needs to be easily updated so that it can be used efficiently within the simulated annealing framework. With thousands of swaps being performed, re-initiating a parameter calculation after each swap becomes cpu-limiting.

Various definitions of statistical parameters were investigated. The significance of the parameters was determined by noting the strength of the relationship between the statistical parameters and waterflood performance parameters. For instance, a coefficient of determination $\left(R^{2}\right)$ of $71 \%$ was obtained when predicting cumulative WOR's using a linear combination of $\mathrm{CV}_{\mathbf{k}^{*}}$ and $\mathrm{k}_{\mathrm{mD}}$. $A$ good correlation between WOR and the two statistical parameters was found to exist over a wide range of waterflood conditions. The parameter $\mathrm{CV}_{\mathbf{L}^{*}}$ is defined as the coefficient of variation of a distancenormalized flow pattern segment permeability: 


$$
C V_{k^{*}}=\frac{\sigma_{k^{*}}}{\bar{k}^{*}}
$$

where:

$$
k^{*}=\frac{k_{s e g}}{I_{D}^{2}}
$$

and

$$
\bar{k}^{*}=\frac{1}{n s e g} \sum_{n=1}^{n s e g} \frac{k_{s e g}(n)}{l_{D}^{2}(n)}
$$

$$
I_{D}(n)=\frac{I(n)}{I(4)}
$$

The flow pattern is subdivided into 8 segments (nseg $=8$ ) and $1(n)$ represents the average length of flow pattern segment $n$. Since the flow pattern geometry used is based on the assumptions of equal rate, unit mobility and an aspect ratio of 1, symmetry exists along both 5-spot diagonals. Therefore, flow pattern segments 4 and 5 are of equal length. High permeability streaks existing directly between the injection well and the producer dominate waterflood response more so that indirect high permeability paths. As a result, the distance-normalized permeability term correlated much better with waterflood performance parameters than flow pattern permeability by itself. Flow pattern segment permeability is calculated using a geometric average of the grid block permeabilities 
within the segment. Once again, a geometric mean is used because it can be updated using just a few calculations.

The other flow pattern term, $k_{m D}$, is defined as the ratio of the mid-pattern average permeability to the flow pattern average permeability:

$$
k_{m D}=\frac{k_{m}}{\bar{k}}
$$

The flow pattern average permeability is the geometric mean permeability of the entire quarter of a 5-spot pattern. The mid-pattern area is defined as the area enclosed by the 4 flow pattern segments closest to the diagonal connecting the injection well and the producer, minus the near well area. The near well area was excluded from the midpattern area because it contributes more to the well effective flow capacity than waterflood efficiency. The optimum radius for defining the near well area was determined empirically to be equal to $1 / 3$ of the pattern side length. 

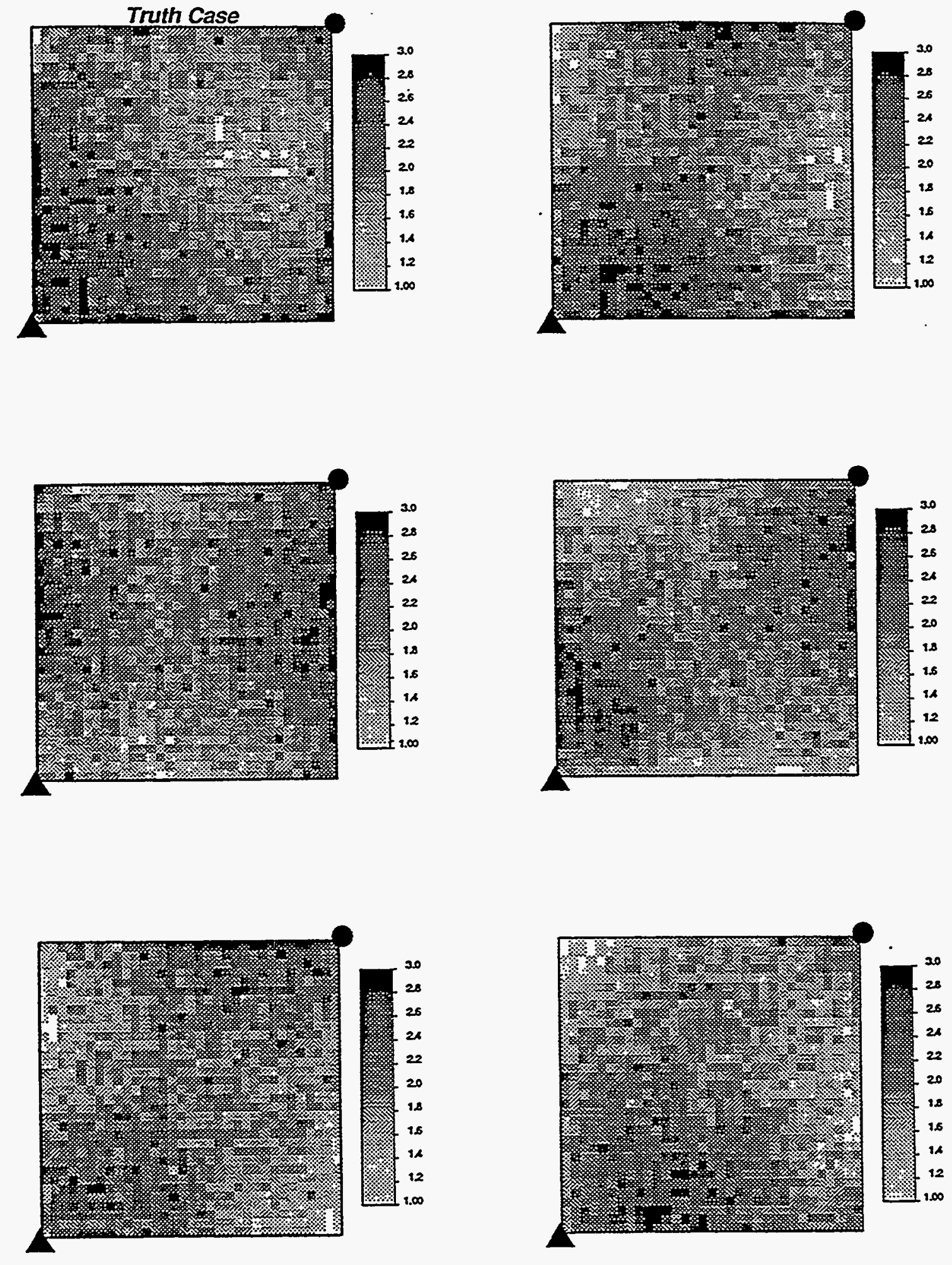

Figure 1 - Greyscale maps of $\log (\mathrm{k})$ for Base Case No. 1. 

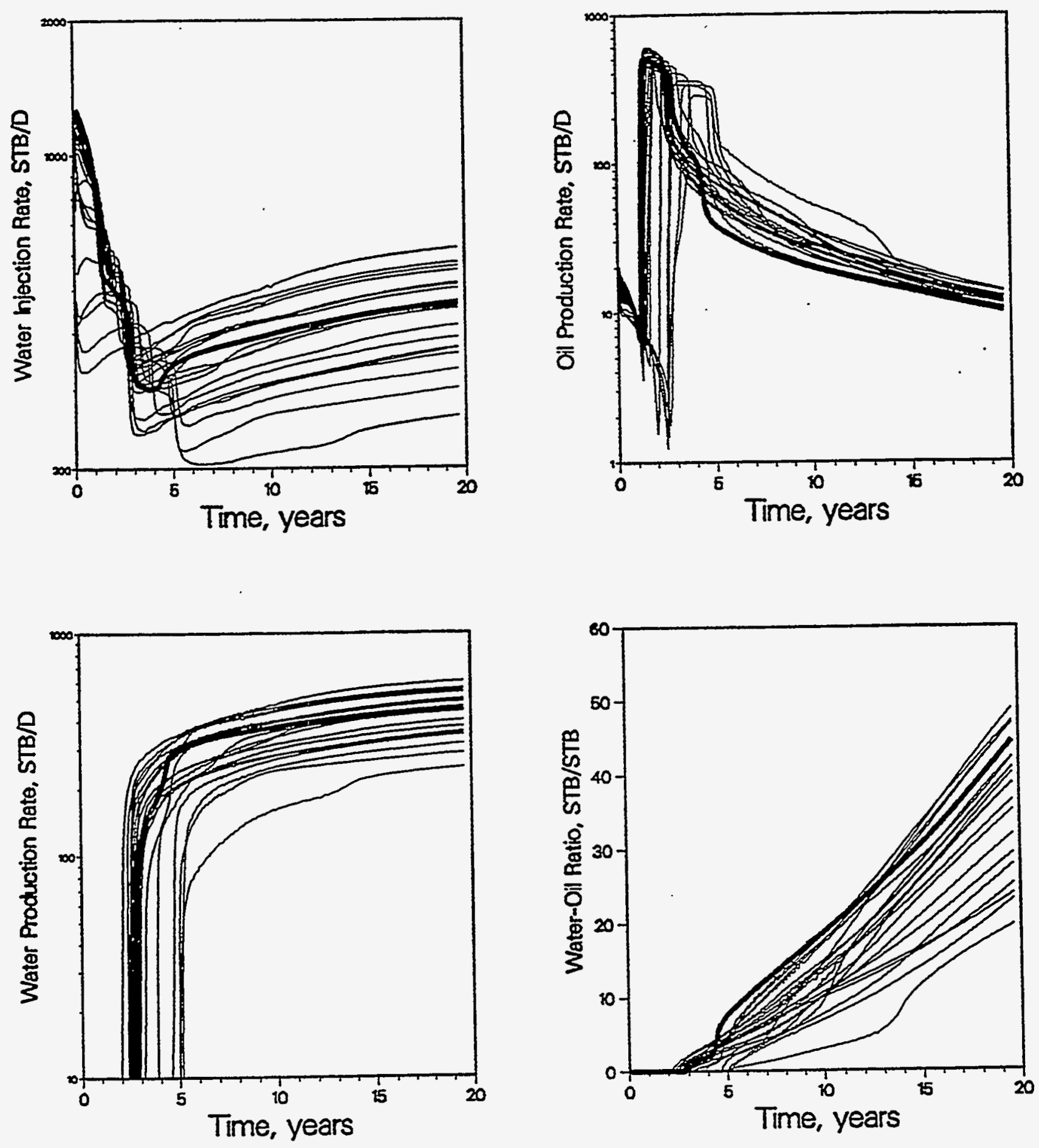

Figure 2 - Waterflood performance plots for Base Case No. 1: $\mu=2.0, \sigma_{\text {log(k) }}=0.4$, conventional simulated annealing (truth case highlighted). 

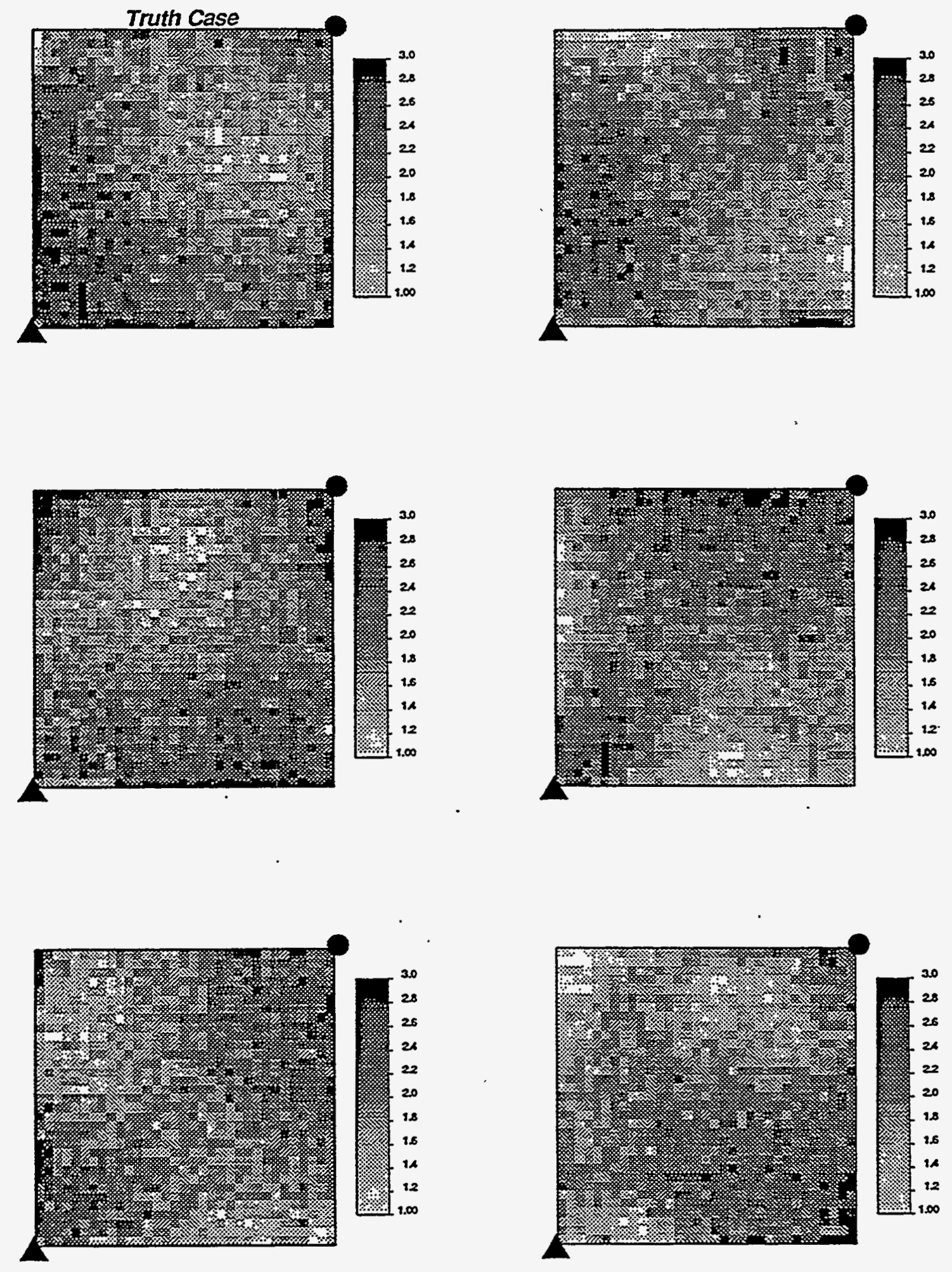

Figure 3 - Greyscale maps of $\log (\mathrm{k})$ for Case Study No. 1. 

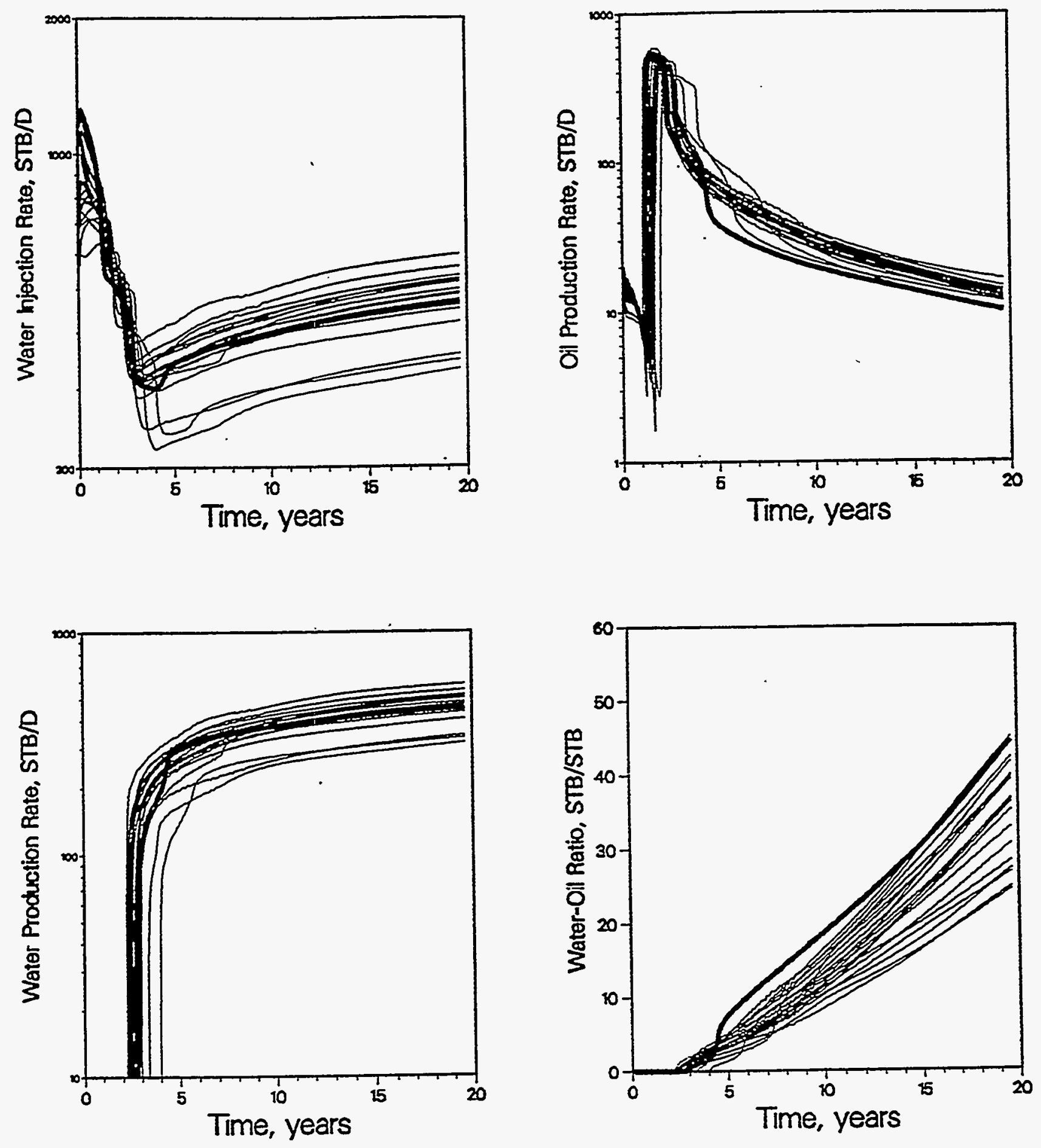

Figure 4 - Waterflood performance plots for Case Study No. 1: $\mu=2.0, \sigma_{\log (x)}=0.4$, simulated annealing with connectivity. $\mathrm{p}_{\mathrm{tH}}=0.319$ (truth case highlighted). 

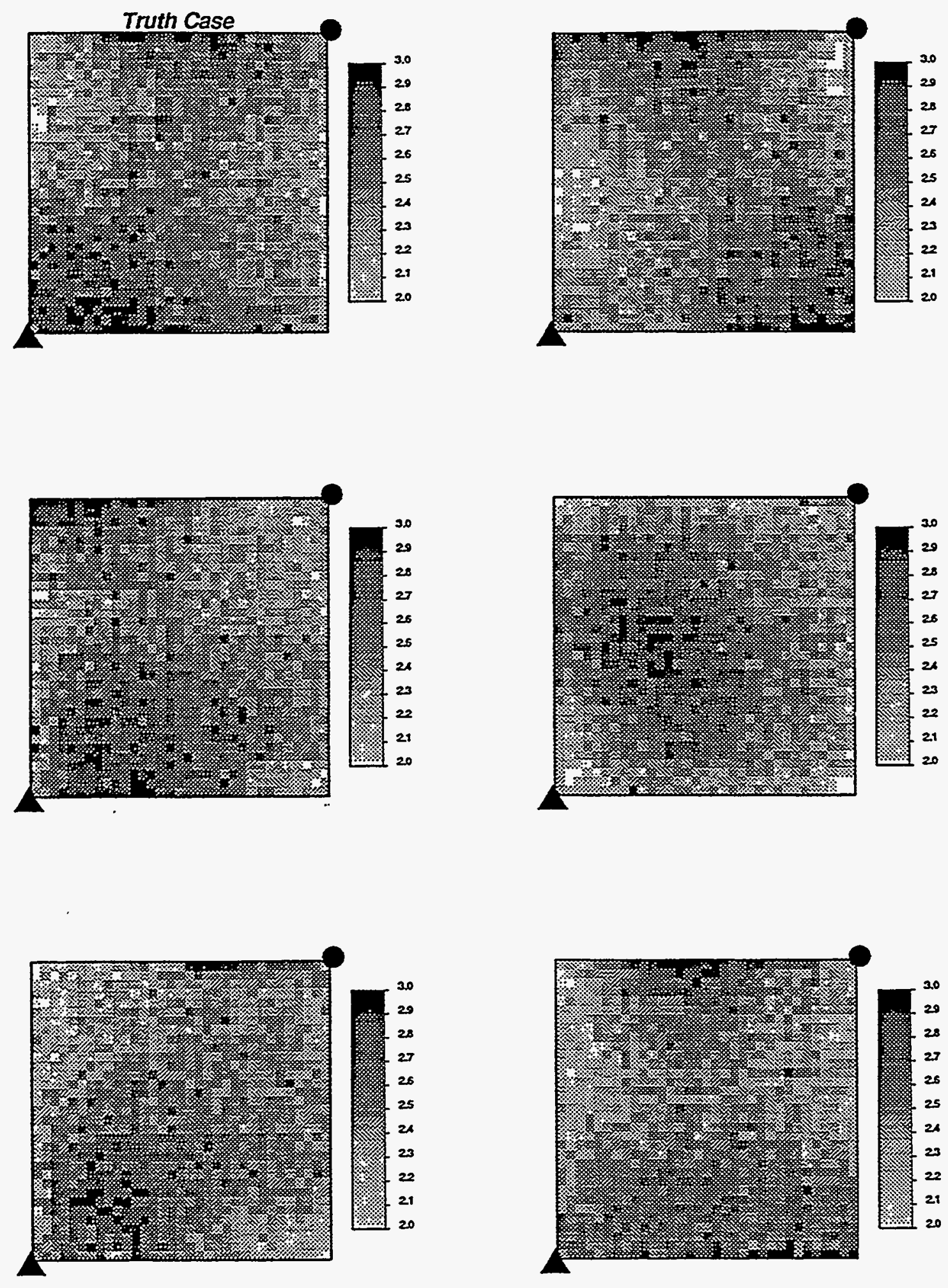

Figure 5 - Greyscale maps of $\log (k)$ for Base Case No. 2. 

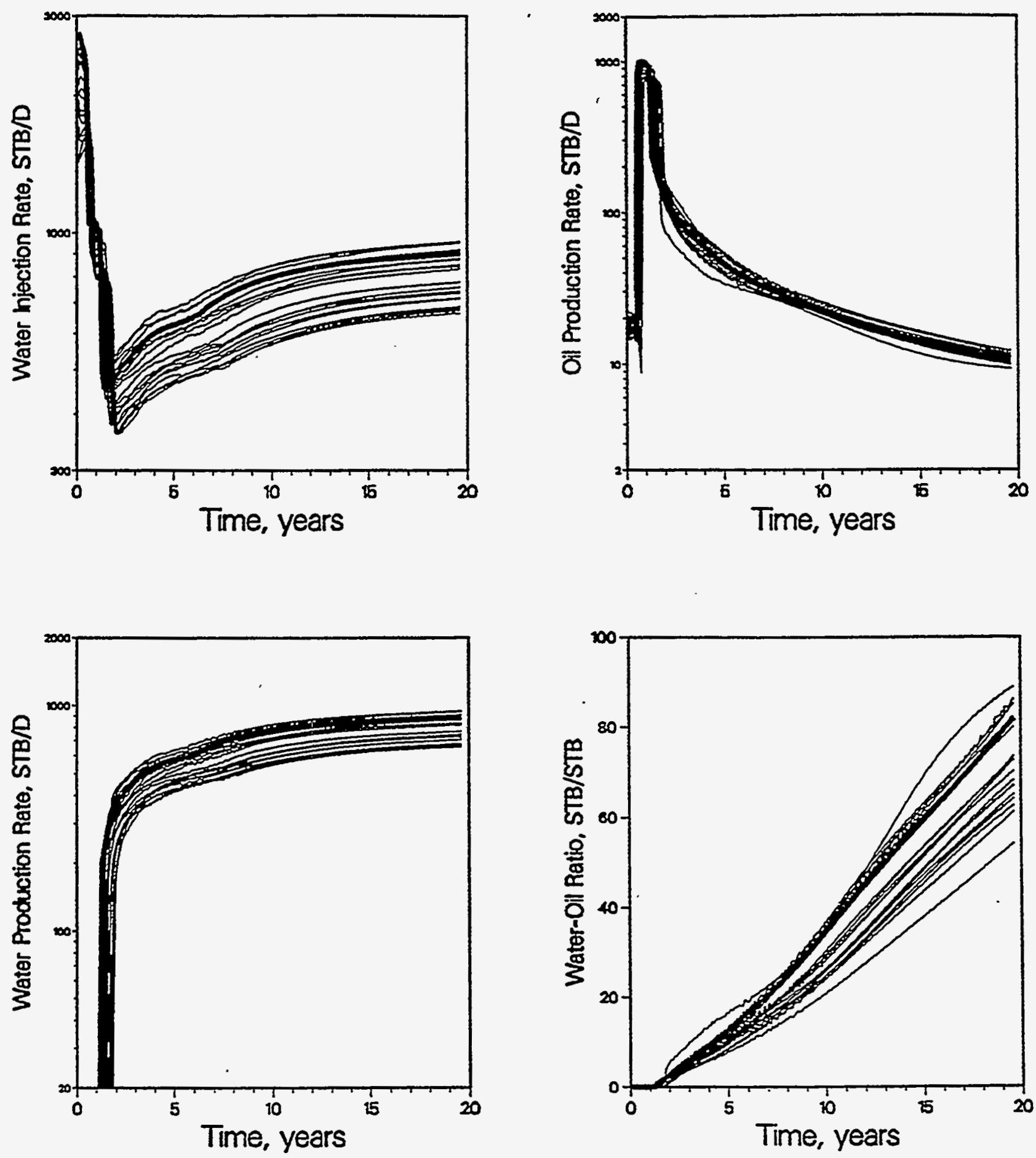

Figure 6 - Waterflood performance plots for Base Case No. 2: $\mu=2.5, \sigma_{\log (\mathrm{k})}=0.2$, conventional simulated annealing (truth case highlighted). 

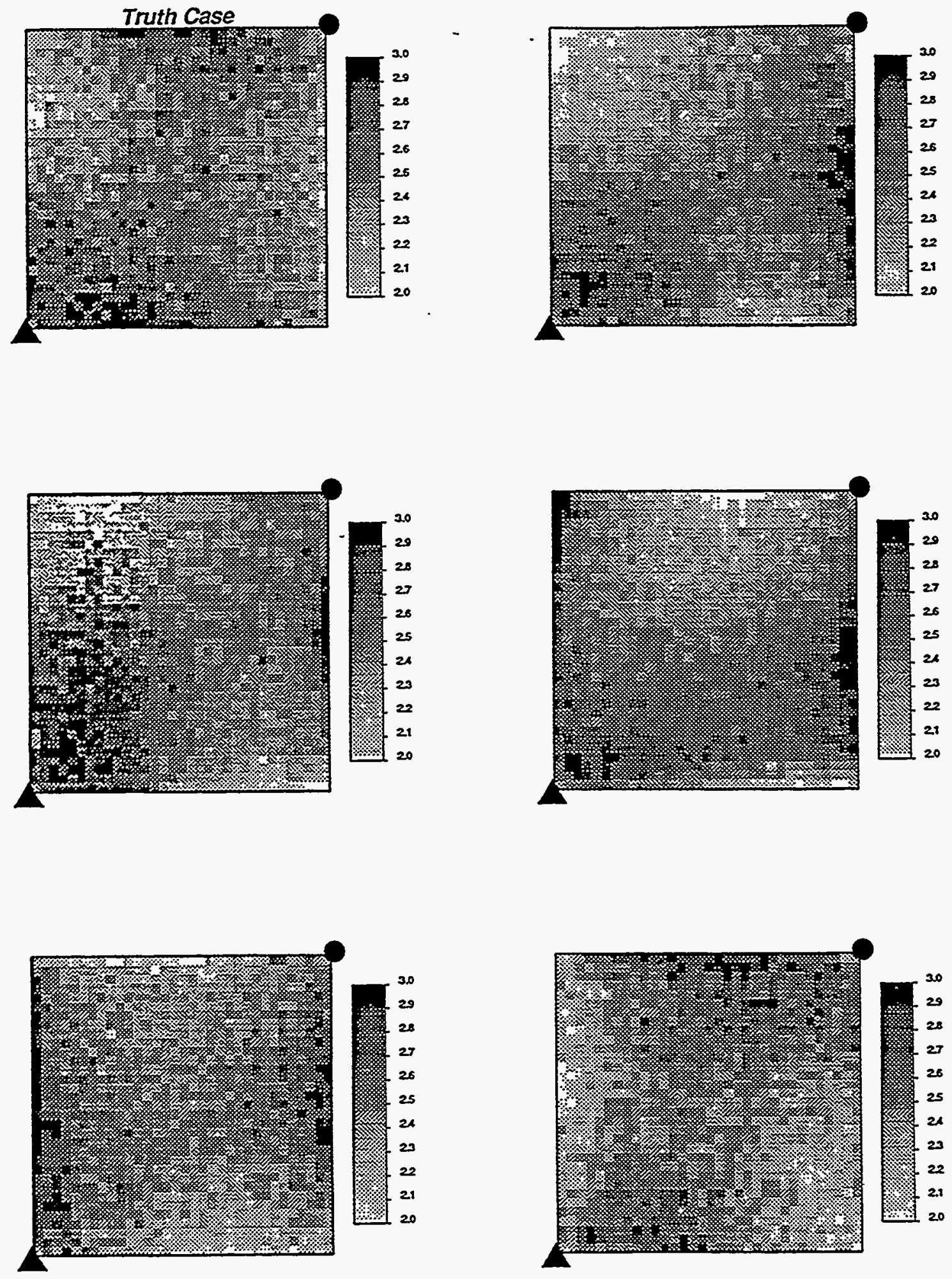

Figure 7 - Greyscale maps of $\log (\mathrm{k})$ for Case Study No. 2. 

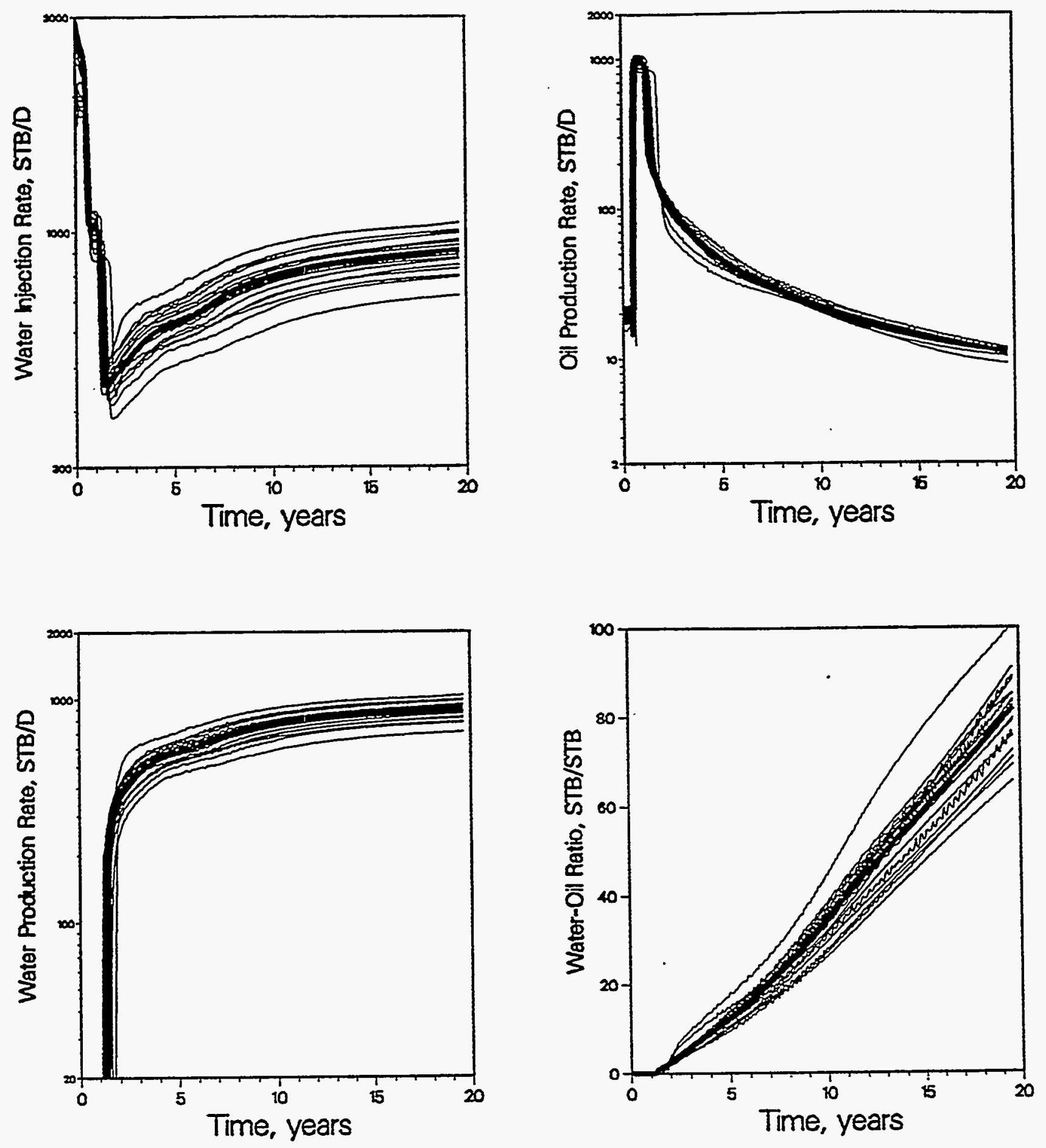

Figure 8 - Waterflood performance plots for Case Study No. $2: \mu=2.5, \sigma_{\log (x)}=0.2$. simulated annealing with connectivity. $\mathrm{p}_{\mathrm{tH}}=0.298$ (truth case highlighted). 

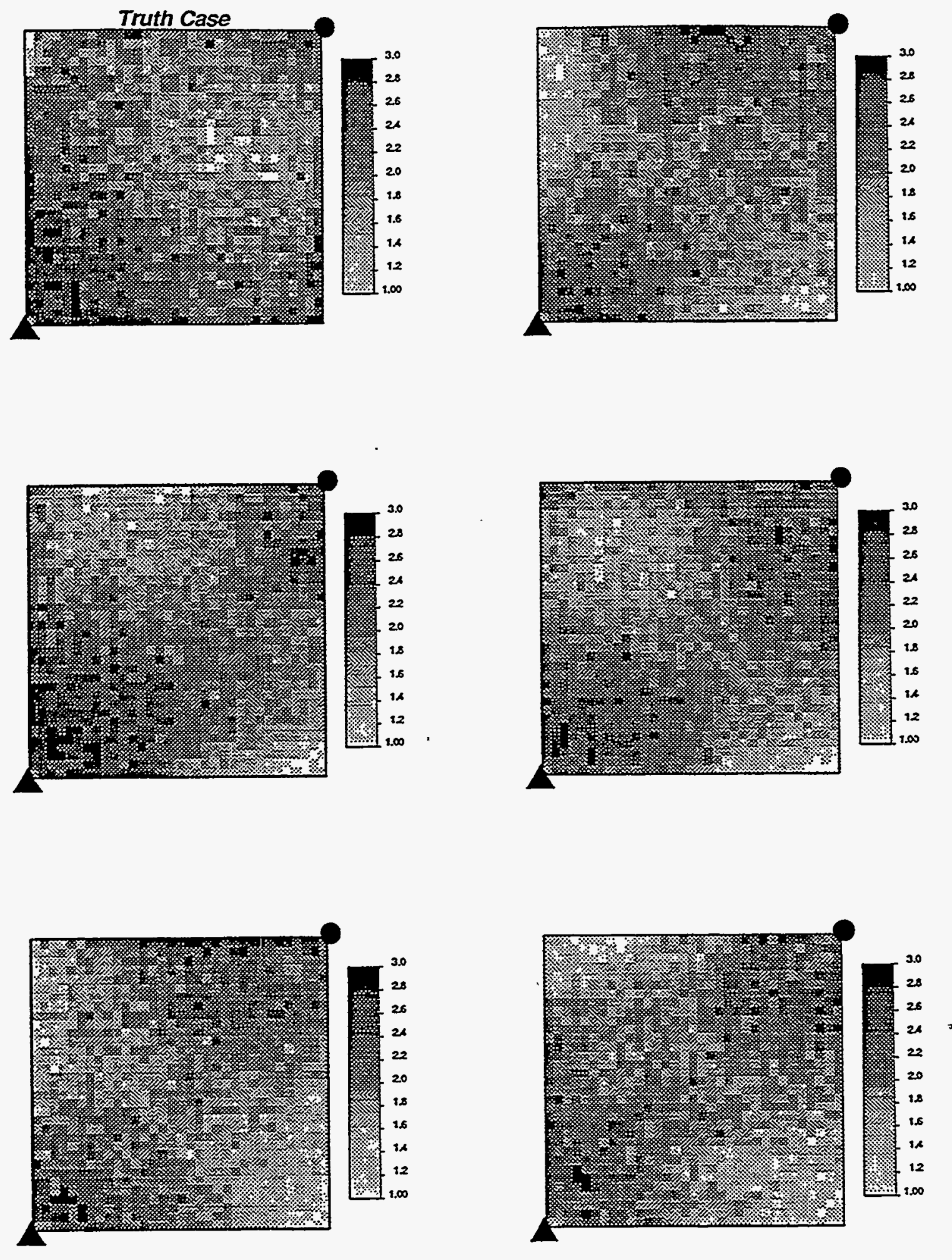

Figure 9 - Greyscale maps of $\log (k)$ for Case Study No. 3. 

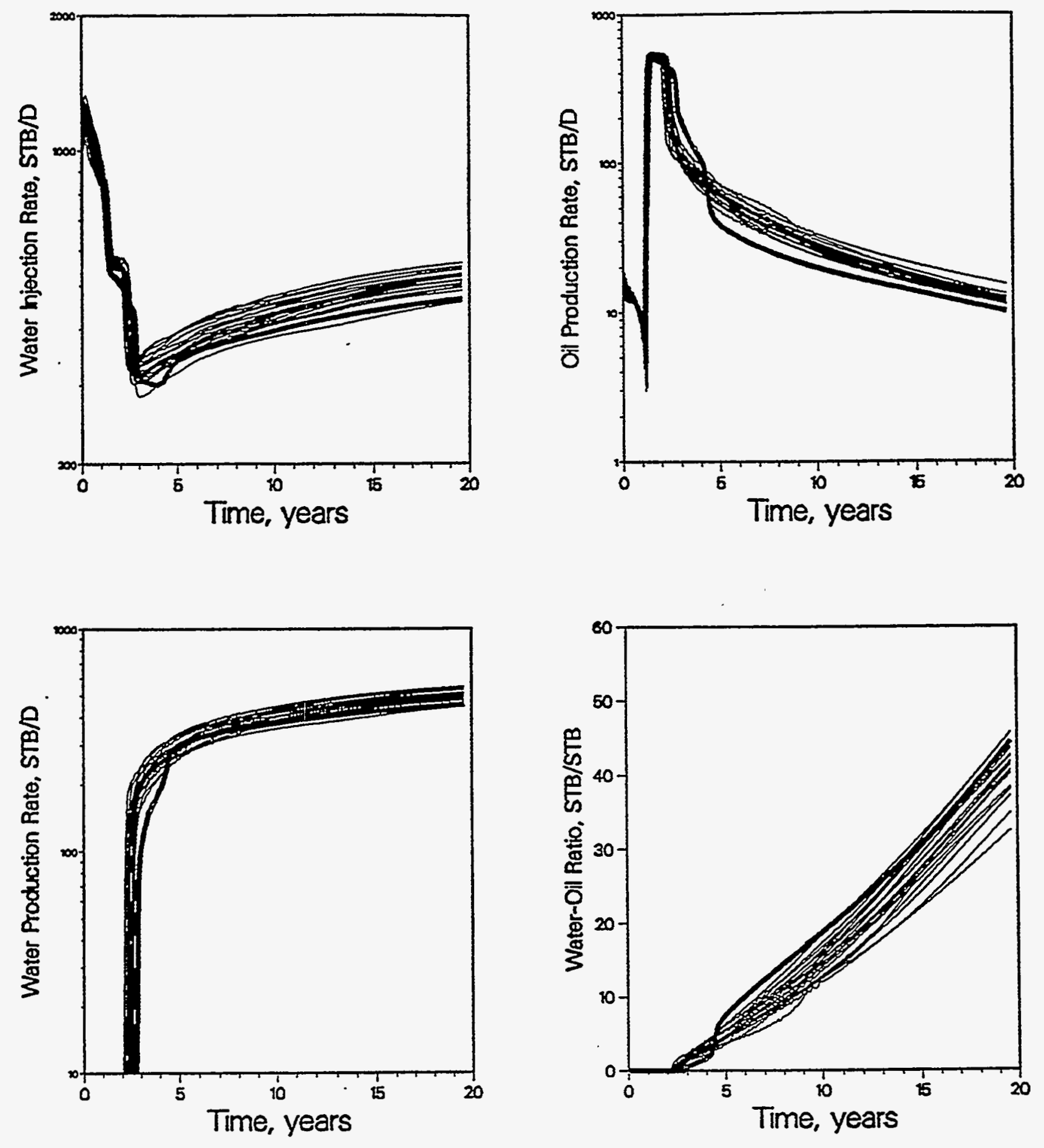

Figure 10 - Waterflood performance plots for Case Study No. 3: $\mu=2.0, \sigma_{\text {log(t) }}=0.4$, simulated annealing with parameter constraints $\mathrm{p}_{\mathrm{tH}}=0.319, \log \left(\mathrm{k}_{\mathrm{wW}}\right)=2.57$, $\log \left(k_{\mathrm{Nw}_{\mathrm{p}}}\right)=2.28$ (truth case highlighted). 

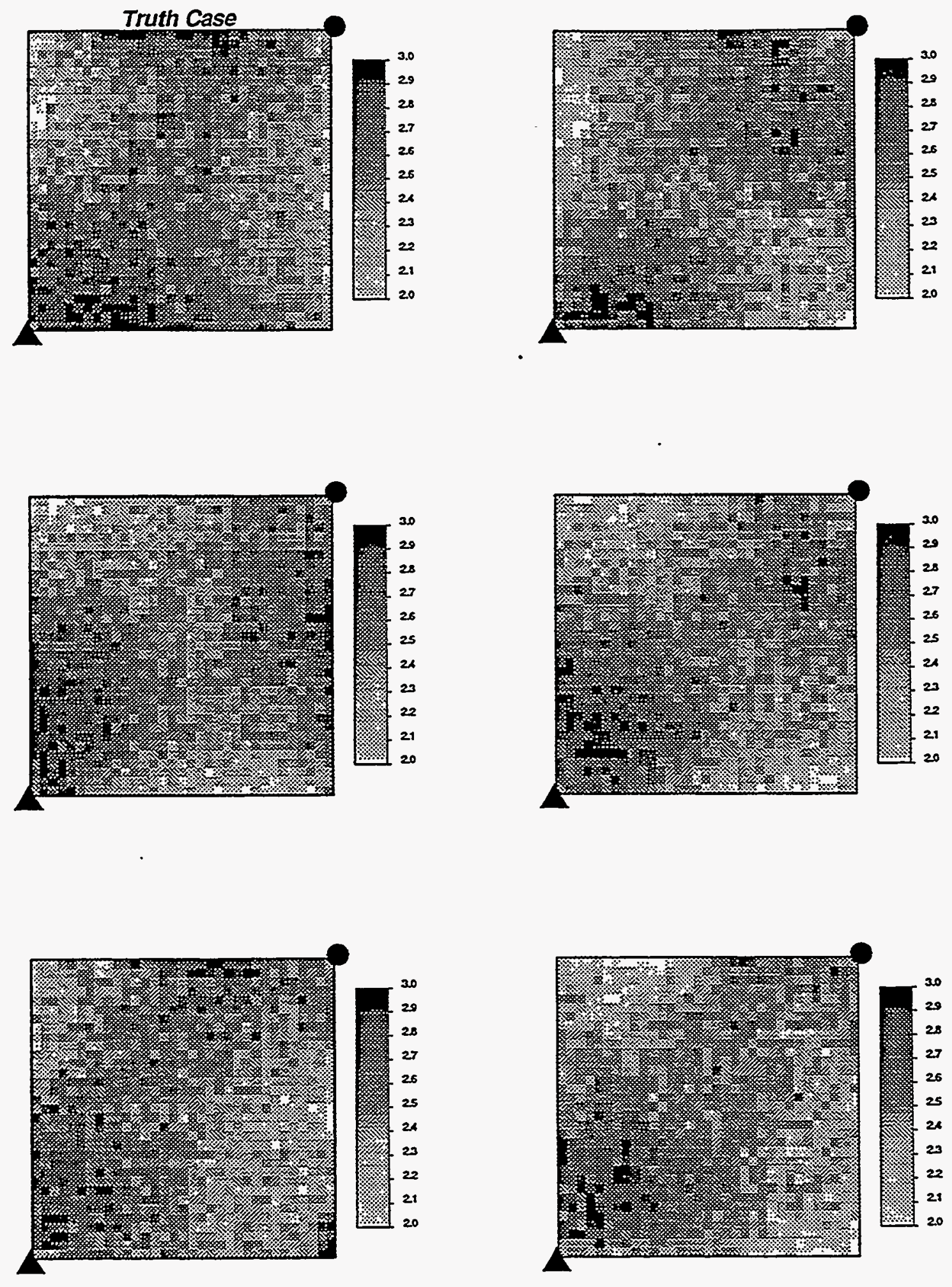

Figure 11 - Greyscale maps of $\log (\mathrm{k})$ for Case Study No. 4. 

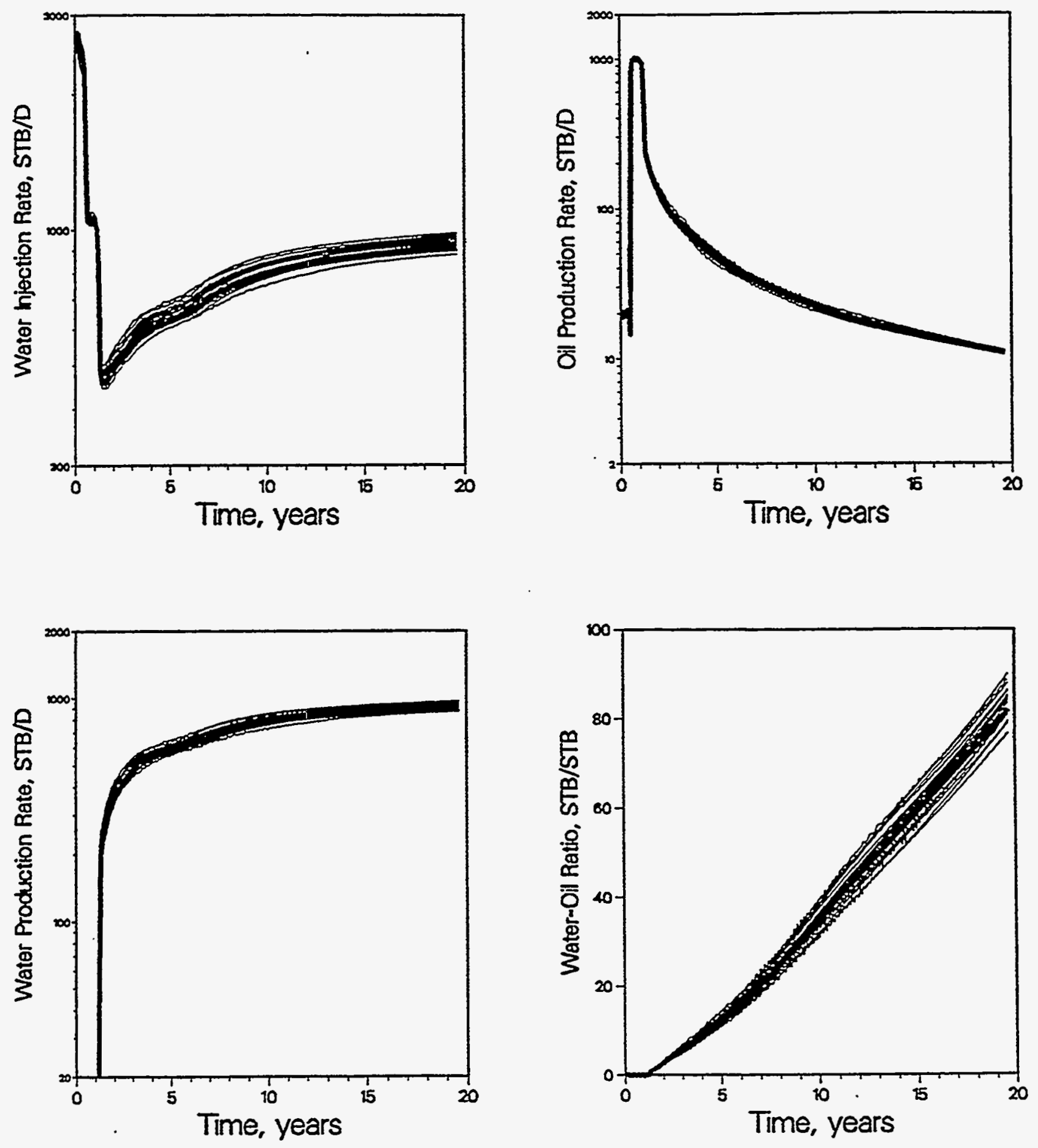

Figure 12 - Waterflood performance plots for Case Study No. 4: $\mu=2.5, \sigma_{\text {logk }}=0.2$, simulated annealing with parameter constraints $p_{\mathrm{LH}}=0.298, \log \left(k_{\mathrm{N}{ }_{\mathrm{i}}}\right)=2.76$. $\log \left(k_{N r_{p}}\right)=2.53$ (truth case highlighted). 

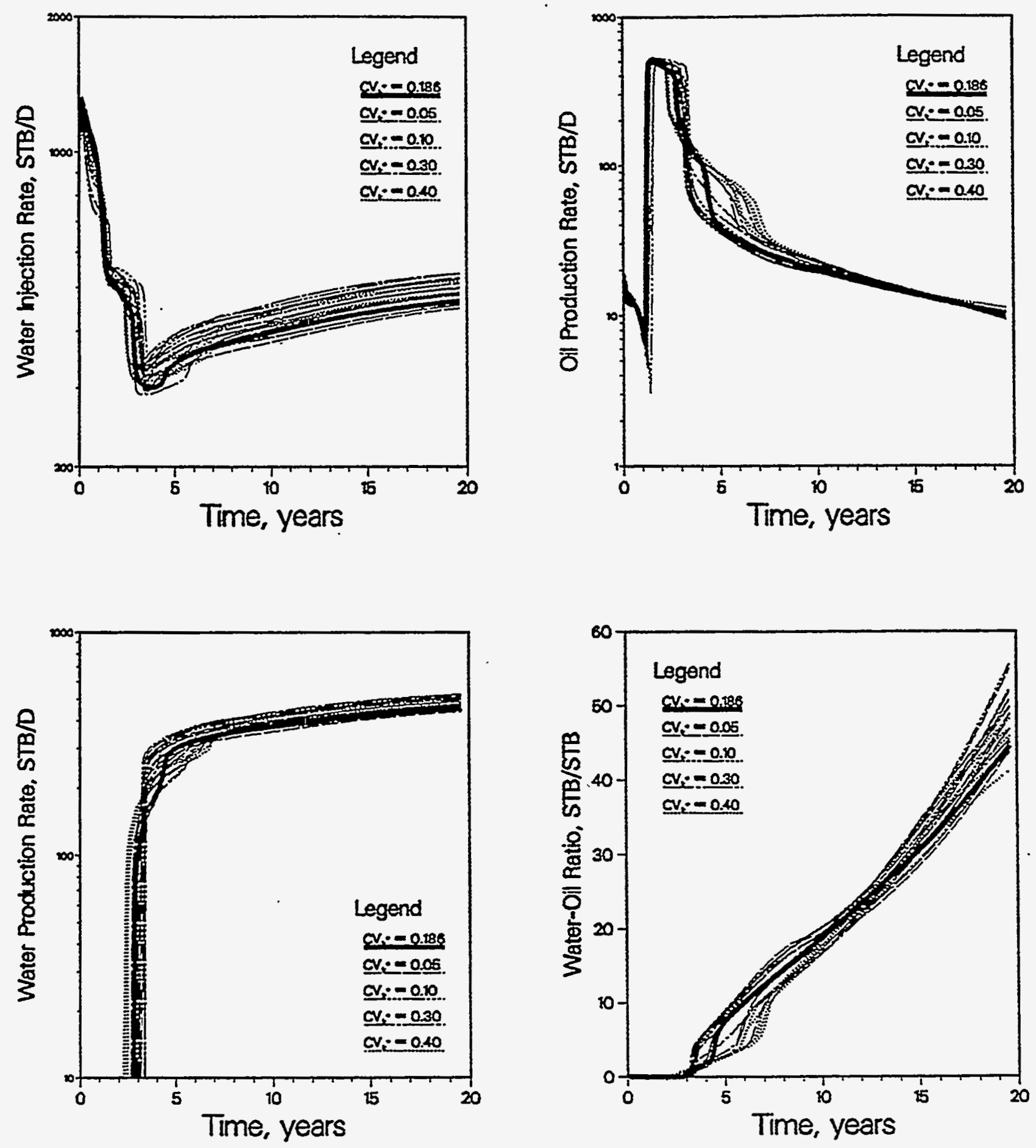

Figure 13 - Waterflood performance plots for Case Study No. $5: \mu=2.0, \sigma_{\text {log }(k)}=0.4$. $C V_{k} \cdot$ sensitivity $(0.05,0.10,0.186,0.30,0.40) . p_{\imath H}=0.319, \log \left(k_{N r_{1}}\right)=2.57, \log \left(k_{N v_{p}}\right)=2.28$, $\mathrm{k}_{\mathrm{mo}}=0.610$ (truth case highlighted). 

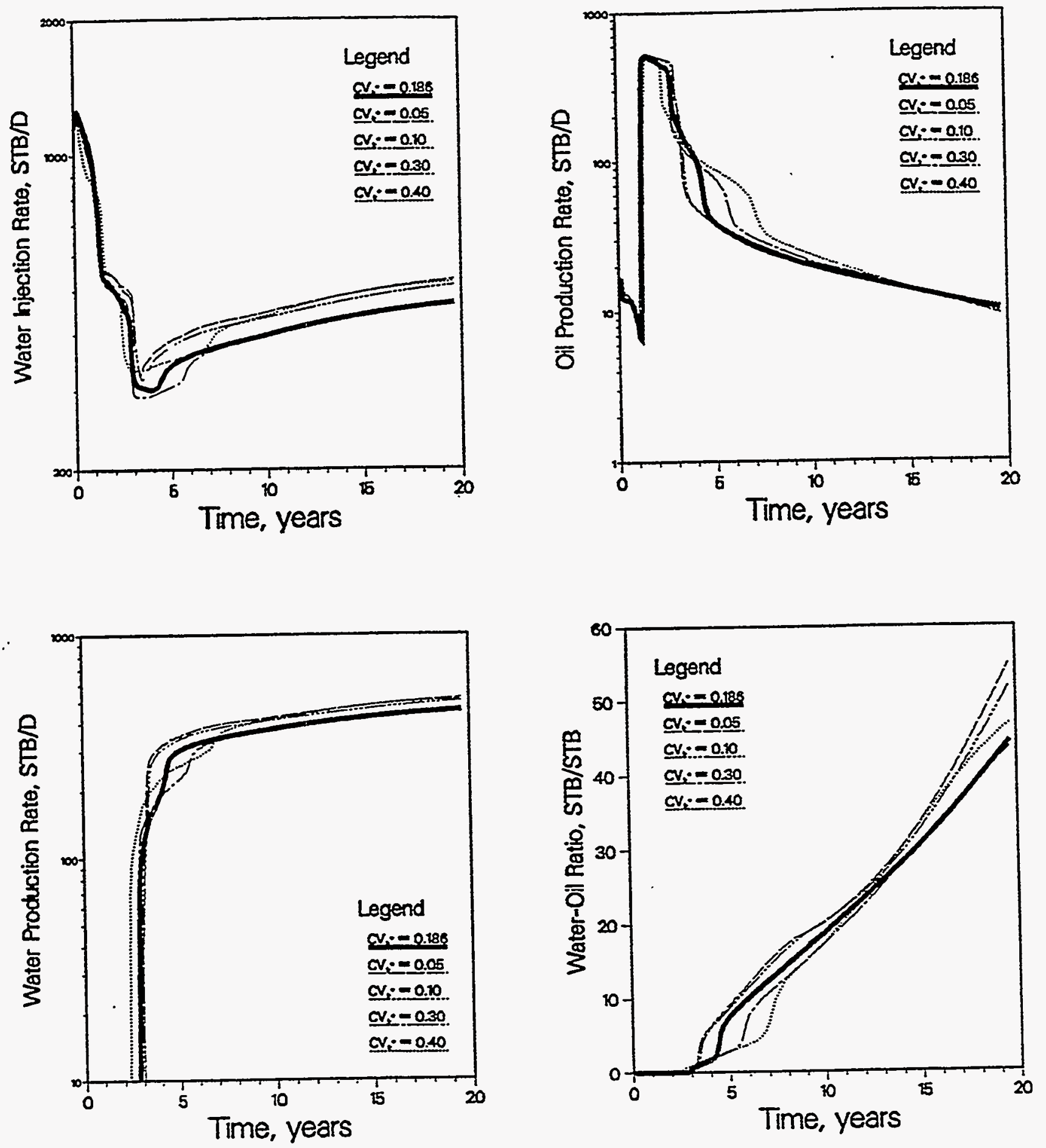

Figure 14 - Waterflood performance plots for Case Study No. $5: \mu=2.0, \sigma_{\log (x)}=0.4$, CV - sensitivity $(0.05,0.10 .0 .186,0.30,0.40), \mathrm{p}_{\mathrm{LH}}=0.319, \log \left(\mathrm{k}_{\mathrm{NW}}\right)=2.57, \log \left(\mathrm{k}_{\mathrm{N} \mathrm{p}_{\mathrm{p}}}\right)=2.28$, $\mathrm{k}_{\mathrm{m}}=0.610$ (truth case highlighted) - one realization per sensitivity. 


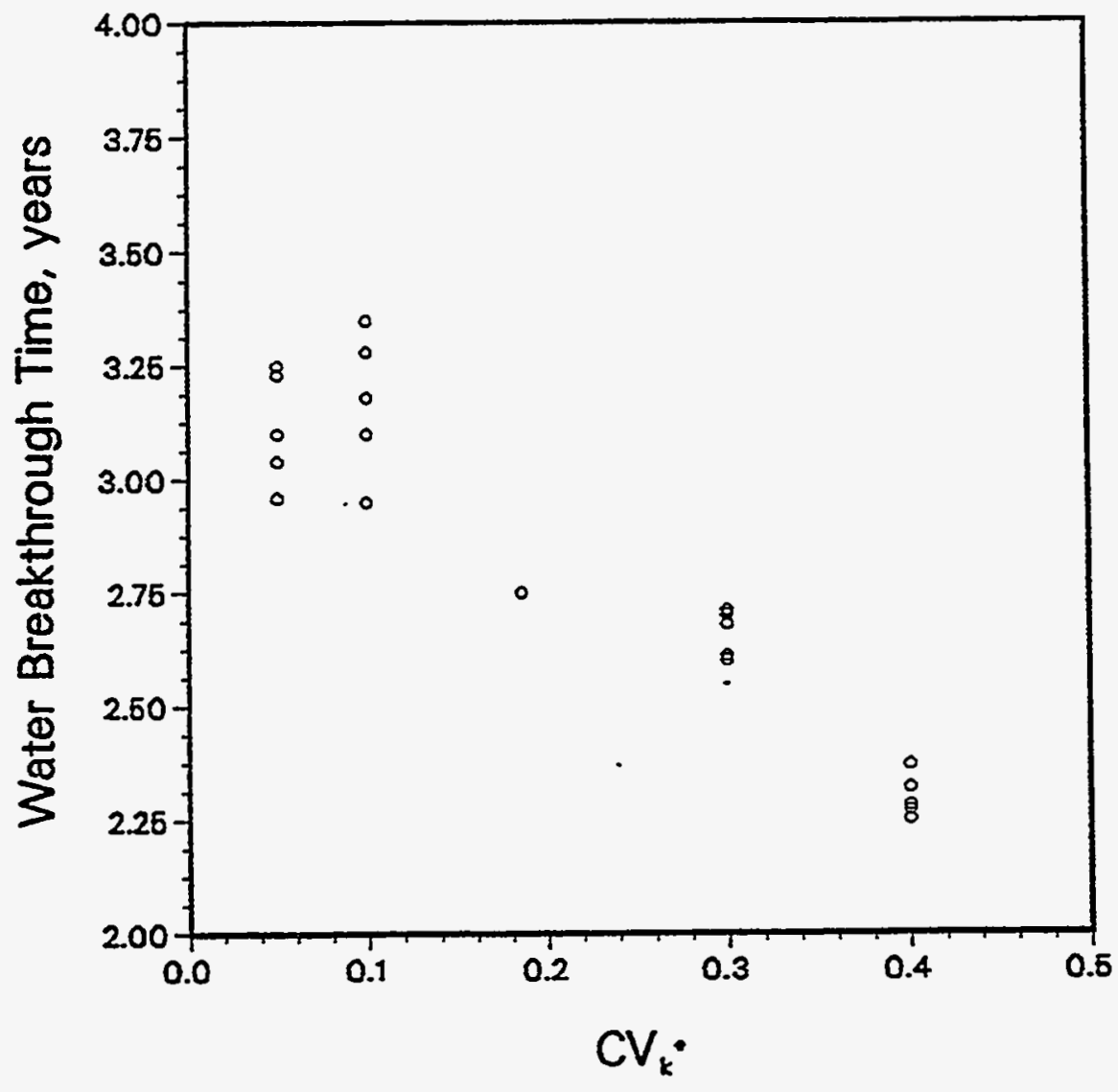

Figure 15 - Correlation between $\mathrm{CV}_{\mathrm{k}}{ }^{\cdot}$ and water breakthrough time. 

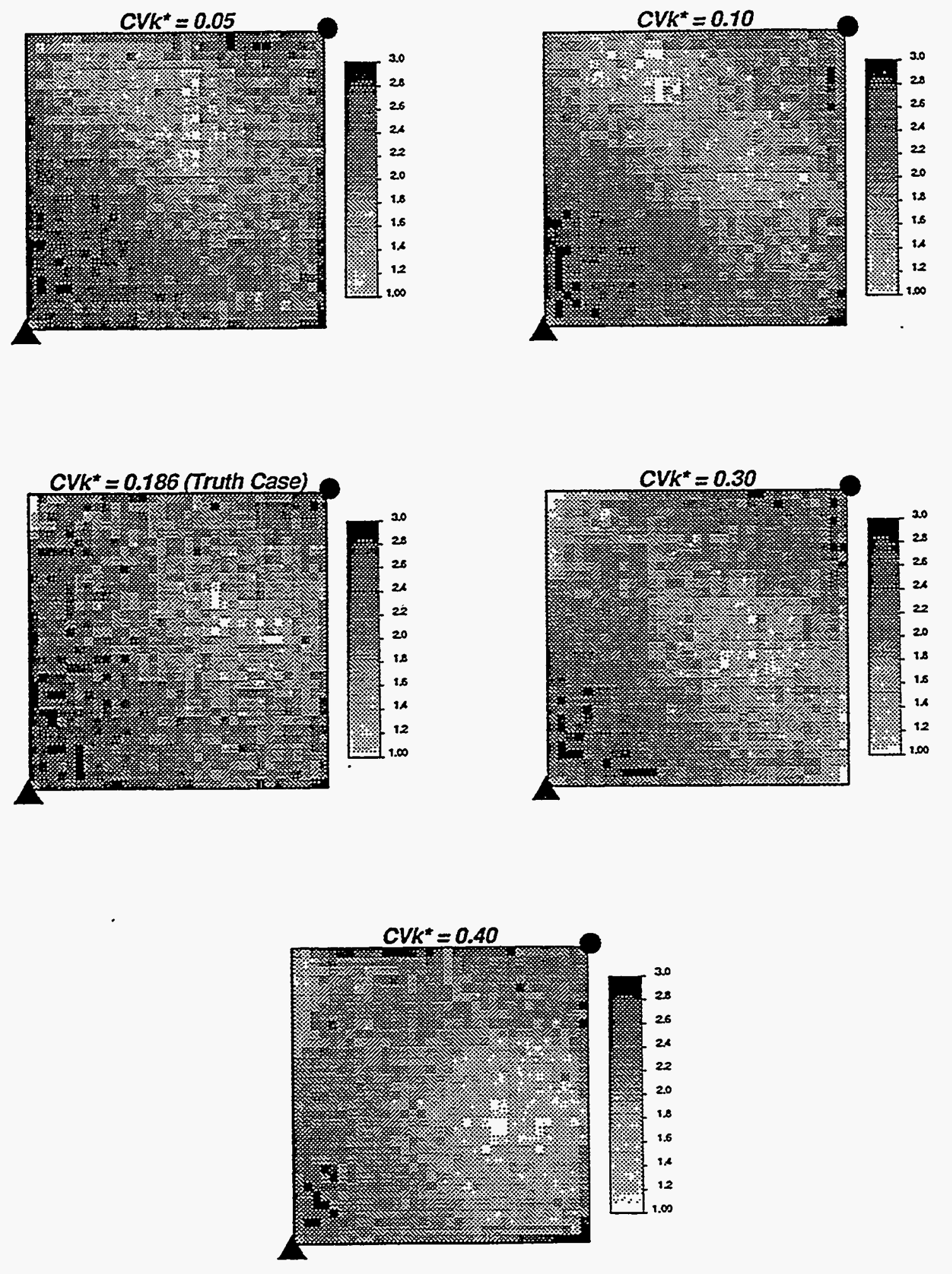

Figure 16 - Greyscale maps of $\log (\mathrm{k})$ for Case Study No. 5. 

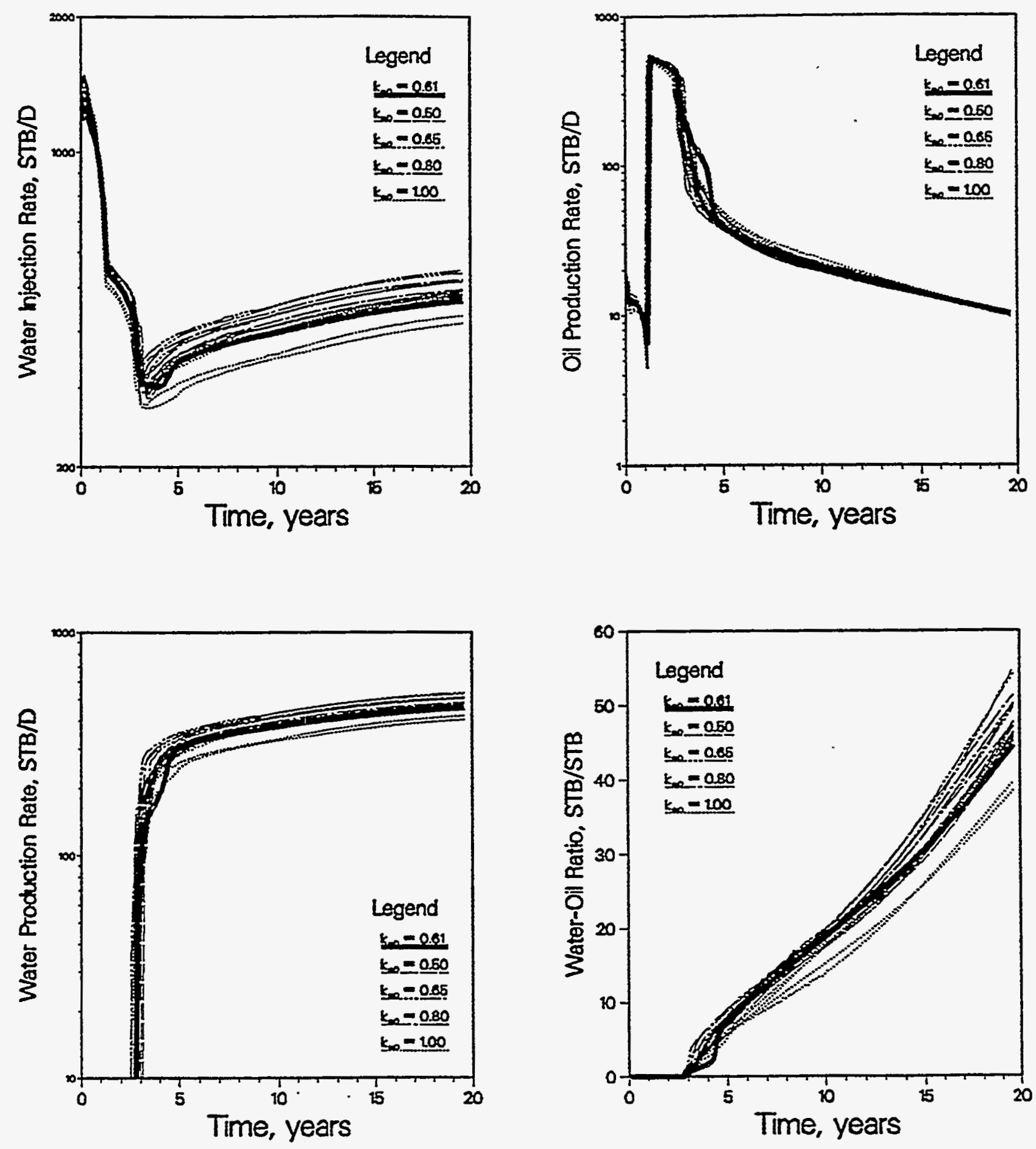

Figure 17 - Waterflood performance plots for Case Study No. 5: $\mu=2.0, \sigma_{\text {log(k) }}=0.4$, $k_{m D}$ sensitivity $(0.50,0.61,0.65,0.80,1.0), p_{t H}=0.319, \log \left(k_{N \times s}\right)=2.57, \log \left(k_{N Y_{p}}\right)=2.28$, $\mathrm{CV}_{\mathrm{k}}^{\circ}=0.186$ (truth case highlighted). 

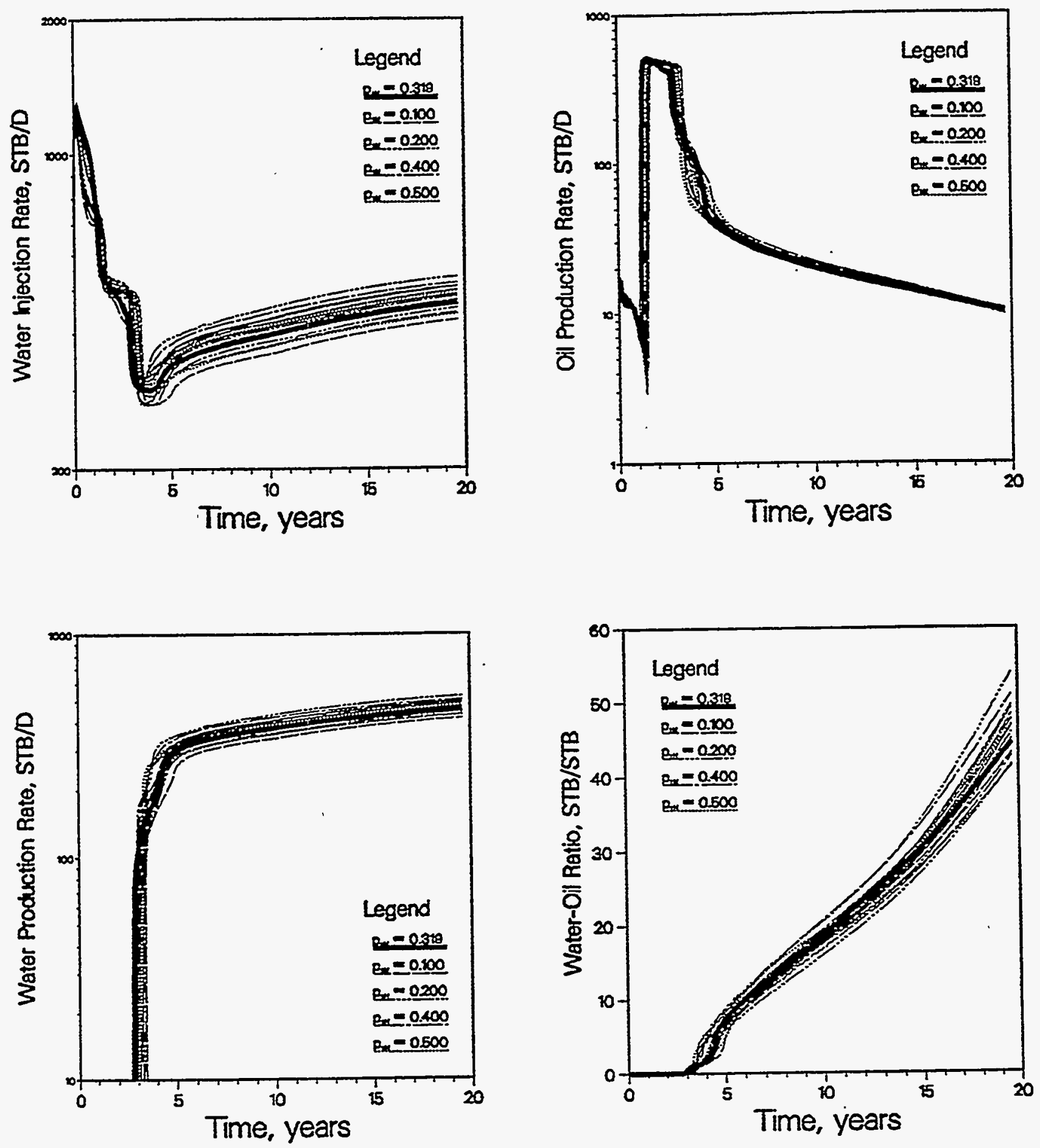

Figure 18 - Waterflood performance plots for Case Study No. $5: \mu=2.0, \sigma_{\log (k)}=0.4$, $p_{u}$ sensitivity $(0.10,0.20,0.319,0.40,0.50), \log \left(k_{N \times 1}\right)=2.57, \log \left(k_{N \times p}\right)=2.28, k_{m D}=0.610$ $\mathrm{CV}_{k^{\circ}}=0.186$ (truth case highlighted). 

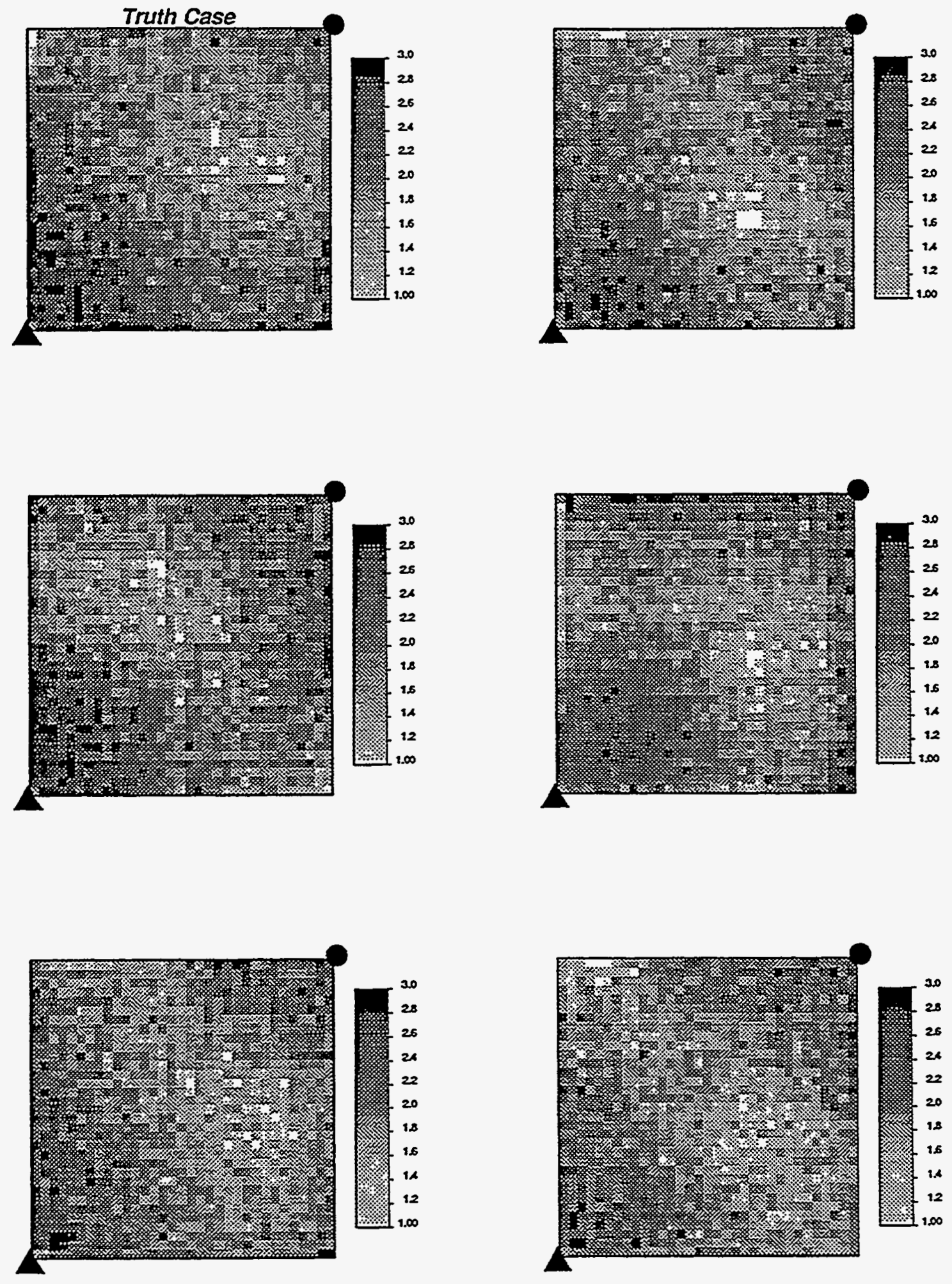

Figure 19 - Greyscale maps of $\log (\mathrm{k})$ for Case Study No. 6. 

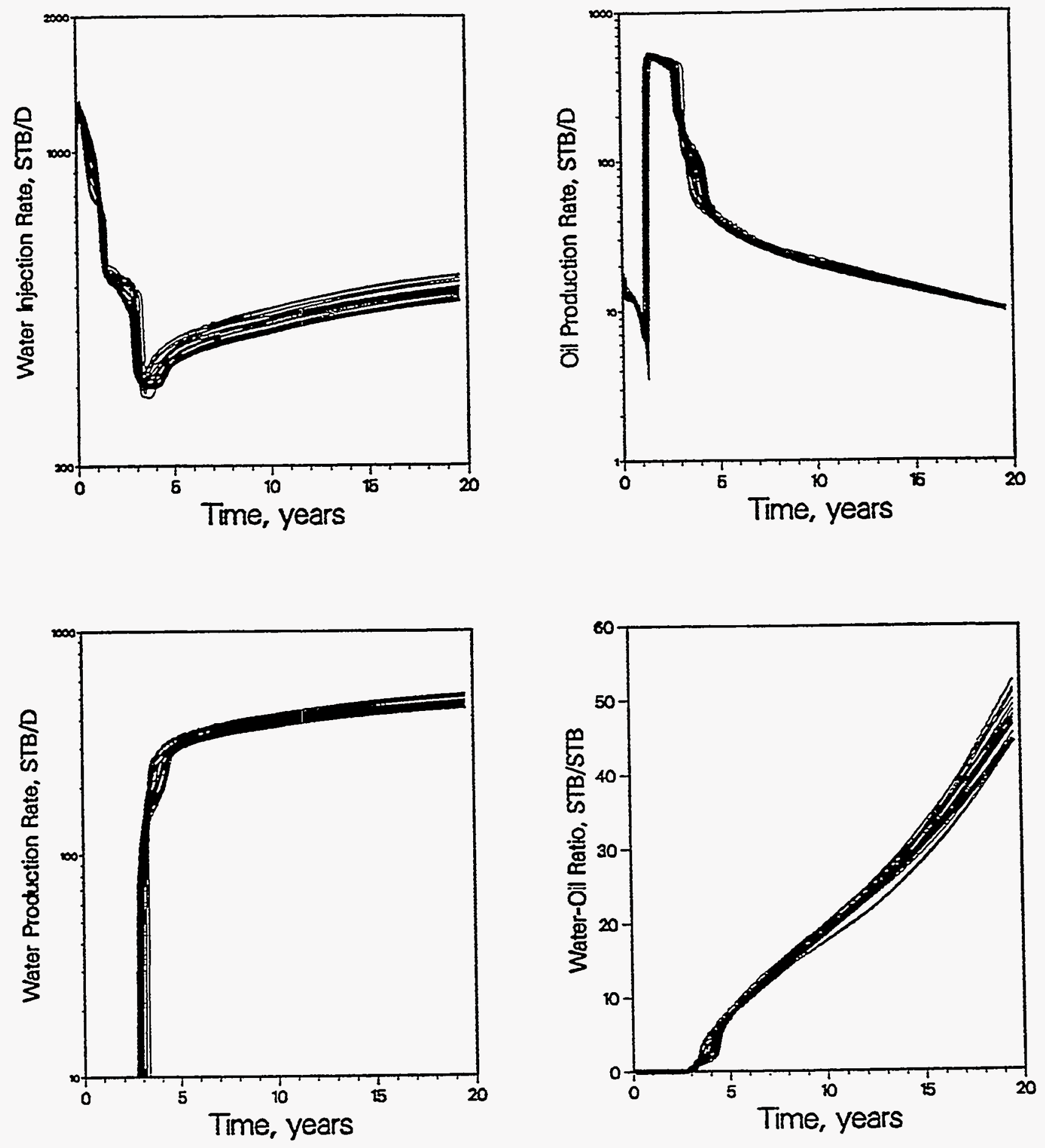

Figure 20 - Waterflood performance plots for Case Study No. 6: $\mu=2.0, \sigma_{10 g(x)}=0.4$. simulated annealing with parameter constraints $p_{t H}=0.319, \log \left(k_{w x_{i}}\right)=2.57$. $\log \left(\mathrm{k}_{\mathrm{N} \mathrm{r}_{\mathrm{p}}}\right)=2.28, \mathrm{k}_{\mathrm{mD}}=0.610, \mathrm{CV}_{\mathrm{r}^{\circ}}=0.186$ ( $\mathrm{truth}$ case highlighted). 

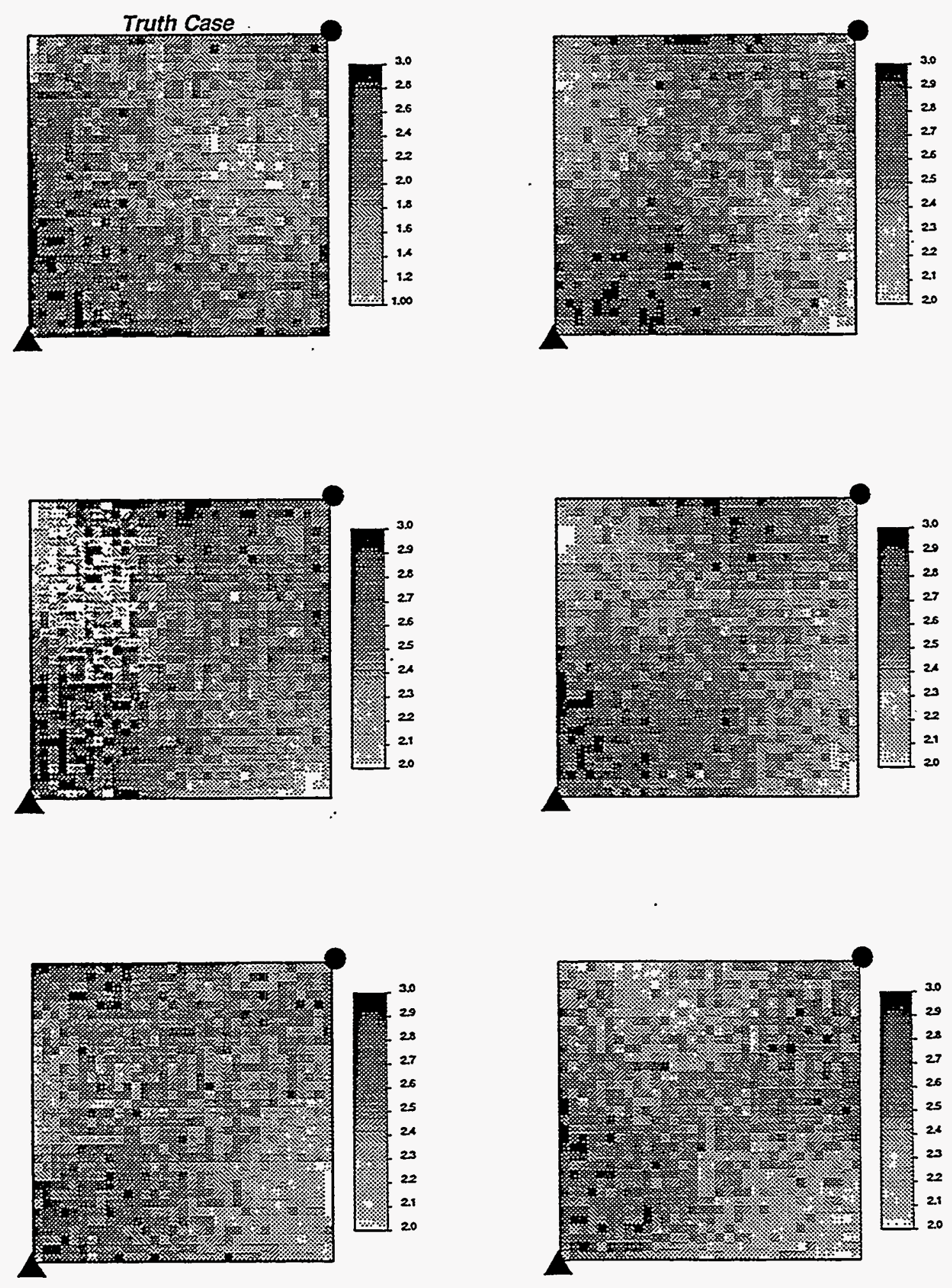

Figure 21 - Greyscale maps of $\log (\mathrm{k})$ for Case Study No. 7. 

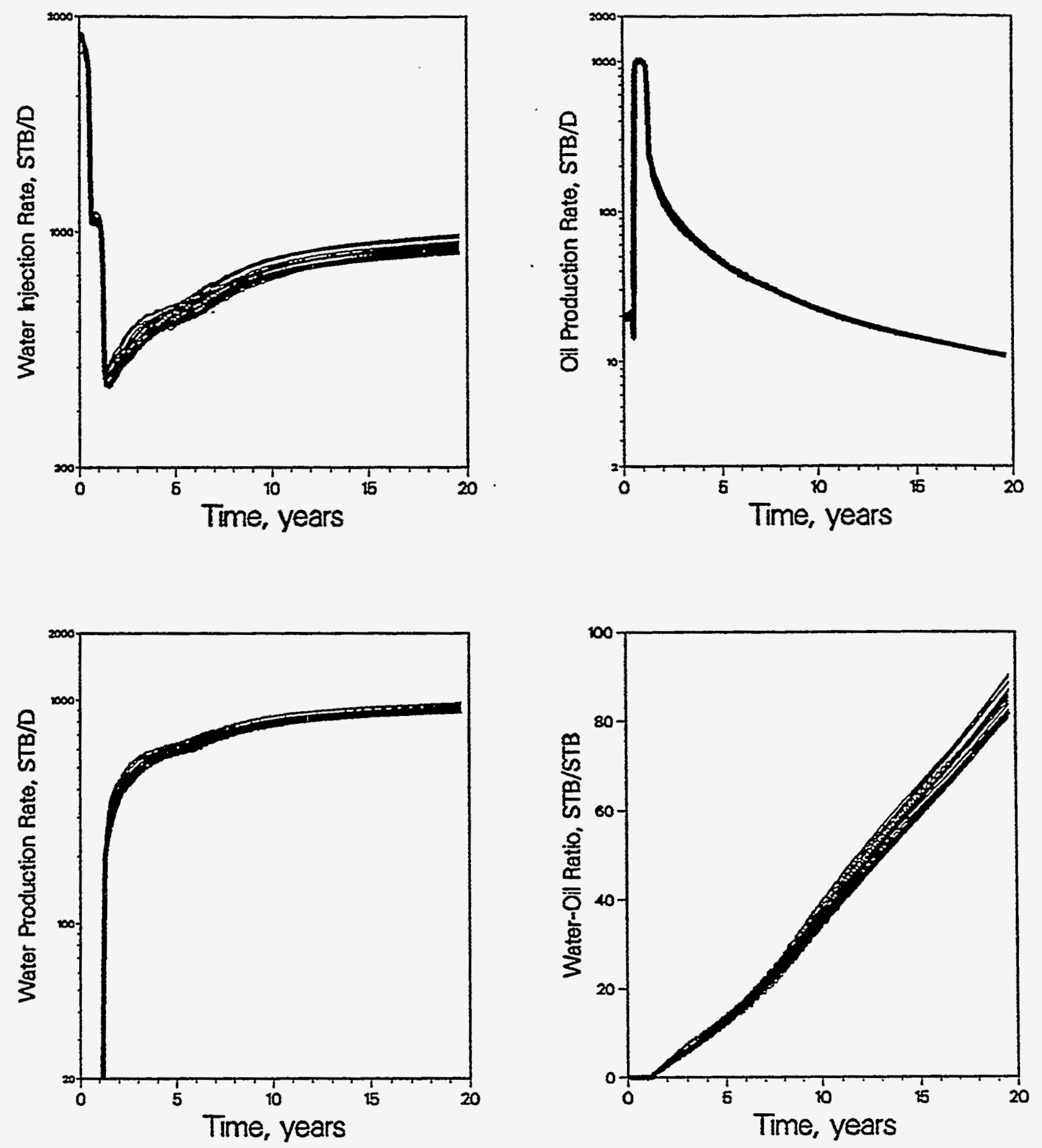

Figure 22 - Waterflood performance plots for Case Study No. $7: \mu=2.5, \sigma_{\text {logk }(k)}=0.2$. simulated annealing with parameter constraints $p_{2 \mathrm{H}}=0.298, \log \left(\mathrm{k}_{\mathrm{N} \mathbf{v}_{\mathrm{i}}}\right)=2.76$. $\log \left(\mathrm{k}_{\mathrm{N} \mathrm{r}_{\mathrm{p}}}\right)=2.53, \mathrm{k}_{\mathrm{mD}}=1.02, \mathrm{CV}_{\mathrm{k}^{\circ}}=0.300$ (truth case highlighted). 

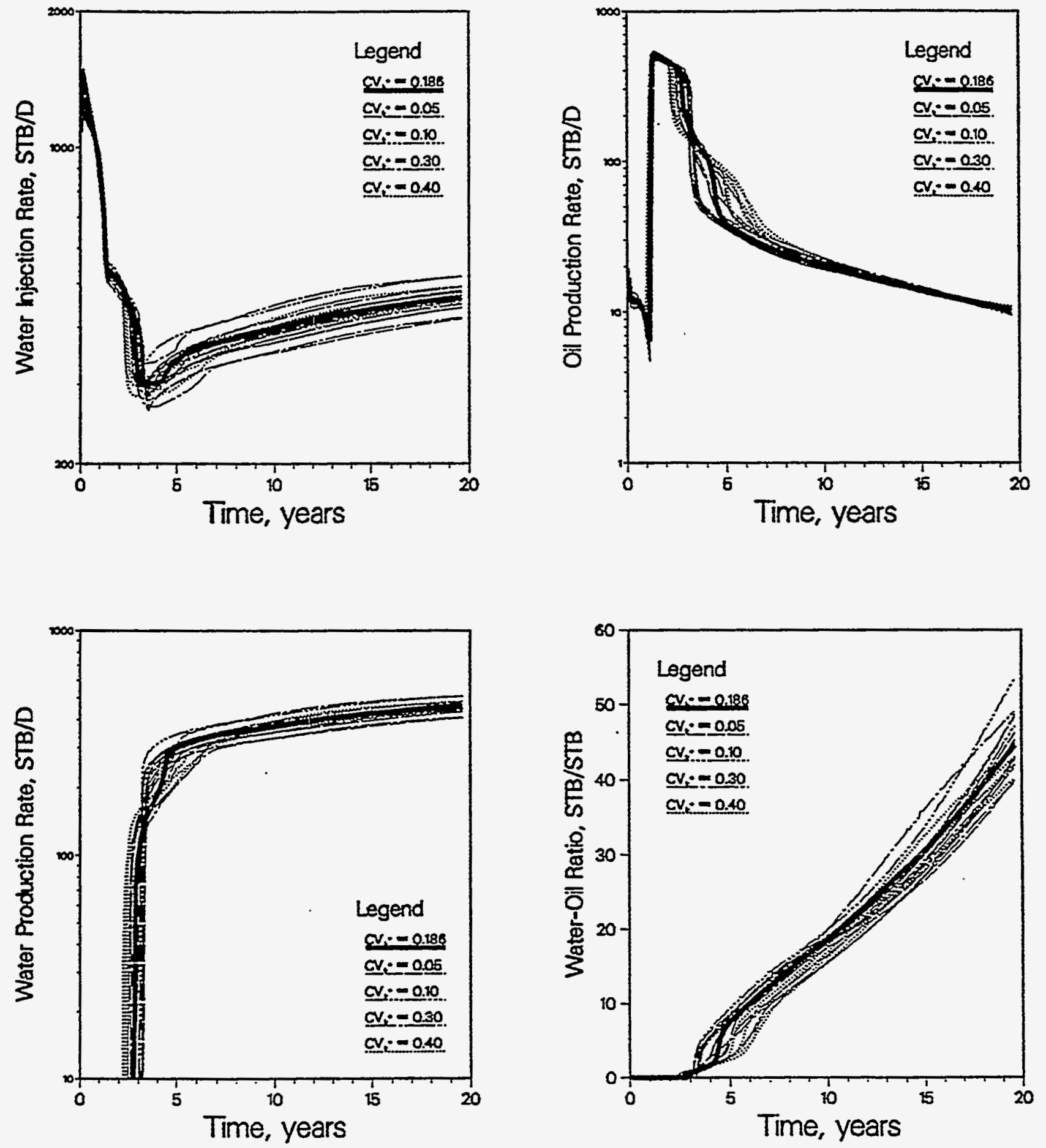

Figure 23 - Waterflood performance plots for Case Study No. 8: $\mu=2.0, \sigma_{10 g(k)}=0.4$,

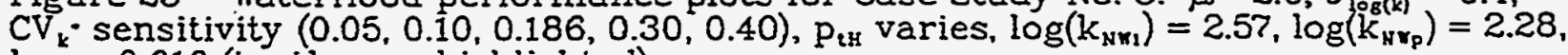
$\mathrm{k}_{\mathrm{mD}}=0.610$ (truth case highlighted). 